Fis.

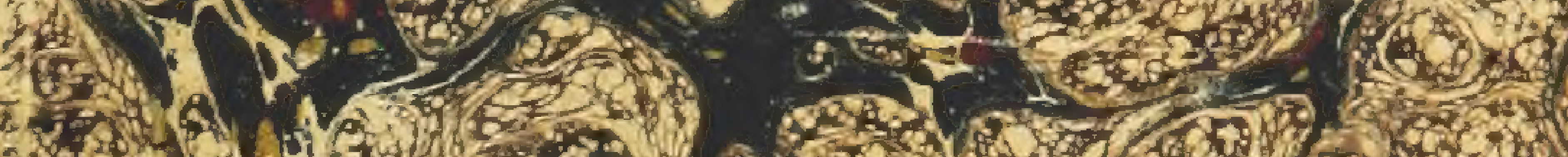

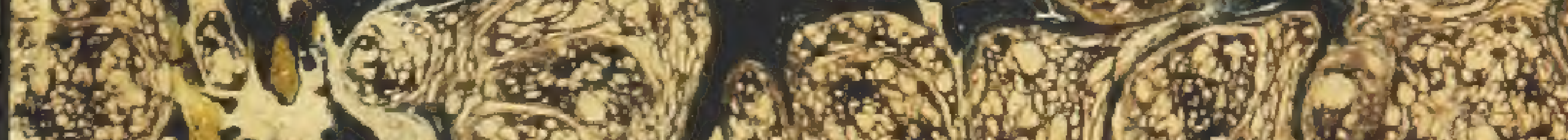

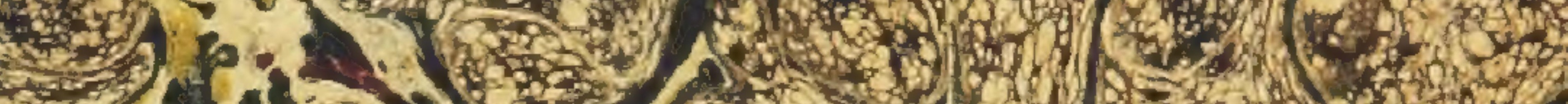

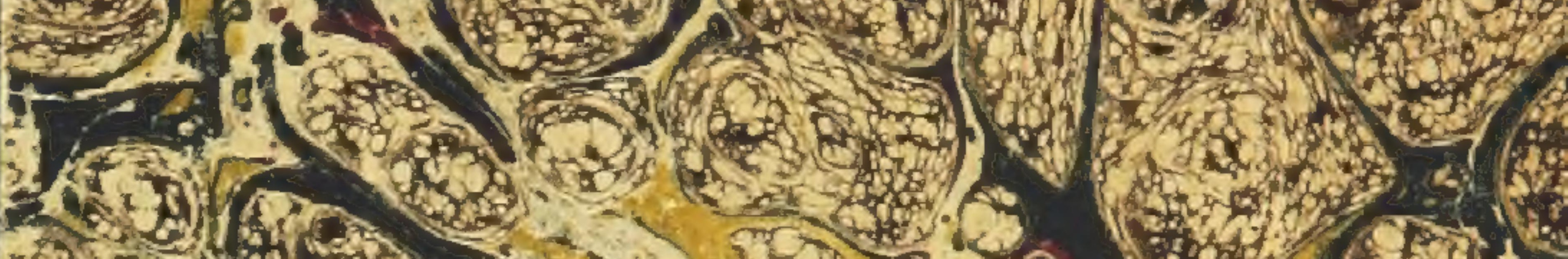

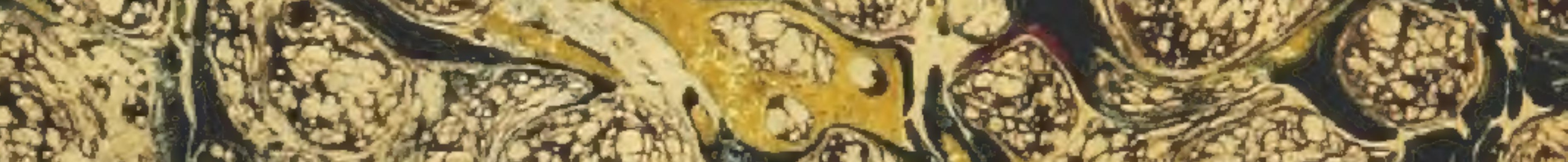

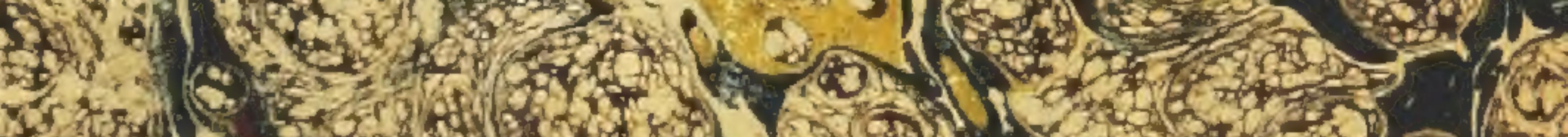

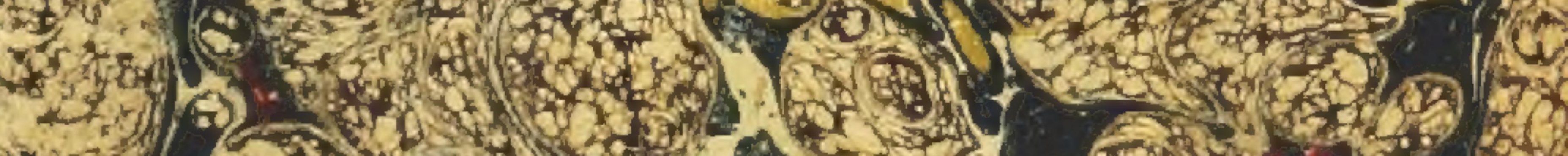

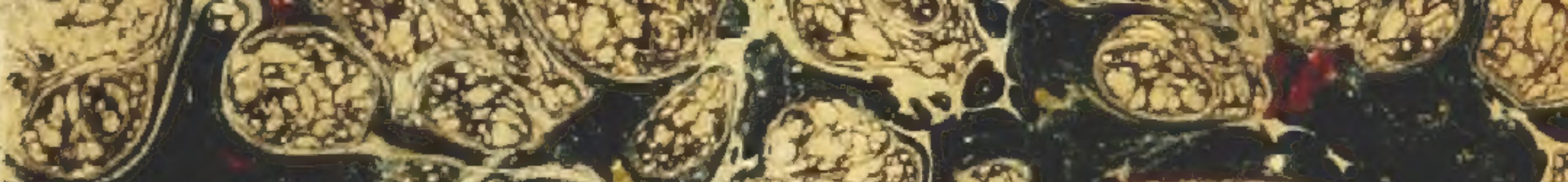

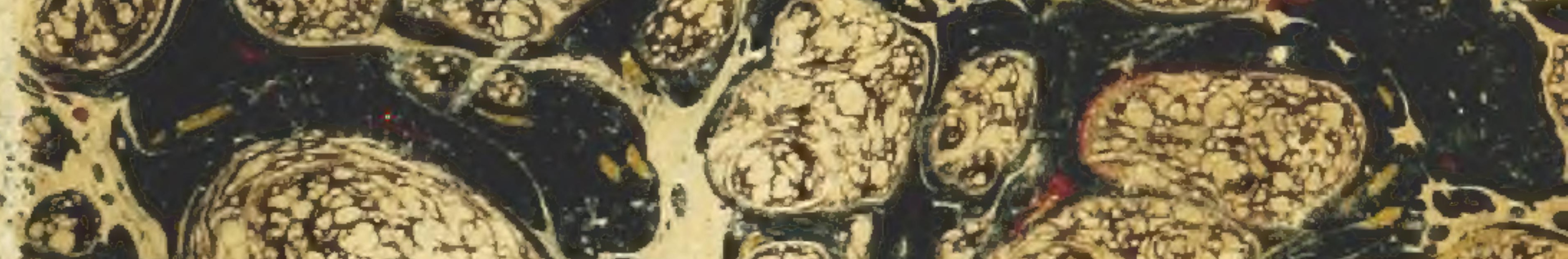

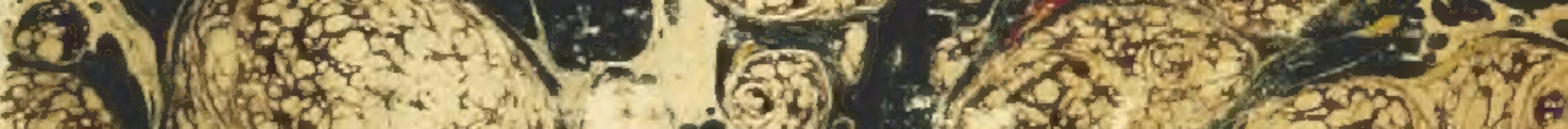

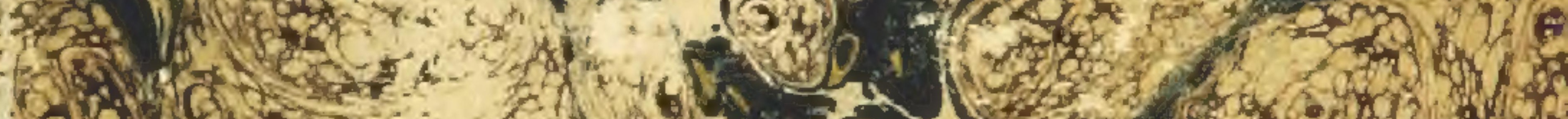

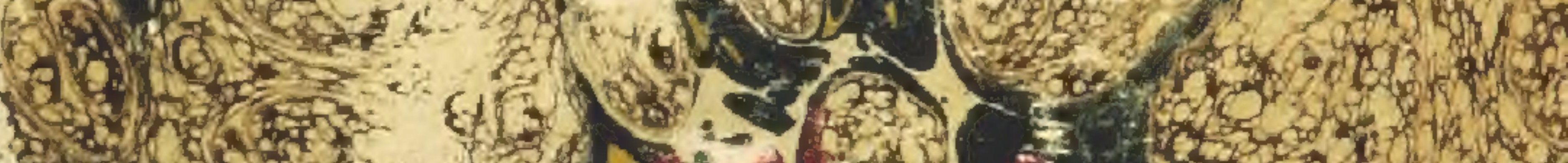
15.

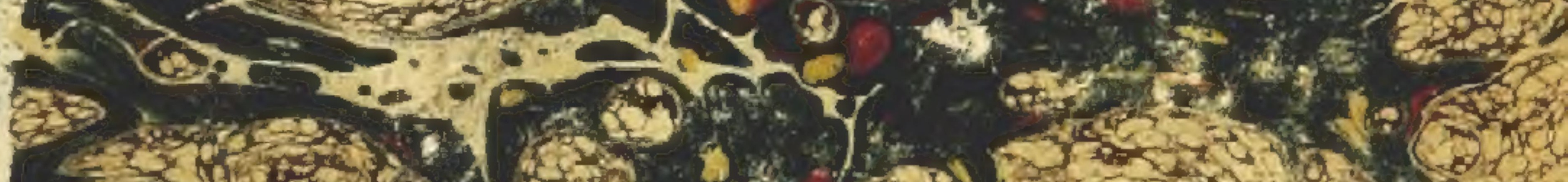

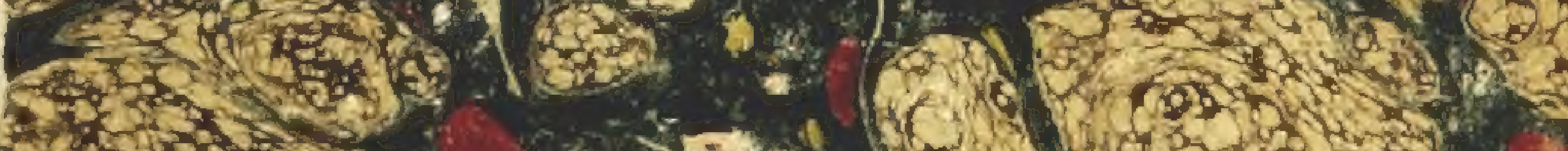

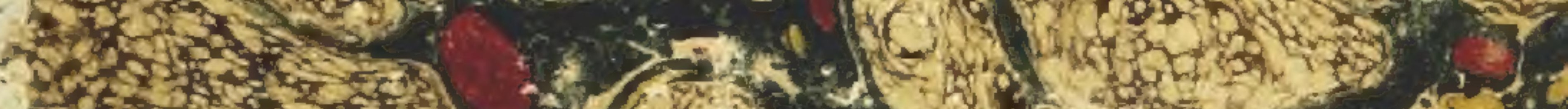

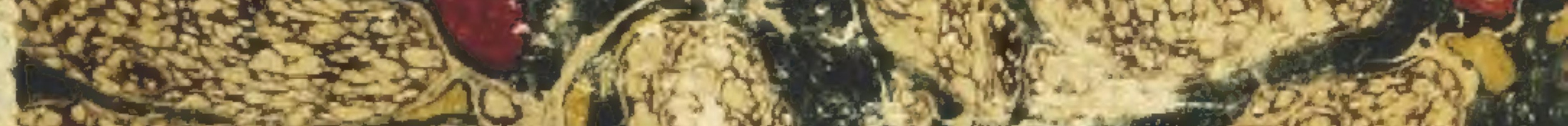

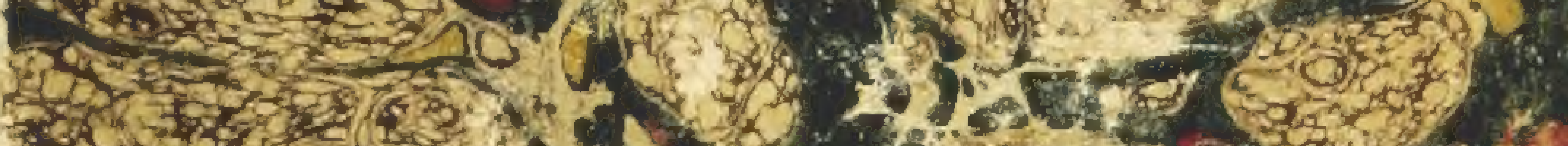

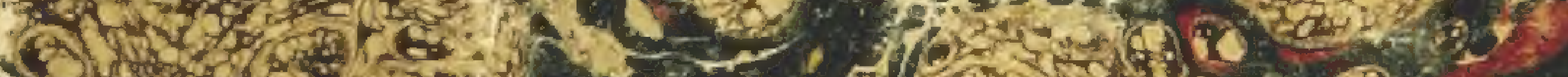

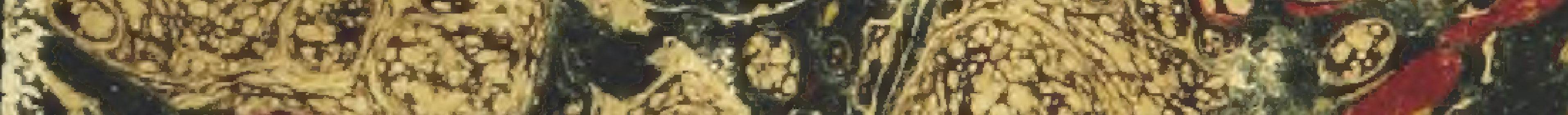

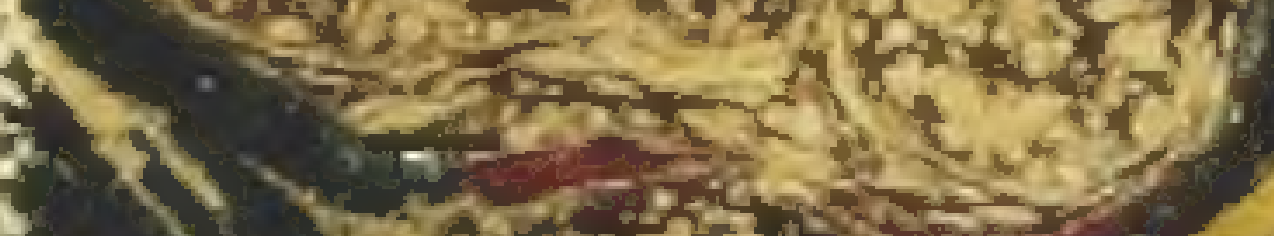

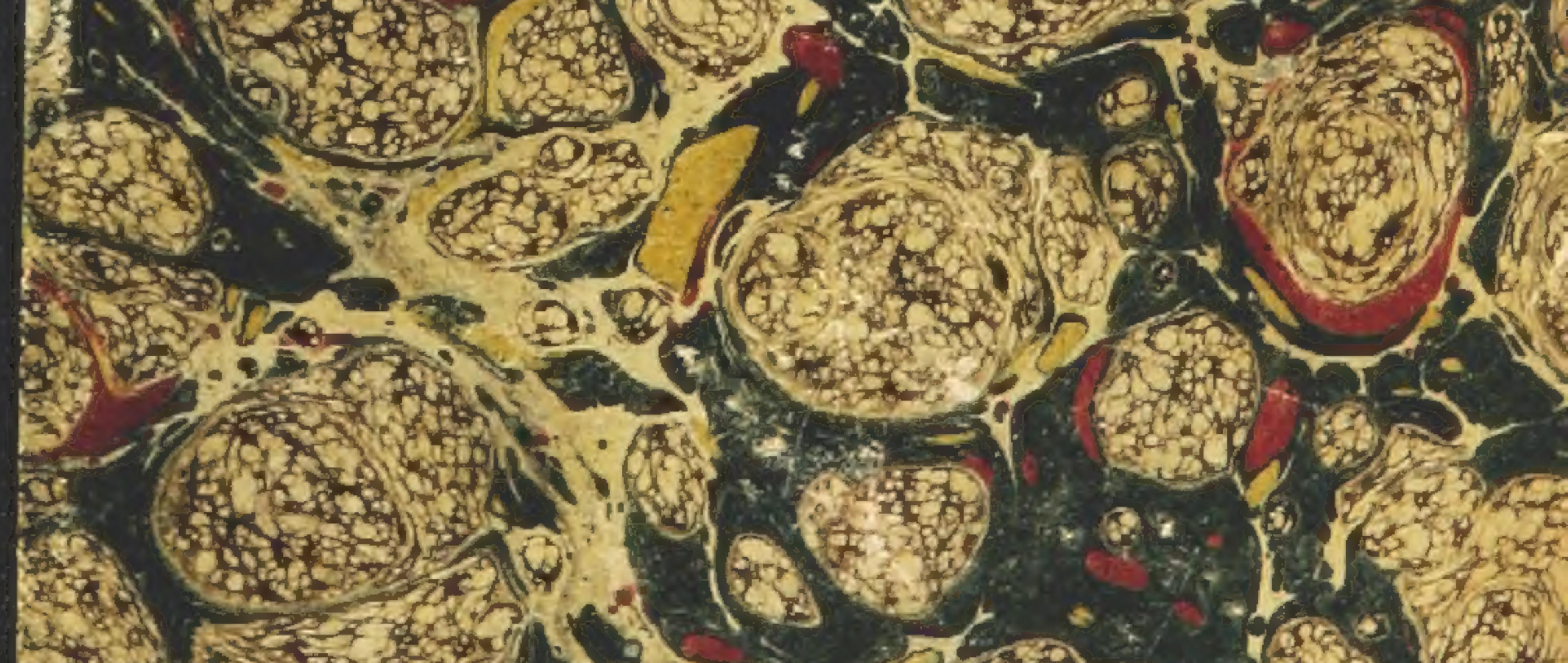

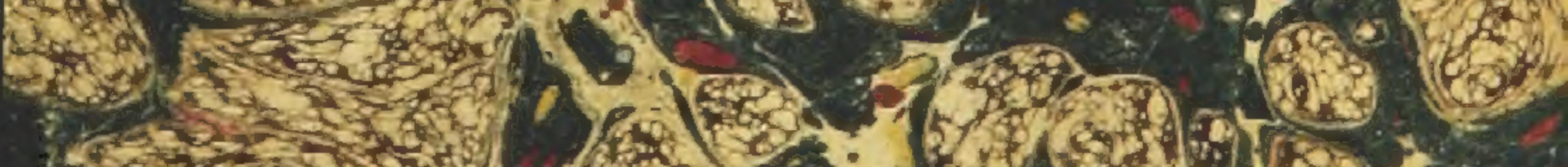

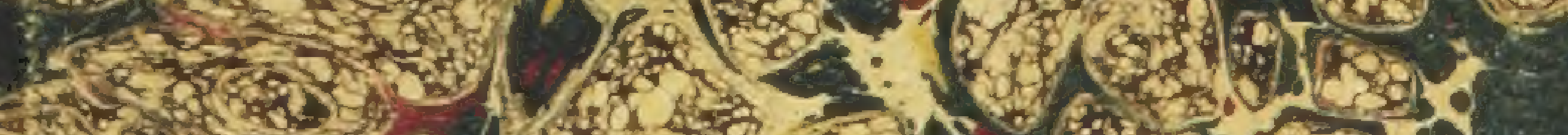

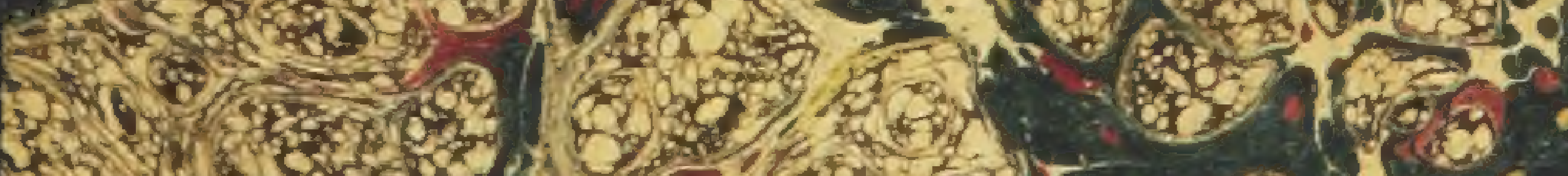




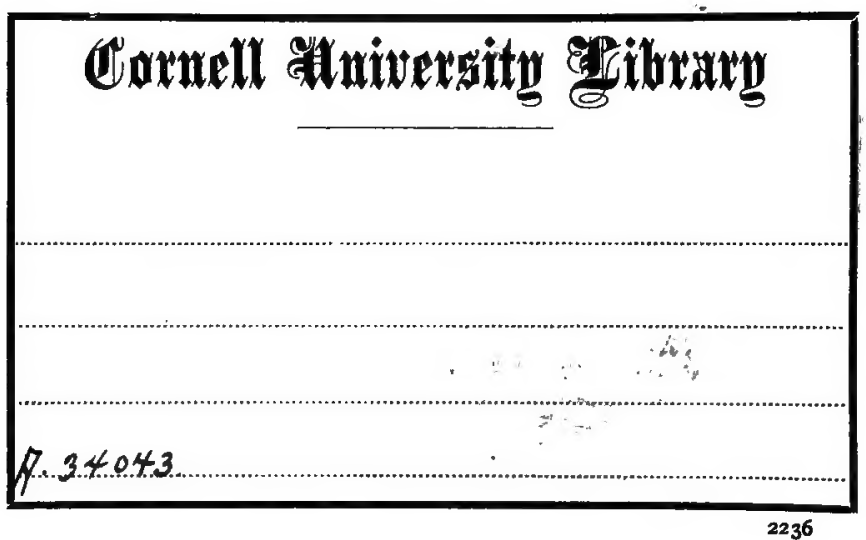




\section{arV17433}

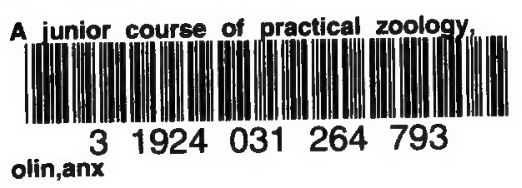




\section{Cornell University Library}

The original of this book is in the Cornell University Library.

There are no known copyright restrictions in the United States on the use of the text.

http://www.archive.org/details/cu31924031264793 
PRACTICAL ZOOLOGY 
Sccond Edition, Revised and Tllustrated, crown 8vo. 3s.6d.

\section{THE FROG:}

\section{AN INTRODUCTION TO ANATOMY AND HISTOLOGY.}

BY

A. MILNES MARSHALL, M.D., D.Sc., M.A., F.R.S.

Profegsor in the Victoria University; Beyer Professor of Zoology in Owens College; late Fellow of St John's College, Cambridge.

London: SMITH, ELDER, \& CO., 15 Waterloo Place. Manchester: J. E, CC 


\section{JUNIOR COURSE}

OF

\section{PRACTICAL ZOOLOGY}

BY

A. MILNES MARSHALL, M.D., D.Sc., M.A., F.R.S.

PROFESSOR IN THE VICTORIA UNIVTRSITY : BEYEK PBOFESSOR OF

ZOOLOGX IN OWEXS COLLEGE: LAATE FELLOW OF

ST JOHN'S COLLEGE, CAMBRIDGE

ASSISTED BY

C. HERBERT HURST

DEMONSTRATOR AND ASSISTANT LECTURER IN ZOOLOGY IN OWINS COJLEG

SECOND EDITTON

LONDON

SMITH, ELDER, \& CO., 15 WATERLOO PLACE 1888

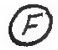





\section{TO THE \\ STUDENTS OF OWENS COLLEGE}

\section{THIS BOOK}

IS DEDICATED

BY THEIR SINCERE WELL-WISHERS

THE AUTHOR, 



\section{PREFACE}

To

\section{THE SECOND EDITION.}

IN the preparation of this second edition advantage has been freely taken of corrections and suggestions received from many sources. The whole book has been carefully revised, and several new figures have been added.

Owens Coldege, April 1888. 



\section{PREFACE}

To

\section{THE FIRST EDITION.}

ThIs воок has been written in the hope that it may meet the wants of those who desire to obtain a practical acquaintance with the elements of animal morphology, and who find the existing manuals insufficient for their purpose.

The animals selected for description are those which are generally accepted as suitable types for a junior laboratory course. They are of convenient size, and can all be obtained readily and at small cost: they include characteristic representatives of the more important of the great groups of animals; and they give opportunity for very varied methods of examination. A student who works conscientiously through the book will thus acquire a good insight into the leading facts of animal structure, and a technical knowledge of the principal methods of research.

In the mode of treatment adopted the practical character of the book has been made paramount. Directions for dis. section have throughout been printed in italies, and a system of indentation has been adopted to render the subdivisions more distinct. In almost all cases the descriptions are so arranged that the whole dissection can be performed on a single specimen : if more than one can be obtained, the order in which the several sections are taken may be varied; but in every case each of the main systems - vascular, nervous, etc.-should be dissected in its entirety, for in this way alone can a proper idea of its relations be obtained. 
No attempt has been made to write exhaustive descriptions of the several animals; neither has strict uniformity of treatment been specially aimed at. Portions of the subject which experience has shown to present special difficulties are treated at what may appear undue length; while, on the other hand, the limits of time ordinarily available for laboratory work have led to the almost entire omission of systems, such as the muscular, which are of subordinate educational value.

Although this is essentially and professedly a laboratory text-book, yet morphological explanations have been freely admitted; and this from a conviction that a student best grasps the meaning of anatomical facts if the explanation is given him while the facts are actually before his eyes.

Illustrations have been introduced somewhat sparingly, for it is of the utmost importance that they should not be allowed to replace the drawings which a student must make from his own dissections. The majority of the figures here given are new, and have been drawn expressly for the book by Mr. Hurst or myself. The drawings on the wood were made by Mr. P. Hundley, and the blocks engraved by Mr. G. Pearson: both these gentlemen have taken great pains to render the figures at once faithful and artistic.

Throughout the whole time of preparation of this volume I have had the constant co-operation and assistance of my friend Mr. C. H. Hurst. Several of the chapters were originally drawn up by him, and in all I have had the advantage of his aid, but for which the publication of the book might have been greatly delayed. Considerable pains have been taken to avoid mistakes, but it is curiously difficult to succeed in this, and corrections or suggestions from those who use the book will be very gratefully received.

Owens College: December 1886. 


\section{CONTENTS .}

\section{INTRODUCTION.}

The observation of animals during life-Drawing - The methods of killing animals-Dissection-Injection-The use of reagentsMicroscopical examination-Section cutting-The mounting of microseopical objects-Measuring of microscopical objectsThe preparation of skeletons . . . . . . . xvii

\footnotetext{
CHAPTER I.

The Protozon.

Amœba-Paramecium-Opalina_Vorticella . . . . . 1

CHAPTER II.

HYDRA.
}

Examination of a living specimen-Examination of transverse sections . . . . . . . . . . .

\section{CHAPTER III.}

The Liver-Fluke of THe Sheep. Fasciola (Distomum) hepatica.

The mature liver-fluke-The life-history of the liver-fluke . . 26 


\section{CHAPTER IV.}

THE LEECH. Hirudo medicinalis.

PAG I

External characters-Dissection-Fxamination of transverse sections.

\section{CHAPTER V.}

The Earthworm. Lumbricus terrestris.

External characters-Dissection-Examination of transverse sections

\section{CHAPTER VI.}

The Freshwater Mussel. Anodonta cygnea.

The shell-Dissection of the mussel-Examination of transverse sections

CHAPTER VII.

The Edible Snarl. Helix pomatia.

External characters-Dissection of the snail. . 103

\section{CHAPTER VIII.}

The Crayfish. Astacus fluviatilis.

External characters-Dissection-The arteries as seen in an injected specimen .

\section{CHAPTER IX.}

The Cockroach. Periplaneta Americana. 


\section{CHAPTER X.}

The Lancelet. Amphioxus lanceolatus.

PAGE

External characters-Anatomy-Examination of transverse sections.

- 170

\section{CHAPTER XI.}

THE Dog-FISH. Scyllium canicula.

External characters-The skeleton-The abdominal viscera-Dissection of the digestive system-Dissection of the respiratory system-Dissection of the circulatory system-Dissection of the renal and reproductive systems-Dissection of the nervous system-Dissection of the sense-organs . . . . . 196

\section{CHAPTER XII.}

The Skeleton of the RabBit. Lepus cuniculus.

The axial skeleton-The appendicular skeleton .. . . . 264

\section{CHAPTER XIH.}

Dissection of the RabBit. Lepus cuniculus.

External characters-Dissection of the buccal cavity-The abdominal viscera-Dissection of the digestive system-The thoracic viscera-Dissection of the circulatory system-Dissection of the renal and reproductive systems-Dissection of the neckDissection of the brain . . . . . . . . 304

\section{CHAPTER XIV.}

The Skeleton of the Fowl. Gallus bankiva.

The axial skeleton-The appendicular skeleton . . . . 367 


\section{CHAPTER XV.}

Dissection of the Pigeon. Columba livia.

External characters-Dissection of the pectoral museles-Dissec-

PAGI tion of the air-sacs-Dissection of the digestive system-Dissection of the circulatory system-Dissection of the trachea and lungs-Dissection of the renal and reproductive systems -Examination of the brain-The sense-organs . . . 395

\section{APPENDIX.}

Reagents for killing, hardening, and preserving-Reagents used in staining-Clearing reagents-Mounting media-Microtomists' media - Cements for fixing down sections eut in paraffin-Injection fluids . 


\section{LIST OF ILLUSTRATIONS.}

FIGURE

1-8. Amaba

9. Paramecium aurelia . . . . . . . . 4

10. Vorticella. . . . . . . . . . . . . . 9

11. Hydra : Diagrammatic longitudinal section . . . . . . 14

12. " Section of the body-wall, highly magnified . . . 16

13. Fasciola hepatica: Diagrammatic figure of the digestive, excretory, and nervous systems . . . . 28

14. " The reproductive system . . . . 30

15-19. " A series of stages in the life-history. . . . 34

Fig. 15. The free-swimming embryo

, 16. A sporocyst, containing rediæ

"17. A young redia

" 18. An adult redia, containing a daughter-redia and cercarie

19. A free cercaria

20. Hirudo medicinalis : The ventral surface . . . . . 40

21. " Diagrammatic figure of the vascular, renal, reproductive, and nervous systems . .

22. " Diagrammatic transverse section through the middle of the body . . . . . .

23. Lumbricus terrestris: Longitudinal vertical section through

the anterior part of the animal .
24.
25.

26. Anodonta cygnea : Dissection from the right side, to show the heart and gills . . . . . . .

27. " Dissection from the right side, to show the digestive, circulatory, and renal systems . . . 91

28. " Diagrammatic transverse section through the middle of the ventricle. . . . . 101

29. Helix pomatia: Dissection from the right side to show the mantle-cavity . . . . . . 109

30. " Dissection from the right side, to show the digestive and reproductive systems . $\quad . \quad 114$

31. " Section along the axis of the shell, transverse to the foot 123

32. Astacus fluviatilis: Transverse section through the abdomen . 127

33. ", The oral appendages . . . . 132

34. " " Diagrammatic transverse section through the

. thorax . . . . . 136

35. " Dissection of a male from the right side * . 140 
36. Periplaneta Americana: The head, front view . . . . 155

37. "The head, from behind. . . . 155

38. " " Dissection of a male from the right side . . . 160

39. " Dissection of the hinder part of the abdomen of a female

40. Amphioxus lanceolatus: A young specimen, seen from the right side . . . . . . 172

41. " Transverse section through the anterior part of the pharynx . . . . 188

42. " Transverse section through the posterior part of the pharynx . . . . . . . 191

43. " Transverse section passing through the atrial pore . . . . . 194

44. "Transverse section passing through the anus . 195

45. Scyllium canicula: The skull and visceral skeleton from the right side

46. "The skull and visceral skeleton from the ventral surface . . . . . . . 210

47. " The pelvic girdle and fins from the ventral surface

48. " The pectoral girdle and fins from the ventral surface . . . . . . 216

49. " Longitudinal vertical section through the head and anterior part of the body . . . . . 224

50. " The venous system . . . . . . . 226

51. " " The branchial blood-ressels. . . . . 231

52. "Transverse section passing through the posterior dorsal fin . . . . 235

53-54. ", Dissection of the renal and reproductive systems of the female

55-56. " Dissection of the renal and reproductive systems of the male . . . . . . . . 244

57. Lepus cuniculus: Selected vertebræ . . . . . . 268

58. " The skull from the right side . . . . . 276

59. " " Dissection of a male from the left side $\quad . \quad . \quad .308$

60. " Diagrammatic transverse section through the head, showing the tympanic cavity and auditory ossicles

61. " Longitudinal vertical section of the brain : * 353

62. " " The brain from the ventral surface . . . . 356

63. " Transverse section of the brain, passing through the third ventricle . . . . . 362

64. "Transverse section of the brain, passing through the cerebellum . . . . . 365

65. Gallus bankiva: The left half of the skeleton, from the right side . . . . . . . 368

66. " The skull from the right side . . . . . . 376

67. Columba livia : Dissection from the right side $\quad . \quad$ * 406 


\section{INTRODUCTION.}

THe following pages contain a brief summary of the methods ordinarily employed in the dissection and microscopical examination of animals. They are not intended to form an exhaustive account of anatomical technology; and in many cases they are repetitions of the practical directions given in other parts of the book.

I. ON THE OBSERVATION OF ANIMALS DURING LIFE.

All animals should be carefully observed alive before they are dissected, for by this means alone is it possible to obtain a clear idea of the uses and mode of action of many organs, such as the tentacles of $B y d r a$, the odontophore of a snail, the scaphognathite of a crayfish, etc.

\section{ON DRAWING.}

Careful drawings must always be made, both of the living animal and of all dissections and preparations. These drawings should be made to scale, in a book kept for the purpose, and on one side of the page only.

Correctness of outline is of more importance than minute detail ; and the usefulness of the drawings is greatly increased by the systematic use of diagrammatic colouring. A separate colour should be employed for each system of organs, the several parts of which may be indicated by gradations of tint.

As a rule, the more bulky organs, as the liver, should be coloured with dull tints, and the brighter colours reserved for the smaller and less conspicuous parts. Arteries are usually coloured red, and veins blue. 


\section{ON THE METHODS OF KILLING ANIMALS.}

The method of killing is of considerable importance, and careful attention should be paid to the directions given at the commencement of each chapter.

It is important in most cases that the animal should die in an expanded or relaxed condition. This is most readily secured in the case of very minute animals, as Hydra and the Protozoa, by suddenly deluging them with osmic acid, which kills them instantaneously, before contraction can occur. With animals of larger size, chloroform affords the most convenient means for attaining the same end.

Small animals, as the leech, earthworm, etc., may be readily killed by dropping them into solutions of corrosive sublimate, of chromic acid, or of picric acid, or into alcohol or chloroform. Crayfish may be killed by momentary immersion in boiling water; and snails should be drowned, when they die fully expanded.

Many other methods are in everyday use, and the purpose for which the animal is intended determines in many cases the method of killing.

\section{ON DISSECTION.}

The object of dissection is to separate the several organs from one another, so far as is necessary to define their boundaries, and display their relations to one another. It consists mainly in the removal of the connective tissue which binds the parts together and obscures their outlines.

The necessary instruments are the following:-

1. Two or three scalpels, of different sizes.

2. Two pairs of forceps; one large and one small. Both pairs should be straight, and should have roughened tips to secure a firmer hold.

3. Two pairs of scissors; one pair large and strong for general dissection, the other pair small for finer work. The latter pair should have the blades either bent at an angleelbow 'scissors-or else curved. In selecting scissors, care 
should be taken to see that they cut quite up to the points of the blades.

4. A pair of bone-forceps or very stout scissors, for cutting bone and other hard substances.

5. A pair of stout needles, firmly mounted in handles.

6. A pair of the finest sewing-needles, mounted in handles. Only about a quarter of an inch of the needle should project. They are used for teasing histological preparations.

7. A seeker, i.e. a blunt needle mounted in a handle, and bent at an angle half an inch from the end.

8. A pocket-lens, containing two or three lenses mounted in a handle, and giving when combined a magnifying power of at least six diameters.

9. A razor, and some means for keeping it sharp.

10. A blow-pipe of metal or glass, with the end bent at an angle.

11. A glass tube drawn out to a point at one end, and fitted with an india-rubber cap at the other: for use as a syringe, as described on $p$. xxi.

For the dissection of the larger animals, as the dog-fish or rabbit, an ordinary deal board, about two feet long by a foot and a half wide, may be used; to this the animal should be fastened by pins, those with large round heads being especially convenient.

Smaller animals, and special parts of larger ones, should be dissected under water, which supports the parts and greatly facilitates the operation. For this purpose an ordinary white pie-dish with sloping sides is well adapted, the bottom being fitted with a soft deal board weighted with lead, or a sheet of cork cemented in with marine glue. A similar but smaller dish may be used for dissecting under spirit.

Animals, such as the cockroach, which are difficult to fix with pins, may be cemented down with melted wax, or they may be half imbedded in a plate of paraffin, and the plate then fixed down with pins in the dissecting-dish.

For fine work a dissecting microscope affords great assistance. The pocket-lens may readily be turned into one by fitting one end of an ordinary wine-cork into the handle or 
case of the lens, and passing a stout pin transversely through the other end. The pin should be stuck upright into the dissecting-board, with the lens over the part to be dissected: focussing is effected by sliding the cork up and down the pin.

The following rules for dissection should be carefully observed :-

1. Before commencing a dissection, fix the animal down firmly to the dissecting-board or dish.

2. In fixing an animal with pins, stick them in obliquely, so that their heads do not get in the way or obscure the dissection.

3. Dissect under water, unless the animal is too large. Change the water as soon as it gets dirty. A gentle stream of water allowed to play upon the dissection is often a valuable aid.

4. Never cut away anything until you are quite certain what it is you are removing.

5. Put the part you are dissecting slightly on the stretch. This applies more particularly to blood-vessels, nerves, ducts, and muscles.

6. In cleaning blood-vessels, nerves, etc., dissect along them, and not across them; and avoid laying hold of them with the forceps.

7. The dissection is in many cases greatly facilitated by placing the specimen in spirit for a day or so before dissecting it. In some cases the dissection may with advantage be performed under spirit.

8. Always keep your instruments clean and sharp. Be careful not to blunt your fine scissors or scalpels by using them for cutting hard parts.

9. If you get in a muddle, stop, and wash the dissection thoroughly under the tap before proceeding further.

Successive slices cut from an animal, or part of an animal, with a razor are often exceedingly instructive; this is especially the case with the mussel and snail, and with the brains of the rabbit and pigeon. The specimens must be previously hardened with spirit or other reagent, and the slices should be examined in water or spirit. 


\section{ON INJECTION.}

The injection of coloured fluids into the blood-vessels or ducts of an animal renders them much easier to see, and to follow to their distribution.

The colouring matter used must not be soluble in, or affected by, any of the fluids in which the specimen is afterwards to be dissected, hardened, or preserved. The most convenient are French blue, Prussian blue, vermilion, and carmine.

In the case of the larger animals, freshly prepared plaster of Paris forms, if coloured, a convenient substance for injection: it solidifies in the vessels, and so does not escape if a vessel is accidentally cut during the dissection.

For smaller animals, thick gum-water or white of egg may be injected cold, or a jelly made of gelatin and water injected warm: the animal should afterwards be put into alcohol to harden the injection. If the animal is not to be dissected after injection, water coloured with any of the above pigments may be used with advantage, and this method is particularly useful for the alimentary and excretory systems of the liver-fluke.

For injecting small animals, a suitable syringe consists of a glass tube with an india-rubber cap fitted on one end, the other end being drawn out to a point sufficiently fine to enter the vessel to be injected. After the tube is drawn out in the flame and cut off, its sharp edges must be slightly rounded off by holding for a moment in the flame. Several such cannulæ of various sizes should be kept ready.

For injecting larger animals, such as a rabbit, with plaster of Paris, the blood must first be washed out of the vessels by injecting warm salt-solution. The following apparatus is necessary, and must all be placed on the table ready for use before the animal is killed; for the blood coagulates after death so rapidly as to allow no time to look for instruments.

1. Cannulæ: glass ones are the most convenient; they should be of various sizes, to fit the several vessels which it is proposed to inject, and each should have a slight constriction 
near the tip, so that it may be firmly secured in the vessel by a ligature. The largest cannula that will enter the vessel should be used.

2. A glass syringe, capable of holding about an ounce of fluid. A larger syringe is liable to get blocked up.

3. Several pieces of strong india-rubber tubing, about an inch long, to connect the nozzle of the syringe with the cannula, both of which they must fit rather tightly.

4. Two or three glass plugs to fit the india-rubber tubing.

5. A dish of water or salt-solution, in which all the cannulæ and india-rubber connections are laid, to displace the air from them.

6. Fine plaster of Paris.

7. A mortar and pestle.

8. Colouring matter, such as carmine previously rubbed up with water : it must be well shaken before use.

9. A piece of muslin to strain the injection through. It must be well wetted before use.

10. A jar into which to strain the injection.

11. A jar containing about half a pint of salt-solution ( 75 per cent.), at a temperature of $100^{\circ} \mathrm{F}$.

12. Dissecting-board, pins, scalpels, scissors, two pairs of forceps, seeker, thin string or thread, and two or three pairs of 'bull-dogs.' These last are very short spring-forceps, the spring being so arranged as to close the forceps. They are convenient to stop the escape of blood or injection from any vessel that may have been cut.

The injection should be performed close to a large sink, over which is a water-tap with a foot or two of wide indiarubber tubing attached.

When everything is ready, kill the animal, and as soon as it is dead, lay it open, cutting as few blood-vessels as possible. Expose the root of the aorta or other vessel from which it is intended to inject the animal; choose a cannula of the right size : fit it with an india-rubber connection; fill it with saltsolution, and stop the end of the connection with a glass plug. Pass a ligature round the vessel: make a longitudinal slit in the vessel; insert the cannula; tighten the ligature upon it, 
and tie it with a bow. If the ligature be too tight it will cut the vessel. Fill the syringe with the warm salt-solution: remove the plug from the cannula: press the body of the animal slightly, to remove some of the blood from its vessels, and to get rid of any clot that may have formed close to the cannula. Inject the salt-solution, to force the remaining blood from the vessels before it can coagulate; and wash or sponge the blood away. If the arteries are being injected, the vena cava and portal veins should be cut open to allow free escape of the blood, and vice versa.

Mix the plaster of Paris in the mortar, stirring in the colour, and making the plaster thin. Strain it rapidly through the muslin, and inject immediately with the syringe. When the vessels appear to be well injected, remove the syringe and insert the glass plug, and wash the animal to get rid of blood and any injection that may have escaped.

Allow the animal to remain two or three hours in cold water before dissecting it or putting it into spirit.

\section{ON THE USE OF REAGENTS.}

Reagents are used for hardening, staining, and preserving specimens. Those required for general use are but few in number, and directions for their preparation will be found in the appendix.

1. Hardening is necessary in the case of soft animals or tissues of which it is proposed to cut slices. For the mussel a $\frac{1}{4}$ per cent. solution of chromic acid in water serves well. For the brain of a rabbit or pigeon ordinary methylated spirit is very convenient and effective. The specimen must be left in the hardening fluid two or three days, and a large bulk of the fluid must be used, or else it must be frequently changed.

Other methods are used when the specimen is to be cut into microscopical sections. (See Appendix.)

2. Staining with some colouring fluid renders the various parts much more distinet, and is useful when the object is to be examined microscopically, and especially when it has to be cut into sections. 
Magenta is useful for staining fresh specimens, but the stain is not permanent.

Carmine is perhaps the most useful of all stains. It affects different cells and parts of cells differently, and the stain is ! permanent. The most useful preparation of it is Grenacher's borax-carmine: this should be used warm, and the specimens after removal from it should be placed in acidalcohol for a few minutes or hours, according to their size.

Picro-carmine has the advantage of being an aqueous stain, and may therefore be used either for fresh or hardened tissues. Immersion of the specimen in acid-alcohol for some time after staining improves the effect.

Hæmatoxylin (Kleinenberg's solution) gives excellent results if used with due care, but a trace of chromic or other acid may completely destroy the colour after the specimen is mounted.

Osmic acid, of which a 1 per cent. or $\frac{1}{2}$ per cent. aqueous solution is used, is both a hardening and a staining reagent. It is only used with fresh tissues, and only with small specimens. It kills Protozoa instantly, and after a time stains their nuclei, though only faintly. Objects hardened with osmic acid may be stained with picro-carmine. A mixture of chromic and osmic acids forms a useful hardening reagent.

Acetic acid, 1 per cent. solution, is useful for rendering the nuclei of cells more distinct: it is used with fresh specimens.

\section{ON MICROSCOPICAL EXAMINATION.}

The microscope affords the means of investigating the structure of minute animals, and the finer details of those of larger size. The microscopical examination of the special organs of the larger animals is of great importance, and must on no account be neglected.

The microscope consists of a body and a stand. The body is a tube of metal carrying the lenses, which are the essential part of the instrument.

The stand supports the body, in such a way that the latter may be moved up and down without any lateral move- 
ment. It also supports a stage, which ought to be horizontal, to hold the object to be examined. The stage has a round hole in its centre, through which light may be refiected on to the object by a mirror fixed below the stage. This aperture is usually much larger than is necessary; but the excess of light, and especially the light far from the optical axis of the lenses, is cut off by means of diaphragms, of which there is a series, of various sizes, fixed in such a way as to allow of their being easily changed.

The combination of lenses at the upper end of the body is the eye-piece; the combination at the lower end is the objective.

One or two eye-pieces, and two objectives of different magnifying powers, $\frac{1}{2}$ inch and $\frac{1}{8}$ inch, are required.

An object can be seen clearly through a microscope only when it is at a certain definite distance from the objective; and this distance varies with different objectives and eyepieces, and to a slight extent with different observers. An objective of high power requires to be nearer to the object than does one of low power. The regulation of this distance is called focussing, and is effected in two ways.

The coarse adjustment of focus is made by simply sliding the body up or down, with a'slight twisting movement, through the tube of the stand in which it is supported, or else by a rack and pinion worked by a milled head.

The fine adjustment is effected by means of a screw, the position of which differs in different microscopes; but which should be so placed that it can readily be worked with either hand. By turning the head of the serew from left to right, in the direction of the hands of a watch, the body of the microscope is lowered, and the objective brought nearer to the object: by turning in the reverse direction, the objective is raised.

In using the microscope the following rules are to be carefully observed :-

1. Examine every object with the low power first. Having adjusted the eye-piece and the objective, turn the mirror so as to reflect the light up the body of the microscope: place the 
object on the stage under the objective, and carefully lower the body with a screwing motion till the objective is about an quarter of an inch from the cover-glass: then look through the eye-piece, and gradually raise the body till the object becomes distinctly visible. Focus accurately with the fine adjustment screw. With the high power, begin with the objective close to the cover-glass, and then proceed as before.

2. Never examine an object with the high power unless it. is protected by a cover-glass. Take extreme care never to let the objective touch the cover-glass; and never to touch the face of the objective or allow any dirt to get on it. The face of an objective cannot be cleaned without doing harm to it.

3. Keep both eyes open when looking through the microscope. Also get into the habit of using either eye.

4. When examining an object, keep one hand on the fine adjustment, and keep screwing it up and down slightly. In this way parts of the object at different depths are brought. into focus, and a much clearer idea of the object is obtained.

5. With a high power, use a small diaphragm : the amount of light will be somewhat diminished, but the clearness and definition much increased.

6. See that the body of the microscope slides smoothly in its tube. If it does not, remove it, and clean it by rubbing with a few drops of olive oil : wipe off the oil before replacing in the tube. Clean the inside of the tube in the same way.

7. The object may appear indistinct from dirt in any of the following places, i.e. on the eye-piece, the objective, or the cover-glass. If it be on the cover-glass the dimness varies when the slide is moved; if on the eye-piece, it varies when this is rotated; if not on either of these, it must be on or in the objective.

The eye-piece and the lower surface of the objective may be cleaned with chamois-leather or silk. If the objective is smeared with glycerine, wash it carefully, then dry with a soft handkerchief. Canada balsam, which sometimes gets on the objective, may be removed with a drop of turpentine on a handkerchief. It is, however, safer to leave this to an optician or to the demonstrator, as a very small quantity of turpentine 
getting inside the rim which carries the lens, may lead to the separation of the two lower lenses from each other, rendering. the objective useless.

\section{ON CUTTING SECTIONS.}

Many points in anatomy and histology may be best made out by examination of microscopical sections, that is, slices cut so thin as to allow of their being examined under the microscope by transmitted light.

The hardened object, supported, if necessary, between two. pieces of carrot, may be held between the fingers and thumb and sections sliced off it freehand with a sharp razor.

The microtome or section-cutting machine, however, gives. better results. It consists essentially of three parts : a clamp or other contrivance for holding the object to be cut; means of guiding the razor; and a 'feeding' arrangement. These parts are so arranged that the razor can be drawn smoothly across the object to be cut, taking off a section at each stroke, the feeding arrangement bringing the object into the path of the razor, and the amount of 'feed' determining the thickness of the section.

In the simplest form of microtome the razor is drawn by hand over a smooth plate, the object to be cut being pushed. up through a hole in the centre of the plate by means of a screw or other 'feeding' arrangement placed below.

More complicated microtomes have been devised to obviate the wearing of the edge of the razor by friction upon the plate; to render it possible to cut the object in any desired direction ; to cut more evenly, more easily, and more rapidly; and to yield sections in a string or ribbon so as to simplify and expedite the mounting of large series of sections. By means of a Threlfall-Caldwell microtome, sections may be obtained cohering at their edges to form a continuous ribbon at the rate of two or three hundred a minute or more.

The preparation of an object for cutting in a microtome depends to some extent upon the nature of the object and the number of sections required, as well as the purposes for which they are intended. The object has in any case to be supported. 
by impregnating it with some homogeneous substance which is firm enough without being brittle or too hard. The freezing method is the simplest of all, but it is not easily applicable when a large number of sections are required mounted in consecutive series, nor when very thin sections are required. The tissue to be cut is first soaked in gum-water, then placed on the freezing-plate of the microtome and cooled by an ether-spray or a freezing mixture underneath. The frozen mass is eut when just thawing.

The imbedding method is more complicated but has many advantages. The specimen, which may be stained previously, is dehydrated by soaking in absolute alcohol; the alcohol is then removed by soaking in turpentine, xylol, oil of eloves or some similar substance, and the object is then transferred to paraffin kept just melted in a water-oven, the temperature not being allowed to rise above $60^{\circ} \mathrm{C}$. Paraffin melting at $55^{\circ}$ or $56^{\circ} \mathrm{C}$ is the best, though a softer paraffin may be used in a cold room, if it be found that the hard paraffin crumbles before the razor. When the object is thoroughly permeated, it is transferred to a small trough of paper with just enough paraffin to form on cooling a block completely enclosing it. It must be cooled as quickly as possible by placing the trough in a dish containing cold water, as the paraffin is liable to crystallise if allowed to cool slowly. A couple of L-shaped blocks of metal, resting on a plate of glass or metal so as to form a box of adjustable size, may be used in place of the paper trough. The block containing the object is fixed in the microtome ; the most efficient of all contrivances for this purpose is, perhaps, a grooved metal plate which is simply warmed slightly and pressed against the end of the block, and then cooled quickly as soon as the block has begun to melt. The further procedure depends entirely upon the particular microtome used. The sections are to be mounted as described in section IX.

\section{ON MOUNTING MICROSCOPICAL OBJECTS.}

The most convenient slide for microscopical objects is a slip of glass three inches long and one inch wide, or for large 
series of sections the slide may be $1 \frac{1}{2}$ inch wide. The glass should be free from air-spaces and other flaws, and the edges should be ground.

Cover-glasses must be very thin (No. 1.) and free from flaws. For single objects circular covers $\frac{1}{2}$ to $\frac{7}{8}$ inch in diameter are best, and for series of sections square or oblong covers which should not come within $\frac{1}{16}$ inch of the edge of the slide; space should also be left at one end for a label.

Cells are required to protect thick objects from the pressure of the cover-glass. Thin flat rings of tin are good, and should be cemented to the slide with gold size or "brown cement "; or a ring of the cement painted on with a brush and allowed to dry may be used as a cell for thinner objects. No cell is required for sections. Copper, brass, and vulcanite cells are to be avoided.

An object may be mounted dry by fixing it to the slide in the centre of a cell with a very small quantity of collodion or gum-water, or of solution of shellac in creasote or alcohol. When the cement is dry, a cover of the same diameter as the cell is to be laid over it, and secured by varnishing at the edge with any of the cements sold for the purpose.

More usually it is advisable to mount an object in a liquid or solid medium. Such are glycerin, which is liquid; Farrant's medium, and Canada balsam, which are liquids becoming solid; and glycerin-jelly, which is solid when cold.

An object, stained or otherwise, to be mounted in glycerin or Farrant's medium may be transferred to it direct from water-great care being taken to avoid introducing air. The drop of glycerin, etc., used should be just large enough to spread out to the edge of the cover. When glycerin is used, the cover must be fixed by means of gold-size or other cement applied round the edge with a small paint-brush.

To mount in glycerin-jelly the object is first soaked in glycerin and then in melted glycerin-jelly (see Appendix), it is then transferred to a drop of the melted glycerin-jelly upon the slide and the warm cover, previously wetted with the melted jelly to aid in excluding air, is immediately placed upon it and held in position till the jelly solidifies. Avoid 
heating more than is necessary. The edges of the cover may be cemented as above, but this is not absolutely necessary.

To mount an object in Canada balsam, it must, after staining, be dehydrated by soaking in alcohol; then soaked in oil of cloves or other essential oil, or in xylol, benzin, chloroform, ether, etc., till the alcohol is removed; and, lastly, placed in the drop of balsam upon the slide and covered.

Sections are mounted as follows:-1. Sections cut frozen are transferred direct to the glycerin on a slide. A thin and delicate section cut frozen cannot well be mounted in balsam, but a moderately thick and tough one may be treated like any other object as described in the previous paragraph.

2. The methods of mounting in balsam sections cut in paraffin are very numerous. The following answers well, and is especially adapted for mounting sections in series.

Dry the slide thoroughly at about $60^{\circ} \mathrm{C}$. While still warm, coat it on one side with a delicate layer of shellac-this is done by dipping a glass rod into an alcoholic solution of shellac (see Appendix), and then holding the rod horizontal, laying it flat upon the slide and drawing it evenly over the surface, when it will leave a thin layer of the solution upon he slide, and the alcohol evaporating immediately leaves an almost imperceptible layer of shellac on the surface. Just before placing the sections upon this, smear it very slightly with oil of cloves; lay the sections perfectly flat upon the still moist slide, and put the slide for fifteen minutes into an oven at a temperature of $60^{\circ} \mathrm{C}$. Remove the slide and pour turpentine upon it, or immerse the warm slide bodily in the turpentine for a minute or two, to dissolve the paraffin out of the sections; lift out of the turpentine; drain about fifteen seconds, drop the balsam upon the sections while they are still wet with turpentine; and cover immediately.

\section{ON MEASURING MICROSCOPIC OBJECTS.}

The eye-piece micrometer is a plate of glass, the upper surface of which is either engraved with a scale, or is divided into a number of equal squares by cross-lines ruled upon it. This is placed between the lenses of the eye-piece of the 
microscope in such a position that the scale can be seen distinctly on looking through the microscope.

The stage micrometer is a slide on which a scale is engraved, divided to, say, hundredths and thousandths of a millimeter.

To measure a minute object, focus it under the microscope with the eye-piece micrometer in position; read off the apparent size on the scale of the micrometer; replace the slide by the stage micrometer and read off upon that the actual value of the noted number of divisions of the eye-piece micrometer.

The value of each division on the eye-piece micrometer will obviously vary when the objective is changed or the length of the microscope tube is altered.

By means of a camera lucida a sheet of paper laid on the table at the side of the microscope is visible at the same time as an object under the microscope, and if a scale be drawn upon this coinciding with the apparent image of the stage micrometer scale seen through the microscope, the paper may be used to measure directly the size of an object whose image is superimposed upon it.

Eye-pieces and stages are also constructed in which the measurement is accomplished by means of a fine screw with a divided head. To use the micrometer stage, the slide bearing the object is laid upon it, one edge of the image is made to coincide with a mark upon the eye-piece micrometer or a slip of glass in the same position. The screw of the stage is then turned till the opposite edge of the image coincides with the same mark, the distance through which the slide has been moved is then read off on the divided head of the screw and this distance is, of course, the diameter of the object.

\section{ON PREPARING SKELETONS.}

Skeletons of rabbits, fowls, etc., may be prepared by maceration or by boiling. Before macerating the bones should have most of the flesh cut off ; they are then to be put into cold water and left for several weeks for the remaining flesh 
to rot away. They will afterwards require copious washing, and may then be bleached by exposure to direct sunlight for two or three weeks, during which time they should be repeatedly dipped into water and then left to dry. This same process also removes much of the offensive smell of freshly macerated bones. Both these effects may, however, be more quickly produced by steeping the bones in a clear solution of bleaching powder, and afterwards washing them in running water.

Boiling the bones and then picking off the flesh yields skeletons serving all the purposes of the student, though not so white as those obtained by maceration.

The preparation of cartilaginous skeletons is described at page 200 . 


\title{
PRACTICAL ZOOLOGY.
}

\author{
$-$ \\ Chapter I. \\ THE PROTOZOA.
}

A PROTOzOon is an animal which consists of only one cell, the equivalent of a single cell of any of the tissues of a higher animal.

Protozoa are always of small size, and are usually microscopic.

\section{AMOBBA.}

This protozoon is found at the bottoms of freshwater pools, and in damp situations. Its indefinite and ever-changing shape is characteristic, and has gained for it the name 'proteus animalcule.' There are several forms, the largest of which are just visible to the naked eye, while others are exceedingly minute.

Place a drop of water containing Amoba on a slide: cover; and search for the animal with a low power of the microscope. When found, examine it with a high power.

\section{A. General appearance.}

The animal is an irregular mass of protoplasm, running out into blunt processes or pseudopodia, the protrusion and retraction of which cause great changes in the form of the cell. The pseudopodia may branch slightly. 
B. Structure.

The whole body is a naked mass of protoplasm, which is of two kinds.

1. The ectosare is the clearer outer portion of the body.

2. The endosarc is the granular and usually more fluid central portion. Its flowing movements, which accompany the protrusion and retraction of the pseudopodia, are rendered obvious by the granules contained in it.

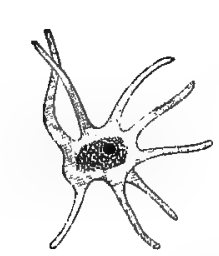

Fig. I.
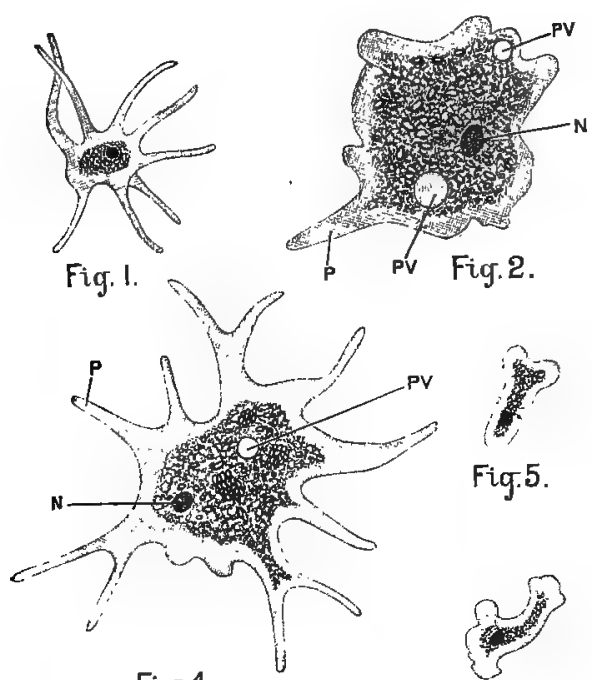

Fig. 4 .

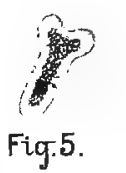

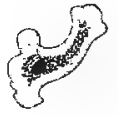

Fig. 7.
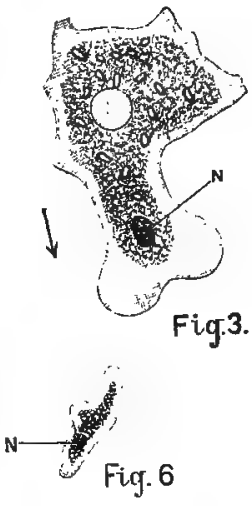

Fits. 1 to 8. - Amcoba; $\times 400$. (с. н. н.)

Figs. 2 and 3 were drawn from the same specimen, which was moving. in the direction shown by the arrow. Fig. 4 is a form with branching pseudopodia. Figs. 5 to 8 were drawn from one specimen at intervals of about twenty seconds.

$\mathbf{N}$, nucleus. $\mathbf{P}$, pseudopodium. PV, pulsating vacuole.

Within the endosarc make out the following structures.

a. Vacuoles are spaces filled with fluid, which is less dense than the surrounding protoplasm. They contain in many cases foreign particles taken in as food. 
b. The pulsating vacuole is a space filled with a watery fluid. It is fairly constant in position, and pulsates rhythmically. More than one may be present. Time its pulsations.

c. The nucleus is a spherical or ovoid body, denser than the rest of the endosarc and highly refractive. During life it is not easily seen, but it is rendered obvious by killing the animal with acetic acid (1 per cent.), or by staining with magenta. More than one may be present.

\section{Movements.}

The constant but slow change of form already referred to is known as 'amœboid movement.' Pseudopodia are protruded from any point of the surface, and can be completely withdrawn, and a slow crawling movement of the whole animal can be effected by their means. Pseudopodia may also surround particles of food and then be withdrawn, bringing the food-particles into the body.

Draw a specimen of Amoeba several times at intervals of half a minute, indicating the direction of flow of the particles by means of arrows. Compare the drawings, and note the differences of form even in sluggish specimens in which move. ment was not obvious.

Feed with indigo or carmine, or other finely divided pigment, and note that food is taken in at all parts of the surface.

\section{Reproduction.}

Amceba multiplies by fission; that is, the whole mass divides into two, each containing a portion of the nucleus, of the endosare, and of the ectosare of the original cell.

\section{PARAMECIUM AURELIA.}

This is a free-swimming freshwater protozoon found abundantly amongst decaying vegetable matter. It differs from Amceba in its more definite shape; in the more marked differentiation of ectosare and endosarc; in the presence of cilia and the absence of pseudopodia ; in its active locomotion 
by means of the cilia; and in the possession of a definite mouth, and of definite dorsal and ventral surfaces, and anterior and posterior ends.

Put a drop of water containing Paramecia on a slide; spread a very little cotton wool over it, to limit the movements of the animals; cover, and examine with low and high powers.

\section{A. General appearance.}

The animal is an elongated, somewhat flattened body about $\frac{1}{100}$ inch in length, rounded at its narrower anterior end, and bluntly pointed at the broader posterior end.

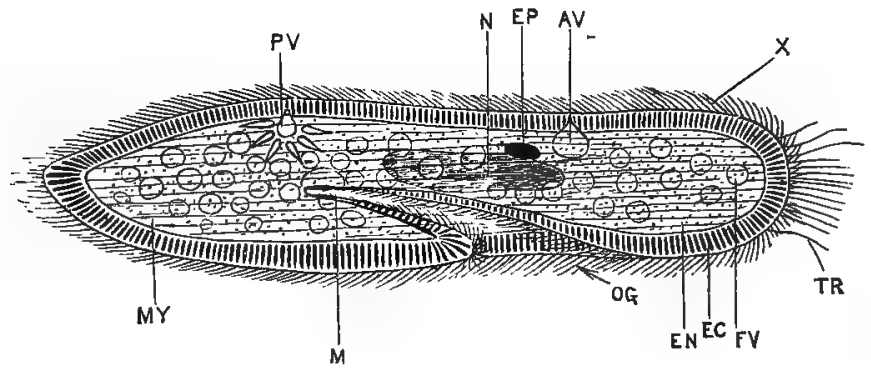

Fig. 9.-Paramecium aurelia, seen from above and slightly from the right side; $\times 300$. (с. ‥ ‥)

$\mathbf{A V}$, anterior pulsating vacuole. $\mathbf{E C}$, ectosare : the lines ruming across it indicate the trichocysts. $\mathbf{F N}$, endosare. $\mathbf{F P}$, paranueleus. $\mathbf{F} \mathbf{V}$, foodvacuole. M, mouth. MY, 'myophan' striation. $\mathbf{N}$, nucleus. $\mathbf{O G}$, oral groove. PV, posterior pulsating vacuole, in systole. TR, discharged trichocyst threads. $\mathbf{X}$, cilia.

It swims actively by means of cilia distributed over its entire surface. Near the middle of the ventral or oral surface is an oblique groove leading to the mouth.

\section{B. Structure.}

The two layers, ectosare and endosarc, are much more sharply defined than in Amœba.

1. The ectosarc is the comparatively firm outer layer, the elasticity of which preserves the general form of the body. Its deeper part is marked by longitudinal or oblique 'myophan' striations, which vary in dis- 
tinctness with the movements of the animal, and are probably due to longitudinal wrinkling of the inner surface of the ectosarc.

a. The cuticle is the delicate superficial and stiffer layer, serving as a protective covering for the underlying protoplasm, of which it is the differentiated external layer.

b. The cilia are very numerous delicate vibratile filaments arising from the layer of ectosare immediately beneath the cuticle, through which latter they project. They are of uniform size over the entire surface, and extend into the oral groove. Their rapid movements serve both for locomotion and for the ingestion of food.

c. The trichocysts are minute oval sacs arranged side by side in the deeper part of the ectosare, perpendicular to the surface, and in such numbers as to form an obvious layer. When the animal is irritated a stiffish thread can be shot out from each of these trichocysts, and project on the surface beyond the cilia. They are protective and offensive weapons.

d. Two pllsating vacuoles are situated in the substance of the ectosare of the dorsal, or aboral region, one at about a third of the animal's length from each end. In diastole they are nearly spherical, but at the moment of systole, or contraction, they become stellate, and canals can then be: seen radiating from them. They also open to the exterior at the same moment.

e. The oral groove runs obliquely backwards along the ventral surface from near the anterior end to the mouth, which is placed a little behind the middle of the length of the animal. Its cilia are directed towards the mouth, and drive water and foodparticles into it. 
f. The mouth is an oval aperture in the ectosarc at the hinder end of the oral groove, through which the food passes into the endosarc.

g. The anus is an aperture between the mouth and the hinder end of the body. It is visible only at the moment of extrusion of fæcal matter.

2. The endosarc is the more fluid granular protoplasm forming the central portion of the cell-body.

a. The food-vacuoles are spherical spaces in the endosare filled with water containing foodparticles.

b. The circulation of the endosarc is rendered obvious by the food-vacuoles and the granules, which are carried round in a definite direction.

c. The nucleus is an elongated ovoid body near the centre of the cell-body.

d. The paranucleus is a much smaller body applied to one side of the nucleus, and resembling it in appearance. In some cases both nucleus and paranucleus appear to be situated in the ectosarc rather than in the endosarc.

\section{Reproduction.}

1. Fission is the most usual method, and is effected in the following manner. A transverse constriction appears on the surface of the animal, and deepens till the body is divided into two, each having a portion of the original nucleus and paranucleus, and each becoming a perfect Paramecium.

2. Conjugation is not a mode of reproduction but is closely connected with it. Two Paramecia become united by their ventral surfaces, and, after swimming about together for some time, separate and become independent again. During and after 
this union certain changes occur in the nuclei and paranuclei of both individuals, resulting in the so-called 'rejuvenescence of the nuclei,' and followed by rapid and repeated fission of the two Paramecia.

\section{OPALINA}

Opalina is a protozoon usually found living in large numbers in the large intestine of the frog. From its large size and the ease and certainty with which it can be obtained it is a very convenient form for examination.

Slit up the large intestine of a freshly-pithed frog; scrape away the contents together with the mucous membrane of the intestine, and dilute with salt solution ( 75 per cent.). Examine a drop of the mixture microscopically.

\section{, A. General appearance.}

Opalina is a flattened, asymmetrically oval body, about $\frac{1}{36}$ inch long, covered with cilia by means of which it swims actively. It has neither mouth nor anus.

\section{B. Structure.}

1. The ectosarc is thinner than in Paramecium. There are no pulsating vacuoles, and no trichocysts.

a. The cuticle, if present at all, is exceedingly thin.

b. The cilia are of equal size over the whole surface.

c. 'Myophan' striations are obvious: they are mainly longitudinal.

2. The endosare contains no food-vacuoles, owing to the fluid nature of the food: this also accounts for the absence of a mouth, the nutriment being absorbed by the whole surface of the cell-body.

a. The nuclei are numerous.

\section{Reproduction.}

This is effected by oblique or transverse fission 


\section{VORTICELLA.}

Vorticella, which is just recognisable with the naked eye, differs from the two preceding infusorians in being attached by a contractile stalk to plants or other objects, often to other aquatic animals; it may, however, under certain conditions become detached from its stalk and swim freely.

Both freshwater and marine forms of Vorticella are known. The following description applies more particularly to some of the larger freshwater species, but most of the marine forms closely resemble these.

Mount a specimen in a drop of water together with a bit of the weed or other substance to which it is attached. Examine it microscopically.

\section{A. General appearance.}

1. The form of the fully expanded animal is bell-shaped, the mouth of the bell being closed by the circular dise, and the handle of the bell represented by the slender stalk by which the animal is attached. The disc is placed somewhat obliquely: it is in contact with the edge of the bell at the 'dorsal' surface, but separated from it in most forms by a groove at the opposite or ' ventral' edge.

2. The peristome is the thickened everted margin of the bell.

3. A fringe of cilia runs round the margin of the disc, in the groove separating it from the peristome.

4. The vestibule is a conical cavity opening exteriorly between the ventral margins of the disc and peristome. The cilia with which it is provided cause a rapid current of water to flow in and out of it.

If this current be not visible, run under the cover-glass a little water containing some insoluble pigment, e.g. carmine or indigo. Indicate the direction of the current in the vestibule by arrows on your drawing. 


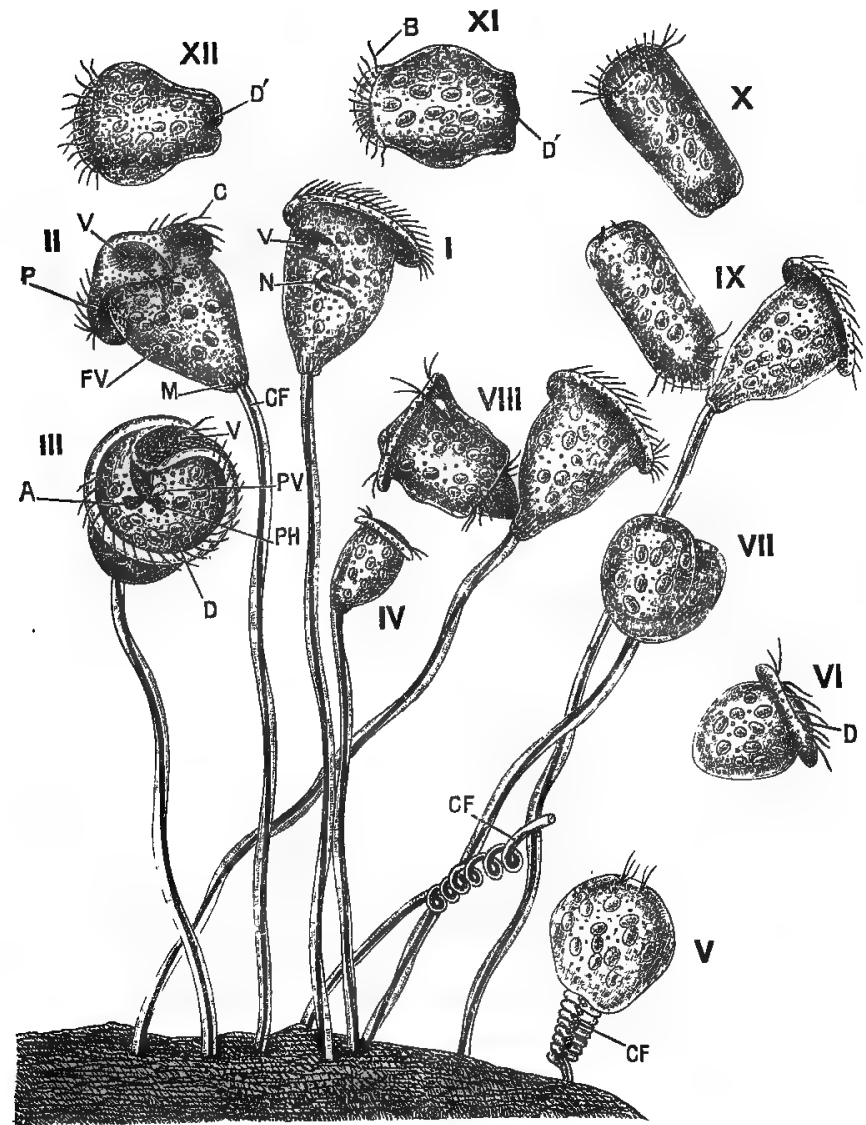

FIG. 10.-A group of Vorticellæ; $\times 220$ (с. н. н.)

Figs. I, II, and III show the animal in various positions. IV is a much smaller specimen drawn to the same scale. $V$ shows a specimen made to contract by action of alcohol. VI is detached from its stalk, and swimming away freely, disc forwards. VII, VIII, IX show three stages of fission. $\mathbf{X}, \mathbf{X I}$, and $\mathbf{X} I I$ show the separated individual swimming by means of the aboral circle of cilia : $X I$ is slightly contracted, XII strongly contracted after the cover-glass has been tapped.

A, Food vacuole discharging contents at anus. C, Peristomial cilia. CF, contractile fibre of stalk. D, dise. $D^{\prime}$, disc retracted. FV, food vacuole. PH, pharynx. PV, pulsating vacuole. P, peristome. $\mathbf{M}$, myophan striation. iN, nucleus. $\mathbf{V}$, vastibule. 
5. The pharynx is the narrower continuation of the vestibule inwards. Its cilia drive water, bearing foodparticles, into the endosare.

6. The anus opens into the vestibule, but is only visible at the moment of extrusion of fæcal matter.

7. The contractility of the animal is exhibited when it is irritated, as by lightly touching the cover-glass with a needle. The peristome is then suddenly turned in, concealing the disc and cilia, and at the same moment the stalk is suddenly coiled up into a corkscrew spiral. If the irritation be discontinued, the stalk gradually straightens out, the disc and peristome become everted, and the ciliary movement is resumed.

B. Structure.

1. The ectosarc is the external layer of the cell-body: it also lines the vestibule and pharynx. The stalk is a prolongation of the ectosare only.

a. The cuticle forms a protective covering for the whole surface. It is thickest on the stalk, and the straightening out of the stalk after contraction probably depends upon its elasticity.

b. Cilia are restricted to the margin of the disc, the vestibule, and the pharynx. They produce a current which runs round the dise in the direction opposite to that of the hands of a watch, then down the vestibule near its ventral surface, and, after a turn in the upper part of the pharynx, up along the dorsal wall of the vestibule to the exterior. The appearance of a long vibratile 'vestibular seta' in the dorsal part of the vestibule is probably due to the movements of the vestibular cilia.

c. A longitudinal ' myophan' striation is visible in the deeper layer of the ectosarc, especially in the lower part of the body close to the stalk.

d. The contractile band running down inside the cuticular sheath of the stalk is continuous above 
with the deeper, 'myophan,' layer of the ectosare of the body. The spiral shortening of the stalk. is due to its contraction. In a specimen in which this band had been broken, it was seen to be drawn up suddenly towards the body at each inversion of the disc, the stalk, however, remaining straight.

2. The endosarc is the more fluid granular central portion of the cell-protoplasm. It does not extend into the stalk.

a. The food vacuoles circulate with the endosarc.

b. The nucleus is an elongated curved band in the upper part of the body near the pharynx.

c. The single pulsating vacuole is spherical, and situated between the disc and the vestibule, into. which latter it opens.

\section{Reproduction.}

1. Fission. The cell-body becomes widened laterally, and a constriction appears, running across its distal surface. This constriction extends downwards till it reaches the base of the bell close to the stalk, dividing the cell-body into two halves: of these one is like the parent, and remains attached to the stalk. The other, while still attached to the base of the first, develops an aboral circle of cilia near its base, becomes detached, and swims away by means of the aboral cilia. After a time it attaches itself by its base to some submerged object, loses its aboral cilia, and forming a stalk by elongation of its base becomes a Vorticella similar to the parent from which it has been cut off. The process of fission may be completed in from one to two hours.

2. Conjugation occurs sometimes, but it is unlike the conjugation of Paramecium in two important points. Firstly, the conjugation is between two dissimilar forms, an ordinary, large, stalked form, and a much 
smaller free-swimming form which has originated from repeated division of a large form. Secondly, the union of the two is a complete and permanent fusion, the smaller being absorbed into the larger.

This permanent fusion of a small active cell with a relatively large fixed cell, followed by division of the fused mass, bears a striking analogy to the process of sexual reproduction occurring in higher animals.

3. Encystment may occur after conjugation, and apparently as the result of it. The disc becomes retracted; the cell-body usually separates from its stalk, becomes spherical, and secretes a cyst around itself; the nucleus enlarges and becomes greatly elongated and then moniliform, and finally breaks up into a number of 'spores.' These are ovoid bodies, each with a circlet of cilia. The cyst ruptures and the spores escape and swim freely, multiplying by fission; later on each becomes attached by the end surrounded by the cilia and develops a disc at the opposite end, loses its original zone of cilia, and grows up into an ordinary Vorticella.

This process is not of frequent occurrence.

\section{Action of Reagents.}

1. Kill some Vorticella with acetic acid (1 per cent. or weaker). Stain with magenta or carmine, and mount in glycerine, or balsam.

The nucleus will be stained, and its shape and relations can be well studied.

2. Weak solutions of poisons such as acetic acid, corrosive sublimate, etc., cause the animal to retract its disc, and the stalk to gradually coil up into a close spiral, and then break off close to the base of the bell. In the natural condition the cell-body may separate in the same way from the stalk and swim away to a fresh resting-place, when scarcity of food or other adverse circumstance renders it advantageous. 


\section{Chapter III.}

\section{HYDRA.}

HYDra is a small freshwater animal, abundant in ponds and ditches and in slowly-moving streams.

It consists of a tubular body about a quarter of an inch in length, one end of which is closed while the opposite end is open and forms the mouth, a short distance below which is a circle of tentacles usually six to eight in number.

Both the tentacles and the body are extremely contractile. The former, when the animal is disturbed, can be almost completely withdrawn, and the latter may shrink up so as to become a mere knob or button.

Hydra is usually attached by its closed basal end or foot to water-weeds or other foreign bodies. By contractions of the foot it can crawl along slowly, and it can also progress more rapidly by fixing itself alternately by the mouth and foot, arching the body with a looping movement like a caterpillar. It is carnivorous, and by means of the nematocysts with which its tentacles are studded can paralyse and kill animals nearly as big as itself and of active habits.

Hydra receives its name from its remarkable power of recovery from injury. A specimen may be cut into two or more pieces, either transversely or longitudinally, and each fragment will not only survive but within a short time become a complete Hydra. The entire animal may even be regenerated from a single tentacle.

At least three species of Hydra are described as occurring commonly in this country. Of these $H$. viridis is distinguished by its green colour and its smaller size, $H$. fusca is brown in colour, and $H$. vulgaris almost colourless. 
It is uncertain, however, how far these spécies are really distinct from one another, and the following description will apply to any of them.

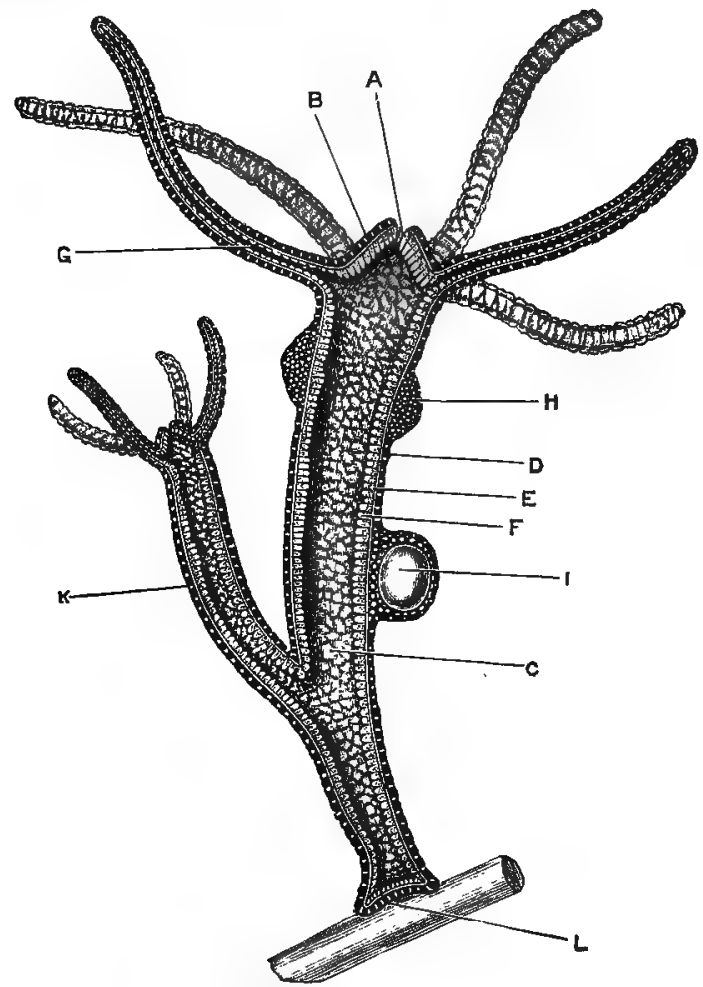

Fig. 11.-Hydra. A diagrammatic longitudinal section of a specimen with ripe reproductive organs, and with a fully-formed bud; $\times \mathbf{1 2}$. (A. M. M.)

A, mouth. B, hypostome. C, enteron, or digestive cavity. D, ectoderm. $\mathbf{F}$, supporting lamella. $\mathbf{F}$, endoderm. $\mathbf{G}$, cavity of one of the tentacles. $\vec{H}$, testis. $\mathbf{I}$, ripe ovum in the ovary. $\mathbf{K}$, fully-formed bud, with rnouth and tentacles. I, foot by which the Hydra is attached to a piece of weed.

\section{EXAMINATION OF A LIVING SPECIMEN.}

Cut off a small piece of weed with a Hydra attached to it; place it on a slide in a drop of water, and put on a cover- 
glass. If the Hydra is detached or fixed to the side of the tank, take it up with a dipping tube, and place it on a slide in a drop of water, with a small piece of weed for the animal to attach itself to, and to protect it from pressure. Cover as before.

\section{A. General appearance.}

Leave the slide until the Hydra has fully expanded, and then examine it with a low power.

1. The body is tubular: its cavity, the enteron, opens to the exterior at the mouth, and serves as the digestive cavity of the animal.

The shape of the body varies very greatly according to the degree of elongation or contraction. - It is usually more slender in its proximal or basal half than in the distal portion, but when fully extended is of nearly uniform diameter throughout. It is often distended locally by food.

a. The foot is the closed proximal end of the body which forms a kind of sucking disc for attachment to foreign bodies.

b. The mouth is a small aperture at the free or distal end of the body. It can be greatly dilated so as to allow the entrance of food-masses of considerable size.

c. The hypostome is the part of the body above the tentacles. It is conical in shape, with the mouth at its summit.

2. The tentacles are hollow processes of the body-wall, usually six to eight in number, and arranged in a single whorl round the base of the hypostome, which is the widest part of the body. Their cavities open into the general digestive cavity of the animal.

The tentacles, which have a warty appearance, are extremely contractile. When fully extended they are two or three times the length of the body: when fully contracted they are scarcely visible. 
They are capable of free and active morement, and are used for capturing and paralysing the prey, which they then, by their contraction, transfer to the mouth.

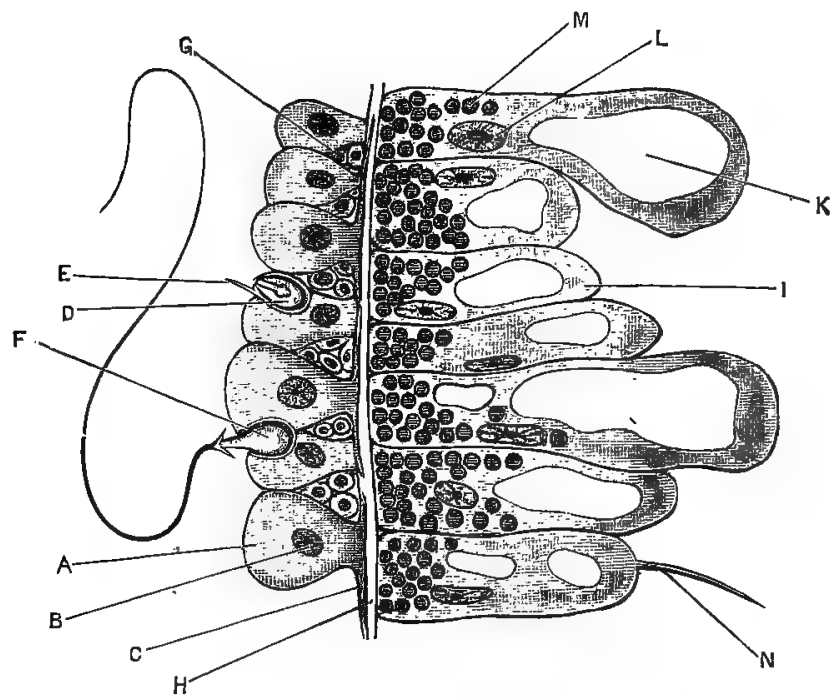

FIG. 12.-Hydra viridis. A small portion of a longitudinal section through the body-wall; $\times 800$. (A. M. M.)

$\mathbf{A}$, one of the large ectoderm cells. $\mathbf{B}$, nucleus of a large ectoderm cell. C, muscular tail of a large ectoderm cell. D, an undischarged nematocyst lying within its parent cell, or cnidoblast. $\mathbf{F}$, cnidocil. $\mathbf{F}$, a nematocyst with discharged thread. G, interstitial cell. H, supporting lamella. I, endoderm cell. $\mathbf{K}$, vacuole of endoderm cell. $\mathbf{L}$, nucleus of endoderm cell. $\mathbf{M}$, chlorophyll corpuscle. N, flagellum of endoderm cell.

\section{B. Structure.}

\section{Structure of the body-wall.}

Examine the body-wall more closely with the low power, and note its division into layers.

a. The ectoderm is the outermost layer of the body. It is colourless, and forms about a third of the entire thickness of the wall. 
b. The endoderm is the inner layer, lining the digestive cavity, and forming about two-thirds of the thickness of the body-wall. It is coloured green or brown according to the species of Hydra examined.

c. The supporting lamella, or mesogloea, is a very thin gelatinous layer between the ectoderm and the endoderm, and hardly visible with the low power.

\section{Structure of the tentacles.}

Examine one of the tentacles with a high power, and note its division into layers corresponding to those of the body-wall. Examine (1) the middle of the width of the tentacle, altering the focus so as to bring the several layers into view in turn; and (2) the edge of the tentacle, where by proper adjustment of the focus a profile view of the cells can be obtained.

a. The ectoderm is a cellular layer made up as follows.

i. The large ectoderm cells form a single layer covering the whole surface of both the tentacles and the body. They are somewhat conical in shape. Their outer ends are closely fitted together, forming a mosaic pattern very clearly visible in surface views of the tentacle. Their inner ends, which rest directly on the supporting lamella, are narrower, and separated from one another by intervals of varying extent.

The shape of these large ectoderm cells varies very greatly with elongation and contraction of the tentacle. When contracted they are widened transversely, and their outlines are very distinct; when expanded their length considerably exceeds their width.

ii. The interstitial cells are small rounded cells placed in little clusters between the inner or basal ends of the large ectoderm cells. 
iii. The nematocysts or 'thread cells' are sharply defined oval capsules, filled with fluid and enclosing a long hollow spirally-wound filament, formed by doubling in of the wall of the capsule at one pole. They develop within certain of the interstitial cells called cnidoblasts, and lie when mature in and between the large ectoderm cells, causing marked projections on the surface.

The cnidoblast, or cell within which the nematocyst is developed, persists as a capsule surrounding it, and is produced at its outer or free surface into a small process, the cnidocil.

These nematocysts can be discharged or exploded, the contained hollow filament being shot out with great force and velocity. In this process of discharge, which is probably brought about by contraction of the cell in which the nematocyst lies, the basal part of the thread is discharged first, and, being provided with barbs, of which there are three principal and several smaller ones, fixes itself at once in the body against which the thread is shot, and into which the remainder of the filament penetrates with great rapidity.

The discharge of these filaments appears to be under the control of the Hydra: they exert a very marked numbing or paralysing action on animals into which they are shot, and are the means by which the Hydra captures its prey. A nematocyst once discharged cannot be used again, and is cast off.

Besides these large nematocysts, much smaller ones, with short thick threads devoid of barbs, occur in large numbers in the tentacles. Their function is unknown.

Nematocysts of both the larger and the 
smaller kinds occur in the body-wall, though less abundantly than in the tentacles. iv. The nerve-cells. Certain small stellate cells found in the ectoderm are described as nervecells. A connection between them and the cells enclosing the nematocysts has been traced, and it is possible that they are concerned in the discharge of the latter.

b. The endoderm consists of a single layer of large granular cells lining the cavity of the tentacle. Several of these bear flagella, by which currents are caused along the tentacle.

Focus the middle of the thickness of the tentacle: note the movements of the flagella of the endoderm cells, and the stream of nutrient particles up and down the cavity of the tentacle.

c. The supporting lamella is the thin gelatinous layer between the ectoderm and the endoderm.

Focus the side of the tentacle at the middle of its thickness, and the supporting lamella will be seen as a thin transparent layer between the ectoderm and the endoderm.

Sharply tap the microscope so as to cause the Hydra to retract its tentacles.

In the contracted condition of the tentacle the ectoderm and endoderm are both strongly wrinkled transversely, but the supporting lamella is not. This shows that the latter is the contractile layer; or at any rate that it contracts to a greater extent than either ectoderm or endoderm.

\section{d. Discharge of the nematocysts.}

Focus one of the tentacles with the high power: then place $a$ drop of acetic acid on the slide and allow it to run under the cover-glass.

As the reagent reaches the tentacles these will be retracted and their nematocysts discharged 
in consequence of the irritation. Some of the nematocysts will remain in the ectoderm with their threads projecting freely; others will separate completely.

Examine, and draw the nematocysts, showing their shape, the threads with their enlarged basal portions, and the barbs. Search for specimens with partially discharged threads; and note that the threads are turned inside out in the process of discharge.

C. Reproduction.

Hydra reproduces asexually and sexually.

1. Asexual reproduction.

a. Gemmation or budding consists in the formation of a hollow outgrowth from the side of the body, which acquires a mouth and tentacles at its distal end, and ultimately constricts at its base, separates from the parent, and becomes an independent animal.

The rate of budding depends largely on the supply of food and on temperature. If a rapidly budding Hydra be transferred to water in which there is little or no food to be obtained the formation of the buds will be stopped, and buds already formed may even be absorbed.

A single Hydra may give rise to more than one bud at once, and these may develop secondary buds before separating from the parent animal. In this way temporary colonies may be formed, which, however, sooner or later break up into their component units.

b. Fission. A Hydra may be cut in two, and each half will live and become a perfect animal. The process of fission, however, is not known to occur naturally.

2. Sexual reproduction.

Hydra is hermaphrodite, the same animal having 
both testes and ovaries. Contrary to the rule among hermaphrodite animals, the reproductive organs are extremely simple in structure, and the animals are capable of fertilising their own ova.

a. The testes are conical or spherical swellings of the body-wall, varying in number from one or two up to twenty. They are usually situated near the oral or distal end of the body, not far below the tentacles; but when numerous they may extend along the greater part of the length of the body. Each testis is formed by local proliferation of the interstitial cells of the ectoderm, giving rise to small conical heaps, which project externally and are covered on their outer surfaces by capsules formed from the large ectoderm cells.

The interstitial cells become directly converted into spermatozoa by modification of their nuclei to form the heads, and elongation of their protoplasm to form the long swimming tails. When fully formed they are discharged by rupture of the capsule at its most prominent point.

Select a Hydra in which the testes are of large size; put it in a drop of water on a slide; cover it; and, by gentle pressure on the cover-glass, rupture one of the testes, and so discharge the spermatozoa. Examine with a high power.

The spermatozoa are filiform bodies about $\frac{1}{500}$ inch in length. Each consists of a small oval highly refractive head, containing the nucleus, and a long actively moving thread-like tail.

b. The ovaries usually develop a little later than the testes. In Hydra viridis there is seldom more than a single ovary present at a time, but in the brown species there may be as many as eight. They form, when ripe, spherical projections of the body-wall considerably larger than the testes, and situated as a rule nearer the basal end of the animal. 
In each ovary only a single ovum is produced. The ovary is formed like the testis by multiplication of the interstitial cells to form a small swelling: one of these cells, near the centre of the heap, soon becomes larger than the rest and becomes the ovum. This grows rapidly at the expense of the other cells of the ovary, which form a capsule around it and supply it with nutriment. The ovum is at first amœboid so as to come into. contact with as many of the nutrient cells as possible. When it has reached its full size it becomes spherical or ovoid; the capsule then thins aways and ruptures at its summit, and, shrinking back, leaves the outer half of the ovum exposed to the water, and ready to receive the spermatozoa.

After fertilisation the ovum segments, forms a hard capsule around itself, becomes detached from the parent, and, falling to the bottom of the tank or pond in which the animal is living, develops into a young Hydra.

\section{EXAMINATION OF PREPARED SPECIMENS.}

\section{A. Teased Specimens.}

Tease thoroughly in a mixture of equal parts of glycerine and water a small portion of the body-wall of a Hydra that has been treated for a day either with Müller's fluid or with a mixture of osmic and acetic acids. Cover, and examine with a high power.

1. The ectoderm cells.

a. The large ectoderm cells will be found isolated in various parts of the preparation. They are somewhat conical in shape, their outer ends being broad and containing the large nuclei. Their inner ends are much narrower, and are produced into slender muscular processes or tails. These 
tails lie on the outer surface of the supporting lamella, and partially imbedded in its substance. Their direction is mainly longitudinal, i.e. parallel to the long axis of the animal, and it is probably on them that the contractility of the supporting lamella (p. 19) depends.

b. The interstitial cells are much smaller, and usually remain in small clusters. Within some of them the early stages of development of the nematocysts may be seen.

c. The nematocysts. In specimens killed with Müller's fluid, nematocysts are frequently met with in which the thread is only partially protruded, and in which the relations of the capsule to the thread can be readily determined.

2. The supporting lamella may be seen in teased preparations in the form of transparent shreds or sheets of greater or less extent, on the outer surface of which a distinct longitudinal striation is present, caused by the muscular tails of the large ectoderm cells.

On the inner surface of the supporting lamella transverse fibres occur which are described by some writers as connected with the endoderm cells, and are regarded as muscular in character.

3. The endoderm cells are very variable in shape. Each commonly contains one or more vacuoles which may be so large as to reduce the protoplasm of the cell to a very thin peripheral lamella. The outer end of the cell, next the supporting lamella, contains in Hydra viridis a number of small spherules coated with chlorophyll, to which the green colour of the animal is due. In the brown species of Hydra similar bodies are present, but devoid of chlorophyll.

The endoderm cells may also contain, in addition to particles of food, little clusters of brown or black granules, probably formed by breaking down of the chlorophyll grains. The flagella are seldom preserved in teased specimens. 


\section{B. Transverse Sections.}

Examine a series of transverse sections through the body of Hydra. To prepare these kill the animal in the expanded condition by suddenly irrigating it with osmic acid; wash it well in distilled water; transfer to alcohol; stain with picrocarmine, and then imbed in paraffin and cut with a microtome.

1. The ectoderm.

a. The large ectoderm cells are well seen in such sections; their muscular tails, which are mainly longitudinal in direction, are cut transversely, and appear as a row of highly refractive dots on the outer surface of the supporting lamella.

At the basal end or foot of the Hydra the ectoderm cells are more columnar in shape, contain longitudinal rows of granules, and are probably glandular.

On the outer surface of the ectoderm cells there is a thin cuticular layer, more obvious in the brown than in the green Hydra.

b. The interstitial cells are well, seen in sections through the body or tentacles, but are absent in the foot.

c. The nematocysts are very abundant in the tentacles, less numerous in the body, and absent in the foot. In specimens killed with osmic acid, few, if any of them will be discharged.

2. The supporting lamella is seen in transverse sections as a very thin transparent layer, between the ectoderm and endoderm.

3. The endoderm cells vary greatly in shape in different parts of the body and at different times. During life they are amceboid, and may extend so far inwards as to almost obliterate the cavity of the animal. They contain vacuoles, which are often so large that the cell-substance is reduced to a thin peripheral 
lamella. The nucleus is usually nearer the outer or basal end, and is flattened in shape. The flagella can very seldom be seen in sections.

In the foot the endoderm cells are much smaller than in the body, and form a row of short columnar or almost cubical cells containing granular concretions. 


\section{Chapter III.}

\section{THE LIVER-FLUKE OF THE SHEEP.}

Fasciola (Distomum) hepatica.

THE adult liver-fluke is a flat unsegmented worm, about an inch and a half long, living in the bile-ducts of certain domestic animals, notably in those of the sheep, in which it gives rise to the destructive disease known as liver-rot. It may occur in man.

The animal is hermaphrodite, and its eggs, which have thick chitinous shells, are deposited in enormous numbers in the bile-ducts of the sheep or other host, from which they pass into the alimentary canal, ultimately escaping with the fæces. From these eggs, if deposited in damp places or in water, embryos are produced which lead a free existence for a short time, but very soon become parasitic within water-snails. Two or more asexually produced generations now succeed; and the last of these, leaving the water, encyst on grass. These encysted forms are swallowed with the grass by sheep, and passing into their bile-ducts become the adult sexually mature flukes.

This alternation of sexual and asexual generations, living parasitically within different hosts, is a very characteristic feature in the life history of the typical parasitic worms.

\section{THE MATURE LIVER-FLUKE.}

Slit open the bile-ducts in the liver of an infected sheep, and transfer the living flukes to a dish of salt-solution ( $\cdot 75$ per cent.) to clean them.

$\S 1$. Inject the excretory system of one fluke, and the 
alimentary canal of another, with a thin injection such as finely-powdered carmine, or freshly-precipitated Prussian blue, suspended in water. To do this make a very small incision with a sharp-pointed knife near the hinder end of the animal - in the middle line for the excretory system, and about $1 \mathrm{~mm}$. from it for the alimentary canal. Inject the coloured fluid into the opening by means of a very fine glass cannula with an indiambber cap. Place the animals, still alive, between glass slips, and tie the slips together with cotton, so as to slightly flatten the animals. Put the slips with the animals between them into alcohol (90 per cent.) for twelve hours or more, when the animals may be dehydrated, cleared, and mounted in balsam.

\$ 2. Squeeze a third specimen somewhat more tightly between two slips, and leave it in alcohol (90 per cent.) for twenty-four hours. Stain it faintly with weak boraxcarmine, and mount it in the ordinary way.

\section{A. External Characters.}

1. In form the animal is flat and oval, with a blunt triangular projection from its broad anterior end. Its length is about an inch and a half, its breadth about half an inch.

2. The mouth is an oval aperture at the anterior end, in the middle of the cup-shaped anterior sucker.

3. The ventral sucker is a muscular cup in the mid-ventral line, near the junction of the triangular anterior portion with the broader part of the body.

4. The genital aperture, through which the penis may be protruded, is on the ventral surface between the two suckers, and slightly nearer the posterior one.

5. The cuticle is a thin layer covering the whole animal. Its surface is covered with minute backwardly-directed spines, which are best seen in a specimen kept in spirit, and dried at the moment of examination.

\section{B. The Alimentary Canal.}

1. The pharynx is an oval muscular mass immediately belind the mouth. 


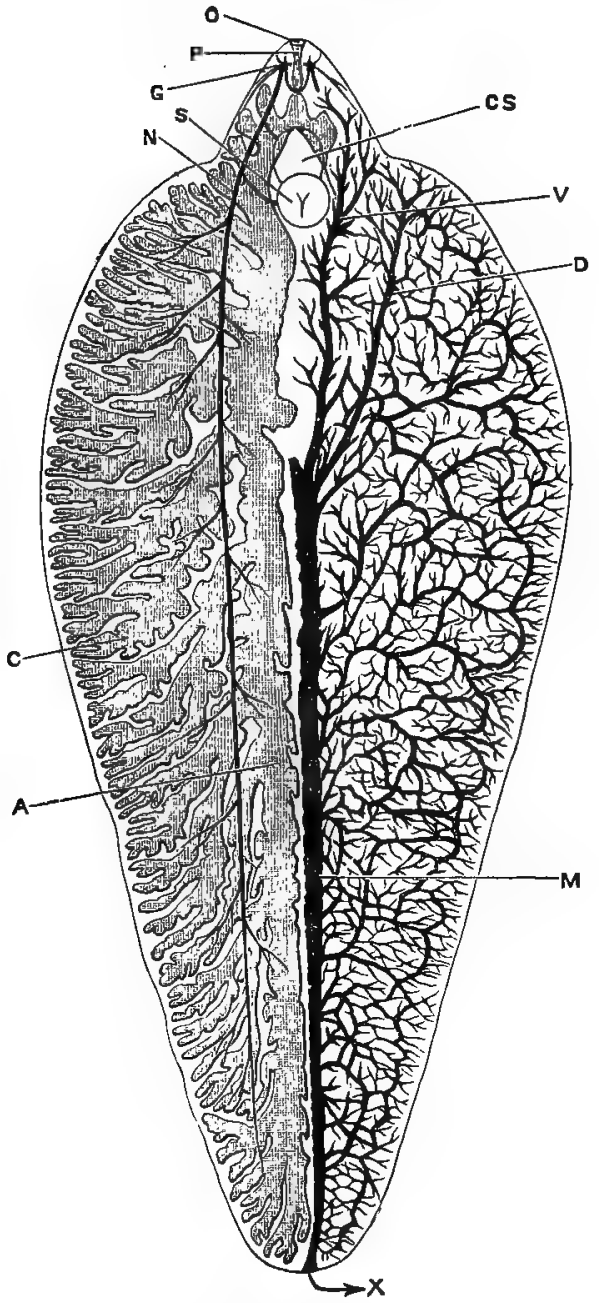

FIg. 13.-Fasciola hepatica from the ventral surface; $\times 4$. (After Sommer.)

The alimentary and nervous systems only are shown on the left side of the figure; the excretory system alone on the right side of the figure.

A, 'right main division of the alimentary canal. C, one of its diverticula. CS, cirrus-sac. D, left anterior dorsal trunk of excretory system. G, lateral ganglion. $\mathbf{M}$, main trunk of excretory system. $\mathbf{N}$, lateral nerve. $\mathbf{O}$, mouth. $\mathbf{P}$, pharynx. $\mathbf{S}$, ventral sucker. $\mathbf{V}$, left anterior ventral trunk of excretory system. $\mathbf{X}$, excretory pore. 
2. The cesophagus is a very short straight thin-walled tube behind the pharynx.

3. The intestine, into which the esophagus opens just in front of the genital aperture, divides immediately into a right and a left limb, each of which runs to the hinder end of the body, giving off numerous small cæca on its inner side, and many large branched ones on its outer side; the whole intestine forming a series of cæcal tubes ramifying all over the body without anastomoses.

\section{The Excretory System.}

This is almost if not quite invisible except in injected specimens. It consists of a network of small much-branched ducts, which commence with ciliated funnel-like mouths opening into the lacunar spaces representing the body-cavity. These small ducts freely anastomose with one another, and open into larger transverse ducts, which in turn open into the main duct.

The main duct is a median tube of considerable size, formed by the union of four anterior ducts, a dorsal and a ventral on each side, at about a quarter of the animal's length from the anterior end : from this point the main duct runs directly backwards to open to the exterior by a terminal median pore.

\section{The Reproductive System.}

This is complicated, as in most hermaphrodite animals.

1. The male organs.

a. The testes are much branched, and lie in the middle part of the body, extending over about half its length and half its width. The two testes are about equal in extent, one lying behind the other.

b. The vasa deferentia are two in number, one arising near the middle of each testis. They run forwards as far as the ventral sucker, where they open into the vesicula seminalis. 
c. The vesicula seminalis is an elongated sac Iying above, and somewhat in front of, the ventral sucker.

d. The ductus ejaculatorius is a fine tube running

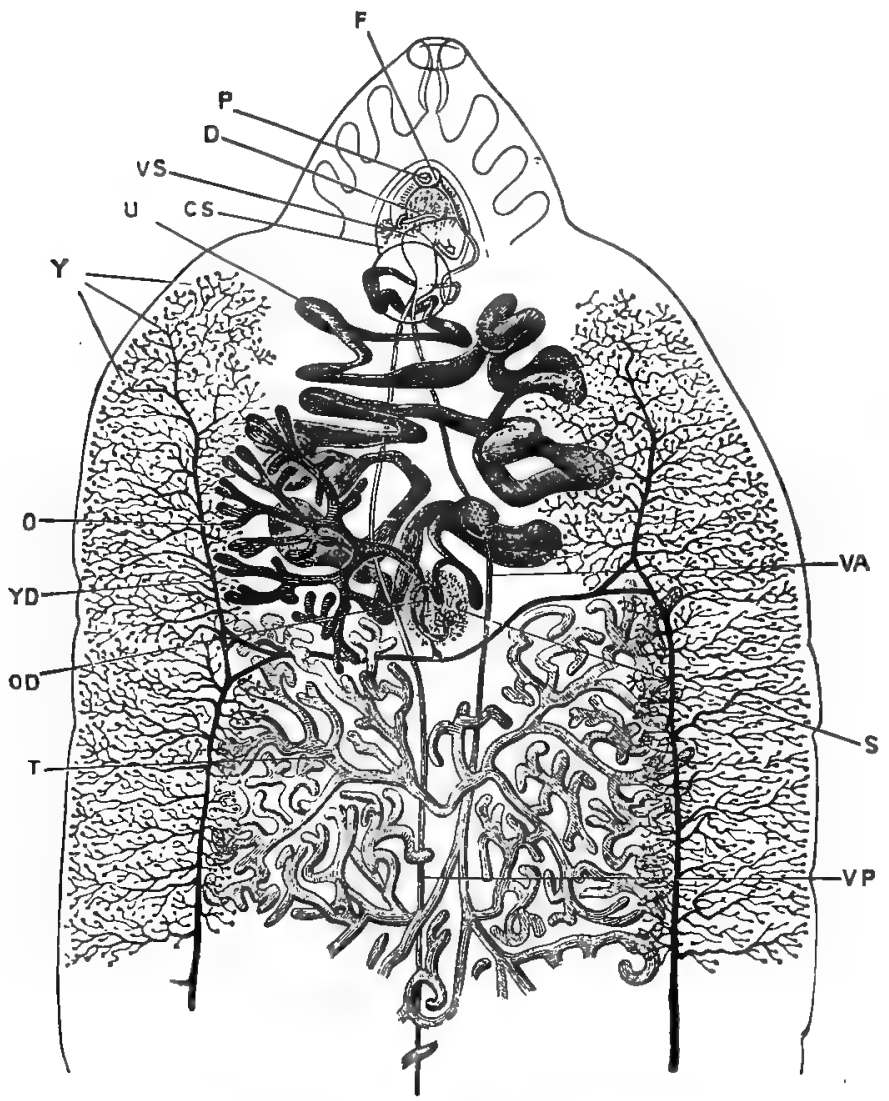

FIG. 14,-Fasciola hepatica. Reproductive organs, from the ventral surface ; $\times 6$. (After Sommer.)

C8, cirrus-sac. D, ductus ejeculatorius. FI, female serual aperture. 0 , ovary. OD, ovarian duct. $\mathbf{P}$, penis. $\mathbf{8}$, shell-gland. $T$, anterior testis. U, uterus. VA, vas defereng from anterior testis. VP, vas deferens from posterior testis. V8, vesicula seminalis. $\mathbf{Y}$, yolk-gland. $\mathbf{Y D}$, duct of yolk-gland. 
from the vesicula seminalis to the end of the penis. When the penis is withdrawn this duct is thrown into convolutions.

e. The penis is a large muscular organ which lies, when withdrawn, within the cirrus-sac, a space in front of the ventral sucker. It can be protruded by evagination, and the ejaculatory duct then lies within it.

f. The cirrus-sac is situated between the ventral sucker and the genital aperture. The penis lies in it when withdrawn, as do also the vesicula seminalis and a small accessory gland which surrounds the ejaculatory duct.

\section{The female organs.}

a. The ovary is branched and tubular : it lies on the right side in front of the anterior testis, and its branches unite to form the narrow ovarian duct.

b. The yolk-glands are very numerous small rounded masses, scattered along two areas extending along the sides of the body from end to end, and each nearly one quarter of the width of the whole worm.

c. The vitellarian ducts, or ducts of the yolk-glands, unite to form an anterior and a posterior duct on each side. These unite about the junction of the anterior and middle thirds of the animal, forming a transverse duct, which runs inwards to open into the median yolk-reservoir: from this a single median vitellarian duct runs a short distance forwards and unites with the ovarian duct to form the oviduct.

Close to the junction is the opening of a short duct, the 'vagina' or ' canal of Laurer,' which is said to open to the exterior on the dorsal surface.

d. The shell-gland is a median glandular mass surrounding the junction of the ovarian and median vitellarian ducts. 
e. The oviduct, or 'uterus,' formed by the union of the ovarian and vitellarian ducts in the midst of the shell-gland, is a wide much-convoluted tube lying between the shell-gland and the genital aperture. Its convolutions extend half-way across the animal, and numerous eggs can be seen within it.

f. The aperture of the oviduct is at the base of the penis, and on its left side. When the penis is fully withdrawn a slight cavity, the 'genital sinus,' is formed, into the left side of which the oviduct then opens.

\section{E. The Nervous System.}

This cannot be made out with certainty in specimens prepared as above. It can be seen in a specimen rendered transparent by caustic potash, though such preparations are very perishable. It may also be made out in sections.

It consists of a nerve-collar around the pharynx, with two lateral ganglia and a median ventral one. From these arise nerves, of which two large lateral ones are the chief. These two run backwards, one on each side of the body (fig. 13.)

\section{LIFE-HISTORY OF THE LIVER-FLUKE.}

The development of the liver-fluke can only be studied under special conditions.

By removing eggs from the bile-ducts of an infected sheep and hatching them in an aquarium, the free-swimming embryos can be readily obtained. Sporocysts and rediæ may be obtained from specimens of Limnaa truncatula, kept in the same aquarium.

The main features in the life-history are as follows.

A. The First Generation is produced sexually but is itself asexual.

1. The eggs, laid in large numbers in the bile-ducts of the sheep or other infected mammal, pass with the bile into the intestine, and so escape from the body. 
They are ovoid bodies, $\cdot 13 \mathrm{~mm}$. long and $08 \mathrm{~mm}$. broad, enclosed in smooth brownish chitinous shells. Within each shell are a single germ-cell and a large number of yolk-cells. The germ-cell segments before the egg is laid, but no further development occurs till after its escape from the body of the host. Afterwards an embryo is formed, and a circular operculum at one end of the shell opens to allow it to escape.

2. The free embryo is conical, with a short papilla at its broad anterior end; the whole surface is covered with long cilia, enabling the embryo to swim rapidly. Two eye-spots are present, and two ciliated funnels, probably excretory. The ectoderm is a single layer of flattened cells, usually arranged in five transverse bands, within which is a mass of granular cells. When this embryo meets with Limncea truncatula, a small freshwater snail, the head-papilla becomes elongated and by means of it the embryo bores its way into the snail. The free life of the embryo is usually of about eight hours' duration.

3. Development of the sporocyst. Within the snail, usually in its pulmonary chamber, the ectoderm cells of the embryo swell and lose their cilia; the embryo grows rapidly, and in two or three weeks becomes an elongated sac, $6 \mathrm{~mm}$. long. This sac, the sporocyst, has an outer structureless cuticle, a thin muscular layer, and an epithelial layer lining the cavity.

The eye-spots, though losing their form, persist; and ciliated excretory funnels are present. Such sporocysts sometimes, though rarely, multiply by transverse fission in the early stages of their development.

B. The Second Generation consists of rediæ, which are produced asexually within the sporocyst, and are themselves asexual.

1. From the epithelium of the sporocyst, cells are budded off, which segment to form solid masses or morulæ lying 

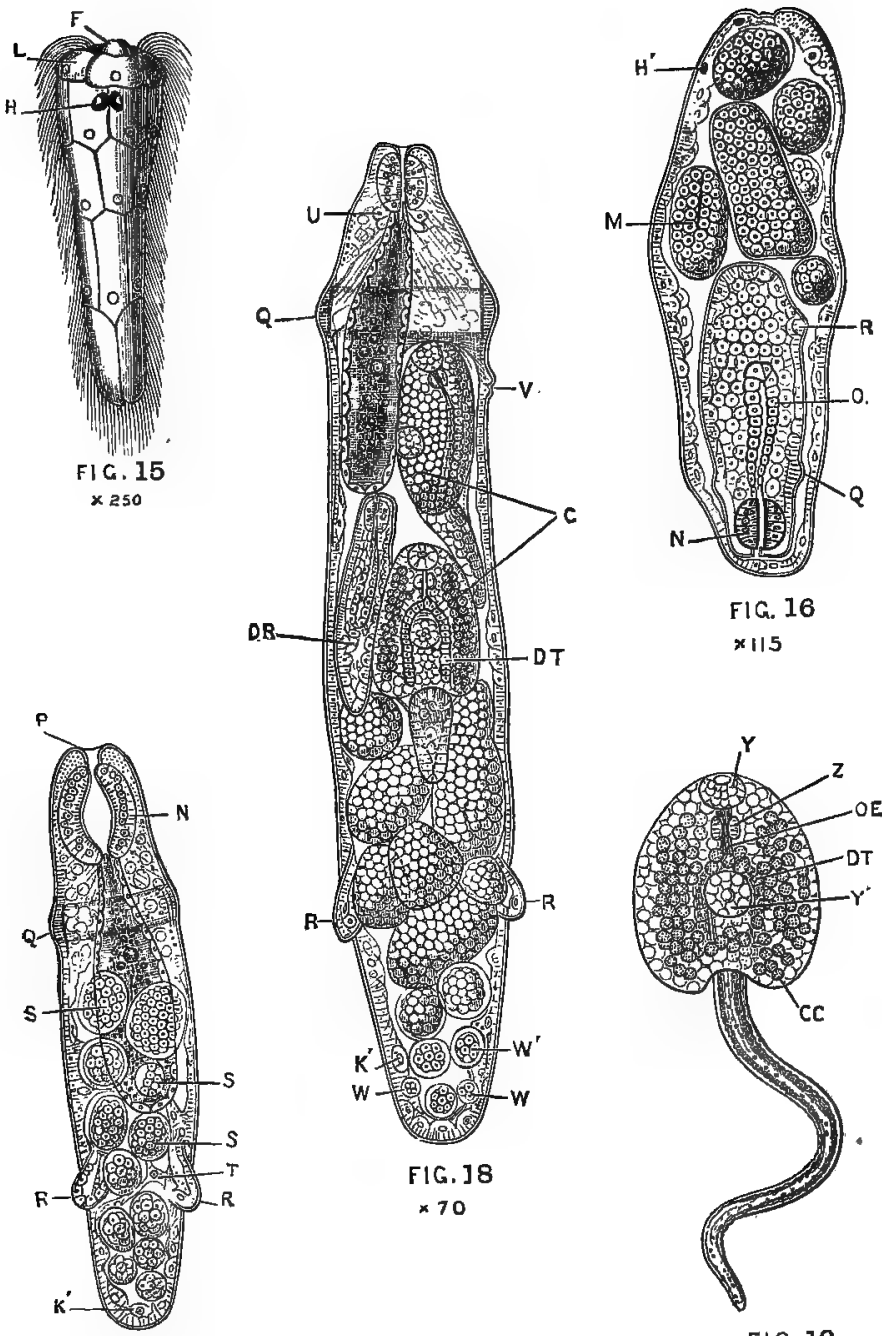

$F[G, 18$

$\times 70$

FIG. 17

$\times 115$

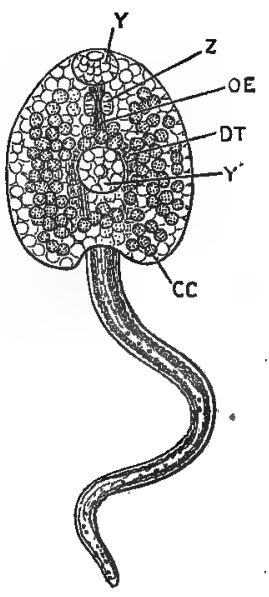

FJ G. 19

$\times 80$ 
in the cavity of the sporocyst. Each of these becomes flattened on one side, and then invaginated to form a gastrula : this increases in size, elongates, and develops into a redia. The rediæ so formed force their way out of the sporocyst and become free: the wound in the sporocyst heals, and other rediæ are formed in the same way within it.

2. The free rediæ wander about in the snail, increasing in size and being especially abundant in the liver. The adult redia (fig. 18) is a cylindrical body about 1.5 $\mathrm{mm}$. long, with a collar-like ridge running round it near the anterior end, and with a pair of blunt processes projecting from the hinder part of the ventral surface, which aid in locomotion. The body-wall resembles that of the sporocyst in structure, but is more muscular, and has definite excretory canals which commence as funnels with ' flame-shaped ' cilia.

The alimentary tract is a comparatively short blind sac, with walls one cell thick: the mouth is at the anterior end, and behind it the wall of the sac is thickened to form a strong muscular pharynx.

From the inner surface of the body-wall of the redia, cells are budded off which develop into gastrulæ

FIGs. 15 to 19.-Fasciola hepatica. Five stages in the life-history. (After Thomas.)

FIG. 15.-The free-swimming embryo.

FIG. 16.-A sporocyst, containing developing rediæ.

Fia. 17.-A young redia. The shaded area represents the digestive sac. Fig. 18.-An adult redia, containing one daughter-redia, two cercarim approaching maturity, and germs in various stages. The shaded area represents the digestive sac.

Fra. 19.-A free cercaria.

The following letters have the same signification in the five flpures : $\mathbf{C}$, nearly ripe cercarize. CC, cystogenous cells of cercaria. DR, daughter-redia. $\mathbf{D T}$, limbs of the digestive tract. $\mathbf{F}$, head-papilla. $\mathbf{H}$, eye-spots. $\mathbf{H}^{\prime}$, the same degenerating. $\mathbf{K}^{\prime}$, germinal cell. $\mathbf{L}$, epaulet-like cells of first row of ectoderm cells. $\mathbf{M}$, ombryo in optical section; gastrula stage. $\mathbf{N}$, pharynx of redia. $O$, digestive sac. $O E$, œsophagus of cercaria. $P$, lips of redia, $\mathbf{Q}$, collar of redia. $\mathbf{R}$, processes of redia serving as rudimentary feet. $\mathbf{S}$, embryos between the morula and gastrula stages. $T$, trabecula crossing body. cavity of redia. $\mathbf{U}$, cells in redia supposed to be glandular. $\boldsymbol{\nabla}$, birth-opening, by which cercarix and daughter-redize escape from the redia. $\mathbf{W}$, morula, still retained in the body-wall. $\mathbf{W}^{\prime}$, larger morula. $\mathbf{Y}$, oral sucker of corcaria. $\mathbf{Y}^{\prime}$, ventral sucker. $\mathbf{Z}$, pharynx of cercaria. 
as in the sporocyst : of these some become rediæ like the parent, while others develop into cercariæ.

C. The Third Form is a cercaria (fig. 19). These are not necessarily the third generation, for several generations of redix may intervene between them and the sporocyst.

1. Within the parent-redia the embryo develops a long tail near its hinder end, an anterior sucker round the mouth, and a posterior sucker on the ventral surface. Its alimentary tract, which is at first solid, becomes bifurcated to form the two limbs of the intestine, the portion in front of the bifurcation forming a pharynx and a short œsophagus, A single redia may contain about twenty such cercariæ at one time.

2. The ripe cercariæ, which measure nearly $1 \mathrm{~mm}$. in length, including the tail, escape from the redia by an aperture just behind the collar. At first they are very active, and work their way out of the snail : as this snail, Limnea truncatula, is amphibious, they may be set free either in water or on damp grass. In either case they shortly lose their tails, and encyst on grass or some other plant.

3. In the encysted condition the cercarix are swallowed by sheep with the grass. They then again become active, escaping from their cysts, and working their way along the bile-ducts to the liver, where they grow rapidly, and develop in about six weeks into sexually mature flukes. 


\section{Chapter IV.}

\section{THE LEECH. Hirudo medicinalis.}

The leech is an elongated flattened worm, from three to five inches in length, and provided with a muscular sucker at each end. The body is marked externally by a series of transverse constrictions dividing it into rings or annuli; and is capable of considerable elongation and contraction.

Leeches occur in freshwater pools and marshes in this country, but far more abundantly on the Continent. They either swim freely by vertical undulations of the body, or progress in a looping manner, attaching themselves alternately by the anterior and posterior suckers. They live on the blood of higher animals ; and they lay their eggs in cocoons, which are buried in holes in the banks of the ponds they inhabit.

Leeches are 'segmented animals,' i.e. several of the organs are repeated, usually in pairs, at regular intervals along part or the whole of the length of the body. The segments or somites, as indicated by the internal organs, are much less numerous than the annuli, five of these latter corresponding to each somite, except at the ends of the body.

This segmental arrangement affects markedly the nervous, excretory, and reproductive systems, and to a less degree the circulatory and digestive organs. It appears to result from a definite arrangement of parts which, in the ancestors of leeches, were scattered irregularly through the body much as in Fasciola.

Leeches intended for dissection should be killed with chloroform. 


\section{EXTERNAL CHARACTERS.}

1. The shape varies greatly with the degree of elongation or contraction. The body is widest a little way behind the middle of its length, and is oval in transverse section, the dorsal surface being more convex than the ventral.

2. The annuli, or rings, into which the body is divided by a series of transverse grooves, are about ninety-five in number, and occur throughout the whole length of the body except its extreme ends. They bear small papillæ which are more obvious when the body is in the contracted condition.

8. The colour varies greatly in different individuals. The dorsal surface is darker than the ventral, and is usually marked by three pale longitudinal stripes on each side, the middle and outer of which are interrupted by dark dots. These dots are markedly larger, especially in the middle stripe, at every fifth annulus, the annulus so marked being the hindmost of a somite. The most anterior of the five annuli making up a somite bears a transverse ring of small white dots. Towards the two ends of the body the somites are shorter and contain fewer annuli.

4. The suckers.

a. The anterior sucker is oval, with the longer axis longitudinal. It is placed on the ventral surface of the anterior end, and is cupped in the centre to form the buccal cavity. It may be regarded as formed by the fusion of the prostomium, a part in front of the mouth, with the first two annuli of the first somite.

The prostomial portion is very commonly folded down over the mouth as a kind of lip.

b. The posterior sucker is circular, and larger than the anterior one. It is separated from the body by a slight constriction and is imperforate. 


\section{External openings.}

a. The mouth is a funnel-like depression in the anterior sucker, at the bottom of which are the three jaws.

b. The anus is a very small aperture on the dorsal surface in, or just in front of, the constriction separating the posterior sucker from the body.

c. The genital apertures. The leech is hermaphrodite: the male and female apertures are separate, and are both on the ventral surface in the median line.

i. The male aperture is a small hole with tumid lips at the hinder border of the twenty-fourth annulus, i.e. in the second annulus of the sixth somite. From it the muscular penis is often seen protruding.

ii. The female aperture is less conspicuous than the male, and is placed one somite further back, i.e. at the hinder border of the twentyninth annulus, the second annulus of the seventh somite.

d. The apertures of the nephridia, or excretory organs, are minute paired openings on the ventral surface of the body, in the hindmost annulus of each somite from the second to the eighteenth inclusive.

Hold the leech in your hand with the ventral surface upwards. Dry it with a cloth, and then gently squeeze it: minute drops of fluid will exude from the nephridial apertsures, which are thus rendered clearly visible.

\section{DISSECTION OF THE LEECH.}

Stretch the leech slightly with the fingers and fix it under water with the dorsal surface upwards, putting the pins through the sides of the suckers so as to avoid injury to the nerve-ganglia.

Mate a longitudinal incision through the stoin a little to one side of the middle line, taking care not to damage the 

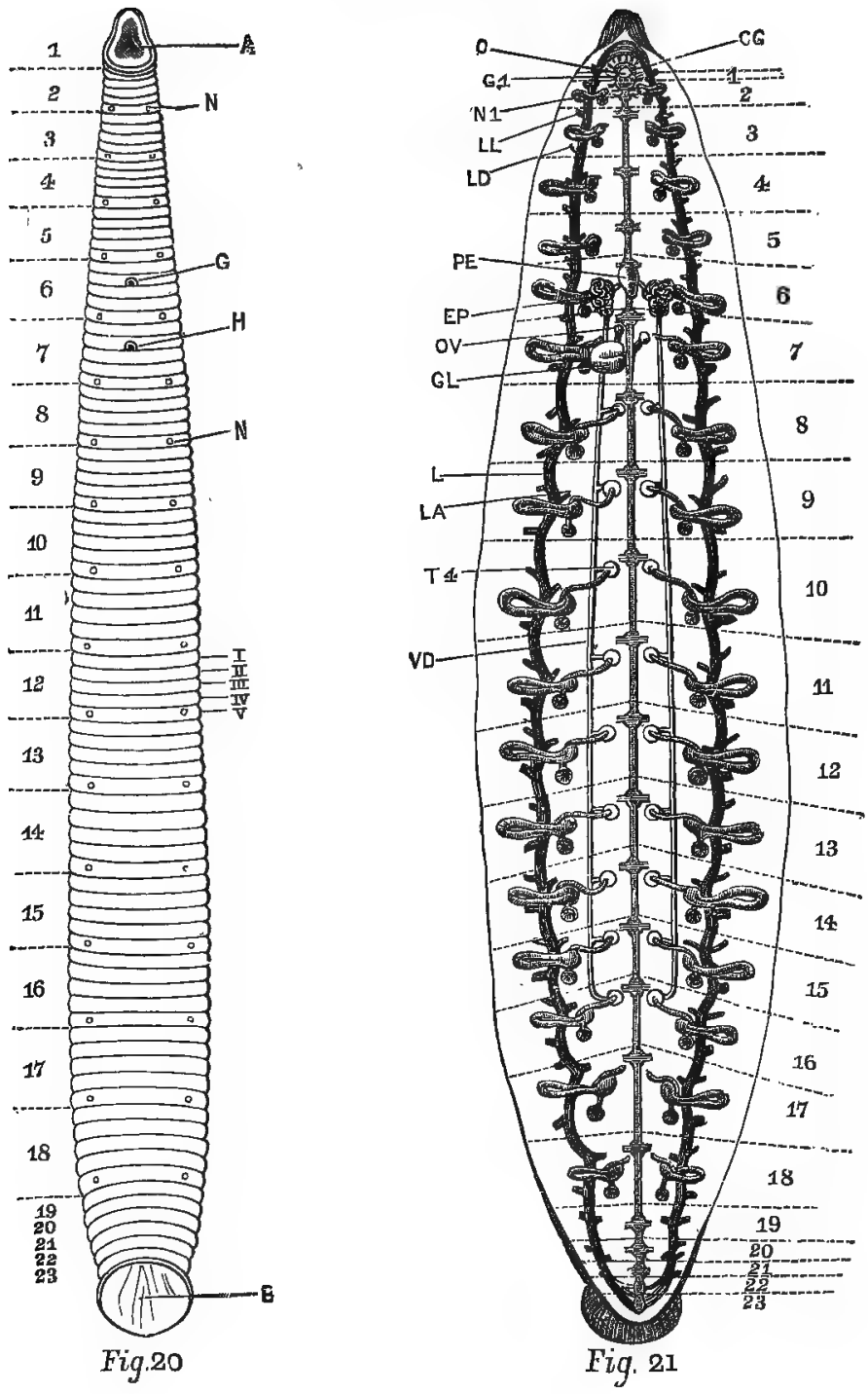
alimentary canal, which lies close beneath the skin. Carefully dissect off the integument from the alimentary canal along the whole length of the animal, and pin out the flaps right and left. Wash thoroughly under the tap. Note at once the dorsal blood-sinus, which runs along the dorsal wall of the alimentary canal.

\section{A. The Digestive System.}

The alimentary canal of the leech runs straight from mouth to anus. Along the greater part of its length it is a wide tube, whose capacity is much increased by paired lateral diverticula, and is further capable of great distension.

A leech can draw as much as three times its own weight of blood, a great part of which is often spontaneously discharged. The digestion of a full meal may take as long as nine months in an adult animal.

Dissect clean the dorsal wall of the alimentary canal along its whole length, taking care not to damage the supraasophageal ganglia. If the canal is empty its walls are white and readily distinguished; if full, it appears red from the contained blood, and is less easy to dissect. Wash frequently under the tap during the dissection.

1. The mouth is a conical depression in the anterior sucker, at the bottom of which are the jaws. These are three laterally compressed muscular cushions arranged in

FIG. 20.-Hirudo medicinalis, seen from the ventral surface. (After A. G. Bourne.)

The numbers 1 to 23 indicate the somites; the numbers I. to V. the five annuli of the twelfth somite.

$\mathbf{A}$, anterior sucker, with the mouth. $\mathbf{B}$, posterior sucker. $\mathbf{G}$, male aperture. $\mathbf{H}$, female aperture. $\mathbf{N}$, nephridial apertures.

FIG. 21.-Hirudo medicinalis. A diagrammatic figure of the renal, nervous, and reproductive systems. The animal has been opened along the mid-dorsal line, pinned out, and the alimentary canal removed. (After A. G. Bourne.)

The somites are numbered 1 to 23 , and their boundaries are indiested by the dotted lines.

CG, supra-csophageal ganglion. EP, left epididymis. G 1, the first of the twenty-three post oral ganglia. GI, glandular enlargement of the oviducts. $\mathbf{L}$, lateral vessel. $\mathbf{L A}$, latero-ventral branch of lateral vessel. ID, latero-dorsal branch. IL, latero-lateral branch. IN 1 , the first of the seventeen nephridia of the left side. $O$, nerve-collar. $\mathbf{O V}$, ovisac containing the left ovary. PE, penis. T 4, the third testis of the left side. VD, vas deferens. 
a triradiate manner, one being dorsal and anterior, and the other two ventro-lateral: each cushion is covered by a thin chitinous cuticle, which is thickened along the free edge of the jaw, and notched into the sharp teeth by which the bite of the leech is produced. The mouth leads, by a very small aperture, into the pharynx.

Snip anvay the margin of the anterior sucker so as to expose the jaws. Examine them from the ventral surface with a pocket lens: remove one of them and examine it with a low power of the microscope to see the teeth.

2. The pharynx is an oval sac with very muscular walls. It is connected with the body-wall by strong radial muscles, which give it a villous appearance, and by their contraction dilate its cavity and produce a sucking action.

3. The salivary glands are very large granular pyriform cells surrounding the pharynx. Each cell is a gland in itself, and is produced into a long stalk or ductule opening on the side of one of the jaws. The secretion has the power of preventing coagulation of the blood, and so facilitates very greatly the act of suction.

Remove part of the wall of the pharynx, and tease it on a slide in salt solution. The large pyriform gland-cells, with their long ductules, will be readily seen with a low power.

4. The oesophagus is a short narrow tube leading from the pharynx to the crop.

5. The crop is by far the largest part of the alimentary tract. It is a straight thin-walled tube lying in the somites from the fourth to the fourteenth, and giving off eleven pairs of lateral diverticula corresponding to these somites.

Of these diverticula the anterior two or three pairs are small ; the remainder increase gradually in size from before backwards; and the hindmost pair 
are much larger than any of the others, extending. backwards through several somites, and lying alongside the intestine.

The crop and its diverticula have their walls thrown into very numerous folds, projecting internally.

Slit up the crop along the mid-dorsal line, wash out its contents thoroughly, and pass a seeker into the several diverticula on one side, slitting them open along their whole length.

6. The stomach is a small spherical slightly bilobed dilatation immediately behind the crop, lying between the basal portions of the backwardly directed last pair of diverticula, and opening behind into the intestine.

It is probable that absorption of the food takes place entirely in the stomach, and its small size will explain the very slow rate at which this absorption is effected.

7. The intestine is a narrow straight tube running from the stomach to the anus. Its inner wall projects as a spiral fold into the cavity.

Slit open the intestine with scissors along the mid-dorsal line; wash it thoroughly, and note the spiral folding of its wall.

\section{B. The Cœlom and the Blood-vessels.}

In the leech there is no obvious body-cavity, the space between the integument and the alimentary canal being filled up by muscle, connective tissue, and other structures.

A complicated system of tubular channels runs through all parts of the body, and is filled with a red fluid, the blood, which contains numerous colourless corpuscles. The principal channels are of two kinds, some having muscular, others non-muscular walls. The former are to be regarded as the true blood-vessels, and the latter, which may be spoken of as sinuses, as parts of the body-cavity or cœlom, which was originally more extensive, but has become reduced to a system of canals which have acquired secondary communications with the blood-vessels. 
The arrangement and relations of these vessels and sinuses are best determined by the microscopical examination of sections, and will be described later on: the broad features, most of which can be made out without difficulty by dissection, are as follows.

1. The Sinuses, or parts of the celom.

a. The dorsal sinus is a longitudinal tube running along the dorsal surface of the whole length of the alimentary canal. It communicates posteriorly with the ventral sinus by fine vessels which pass between the intestine and the terminal diverticula of the crop. It has already been seen.

Dissect away the alimentary tract completely, from the cesophagus backwards, taking great care not to damage the underlying organs. The further dissection is greatly facilitated by putting the leech into strong spirit for ten minutes or so.

b. The ventral sinus is a median tube running along the body beneath the alimentary canal. Within it the ventral nerve-chain lies. It communicates with a series of peri-nephrostomial sinuses, lying on the dorsal surfaces of the testes; and also with the capillary system on the walls of the crop.

\section{The Blood-vessels.}

a. The lateral bload-vessels are a pair of longitudinal vessels running in a sinuous course along the sides of the body. They are connected together by transverse vessels which pass across beneath the ventral sinus. They send branches to the intestine, the nephridia, and the reproductive organs.

b. The cutaneous plexuses are very abundant, and connect the sinuses and blood-vessels in various places, putting them in free communication with one another. Their microscopical characters will be described later on. 


\section{The Excretory System.}

The excretory organs of the leech consist of a series of seventeen pairs of nephridia or segmental organs, lying in the somites from the second to the eighteenth inclusive. They lie at the sides of and below the alimentary canal, and open to the exterior by paired apertures on the ventral surface.

1. Structure of a nephridium (figs. 21 and 22).

Each nephridium is a cellular rod, thicker in the middle than at the ends, and bent on itself so as to form a loop, the two limbs of which are anterior and posterior respectively. The looped portion lies at the side of the alimentary canal, extending up into the dorsal part of the body : the ends lie in the ventral region, that of the anterior limb being in most cases prolonged inwards almost to the median plane, and that of the posterior limb being rolled up on itself. From the anterior limb a duct runs back to a vesicle with muscular walls, which opens to the exterior on the ventral surface.

The cellular rod is traversed by a complicated system of ducts, which perforate the individual cells, and receive minute ductules ramifying in the substance of the cells.

The whole nephridium is invested by a pigmented connective-tissue capsule, and receives a very abundant supply of blood from branches of the lateral vessels, the efferent stream passing inwards through the peri-nephrostomial sinus to the ventral sinus.

Dissect carefully one of the nephridia about the middle of the body, noting its several parts, and their relations to one another and to other organs.

Remove another nephridium from the body, and dissect it on a slide in water: examine it with low and high powers of the microscope.

Snip off with fine scissors the dorsal half of one of the testes, with the testis-lobe of the nephridium: mount it on a 
slide in a drop of water, and examine with low and high powers. parts.

The entire nephridium consists of the following

a. The testis-lobe forms the ventral half of the anterior

limb of the loop. It is a cylindrical rod. the inner end of which lies in the peri-nephrostomial sinus on the dorsal surface of the testis. This end is slightly enlarged, and has a cauliflowerlike appearance, the cells covering it being spongy and ciliated, and often depressed at their ends.

This inner end of the testis-lobe probably corresponds to the funnel-like opening of the nephridium into the body-cavity in the earth. worm.

b. The main lobe is the thickest part of the rod : it is continuous with the outer end of the testis-lobe, and forms the anterior limb, the bend, and half of the posterior limb of the loop.

c. The apical lobe is separated by a slight constriction from the posterior limb of the main lobe. It runs downwards and inwards, diminishing slightly in thickness, and ends in a coiled-up extremity, the apex of which is closely attached to the junction of the testis-lobe and the main lobe.

d. The vesicle-duct is a short narrow tube arising from the anterior limb of the main lobe at its junction with the testis-lobe, and running backwards beneath the apical lobe to the vesicle.

e. The vesicle is a somewhat flattened sac with muscular walls: it lies behind the rest of the nephridium, and opens to the exterior by a short duct, the opening being on the ventral surface of the body and in the hindmost annulus of the somite in which the nephridium lies.

2. Microscopic structure of a nephridium (fig. 22). Remove a nephridium from a freshly-killed specimen; 
place it on a slide in a drop of 0.75 per cent. salt solution, and spread it out with needles so as to show the mutual relations of its several parts.

Remove another nephridium in the same way, and tease it slightly.

Cover the specimens and examine with low and high powers.

a. The testis-lobe consists of a spongy mass of cells, traversed in an irregular manner by ductules.

b. The main lobe consists of large granular cells, traversed in a very complicated manner by fine branching ductules which pass across from cell to cell, and are lined by a cuticular layer.

c. The apical lobe consists of cells traversed by much larger ductules, most of the cells being merely hollow cylinders placed end to end to form tubes.

d. The main duct of the gland is also formed of cylindrical cells placed end to end, the passage being intracellular. It commences at the apex of the apical lobe, passes back along this lobe and along the posterior and anterior limbs of the main lobe, enters the apical lobe again, and goes right round the loop a second time, and then passes as the vesicle-duct to the vesicle.

e. The vesicle, which is extremely dilatable, possesses a muscular coat, and is lined by a ciliated epithelium, as is also the short passage leading from it to the exterior.

f. The blood-vessels form a very fine capillary plexus lying between the individual cells of the nephridium.

\section{Special characters of the nephridia.}

a. The nephridia in the second to the seventh somites, in which there are no testes, have the testis-lobe short or absent, and devoid of the cauliflowerlike end. These anterior nephridia are smaller than the succeeding ones. 
b. From the eighth to the sixteenth somites, i.e. those in which the testes are situated, the nephridia. have the characters detailed above.

c. The two hindmost pairs of nephridia, in the seventeenth and eighteenth somites respectively, have well-developed testis-lobes, with cauliflower heads lying in peri-nephrostomial sinuses.

\section{The Reproductive System.}

Leeches are hermaphrodite, but do not impregnate their own ova.

1. The male organs.

a. The testes are nine pairs of spherical sacs lying in the somites from the eighth to the sixteenth inclusive. They are situated in the ventral region of the body at the sides of the ventral sinus. Each pair lies mainly in the second, but partly in the first annulus of the somite in which it occurs.

The segmental repetition of the testes is noteworthy. Sometimes a tenth and even an eleventh pair of testes may be present.

Remove one of the testes, and tease it on a slide in salt solution. Cover, and examine with low and high powers.

Spermatozoa in various stages of development will be seen.

b. The vasa efferentia are a series of short sinuous ducts which run outwards from the testes, and open into the vasa deferentia.

c. The vasa deferentia are a pair of longitudinal tubes which run forwards in a sinuous course along the ventral body-wall, about midway between the testes and the vesicles of the nephridia.

In front of the first pair of testes they continue their course through the seventh somite, and in the sixth enter the vesiculæ seminales. 
d. The epididymes are a pair of convoluted tubular bodies with thick white walls, lying in the sixth somite. They secrete a substance by which the spermatozoa are aggregated into spermatophores. From the inner side of each a short duct leads to the base of the penis, the duct of the right side passing under the nerve-chain.

e. The penis is a pyriform body lying in the sixth somite. It is wide at its base, narrow and tubular in its distal part, and can be protruded some distance through the male genital aperture in the second annulus of this somite.

2. The female organs lie entirely in the seventh somite.

a. The ovaries are a pair of minute filamentous bodies enclosed in small spherical ovisacs, and lying close to the mid-ventral Jine.

b. The oviducts are a pair of slender tubes leading from the sacs of the ovaries, which unite to form a much convoluted duct surrounded by unicellular gland cells, and opening into the vagina. The right oviduct passes under the nerve-chain.

c. The vagina is a thick-walled muscular tube, opening to the exterior at the female genital pore in the second annulus of the seventh somite.

\section{E. The Nervous System.}

The nervous system consists of a pair of supra-œsophageal ganglia, a nerve collar, and a double ganglionated cord extending the whole length of the ventral surface. From the ganglia nerves run outwards to the various parts of the body.

1. The supra-cesophageal ganglia are a pair of closely apposed bodies in the dorsal wall of the anterior end of the pharynx, immediately behind the jaws. They supply the jaws, the eyes, and other parts of the anterior end of the body.

2. The nerve collar is very small, and closely surrounds the anterior end of the pharynx. 
3. The ventral nerve-chain lies in the ventral sinus, which must be slit open to expose it. It consists of two halves very closely apposed in the median plane. There are twenty-three pairs of ganglia, lying in the first annuli of their respective somites. The successive pairs of ganglia are much closer together at the two ends of the body than they are along the middle portion of its length.

The first pair, or sub-œsophageal ganglia, are much the largest, and give off five pairs of nerves.

The last, or twenty-third pair, are also large, and give several branches to the posterior sucker.

From each of the other pairs of ganglia two pairs of nerves are given off, supplying the corresponding somite.

\section{F. Sense Organs.}

1. Eyes. The leech has ten eyes, which are visible to the nalked eye as minute black dots, arranged round the edge of the anterior sucker. Each consists of a pigmented cup, filled with rod-like bodies, and receiving a nerve at its base. The eyes differ very little from smaller cup-like tactile bodies found distributed over the anterior part of the body.

Their structure can only be made out by examination of microscopical sections.

\section{EXAMINATION OF TRANSVERSE SECTIONS.}

Many points in the anatomy of the leech can only. be determined by examination of microscopical preparations; and for this purpose transverse sections of the body are the most instructive. Of these half a dozen or more should be examined, as the appearances will necessarily differ according to the particular organs and parts through which the section happens to pass.

The following description is a general one, and will apply to any section through the middle half of the body. 


\section{A. Shape.}

A transverse section of the body of a leech is oval in outline, the transverse diameter being the greater, and the ventral surface being flatter than the dorsal.

\section{B. The Integument.}

1. The cuticle. The most superficial layer is a thin structureless elastic cuticle, which is secreted by the underlying epidermis, and is shed and renewed at frequent intervals during life.

2. The epidermis consists of a single layer of columnar nucleated cells, wider at their outer than at their inner ends.

a. The epidermal glands. Certain of the epidermal cells are modified to form unicellular glands. These may remain in the epidermal layer as mucous glands; or they may sink down into the muscular layers, as in the case of the salivary glands, and of the clitellar glands of the fifth to the seventh somites, which latter secrete the cocoon in which the eggs are laid.

In all cases each cell is a complete gland in itself, and preserves its communication with the surface by means of a long tubular stalk.

3. The dermis is a layer of some thickness lying between the epidermis and the muscular coats. It consists of a jelly-like matrix containing numerous branched corpuscles. It is traversed by pigmented fibres, which branch and anastomose very freely, and penetrate between the epidermal cells : to these the colour of the skin is due. There are also in the dermis irregularly arranged muscle-fibres, chiefly transverse in direction, and a very abundant capillary plexus, the branches of which penetrate between the inner ends of the epidermal cells. It is by means of this cutaneous capillary system that respiration is effected. 


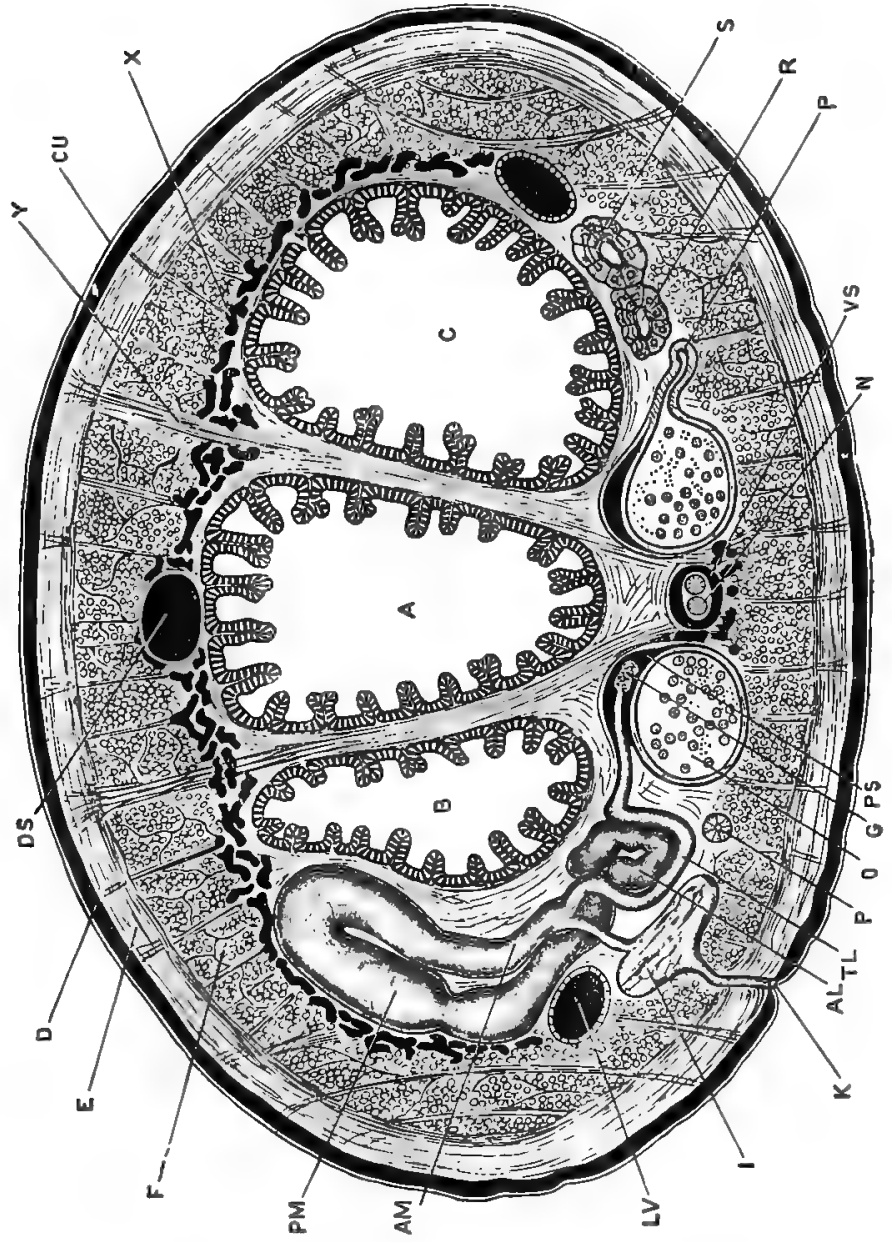




\section{The Muscles.}

The muscles of the leech consist of long fusiform cells arranged either singly or in bundles. Each muscle-cell consists of an outer cortical layer which is striated longitudinally, and a central medullary portion composed of granular protoplasm and containing the nucleus. The cortical layer is sharply marked off from the medullary portion, so that in a transverse section of a muscle-cell it appears as a ring, radially striated, and surrounding a central finely granular mass.

1. The outer or circular layer of muscles consists of fibres which encircle the body transversely. The layer itself is not much thicker than the epidermis, but the transverse muscles of the dermis should, perhaps, be grouped with it.

2. The middle or oblique layer of muscles is more deeply placed, and is separated from the circular layer by a stratum of connective tissue. The fibres run obliquely round the body.

3. The inner or longitudinal layer of muscles lies immediately within the oblique layer, and is by far the thickest of the three layers.

The fibres run longitudinally, and are therefore seen in transverse section. They are arranged in bundles, and have a very characteristic appearance, looking, for the reason noticed above, like rings of various sizes packed together in groups, and usually

FIg. 22.-Firudo medicinalis. A diagrammatic transverse section through the middle of the body. On the left side of the figure the whole of a nephridium is drawn in order to show its relations: the anterior surface of the section is towards the spectator, and the two limbs of the main lobe of the nephridium are slightly displaced, being represented as inner and outer, instead of anterior and posterior respectively. (A. M. M.)

$\mathbf{A}$, crop. AL, apical lobe of nephridium. AM, anterior limb of main Iobe of nephridium. $\mathbf{B}$, right diverticulum of crop. $\mathbf{C}$, left diverticulum of crop. CU, cuticle. $\mathbf{D}$, epidermis. DS, dorsal sinus. $\mathbf{E}$, layer of circular and oblique muscles. $\mathbf{F}$, longitudinal muscle-layer. $\mathbf{G}$, cauliflower head of nephridium. I, vesicle of nephridium. $\mathbf{K}$, external aperture of nephridium. $\mathbf{I} \mathbf{V}$, lateral vessel. $\mathbf{N}$, nerve-cord. $\boldsymbol{O}$, testis with developing spermatozos. $\mathbf{P}$, vas deferens. $\mathbf{P} \mathbf{M}$, posterior limb of main lobe of nephridium. PS, perinephrostomial sinus. $\mathbf{R}$, section of nephridium. $\mathbf{S}$, intracellular ductules of nephridium. TI, testis Iobe of nephridium. VS, ventral sinus. $\mathbf{X}$, botryoidal tissue. $\bar{Y}$, dorso-rentral muscles. 
more or less polygonal from mutual pressure. Each ring is really a transverse section of a single musclecell.

4. The dorso-ventral muscles are bundles of muscle-fibres crossing the body more or less obliquely from the dorsal to the ventral surface. They, pass between the bundles of longitudinal muscles and through the oblique and circular layers, their ends spreading out just beneath the epidermis.

\section{The Alimentary Canal.}

A section across the middle portion of the body will cut the crop, of which the median portion and the lateral diverticula will in most sections appear separate from each other.

The walls consist of an outer connective-tissue layer with a few muscular fibres, and an inner lining formed of a single layer of short columnar epithelial cells. The folds into which the inner surface is thrown involve the connective-tissue layer as well as the epithelium.

\section{E. The Blood-vessels and Sinuses.}

These two systems, corresponding to the vascular and coelomic systems of higher animals, are in free communication with each other. They are readily recognised in sections, owing to the deeply staining blood-clots with which they are filled.

1. The dorsal and ventral sinuses lie in the median plane, above and below the crop respectively. They have thin non-muscular walls, and the ventral one encloses the nerve-chain.

2. The peri-nephrostomial sinuses are easily recognised in sections that pass through the testes, on the dorsal surfaces of which they lie.

3. The lateral vessels are of large size, and have distinct muscular walls.

4. The capillaries are very thin-walled tubes, the distribution of which can be readily followed owing to the contained blood. The intercellular plexuses of the 
epidermis and of the nephridia should be specially noticed.

The capillaries are directly continuous in various parts of the body with brown pigmented fibres, similar to those of the dermis already described.

5. The botryoidal tissue consists of a network of vessels, the channels of which are irregular in width and the walls formed of large granular pigmented cells which project as irregular rounded swellings.

These euriously swollen vessels occur in great abundance around the crop, immediately within the longitudinal muscle-layer. They are in free communication with the ordinary blood-capillaries.

\section{F. The Excretory Organs.}

The nephridia have already been fully described. The details of their structure, and more especially the intracellular network of ductules, and the intercellular capillary plexuses, can be very readily made out in transverse sections, and should be carefully studied. The structural differences in various parts of the organ should be noted, and particularly the head of the testis-lobe, which lies in the peri-nephrostomial sinus.

\section{G. The Reproductive Organs.}

1. The testes are seen, if the section happens to pass through them, as spherical sacs lying at the sides of the ventral sinus, and containing groups of spermatozoa in various stages of development.

2. The vasa deferentia lie to the outer sides of the testes : each is a thick-walled tube, with a small central lumen.

\section{H. The Nervous System.}

The nerve-chain lies in the ventral sinus, and is readily recognised. Its double character is very obvious in transverse section, and if the section passes through a ganglion the large pyriform nerve-cells can be well seen. Between and below the two halves of the ventral chain lies a slender longitudinal nerve, the intermediary nerve. 


\section{Chapter V.}

\section{THE EARTHWORM. Lumbricus terrestris.}

THE earthworm lives in burrows in moist earth, through which it makes its way by alternate elongation and contraction of its body, aided by small stiff setæ projecting from its surface.

It is a pinkish worm about six inches long, cylindrical in front, flattened posteriorly. Its food consists of organic matter derived chiefly from the soil, which it swallows in enormous quantities. The worm is hermaphrodite, and lays its eggs in a chitinous cocoon, which also contains packets of spermatozoa and an albuminous fluid.

The body is marked by transverse grooves dividing it into annuli, of which there are about one hundred and fifty. This external annulation corresponds with an internal segmentation, the body-cavity being divided into chambers by transverse septa, which are placed opposite the external grooves. Nearly all the organs exhibit a corresponding segmental arrangement, there being one pair of nephridia and one pair of nerve-ganglia in each segment or somite throughout the greater part of the animal's length, and the blood-vessels, muscles, and setæ being also disposed segmentally.

Several species of Lumbricus occur in this country. These differ from one another in the position and arrangement of certain of the reproductive organs, and in other minor points. The following description, taken from the largest of the commoner species, will however apply with but slight alterations to any of the others.

The worm is most conveniently killed by dropping it into spirit for a few minutes before dissection. 


\section{EXTERNAL CHARACTERS.}

1. Form. The body is cylindrical along the greater part of its length, flattened in its hinder part. It is pointed in front, blunt behind, and is thickest about one-third of its length from the anterior end.

2. The general colour of the animal is pinkish, darker in the dorsal and anterior part of the body, paler on the ventral surface and in the hinder part of the body ; and with a dark-coloured band down the middle of the back. The dorsal blood-vessel can be seen through the integument, appearing as a dark-coloured wavy line along the dorsal surface.

3. The annuli into which the body is divided externally by transverse grooves, are larger and more obvious in front than behind. Each annulus corresponds to a segment, and is subdivided by smaller grooves, the number of which varies in different regions of the body, and also on the dorsal and ventral surfaces.

4. The clitellum, or cingulum, is the thickened integument of the dorsal and lateral surfaces of the thirty-second and five following segments, but varies slightly both in position and extent in different specimens and at different times of the year. In other species of earthworm it occupies other segments.

5. The cuticle is a delicate chitinous membrane investing the whole body of the worm. It may usually be detected by its iridescence, and can be readily stripped off a worm which has been killed with chloroform, or one which has been macerated a few hours in water. Examined microscopically it shows a series ofoblique lines, intersecting each other at right angles ; and minute highly refractive thickenings at many of the points where the lines intersect.

6. The setæ are short chitinous spines implanted in the body-wall, and arranged in two double rows along each side of the body, each segment except the first and last bearing four pairs. They may be felt on 
drawing the worm through the fingers from tail to head. The setæa are to be regarded as special localised thickenings of the cuticle.

The setce are easily isolated by boiling a bit of a worm in a solution of caustic potash: they should then be washed and mounted in glycerine, or dried and mounted in balsam.

Each seta is sigmoidally curved : it is thickest at its middle, and tapers towards each end.

\section{External apertures.}

a. The mouth is a small crescentic aperture on the ventral surface of the first segment. It is overhung by the prostomium or cephalic lobe.

b. The anus is an oval aperture at the hinder end of the body.

c. The genital apertures are four pairs.

i. The apertures of the vasa deferentia are situated on the ventral surface of the fifteenth segment, one on each side, slightly to the outer side of the inner double row of setæ. They have tumid lips.

ii. The apertures of the oviducts are similarly situated in the fourteenth segment. They are minute transverse slits not seen so easily as. the male apertures, their lips not being tumid. iii. The apertures of the spermathecæ or receptacula. seminis, of which there are two pairs, are situated in the grooves between the segments and in the line of the outer row of setæ, the first pair between segments IX. and x., the posterior pair between segments $\mathrm{x}$. and $\mathrm{xI}$.

d. The excretory apertures are difficult to see. Each segment, except the first three and the last, hasone on each side, on the ventral surface a little in front of the outer seta of the inner double row.

To see these apertures, strip off the cuticle from the anterior part of the worm, and examine the worm with a 
pocket lens, squeezing it slightly so as to cause the colomic fluid to exude from the apertures.

e. The dorsal pores, though small, are often visible to the naked eye. They open mid-dorsally in the grooves between the segments, and place the colom in communication with the exterior.

The first one is between segments $\mathbf{x}$. and $\mathrm{Xr}$. and opens into segment $x I$. Behind this point they occur regularly in every intersegmental groove.

\section{DISSECTION OF THE EARTHWORM.}

Extend the animal under water with the dorsal surface upwards, fixing it down by two pins through the sides of the fourth segment and two near the hinder end. Cut through the body-wall from end to end, close to the mid-dorsal line. Gently raise the flaps with the forceps, and note the transverse septa dividing the body-cavity into segments. Carefully cut through these septa close to the body-wall, and pin out the flaps right and left with fine pins. If the worm has been freshly killed, immerse it for a few minutes in spirit.

\section{A. The Septa.}

These are incomplete transverse membranous partitions, connecting the alimentary canal with the body-wall and dividing the body-cavity into segments. They are placed opposite the grooves on the external surface throughout the whole length of the body, except in two regions : (1) in front of the fourth segment, where they are absent; (2) in the region of the gizzard, where they are shifted backwards so that they are less numerous than the external rings, while immediately behind it they are more numerous (fig. 23).

\section{B. The Digestive System.}

The alimentary canal is a straight tube running from the mouth to the anus, and is divisible into the following regions.

1. The buccal cavity is small, and is situated in the first three segments. The opening of the mouth in the ventral part of the first segment has soft lips and no teeth. 
2. The pharynx, which follows the buccal cavity, has thick muscular walls connected with the body-wall by strands of muscle which run obliquely backwards, perforating the anterior septa. The pharynx extends backwards about as far as the sixth or seventh annulus when the animal is in an average condition of extension; but it lies entirely in front of the septum dividing the fifth segment from the sixth (fig. 23).

3. The cesophagus is a straight tube extending from the pharynx to the thirteenth or fourteenth segment. When the animal is not fully extended the œsophagus appears dilated in each segment, and constricted where it perforates the septa.

a. The esophageal pouches are a pair of short lateral. diverticula of the csophagus "in segment x. At certain seasons they contain concretions of more or less crystalline calcareous matter, chiefly calcium carbonate.

To see these cesophageal pouches press aside the large white vesicula seminales which hide them.

b. The cesophageal glands are two pairs of lateral protuberances on the sides of the csophagus in segments XI. and XII. They are hollow, and their cavities, which are subdivided by a large number of horizontal lamellæ, contain a milky calcareous fluid. They are local thickenings of the glandular walls of the csophagus, and their numerous cavities open into the cesophageal pouches.

4. The crop is a large dilatation of the alimentary canal in segments XIII. to XVI., but when filled it encroaches upon the segments in front, pushing the septa before it (fig. 23).

5. The gizzard extends from the sixteenth segment backwards to about the twentieth. Its walls are very thick and muscular, and it has a thick chitinous lining. 


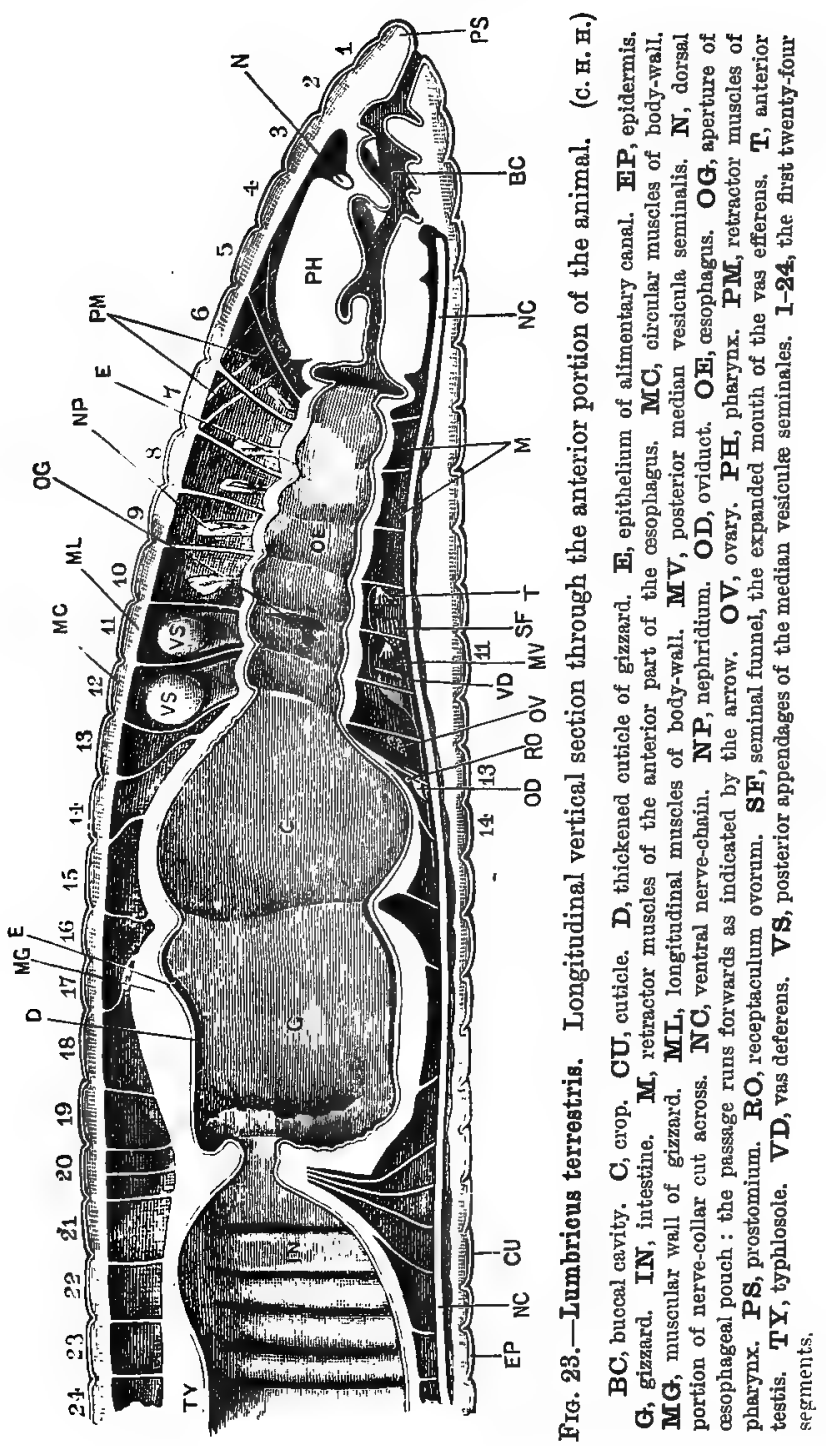


6. The intestine is a thin-walled sacculated tube running straight backwards from the gizzard to the anus.

a. The 'hepatic cells' are a layer of yellow cells surrounding the intestine and in close relation with the blood-vessels. Their function is unknown.

Cut the intestine open along one side, and wash out its contents.

b. The typhlosole is a prominent median longitudinal ridge projecting into the cavity of the intestine from its dorsal wall, of which it is a fold. "Its surface is marked by transverse folds.

\section{The Circulatory System.}

The blood is a fluid coloured bright red by hæmoglobin and containing colourless corpuscles. Several of the larger vessels contract rhythmically, and especially the five pairs of ' hearts.'

For the dissection of the circulatory system a fresh worm should be taken. Owing to the dark red colour of the contained blood the vessels can be readily traced. The dissection is best performed under spirit.

1. The dorsal vessel is a large median vessel running along the dorsal surface of the alimentary canal throughout its whole length; and dividing in front into branches ramifying over the pharynx. It can be seen through the skin in the living animal, and the flow of blood in it is from behind forwards.

A large lateral cesophageal vessel runs along each side of the œsophagus, and communicates with the dorsal vessel in the tenth segment. Branches of it ramify over the pharynx and over the œsophageal glands and pouches.

From the alimentary canal the blood is returned to the dorsal vessel by intestinal vessels, of which there are in the region of the intestine two to each segment. 
2. The ventral vessel is a median vessel running backwards below the alimentary canal the whole length of the animal.

3. The 'hearts ' are five pairs of large vessels lying in segments vIr. to xI., and connecting the dorsal with the ventral vessel. During life they are rhythmically contractile, the contractions passing along them from above downwards, and commencing with the hindmost pair. In the fresh state each has frequently a moniliform appearance.

4. Neural vessels. These are in close relation with the nerve-cord which runs along the floor of the bodycavity in the mid-ventral line (see p. 74).

a. The subneural vessel is median, and lies immediately beneath the nerve-cord along its whole length.

b. The lateral neural vessels are a pair of smaller longitudinal vessels lying along the sides of the nerve-cord.

5. The nephridial vessels supply the nephridia or excretory organs (see p. 64).

a. The nephridial arteries arise from the ventral vessel, one pair in each segment, and run outwards to the nephridia.

b. The nephridial veins return the blood from the nephridia to the subneural vessel.

6. The parietal vessels. In each segment the subneural and dorsal vessels are connected by a pair of parietal vessels, which run on the inner surface of the bodywall, and receive branches from it.

\section{The Excretory System.}

This consists of a series of tubular nephridia or " segmental organs,' of which each segment, except the first three and the last, contains a pair. They are largest in the region of the cosophagus. 
Each nephridium is a long tube thrown into loops, which are closely applied together and suspended in the body-cavity close to its wall. The tube commences with a minute ciliated mouth-the nephrostome-opening into the body-cavity; then follows a long and very slender portion, to which succeeds: a shorter and wider part; the terminal portion is still wider and has muscular walls, and opens distally to the exterior at the aperture already seen on the surface.

\section{Examination of a nephridium in situ (fig. 25).}

Remove an inch or so of the intestine, so as to expose the nephridia fully. Wash gently under the tap, and then examine under spirit with a pocket lens or dissecting microscope.

The nephridia are seen as opaque white loops lying along the inner surface of the body-wall, and extending from near the mid-ventral line almost to the mid-dorsal line. Each is in relation with two segments, the funnel and a very short length of the tube lying in the anterior of the two, and the rest of the tube with the external opening in the posterior.

Examine one of the nephridia more closely, noting its parts.

a. The nephrostome is a minute funnel lying in the body-cavity close to the mid-ventral line, and connected by a short stalk-the first part of the nephridial tube-with the septum behind it.

b. The looped portion of the tube lies in the segment behind that containing the funnel. Three limbs can readily be made out in the loop, of which the middle one is the longest and reaches almost to the mid-dorsal line; while the outer one is the widest and leads to the external aperture.

\section{Microscopical structure of a nephridium.}

Carefully remove an entire nephridium with a very small part of the septum which it traverses. Stain it, dehydrate with alcohol, clear with oil of cloves, and mount in balsam.

Examine with low and high powers. 
a. The funnel is ciliated, its border consisting of a row of large cells with very distinct nuclei. The nephrostome or open mouth of the funnel can be readily seen.

b. The first part of the tube is very slender and difficult to make out: it lies alongside the inner and middle of the loops formed by the wider part of the tube. The canal of the tube is here an intracellular one, traversing the individual cells.

c. The second part of the tube is much wider, the change in diameter being abrupt, and the canal having from this point proper cellular walls. It runs up and down the middle loop of the three, at the dorsal end of which it is considerably dilated; then up and down the shorter inner loop ; and finally across to the outer loop, where it forms the terminal portion of the tube. The first portion of the tube is lined by a ciliated epithelium; the distal portion has glandular walls, and is very vascular.

d. The terminal portion of the nephridium is much wider than the rest: its walls are non-glandular, and composed largely of interlacing muscular fibres. It opens to the exterior by the aperture already seen, in front of the outer seta of the inner double row.

\section{E. The Reproductive System.}

The earthworm is hermaphrodite. The reproductive organs are lodged in segments IX. to $\mathrm{xV}$., i.e. in the region of the œsophagus.

1. The female organs consist of a pair of ovaries ; a pair of oviducts, with a receptaculum ovorum opening into each ; and two pairs of spermathecæ.

a. The ovaries, which lie in segment xIII., are a pair of white pyriform bodies attached to the septum forming the anterior wall of the segment, and 
hanging freely from it into the coelom below the œsophagus.

To see the ovaries, carefully cut across the cesophagus in this segment, and gently press apart the septa bounding it in front and behind.

b. The oviducts are a pair of short tubes, the wide mouths of which are placed in the hinder part of segment XIII., one opposite each ovary.

From the points where they perforate the septum, the oviducts run outwards and slightly backwards to their external openings on the ventral surface of segment xIv.

To see them, cut through the oesophagus in segment xIV.

c. The receptacula ovorum are a pair of reniform sacs, attached to the anterior wall of segment xiv. just above the oviducts, into the upper part of the funnel-like mouths of which they open.

d. The spermathecæ or receptacula seminis are two pairs of globular sacs lying in the hinder parts of segments Ix. and $\mathbf{x}$. respectively. Their ducts, which are short, run backwards and open to the exterior in the grooves between the ninth and tenth, and the tenth and eleventh segments respectively, just within the line of the outer double row of setæ.

In these the seminal fluid received from another worm is stored, and from them it is poured out into the egg-case or cocoon in which the eggs are laid.

Some species of earthworm have the spermathecæ in segments $\mathrm{x}$. and XI., their ducts passing forwards to open in the same position as above.

2. The male organs consist of two pairs of testes; two pairs of seminal funnels, leading into a pair of vasa deferentia; and median and lateral vesiculæ seminales. 
a. The vesiculæ seminales vary greatly in form and size with the season of the year. When fully developed they form conspicuous milk-white bodies at the sides of the csophagus, and overlapping it.

i. A pair of anterior lateral vesiculæ seminales lie

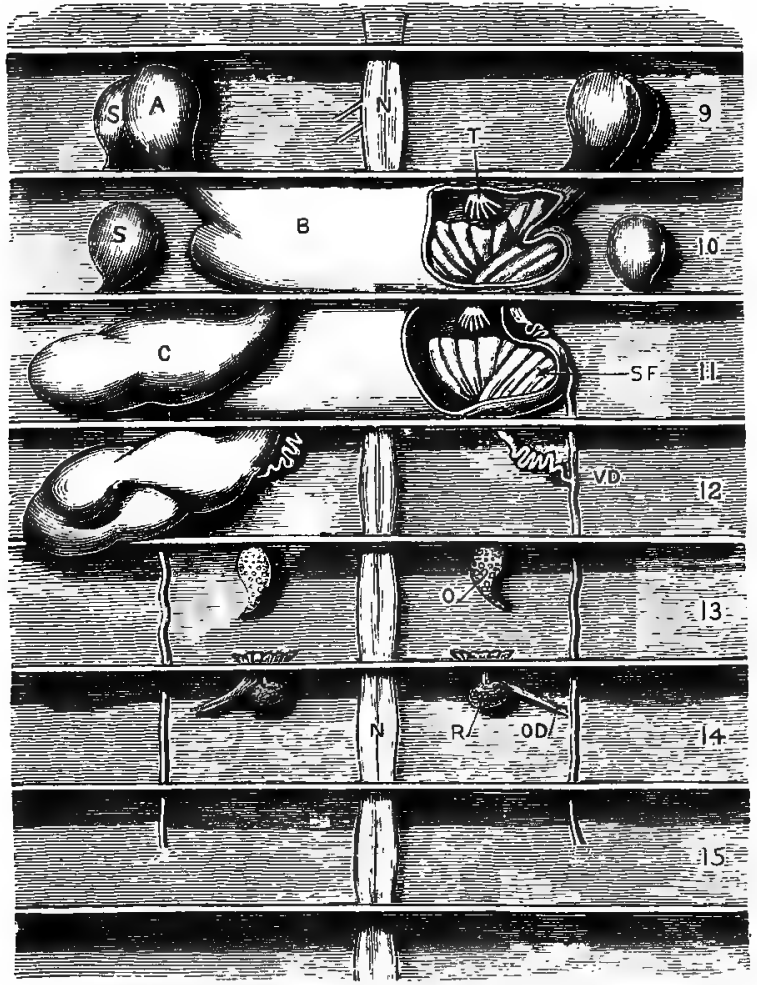

FIG. 24.-Lumbricus terrestris. Plan of the reproductive organs as seen from above after removal of the alimentary canal. The body-wall is pinned down flat. (C. H. H.)

$\mathbf{A}$, anterior lateral vesicula seminalis. $\mathbf{B}$, first median vesicula seminalis. $\mathbf{C}$, posterior lateral vesicula seminalis. $\mathbf{N}$, nerve-chain, $\mathbf{O}$, ovary. $\mathbf{O D}$, oviduct. $\mathbf{R}$, receptaculum ovorum. $\mathbf{S}$, spermathecæ. $\mathbf{S F}$, seminal funnel lying in the median vesicula seminalis. T, testis. VD, vas deferens: the reference line indicates the point where the posterior vas efferens opens into it. 9 to 15 , ninth to fifteenth segments. 
in segment Ix. They are opaque white bodies attached to the posterior wall of the segment, one at each side of the cosophagus.

Some species of earthworm have a similar pair in segment $x$. , but in Lumbricus terrestris these are not developed.

ii. Two pairs of posterior lateral vesiculæ seminales lie in segments XI. and XII. respectively. They are very much larger, than the anterior vesiculæ, especially at certain seasons, and are attached to the anterior septa of their respective segments, somewhat nearer the median plane than the anterior vesiculæ.

iii. A thin-roofed median vesicula seminalis, of considerable size, is situated in each of the segments $\mathbf{x}$. and $\mathrm{xI}$. below the œsophagus, which must be removed to expose them. Within each are a pair of testes and a pair of seminal funnels. With the anterior of these median vesiculæ the anterior lateral vesiculæ and the first pair of posterior lateral vesiculæ communicate. The second pair of posterior lateral vesiculæ open in similar fashion into the second median vesicula.

b. The testes are two pairs of flattened digitate bodies of small size, attached to the septa in front of segments $\mathrm{x}$. and $\mathrm{xI}$. respectively, and projecting freely into the cavities of the median vesiculæ seminales, not far from the nerve-cord.

To see the testes, open the two median vesicula seminales without injuring the structures within them. Wash carefully.

c. The seminal funnels are large, opaque, white, muchfolded membranous funnels, of which one pair is situated in each median vesicula seminalis, the open mouths being turned towards the testes, and almost enclosing them. Their narrow ends 
perforate the hinder septa of their respective segments (x. and $\mathrm{xI}$.), and are continuous with the vasa efferentia.

d. The vasa deferentia. From the posterior end of each seminal funnel a fine convoluted tube, the vas efferens, runs outwards and backwards on the ventral body-wall. The two vasa efferentia of each side unite in segment xII., and the vasa deferentia so formed run back to open to the exterior by the apertures already seen on the ventral surface of segment $\mathrm{xV}$. The vasa deferentia are slightly imbedded in the body-wall, and their exposure requires care.

\section{Microscopical examination of the reproductive organs,} and of their contents.

a. The ovaries. Remove an ovary entire; stain with carmine or magenta, and mount in glycerine; or dehydrate, clear, and mount in balsam.

The proximal and thicker end of the ovary is a solid mass of cells from which the ova are formed: the distal portion contains ripe ova. Each ovum is a large cell with very distinct nucleus and nucleolus. The nucleolus is stained by carmine more deeply than the rest of the cell.

b. The receptacula ovorum. Remove one and examine it as above.

Ova may be seen in it at certain seasons.

c. The contents of the spermathecæ. Remove a spermatheca and transfer it to a slide. Tease it in water and examine with low and high powers.

The seminal fluid in the spermathecr contains innumerable mature spermatozoa, each of which is an exceedingly fine filament with a slightly thickened rod-like head at one end.

d. The testes. Remove a testis; stain, dehydrate and 
clear it, and tease it in balsam: mount another in water. Examine with a high power.

The spermatospores are cells, each of which by division gives rise to a large number of spermatozoa. Only the earliest stages of the division occur in the testes, the spermatospores leaving them as ovoid masses with numerous nuclei, and tuberculated surfaces.

e. The contents of the resiculæ seminales. Tease in water some of the white mass filling the vesiculce. Stain another portion, and mount it in glycerine.

The vesiculæ contain spermatozoa in all stages of development:-(1) the morula-like spermatospore as it leaves the testis; (2) a stage in which the tubercles, or spermatoblasts, are more distinet and are produced outwards into filaments; (3) later stages in which the filaments are elongated to form the tails of the spermatozoa, while the bodies of the spermatoblasts form their rod-like heads.

A part of the spermatospore remains unchanged in the centre of the mass, while the peripheral part becomes converted, as described above, into a wisp-like tuft of spermatozoa.

\section{F. The Nervous System.}

This consists, as in the leech, of a double ventral cord, swollen slightly in each segment; and a pair of dorsal ganglia in front, connected with the ventral chain by a pair of connectives running round the sides of the alimentary canal.

1. The nerve-collar is a small ring of nervous substance surrounding the buccal sac just in front of the pharynx, in the third segment. It consists of a pair of pyriform supra-œsophageal ganglia, united in the median plane; a pair of lateral connectives running round the sides of the buccal cavity; and a pair of 
closely apposed ventral ganglia, the first pair of the ventral chain.

\section{The ventral nerve-chain.}

Remove the alimentary canal, from the pharynx backwards, without injuring the nerve-collar and chain.

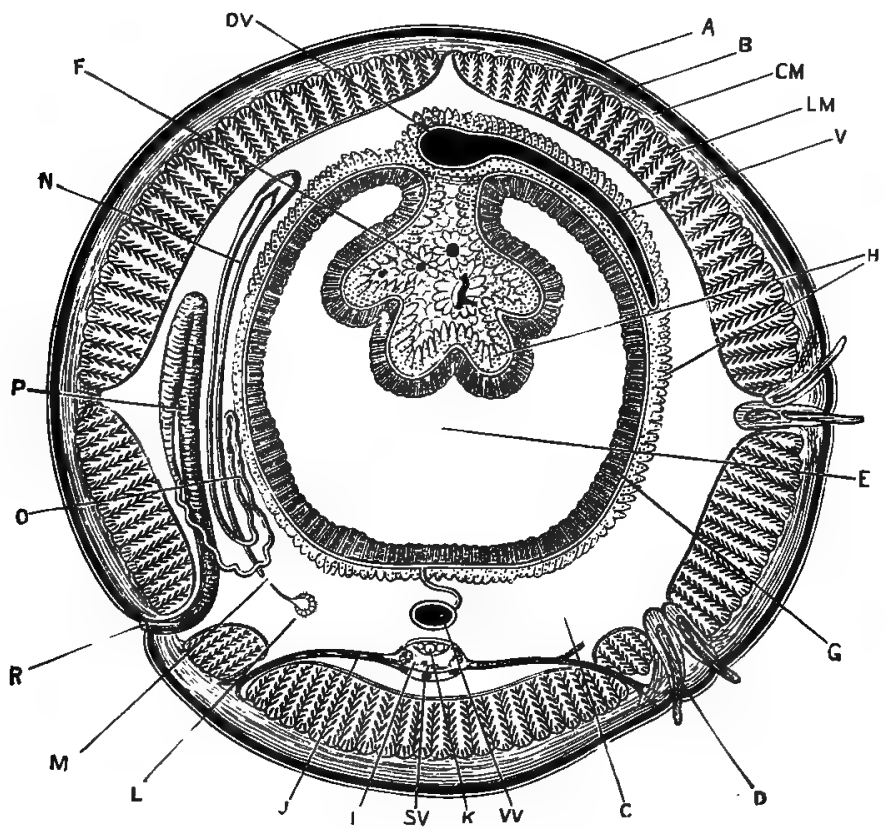

Fig. 25.-Lumbricus terrestris. A diagrammatic transverse section through the middle of the body. On the left side of the figure a nephridium is shown, and on the right side the setæ. The septa are not shown, and parts only of the circulatory system are represented. (A. M. M.)

A, cuticle. B, epidermis. C, ccelom. CM, layer of circular muscles. $\mathbf{D}$, sets. DV, dorsal vessel. $\mathbf{E}$, cavity of intestine. $\mathbf{F}$, typhlosole, filled with 'hepatic' cells. G, epithelium of intestine. $\mathbf{H}$, ' hepatic ' cells. $\mathbf{I}$, ventral nerve-cord. $\boldsymbol{J}$, nerve. $\mathbf{K}$, 'giant fibres.' $\mathbf{L}$, nephrostome. $\mathbf{L M}$, layer of longitudinal muscles. $\overrightarrow{\mathbf{M}}$, the point where the nephridium perforates the septum between two segments : between the two cut ends a considerable length of very fine tube lies, which is not shown in the figure. $\mathbf{N}$, the first or middle loop of the nephridium. $O$, the second or inner loop of the nephridium. $\mathbf{P}$, the third or outer loop of the nephridium. $\mathbf{R}$, external aperture of the neplridium. $\mathbf{S V}$, subneural vessel. $\mathbf{V}$, intestinal vessel. $\mathbf{V}$, ventral vessel. 
The chain runs along the ventral body-wall in the middle line, giving off three pairs of nerves in each segment along nearly the whole length of the body. The cord is slightly swollen opposite the middle of the segments, the swellings or ganglia being more conspicuous in the hinder part of the body.

\section{EXAMINATION OF TRANSVERSE SECTIONS OF THE INTESTINAL REGION.}

The structure of the body-wall and alimentary canal, and many other points in the anatomy of the earthworm, are best understood from a microscopical examination of transverse sections of the whole animal.

To prepare these sections kill a worm with cold saturated solution of corrosive sublimate; out out about half an inch from the middle of its length, and leave this in the sublimate for twenty-four hours. Wash it well, and transfer to spirit. Carefully syringe out the contents of the intestine. Stain; imbed, and cut into transverse sections with a microtome. Mount the sections in order.

Examine with low and high powers.

\section{A. Shape.}

The section is nearly circular in outline. The dorsal surface is indicated by the typhlosole, the ventral by the nerve-cord.

\section{B. The Integument.}

1. The cuticle is a thin structureless superficial layer, often separated by the action of the reagents from the underlying epidermis.

2. The epidermis lies immediately below the cuticle. It consists of a single layer of columnar cells, set side by side at right angles to the surface, with nuclei near their bases. Many of these cells have the character of 'goblet cells,' being distended by a granular secretion formed within them; other cells 
lying between these are extremely slender. In the clitellum the epidermis is greatly thickened, and blood-vessels penetrate between the cells.

3. The dermis is an exceedingly thin layer immediately beneath the epidermis.

4. The setæ are lodged in sacs, which are invaginations of the skin extending deep into the body, through both the muscular layers. They are cuticular structures, and are moved by special muscles. Setæ in various stages of development may be seen within the sacs.

\section{The Muscular Layers of the Body-wall.}

1. The external layer, which is rather thicker than the epidermis, consists of transverse fibres running round the body : it is completely interrupted at the grooves between the segments. The connective tissue between the muscle-fibres is pigmented slightly : in the dorsal wall of the œsophageal region this pigmentation is very marked.

2. The internal layer is very much thicker than the external. It consists of longitudinal fibres, arranged on the sides of a series of radial longitudinal sheets, and having a feather-like appearance in transverse section.

\section{The Cœlom, or Body-cavity.}

This is the space around the intestine, between it and the body-wall. It is lined by a thin peritoneal epithelium.

\section{E. The Intestine.}

This occupies the middle of each section. Its diameter is about half that of the body of the worm.

1. The typhlosole is a fold of the dorsal wall extending into the intestine from above, narrowing its cavity, and making it crescentic in transverse section.

2. The epithelium lining the intestine consists of a single layer of columnar ciliated cells.

3. The muscular layer of the intestinal wall is thin. The fibres, which are nearly all transverse in direction, are imbedded in a connective-tissue layer. 
4. The 'hepatic' cells lie outside the muscular layer, around the vessels which return the blood from the intestine to the dorsal vessel. They are elongated granular cells of large size, and generally arranged radially to the surface of the intestine. They are very abundant in the cavity of the typhlosole, which they almost completely fill.

\section{F. The Nephridia.}

These lie at the sides of the alimentary canal. Their appearance in transverse sections varies greatly according to the part of the nephridium through which the section happens to pass. By reference to the description of a nephridium, on page 64, it will be fairly easy to identify the parts present in any particular section that may be examined.

\section{G. The Septa.}

Owing to their obliquity, parts of the septa are present in nearly all transverse sections, lying in the body-cavity between the body-wall and the alimentary canal. Each septum is a thin sheet of connective tissue, in which are circular and radial muscles. The central part of the ventral edge of the septum is not attached to the body-wall, a gap being left through which the cœlomic fluid can pass from one segment to another.

\section{H. The Nerve-cord.}

This lies near the ventral surface, just within the longitudinal muscle-layer, and is enclosed in a double connectivetissue sheath, between the two layers of which is a stratum of longitudinal muscle-fibres.

In the sheath lie three longitudinal blood-vessels, a subneural below the cord, and a pair of lateral neural vessels at the sides. Along the dorsal surface of the cord are three 'giant-fibres ' of unknown function. The fibrous connective tissue of the sheath extends into the cord, dividing its fibres into two main bundles, and subdividing each of these into smaller ones. Nerve-cells of large size, each with distinct nucleus and nucleolus, form a layer along the whole length of 
the ventral and lateral surfaces of the cord: they are more abundant in the ganglionic enlargements.

\section{The Blood-vessels.}

These are readily recognised by the deeply staining bloodclots with which they are filled. The larger vessels have muscular walls. Besides the neural vessels just mentioned the following may be recognised.

1. The dorsal vessel lies in the body-cavity above the intestine. It is of large size, and has masses of 'hepatic" cells at its sides.

2. The intestinal vessels lie in the walls of the intestine: they are surrounded by 'hepatic' cells, and may in some sections be seen to open into the dorsal vessel.

3. The ventral vessel lies between the nerve-cord and the intestine, and is connected with the latter by a median sheet of vascular connective tissue. 


\section{Chapter VI.}

\section{THE FRESHWATER MUSSEL. Anodonta cygnea.}

THe body of the mussel is enclosed in a shell, the two halves or valves of which cover the right and left sides of the body respectively. These valves, which are hinged together along the median dorsal line, can be opened to a slight extent by means of an elastic ligament along the hinge-line, and closed by powerful adductor muscles. When the valves are closed they completely conceal all parts of the body.

Mussels vary a good deal in size according to their age; a fair-sized specimen is about four and a half inches long, one and a half thick, and two and a half from the dorsal to the ventral edge.

The mussel lives in freshwater streams, with its anterior end buried somewhat obliquely in the mud of the bottom, and the posterior end projecting up into the water. By means of a muscular foot it can plough its way slowly along the bed of the stream. Its food consists of minute organisms, both animal and vegetable, which are swept in at the hinder end of the shell by eiliary currents and carried forwards to the mouth, the stream of water so kept up serving for respiration as well as nutrition.

The sexes are distinct. The young embryos are retained within the mother during their early stages of development; they then hatch and attach themselves to fish. While attached they increase considerably in size, and undergo a distinct metamorphosis. Finally they detach themselves, and adopt the mode of life of the adult. 


\section{THE SHELL.}

The shell is a cuticular product formed by the underlying epidermis. It is, therefore, entirely outside the body, and incapable of growth except by addition of new rings or new layers to the part already formed. It consists of an organic basis impregnated with calcareous salts.

\section{A. The Outer Surface of the Shell.}

1. Shape. The anterior end of the shell is rounded, the posterior more pointed, and the dorsal hinge-line straight. In the female the shell is more convex than in the male.

2. The umbo is the oldest part of each valve. It is close to the hinge-line, and about a quarter of the length of the shell from its anterior end. It has usually a pearly white appearance, owing to the outer layer of the shell having been rubbed off.

3. The lines of growth are the series of concentric rings on the surface of the shell, marking successive stages in its growth. Each of these lines was at one time the actual margin of the shell.

The shell does not grow uniformly in all directions. Growth is much more rapid towards the ventral than the dorsal surface, and more rapid posteriorly than anteriorly; hence the umbo or oldest part is close to the dorsal edge, and nearer the anterior than the posterior end.

4. The ligament connects the two valves together along the mid-dorsal line; it is really a median, imperfectly calcified part of the shell itself. It is very narrow in the anterior part of the hinge-line, and much broader behind. As the valves are only kept closed by contraction of the adductor muscles, they will open as soon as these muscles relax. Hence in dead specimens the valves always gape, owing to the unopposed action of the elastic ligament. 


\section{B. The Inner Surface of the Shell.}

Wedge open the valves of the shell with the handle of a scalpel or other instrument, so that the ventral edges of the valves are about half an inch apart, and the adductor muscles can be clearly seen. Note the mantle-lobes or flaps lining the shell-valves, and separate with a scalpel the right mantle-lobe from the shell.

Identify the positions of the adductor muscles by comparison with fig. 26, and detach with a scalpel first the anterior, then the posterior adductor muscle from the right valve, keeping the knife close to the shell. As soon as this is done the valves will gape open, about half or three-quarters of an inch, owing to the action of the ligament. Bend back the right valve of the shell, and remove it completely, cutting through the ligament along the hinge line. Clean the detached valve, and examine it.

1. The inner surface has a white pearly appearance, except round the edge. The lines of growth are visible, but not nearly so plainly as on the outside.

2. The hinge is a smooth longitudinal ridge extending backwards from the umbo along the dorsal edge of the shell. When the valves are closed, the two hinges are in contact opposite the umbo, but gradually diverge from each other behind it.

3. The ligament, which is very strong and elastic, commences in front of the umbo, and extends back along the straight dorsal edge of the shell, being very narrow in front, but wider behind.

4. The muscular impressions are slight depressions of the shell, to which the adductor and other muscles are attached.

a. The anterior adductor impression is a large oval slightly depressed area, with its long axis vertical, close to the anterior end of the shell, and nearer the dorsal than the ventral edge. 
b. The anterior retractor impression is small, and continuous with the posterior border of the anterior adductor impression.

e. The protractor impression is a separate small impression, about $\frac{1}{8}$ inch behind the lower part of the anterior adductor impression.

d. The posterior adductor impression is near the dorsal edge and posterior end of the shell, below and behind the hinder end of the ligament. It is rather larger than the anterior adductor impression, and is oval in shape, with the longer axis horizontal.

e. The posterior retractor impression is small, and continuous with the upper and anterior edge of the posterior adductor impression.

f. The pallial line, or line of attachment of the mantle to the shell, runs nearly parallel to the ventral edge of the shell, and about half an inch from it. It connects the hinder border of the posterior adductor impression with the ventral edge of that of the anterior adductor.

5. The paths of shifting of the muscles are faint triangular depressions, converging from the margins of the several muscular impressions towards the umbo. They mark the boundaries of the paths along which the muscles have shifted during the growth of the shell. In the young state, when the umbo and the part immediately round it form the whole valve, adductor and other muscles are present in the same relative positions as in the adult: during the growth of the shell by addition of new matter round its margin the muscles gradually shift outwards, increasing in size as they do so.

\section{The Structure of the Shell.}

If the shell be broken across, and the edge examined with a pocket lens, it is seen to consist of three layers. 
1. The periostracum or outer layer is thin, horny, and uncalcified. To it the brownish-green colour of the shell is due. It is commonly, but erroneously, spoken of as the 'epidermis ' of the shell.

In the growth of the shell this layer is the first to be formed, and the free edge of the shell, especially round the ventral border and the posterior end, consists of it alone. It is formed by the thickened edge of the mantle-fold.

The periostracum is reflected over the edge of the shell, and lines a narrow strip of its inner surface round the ventral border and at the two ends.

2. The middle or prismatic layer forms about half the thickness of the shell : it is very densely calcified, and consists of numerous polygonal prisms placed side by side, and obliquely to the surface of the shell.

Like the periostracum, it is formed by the edge of the mantle. It is visible on the outer surface of the shell at places where, as at the umbo, the periostracum has been rubbed off. It can also be seen round the margin of the shell on its inner surface.

3. The inner, nacreous or pearly layer lines the whole of the inner surface of the shell except the marginal rim. It varies in thickness, but is in most parts about as thick as the prismatic layer, and consists of a number of superposed laminæ laden with calcareous particles. It is formed by the surface of the body and mantle in contact with the shell.

Pearls are formed in animals closely allied to the mussel by concentric deposits of this nacreous layer around foreign bodies, such as grains of sand. 


\section{DISSECTION OF THE MUSSEL.}

In dissecting the mussel, a small brush may with great advantage be used to remove the mucus and to clean the several parts.

Leave the animal in the left valve of the shell, and fix it down firmly to the dissecting-board under water. Pins can readily be passed through the ligament.

\section{A. External Characters.}

1. The muscles. The anterior and posterior adductors are readily recognised, and form useful landmarks. Their fibres run transversely from one valve to the other, and by their contraction close the valves.

Close to the adductors are the smaller protractor and retractor muscles of the foot, the origins of which have already been seen on the inner surface of the shell.

The protractor is behind the lower border of the anterior adductor, and the anterior retractor is behind the middle of the same muscle. The posterior retractor is continuous with the upper and anterior border of the posterior adductor.

2. The right mantle-lobe is the flap of integument which covers the side of the body: it is in contact during life with the inner surface of the shell, to which it is attached along the pallial line. Its inner surface is ciliated.

Dorsally it is continuous with the body-wall, of which it is a prolongation. Its ventral border is free and grooved, this free border secreting the outer and middle layers of the shell.

The mantle-lobe begins in front above the middle of the anterior adductor muscle, and runs round its anterior border as a narrow fringe. Opposite the part of the body between the two adductor muscles the mantle-lobe is very wide, extending from near the dorsal border to the ventral edge of the shell. 
At the hinder end of the animal, below the posterior adductor, the margin of the mantle which here bounds the inhalent aperture, is very greatly thickened and pigmented, and bears a fringe of tentacular processes.

Immediately behind the posterior adductor the mantie-lobe fuses with its fellow of the opposite side, and the fused borders run forwards along the dorsal surface of the posterior adductor for about half an inch. They then separate and continue forwards as a pair of narrow fringes, bounding a shallow groove, for about half an inch further, where they cease.

3. The left mantle-lobe, which can as yet be only imperfectly seen, agrees exactly with the right, the animal being bilaterally symmetrical.

4. The pallial or mantle-cavity is the space between the right and left mantle-lobes. In it lie the foot and the greater part of the visceral mass, the gills, and other organs.

It is divided by a horizontal partition, formed by the bases of the gills, into two chambers of very unequal size;-(1) the large ventral branchial chamber; (2) the smaller dorsal supra-branchial chamber, the hinder end of which forms the cloacal chamber.

5. The pallial openings. If a mussel be placed in a vessel of water with a layer of mud or sand at the bottom, and left to itself, it will assume the position described above, the anterior end being buried somewhat obliquely in the mud, and the posterior end projecting into the water. The valves will be slightly opened, and streams of water, which can be rendered clearly visible by adding a little colouring matter, will pass in and out of the posterior end, the ingoing or inhalent stream being ventral, the exhalent dorsal.

This current is maintained by the cilia of the 
mantle, gills, and palps: it serves to bring water for nutrition and respiration, and to carry away the fæcal matter and the excretory and reproductive products.

a. The inhalent aperture, through which the inhalent stream enters the branchial chamber, is a vertical slit at the hinder end of the body: it is bordered laterally by the thickened posterior edges of the mantle-lobes, which bear the tentacular fringes. Below, it is incompletely closed by the approximation of the edges of the mantle-folds; and above, it is separated from the exhalent aperture by a horizontal partition formed by the fusion of the gills of the two sides with each other.

b. The exhalent or cloacal aperture is much smaller than the inhalent opening, and lies immediately dorsal to it, and behind the posterior adductor muscle. It is bounded laterally by the thickened mantle-borders, which have here no tentacles.

Pass a seeker into the cloacal opening, and forwards along the supra-branchial cavity above the gills. Note the partition between the exhalent and inhalent apertures.

6. Organs lying in the branchial chamber.

Turn back the right mantle-lobe as fully as possible.

a. The foot and visceral mass form a large laterally compressed oblong mass, about half the length of the shell, and lying between the two adductors. The upper two-thirds, which are paler in colour, form the visceral mass : the lower or ventral third forms a powerful muscular foot, reddish in colour, which can be protruded from between the valves in front, and is used by the animal for working its way along the bed of the stream in which it lives.

b. The gills are two pairs of large lamellar organs lying at the sides of the visceral mass, between it and the mantle, and extending back beneath the posterior adductor to the hinder end of the 
mantle-cavity. The outer gill is, in the female, often greatly distended with embryos.

c. The labial palps are two pairs of triangular folds lying at the sides of the anterior part of the visceral mass, between the anterior adductor and the gills. Each pair consists of a couple of lamellæ, of about equal size, attached along their dorsal borders to the body-wall, and marked on their apposed surfaces by close-set transverse ridges.

\section{The external apertures.}

a. The mouth is in front, between the anterior adductor and the anterior border of the foot.

Pass a seeker into the mouth, and note that it passes vertically upwards behind the anterior adductor, into the stomach.

The mouth is bordered by anterior and posterior lips, formed by the labial palps. The outer palp is continuous with its fellow of the opposite side, forming a low ridge-like anterior lip, immediately behind the anterior adductor. In a similar manner the two inner palps are continuous with each other and form a posterior lip, in front of the foot.

The deep groove between the outer and inner palps of each side thus leads directly into the mouth. The palps are richly ciliated, so that any food-particles that come within the range of their action are swept into the groove and carried forwards to the mouth.

b. The anus opens into the cloacal cavity, close to its. external opening.

Insert a seeker into the groove between the margins of the mantle-lobes above the posterior adductor, and pass it back over the adductor into the cloacal cavity. Slit up this canal so as to expose thoroughly the hinderipart of the rectum, and the anus.

Insert a seeker into the anus and pass it upwards and 
forwards along the rectum as it lies on the dorsal surface of the posterior adductor.

\section{B. The Gilis.}

Cut away the right mantle-lobe completely with scissors, cutting along the base of the palps, round the anterior end of the gills, and back along the attached base of the outer gill. Take care not to damage the parts to which the mantle is attached.

Each gill consists of a pair of lamellæ, united along their ventral borders. Each lamella is formed of very numerous

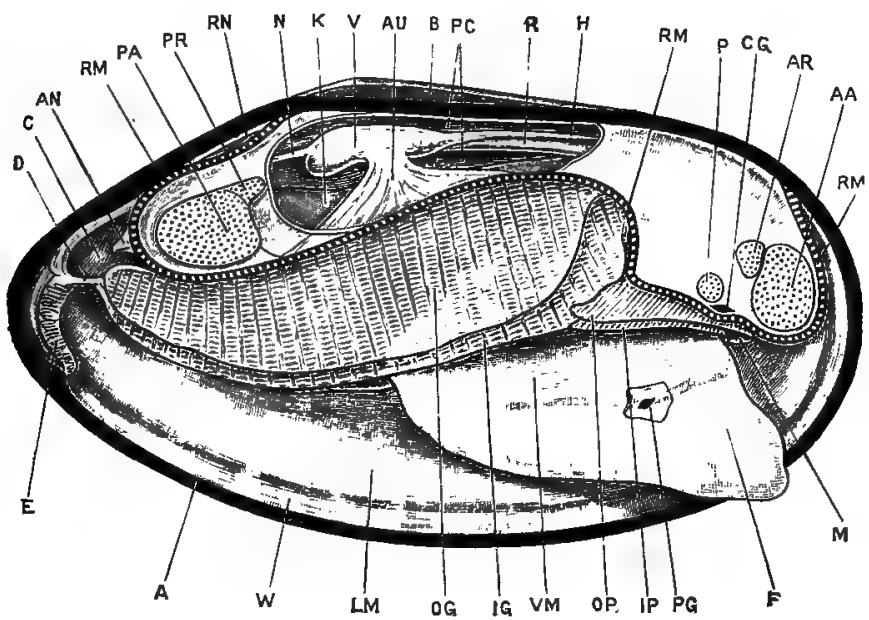

Frg. 26.-Anodonta cygnea. Diagrammatic view from the right side. The right valve of the shell and the right mantle-lobe have been removed, and the pericardial cavity opened from the right side. The line of attachment of the right mantle-lobe is indicated by the strong dotted line. (A. M. M.)

A, margin of the left valve of the shell in which the animal is lying. AA, anterior adductor muscle. AN, anus. AR, right anterior retractor muscle. AU, right auricle. $\mathbf{B}$, ligament of the shell. $\mathbf{C}$, closcal cavity. $\mathbf{C G}$, right cerebral ganglion. $\mathbf{D}$, partition between the inhalent and exhalent apertures. F, tentacles borderitig inhalent aperture. F, foot. $\mathbf{H}$, anterior gorta IG, inner gill of right side. IP, inner labial palp of right side. $\mathbf{K}$, kidney. $\mathbf{L} \mathbf{M}$, left mantle-lobe. $\mathbf{M}$, mouth. $\mathbf{N}$, posterior aorta. $\mathbf{O G}$, outer gill of right side. $\mathbf{O P}$, outer labial palp of right side. $\mathbf{P}$, right protractor muscle. PA, posterior adductor muscle. PC, pericardial cavity. $\mathbf{P G}$, right pedal ganglion. $\mathbf{P R}$, right posterior retractor muscle. $\mathbf{R}_{\text {, rectum. }}$ RM, line of attachment of right lobe of mantle. RN, space between right and left lobes of mantle above the posterior adductor muscle. $\nabla$, ventricle. $\mathbf{\nabla} \mathbf{M}$, visceral mass. $\mathbf{W}$, edge of left mantle-lobe. 
vertical and horizontal bars, united to form a kind of trelliswork with very small meshes, the greater number of which are blocked up by fusion of the deeper surfaces of the bars with one another.

The two lamellæ of each gill are further united at intervals by thick vertical ridges, parallel to the vertical bars. In the case of the inner gill these ridges are at intervals of about twenty bars; in the outer gill they are more numerous, occurring at about every seventh bar.

1. The outer gill.

a. The outer lamella of the outer gill is attached along the whole length of its dorsal border to the inner surface of the mantle, close to its line of origin from the body-wall. This line of attachment of the gill runs obliquely across the body, its anterior end being near the dorsal surface a little below and behind the umbo, while its posterior end is below and behind the posterior adductor.

b. The inner lamella of the outer gill is attached along its whole length to the outer lamella of the inner gill, and to the body-wall.

Turn the outer gill back, so as to see its attachment to the inner gill.

2. The inner gill.

a. The outer lamella of the inner gill is attached along its whole length, as just noticed, to the inner lamella of the outer gill.

b. The inner lamella of the inner gill varies in its attachment at different parts of its length.

Turn the inner gill back so as to expose the dorsal border of its inner lamella.

i. The anterior part of the dorsal border, about a quarter of its total length, is attached to the side of the visceral mass.

ii. The middle portion has a free edge, and extends back as far as the hinder border of the foot. 
iii. The posterior part, behind the foot, is fused with the corresponding lamella of the gill of the opposite side, forming the partition between the inhalent and exhalent chambers of the mantle-cavity.

3. The supra-branchial or exhalent passages are wide longitudinal channels running just above the bases of the gills, and continuous with the interlamellar spaces of the gills.

Alongside the foot there are two such passages on each side, one above the outer gill, and one above the inner gill. The latter communicates with the large branchial chamber through the longitudinal slit between the inner lamella of the gill and the hinder part of the visceral mass.

Behind the foot there are only three passages, the two inner ones becoming continuous with each other; and beneath the posterior adductor the three unite to form a wide shallow passage, opening behind into the cloacal chamber.

Pass a seeker into the cloacal cavity, and then forwards along the supra-branchial passages above the bases of the gills. Lay open first the outer passage of the right side, and then the inner one; taking care not to damage the kidney, which lies immediately above them. Note that the inner passages of the two sides are continuous with each other behind the foot, and that they communicate with the branchial cavity through the slits between the inner gills and the body.

The respiratory stream of water, kept up by the cilia on the mantle and gills, enters at the inhalent aperture, passes forwards along the branchial cavity and then makes its way through the meshes of the gills into the supra-branchial passages. It then passes back along the supra-branchial passages to the cloacal cavity, and so out.

Respiration is probably effected mainly by the inner surfaces of the mantle-lobes, and not by the gills. 


\section{The structure of the gills.}

Cut the gills across, and examine the cut edges with a pocket lens. Separate the two lamella from each other; and mount small pieces of them and examine with the microscope.

For a more minute study of the structure of the gills microscopical sections should be made through various parts.

The individual bars are slender, vertical, laterally compressed rods, the inner edges of which, i.e. those turned towards the space between the two lamellæ of the gill, are in most cases expanded laterally, and fused with their neighbours.

The bars are elothed with a single layer of ciliated epithelial cells, which are cubical over the greater part of the surface, but columnar along the outer edge, a row of cells along each side of this outer edge having peculiarly long cilia. Each bar is strengthened by a double chitinous rod, which lies close to its outer border.

The inner or deeper parts of the bars consist chiefly of lacunar tissue, i.e. a loose network of branched cells, the meshes of which contain blood. Distinct blood-vessels are comparatively few in number; they are of large size, and lie principally in the interlamellar rods which bolt together the two lamellæ of each gill.

\section{The Circulatory System.}

1. The pericardial cavity is an elongated space of considerable size lying along the dorsal surface of the animal, ventral to the ligament, and above the bases of the gills. It contains the heart, and is traversed by the rectum. Its walls are thin and semitransparent behind; thicker and spongy in front. Through them the pulsations of the heart can be seen.

Open the pericardial cavity by a longitudinal incision along the right side, and cut away as much of its walls as is necessary to expose the heart fully. 
2. The heart consists of three chambers, a median ventricle, and right and left auricles; the latter returning to the ventricle the blood from the mantle-lobes and gills of the right and left sides respectively.

a. The right auricle is a triangular sac with very thin transparent walls. The base of the triangle lies along the dorsal border of the gills of the right side, from which it receives blood; the apex is attached to the side of the ventricle about the middle of its length, and opens into it by a valved aperture.

To see the auricle, press the gills down gently.

b. The left auricle is similar to the right, and returns the blood from the left gills and mantle-lobe.

c. The ventricle is an elongated thick-walled muscular sac of a reddish colour, lying in the middle of the pericardial cavity, and receiving the auricles laterally. Its posterior end is enlarged and bilobed.

The ventricle surrounds the rectum, which runs straight through the pericardial cavity from end to end, apparently traversing the ventricle, though not doing so in reality. In the early stages the ventricle lies completely below the rectum, but during development its anterior end extends up at each side, and the two processes meet each other and unite above the rectum, completely surrounding it.

3. The arteries.

There are two main arteries, one arising from each end of the ventricle, which convey the aërated blood from the heart to all parts of the body. Their main branches may be followed by injecting them from the ventricle.

a. The anterior aorta is a continuation of the anterior end of the ventricle: it runs forwards along the dorsal surface of the rectum, enters the visceral 
mass, and divides into visceral and pedal arteries, which supply the anterior two-thirds of the animal.

b. The posterior aorta arises from the posterior end of the ventricle, and runs backwards beneath the rectum: it divides into right and left branches, which supply the hinder part of the body.

4. The veins, except the largest ones, cannot be dissected without injecting them, and even then they are difficult to follow.

a. The vena cava is a median longitudinal vessel lying beneath the floor of the pericardium : it receives, in front, large veins returning blood from the foot and visceral mass; and behind, much smaller vessels from the posterior part of the body.

b. The afferent branchial veins are a pair of large vessels, running along the lines of attachment of the outer and inner gills to each other. Vessels arise from them which carry blood to the gills.

c. The efferent branchial veins run along the bases of the outer lamellæ of the outer gills: they return to the auricles the blood from the gills, and also receive at their anterior and posterior ends large vessels returning aërated blood from the mantle-lobes.

\section{The course of the circulation.}

The ventricle receives arterial blood from the auricles, and drives it through the arteries all over the body. The blood from the foot and viscera is returned to the vena cava, from which it passes through the kidneys to the gills. From the gills it is returned by the efferent branchial veins to the auricles. The blood from the mantle-lobes is returned direct to the auricles, and does not pass through either the kidneys or the gills.

The circulation is in great part carried on through irregular channels or lacunæ. 


\section{The Kidneys.}

The bidneys, or 'organs of Bojanus,' are paired, and lie side by side just beneath the pericardium.

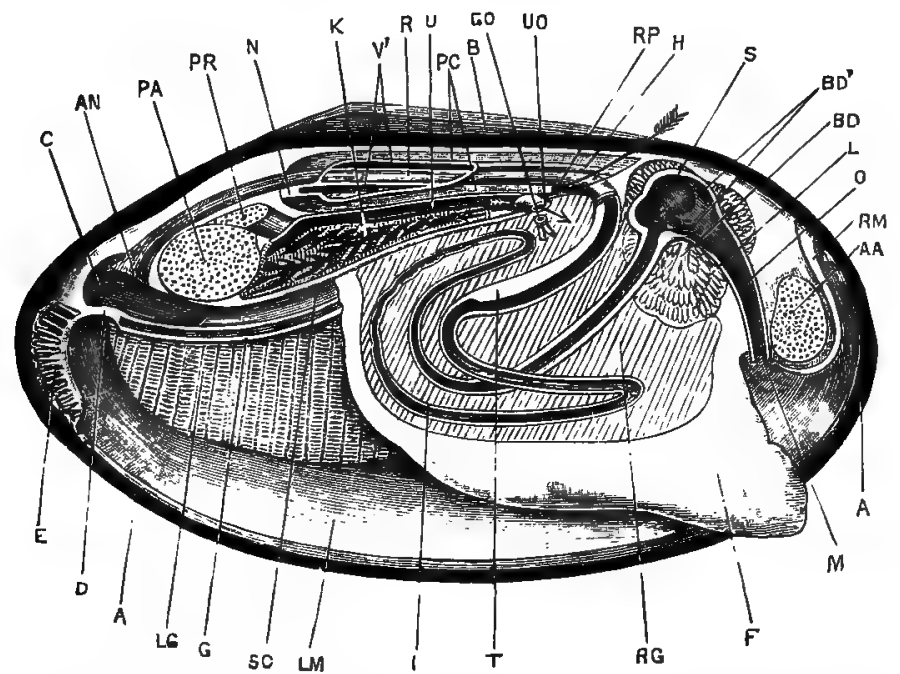

FIG. 27.-Anodonta cygnea. Diagrammatic view from the right side. The right valve of the shell, the right mantle-lobe, the right gills, and the right auricle, have been removed. The alimentary canal has been laid open from the right side as far as the point where it enters the pericardial cavity. The pericardial cavity, the ventricle, and the right kidney and ureter have been laid open from the right side. The longer.arrow passes from the pericardial cavity through the right reno-pericardial aperture into the right kidney. The shorter arrow passes through the external aperture of the right ureter. (A, M. M.)

$\mathbf{A}$, margiu of the left valve of the shell in which the animal is lying. $\mathbf{A} \mathbf{A}$, anterior adductor muscle. AN, anus. B, Iigament of the shell. $\mathbf{B D}$, bileduct. $\mathbf{B D}^{\prime}$, openings of bile-ducts into stomach. $\mathbf{C}$, cloacal cavity. $\mathbf{D}$, septum between the closcal and branchial cavities. $\mathbf{E}$, tentacles bordering inhalent aperture. $\mathbf{F}$, foot. $\mathbf{G}$, line of fusion of inner lamellæ of right and left inner gills. GO, genital aperture. $\mathbf{H}$, anterior aorta. I, intestine. $\mathbf{K}$, kidney. $\mathbf{L}$, liver. $\mathbf{L} \mathbf{G}$, inner gill of left side. $\mathbf{L M}$, left mantle-lobe. $\mathbf{M}$, mouth. $\overrightarrow{\mathbf{N}}$, posterior aorta. $\mathbf{O}$, cesophagus. PA, posterior adductor muscle. $\mathbf{P C}$, pericardial cavity. $\mathbf{P R}$, right posterior retractor muscle. $\mathbf{R}$, rectum. $\mathbf{R G}$, genital gland-ovary or testis. RM, line of attachment of right lobe of mantle. RP, right reno-pericardial aperture. $\mathbf{S}$, stomach. $\mathbf{S C}$, median supra-branchigl canal, lying above the inner-gills. $T$, typhlosole. $U$, right ureter. UO, external opening of right ureter. $\mathbf{V}^{\prime}$, cavity of ventricle. 
Each kidney is a wide thin-walled tube, doubled on itself so that its two ends are closely approximated. These ends are anterior, and placed opposite the anterior end of the pericardium, while the loop is posterior and lies against the posterior adductor muscle.

The two limbs of the loop are very different: the ventral limb, or kidney proper, has spongy walls, and is lined throughout by a darkly pigmented glandular epithelium, so that the whole limb appears black. The dorsal limb, or ureter, is a wide thin-walled tube which conveys the excretory products forwards to the external opening.

Dissect the right kidney as described below, examining first the ureter, and then the glandular portion.

1. The external opening of the ureter is on the side of the body between the two lamellæ of the inner gill, and a short distance in front of the point at which its inner lamella becomes free from the body.

Turn back the gills of the right side; and pass one blade of a fine pair of scissors between the lamella of the inner gill, through the anterior end of the slit between the inner lamella and the body. Cut forwards through the inner lamella parallel to its base, and about an eighth of an inch from it. Separate the cut edges of the lamella, and gently brush the parts clean.

The opening of the ureter is a small hole, with rather prominent white lips. Almost immediately below it is a smaller and less conspicuous opening, the aperture of the genital duct, which should be recognised at once.

\section{The ureter.}

Remove the gills of the right side completely, cutting along their lines of attachment to the body and mantle. Inflate the ureter through its opening, and slit it up along its whole length.

The ureter is a wide thin-walled passage lying above and to the outer side of the kidney. Its roof 
is continuous with, and inseparable from, the floor of the pericardium; and its floor is continuous with the roof of the kidney. On its inner side it is separated from the ureter of the other side by the vena cava. The two ureters communicate with each other through a large slit-like opening near their anterior ends.

3. The kidney appears as a black sausage-shaped body lying beneath the ureter. Posteriorly it enlarges considerably, and is in close contact with the posterior adductor muscle, which it partially embraces.

Lay open the kidney longitudinally with scissors.

Its walls are thrown into thick spongy folds, clothed with a black glandular epithelium. The enlarged posterior end opens into the hinder end of the ureter.

4. The reno-pericardial openings are a pair of crescentic slits at the anterior end of the pericardial cavity, in the angle between the rectum and the floor of the pericardium; they lead from the pericardial cavity into the anterior ends of the right and left kidneys respectively.

Find the reno-pericardial opening on the right side, and pass a seeker through it, and backwards along the kidney. Slit open the passage, using the seeker as a guide.

The pericardial cavity is developmentally a part of the body-cavity or cœlom. Hence in the mussel, as in the earthworm and in other animals generally, the kidneys are tubular organs with glandular walls, leading from the body-cavity to the exterior.

From the external apertures the excretory products are carried back by the exhalent respiratory stream along the supra-branchial passages to the cloacal cavity, and so out.

\section{E. The Nervous System.}

The nervous system of the mussel consists of three main pairs of ganglia widely distant from one another, but united 
by nerve-connectives, and giving off nerves which supply the various organs.

The ganglia are about the size of large pin-heads, and are of a bright orange colour which renders them easy to see.

1. The cerebral ganglia are placed one on each side of the hinder border of the mouth, just above the line of attachment of the mantle-lobe, and below and in front of the protractor muscle. The ganglion lies very close to the surface, just beneath the skin, and is readily exposed if its exact position be determined first.

The two cerebral ganglia are connected together by a commissure which runs round the front of the mouth. Each ganglion also gives nerves to the palps, the anterior adductor muscle, and other adjacent parts, and is united by nerve-connectives with the other ganglia of its side.

The cerebral ganglia of the mussel probably correspond to the cerebral (supran-œsophageal) and pleural ganglia of other molluses, such as the snail.

2. The pedal ganglia are a pair of closely apposed ganglia about the same size as the cerebral. They are placed in the foot, along the line of junction of the visceral portion with the foot proper, and about a third of the length of the foot from its anterior end.

To find the pedal ganglia split the anterior part of the foot with a scalpel in the median plane, and dissect the two halves apart until the ganglia are met with. The junction of the muscular and visceral portions is very clearly defined, and the ganglia are placed close to it.

Each pedal ganglion gives nerves to the foot, one of these supplying the auditory organ or otocyst, which is placed a little behind and below the ganglion.

3. The cerebro-pedal connectives are a pair of nerve-cords connecting the cerebral ganglia with the pedal ganglia. They run in an almost straight course between the two ganglia, and are easily exposed. 
4. The visceral or parieto-splanchnic ganglia are a pair of closely apposed ganglia, situated on the under surface of the posterior adductor muscle about the middle of its length.

Pass a seeker into the cloacal cavity, and along the median supra-branchial passage, and lay open this latter, if it has not already been done. The visceral ganglia are then readily found in the position noted above.

From the ganglia nerves are distributed to the posterior adductor, the mantle, and other parts.

5. The cerebro-visceral connectives are a pair of long nerve-cords which connect the cerebral with the visceral ganglia.

From each cerebral ganglion the connective curves upwards and backwards through the visceral mass, lying close to the surface. It then runs straight back along the inner surface of the kidney to the visceral ganglion. The part in relation with the kidney is very easy to disseet: in front of the kidney the dissection is more troublesome, but presents no serious difficulty.

\section{F. The Digestive System.}

1. The alimentary canal is a convoluted tube the greater part of which lies in the visceral mass, imbedded in the liver and generative organ, while the terminal portion runs back through the pericardial cavity to the anus.

Insert a seeker into the mouth, and using this as a guide slit up the oesophagus and stomach with scissors. Slit open in a similar fashion the whole length of the intestine, beginning at the rectum and working backwards to the stomach (cf. fig. 27, p. 91).

a. The mouth lies immediately behind the anterior adductor, and between the lips formed by the palps : there are no jaws. The food, which consists of minute organisms of various kinds, both 
animal and vegetable, is swept in at the hinder end of the shell by the inhalent stream of water, and carried forwards to the mouth by the action of the cilia covering the palps.

b. The cesophagus is a short straight tube passing almost vertically upwards behind the anterior adductor.

c. The stomach is a slightly dilated chamber whose cavity is partially subdivided by folding of its walls.

d. The intestine arises by a small orifice from the ventral surface of the stomach, and runs backwards and downwards into the visceral mass, where it is closely surrounded by the generative gland. It follows the course shown in fig. 27, and finally passes into the rectum.

e. The rectum, which is rather wider than the intestine, runs upwards through the visceral mass to the anterior end of the pericardial cavity, through which it runs back horizontally, surrounded by the ventricle. Behind the pericardial cavity it turns slightly downwards, runs over the posterior adductor, and opens into the cloacal cavity by a slit-like anus.

The ventral wall of the rectum is folded so as to form a prominent longitudinal ridge, the typhlosole, projecting into its cavity. This typhlosole is largest at the junction of the rectum with the intestine.

2. The liver is a large many-lobed gland surrounding the stomach and adjacent parts of the cesophagus and intestine, and opening into the stomach by several bile-ducts.

\section{G. The Reproductive System.}

The sexes are separate. The reproductive organs are simple, and very similar in the two sexes. 
The ovary, or testis, is very large, and, when the sexual products are ripe, fills up the greater part of the visceral mass between the foot and the kidneys. The ducts converge on each side to the genital aperture, which lies on the side of the body immediately below the opening of the ureter.

Tease a small portion of the ovary, or testis, in salt solution or glycerine; cover, and examine it with low and high powers.

In the female the eggs, after they escape from the genital aperture, pass in large numbers into the space between the two lamellæ of the outer gill, which they distend very greatly. Here they develop into embryos known as glochidia, which are in many respects very unlike the parent. They have bivalved shells, each valve of which is triangular and incurved at its apex so as to form a sharp tooth, the teeth of the two valves constituting an efficient pair of pincers. There is only a single adductor muscle; the gills are absent or rudimentary, as also is the foot. A long coiled filament, the byssus, serves to anchor the glochidium to the gill.

Take a female in which the outer gills are distended with embryos : cut the gill across; remove some of the embryos, mount them on a slide in water, and examine them with a microscope.

\section{EXAMINATION OF TRANSVERSE SECTIONS.}

Several points in the anatomy of the mussel, and notably the relations of the gills, kidneys, and heart, are best studied by means of a series of transverse sections.

For this purpose take a good-sized specimen, and put it into $\frac{1}{4}$ per cent. chromic acid, wedging the valves slightly open so that the acid may have free access to the branchial cavity. After a couple of days transfer it to spirit and keep it till wanted.

To make the sections, remove both valves of the shell carefully; place the animal on a board, and cut it transversely 
with a razor into a series of slices about a quarter of an inch thick; arrange the sections in order in a dissecting dish under water; examine and draw them.

The most instructive sections are those passing, (1) through the renal and reproductive apertures; (2) through the middle of the ventricle; (3) through the posterior adductor. The chief features of these sections are as follows.

\section{A. Transverse Section through the Renal and Reproductive Apertures.}

The section passes through the widest part of the body, a little way behind the umbo.

1. The mantle-folds arise very close to the dorsal surface, one on each side of the pericardial cavity. The folded margin which secretes the two outer layers of the shell should be noticed.

2. The visceral mass is large and laterally compressed, and lies in the middle of the section between the two mantle-lobes. The chief part of its substance is made up of the lobes of the ovary, or testis. The ventral edge of the visceral mass is produced into the wedge-shaped muscular foot.

3. The alimentary canal. The coils of the intestine are seen in the visceral mass, imbedded in the generative gland, and cut across about half a dozen times. Lying in the pericardial cavity in the median line immediately beneath the dorsal surface is the rectum, in which the typhlosole or fold of its ventral surface is well seen.

4. The gills lie in the dorsal half of the mantle-cavity, between the visceral mass and the mantle. The attachments of their lamellæ to the body-wall are well seen. The inner lamella of each inner gill is attached to the base of the visceral mass.

At the base of each gill is the supra-branchial canal, along which the respiratory stream of water flows backwards to the cloacal cavity. 


\section{The circulatory system.}

a. The pericardial cavity is nearly circular in outline; its roof and sides are thick and spongy, forming what is spoken of as Keber's organ.

b. The anterior aorta lies on the dorsal surface of the rectum, and its cavity is slit-like in section.

c. The vena cava lies in the median plane between the excretory organs and the visceral mass.

6. The excretory organs are paired, and lie between the pericardium and the visceral mass.

a. The glandular portions, or kidneys proper, are cut across close to their anterior ends. They are seen as a pair of small tubes, with glandular longitudinally folded walls, lying side by side just above the visceral mass and opposite the bases of the inner gills.

If the section has passed a little in front of the excretory apertures, the passages from the pericardial cavity to the anterior ends of the kidneys are well seen.

b. The ureters are eut through at the part where they communicate with each other across the median plane: they appear as a wide cavity immediately below the pericardium and above the kidneys. The external openings are to the outer sides of the kidneys, between the two lamellæ of each inner gill.

7. The reproductive apertures are immediately ventral to the excretory apertures; they may be followed with a seeker into ducts leading from the genital gland.

B. Transverse Section through the Middle of the Ventricle.

1. The mantle-lobes have the same appearance as in the previous section.

2. The visceral mass is cut across close to its posterior end, and hence is much smaller than in the previous section : it is occupied almost entirely by the ovary, or testis. 
3. The alimentary canal. The intestine is cut across twice in the visceral mass; and the rectum with the typhlosole is cut across as it lies in the pericardial cavity.

4. The gills. The attachments of the gills are the same as in the previous section, except that the inner lamella of the inner gill ends in a free edge above.

At the base of each gill, between its lamellæ, is a wide supra-branchial canal. The inner canal of each side communicates with the branchial cavity through the slit between the unattached inner lamella of the gill and the base of the visceral mass.

\section{The circulatory system.}

a. The pericardial cavity is very much larger and has thinner walls than in the previous section, and is triangular in shape.

b. The ventricle surrounds the rectum : its outer wall is thick and muscular, its inner wall thin.

c. The auricles are a pair of very thin-walled sacs lying at the sides of the pericardial cavity. Each is attached along its outer side to the wall of the cavity, and by its inner border to the ventricle, into which it opens by a slit-like valved aperture.

d. The vena cava is a median thin-walled tube lying in the floor of the pericardium.

6. The excretory organs.

a. The kidneys are two wide tubes with thick spongy walls, whose epithelium is glandular, pigmented, and folded. They lie below the pericardium, above the visceral mass, and opposite the bases of the inner gills. Their inner walls are in contact with each other below ; and are separated dorsally by the vena cava, from which large sinuses enter them.

b. The ureters are a pair of thin-walled tubes lying along the dorsal surface and outer sides of the 
kidneys, immediately below the pericardium. They are separated from each other in the median plane by the vena cava.

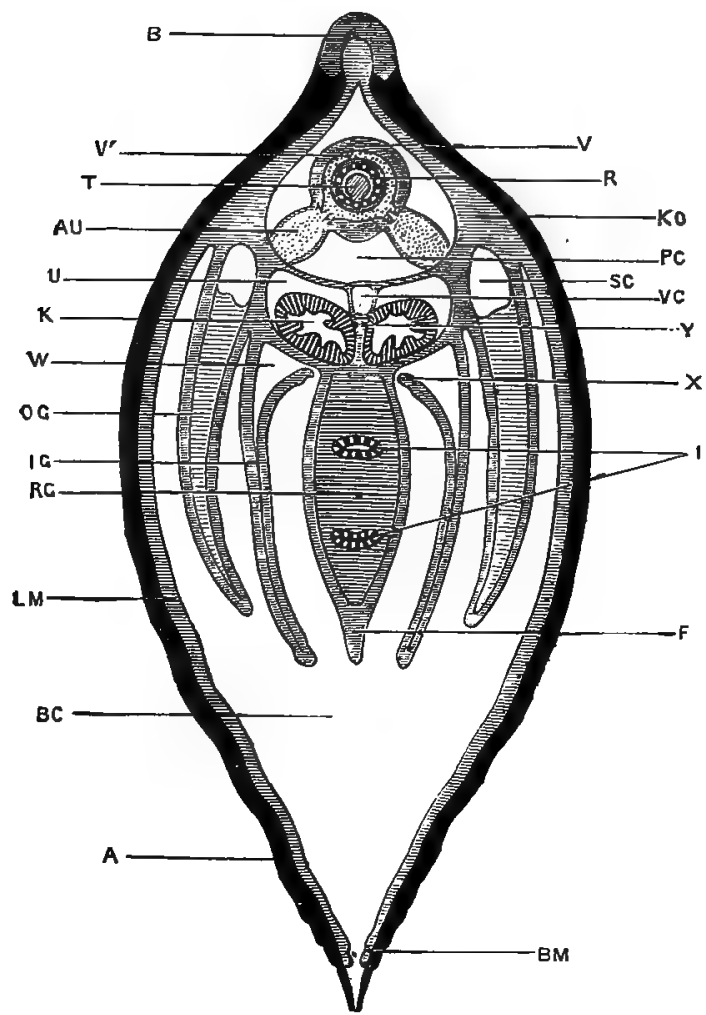

FIt. 28.-Anodonta cygnea. Transverse section of the animal passing through the middle of the length of the ventricle. The cavity of the heart is dotted. (A. M. M.)

A, shell. AU, left auricle. $\mathbf{B}$, ligament. BC, branchial cavity. $\mathbf{F}$, foot. $\mathbf{I}$, intestine. IG, left inner gill. $\mathbf{K}$, left kidney. KO, Keber's organ of the right side. IMM, left mantle-lobe. OG, left outer gill. $\mathbf{P}$, pericardial cavity. $\mathbf{R}$, cavity of rectum. RG, genital gland, ovary or testis. RM, edge of right mantle-lobe. SC, supra-branchial canal of right outer gill. T, typhlosole. $\bar{U}$, left ureter. $\mathbf{V}$, wall of ventricle. $\nabla^{\prime}$, cavity of ventricle. VC, vena cara. W, supra-branchial canal of left inner gill. $\mathbf{X}$, free upper edge of the inner lamella of the right inner gill : between this edge and the body is the slit through which the branchial cavity communicates with the supra-branchial canal of the inner gill. $\mathbf{Y}$, the right cerebro-visoersl connective. 


\section{The nervous system.}

The connectives between the cerebral and visceral ganglia lie side by side, between the two kidneys and immediately below the vena cava.

C. Transverse Section through the Middle of the Posterior Adductor Muscle.

1. The posterior adductor is a large mass of transverse muscular bands running across the dorsal part of the section.

2. The gills are cut across close to their hinder ends, and are small. The inner lamellæ of the two inner gills are united with each other in the median plane, forming a horizontal septum which separates the large branchial chamber below from the wide but shallow suprabranchial or exhalent chamber.

3. The rectum lies immediately dorsal to the adductor muscle. The typhlosole is still present.

4. The visceral ganglia lie side by side on the ventral surface of the adductor. 


\section{Chapter VII.}

\section{THE EDIBLE SNAIL. Helix pomatia.}

THE snail is a terrestrial animal, feeding on leaves and other vegetable matter which it rasps off with its toothed tongue or odontophore. The dorsal surface of the body carries a spirally coiled univalve shell, which lodges most of the viscera, and within which the entire animal can be withdrawn for protection. It breathes air by means of a pulmonary chamber, formed by a fold of skin - the mantle-on the dorsal surface, It is hermaphrodite, but incapable of self-fertilisation.

The edible snail occurs in many parts of England, and can be readily obtained from dealers in Paris. On account of its large size it is preferable to the commoner English species, $H$. aspersa, to which, however, the following description will apply with but slight modification.

Snails are best killed by drowning them in water, when they die in an expanded condition in about two days. In winter the mouth of the shell is closed by a plate of calcified mucus, the epiphragm, which must be removed before putting the snail in water. When dead the snail should be put into spirit for a short time before it is dissected, in order to coagulate the mucus.

\section{EXTERNAL CHARACTERS.}

\section{A. Before Removal of the Shell.}

1. The shell is a right-handed conical spiral, each turn of which overlaps and conceals the greater part of the preceding turn. In the fully formed shell there are about four and a half turns in the spiral. 
The shell is placed obliquely on the back of the animal with its apex to the right: its mouth is in front, and faces downwards and to the right. Like the shell of the mussel it is a cuticular structure, i.e. it is outside the epidermis. It has therefore no power of interstitial growth, but can only increase in size by the addition of new shell-matter round its free edge or mouth.

The shell will be more fully described after its removal from the body.

2. The part of the animal outside the shell. This consists chiefly of the head and the foot, and will vary greatly according to the degree of protrusion of the animal from the shell. It has a mammillated surface.

a. The head is the rounded anterior extremity of the animal. It bears the two pairs of tentacles and the mouth.

i. The posterior or dorsal tentacles are a pair of long fleshy cylindrical processes of the dorsal surface of the head. They are very freely movable, and can be completely withdrawn by invagination when the animal is disturbed. Each bears an eye at its extremity, which is only visible when the tentacle is fully protruded.

ii. The anterior or ventral tentacles are a pair of much smaller processes arising from the sides of the head, below and in front of the larger tentacles. Like these latter they can be completely withdrawn by invagination.

b. The foot is a large flattened muscular expansion of the ventral wall of the body. It is of an elongated oval shape, commencing in front with a rounded anterior border immediately below and behind the head, and narrowing behind to a long pointed extremity. A median longitudinal groove runs along its ventral surface. 
c. The collar is a thickened fleshy rim surrounding the mouth of the shell, and separated by a deep groove from the body, which it encircles. It is thicker at the sides than in front or behind, and is especially thick on the right side in the angle of the mouth of the shell.

d. The external apertures.

i. The mouth is a small aperture on the ventral surface of the anterior end of the head. It is bordered at the sides by two prominent lateral lips, and is separated from the foot by a less conspicuous inferior lip.

Pass a seeker into the mouth, and then upwards and backwards into the cavity of the buccal mass.

ii. The genital aperture is on the right side of the head, behind and below the larger tentacle, and immediately behind the posterior edge of the right lip. From it a groove runs backwards along the dorsal surface of the body: a similar groove runs along the left side.

Pass a seeker into the genital aperture, and backwards along the genital passage.

iii. The respiratory or pulmonary aperture. On the right side of the collar at its widest part is a deep notch, at the dorsal end of which is a large round hole, the respiratory aperture, leading into the pulmonary chamber.

Wash away the mucus from the collar on the right side; clean the notch in the collar, and pass a seeker through the respiratory aperture into the pulmonary chamber.

iv. The anus is a smaller slit-like hole, immediately below and to the right of the respiratory aperture. From it a spout-like groove runs forwards to the edge of the collar.

Clean the anal opening and groove, and pass a seeker into the anus and back along the rectum. 
v. The aperture of the pedal gland (p. 121) is immediately below the inferior lip, between the head and the foot.

Pass a seeker into the aperture, and backwards along the gland.

\section{B. After Removal of the Shell.}

Twist the animal out of the shell, taking care not to damage the upper part of the body in so doing. The shell: comes off readily if the snail has been put in spirit for a short time after drowning.

1. The shell.

a. The colour varies a good deal in different specimens. The ground colour is a yellowish brown, markedby darker longitudinal bands running round the spiral, and by transverse bands corresponding tothe lines of growth.

b. The nucleus is the oldest part of the shell, at the top of the spiral, corresponding to the umbo of the. mussel-shell.

c. The mouth is oval, and notched posteriorly by the penultimate turn of the shell. Its lip is smooth. and slightly everted.

d. The lines of growth are transverse ridges parallel. to the mouth, and indicating successive positions. of the mouth during the growth of the shell.

e. The umbilicus is an opening on the under surface of the shell leading into the hollow axis orcolumella. It is sometimes closed.

Clip away one half of the shell with bone-forceps, and grind the cut edges flat on a file or stone:

$f$. The columella is the axial portion of the shell round which it is coiled; it is formed by the inner wall of the several turns, each of which overlaps rather more than half of the preceding one.

g. The structure of the shell. The shell, like that of 
the mussel, consists of three layers, of which the outer and middle ones are formed by the collar, and consequently can only increase by additions round the mouth of the shell. The inner layer is formed by the integument covering the visceral mass.

i. The periostracum or outer layer is horny and uncalcified. To it the colour of the shell is due. It is usually rubbed off the uppermost turns of the shell, leaving the middle layer exposed.

ii. The middle layer is much the thickest of the three. It is densely calcified, and has an opaque porcellanous appearance.

iii. The inner or nacreous layer has a glistening, pearly appearance, and a very smooth surface. It is formed by the superposition of a number of delicate, densely calcified laminæ.

2. The visceral hump is the part of the snail which is permanently lodged within the shell. It is the elongated and spirally coiled dorsal part of the animal, and contains the greater part of the viscera. It is covered by a very thin layer of integument which secretes the nacreous layer of the shell, and which is sufficiently transparent to allow the several organs to be seen through it.

For convenience of description the basal or largest turn of the spiral, commencing with the collar, will be spoken of as the first turn, the next as the second, and so on.

Place the snail in a dissecting dish under water, and determine the positions of the organs mentioned below. Note. that the outer side of the spiral corresponds to the animal's left side, and the inner side to the animal's right.

a. The kidney is a large, somewhat triangular body, of a yellowish-white colour and granular appearance, lying about half-way round the first turn of the spiral, opposite to the collar. 
b. The pericardium, enclosing the heart, is an oval sac, lying immediately to the left of the anterior half of the kidney, which is notched to receive it.

c. The mantle, forming the thin roof of the respiratory cavity, extends forwards along the dorsal surface and left side of the body, from the pericardium and kidney to the collar.

d. The liver is a dark reddish-brown mass, which begins immediately beyond the pericardium and kidney, and extends to the top of the spiral.

e. The intestine is a thin-walled tube, much paler in colour than the liver, and appearing on the surface at one or two places in the first and second turns of the spiral.

f. The rectum runs along the right border of the first half-turn of the spiral, just below the ridge-like thickening which borders the right or inner side of the spiral.

g. The albumen gland is a large white or yellowish mass at the junction of the first and second turns of the spiral. It separates the right and left lobes of the liver from each other.

h. The hermaphrodite gland is a small yellowish body on the inner side of the upper half of the second turn of the spiral.

i. The columellar muscle, by which the animal can be retracted within the shell, runs along the right or inner side of the spiral, and is attached to the columella in the upper half of the first turn.

\section{DISSECTION OF THE MANTLE-CAVITY AND ORGANS IN RELATION WITH IT.}

The respiratory or mantle-cavity of the snail lies along the dorsal surface, commencing at the collar, and extending backwards rather more than three-fourths of the way round the first turn of the spiral. It is formed by a transverse fold of skin which, arising from the dorsal surface of the visceral 
mass, grows forwards along the back, ending in front in a thickened rim, the collar. Between the mantle-fold and the back of the animal, is the large mantle-cavity, which at first. opens anteriorly by a wide orifice. Fusion of the collar with the body-wall reduces this orifice to a small round aperture placed on the right side-the respiratory or pulmonary aperture-through which air alternately enters and leaves the cavity during respiration.

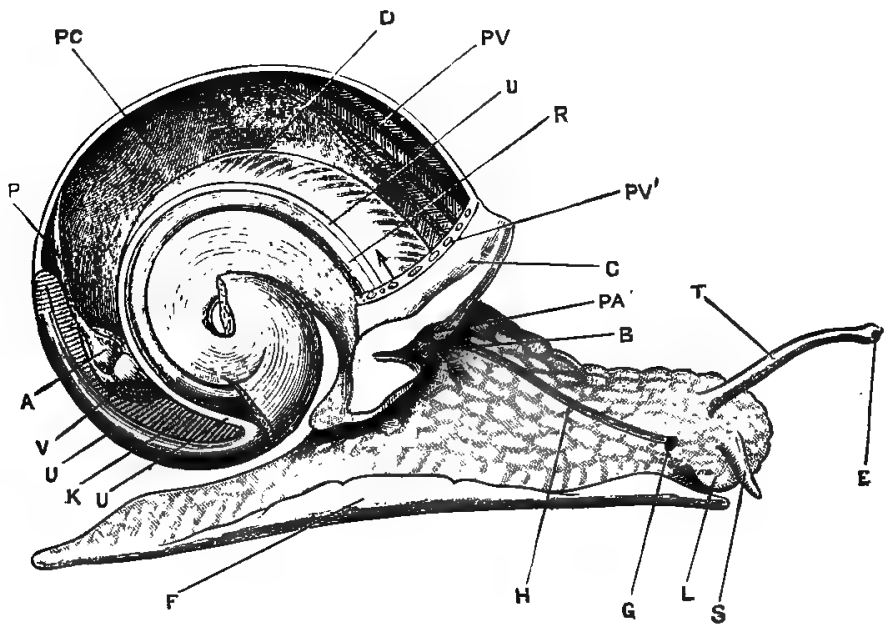

Fra. 29.-Helix pomatia. Dissection from the right side to show the mantle-cavity and organs in relation with it. The arrow passes through the pulmonary aperture into the mantle-cavity. The shell and the upper part of the spiral visceral mass have been removed. (A. M. M.)

A, anricle. B, anus. C, collar. D, dorsal body-wall forming the foor of the mantie-cavity. $\mathbb{E}$, ege. $\mathbf{F}$, foot. $\mathbf{G}$, genital aperture. $\mathbf{H}$, groove leading to genital aperture. $\mathbf{K}$, kidney. $\mathbf{L}$, lip. $\mathbf{P}$, pulmonary vein. $\mathbf{P A}$, pulmonary aperture. PC, pulmonary or mantle-cevity. PV, pulmonary vessels in roof of mantle-cavity. $\mathbf{P V}^{\prime}$, cut ends of pulmonary vessels. $\mathbf{R}_{\text {n }}$ rectum. $\mathbf{S}$, anterior tentacle. T, posterior tentscle., $\mathbf{U}$, ureter. $\boldsymbol{\nabla}$, ventricle.

In close relation with the mantle-cavity are the rectum, the heart, and the kidney and ureter.

Place the snail in a dissecting dish under water, and pin it firmly down through the foot, with the dorsal surface upward. Open the mantle-cavity by a transverse incision 
through its thin roof, immediately behind the collar. Extend this incision backwards along each side of the cavity to its posterior end, cutting along the left side close to the junction of the mantle and body-wall, and along the right side about a quarter of an inch to the left of the prominent ridge that borders this side, midway between this and the right border of the kidney.

Turn back the roof of the mantle-cavity, and pin it down so as to fully expose the pericardium and kidney.

\section{A. The Walls of the Mantle-cavity.}

1. The roof and sides of the cavity are formed by the mantle itself, a thin fold of skin which bears on its under surface a very rich network of large bloodvessels, especially abundant in front and along the right side.

These vessels, in which the blood is aërated, unite to form a large trunk, the pulmonary vein, which takes back the arterialised blood to the heart, running along the left side of the kidney.

The mantle is greatly thickened in front to form the collar : posteriorly it is thickened by the kidney and pericardium, both of which lie in its substance.

2. The floor of the cavity, which is formed by the dorsal wall of the body, is muscular, and strongly arched from side to side. Contraction of its muscles causes flattening of the floor, and thus enlarges the cavity, causing an inrush of air through the pulmonary aperture. On relaxation of the muscles the floor resumes its previous convex shape, diminishing the size of the cavity and effecting expiration.

By alternate contraction and relaxation of the floor of the mantle-cavity the air is continually renewed, and the cavity thus becomes a true lung.

\section{B. The Organs in relation with the Mantle-cavity.}

1. The pericardial cavity and beart.

a. The pericardial cavity, which has already been seen from the exterior, is oval, thin-walled, and half 
an inch or more in length. It lies in the roof of the mantle-cavity at its posterior end and left side and is in close contact with the left side of the kidney about the middle of its length.

Open the pericardial cavity to expose the heart.

b. The heart has two chambers, of about equal size.

i. The auricle is proximal, i.e. nearer the collar, and has thin walls. It receives in front the pulmonary vein, which returns to it aërated blood from the lung.

ii. The ventricle is the pear-shaped distal division of the heart, with thick muscular walls. It receives aerrated blood from the auricle, and drives it through the aorta to all parts of the body.

The blood-vessels will be described later on.

2. The kidney and ureter.

a. The kidney is a large, pale yellow, triangular body in the posterior part of the roof of the mantlecavity. Its left side is notched to receive the pericardium, which lies close against it.

i. The reno-pericardial canal is a narrow ciliated passage leading from the pericardial cavity to the kidney. Its pericardial opening is about the middle of the length of the cavity, opposite the ventricle, and close to the inner or ventral border of the septum between the pericardial cavity and the kidney.

To see the reno-pericardial passage make a small hole into the pericardial cavity, and inject it with carmine or other colouring matter in suspension. This will pass into the kidney along the passage, which can then be readily followed.

ii. The structure of the kidney. The kidney is a sac lined by a glandular epithelium, and with its walls thrown into a large number of lamellar folds projecting into its cavity. 
Slit open the kidney longitudinally: wash it out with a gentle stream of water, and note its structure.

b. The ureter is a thin-walled duct which commences at the proximal end of the kidney and runs back along its right side to the distal end. It then turns sharply forwards, and runs along the right side of the mantle-cavity to its anterior end, lying dorsal to the rectum, and just within the prominent ridge-like fold that marks the junction of the roof and right side of the mantle-carity.

In front it opens into the mantle-cavity immediately above and to the right of the pulmonary aperture. The opening is slit-like, and is continued as two divergent grooves with prominent lips, which run down the right and left sides of the pulmonary aperture, the right groove being continuous below with the spout-like aperture of the rectum.

Insert one blade of a pair of scissors into the pulmonary aperture from the outside, and cut through the collar, keeping close to the left side of the aperture. Turn the right lip of the aperture over to the right side. Pass a seeker into the rectum from the anus so as to identify it. Note the ureter munning along the right side of the rectum, but dorsal to it in the natural position of the parts. Trace the ureter forwards to its opening, and inflate it with a blowpipe. Insert a seeker into the opening; and, using it as a guide, slit up the ureter along its whole length, following it back to the hinder end of the mantle-cavity, and then forwards close alongside the kidney to its anterior end, into which it opens.

3. The rectum is a wide tube running along the right side of the mantle-cavity, ventral to the ureter. It opens in front at the anus, which has already been seen.

Insert a bristle or seeker into the rectum from the anus, and note carefully the relations of the anus and the respiratory and renal apertures. 


\section{DISSECTION OF THE DIGESTIVE SYSTEM.}

Cut away the part of the collar to the left of the pulmonary aperture. Detach the right side of the collar, with the anal aperture, from the body-wall. Separate the rectum from the body by cutting along the right side of the mantle, just below the rectum, as far back as the hinder end of the mantle-cavity. Turn the rectum back, and pin it down.

Make a median longitudinal incision through the floor of the mantle-cavity, and continue it forwards along the head to its anterior end. Dissect the flaps away from the underlying parts, and pin them out right and left.

Carefully dissect off the thin'skin covering the spiral visceral mass; and unravel the reproductive organs, which are white in colour and very bulky, and the alimentary canal, the coils of which are very intimately connected with the liver.

Arrange the reproductive organs on the right, the digestive organs on the left side of the dissection.

The removal of the integument from the visceral mass requires care, but presents no great difficulty. Special care is needed at the posterior end of the mantle-cavity, where the parts are rather firmly bound together.

The dissection is greatly facilitated by frequent washing under the tap.

\section{A. The Alimentary Canal.}

The alimentary canal is a convoluted tube, of nearly uniform size along the greater part of its length, but presenting. special features in its anterior portion.

1. The buccal mass or pharynx is the enlarged anterior end of the canal, into which the mouth opens. It forms. a prominent rounded mass in the head, with stout. muscular walls, and will be more fully examined at a later stage of the dissection. It contains the odontophore (p. 122).

Insert a seeker into the mouth, and note that it pa sses. upwards and backwards into the buccal mass. 


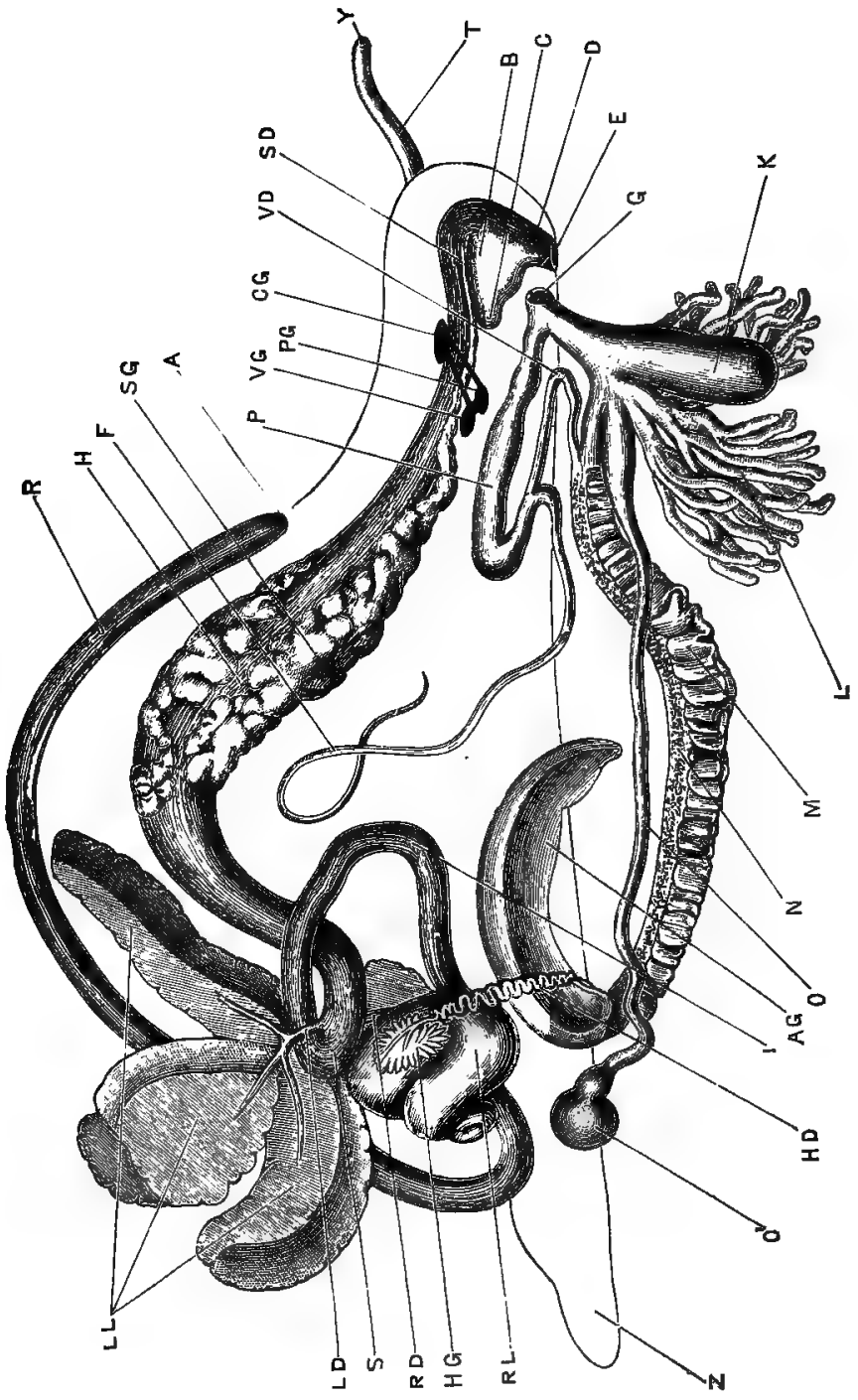


2. The csophagus is a narrow thin-walled tube, which arises from the dorsal surface of the buccal mass and runs backwards, passing into the crop.

3. The crop is a large fusiform thin-walled dilatation of the alimentary canal, lying partly in front of the visceral hump, partly in the first turn of the spire. It is widest about the middle of its length, and gradually narrows behind.

4. The 'stomach' is a slightly dilated loop of the alimentary canal, between the crop and the intestine. It lies close to the surface of the visceral hump, near the commencement of the second turn of the spire, and between the right and left lobes of the liver. Its mucous membrane is thrown into longitudinal folds.

5. The intestine describes a somewhat S-shaped course, and is embedded in the liver, from which it is not very easy to separate it. From the stomach it runs back round the first turn of the spiral, lying in a groove on the outer side of the left lobe of the liver, to the hinder end of the mantle-cavity; where it passes into the rectum. It is superficial along a great part of its course.

6. The rectum has already been traced along the right side of the mantle-cavity to the anus.

\section{B. The Digestive Glands.}

1. The salivary glands are a pair of large white lobulated glands lying on the upper surface and the sides of the crop, to the walls of which they are attached by

Frg. 30.-Helix pomatia. Dissection from the right side to show the digestive and reproductive systems. (A. M. M.)

A, anus, AG, albumen gland. $\mathbf{B}$, buccal mass. $\mathbf{C}$, sac of odontophore. OG, cerebral ganglion. D, jaw. $\mathbf{F}$, mouth. $\mathbf{F}$, flagellum. G, genital aperture. H, crop. HD, hermsphrodite duct. $\mathbf{H}$, hermaphrodite gland. $\mathbf{I}$, intescine. $\mathbf{K}$, dart sac. $\mathbf{L}$, mucous glands. $\mathbf{L} \mathbf{D}$, left bile-duct. $\mathbf{L}$,'left lobes of liver. M, female portion of the common duct. $N$, male portion of the common duct. $O$, receptaculum seminis. $\mathbf{O}^{\prime}$, dilated end of receptaculum seminis. $\mathbf{P}$, penis, retracted. $\mathbf{P G}$, pedal ganglion. $\mathbf{R}$, rectum. $\mathbf{R} \mathbf{D}$, right bile-duct. $\mathbf{R L}$, right lobe of liver. $\mathbf{S}$, 'stomach.' $\mathbf{S D}$, salivary duct. $\mathbf{8 G}$, salivary gland. $T$, tentacle. VD, vas deferens. VG, viscero-pleural ganglion. $\frac{\mathrm{Y}}{\mathbf{y}}$, eye. $\mathbf{Z}$, posterior end of foot. 
numerous strands of connective tissue. Their ducts, which are long and slender, run forwards and open into the buccal mass, one on each side of the csophagus.

2. The liver is a very large bilobed gland, of a reddishbrown colour, which forms a large part of the visceral hump.

a. The left lobe of the liver is the larger of the two, and is partially subdivided into three lobes. It lies in the upper half of the first turn of the spire, immediately behind the mantle-cavity, and is grooved on its outer surface by the intestine. It has three main ducts, which unite to open by a single large aperture into the left side of the stomach.

b. The right lobe of the liver occupies the greater part of the second and upper turns of the spire. It is separated from the left lobe by the posterior end of the crop, the stomach and the commencement of the intestine, and the albumen gland. It has one large duct, which opens into the right side of the stomach, almost opposite to the left. duct, but slightly beyond it.

Slit up the stomach, and wash out its contents. Note the openings of the bile-ducts, and follow these with a seeker into. the lobes of the liver.

\section{DISSECTION OF THE REPRODUCTIVE SYSTEM.}

The snail is hermaphrodite, but does not fertilise its own ova. As is usual in such cases, the reproductive organs are extremely complex.

1. The hermaphrodite gland is a small yellowish body lying on the inner side of the second turn of the spire, and closely imbedded in the right lobe of the liver. Within it both ova and spermatozoa are. produced. 
Place a small piece of the hermaphrodite gland on a slide in a drop of water : tease it slightly: cover; and examine with low and high powers.

The gland consists of a number of finger-like follicles, in each of which ova are developed in the outer wall, and spermatozoa in the more central part. The ova are large round granular cells, with very large reticulate nuclei : the spermatozoa, which are generally aggregated in wisp-like bundles, have small rod-like heads and very long tails.

2. The hermaphrodite duct is a very sinuous duct of a whitish colour, lying along the inner side of the spire close to the columella, and opening into the inner side of the albumen gland.

3. The albumen gland is an elongated compact yellowish white body, varying greatly in size at different times. It lies in the upper half of the first turn of the spire along its inner side, between the stomach and the left lobe of the liver.

4. The common duct arises from the end of the albumen gland, close to the point of entrance of the hermaphrodite duct, and runs forwards through the body almost to the head, lying along the right side of the crop and œsophagus, and slightly ventral to these.

It is very wide, and along the greater part of its length is incompletely divided by a longitudinal septum into two parallel ducts. Of these one, the walls of which are thrown into very numerous and prominent transverse folds, serves for the passage of the ova; while the other, which transmits the spermatozoa, has a comparatively smooth surface, but has its walls thickened by a whitish glandular prostatic mass. At their anterior ends the two ducts separate completely from each other as oviduct and vas deferens respectively.

Slit open the common duct longitudinally; and note the septum, which partially divides it into male and female ducts. 
5. The male duct and its accessory organs.

a. The vas deferens is a slender, slightly convoluted tube of uniform diameter, which runs forwards along the inner side of the female organs, between these and the buccal mass, passes under the retractor muscle of the right upper tentacle, and then runs back to open into the base of the penis.

b. The flagellum is a long tubular diverticulum, which arises from the vas deferens just before it reaches. the penis. It lies along the dorsal surface of the crop and other viscera. In it the spermatozoa are agglutinated together to form a long rod-like body, the spermatophore.

c. The penis is a muscular tube continuous with the vas deferens, and lying somewhat obliquely across the csophagus just behind the buccal mass; it opens to the exterior at the genital pore, through which it can be protruded.

To the penis is attached the retractor penis muscle, a narrow muscular band, which arises. from the floor of the mantle-cavity, and runs downwards and forwards to its insertion.

6 The female duct and its accessory organs.

a. The oviduct is a short thick-walled tube, running forwards from the point at which it leaves the vas deferens.

b. The vagina is a thick-walled tube into which the oviduct opens behind, and which runs forwards to open with the penis at the common genital pore.

c. The mucous glands are two tufts of tubular glands, opening by a pair of apertures into the vagina: each tuft consists of thirty to forty finger-like processes.

d. The dart sac is a large pyriform sac opening into the vagina just beyond the mucous glands. It. 
has very thick walls, and contains a quadrangular calcareous spicule.

e. The spermatheca, or receptaculum seminis, is a long cæcal diverticulum from the hinder end of the vagina. It lies alongside the common duct, and its hinder end is expanded into a globular dilatation just behind the pericardium and kidney. It is of about the same length as the flagellum, and serves to lodge the spermatophore received from another snail.

A small diverticulum is very commonly given off about a third of its length from the vagina. In $H$. aspersa this is largely developed and forms the longer portion of the spermatheca.

Care must be taken not to mistake the spermatheca for the vas deferens, nor to cut away its globular dilatation during the dissection of other parts.

\section{DISSECTION OF THE NERVOUS SYSTEM.}

The nervous system consists of paired ganglionic masses placed above and below the alimentary canal, and joined together by nerve-commissures and connectives so as to form a collar encircling its anterior end. When the head is protruded this collar surrounds the œsophagus immediately behind the buccal mass; but when the head is retracted the buccal mass is pulled back through the collar, which then lies in front of it.

From these principal ganglia, nerves radiate outwards to the various parts of the body. The ganglia are enveloped in a rather dense layer of connective tissue, which must be removed before their shape can be determined.

1. The supra-cesophageal or cerebral ganglia are a pair of closely apposed pyriform masses, forming a broad transverse band across the dorsal surface of the alimentary canal.

From the outer ends of the ganglia large nerves run to both pairs of tentacles and to the lips. 
From their under or ventral surface a pair of buccal nerves run forwards alongside the œsophagus, and end in a pair of small buccal ganglia, which lie at the outer sides of the salivary ducts close to their openings into the buccal mass. The buccal ganglia are united by a transverse commissure, and from them nerves arise supplying the buccal mass.

From the posterior and outer border of each supra-œsophageal ganglion two stout connectives pass downwards and slightly backwards to the subosophageal ganglia. Between these two on each side is the slender auditory nerve.

2. The sub-œsophageal ganglia are a number of closely approximated ganglionic masses, the boundaries of which are difficult to determine. The anterior aorta runs through the centre of the mass, marking the division between the antero-inferior portion, or pedal ganglia, and the postero-superior portion, or visceropleural ganglia.

The pedal ganglia are a pair of closely apposed masses, from which a number of large nerves pass to the foot.

b. The viscero-pleural ganglia consist of at least two pairs of ganglionic masses, closely apposed to each other and to the pedal ganglia. From them large nerves pass outwards to the viscera and to the body-wall.

\section{The structure of the ganglia.}

Remove one of the ganglia; place it in 1 per cent. acetic acid for a short time to soften the connective tissue, and then tease it on a slide in glycerine. Cover; and examine it with low and high powers.

The nerve-cells are large granular cells with very large and distinctly reticulate nuclei, and with long branching processes by which they are connected with one another and with the nerve-fibres. 


\section{THE SENSE ORGANS.}

1. The eyes are placed one at the end of each of the larger tentacles, and are only exposed when these are fully protruded. Each receives a branch from the large nerve supplying the tentacle.

Each eye is a small spherical vesicle, the wall of which is strongly pigmented in its posterior portion, and lined by a layer of retinal cells. The cavity of the vesicle is partially filled by a large cuticular lens, which projects into it from its anterior wall.

2. The auditory organs are a pair of small spherical sacs imbedded in the substance of the pedal ganglia, and containing numerous minute calcareous otoliths. They are placed at the ends of the auditory nerves, which connect them with the supra-œsophageal ganglia.

3. The pedal gland is a tubular sac extending along the greater part of the length of the foot, and opening in front by an aperture just below the head.

It has glandular walls, and is lined by a ciliated epithelium. The cells of the ventral wall resemble sensory cells in character, and the gland has hence been regarded by some writers as, at any rate in part, a sensory organ, possibly olfactory in function.

Pass a seeker into the external aperture of the pedal gland, and lay the gland open along its whole length.

\section{DISSECTION OF THE BUCCAL MASS.}

Remove the nerve-collar. Cut across the oesophagus and salivary glands about a quarter of an inch behind the buccal mass, and fix down the buccal mass firmly by pins throigh its retractor muscles. Note the horny jaw, seen as a dark transverse hoop across the front of the buccal mass; and the sac of the odontophore, which forms a rounded projection from its hinder end.

Slit up the cesophagus with scissors along the mid-dorsal line, and continue the cut forwards along the buccal mass as 
far as the jaw. Separate the two sides so as to expose the cavity of the buccal mass.

1. The odontophore or radula is a brownish-yellow chitinous riband lying on the floor of the buccal mass, and having its free surface produced into an immense number of minute backwardly directed teeth, arranged in very regular transverse rows.

The hinder end of the odontophore is folded longitudinally on itself, and lodged in a tubular sac, which projects freely behind the buccal mass, and within which the odontophore is formed as a cuticular secretion of its epithelial lining. The anterior end is opened out and rests on a cartilaginous cushion. By means of protractor and retractor muscles, the whole organ can be protruded from the mouth, and worked backwards and forwards with a licking or rasping action. As it wears away in front, it is replaced by growth forwards from its hinder end.

Dissect out the odontophore; clean it; and mount it on a slide in a drop of water or glycerine, with its toothed surface upwards. Examine it with low and high powers.

2. The jaw is a semicircular chitinous bar, strongly ridged on its posterior surface, against which the odontophore works.

Dissect out the jaw and examine it with a lens or the microscope.

VIII. THE CIRCULATORY SYSTEM.

The dissection of the circulatory system must be performed on a second snail.

It is greatly facilitated by injecting the vessels with a coloured fluid. The arteries should be injected from the ventricle, and the pulmonary vein and its branches from the auricle. The venous system must be injected from several places; the right pulmonary sinus is readily injected both forwards and backwards, and the pedal sinuses may also be injected. 


\section{The arterial system.}

From the ventricle arises a single large vessel, the aorta. This gives off almost at once a large visceral artery, which runs along the upper surface of the liver to the top of the spire, supplying both lobes of the liver, the intestine, and the reproductive organs.

The aorta itself runs forwards along the right side of the crop, between it and the common duct,

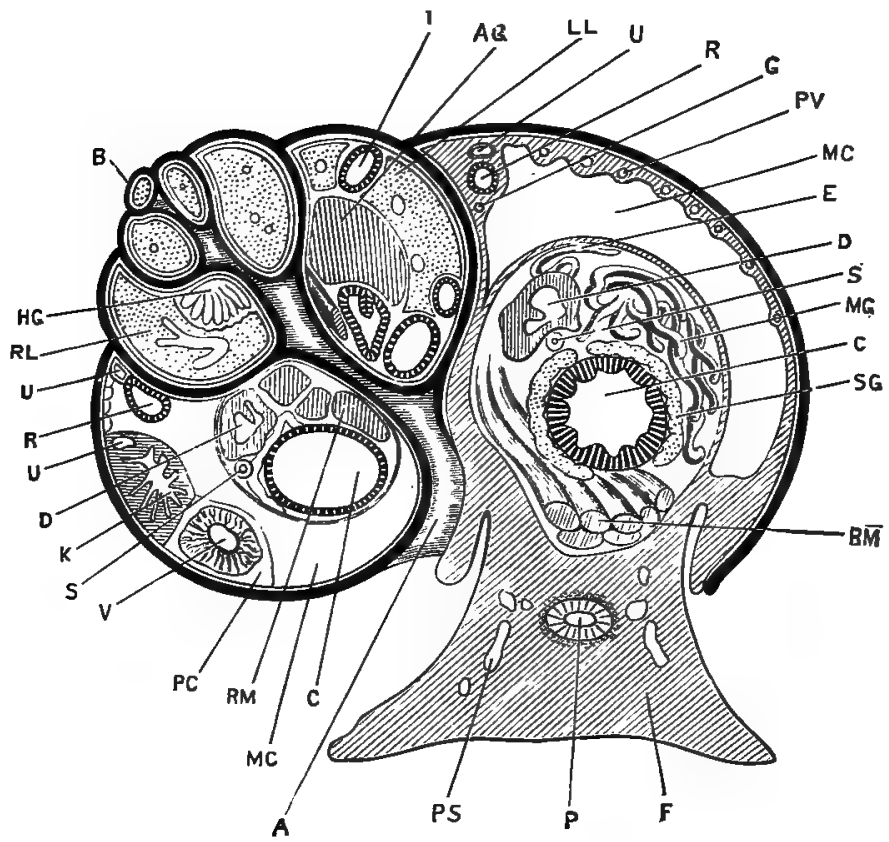

FrG. 31.-Helix pomatia. Section taken along the axis of the shell, and transversely to the foot. The shell is represented by the thick black line. (A. M. M.)

A, umbilicus. AG, albumen gland. B, nucleus of shell. C, crop. D, common duct. $\mathbf{E}$, flagellum. $\mathbf{F}$. foot. $\mathbf{G}$, pulmonary sinus, HG, hermaphrodite gland. I, intestine. $\mathbf{K}$, kidney. IL, left lobe of liver. MC, pnlmonary or mantle-cavity. M G, mucous glands. $\mathbf{P}$, pedal gland. PC, pericardial cavity. $\mathbf{P 8}$, pedal sinus. $\mathbf{P V}$, pulmonary vessels. $\mathbf{R}$, rectum. RI, right lobe of liver. RM, retractor muscles. S, spermatheca. SG, Ealivary gland. U, ureter. $\mathbf{V}$, ventricle. 
giving off large branches to the salivary glands and body-wall. It then continues its course forwards along the under surface of the oesophagus; pierces the sub-œsophageal ganglionic mass, passing between the pedal and viscero-pleural ganglia; gives off a large pedal artery which runs back along the foot; and finally divides into large branches supplying the tentacles and buccal mass.

2. The venous system.

The venous system consists partly of tubular vessels with definite walls, and partly of irregular lacunar spaces, the exact relations of which to the arteries and to the body-cavity are not determined.

Two large irregular pedal sinuses lie in the foot, one on each side of the pedal gland.

A visceral sinus commences at the top of the spire and runs down it, receiving branches from the various organs, to the posterior end of the mantlecavity, where it opens into the pulmonary sinus, a large vein running all round the mantle-cavity at the junction of its sides and floor. Along the right side the pulmonary sinus lies immediately ventral to the rectum, to which it is closely attached, and from which it receives numerous small veins. From the pulmonary sinus the blood is carried to the roof of the mantle-cavity by the afferent pulmonary vessels : these run parallel to one another in the roof of the mantle-cavity, interdigitating with the efferent vessels, with which they are connected by a network of thin-walled vessels in which the blood is aerrated. The efferent vessels unite to form the pulmonary vein, a large trunk running straight back in the roof of the mantle-cavity to the heart. Just before reaching the auricle, the pulmonary vein receives a large renal vein from the kidney. 


\section{Chapter VIII.}

\section{THE CRAYFISH. Astacus furiatilis.}

THE freshwater crayfish is fairly common in many of the streams of England and Ireland, hiding in holes under the banks. It is of a greenish grey colour; and in form, as well as in internal structure, it closely resembles the lobster, to which it is nearly related. It moves about slowly, half walking on its long thoracic legs, half swimming, but when alarmed darts suddenly backwards by violent flexion of its tail or abdomen.

The hard external cuticle of the crayfish is transversely jointed, to allow movements of the body and limbs; and this segmentation affects also those internal organs which are directly concerned with locomotion, i.e. the muscles, and the nerves and blood-vessels supplying them. No segmentation, however, is to be seen in the alimentary canal or its glands, and none in the excretory and reproductive systems: in this respect the crustacea differ widely from such animals as the leech, in which these systems are arranged segmentally.

Each segment of the crayfish, except the last, bears a pair of jointed appendages. These differ greatly in the various regions of the body, but are all modifications of one common type, and serially homologous with one another. The anterior ones form tactile organs, those in the region of the mouth act as jaws, and the remainder as organs of locomotion. The foremost pair of these latter are of great size, and end in powerful pincers used for prehension of food and for offensive and defensive purposes. 
The crayfish is carnivorous, seizing its food, and tearing it to pieces by means of the large pincers, aided by the smaller pincers of the next two pairs of legs, and then cutting it up into little bits with the jaws, which are placed at the sides of the mouth. The real chewing is effected afterwards in the stomach itself.

The sexes are distinct. The male and female are very similar, but the abdomen is broader in the female.

The eggs are comparatively large, and are carried about by the female, attached to the abdominal appendages, until the young are hatched. The newly-hatched crayfish differs considerably in form from the adult, but has the full number of appendages already present. It remains for a time attached by its pincers to the appendages of the mother, but soon takes to independent life. To allow for the growth of the animal the cuticle, which is incapable of interstitial growth, is cast off periodically, and a new one secreted. This ecdysis, or shedding of the cuticle, occurs three or more times during the first year of the animal's life, and afterwards about once a "חо $\mathrm{nr}$ less often. It involves not merely the external inve

gil th of the body and limbs, but the covering of the sense organs, and to a certain extent the lining of ttary canal.

Crayfish may be killed almost instantaneously by dropping them into boiling water.

\section{EXTERNAL CHARACTERS.}

The body of the crayfish is very obviously divisible into an anterior unjointed portion, the cephalothorax, and a jointed and flexible hinder portion, the abdomen.

\section{A. Examination of a Typical Segment of the Body.}

Examine in detail the third or fourth segment of the abdomen, noticing the following points.

1. The cuticular investment or exoskeleton of the segment is hardened by calcification, except the portions at the joints which remain soft to allow of movement. 
a. The tergum is the convex dorsal plate of the exoskeleton.

b. The sternum is the ventral transverse bar between the bases of the limbs.

c. The pleura are a pair of plates projecting down at the sides.

d. The epimera are the portions of hardened cuticle between the pleura and the bases of the limbs. They are very small in this segment.

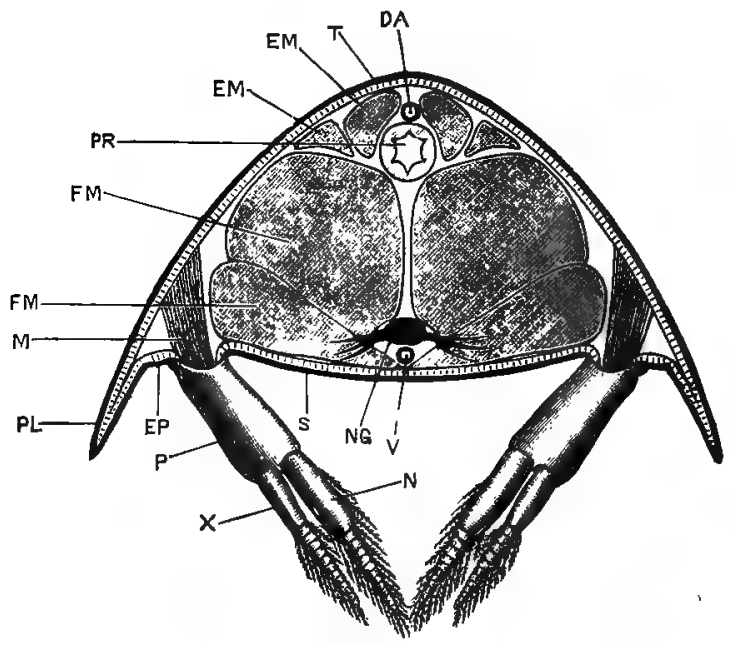

Fra. 32. - Astacus fluviatilis, var. nobilis. Transverse section through the abdomen. (с. н. н.)

DA, dorsal abdominal artery. FM, extensor muscles of the abdomen. FP, epimeron. FM, flexor muscles of the abdomen. $\mathbf{M}$, muscles of the appendage. $\mathbf{N}$, endopodite. $\mathbf{N} G$, nerve-ganglion. $\mathbf{P}$, protopodite : the proximal joint, which is very short, has been almost entirely cut away; its length is indicated by the notch on the outer side of the protopodite. PL, pleuron. PR, proctodoum. S, sternum. T, tergum. V, ventral abdominal artery. $\mathbf{X}$, exopodite.

e. Soft uncalcified portions connect the segment with those in front of and behind it, and allow movement of one segment upon another.

f. The joints between the successive segments are provided with a rounded peg or pivot at each side on the anterior margin of the tergum, near its 
junction with the pleuron. This peg fits into a corresponding depression in the hinder margin of the segment next in front, preventing movement of the abdomen from side to side.

2. The appendages are attached to the sides of the ventral surface of the segment, between the epimera and the ends of the sternum, by soft patches of cuticle, the arthrodial membranes. Each appendage consists of a two-jointed basal portion, the protopodite, supporting distally two many-jointed filaments, the endopodite and the exopodite, the former of which is nearer the median plane.

\section{B. The Body.}

This is obviously composed of two parts, the cephalothorax and the abdomen.

1. The cephalothorax is composed of thirteen segments fused together, the fusion being so complete that the segmentation is not recognisable on the dorsal and lateral surfaces, though it can still be seen below.

The cephalothorax is encased laterally and dorsally by a large shield, the carapace. This is divided into cephalic and thoracic portions, covering respectively the head and the thorax of the animal, by the shallow cervical groove, which runs transversely across its dorsal surface, and obliquely forwards at the sides. The thoracic portion of the carapace arises as an outgrowth from the hinder border of the head, which grows back over the thoracic segments, fusing with them more or less completely.

a. The head, or part in front of the cervical groove, is composed of five segments. In it are to be noticed the following structures.

i. The rostrum is a median pointed prolongation of the carapace, extending forwards from its anterior end. 
ii. The eyes are paired and mounted on movable stalks, one at each side of the rostrum.

iii. The mouth is far back on the ventral surface of the head, and almost hidden by the jaws.

b. The thorax, or part behind the cervical groove, is composed of eight segments. The following points are to be noticed in it.

i. The thoracic portion of the carapace is divided by two longitudinal grooves about half an inch apart, the branchio-cardiac grooves, into a median dorsal portion covering the heart, and two large plates, the branchiostegites, which extend down at the sides of the thorax and cover the gills.

ii. The sterna of the thoracic segments are all fused together, except the hindmost, which is movable.

2. The abdomen is composed of seven movable segments, united by peg and socket joints, which allow of movement in a vertical plane only, the limit of extension possible being nearly a straight line.

a. The first abdominal segment is smaller than the succeeding ones, and is devoid of pleura.

b. The segments from the second to the sixth are essentially like the typical segment already examined.

c. The seventh abdominal segment or telson is much flattened dorso-ventrally, and is devoid of pleura and of appendages: it is divided transversely by an imperfect hinge, and its ventral surface, which is only slightly calcified, is perforated by the longitudinal slit-like anus.

\section{The Appendages.}

These are all reducible to a common type composed of the three parts, protopodite, exopodite, and endopodite, already seen in the appendage of the typical segment, together with 
an epipodite, which may be a simple plate or may be modified to form a gill. From such a typical form all the appendages of the crayfish can be derived by variations in form and relative size of the various parts, or by total suppression of one or more parts.

In the following description the appendages are numbered from before backwards, and are distinguished by the large Roman numerals I. to XIX.

Remove the appendages of the right side one by one, beginning with the hindmost, and taking special care to remove each appendage entire. The characters of the smaller ones can be best made out by examination in a drop of water, between two slips of glass.

1. The abdominal appendages, excepting the hindmost, are of small size, and serve in the female to carry the eggs. The last or twentieth segment has no appendages.

XIX. The sixth abdominal appendages are broad and lamellar, and form with the telson the powerful tail-fin.

i. The protopodite is short, broad and undivided.

ii. The endopodite is a flat oval plate, fringed at its free edge with setæ.

iii. The exopodite is similar, but larger, and divided by a transverse hinge into two parts.

XVIII. The fifth abdominal appendage is a small swimmeret.

i. The protopodite is two-jointed, the small proximal joint being named coxopodite, the longer distal joint, the basipodite.

ii. The endopodite is a many-jointed filament covered with setæ. Its first joint is the largest.

iii. The exopodite is similar, but smaller.

XVII. and XVI. These two pairs of appendages closely resemble XVIII. 
$\mathbf{X V}$. In the female this resembles XVIII., but is liable to reduction, and may be absent.

In the male it is normally turned forwards and is specially modified for transferring the spermatozoa to the female. The protopodite and exopodite resemble those of XVIII., but the large proximal joint of the endopodite is produced distally on its inner side into a short plate, rolled longitudinally upon itself to form an imperfect tube: the remainder of the endopodite is a small jointed rod as in XVIII.

XIV. In the female this is reduced or absent.

In the male it is modified for the same purpose as XV. It is an unjointed rod, the distal portion of which is flattened and rolled upon itself to form an imperfect tube. It is probably formed of the protopodite and endopodite fused together.

2. The thoracic appendages, of which there are eight pairs, fall readily into two groups: a posterior group of five pairs of large appendages, XIII. to IX., used for walling and prehension, and devoid of exopodites; and an anterior group of three pairs of maxillipedes or foot-jaws, VIII. to VI., which possess both exopodites and endopodites, and aid in passing food to the mouth.

XII. The last but one of the thoracic legs may be taken as a type of the series.

i. The protopodite consists of two joints, of which the first, or coxopodite, is short and broad, and bears a large gill and a tuft of long fine hairs, the 'coxopoditic setæ.' The second joint or basipodite is much smaller, and bears the endopodite.

ii. The endopodite consists of five joints named as follows, beginning at the proximal end :ischiopodite, meropodite, carpopodite, pro- 

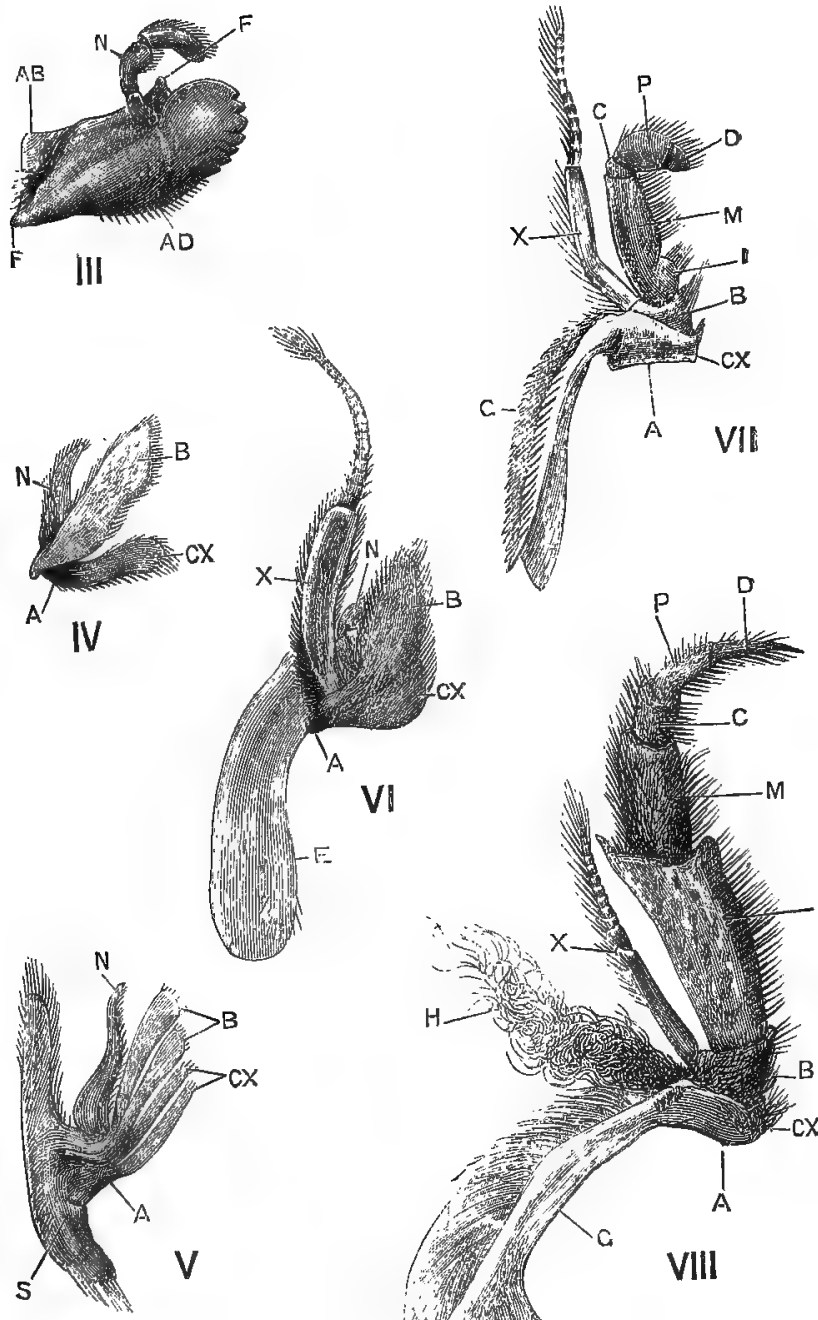
podite, and dactylopodite. Of these the meropodite and the propodite are the longest, and the dactylopodite is clawlike.

XIII. This, the last thoracic leg, differs from XII. in having no gill. In the male the coxopodite is perforated at its inner angle by the genital pore.

XI. This resembles XII., but the propodițe is prolonged distally so as to form with the dactylopodite a pair of pincers or chelæ. The inner angle of the coxopodite is perforated by the genital pore in the female.

$\mathbf{X}$. This is like $\mathbf{X I}$., but has no genital pore.

IX. This is similar to $\mathbf{X}$, but much larger and stouter, the terminal chelæ being especially large and powerful. The basipodite and ischiopodite are firmly fused together, as happens occasionally in other limbs of the series.

The anterior group of thoracic appendages, or maxillipedes, can be more easily removed if the anterior portion of the branchiostegite be first cut away.

VIII. The third maxillipede is directed forwards under the five appendages in front of it.

i. The protopodite consists of coxopodite, bearing a gill and coxopoditic setæ; and basipodite, bearing endopodite and exopodite.

ii. The endopodite consists of five joints, named as in the legs. The first joint or ischiopodite is the largest and is fused with the basipodite.

FIG. 33.-Astacus fluviatilis var. nobilis. The oral appendages of the right side seen from below and behind; $\times 2 \frac{1}{2}$. (с. H. н.)

III, the mandible. IV and V the first and second maxillæ. VI, VII, and VIII, the three maxillipedes.

A, region of attachment to body-wall. $\mathbf{A} \mathbf{B}$ and $\mathbf{A D}$, points of insertion of abductor and adductor muscles of mandible. $\mathbf{B}$, basipodite. $\mathbf{C}$, carpopodite. $\mathbf{C X}$, coxopodite. $\mathbf{D}$, dactylopodite. $\mathbf{E}$, epipodite. $\mathbf{F}$, pivots of mandible. $\mathbf{G}$, gill. $\mathbf{H}$, coxopoditic setæ. I, ischiopodite. $\mathbf{M}$, meropodite. $\mathbf{N}$, endopodite. $\mathbf{P}$, propodite. $\mathbf{S}$ scaphognathite. $\mathbf{X}$, first joint of exopodite. 
iii. The exopodite is slender, and consists of a long. proximal joint and a distal many-jointed filament.

VII. The second maxillipede is smaller and less firmly calcified than VIII., which it resembles in form ; but the exopodite is larger than in VIII., and the endopodite smaller, its largest joint being the meropodite, and all its joints being movable.

VI. The first maxillipede is smaller and softer than VII.

i. The two joints of the protopodite are expanded on the inner side, forming a large two-lobed lamella. A broad plate, the epipodite, takes. the place of a gill.

ii. The endopodite is very small and two-jointed.

iii. The exopodite resembles that of VII.

3. The head appendages consist of three pairs of oral appendages or jaws, V. to III., placed at the sides of the mouth, and two pairs of pre-oral appendages, II. and I., at the front of the head.

J. The second maxilla consists of a protopodite, an endopodite, and on the outer side an organ subservient to respiration, the scaphognathite.

i. The protopodite consists of two joints, coxopodite and basipodite, each of which is expanded on its inner side into a bilobed lamella.

ii. The endopodite is small, pointed, and unjointed. iii. The scaphognathite is an elongated plate attached by the middle of its inner edge to the outer side of the protopodite. It may be regarded as the exopodite and epipodite fused together. It serves to keep a current of water flowing through the gill-chamber (p. 139).

IV. The first maxilla is small. The two joints of the protopodite extendinwards as thin curved lamellæ, 
and the endopodite is a small plate to their outer side. There is no exopodite.

III. The mandible consists of a very stout undivided protopodite, forming the powerful jaw at the side of the mouth; and an endopodite, which forms a small three-jointed palp, lying in a groove on the front of the protopodite.

Before removing the mandible, note the position of the two pivots about which it turns, and the points of insertion of the tendons of its abductor and adductor muscles.

Immediately in front of the first maxilla and behind the mandible is a small unjointed plate, leaflike in form. It is not regarded as a true appendage, but as an outgrowth of the metastoma, which forms the posterior and lateral borders of the mouth.

II. The antenna consists of protopodite, exopodite, and endopodite, the last of which forms the long ' feeler.'

i. The protopodite consists of two joints; the first, or coxopodite, being very broad, and projecting only very slightly from the surface of the head: on its ventral surface is a tubercle perforated posteriorly by the renal aperture. The second joint, or basipodite, is also short and stout, and bears the endopodite and exopodite.

ii. The endopodite has two stout basal joints and a long slender many-jointed feeler.

iii. The exopodite is a stout sharp-pointed blade, the scaphocerite.

I. The antennule is smaller than the antenna.

i. The protopodite is three-jointed, the basal joint being much the largest, and containing the auditory organ, which opens on its dorsal surface. The aperture is three-cornered, and is guarded by a series of plumose setæ, which extend across it from its outer lip. 
ii. The endopodite and exopodite are two manyjointed filaments, the exopodite being slightly the larger.

\section{The Gills and Gill-chambers.}

The gills are highly vascular external outgrowths from the" sides of the thorax and from its appendages. In them respiration occurs, this process consisting in an interchange,

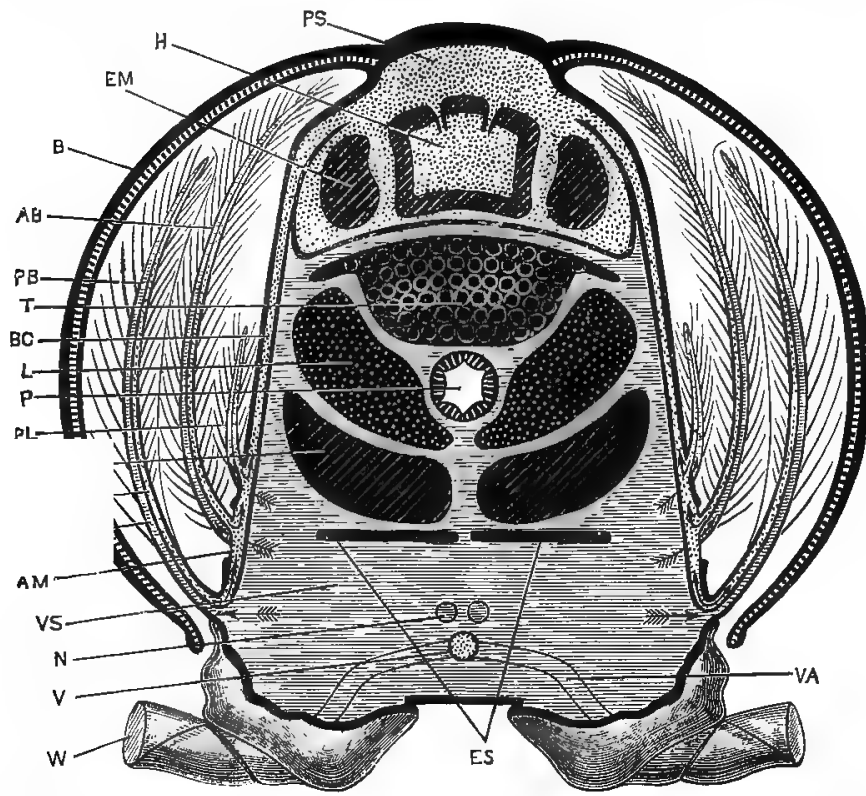

Fra. 34.-Astacus fluviatilis. Diagrammatic transverse section through the thorax. The dotted spaces contain arterial blood. The spaces - shaded with horizontal lines contain venous blood. The arrows indicate the direction of the blood circulation. (c. н. н.)

AB, arthrobranchia : only one is figured on each side. $\mathbf{A} \mathbf{F}^{2}$, afferent vessel of gill. AM, arthrodial membrane. B, brancliostegite. BC, branchio-cardiac canal. FF, eflerent vessel of gill. FM, extensor muscle of the abdomen. ES, endophragmal skeleton, H, heart, showing the dorsal ostia, with their valves. L, liver. $\mathbf{M}$, flexor muscle of the abdomen. $\mathbf{N}$, nerve-connective. $\mathbf{P}$, proctodcum. PB, podobranchia. PL, pleurobranchia. PS, pericardial sinus. $T$, testis, with roots of vasa deferentia. $\mathbf{V}$, sternal artery. VA, artery supplying leg. VS, ventral blood-sinus : it contains numerous large muscles moving the limbs, which are not represented in the figure. $\mathbf{W}$, one of the legs, cut off short. 
by diffusion through the thin cuticle, of the gases dissolved in the water and in the blood. The gills are enclosed and protected by downward growths of the carapace at the sides of the thorax, the branchiostegites.

1. The branchial chambers are situated one at each side of the thorax, between the body and the branchiostegite.

Expose the left branchial chamber by cutting away the branchiostegite of that side; and fix the animal on its right side under water.

The chamber is bounded on its inner side by the thoracic epimera, and on its outer side by the branchiostegite. It is open below and behind, but the former opening is partially stopped by the basal joints of the legs. The greater portion of the chamber is occupied by the gills; but in front of them and just behind the line of the cervical groove, is a channel, the cervical canal. In this canal the scaphognathite works to and fro with a sculling movement, driving the water forwards out of the gill-chamber, and discharging it in front just below the renal aperture. (Cf. Section E, p. 139.)

2. The gills may:be described in three groups according to their position of attachment.

a. Podobranchiæ are gills attached to the coxopodites of appendages VII. to XII. The coxopodite of VI. bears a large epipodite in place of a gill.

b. Arthrobranchiæ are gills arising from the arthrodial membranes at the bases of the thoracic appendages. At the base of each of the limbs VIII. to XII. are two arthrobranchiæ, an anterior and a posterior one; and attached to the arthrodial membrane of VII. is a single one.

c. Pleurobranchiæ are gills arising from the side-wall of the thorax, above the ridges which form the dorsal boundaries of the arthrodial membranes. The crayfish has only one well-developed pleuro- 
branchia on each side, on the thirteenth segment. but rudiments are found on the next two or three. segments in front.

The following table shows the number and arrangement of the gills in the crayfish :

Rud.=mdiment. Ep.=Epipodite.

\begin{tabular}{|l|c|c|c|c|c|c|c|c|c|}
\hline \multicolumn{1}{|c|}{ Segment } & VI. & VII. & VIII. & IX. & X. & XI. & XII. & XII. & Totals \\
\hline Podobranchiæ & Ep. & 1 & 1 & 1 & 1 & 1 & 1 & 0 & $=6+$ Ep. \\
Arthro. anterior & 0 & 1 & 1 & 1 & 1 & 1 & 1 & 0 & $=6$ \\
Arthro. posterior & 0 & 0 & 1 & 1 & 1 & 1 & 1 & 0 & $=5$ \\
Pleurobranchiæ & 0 & 0 & 0 & 0 & Rud.) & Rud. & Rud. & 1 & $=1+3$ Rud. \\
\hline
\end{tabular}

Verify the above table by carefully counting the gills, and turning them down as they are checked off in the table.

3. The structure of the gills is different in the different. groups.

Remove and examine in turn a pleurobranchia, an arthrobranchia, and a podobranchia.

a. The pleurobranchiæ and arthrobranchiæ resemble bottle-brushes, each consisting of a series of delicate branchial filaments arranged upon a central stem, which is traversed by afferent and efferent bloodvessels. It is in the branchial filaments that respiration is effected, diffusion taking place very readily through the thin cuticle covering them.

b. A podobranchia is more complicated, and consists of, (i.) a basal plate arising from the outer surface of the coxopodite, and covered with finely plumose setæ; (ii.) a stem arising from the dorsal border of the basal plate close to its apex; (iii.) a lamina, which is a corrugated plate borne on the distal end of the stem, doubled longitudinally upon itself, and beset with small hooked setæ; and (iv.) a. plume, which arises from the apex of the stem. and resembles an arthrobranchia. The plume and the outer face of the stem are covered with.

- branchial filaments. 


\section{E. Demonstration of the Respiratory Current of water through the gill-chamber.}

Place a living crayfish in a shallow dish of water; and, when the animal is at rest, run into the dish close to the bases of the hinder legs, by means of a pipette, a few drops of water coloured with suspended carmine or other pigment. Watch the currents entering under the edges of the branchiostegites behind, and issuing in front from the mouths of the cervical canals.

Open the cervical canal of one side by making two cuts, one immediately behind the cervical groove, the other parallel to it and a quarter of an inch further back; and removing the strip between the two cuts. Lay the animal on its side in the water, and observe the sculling movements of the scaphognathite, driving the water forwards out of the cervical canal.

\section{DISSECTION OF THE CRAYFISH.}

The several systems are described below in the order in which it will be most convenient to dissect them: this order may be varied if found desirable.

\section{A. The Circulatory System.}

The blood of the crayfish, which is almost colourless, is carried from the heart to the various parts of the body by arteries which open into large lacunar spaces bathing the several organs : from these it passes to the gills, in which it is aërated, and from which it is conveyed through the branchiocardiac canals to the pericardial sinus. From the sinus it enters the heart through the apertures in its walls.

\section{The pericardial sinus and heart.}

With stout scissors carefully cut through the thoracic portion of the carapace along the outer side of each branchiocardiac groove, taking care not to injure the structures beneath. Connect the two cuts in front by a transverse cut along the cervical groove, and remove the portion of carapace so isolated.

Cut through the skin underlying the carapace, and turn it 


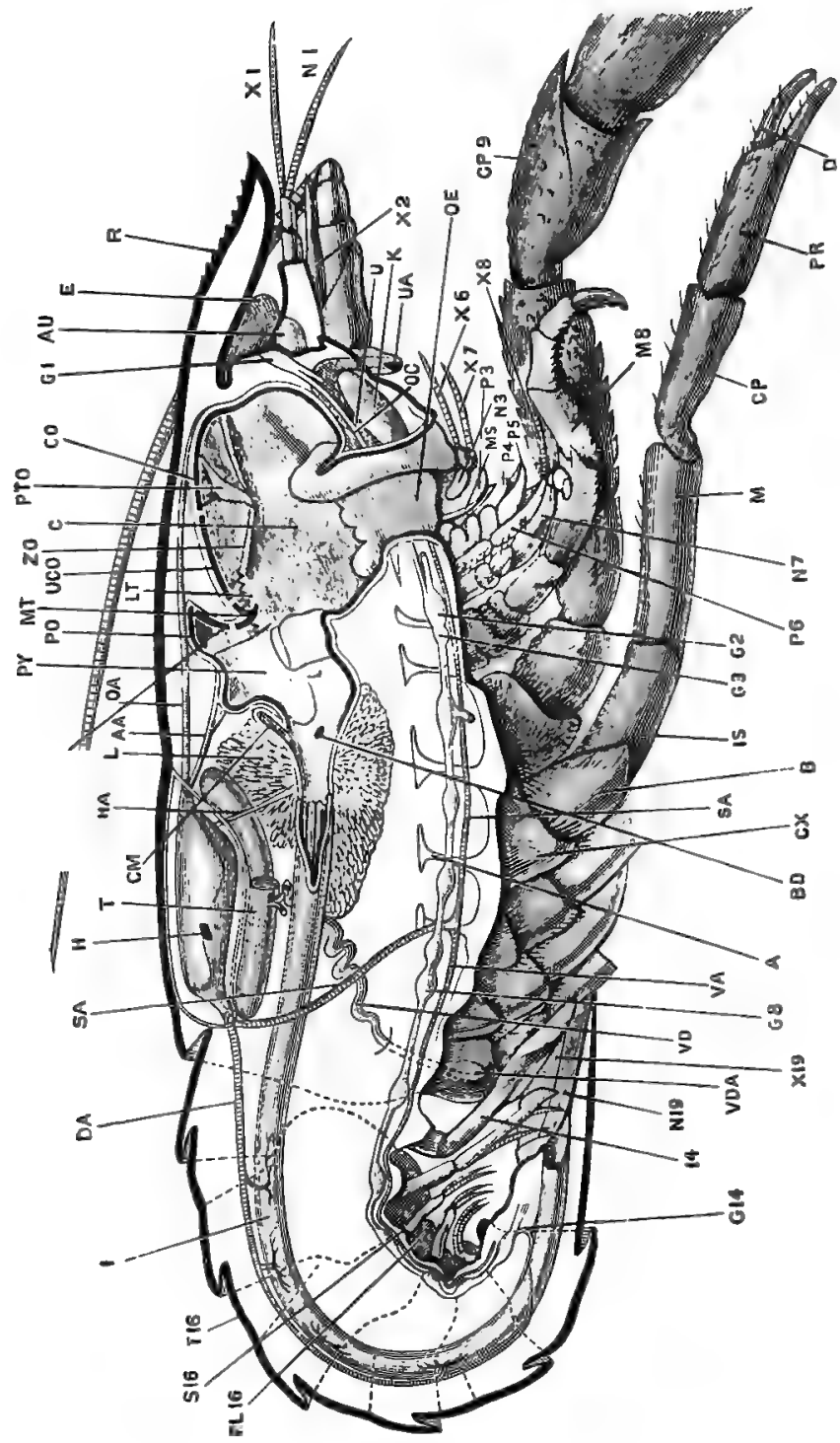


aside, thus laying open the pericardial simus and exposing the heart.

a. The pericardial sinus is a cavity of considerable size, lying in the dorsal portion of the thorax and receiving the arterial blood from the gills.

b. The heart is a polygonal, thick-walled, muscular sac lying in the pericardial sinus. If living it will be seen to contract rhythmically. It receives blood from the pericardial sinus by three pairs of valvular apertures, the ostia, of which the dorsal pair will be readily seen. The lateral and ventral pairs will be better seen at a later stage of the dissection.

c. The roots of the arteries are rather small and transparent, and hence difficult to see. They arise from the two ends of the heart, and should be noticed at the present stage in the dissection, though the arteries themselves are best seen in

FIa. 35.-Astacus fluviatilis, var. nobilis. Dissection of a male from the right side. The right side of the body-wall with its appendages, and the right side of the cesophagus, stomach, and mesenteron, have been removed. The thick black line represents the cut edge of the cuticle. (с. н. н.)

A, endophragmal skeleton. A.A, antennary artery. AU, auditory sac. $\mathbf{B}$, basipodite of the tenth appendage. BD, aperture of left bile-duct into the mesenteron. C, cardiac chamber of the stomach. CM, cæcum. CO, cardiac ossiole. CP, carpopodite of tenth appendage. CP 9, carpopodite of ninth appendage. ' $\mathbf{C X}$, coxopodite of tenth appendage. D, dactylopodite of the same. DA, dorsal abdominal artery. F, eye. G 1, supra-0esophageal ganglion. G 2, sub-cesophageal ganglion. $\mathbf{G} 3$, second post-cesophageal ganglion. G 8, last thoracic ganglion. G 14, last abdominal ganglion giving off posterior visceral and other nerves. H, right lateral ostium of the heart. HA, hepatic artery. I, proctodoum. IS, ischiopodite of the tenth appendage. $\mathbf{K}$, kidney. $\mathbf{I}$, liver. $\mathbf{L} \mathbf{I}$, lateraI tooth. $\mathbf{M}$, meropodite of the tenth appendage. MS, metastoma. MT, median tooth. 'M 8, meropodite of the eighth appendage. $\mathbf{N} 1, \mathbf{N} 3$ etc. endopodites of first, third, etc. appendages. $\mathbf{O A}_{\mathbf{A}}$, ophthalmic artery. OC, left para-cesophageal connective ; the greater part of the right one has been removed. OF, cesophagus. PL 16, pleuron of the sixteenth segment. PO, pyloric ossicle. PPO, prepyloric ossicle. PR, propodite of the tenth appendage. PTO, pterocardiac ossicle. PY, pyloric chamber of the stomach. $\mathbf{P} 3, \mathbf{P} 4, \mathbf{P} 5$, protopodites of the third, fourth, and fifth appendages. $\mathbf{R}$, rostrum. SA, sternal artery. $\mathbf{S} 16$, sternum of the sixteenth segment. T, testis : the right anterior lobe has been removed. T 16, tergum of the sixteenth segment. U, ureter. UA, aperture of the ureter. UCO. urocardiac ossicle. VA, ventral abdominal artery. VD, left vas deferens the right one has been cut off short. VDA, aperture of the left vas deferens. $\mathbf{X} 1, \mathbf{X} 2$, etc., exopodites of first, second, etc., appendages. $\mathbf{Z O}$, zygocardiac ossicle. 14, the fourteenth appendage. 
an injected specimen, and will be described later (p. 151).

i. From the anterior end of the heart arise a median ophthalmic artery and a pair of antennary arteries; and behind and below these a pair of hepatic arteries.

ii. From the hinder end of the heart arises the large median dorsal abdominal artery, and immediately below this the large median sternal artery, which latter can be better seen at a later stage.

d. The alæ cordis are three pairs of fibrous bands which hold the heart in position by connecting it with the walls of the pericardial sinus.

Remove the branchiostegite and turn down the gills of the left side, unless this has already been done.

2. The branchiocardiac canals are a series of channels in the body-wall, conveying blood from the gills to the pericardial sinus. They can be seen through the transparent body-wall running from the bases of the gills, and converging to open into the sides of the pericardial sinus.

Cut a gill off close to its base, and blow into the cut end of the efferent blood-vessel with a blowpipe, or inject some coloured fluid into it, and observe the air or coloured fuid entering the pericardial sinus.

\section{B. The Reproductive System.}

Carefully remove the side-wall of the thorax, and expose the reproductive organs.

\section{a. In the male.}

i. The testis is a soft white body lying immediately below the pericardial sinus, and extending backwards a little beyond it. It consists of a pair of anterior lobes, and a median posterior lobe. 
ii. The vas deferens of each side is a long thick convoluted tube, runing from the junction of the anterior and posterior lobes of the testis to the external aperture on the coxopodite of the last thoracic leg. Before the breeding season it will be found filled with an opaque white seminal mass.

Tease a small piece of the mass in water or glycerine on a slide; cover, and examine it with low and high powers.

iii. The spermatozoa are flattened spheroidal cells from which project tangentially a number of stiff immobile curved processes.

\section{b. In the female.}

i. The ovary is situated below, and at the sides of the pericardial sinus, and extends some distance behind it. Like the testis it consists of two anterior lobes and a posterior one.

ii. The oviduct of each side is a short, wide, almost straight tube, running from about the middle of the length of the ovary to the external opening on the coxopodite of appendage XI., the last thoracic leg but two.

\section{The Digestive System.}

The alimentary canal of the crayfish is a tube running in a nearly straight line from the mouth to the anus. Of this tube the middle portion or mesenteron, which is very short, is alone formed from the primitive alimentary tract of the embryo, and the 'liver' is an outgrowth of it. The stomatodoum or anterior portion of the canal and the proctodoum or hind portion, which together form almost the whole length of the canal, are both formed by invagination of the external surface of the body; and both have a chitinous lining which is continuous, at the mouth and anus respectively, with the chitinous external covering of the body. 
1. The digestive glands.

a. The 'liver' is a large bilobed body, of a brown or greenish colour, lying in the thorax at the sides of the alimentary canal. Each lobe is much branched and hollow, and its secretion enters the mesenteron by a wide bile-duct, which will be seen later on in the dissection.

\section{The alimentary canal.}

Remove the genital duct and the greater. part of the liver of the left side, and as much of the muscles and cephalic carapace as is necessary to expose the alimentary canal.

a. The stomatodoum, or first portion of the alimentary canal, has a chitinous lining.

i. The mouth is an oval aperture on the ventral surface, between the jaws.

ii. The cesophagus is a short wide tube, running vertically upwards from the mouth.

iii. The stomach is a spacious dilatation of the alimentary canal, occupying the greater part of the head, and extending a short distance into the thorax. It consists of a larger cardiac chamber in front, and a smaller pyloric chamber behind, separated from each other by a constriction. Its chitinous lining is thick, and some parts of it are especially hardened, forming the gastric mill, a complex mechanism of 'ossicles' supporting three large and strongly calcified teeth. These structures will be seen at a later stage of the dissection.

b. The mesenteron or mid-gut, which follows the stomach, is very short and devoid of chitinous lining. On each side it receives a wide bile-duct from the liver, and its dorsal wall is produced into a small cæcum.

c. The proctodoum or intestine is a long narrow straight tube, running from the hinder end of the 
mesenteron to the anus. It has a chitinous lining throughout, and its inner surface presents longitudinal and slightly spiral ridges beset with small papillæ.

The anus is a longitudinal slit on the ventral surface of the telson: it can be closed by approximation of its sides.

Remove a piece of the intestine, and examine its inner surface under water with a low power of the microscope, to see the ridges and papilla.

d. The gastric mill is a complex structure made up of numerous sclerites, or hardened portions of the cuticle lining the stomach, by means of which the chewing of the food is effected: the principal sclerites will alone be described here, viz. five median dorsal pieces and two pairs of lateral pieces, the others being small and of less importance.

Remove the entire stomach from the body, avoiding injury to the structures around the cesophagus; place it in a dish under water, and carefully clean away the muscles.

i. The cardiac ossicle is a broad transverse sclerite in the roof of the cardiac chamber of the stomach.

ii. The urocardiac ossicle is a median dorsal sclerite in the hinder wall of the cardiac chamber. Its upper end is broad, and hinged to the hinder edge of the cardiac ossicle; its lower end is narrow, and directed downwards and backwards.

iii. The pyloric ossicle is a narrow transverse sclerite in the anterior part of the dorsal wall of the pyloric chamber.

iv. The prepyloric ossicle lies in the anterior wall of the pyloric chamber, and is hinged above to the anterior border of the pyloric ossicle. 
v. The median tooth is a strong calcified sclerite, at the junction of the cardiac and pyloric cham. bers. With its upper surface are articulated the urocardiac ossicle in front and the prepyloric behind.

vi. The pterocardiac ossicles are attached one to each end of the cardiac ossicle by oblique hinges. Their lower ends are curved backwards.

vii. The zygocardiac ossicles are a pair of stout sclerites, running from the outer ends of the pyloric ossicle forwards, downwards, and outwards, to the ventral extremities of the pterocardiacs. Their ventral margins are curved inwards to form a pair of stout plates, calcified at their inner borders to form the lateral teeth. The inner edge of each lateral tooth bears a row of denticles, which decrease in size from before backwards.

This gastric mill is put into action by muscles arising from the carapace and attached to the cardiac and pyloric ossicles, which, by their contractions, they pull away from each other. The cardiac ossicle moving forwards pulls the urocardiac and the median tooth with it, and the prepyloric ossicle rotates forwards with them, giving a downward movement to the hinder part of the tooth, and causing its apex to rotate forwards. The same movement of the cardiac ossicle forwards, and of the pyloric backwards, causes the zygocardiacs to pull back the pterocardiacs, which rotate about the oblique hinges, pressing the zygocardiacs inwards, and bringing the lateral teeth together in the median plane.

Pull the cardiac and pyloric ossicles forwards and backwards respectively with forceps, when the three teeth will be scen to come together sharply. 


\section{The Excretory System.}

1. The kidneys, or 'green glands,' are a pair of rounded and somewhat flattened bodies, of a light green colour, lying in the ventral part of the head, in front of the mouth.

2. The ureters are a pair of wide thin-walled sacs, lying one on the dorsal surface of each kidney. Each ureter communicates directly with the cavity of its kidney, and opens to the exterior by an aperture already seen on the hinder surface of a tubercle on the basal joint of the antenna.

- Inflate the ureter by blowing air in at its external aperture.

\section{E. The Endophragmal Skeleton. (Fig. 35, p. 140.)}

In the thorax a series of calcified plates project inwards from the cuticle of the ventral surface, forming an internal skeleton, which gives origin to the muscles of the legs and to the powerful flexor muscles of the abdomen.

\section{F. The Nervous System.}

1. The central nervous system consists of a series of ganglia arranged in pairs, and united by paired bundles of nerve-fibres, or connectives, to form a chain, which runs along the whole length of the body close to the mid-ventral line. The two ganglia of each pair are usually fused indistinguishably.

a. The pre-cesophageal ganglionic mass, or 'brain,' is a white mass of considerable size, situated just behind and slightly above the bases of the antennules. It gives off nerves supplying the eyes, the antennules, and the antennæ.

b. The para-cesophageal connectives are a pair of long nerve-cords, one on each side of the oesophagus, connecting the 'brain' with the hinder part of the nervous system. They are connected with each other by a transverse commissure, immediately behind the œsophagus. 
c. The post-œsophageal ganglionic mass is an ovoid mass, lying a short way behind the mouth. From it arise the nerves supplying the mandibles, both pairs of maxillæ, and the first and second maxillipedes.

d. The thoracic nerve-chain consists of six ganglionic masses, united by paired connectives, and supplying the appendages from the third maxillipedes to the last thoracic legs. The first one is situated immediately behind the post-œsophageal ganglionic mass, with which it is closely connected. The connectives between the fourth and fifth ganglionic masses diverge slightly, and the sternal artery (p. 152) passes between them.

The thoracic nerve-chain lies beneath the endophragmal skeleton, which must be removed to expose it.

e. The abdominal nerve-chain consists of six ganglionic masses, joined by connectives, and supplying the abdominal segments and their appendages. The hindmost ganglionic mass is larger than the others, and supplies both the nineteenth segment and the telson.

\section{The visceral nervous system.}

This can only be dissected in a specimen in which the alimentary canal has been left undisturbed.

a. The anterior visceral nerve is a median nerve formed, just in front of the cesophagus, by the union of two median nerves from the "brain " with two pairs of nerves arising from ganglionic swellings on the para-œsophageal connectives. From the union of these, the nerve runs up in front of the stomach to branch out on its dorsal wall.

b. The posterior visceral nerve arises from the hindmost ganglionic mass of the abdominal nervechain, and runs forwards along the ventral surface of the intestine. 


\section{G. The Organs of Special Sense.}

Besides tactile organs, i.e. the antennules, antennæ, and the palps of the oral appendages, the crayfish has eyes, ears, and olfactory organs.

1. The eyes, on movable stalks, have already been seen at the sides of the rostrum.

a. External characters of the eye.

Remove one of the eye-stallis, and examine it carefully with a lens.

The 'cornea' is the transparent, apparently black, patch of uncalcified cuticle covering the end of the eye-stalk, and bounded by an oval outline. Its surface is divided into quadrangular facets by lines crossing each other nearly at right angles, each facet corresponding to one of the elements of which the compound eye consists.

b. Structure of the eye as seen in a longitudinal section.

Carefully divide the eye-stalk and eye longitudinally into two halves. The calcified portion of the cuticle is best cut with scissors, the remainder with a sharp knife. Examine one of the cut surfaces with a strong lens.

The eye consists of a number of conical bodies arranged in a radiate fashion, their bases being turned outwards and formed by the corneal facets, and their apices being directed inwards and connected with the optic ganglion. Each of these component elements is isolated optically from its neighbours by pigment.

i. The cornea is thin and semicircular in section, and the strips of cuticle separating its facets scarcely differ from the facets themselves.

ii. The crystalline pyramids are transparent conical bodies arranged radially and abutting upon the cornea, each pyramid corresponding 
to one corneal facet. They are separated from one another by layers of pigment near their outer ends.

iii. The inner ends of the pyramids are imbedded in a black pigmented layer, in which lie a series of radially arranged rods and fusiform bodies, each of the latter being surrounded by a layer of brilliant pigment, either white or pink.

iv. The optic ganglion occupies the centre of the eye. Its surface is deeply pigmented.

v. The optic nerve lies in the axis of the eye-stalk, and connects the optic ganglion with the 'brain.'

2. The auditory organs are lodged in the basal joints of the antennules, and open to the exterior on their dorsal surfaces by three-cornered apertures, guarded by fringes of plumose setæ.

Remove an antennule entire. Holding it firmly between the fingers, carefully remove with scissors the inner and outer walls of the basal joint, taking care not to injure the auditory sac itself. Clear away the muscles surrounding the sac, and the sete from the auditory aperture.

With a fine-pointed syringe wash out the contents of the auditory sac-a collection of sand and other foreign matter from the water in which the animal lives, to which the name ' otolith' has been given.

Examine the sac in water or glycerine on a stide under the low power of the microscope.

a. The auditory sac is a transparent ovoid chitinous sac, continuous at its opening with the cuticle of the antennule.

b. The auditory setæ are nearly straight, simple, pointed setæ varying greatly in size, the outer ones being the largest. The larger ones are arranged in parallel curved lines along the floor and inner wall of the sac; of these there are two 
rows behind, the number increasing to five or more in front. Parallel to these runs a row of very minute setæ on the inner side.

c. The auditory nerve supplying these rows of setæ may have been removed with the muscles, but should be looked for.

3. Olfactory setæ. On the ventral surface of each joint of the exopodite of the antennule are two tufts of fine sensory setæ. Under a low power of the microscope each is seen to consist of two joints, of which the distal one is much the longer, and is flattened. They are probably olfactory in function.

III. THE ARTERIES AS SEEN IN AN INJECTED SPECIMEN.

Inject from the ventricle with water coloured with carmine.

Kill the animal by dropping it into boiling water. Dissect from the left side, removing the left half or more of the carapace, and such parts of the liver, genital gland and duct, and of the side-wall and muscles of the abdomen as may be necessary to expose the following arteries.

1. The ophthalmic artery is a median artery arising from the anterior end of the heart and running forwards over the stomach, then curving downwards in front of the stomach and dividing to supply the eyes.

2. The antennary arteries arise from the anterior end of the heart, one on each side of the ophthalmic artery. Each runs forwards, outwards, and downwards over the side of the stomach, to which it sends a gastric artery ; and then forwards to the anterior end of the head, supplying the kidney, antenna, and antennule, and sending a branch to the rostrum. On its way it gives off numerous small arteries to the muscles of the regions which it traverses.

3. The hepatic arteries arise from the ventral surface of the heart near its anterior end, and supply the liver, in which they are buried. 
4. The sternal artery is single, and arises from the hinder end of the heart, whence it runs directly downwards, passing to the right or left of the intestine, and between the connectives uniting the fourth and fifth ganglion-pairs of the thoracic nerve-chain (p. 148). It then turns forwards beneath the nerve-chain, supplying the sternal region of the thorax, and the appendages from the hindmost ambulatory legs to the mandibles.

a. The ventral abdominal artery is a large median vessel given off by the sternal artery on reaching the ventral region of the body. It runs backwards beneath the nerve-chain, and supplies the ventral region of the abdomen and the abdominal appendages.

5. The dorsal abdominal artery is a large median artery arising from the ventral part of the binder end of the heart, and running backwards just above the intestine to the hinder end of the body, supplying the intestine, muscles, and other parts of the dorsal region of the abdomen. 


\section{Chapter IX.}

\section{THE COCKROACH. Periplaneta Americana.}

THE common cockroach or 'black-beetle' of our kitchens and bakehouses-Periplaneta (Blatta) orientalis-is not a true beetle, but an insect belonging to the order Orthoptera, of which grasshoppers, locusts, and crickets are also members. This species differs only slightly from $P$. Americana, which has been chosen for description because it is larger and more easily dissected than the common form. P. Americana may be found in large numbers on board ships and at most docks.

Cockroaches are active insects, of nocturnal habits, hiding away in the daytime and coming out to feed at night. They will devour almost any animal or vegetable substance.

The whole animal is covered by a chitinous cuticle which is thick and hard, and of a dark brown colour, except at the joints, where it is soft and paler. By these joints the body is divided transversely into segments, which are movable on one another except in the head. As in the crayfish the segmentation affects certain of the internal organs, as the muscular and nervous systems, but is not shown by the digestive, excretory, or reproductive organs.

The female lays its eggs in a hard oblong capsule, in which they are placed side by side. The young develop without metamorphosis, i.e. when newly hatched they resemble the adult in form and habits, though they are devoid of wings. During growth the cuticle is shed several times. The wings appear at the last ecdysis, when the animal attains sexual maturity: the female of $P$. orientalis is, however, wingless. 


\section{HABITS.}

Watch the living animal in a glass vessel, noting especially the positions of its legs and head; also the mode of using the long antenne, and the maxillary and labial palps.

Watch the respiratory movements of the abdomen. Feed with sopped bread, and watch the lateral movement of the jaws.

\section{EXTERNAL CHARACTERS.}

Kill the animal by pouring two or three drops of chloroform upon it.

\section{A. The Body.}

The body is divided into head, thorax, and abdomen, each of which is composed of several segments. A small neck connects the head with the thorax.

1. The head is broad from side to side, and short from before backwards, and is produced downwards into a broad process bearing the jaws. Owing to its shortness and vertical elongation, it has a deceptive appearance of being bent downwards and backwards beneath the thorax.

a. The epicranium covers the dorsal and posterior surfaces of the head. It is divided into right and left halves by a median suture which divides in front into two, running obliquely outwards to the fenestræ.

b. The clypeus is a broad plate covering the front of the head, below the epicranium.

c. The labrum is a smaller plate hinged to the lower edge of the clypeus, and forming the anterior or ' upper' lip.

d. The genæ, or 'cheeks,' are a pair of vertical plates covering the sides of the head, behind and below the eyes.

e. The eyes are large black reniform elevations, one on each side of the head. They are compound, each having very numerous hexagonal facets.

The fenestræ are a pair of small white oval patches to the inner sides of the bases of the antennæ. 
2. The neck is short and slender: its cuticular covering is soft and white, with the exception of seven thickened patches or sclerites which are hard and brown. Of these sclerites two are slender transverse bars on the ventral surface; a third is median and dorsal, and almost divided into two longitudinally; the remaining four are much larger, and form two pairs at the sides of the neck.

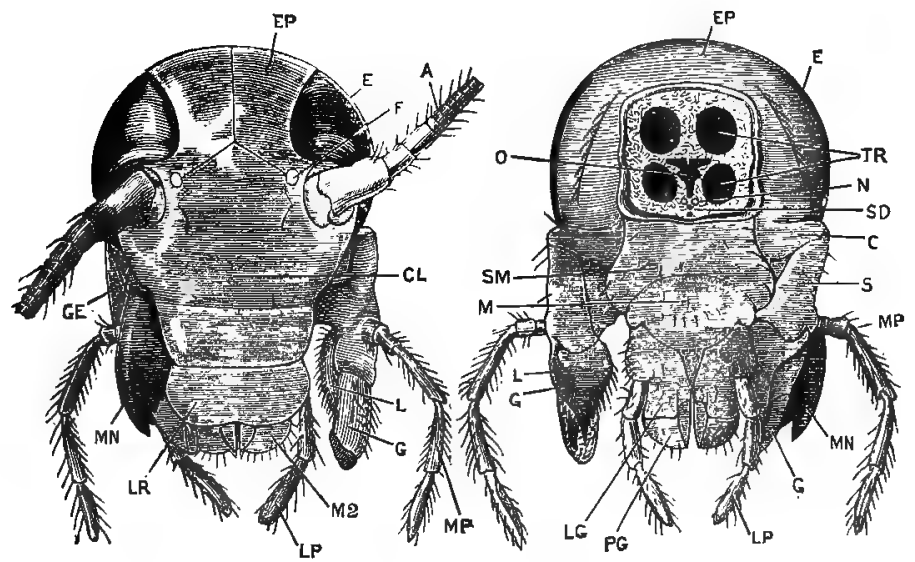

Fia. 36.-Periplaneta Americana. The head, front view. (C. н. ․)

A, antenna. CI, clypeus. E, eye. EP, epicranium. F, fenestra. G, galea.

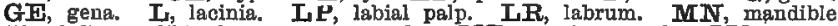
(the left mandible has been removed). MP, maxillary palp. M2, second maxilla.

FIG. 37.-Periplaneta Americana. The head from behind. (с. н.н.)

F, FP, $G, I, L P, M N$, and $M P$ as in the preceding figure.

C,' cardo. $\mathbf{L G}$, lignala. $\mathbf{M}^{\text {, mentum. }} \mathbf{N}$, nerve-connective. $O$, esophagus. $\mathbf{P G}$, paraglossa. $\mathbf{S}$, stipes. $\mathbf{S} \mathbf{D}$, salivary ducts. $\mathbf{S M}$, submentum. TR, vical tracheæ.

3. The thorax consists of three segments.

a. The prothorax is the first and longest segment of the thorax. Its tergum, the pronotum, is prolonged forwards over the neck.

b. The mesothorax is smaller, and its tergum bears at its anterior corners a pair of elytra, or wing-covers. 
These are folds of skin and cuticle which extend backwards beyond the hinder end of the abdomen, and are movably articulated to the mesothoracic tergum or mesonotum.

[The elytra are rudimentary in the female of P. orientalis.]

c. The metathorax, or hindmost segment of the thorax, bears a pair of wings, which are membranous outgrowths of the body-wall, like the elytra, but broader, thinner, and more movable. By means of these the animal is able to fly. When not in use they are folded longitudinally like a fan and covered by the elytra.

[The female of $P$. orientalis has no wings.]

4. The abdomen, which forms rather more than half the length of the body, is broad from side to side, especially in the female, and is flattened dorsoventrally. Its cuticular investment is softer than that of the head and thorax. It is composed of ten distinct segments, but the hinder ones are telescoped within one another, so that the full number are not seen at once. The podical plates may represent the tergum of an eleventh segment.

a. The terga are cuticular plates covering the dorsal surface of the abdomen and overlapping one another from before backwards. They are broad from side to side, but short from before backwards. Of the ten only eight are obvious, the eighth and ninth being hidden by the seventh through the telescoping of this part of the body. The tenth is prolonged backwards into a thin horizontal plate, deeply notched in its posterior border.

[In $P$. orientalis the eighth and ninth terga are hidden by the seventh in the female only.]

b. The podical plates are a pair of small cuticular plates at the sides of the anus.

To see the podical plates, lift up the tenth tergum with the forceps. 
c. The cerci are a pair of many-jointed styles, attached under the lateral edges of the tenth tergum and projecting backwards.

d. The sterna on the ventral surface resemble the terga on the dorsal surface. The first is rudimentary.

In the male the ninth sternum bears a pair of styles.

In the female the seventh sternum is produced backwards into a large boat-shaped process, which forms the lower wall of the large genital pouch. The hinder sterna are modified in relation with the sexual apparatus, and are completely hidden by the seventh.

\section{B. The External Apertures.}

1. The mouth opens on the ventral surface of the head, behind the labrum and between the jaws.

2. The anus is at the hinder end of the body, beneath the tenth abdominal tergum and between the podical plates.

3. The genital aperture is single, and is placed at the hinder end of the body, ventral to the anus. It is larger in the female than in the male, and will be better seen at a later stage of the dissection.

4. The stigmata, or spiracles, are the respiratory apertures, twenty in number. There are two large thoracic ones on each side, one between the prothorax and the mesothorax, and the other between the mesothorax and the metathorax. A pair of abdominal stigmata are situated between the lateral margins of the tergum and the sternum of each of the first eight abdominal segments.

\section{The Appendages.}

1. The head appendages are four pairs.

a. The antennæ are very long slender many-jointed and freely movable filaments, which arise from a pair of oval arthrodial membranes just in front of and below the eyes. 
To see the next three pairs of appendages, fix the animal down on its back by means of a pair of small pins passed through the sides of its prothorax. Bend the ventral part of the head forwards and fix it in that position by means of crossed pins.

Examine the appendages in situ, noting their positions.

Remove the whole of the labium (s M to P G, fig. 37) without injury to the structures beneath it. Mount it in glycerine.

Similarly remove and mount the right first maxilla and the right mandible.

b. The mandibles are a pair of stout jaws, strongly toothed on their inner margins, and working sideways. They lie immediately below the genæ, and articulate with the epicranium and with the clypeus.

c. The first maxillæ are situated behind the mandibles. Each consists of the following parts.

i. The protopodite consists of two joints, the cardo or proximal piece, and the stipes or distal piece.

ii. The endopodite has two divisions, an inner and an outer. The inner division, or lacinia, is a hard blade pointed at the end, and provided with stiff setæ along its inner border. The outer division, or galea, is softer and is elongated: on the inner surface of its distal end is an oval cushion covered with short curved stiff setæ, and notched to receive the point of the lacinia.

iii. The exopodite or maxillary palp is a five-jointed palp, borne on the outer side of the distal end of the stipes. Its first two joints are short.

d. The second maxillæ are similar in shape to the first maxillæ, but smaller. They are fused together in the median plane by their protopodites, and together constitute the labium or posterior lip. 
i. The two protopodites are fused in the median plane to form a two-jointed plate. The submentum or proximal joint is the larger of the two, and is very broad from side to side. The distal joint, or mentum, is smaller, and bears the endopodites and exopodites.

ii. The endopodites are together sometimes called the ligula. Each is divided into an outer division, the paraglossa, corresponding to the galea of the first maxilla; and an inner division, corresponding to the lacinia.

iii. The exopodites are three-jointed palps corresponding in form and position to those of the first maxillæ. The stump supporting each is 'called the palpiger.

2. The thoracic appendages are three pairs of legs, borne by the sterna of the thoracic segments. They are very similar to one another, but the hindmost are rather the largest. Each leg consists of the following parts.

a. The coxa is the stout, flat, proximal joint of each leg.

b. The trochanter is a very small piece attached to the distal end of the coxa.

c. The femur resembles the coxa in form, but is more slender.

d. The tibia is a slender straight joint armed with strong spines.

e. The tarsus, or foot, is six-jointed, each joint having a soft white setose patch under its distal end, giving the animal a foothold in climbing steep or slippery surfaces. The terminal joint or pulvillus is small and bears a pair of claws.

3. Abdominal appendages are very rare amongst adult insects. The anal cerci already described, and possibly also the styles on the ninth sternum of the male, may be appendages, as also may the gon- 


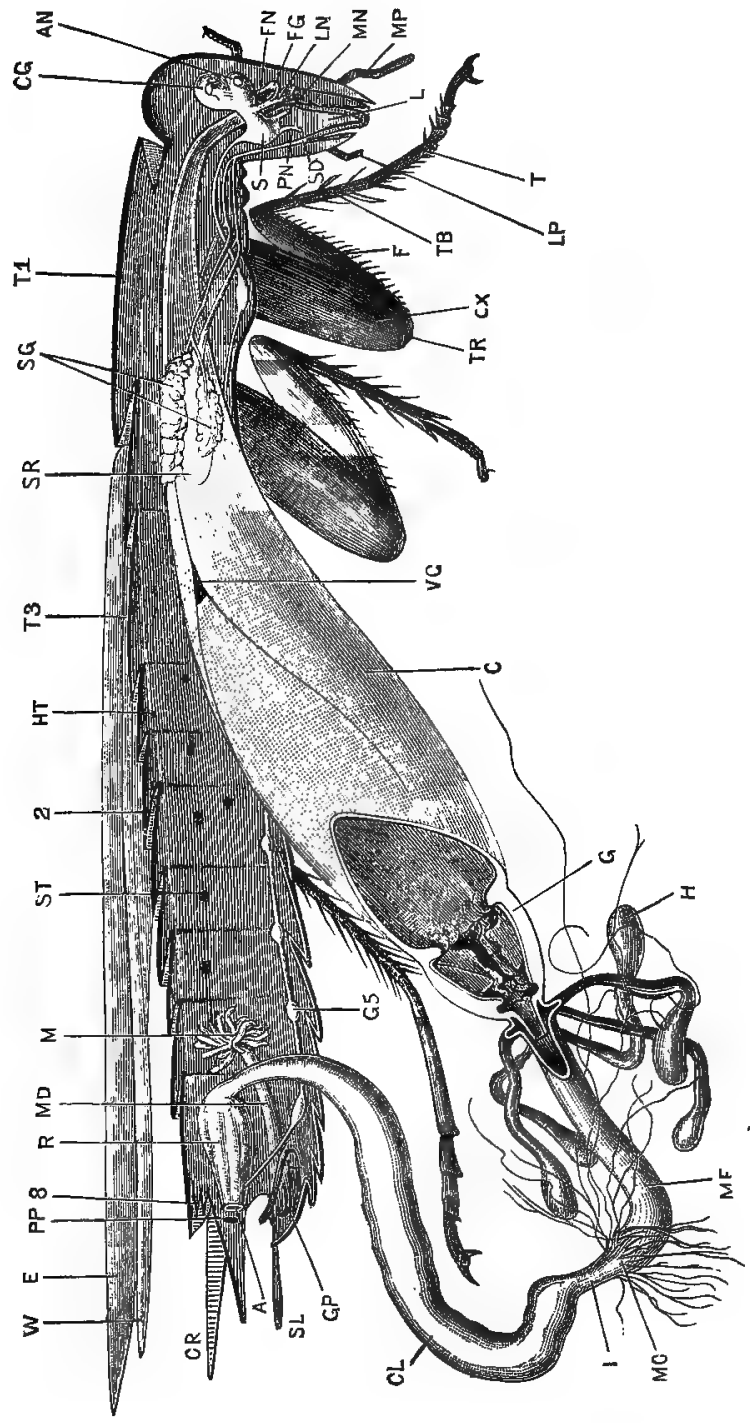


apophyses; but it is doubtful whether these really belong to the same category as the appendages of the head and thorax.

The gonapophyses belong to the genital apparatus, and will be described later.

\section{DISSECTION OF THE COCKROACH.}

Fix the animal down with the dorsal surface upwards. This may be done by drying it with blotting paper, and immersing its under surface in melted paraffin or wax, and holding it in this position till the wax is cool; or the animal may be fixed to a board by means of small pins passed through the lateral regions of the mesothorax and of the abdomen.

Cut off the elytra and the wings. Carefully cut through the terga of the abdomen and thorax close to each side, and remove the terga, avoiding injury to the heart, which lies just beneath.

\section{A. The Circulatory System.}

1. The heart is a straight chambered tube running along the mid-dorsal line of the abdomen and thorax. It receives blood through lateral paired openings, arranged segmentally, and drives it forwards by rhythmical contractions.

FIG. 38.-Periplaneta Americana. The male dissected from the right side. The testes, which are very small in the adult, are not figured. (c. H. в.)

A, anus. AN, antennary lobe of supra-cesophageal ganglion, with antennary nerve cut short. C, crop. CG, supra-cesophageal ganglion. CI, colon. $\mathbf{C R}$, anal cercus. $\mathbf{C X}$, coxa. $\mathbf{E}$, elytron. $\mathbf{F}^{\mathbf{1}}$, femur. $\mathbf{F G}$, frontal ganglion. FN, visceral nerve. $G$, gizzard. $G P$, one of the gonapophyses. $G 5$, fiftl abdominal ganglion. H, hepatic creum. HT, heart. I, ileum. I, lingua. LN, nerve to labrum. LP, labial palp. M, mushroom-shaped gland. MC, Malpighian cæc opening into junction of mesenteron and ileum : they are much longer and moxe numerous than indicated. MD, ejaculatory duct. MF, mesenteron. MN, maxillary nerve. MP, maxillary palp. PN, labial nerve. $\mathbf{P P}$, podical plate, diagrammatically drawn. $\mathbf{R}$, rectum. $\mathbf{S}$, sub-cesophageal ganglion. SD, salivary duct just above its opening into the mouth. SG, salivary glands. SL, style borne by ninth sternum. SR. salivary receptacle. $8 T$, one of the abdominal stigmata. $T$, proximal joint of the tarsus. TB, tibia. TR, trochanter. T 5, first thoracic tergum, or pronotum. T 3, third thorsoic tergum, or metanotum. VG, visceral ganglion. W, wing. $\mathbf{2}$, tergux of second abdominal segment. $\mathbf{8}$, tergum of eighth abdominal segment. 


\section{B. The Digestive and Excretory Systems.}

The alimentary canal, with its glandular appendages, is situated almost entirely in the abdomert, and is enveloped in an opaque-white, dendritic mass, the fat-body.

Expose and unravel the alimentary canal and its appendages. In dissecting the salivary ducts great care must be taken not to injure the nervous system in the head.

1. The alimentary canal is short, and slightly convoluted in its hinder part. It may be divided according to its mode of development into three regions: viz. the stomatodceum and the proctodoum, which are invaginations of the anterior and posterior ends of the embryo respectively; and the mesenteron, or middle portion, which alone is formed from the primitive alimentary tract of the embryo.

a. The stomatodoeum has a chitinous cuticular lining, continuous with the cuticular investment of the head; and is divided into the following regions.

i. The buccal cavity is lodged in the head, and its posterior wall is raised up to form an elongated fleshy tongue or lingua.

The salivary duct opens by a median aperture in the angle between the lingua and the labium (fig. 38).

ii. The osophagus is a narrow tube running from the mouth through the nerve collar and along the neck. It is laterally compressed in the cervical region.

iii. The crop is a large thin-walled pyriform sac, extending through the thorax and far into the abdomen. It is not separated by any distinct line of demarcation from the osophagus. iv. The gizzard, or proventriculus, is a pyriform sac with thick muscular walls. Within it is a series of six large cuticular teeth, and behind them six small elevations covered with setæ.

Lay the gizzard open to expose the teeth. 
b. The mesenteron is a short narrow tube extending backwards from the hinder end of the gizzard.

Lay the mesenteron open and note the funnel-like prolongation of the gizzard extending into it.

c. The proctodœum is the convoluted hinder portion of the alimentary canal, and is lined by a thin chitinous cuticle. It is divided into the following regions.

i. The ileum is a short narrow tube, the commencement of which is marked by the attachment of the numerous fine thread-like Malpighian tubules.

ii. The colon is much wider and longer than the ileum, and is constricted at its hinder end.

iii. The rectum is the short dilated terminal portion of the alimentary canal. Its wall has six longitudinal ridges, projecting internally. It ends at the anus, between the podical plates.

2. The digestive glands.

a. The salivary glands are two pairs of diffuse white glands on the sides of the anterior part of the erop.

The salivary receptacles are a pair of thinwalled sacs, one on each side of the crop, between the two glands of its side.

The ducts of the receptacles run forwards and unite beneath the nerve-cord at the hinder end of the neck to form a median duct, which runs forwards to open into the mouth, behind the base of the lingua.

The ducts of the salivary glands unite to form one duct on each side: the two ducts thus formed run forwards and unite beneath the nerve-cord in the hinder part of the neck to form a median duct: this runs forwards between the nerve-cord and the median duct of the receptacles, and opens into the latter at the anterior end of the neck. 
Having made out the anatomical relations of the salivary glands, receptacles, and ducts, remove the whole apparatus. Stain it with carmine; mount it in glycerine, and examine with the microscope.

Nuclei are visible in the walls of the ducts, and in the secretory cells of the glands.

The chitinous lining of the ducts shows a spiral thickening.

b. The 'hepatic' cæca are eight or seven cæcal diverticula of the anterior end of the mesenteron. They are convoluted and club-shaped.

Note the openings of the hepatic caca into the mesenteron.

3. The excretory system consists of sixty or more Malpighian tubules, which are fine filamentous diverticula of the anterior end of the ileum, arranged in six bundles. They are very long and convoluted, and are interlaced among the trachex and the filaments of the fat-body all through the abdomen.

\section{The Respiratory System.}

The respiratory system consists of a series of tracheal tubes: containing air. These commence at the respiratory apertures, or stigmata, on the sides of the thorax and abdomen, and ramify through all parts of the body. Expiration is effected by contraction of the muscles of the body, which compress the tracheæ ; inspiration, by dilatation of the tracheæ through the elasticity of their cuticular lining.

The readiness with which air is carried to all parts of the body, through the great perfection of the respiratory system, accounts for the extreme imperfection of the circulatory system in an animal which is otherwise very highly organised.

1. The tracheal tubes when full of air are silvery white.

Remove a large trachea, and examine it microscopically in water.

a. The cuticular lining has a very strong spiral thickening, which gives great elasticity to the tube. 
b. The cells which form the organised wall of the tube, and secrete the chitinous lining, are thin and not easily seen till stained.

Stain with carmine, and note the nuclei of the cells.

Remove small portions of muscle from the leg, of the fatbody, of the salivary gland, etc., and note and examine the fine tracheal tubes ramifying through them. Stain as above.

\section{The Nervous System.}

The nervous system consists of supra-œsophageal and sub-œsophageal ganglia, and para-œsophageal connectives in the head; a double ventral chain in the thorax and abdomen; and a visceral nervous system in relation with the alimentary canal. (Fig. 38, p. 160.)

1. The cephalic nervous system.

If the buccal cavity has already been exposed, a second specimen will probably be necessary for the dissection of the nervous system of the head.

Fix the head, with the anterior surface upwards, by means of a fine pin through the upper part of the epicranium and another between the mandibles.

Remove the clypeus and the anterior portion of the epicranium with scissors or the point of a scalpel, taking care not to injure the ganglia, which lie close beneath the clypeus. Carefully expose and clean the following structures, avoiding injury to the cephalic portion of the visceral nervous system.

a. The supra-cesophageal, or 'cerebral,' ganglia are a pair of large closely apposed ganglia close to the anterior surface of the head. They are supported by an internal chitinous framework, the tentorium.

i. The 'hemispheres' are the large rounded upper parts of the ganglia. From them arise the large optic nerves.

ii. The antennary lobes are the smaller and lower portions of the ganglia. From them the antennary nerves arise. 
Turn the head and fix it with the right side upwards. Remove the gena and mandible, and as much as is necessary of the first maxilla, submentum, and mentum, to expose the right half of the following structures.

b. The para-csophageal connectives are a pair of short thick bands of nervous tissue, running round the sides of the csophagus, from the lower part of the supra-cesophageal ganglia to the sub-œsophageal ganglia. From each a nerve arises supplying the labrum, as well as a nerve belonging to the visceral system.

c. The sub-esophageal ganglia are a pair of large ganglia lying between the submentum and the cesophagus. Below they give off several nerves, of which the two largest on each side supply the maxillary and labial palps. Above, they give off connectives, which run back through the neck and form the anterior part of the ventral chain.

\section{The ventral chain.}

Fix the cockroach with the dorsal surface upwards: remove the gizzard, mesenteron, and proctodoum; and turn the crop aside.

The ventral chain consists of a double series of ganglia, with double connectives, running along the ventral wall of the body.

A pair of closely apposed ganglia lie in each of the three thoracic segments, and a pair of smaller ganglia in each of the first six abdominal segments. The last pair are the largest of the abdominal ganglia, and give off nerves to the various parts of the sixth and succeeding segments.

3. The visceral nervous system arises as a pair of nerves, one from the anterior part of each para-œsophageal connective. The two nerves, after giving off a pair of nerves to the labrum, unite in the frontal ganglion, a small median ganglion on the anterior wall of the œsophagus, just below the antennary lobes. From 
this ganglion a median recurrent nerve runs backwards on the œsophagus, beneath the supra-œsophageal ganglia, from which it receives a pair of ganglionated nerve-trunks: it then continues its course backwards, as a median nerve, along the cesophagus and crop to a small triangular ganglion on the dorsal surface of the crop, about the middle of its length. From this ganglion two branches run obliquely backwards over the surface of the crop.

\section{E. Sense Organs.}

1. The tactile organs have already been examined. They are the antennæ, the maxillary palps, the labial palps, and the anal cerci.

2. The eyes are very large compound organs which agree in essential characters with those of the crayfish. The corneal facets are hexagonal.

3. The fenestræ are perhaps sense organs, but their function is unknown.

\section{F. The Reproductive System.}

1. In the male.

a. The testes are readily recognisable in the young, but atrophy in the adult. They are paired organs imbedded in the fat-body below the fifth and sixth terga of the abdomen.

b. The vasa deferentia are a pair of narrow tubes leading from the testes to the vesiculæ seminales.

c. The vesiculæ seminales, together known as the ' mushroom-shaped gland,' are two tufts of white glandular cæca, opening into the slightly dilated anterior end of the ejaculatory duct.

d. The ejaculatory duct is a short muscular tube opening behind to the exterior, just below the anus.

e. The gonapophyses are a series of strong chitinous hooks and plates, forming a complex copulatory organ. They are asymmetrical, and surround the genital aperture. 


\section{In the female,}

a. The ovaries are two sets of elongated tubular organs in the hinder part of the abdomen. Each set consists of eight tubes, united in front and attached by a ligament to the dorsal wall of the thorax. Behind and below they unite to form the oviducts. Each tube has a moniliform appearance, from the bulgings caused by the con-

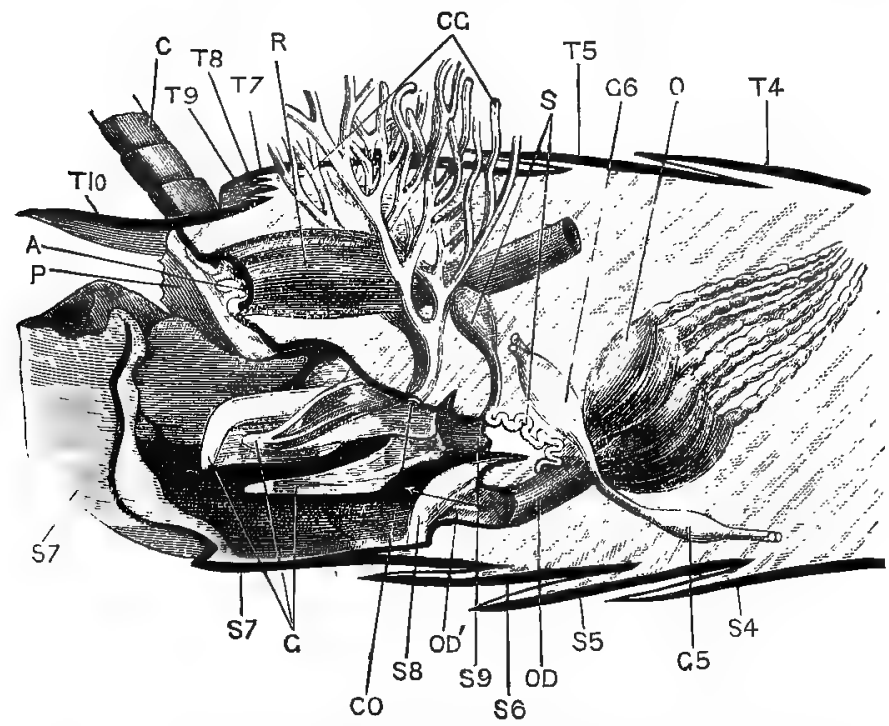

FIg. 39.-Periplaneta Americana. The hinder part of the abdomen of the female dissected from the right side to show the reproductive apparatus. The right ovary with its duct has been removed, and only a small portion of the colleterial glands is shown. The thick black lines represent the cut edges of the terga, sterna, etc. $\dot{x} 8$. (с. В. н.)

A, anus. C, anal cercus. CG, colleterial glands. CO, aperture of ducts of colleterial giands. $\mathbf{G}$, gonapophyses. $\mathbf{G} 5$ and $\mathbf{G} 6$, the last two abdominal nerve-ganglia. $O$, left ovary. $O D$, left oviduct. $O^{\prime}$, median oviduct. $\mathbf{P}$, podical plate. $\mathbf{R}$. rectum. $\mathbf{S}$, the two divisions of the spermatheca. $\mathbf{S} 4$, S5, etc., fourth and following sterna. T 4, T 5, etc., fourth and following terga.

tained ova : in the hinder and lower part of the tube the ova are large and nearly mature; those 
in the upper part of the tube are in various earlier stages of development.

At the time of laying, one ovum escapes from each ovarian tube, and the sixteen are laid side by side in a capsule.

b. The oviducts, formed as above, are a pair of short wide tubes, which unite, and open to the exterior by a median vertical slit in the eighth sternum.

c. The spermatheca consists of two unlike and unequal cæca of small size, opening by a median aperture on the ninth sternum. It lies close behind the last nerve ganglia of the ventral chain.

d. The colleterial glands are a pair of much-branched tubular glands, whose duets open separately, but close together, behind the spermatheca, and between the inner gonapophyses.

e. The gonapophyses are six symmetrically arranged processes, strongly chitinised on their outer surfaces. They are outgrowths of the sternal surface, between the vulva and the anus; and aid in depositing the eggs. 


\section{Chapter $\mathrm{X}$.}

\section{THE LANCELET. Amphioxus lanceolatus.}

AMpHIoxus is a small semi-transparent fish-like animal, about. a couple of inches in length, found in shallow parts of the Mediterranean and other seas. It is of sluggish habits, and. usually remains buried in the sand, either completely or with the anterior end alone protruding; but if disturbed it swims actively by rapid lateral movements of the body.

Though distinctly belonging to the great group of vertebrate animals, Amphioxus is in several important respects simpler and more primitive than any of the more familiar members of the group. Indeed, in spite of its fish-like appearance and habits, the differences between Amphioxus and an ordinary fish, such as a dog-fish or whiting, are of greater morphological importance than those separating fish from the most highly organised vertebrates, such as birds or mammals. It is therefore necessary to divide vertebrates into two main divisions, the first of which, Acrania, includes Amphioxus and a degenerate group, the ascidians; while the second division, Craniota, includes all remaining vertebrates, from fish to. mammals.

\section{EXTERNAL CHARACTERS.}

Take an adult specimen preserved in spirit, place it in a. small dish in weak spirit or water, and examine with a pocket lens or dissecting microscope.

a. The shape. Amphioxus is elongated, pointed at. both ends, and compressed from side to side, 
especially towards the posterior end. There is no obvious head, and no trace of limbs.

b. The fins. A low dorsal fin runs along the middorsal line from end to end of the animal, becoming more prominent at the hinder end as the upper lobe of the caudal fin.

The ventral surface bears a median fin in its posterior third, but in front of this is flattened, so that the body is triangular in transverse section. The sides of this flattened surface are bordered by the lateral fins, or metapleural folds.

c. The myotomes. The sides of the body are marked by a series of $>$ shaped lines, formed by septa of connective tissue which divide the great lateral muscles of the body into segments or myotomes.

d. The mouth is a large oval aperture on the ventral surface of the anterior end of the body, and is fringed on each side by a series of ciliated tentacles or cirri.

e. The anus is on the ventral surface, not far from the hinder end of the body. It is placed a little to the left of the median plane, at the base of the caudal fin.

f. The atrial pore is a median aperture on the ventral surface, bordered by prominent lips. It is in front of the anus, at the junction of the ventral and lateral fins, and about one-third the length of the animal from its hinder end. Through it the water escapes which has been taken in at the mouth for respiration.

\section{THE ANATOMY OF AMPHIOXUS.}

Owing to its small size it is not practicable to determine all the points of its anatomy by dissection. The external characters should be determined by examination of adult specimens preserved in spirit. The general anatomy is best seen in young specimens, not more than half an inch in 

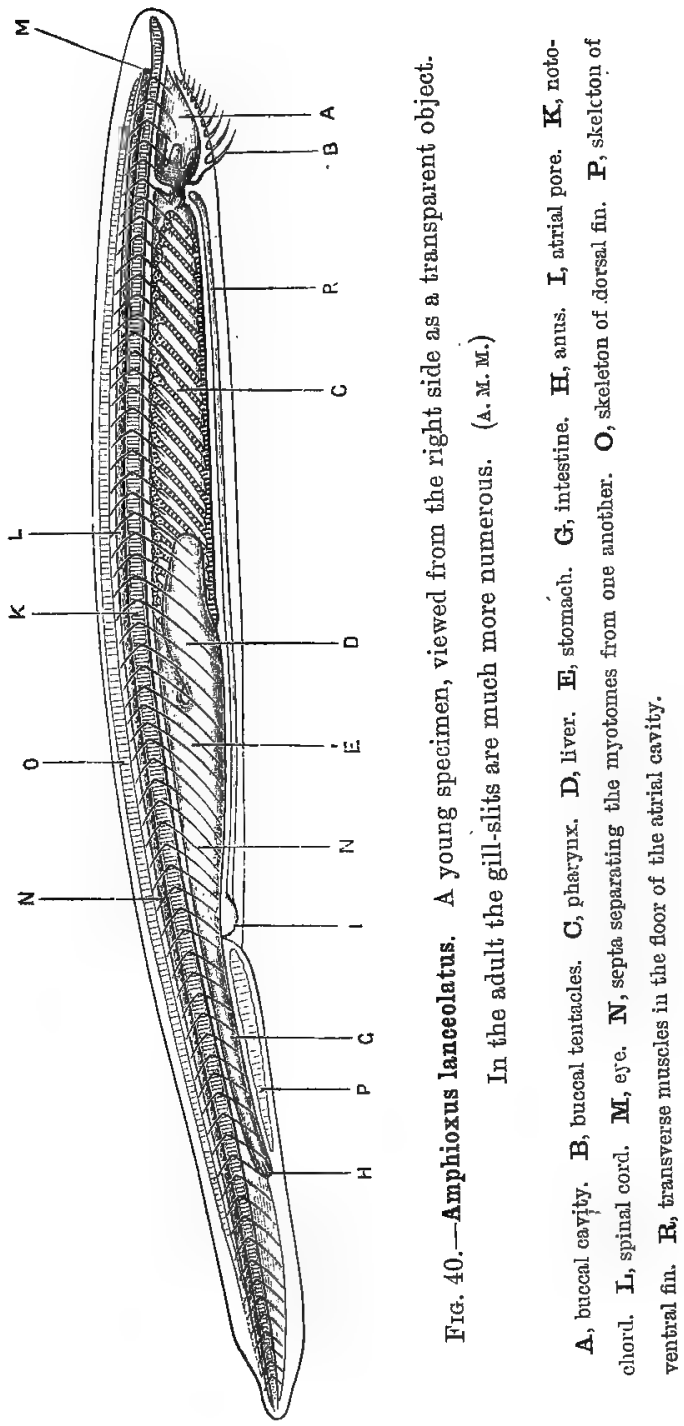
length, mounted whole as microseopical preparations; while the details of structure should be confirmed by microscopical examination of transverse sections through various regions of the body.

Examine under a low power a young specimen of Amphioxus which has been stained and mounted whole.

Examine also a series of transverse sections through various parts of the body of an adult specimen.

The main features in the anatomy of Amphioxus may readily be determined by dissection of an adult specimen which has been macerated in 20 per cent. nitric acid for $a$ day.

\section{A. The Skeletal System.}

The skeleton of Amphioxus is extremely simple. It contains neither bone nor cartilage; but remains throughout life in a condition corresponding to a very early stage of development in higher vertebrates.

1. The notochord is a median elastic rod which traverses. the body from end to end, lying rather nearer the dorsal than the ventral surface, and between the central nervous system and the alimentary canal. It is in contact with the integument at the two ends of the body; and it serves to slightly stiffen the animal.

It consists of cells in which, at an early period, large vacuoles appear : these increase in size, and ultimately almost completely fill the cells, rendering their outlines difficult to trace. On the dorsal and ventral surfaces of the notochord, the cells are comparatively unaltered.

2. The buccal skeleton. At each side of the mouth is a curved bar, resembling the notochord in structure. Each bar is divided transversely into about a dozen short segments, and from these filaments arise which support the buccal tentacles.

3. The skeleton of the median fins. Along the whole length of the dorsal fin, except at its extreme anterior and. 
posterior ends, is a longitudinal series of connectivetissue compartments filled with a gelatinous substance. The compartments are more than twice as numerous as the segments of the body, and their function is uncertain. A similar structure extends along the ventral fin, between the anus and the atrial pore.

4. The branchial skeleton. A series of elastic rods are developed in the walls of the pharynx to support the gill-arches. Their arrangement will be described with the alimentary canal.

5. The connective-tissue skeleton. The connective tissue of Amphioxus is very dense, and forms a kind of skeleton, of which the following are the chief parts.

a. The sheath of the notochord is a thick sheath closely surrounding the notochord.

b. The neural tube surrounds the central nervous system, and is continuous below with the notochordal sheath.

c. The septa are sheets of connective tissue which run outwards from the notochordal and neural sheaths to the integument. They separate the successive muscle-segments, or myotomes, from one another; and their lines of insertion into the integument form the $>$ shaped markings along the sides of the animal. The septa on the two sides of the body are not in pairs, but alternate with one another.

\section{B. The Muscular System.}

1. The lateral muscles form by far the largest part of the muscular system. They embrace the sides of the body along its whole length, and are divided, as noticed above, into muscle-segments or myotomes by the connective-tissue septa. The muscle-fibres run longitudinally, i.e. parallel to the axis of the body; and it is by alternate contractions of these muscles 
on opposite sides of the hody, aided by the elasticity of the notochord, that the lateral undulatory movements of the animal when swimming are produced.

2. The ventral muscles form a sheet covering the ventral surface of the body from the mouth to the atrial pore. The muscle-fibres run transversely from side to side, and by their contraction aid in expelling the water from the atrial cavity.

3. Smaller muscle-bundles are found in relation with the mouth and its tentacles, with the gill-apparatus, and with the anus and atrial pore. Nearly all the muscles are striated.

\section{The Digestive and Respiratory Systems.}

The alimentary canal is a nearly straight tube, the anterior part of which is modified for respiration, as in aquatic vertebrates generally.

1. The buccal cavity is bounded laterally by folds, which are anterior prolongations of the atrial folds : within these and near their free margins are the curved bars which support the tentacles. There are no jaws.

The anterior part of the buccal cavity is lined by a single layer of short columnar epithelial cells, some of which bear short flagella. In the hinder part of the cavity the epithelium is altogether different, the cells being very long and slender, and provided with long flagella. The boundary between these two regions is marked by a sharply defined sinuous line (fig. 40, p. 172).

2. The velum is a muscular diaphragm between the buccal cavity and the pharynx, opposite the anterior angle of the seventh myotome. It is perforated below its middle by an aperture which leads upwards and backwards into the pharynx, and the hinder border of which is fringed with cirri. Below this oral aperture are a pair of small ' hyoidean apertures' which also connect the buccal cavity with the pharynx. 
8. The pharynx is a wide sac, forming about half the length of the alimentary canal. It is attached along its mid-dorsal line to the under surface of the sheath of the notochord, from which it hangs down freely into the atrial cavity. Its sides are perforated by a large number of slit-like apertures-the gill-slits-which run obliquely downwards and backwards. The parts of the pharyngeal wall between the successive slits. are narrow bars-the gill-arches-each of which is. strengthened by an axial rod of a horny substance. These arches are of two kinds, arranged alternately; the axial rods of the second, fourth, etc., arches being forked at their ventral ends, while the alternate rods are unsplit. Each double gill-slit is originally a single one, but becomes divided in the course of development by the downgrowth of the unsplit bar from its dorsal end. The split bars may hence be called primary rods, and the unsplit ones secondary rods. Like the myotomes the gill-arches are not in pairs, but alternate on the two sides of the body.

The successive gill-arches are connected by horizontal bars, of which there are usually three or more to each slit, so that the pharynx has the character of an open meshwork. The inner surface of the pharynx is lined by columnar flagellate cells, which maintain a continual stream of water through the gill-slits from the pharynx to the atrial cavity, the stream serving to aërate the blood in the vessels of the arches as it passes over them.

Along the mid-dorsal line of the pharynx is the deep hyperbranchial groove, lined by a single layer of long columnar ciliated cells. A band of similar cells runs along the mid-ventral wall of the pharynx, and is folded longitudinally in its hinder part to form the hypobranchial groove.

4. The intestine commences at the hinder end of the pharynx, close to the dorsal surface, and runs straight 
to the anus. It is a direct continuation of the hyperbranchial groove, and at its commencement is very narrow: further back it dilates considerably; and this expanded part, which is sometimes called 'stomach,' is produced into a large pouch-like sacthe liver-which runs forwards some distance along the right side of the pharynx, and ends blindly in front.

\section{The Atrial Cavity.}

The boundaries and relations of this cavity can only be determined by the examination of a series of transverse sections.

The atrial or epipleural cavity is the large space which surrounds the pharynx at the sides and below: into it the respiratory stream of water passes from the pharynx through the gill-slits, to escape finally from the atrial pore.

In the early stages of development the gill-slits are exposed on the sides of the body, and the water passes through them directly to the exterior. Later on a longitudinal ridgelike fold of the body-wall appears on each side of the body above the gill-slits, and these atrial or epipleural folds rapidly grow downwards so as to form flaps covering over the gillslits : the space between the atrial folds and the body-wall is the atrial cavity. The flaps ultimately meet each other below the body in the mid-ventral line, and fuse along their whole length, except at the atrial pore, which remains open throughout life. The anterior ends of the folds grow forwards to form the side-walls of the buccal cavity.

The epithelium lining the atrial cavity is supported by a layer of connective tissue, and usually contains a good deal of brown pigment, which renders it easy to trace. It is repre: sented by the thick black line in figs. $41-43$, which show its distribution in different regions of the body. It lines the inner surface of the atrial folds, covers the outer surface of each of the gill-arches, and invests the intestine and liver.

On the primary gill-arches this layer of atrial epithelium. is wider than on the secondary arches, and towards the dorsal 
surface of the pharynx it rises up into folds. Two or more of these folds unite on each side to form the suspensory folds of whe pharynx, which run across from the dorsal part of the sides of the pharynx to the inner surface of the atrial folds, and separate the atrial cavity below from the dorsal cœlomic canals above.

The atrial cavity commences opposite the anterior end of the pharynx, and extends back almost as far as the anus: behind the atrial pore it is confined to the right side of the body.

\section{E. The Colom or Body-cavity.}

Like the atrial cavity this can only be traced by means of sections.

The colom, or body-cavity, is perfectly distinct from the atrial cavity, though its boundaries are not easy to follow, and its relations vary considerably in different regions of the body. It contains during life a coagulable fluid.

1. Behind the atrial pore the cœlom is a cavity of some width, surrounding the intestine and separating it from the body-wall.

2. In front of the atrial pore it becomes greatly reduced, owing to the increased size of the atrial cavity: it is, however, readily recognisable as a narrow space immediately surrounding the intestine and the liver.

3. In the region of the pharynx the cœlom becomes much subdivided and more difficult to trace. Its chief divisions are as follows:-

a. The dorsal cœlomic canals are a pair of irregular spaces lying at the sides of the dorsal part of the pharynx, between the atrial folds and the suspensory folds of the pharynx.

b. The branchial celomic canals are a series of tubular cavities in the primary gill-arches, lying within the folds into which the atrial epithelium is thrown on the outer sides of these arches. These cavities are very small in the ventral portions of the arches, but widen out towards their dorsal 
ends, and open, as shown in figs. 41 and 42 , into the large dorsal coelomic canals above the suspensory folds.

c. A series of colomic spaces surround the reproductive organs.

4. The metapleural canals are a pair of wide spaces running along the lateral or metapleural folds. They are possibly of a lymphatic nature.

\section{F. The Circulatory System.}

The main features of the circulatory system can be made out by examination of a series of transverse sections.

There is no heart, but the general course of the circulation is the same as in other gill-breathing vertebrates. The blood is colourless and contains but few corpuscles.

The principal vessels are as follows.

1. The cardiac aorta is a median longitudinal vessel, running forwards along the ventral wall of the pharynx in the floor of the hypobranchial groove.

2. The aortic arches are a series of vessels, arising from the cardiac aorta, and running up the primary gill-arches. Each aortic arch has at its ventral end a small contractile dilatation, which lies in the fork between the split ends of the axial rod of the gill-arch, the aortic arch itself lying along the inner side of the rod.

By means of vessels in the horizontal bars of the pharynx, the aortic arches of the primary arches are connected with similar vessels in the secondary arches.

The most anterior aortic arch on the right side is much larger than any of the others, and sends branches forwards to the anterior end of the body.

It is while the blood is in these vessels that it becomes aërated by the respiratory stream of water passing through the gill-slits.

3. The dorsal aortæ are a pair of longitudinal vessels into which the aortic arches of both primary and secondary gill-arehes open at their dorsal ends. They lie just 
beneath the notochord one on each side of the hyperbranchial groove, and project into the dorsal cœlomic canals.

The dorsal aortæ carry the arterialised blood backwards: they unite at the hinder end of the pharynx to form a single vessel, which runs back along the dorsal surface of the intestine.

4. The portal veins are vessels running, in the colom, along the ventral surface of the intestine : they collect the blood from the intestine and carry it forwards to the liver, where they break up into capillaries.

5. The hepatic veins are vessels which collect the blood from the liver and carry it backwards along the dorsal surface of the liver. On reaching the junction of the liver and intestine, they unite to form a single vessel, which turn forwards along the ventral surface of the pharynx and becomes the cardiac aorta.

6. A longitudinal vessel runs along the inner side of each atrial fold, and supplies the myotomes and reproductive organs.

\section{G. The Excretory System.}

It is not yet certain whether Amphioxus has any definite excretory organs.

The pigmented canals discovered by Lankester are possibly excretory. These are a pair of short tubes, with deeply pigmented walls, placed in the twenty-seventh segment of the body, opposite the hinder end of the pharynx. They lie, one at each side of the body, in the dorsal cclomic canals above the suspensory folds of the pharynx. Each tube is attached along its outer side to the body-wall, and opens behind into the atrial cavity : in front it is considerably contracted, but appears to open into the ccelomic canal.

Hatschek regards as a kidney' a ciliated canal which lies on the left side of the anterior end of the body. It extends. from the anterior border of the mouth to immediately behind the velum, and is believed to open behind into the pharynx. 


\section{H. The Nervous System.}

The nervous system of Amphioxus consists of:-(1) the central portion, which, as in other vertebrates, is a tube of nervous matter lying in a sheath of connective tissue immediately above the notochord, and extending almost the entire length of the body ; (2) the peripheral portion, or nerves proper, which connect the central portion with the various parts of the body.

The nervous system can be well seen in transverse sections of adults, or by examination of young specimens mounted whole. If fresh specimens can be obtained, the entire nervous system can be isolated by placing them in 20 per cent. nitric acid for three days, then washing thoroughly and leaving in water for a day. By this treatment the connective tissue is so softened and loosened that on shaking the bottle the nervous system is readily and completely separated from all the other parts. It should then be transferred to alcohol, stained, and mounted in the usual manner.

1. The central nervous system or spinal cord is of nearly uniform diameter along the greater part of its length: it diminishes slightly in size towards the anterior end, and much more markedly at the posterior end, which is very slender.

The central canal lies much nearer the ventral than the dorsal surface: it is very small except at the extreme front end, where it expands to form the ventricle, from the anterior end of which a small diverticulum arises which communicates by a pore with a ciliated pit on the dorsal surface and left side of the anterior end of the body. Through this pit the central canal of the nervous system opens directly to the exterior.

At intervals along the spinal cord are spots of black pigment, contained in cells in the floor of the central canal.

2. The peripheral nervous system consists of nerves of two kinds: (1) nerves which arise by single roots, and, 
except in the case of the first pair, from the dorsal surface of the central nervous system; (2) nerves arising by multiple roots, and from the ventral surface of the cord.

a. Nerves arising by single roots. These apparently correspond to the dorsal or sensory roots of the spinal nerves of other vertebrates: they differ from these, however, in having no ganglia, and in the fact that some of their branches are distributed to muscles.

i. The first pair of nerves arise from the ventral surface of the anterior end of the central nervous system, and supply the anterior fin.

ii. The second pair of nerves are much larger. They arise from the dorsal surface of the central nervous system immediately behind the first pair. Each divides into three main branches, supplying the anterior fin.

iii. The third pair of nerves are much smaller than the second, and arise immediately behind them. They supply the parts about the mouth.

iv. The fourth pair of nerves arise from the dorsal surface of the spinal cord, a short distance behind the third pair ; the nerve of the right side being a short distance behind that of the left side.

v. The fifth pair of nerves are still more oblique, the right nerve arising some distance behind the left.

The succeeding nerves, along the whole length of the cord, arise alternately on the two sides. These nerves run outwards along the connective-tissue septa separating the myotomes, and divide into branches supplying the skin and muscles 
b. Nerves arising by multiple roots. These apparently correspond to the ventral or motor roots of the spinal nerves of other vertebrates.

They arise from the ventral surface of the spinal cord along nearly its whole length, alternating with the dorsal nerves; a ventral nerve on one side being opposite a dorsal nerve on the other. Each arises by a large number, thirty or more, of very slender rootlets, placed one in front of another, the entire length of a ventral root being nearly equal to the interval between two successive roots. The several rootlets do not unite, but pass separately through the connective-tissue sheath of the cord, and end in the myotomes.

\section{The Sense Organs.}

The sense organs of Amphioxus are extremely simple in structure, and differ markedly from those of the higher vertebrates in being unpaired.

1. The pit-like depression of the skin that places the central canal of the nervous system in communication with the exterior has been described as an olfactory organ.

2. The 'eye' is a rounded pigment-spot in the anterior wall of the ventricle, i.e. at the extreme anterior end of the central nervous system.

\section{K. The Reproductive Organs.}

The sexes are distinct, but the males and females are alike except as regards the microscopic structure of their reproductive organs. There are no special genital ducts.

\section{The female.}

The ovaries are a series of saccular organs of a horseshoe shape, arranged in a row along the inner surface of the atrial fold on each side of the pharynx, and extending back as far as the atrial pore. They lie in cavities, which are extensions of the coelom into 
the atrial folds: these cavities are readily recognisable while the ovaries are young, but become almost obliterated when the ova ripen.

The ova, when mature, are discharged into the atrial cavity by dehiscence of the proper wall of the ovary and of the atrial membrane. The discharged ova, together with the projecting ovaries, form a bulky mass, which causes great distension of the atrial cavity and distortion of the pharynx and other organs.

The ova, which measure $\frac{1}{250}$ inch in diameter, ultimately pass through the gill-slits into the pharynx, and escape through the mouth; or possibly through the hyoidean apertures.

2. The male.

The testes are similar in form and position to the ovaries; and the spermatozoa, when ripe, are discharged like the ova into the atrial cavity, from which they escape by the atrial pore.

\section{EXAMINATION OF TRANSVERSE SECTIONS.}

Many points in the anatomy of Amphioxus are determined far more readily by an examination of transverse sections of the whole animal than by dissection. For this purpose an entire specimen should be cut by means of a microtome, and the sections mounted on slides in their proper sequence. It. is only necessary to examine in detail a few of the more typical ones.

\section{A. Transverse Section passing through the Buccal Cavity.}

1. General characters. The dorsal surface is indicated by the laterally compressed dorsal fin: the ventral surface by the mouth-opening, which is bordered at the sides by the thickened lips; within the buccal cavity are usually seen sections of the tentacles. About the middle of the section is the notochord, and above this is the spinal cord. At the sides are 
the myotomes, separated from one-another by connective-tissue septa.

2. The skin consists of two layers.

a. The epidermis is a single layer of short columnar cells, with a thick cuticle on their outer ends.

b. The dermis is a very thin layer of connective tissue, underlying and supporting the epidermis.

3. The skeletal system.

a. The notochord is slightly oval, its longer axis being vertical. It is crossed by numerous wavy transverse bands, the spaces between which are occupied by a homogeneous substance: this latter is the coagulated semifluid matter occupying the vacuoles of the notochordal cells, the outlines of which are indicated by the wavy bands.

Near the surface of the notochord, and especially in the dorsal region, cells may be seen which have undergone comparatively little modification.

b. The connective tissue forms a thick deeply-staining investment to the notochord, which is prolonged upwards to enclose the spinal cord, and outwards to form the septa: these latter separate the myotomes from one another, and are continuous at their outer borders with the dermis.

c. The buccal skeleton. Sections of the segmented rods which strengthen the lips are seen bordering the entrance to the buccal cavity: a smaller rod occupies the axis of each tentacle. In structure these rods resemble the notochord.

d. The skeleton of the dorsal fin. The dorsal fin is filled with a gelatinous homogeneous substance, which stains slightly with reagents.

4. The muscles.

a. The myotomes are seen as squarish masses of muscle, lying along the sides of the body in the 
compartments formed by the septa. Owing to the obliquity of the myotomes, three or more are cut in each transverse section, and owing to their > shape each myotome is cut twice. The myotomes of the two sides are not opposite, but alternate with each other.

b. Other much smaller muscles are seen in connection with the buccal skeleton and tentacles.

\section{The atrial cavity.}

The buccal cavity is, strictly speaking, part of the atrial cavity, and not of the alimentary canal, its sides being formed by growth forwards of the anterior ends of the atrial folds. The true mouth of Amphioxus is the aperture in the velum leading into the pharynx, and not the entrance to the buccal carity.

The epithelium lining the buccal cavity consists of a single layer of cells, which in the anterior and ventral part are short and columnar, but in the posterior and dorsal region are much elongated, slender and flagellate.

6. The nervous system.

a. The spinal cord lies in a connective-tissue sheath immediately above the notochord. In transverse section it is somewhat triangular, with rounded angles, and is rather wider from side to side than it is dorso-ventrally.

The central canal lies nearer the ventral than the dorsal surface: it is lined by short columnar ciliated cells, and immediately below it are the large deeply pigmented cells already seen in the whole animal. From the central canal a narrow vertical slit extends upwards to the dorsal surface of the cord, both the canal and the slit being bordered by large nerve-cells. In young specimens the slit is open above, but in adults it is closed by connective tissue, and by processes of the 
nerve-cells growing across it from side to side. The greater part of the cord has a finely punctate appearance, caused by the cut ends of the nerve-fibres.

b. The nerves will only be recognised if the section happens to pass through their roots of origin.

i. The dorsal nerves arise by large single roots from the sides of the cord, about midway between the dorsal and ventro-lateral angles.

ii. The ventral nerves arise by very minute and multiple rootlets from the ventro-lateral angles of the cord.

B. Transverse Section through the Anterior Part of the Pharynx.

1. General characters. The section is triangular in shape, the apex being formed by the dorsal fin, the base by the ventral surface, and the basal angles by the metapleural folds. The pharynx, perforated by the gillslits, and surrounded by the atrial cavity, lies below the notochord.

2. The skin of the ventral surface is thrown into very strong folds, to allow for the distension of the atrial cavity when the reproductive organs attain their full size. Its general characters are the same as in A.

3. The skeletal system is the same as in A, except that the branchial skeleton is present, and that there is no buccal skeleton.

4. The muscular system.

a. The myotomes are similar to those of $\mathrm{A}$, but considerably larger.

b. The ventral muscles form a thin sheet running in the atrial fold of each side from the lowest myotome to the mid-ventral line, where the muscles of the two sides meet in a median raphe.

c. Small muscular bands are present in the suspensory folds of the pharynx and in the gill-arches. 
5. The pharynx is of an inverted heart-shape in transverse section.

a. The branchial bars, or gill-arches. Owing to their obliquity and extreme closeness to one another twenty or more bars may be cut on each side in a single section. Each bar is clothed on its outer surface by the atrial epithelium, which is a single

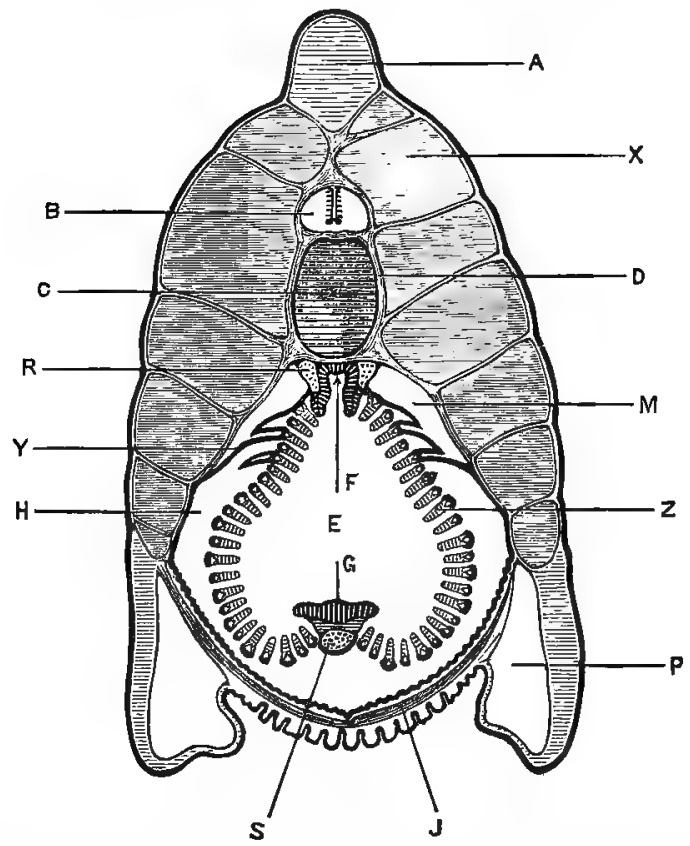

Frg. 41.-Amphioxus lanceolatus. Transverse section through the anterior part of the phargnx of an adult specimen. The boundary of the atrial cavity is indicated by a thick black line. (A. M. M.)

A, skeleton of dorsal fin. B, spinal cord. $\mathbf{C}$, notochord. D, connectivetissue sheath surrounding notochord. $\mathbf{E}$, cavity of pharynx. F, hyperbranchial groove of pharynx. G, hypobranchial band of pharynz, which in this anterior part is flattened out or even conver. H, atrisil cavity. J, transverse muscles in floor of atrial cavity. M, dorsal colomic canal. $\mathbf{P}$, metapleural canal. $\mathbf{R}$, left dorsal aorta. $\mathbf{S}$, cardiac aorta, $\mathbf{X}$, myotome. $\mathbf{Y}$, suspensory fold of pharynx, separating the dorsal ccelomic canal from the atrial cavity. Z. gill-arch or branchial bar ; the white triangular spot represents the cut surface of the skeletal rod of the arch. 
layer of columnar cells. Immediately within this is the skeletal rod, deeply grooved along its inner surface. The rod is ensheathed in connective tissue, which runs inwards from it as a narrow plate clothed on each side by the pharyngeal epithelium, a single layer of very long columnar flagellate cells.

The branchial bars are alternately large and small, the difference being especially marked at their outer edges. The large ones are the primary, and the small ones the secondary bars.

In the large primary bars a small space is visible between the atrial epithelium and the skeletal rod. This space, the branchial cœlomic canal, which is part of the cœlom, is much larger in the dorsal than the ventral bars of the section, and in the most dorsally placed ones it becomes a space of considerable size, the atrial epithelium being prolonged outwards for some distance. The uppermost two or three of these outward prolongations of the atrial epithelium unite together to form the suspensory folds of the pharynx, which sling it to the inner surface of the atrial folds, the branchial colomic canals opening here into the dorsal coelomic canals (fig. 41).

b. The hyperbranchial groove is a deep median groove in the dorsal wall of the pharynx immediately beneath the notochord. It is lined by columnar epithelium.

c. The ventral wall of the pharynx is a flat or slightly convex plate, the hypobranchial band, covered by a layer of columnar epithelial cells. The hypobranchial groove does not extend into this part of the pharynx.

6. The atrial cavity surrounds the sides and ventral surface of the pharynx, lying between it and the atrial folds, and communicating with the cavity of the pharynx 
through the gill-slits. The atrial epithelium consists of a single layer of pigmented columnar cells, and is often much folded on the ventral wall.

7. The colom is much subdivided in this region: its principal divisions are as follows.

a. The dorsal colomic canals are a pair of large irregular spaces at the sides of the hyperbranchial groove, above the suspensory folds of the phargnx, and between these and the atrial folds.

b. The branchial colomic canals are the cavities in the primary branchial bars, between the atrial epithelium and the skeletal rods. They all open at their dorsal ends into the dorsal colomic canals.

8. The metapleural canals are a pair of large spaces, triangular in section, lying in the metapleural folds.

9. The circulatory system. The vessels can be readily recognised in sections, owing to the coagulated blood which they contain.

a. The cardiac aorta is a median vessel lying in the floor of the pharynx.

b. The dorsal aortæ are a pair of vessels lying one on each side of the hyperbranchial groove.

c. The aortic arches lie in the branchial bars, both primary and secondary, along the inner sides of the skeletal rods.

10. The nervous system is the same as in A.

C. Transverse Section passing through the Hinder Part of the Pharynx.

1. General characters. The section is larger than B, passing through the thickest part of the body. It is triangular in shape, the angles being formed by the dorsal and lateral fins respectively. The section passes through the laterally compressed phargnx, on the right side of which is the liver. The reproductive organs project into the atrial cavity from the inner sides of the atrial folds. 
The cutaneous, skeletal, muscular, and nervous systems are practically the same as in B.

2. The digestive system.

a. The pharynx has the same structure as in B, but differs markedly in shape, being strongly compressed laterally, so that its two sides are almost

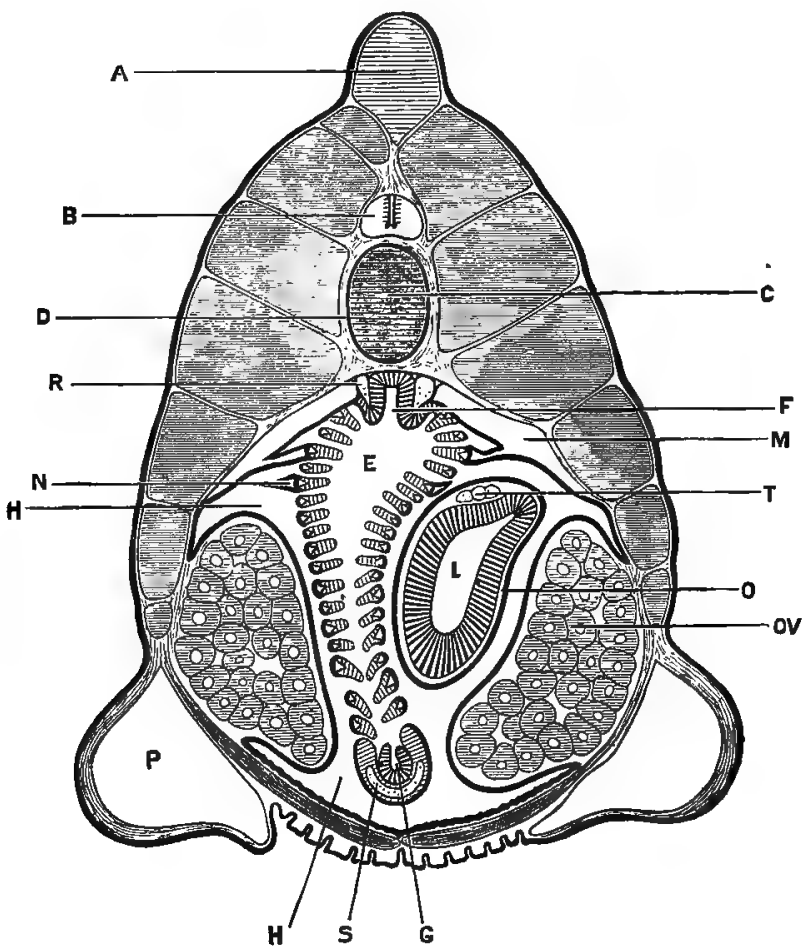

Frc. 42.-Amphioxus lanceolatus. Transverse section through the hinder part of the pharynx of an adult female, passing through the liver and ovaries. The boundary of the atrial cavity is indicated by a thick black line. (A. M. M.)

A, skeleton of dorsal fin. B, spinal cord. C, notochord. D, connectivetissue sheath of notochord. $\mathbf{E}$, cavity of pharynx. $\mathbf{F}$, hyperbranchial groove. G, hypobranchial groove. $\mathbf{H}$, atrial cavity. $\mathbf{L}$, liver. $\mathbf{M}$, dorsal cœlomic canal. $\mathbf{N}$, branchial cœlomic canal. $\mathbf{O}$, colomic'space surrounding liver. $O V$, ovary. $\mathbf{P}$, metapleural canal. $\mathbf{R}$, left dorsal aorta. $\mathbf{S}$, cardiac aorta. T, hepatic veins. 
in contact with each other; and the hypobranchial groove is as well marked as the hyperbranchial.

b. The liver lies at the right side of the pharynx, and is oval in section. Its walls are thick, and consist of a single layer of very long and slender flagellate cells, resting on a thin outer wall of connective tissue.

3. The atrial cavity has the same general relations as in B. The atrial epithelium covers the outer surfaces of the branchial bars, and the suspensory folds of the pharynz; and is continued downwards over the inner surfaces of the atrial folds, covering the reproductive organs, and being thrown into folds along the ventral wall of the atrial cavity. It also forms an investment to the liver.

4. The colom is still more subdivided than in B : its main divisions are as follows.

a. The dorsal colomic canals are a pair of large spaces above the suspensory folds of the pharynx, as in B.

b. The branchial celomic canals are a series of cavities in the primary branchial bars, as in B.

c. A space surrounding the liver, between its proper wall and the atrial epithelium, is part of the cœlom.

d. Paired spaces immediately surrounding the reproductive organs are also extensions of the cœlom.

5. A pair of large metapleural canals lie in the lateral fins.

6. The circulatory system.

a. The cardiac aorta, the dorsal aortæ, and the aortic arches are the same as in $B$.

b. The portal veins lie on the liver, in the colomic space surrounding it. They are often difficult to identify in transverse sections. 
c. The hepatic veins are three or four vessels lying side by side on the dorsal surface of the liver, in the colomic space between the liver and the atrial membrane investing it.

7. The reproductive organs occupy similar positions in the two sexes. They are masses, varying in size according to the degree of ripeness of the ova or spermatozoa, lying on the inner sides of the atrial folds, and projecting into the atrial cavity.

D. Transverse Section passing through the Atrial Pore.

1. General characters. The section is smaller than the preceding one, and the myotomes reach much further down the sides. The intestine is cut across, but the section lies entirely behind the reproductive organs. The metapleural folds are present, but are much smaller than in B or C, and between them in the median plane is the atrial pore.

The skin, and the skeletal, muscular, and nervous systems are practically the same as in B, except that the ventral muscles are absent.

2. The intestine is circular in section. Its walls are thick and, like those of the liver, consist of a single layer of very long and slender epithelial cells, resting on a thin connective-tissue basement membrane.

3. The atrial cavity surrounds the sides and the ventral surface of the intestine, and opens to the exterior at the atrial pore in the mid-ventral line between a pair of thickened and prominent lips, which are prolonged backwards like a spout.

4. The cœlom is reduced to a narrow space surrounding the intestine, between its proper wall and the atrial membrane.

5. The metapleural canals are very greatly reduced in size.

6. The circulatory system.

a. The dorsal aorta is a median vessel, between the notochord and the intestine. 
b. The portal or sub-intestinal veins are three or four vessels lying side by side on the ventral surface of the intestine, in the colomic space surrounding it.

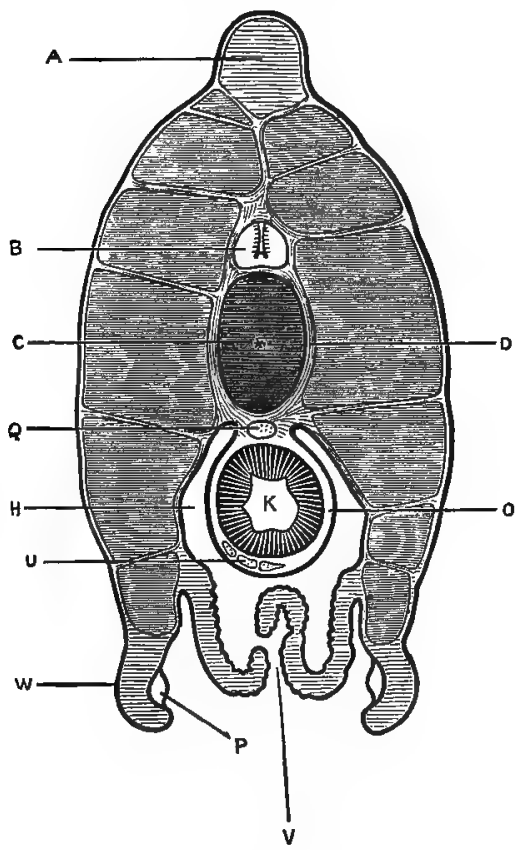

Fig. 43.-Amphioxus lanceolatus. Transverse section of an adult specimen, passing through the atrial pore. The boundary of the atrial cavity is indicated by a thick black line. (A.M. M.)

$\mathbf{A}$, skeleton of dorsal fin. $\mathbf{B}$, spinal cord. $\mathbf{C}$, notochord. $\mathbf{D}$, connectivetissue sheath of notochord. $\mathbf{H}$, atrial cavity. $\mathbf{K}$, intestine. $\mathrm{O}$, ccelomie space surrounding intestine. $\mathbf{P}$, metapleural canal. $\mathbf{Q}$, dorsal sorta. $\boldsymbol{U}$, portal veins. $\mathbf{V}$, atrial pore. W, metapleural folds.

E. Transverse Section passing through the Anus.

1. General characters. The section is much smaller than the preceding ones. It is oval, with median dorsal and ventral fins, and the notochord occupies its centre. 
The section lies behind the atrial cavity, which is therefore entirely absent.

The skin and the skeletal, muscular, and nervous systems are practically the same as in B, except that the ventral muscles are absent.

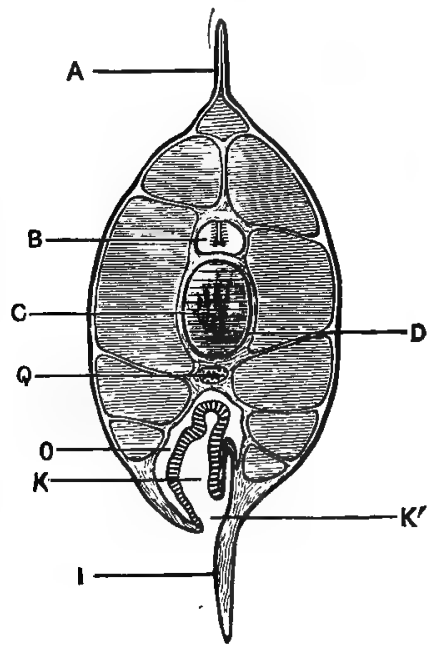

FIa. 44.-Amphioxus lanceolatus. Transverse section of an adult specimen, passing through the anus. (A. … M.)

A, dorsal fin. B, spinal cord. C, notochord. D, connective-tissue sheath of notochord. $\mathbf{I}$, ventral fin. $\mathbf{K}$, intestine. $\mathbf{K}^{\prime}$, anus. $\mathbf{O}$, coelom. $\mathbf{Q}$, dorsal aorta.

2. The intestine lies below the notochord, and the anus is on the left side of the ventral fin.

3. The colom is a space surrounding the dorsal and lateral surfaces of the intestine.

4. The dorsal aorta is a median vessel in the connective tissue below the notochord. 


\section{Chapter XI.}

\section{THE DOG-FISH. Scyllium canicula.}

THE dog-fish, together with the sharks and skates, belongs to the group of fish known as Elasmobranchs, the great majority of which are powerful swimmers, of carnivorous habits, and exceedingly voracious. One of the chief characters of the group is that the internal skeleton remains cartilaginous throughout life, no bone being developed in it : hence Elasmobranchs are frequently spoken of as cartilaginous fish.

There are several kinds of dog-fish found off our own coasts. The following description is taken from one of the most abundant species, but will apply with but slight changes to any of the common forms. Skates differ markedly from dog-fish in external appearance, owing to the enormous size of their pectoral fins, which form the great lateral wing-like expansions of the body: as regards internal structure, however, the differences are comparatively unimportant, and a skate might easily be dissected by following the directions given in this chapter. All the measurements here given are taken from a dog-fish of about two feet in length, which will be found the most convenient size for dissection.

Most dog-fish lay eggs, which are of large size, and enclosed in horny capsules produced at their angles into long spirally twisted strings for attachment. In many cases the eggs are retained within the oviduct during part or the whole of their development; and in some few species a still closer relation is established between the mother and the embryo, similar in its essential features to the placenta of mammals, whereby the embryo obtains during its development a supply of nutriment direct from the mother. 


\section{EXTERNAL CHARACTERS.}

Lay the dog-fish on a board, and determine the following points.

\section{A. General Appearance.}

The body is greatly elongated : it is broadest about the level of the pectoral fins, in front of which it is flattened dorsoventrally, ending in the blunt rounded snout. The hinder part of the body is compressed laterally, and tapers gradually to the end of the long slender tail, the hindmost three or four inches of which are bent up at an angle.

The general colour is grey; paler on the ventral surface, and marked on the dorsal and lateral surfaces with dark brown spots. The entire surface is covered with small scales, and along each side of the head and body there runs a slight longitudinal groove, the lateral line.

\section{B. The Fins.}

The fins are flattened outgrowths arising from various parts of the body. Each is supported by an internal cartilaginous skeleton, and is usually triangular in shape, the base or attached border being very commonly free at its posterior end.

\section{Median fins.}

a. The dorsal fins are two in number, the anterior and larger one being placed about the middle of the length of the body, and the posterior one a short distance further back.

b. The caudal fin forms a vertical fringe around the posterior four or five inches of the tail. Its dorsal portion is narrow, its hinder end truncated, and its ventral portion partially subdivided into a small posterior and a large anterior lobe.

c. The ventral fin is placed opposite the interval between the two dorsal fins.

2. Paired fins, representing the limbs of higher vertebrates.

a. The pectoral fins are the largest of all the fins, and project horizontally outwards from the sides of 
the ventral surface of the body, just behind the head.

b. The pelvic fins are smaller than the pectoral, and are placed on the ventral surface of the body, a little way in front of the middle of its length. Their inner borders touch each other, and in the male are fused together behind.

In the male the inner border of each pelvic fin is separated from the rest of the fin, and specially modified to form a copulating organ or clasper : this is a stout, backwardly directed rod, deeply grooved along its inner and dorsal surface. The groove leads forwards into a sac which lies just beneath the skin of the ventral surface, close to the median line, and ends blindly about an inch in front of the pelvic girdle.

\section{External Apertures.}

\section{Median apertures.}

a. The mouth is a large transverse crescentic opening on the under surface of the head, a short distance from its anterior end.

b. The cloacal aperture is on the ventral surface of the body between the pelvic fins, i.e. in front of the middle of the length of the body. It gives exit to fæcal matter from the intestine, and to the renal and genital products.

\section{Paired apertures.}

a. The nostrils are a pair of large circular apertures on the under surface of the head in front of the mouth, with which they are connected by wide grooves covered by flaps of skin.

b. The spiracles are a pair of small apertures on the sides of the head, just behind the slit-like openings of the eyes. They are really a pair of gillclefts.

Pass a seeker down one of the spiracles into the mouth. 
c. The gill-clefts are a series of five vertical slits on each side of the neck, in front of and slightly above the pectoral fins. They open obliquely backwards, and during life give exit to the water passed from the mouth over the gills for the purpose of respiration.

Pass a seeker through the gill-clefts into the mouth.

d. The abdominal pores are a pair of small apertures opening into pocket-like depressions, at the sides of the cloacal aperture, and between the pelvic fins. They communicate with the abdominal cavity, and are best seen from within (p. 217); they are often closed in young specimens, especially in females.

\section{Scattered apertures.}

a. The apertures of the mucous canals are rows of minute openings arranged symmetrically on the surface of the head, and especially abundant on the snout. They lead into tubes of considerable length-the mucous canals-which lie beneath the skin, and are filled with a transparent gelatinous substance.

Squeeze the head so as to press out the gelatinous substance, and so render the openings of the tubes more obvious.

\section{The Scales and Teeth.}

1. The scales. The whole surface of the body is covered with small placoid scales, which are special developments of the skin.

a. General arrangement. The scales are larger on the dorsal than on the ventral surface, and differ somewhat in shape in different parts of the body. Their projecting points are directed backwards, so that the finger can be readily passed along the body from the head to the tail, but meets with considerable resistance in the reverse direction. 


\section{b. Structure of the scales.}

Cut out a small piece of skin, and boil it for a few mimutes in solution of caustic potash to isolate the scales. Mount them in glycerine, and examine with the microscope.

Each scale consists of a bony base, usually four-lobed, from which a pointed spine of dentine, capped with enamel, projects backwards.

2. The teeth are really the enlarged scales of the skin covering the jaws. They are arranged in several rows, and have their points directed backwards.

\section{THE SKELETON.}

The skeleton of the dog-fish forms an important link between the exceedingly simple condition seen in Amphioxus and the complicated bony framework of the higher vertebrates.

In the embryo a rod-like notochord extends down the back, immediately beneath the central nervous system and dorsal to the alimentary canal, similar to that of Amphioxus, except that it does not reach quite to the anterior end of the body. Around this notochord a sheath of cartilage is formed, which in the head gives rise to the base of the skull, and in the body becomes divided transversely into segments-the centra or bodies of the vertebræ- the Hexibility of this region being thereby increased.

Above the centra neural arches are formed, which surround and protect the brain and spinal cord; and below them hæmal arches, which tend to enclose the body-cavity.

Besides this axial skeleton a visceral skeleton is developed around the anterior part of the alimentary canal, and skeletal elements are also formed in relation with the fins.

The skeleton consists throughout life of cartilage. It is hardened in many places by deposition of calcareous salts, but no true bone is developed in it.

The skeleton of the dog-fish is best prepared by taking a fresh specimen, dipping it in hot water a few degrees below 
boiling point for a few minutes, in order to soften the connective tissue, and then scraping or brushing away the soft parts until the skeleton is clean. Specimens so prepared must not be allowed to dry, but should be kept in weak spirit.

\section{A. The Vertebral Column, or ' Backbone.'}

A typical vertebra consists of a centrum, traversed by the notochord; a dorsal or neural arch, surrounding the spinal cord; and a ventral or hæmal arch surrounding the bodycarity. The caudal or tail vertebræ of the dog-fish are perfectly typical, while the anterior or trunk vertebræ have the hæmal arch modified or absent.

The structure of the vertebral column is best made out by cutting longitudinal, transverse, and horizontal sections through various parts of its length, and comparing these with one another.

1. The centra are short cylinders of cartilage, about as long as they are wide, connected together by intervertebral ligaments of strong connective tissue, which allow slight movement between the successive vertebræ and free flexion of the column as a whole.

Each centrum is a ring, thin at its ends, but thickened. on its inner side in the middle of its length, so that the cavity is hour-glass-shaped (fig. 49, p. 224). The inner surface of the ring is very densely calcified.

The notochord persists throughout the whole length of the column: it is moniliform in shape, being greatly constricted opposite the middle of each vertebra, but dilated almost to the full width of the column in the intervertebral spaces.

2. The hæmal arches.

In the anterior part of the vertebral column the centra are flattened ventrally, and produced laterally into blunt horizontal transverse processes which bear at their ends movably articulated cartilaginous ribs, about half an inch in length. 
Further back the transverse processes become more prominent and project ventrally as hæmal processes, causing the under surface of the column to appear grooved.

In the tail the ribs are absent, and the hæmal processes of each vertebra meet and unite midventrally, thereby completing the hæmal arch.

At the hinder end of the tail, opposite the caudal fins, the hæmal arches are produced downwards into median hæmal spines of some length and breadth.

The hæmal arches develop independently of the vertebral centra, but become connected with these at a very early date.

3. The neural arches are made up of elements of three kinds; the neural processes, neural plates, and neural spines.

a. The neural processes, forming the bases of the neural arches, are blunt processes projecting upwards from the sides of each vertebra opposite its middle.

b. The neural plates are a series of thin hexagonal plates of cartilage which form the sides of the neural canal (fig. 49). On each side there are twice as many neural plates as there are vertebræ, the neural plates being placed alternately opposite the middles of the vertebræ, and opposite the intervertebral intervals. The former or vertebral neural plates are fused in the adult with the tops of the neural processes, and have their posterior borders notched for the passage of the ventral roots of the spinal nerves. The dorsal roots of the nerves pass out through notches in the posterior borders of the intervertebral neural plates.

c. The neural spines are a series of median nodules of cartilage, wedged in between the dorsal ends of the neural plates so as to complete the neural arches dorsally. 
Along the greater part of the length of the column there are two neural spines to each vertebra, but in places the arrangement is less regular.

\section{B. The Skull.}

The skull of the dog-fish remains cartilaginous throughout life, thereby escaping the complications that result from the development of bone : in it, also, the several factors of which the skull is made up are more readily determined than in the higher forms. For these reasons it is peculiarly instructive, affording an important clue to the complex condition met with in higher vertebrates.

The skull consists of, (1) an axial tube, the cranium proper, open at both ends, which lodges the brain, and is to be regarded as an anterior unsegmented continuation of the vertebral column; and (2) the olfactory and auditory sense capsules, which are cartilaginous capsules investing the nose and ear respectively. These latter are at first independent of the cranium, but in the adult fuse with it, forming lateral expansions of its anterior and posterior ends. This fusion of the sense-capsules and cranium is so complete that they will be described together.

\section{General form of the skull.}

The skull is a somewhat oblong box of cartilage, deeply hollowed at the sides to form the orbits for the lodgment of the eyes, and ending in front in a short pointed rostrum formed by three converging rods.

The olfactory capsules are thin-walled lateral expansions at the anterior end, in front of the orbits; and the auditory capsules are more massive projections behind the orbits.

Examine the several surfaces of the skull in succession, identifying the parts described below.

2. The dorsal surface of the skull.

a. The olfactory capsules are a pair of large oval cartilaginous sacs at the anterior end of the skull: 


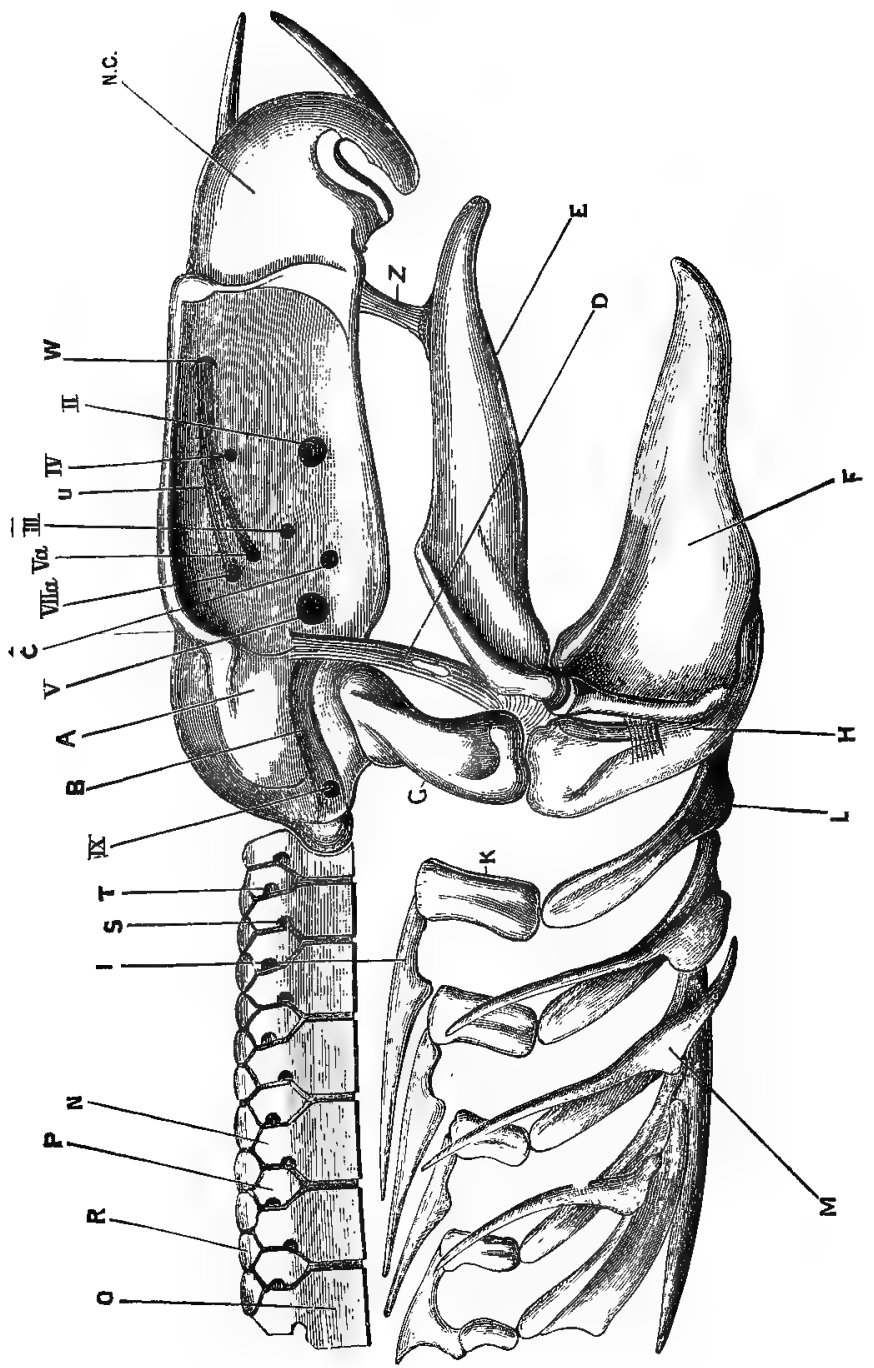


they have very thin walls and are readily torn. Their long axes are placed somewhat obliquely, and they are separated from each other by a thin median internasal septum.

b. The rostrum is formed by three cartilaginous bars, of which two run forwards from the upper surface of the olfactory capsule, converging in front; while the third is an anterior prolongation of the floor of the cranium, or brain-case.

c. The anterior fontanelle is a large, almost circular hole in the roof of the skull, between and behind the olfactory capsules. During life it is closed by a connective-tissue membrane, in connection with the inner surface of which is the pineal body.

d. The supra- orbital crests are prominent curved ridges of cartilage running along the sides of the skull from the olfactory to the auditory capsules, and forming the dorsal boundaries of the orbits. Along the inner side of each crest, between it and the cranium, is a shallow longitudinal groove in which lies a bundle of mucous canals.

e. The foramina for the ophthalmic nerves are a pair of small apertures behind and to the outer sides of the anterior fontanelle, and to the inner sides of the supra-orbital crests. Through them the

Fig. 45.-Scyllium canicula. The skull and visceral skeleton with the anterior part of the vertebral column, seen from the right side. The labial cartilages and gill-rays are omitted. (A. M. M.)

A, auditory capsule. B, post-orbital groove. C, inter-orbital canal. $\mathbf{D}$, pre-spiracular ligament: the white spot below the reference line is the pre-spiracular cartilage. $\mathbf{E}$, upper jaw. $\mathbf{F}$, lower jaw. G, hyo-mandibular cartilage. H, cerato-hyal. I, pharyngo-branchial. K, epi-branchial. I, ceratobranchial. $\mathbf{M}$, extra-branchial. $\mathbf{N}$, vertebral neural plate. $\mathbf{N C}$, olfactory capsule. $\mathbf{O}$, centrum of vertebra. $\boldsymbol{P}$, intervertebral neural plate. $\mathbf{R}$, neural spine. $\mathbf{S}$, foramen for the ventral root of a spinal nerve. $T$, foramen for the dorsal root of the same nerve. U, orbital grooves, lodging the ophthalmic branches of the fifth and seventh nerves. W, aperture at end of orbital groove through which the ophthalmic branches of the fifth and seventh nerves leave the orbit. $\mathbf{Z}$, ethmo-palatine ligament.

II, optic foramen. III, foramen for third nerve. IV, foramen for fourth nerve. $V$, foramen for the main branches of the fifth and seventh nerves, and for the sixth nerve. Va, foramen for the ophthalmic branch of the fifth nerve. VIIa, foramen for the ophthalmic branch of the seventh nerve. IX, foramen for the ninth or glosso-pharyngeal nerve. 
ophthalmic branches of the fifth and seventh nerves pass from the orbit to the dorsal surface of the olfactory capsules.

f. The auditory capsules are large lateral projections at the hinder end of the skull. They lodge the auditory organs, certain parts of which can be recognised without dissection.

i. Between the two auditory capsules there is a median depression, at the bottom of which are a pair of holes. Each hole leads downwards and outwards into a canal, the aqueductus vestibuli, which opens below into the internal ear.

Pass a seeker down the aqueductus vestibuli on one side into the ear.

ii. From the outer side of the aperture of the aqueductus vestibuli on each side, a ridge runs forwards and outwards across the skull-roof and towards the orbit. In this lies the anterior vertical semicircular canal of the ear, which can usually be seen through the cartilage.

iii. A similar ridge, running backwards and outwards from the same point, lodges the posterior vertical semicircular canal.

iv. The external or horizontal semicircular canal can usually be seen through the cartilage, lying to the outer side of the other two canals, and with its convexity directed outwards.

3. The side of the skull.

a. The rostrum and olfactory capsule have already been seen.

b. The orbit is the deep oval concavity at the side of the skull, extending from the olfactory to the auditory capsule, and bounded above and below by the prominent supra-orbital and sub-orbital ridges. It lodges the eyeball and its muscles, and is 
traversed by numerous nerves; the rest of the space being occupied by the large orbital bloodsinus.

Identify the following structures within the orbit.

i. The orbito-nasal foramen is a small round hole at the anterior inferior angle of the orbit, through which the veins of the anterior part of the head communicate with the orbital sinus.

ii. The optic foramen is a large hole about the middle of the length of the orbit and near its ventral border. Through it the optic nerve enters the orbit.

iii. The foramen for the third nerve is a small hole in the inner wall of the orbit, about a quarter of an inch behind the optic foramen and a little above it.

iv. The foramen for the fourth nerve is a very small hole near the dorsal border of the inner wall of the orbit, vertically above or very slightly behind the optic foramen.

v. The foramen for the fifth and seventh nerves is a large hole at the posterior and ventral angle of the orbit, immediately in front of the auditory capsule. Through it the main branches of the fifth and seventh nerves, and also the sixth nerve, enter the orbit.

vi. The aperture of the inter-orbital canal is a small round hole just in front of, and a little below the foramen for the fifth and seventh nerves, and below and behind the foramen for the third nerve. The canal traverses the base of the skull, and places the orbital sinuses of the two sides in communication with each other.

vii. The foramina for the ophthalmic branches of the fifth and seventh nerves. These nerves enter the orbit through two holes near its hinder 
end, just in front of the auditory capsule, and above the foramen for the main branches of the fifth and seventh nerves: the aperture for the ophthalmic branch of the seventh nerve is the larger of the two, and placed slightly above and behind that for the fifth. From these apertures shallow grooves run forwards along the inner wall of the orbit, close to its dorsal border, to the foramen by which the two nerves leave the orbit: this is situated at the antero-superior angle of the orbit, and has already been seen on the dorsal surface of the skull.

c. The articular surface for the hyo-mandibular cartilage is a concave depression on the side of the hinder end of the skull, below the auditory capsule.

d. The post-orbital groove is a deep horizontal groove immediately above the articular surface for the hyo-mandibular cartilage, and overhung by the projecting ledge of the auditory capsule which lodges the horizontal semicircular canal : it lodges during life the venous canal through which the orbital sinus communicates with the anterior cardinal sinus.

The anterior end of the groove is bridged over by the upper end of the stout pre-spiracular ligament.

e. The foramen for the glosso-pharyngeal nerve is at the hinder end of the floor of the post-orbital groove, behind and below the horizontal semicircular canal of the ear.

4. The ventral surface of the skull.

a. The under surface of the skull is a broad flat plate of cartilage, crossed almost transversely a little behind its middle by a pair of grooves in which the carotid arteries lie. At the point of meeting 
of the grooves in the middle line is an aperture through which the internal carotid artery enters the skull.

b. The apertures of the olfactory capsules are large, but are considerably reduced by the nasal cartilages, which are liable to be torn in cleaning the skull. Within the capsules are seen the large foramina, closed in the natural condition by fenestrated membranes, through which the olfactory nerves enter.

\section{The posterior end of the skull.}

To see this properly the stull must be separated from the vertebral column.

a. The foramen magnum is the large median hole through which the spinal cord enters the skull to expand into the brain.

b. The notochord lies immediately below the foramen magnum.

c. The condyles are a pair of rounded prominences at the sides of the notochord, and below the foramen magnum: they articulate with the first vertebra.

d. The foramen for the pneumogastric nerve of each side is at the bottom of a pit-like depression at the side of the foramen magnum, below and to the inner side of the auditory capsule.

\section{The Visceral Skeleton.}

This consists of a series of cartilaginous hoops or arches placed one behind another, and encircling the anterior part of the alimentary canal. In accordance with the shape of the mouth and pharynx, which they surround, the hoops are wide from side to side, and compressed dorso-ventrally.

The first arch gives rise to both upper and lower jaws.

The second or hyoidean hoop is large, and plays an important"part in slinging the jaws to the skull. 


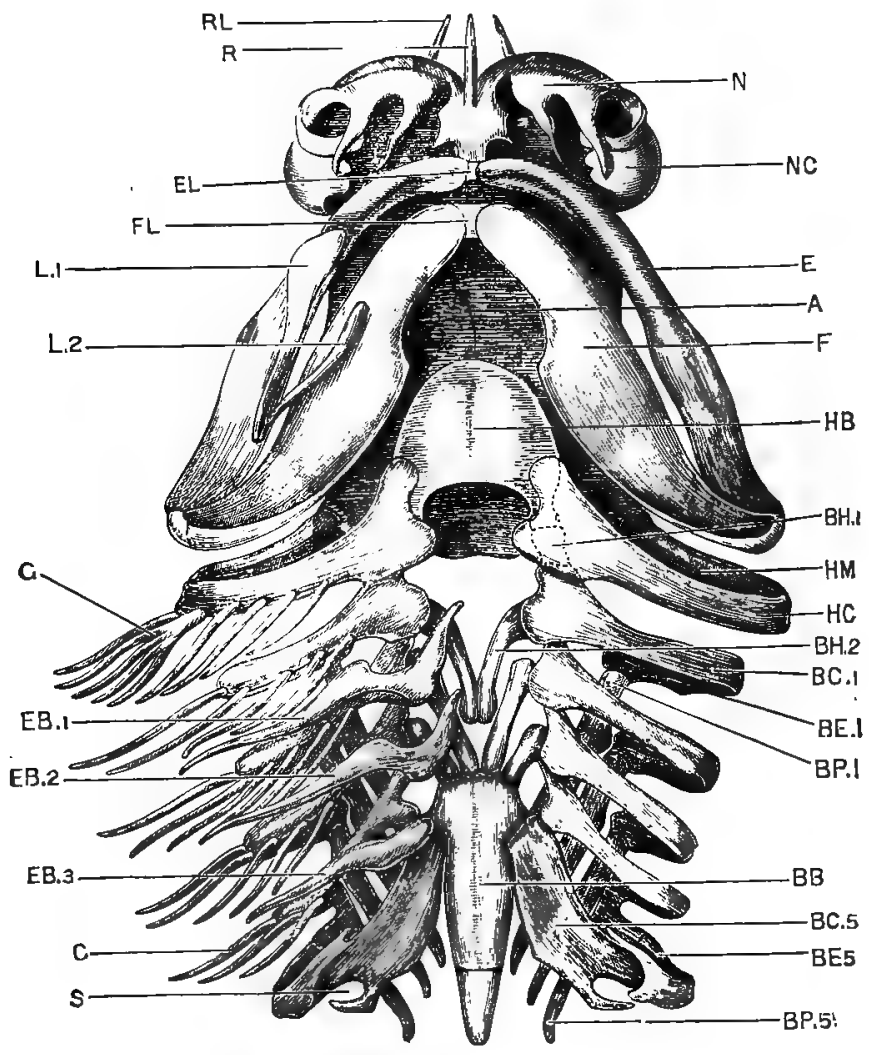

Frg. 46. Scyllium canicula. The skull and visceral skeleton seen from the ventral surface. The labial cartilages and gill-rays of the left side have been removed. (A. M. M.)

A, base of skull. BB, basi-branchiel. BC 1, BC 5, first and fifth cerato. branchials. BE 1, BE 5. first and fifth epi-branchials. BEF 1, first hypobranchial, represented as though seen through the cerato-hyal. BH 2 , second hypo-branchial. BP 1, BP 5, first and fifth pharyngo-branchials. F, upper jaw. EB 1, FB 2, FB 3, first, second, and third extra-branohials. FI, ligament connecting the two halves of the upper jaw. $\mathbf{F}$, lower jaw. FL, ligament connecting the two halves of the lower jaw. G, gill-ray. HB, besi-hyal. HC, cerato-hyal. HM, hyo-mandibular cartilage. I 1, I, 2, labial cartilages. $\bar{N}$, nasal cartilage. NC, olfactory capsule. $R$, median bar of rostrum. RL, lateral bar of rostrum. $\mathbf{S}$, notch for Cuvierian sinus. 
The third' and succeeding hoops are more slender: they surround the pharynx and support the gills.

To see the visceral skeleton properly, a roll of paper should be passed in at the mouth-opening, and back through the several hoops.

1. The first or mandibular arch is very greatly modified; its dorsal part is represented by the pre-spiracular ligament, while its ventral part is large and stout and forms the lower jaw. The upper jaw is formed as an outgrowth from its anterior edge.

a. The pre-spiracular ligament is a strong fibrous band in which is a small nodule of cartilage, and which runs from the anterior border of the auditory capsule to the distal end of the hyomandibular cartilage, where it blends with ligaments connecting the hyo-mandibular cartilage with the upper and lower jaws, close to the angle of the mouth. An additional ligament connects the lower jaw with the cerato-hyal a short way below the angle of the mouth.

b. The upper jaw consists of a pair of stout cartilaginous rods, wide behind and narrowing towards their anterior ends, where they are united across the middle line by ligament. They extend forwards as far as the openings of the olfactory capsules, and are connected by stout ethmo-palatine ligaments with the base of the skull, at the antero-inferior angles of the orbits. They bear teeth along the ventral border of the anterior two-thirds of their length.

c. The lower jaw consists of a pair of wide flattened bars of cartilage, which are movably hinged with the upper jaw behind, and bound together by a median ligament in front. It lies, when the mouth is shut, parallel to and immediately behind the upper jaw. It bears teeth along the dorsal border of the anterior two-thirds of its length on each side. 
2. The second or hyoidean arch is incomplete above: it consists of a pair of rods the dorsal ends of which articulate with the sides of the skull, while the ventral ends are connected together by a median plate of cartilage. Each rod is further divided into an upper and a lower segment.

a. The hyo-mandibular cartilage, or upper segment, is a short stout bar of cartilage, projecting almost horizontally outwards from the skull, but slightly downwards and backwards. Its inner and upper end articulates with a concave surface on the side of the skull, near its hinder end, and immediately below the post-orbital groove : its outer end articulates with the lower segment of the arch, opposite the angle of the mouth. To its hinder border are attached a series of rods of cartilage-the gill-rays-which support the gill-folds.

The hyo-mandibular cartilage in the dog-fish is spoken of as the suspensorium, because it forms the skeletal link between the jaws and the skull.

Skulls, like that of the dog-fish, in which the hyoidean arch forms the suspensorium are spoken of as hyostylic.

b. The cerato-hyal, or lower segment of the hyoidean arch, is a longer and more slender bar which runs: forwards and inwards in the floor of the mouth, just behind the lower jaw, and partly overlapped by it. It bears gill-rays along its posterior border.

c. The basi-hyal is a broad median plate of cartilage, lying in the floor of the mouth behind the lower jaw. It is rounded anteriorly, and produced behind into two horns which are attached by ligaments to the dorsal surfaces of the ceratohyals.

3. The branchial arches are the remaining five visceral arches : they diminish in size from before backwards, and each is divided into four segments on each side. 
a. The pharyngo-branchials, the most dorsal elements, are flattened rods running forwards and outwards in the roof of the pharynx. The anterior ones are connected with their fellows of the opposite side by ligament; and the last two of each side are much smaller than the others, and fused together.

b. The epi-branchials are short broad plates attached to the outer ends of the pharyngo-branchials. With the exception of the hindmost they bear gill-rays along their posterior borders.

c. The cerato-branchials are stout rods running forwards and inwards in the ventral wall of the pharynx, and bearing gill-rays along their posterior borders. The fifth or hindmost cerato-branchial is shorter but much broader than the others; it has no gillrays; and its posterior border is deeply notched to lodge the Cuvierian sinus (p. 227).

d. The hypo-branchials are small rods in connection with the inner ends of the first, second, third, and fourth cerato-branchials. The firsthypo-branchial, which is very small, is directed forwards, and connects the first cerato-branchial with the basihyal. The three hinder hypo-branchials are larger, and are directed backwards.

e. The basi-branchial is an elongated flattened median plate, pointed behind. It is connected with the two hinder pairs of hypo-branchials in front, and at the sides with the fifth cerato-branchials. With these latter, it strengthens and supports the dorsal wall of the pericardial cavity. (Fig. 49, p. 224.)

f. The extra-branchials are three pairs of elongated curved rods lying along the outer sides of, and beneath the second, third, and fourth branchial arches.

4. The labial cartilages are two pairs of slender cartilaginous rods in the folds of skin at the sides of the mouth. 


\section{The Skeleton of the Fins.}

1. The median fins.

The skeleton of the dorsal fins consists of a series of parallel rods of cartilage, the fin-rays, sloping upwards and backwards, and calcified along their axes. The lower or basal ends of the rods often unite together, and the dorsal ends support one or more rows of closely fitting polygonal plates. On each side of this median cartilaginous skeleton is a series of slender elongated horny fibres, derived from the skin.

The other median fins are similar to the dorsal ones, except that the cartilaginous elements may be reduced to a longitudinal basal rod, or may even be completely absent.

2. The paired fins are essentially similar to the median fins, and are probably to be regarded as local enlargements. of lateral fins, which originally extended the whole length of the body.

It is convenient to take the posterior or pelvic fins before the pectoral, as their structure is simpler.

a. The pelvic girdle is a nearly straight bar of cartilage, placed transversely across the ventral surface of the body, about half an inch in front of the cloaca. The ends of the bar are produced forwards into blunt processes, and bear on their posterior surfaces the articular facets for the pelvic fins.

b. The pelvic fins. The cartilaginous skeleton of each fin consists of a slightly curved basal rod, the basi-pterygium, which lies along the inner side of the fin, and articulates in front with the pelvic girdle. From the outer side of the basi-pterygium a series of parallel cartilaginous fin-rays, about fourteen in number, run outwards and backwards, supporting at their ends smaller polygonal plates. The anterior one or two of the fin-rays may articulate independently witl the pelvic girdle. 
In the adult male, the posterior end of the basi-pterygium is produced backwards into a large spout-like process, supporting the clasper.

Besides the cartilaginous skeleton, horny fibres like those of the median fins are present in great numbers on both surfaces of the fin.

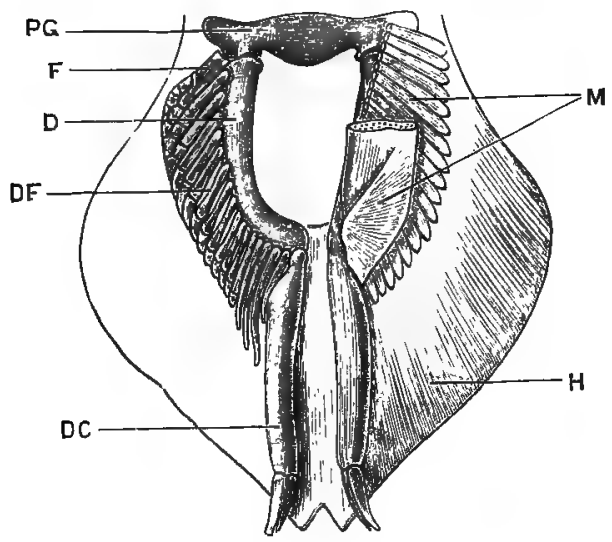

Fig. 47.- Seyllium canicula. The pelvic girdle and fins of a male specimen, seen from the ventral surface. In the right fin the cartilaginous skeleton is shown, in the left the muscles and horny dermal fibres. (A. M. M.)

D, basi-pterygium. DC, posterior end of the basi-pterygium, forming the skeleton of the clasper. DE', fin-ray. F, anterior fin-ray, articulating directly with the pelvic girdle. $\mathbf{H}$, horny fibres. M, muscles. PG, pelvic girdle.

c. The pectoral girdle, which lies immediately behind the last gill-arch, is considerably larger than the pelvic, and consists of a hoop of cartilage incomplete above.

The ventral portion of the hoop is produced forwards into a flattened process, which forms part of the floor of the pericardial cavity, and is hollowed dorsally to receive the ventricle of the heart.

At each side the arch is thickened, and bears on its outer border a triple articular facet for the basal cartilages of the fin. 
d. The pectoral fin has three basal cartilages, the propterygium, meso-pterygium, and meta-pterygium, articulating with the three facets on the pectoral girdle. Of these the pro-pterygium is the smallest, and the meta-pterygium, which extends along the inner or attached border of the fin, and corresponds

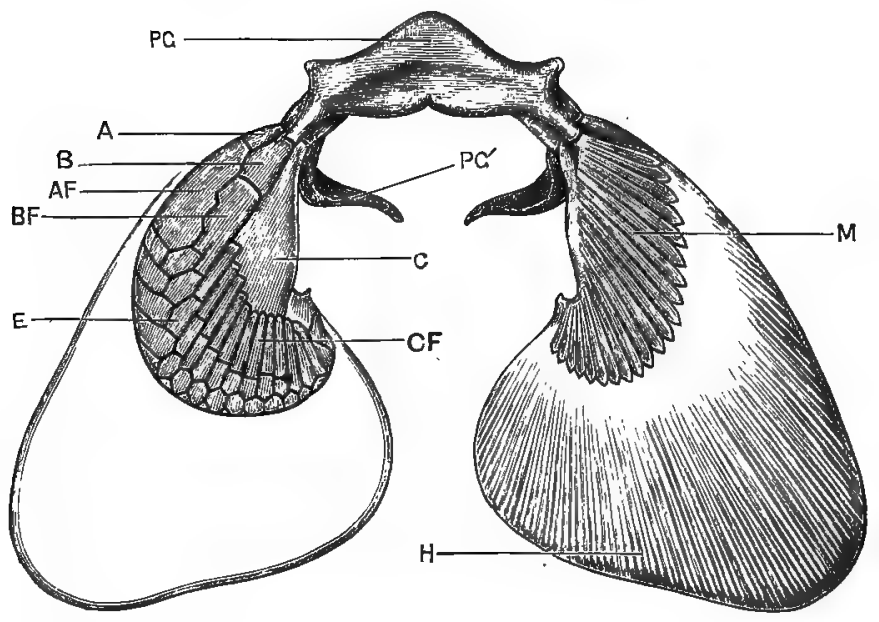

FIG. 48.- Scyllium canicula. The pectoral girdle and fins of a male specimen, seen from the ventral surface. In the right fin the cartilaginous skeleton is shown, in the left the muscles and horny dermal fibres. (A. M. M.)

A, pronterygium. AF, pro-pterygial fin-ray. $\mathbf{B}$, meso-pterygium. BF, meso-pterygial fin-ray. C, meta-pterygium. CF, meta-pterygial fin-ray. $\mathbf{F}$, polygonal cartilaginous plate. $\mathbf{H}$, horny fibres. $\mathbf{M}$, muscles of fin. $\overrightarrow{\mathbf{P G}}$, ventral portion of pectoral girdle. ' $\mathbf{P G}^{\prime}$, dorsal portion of pectoral girdle.

to the basi-pterygium of the pelvic fin, is much the largest.

From the outer borders of the basal cartilages a series of close-set cartilaginous fin-rays diverge: of these the pro-pterygium supports a single large one; the meso-pterygium also supports one, which is, bowever, split distally into two or three; while 
the meta-pterygium supports twelve or more much narrower rays.

To the outer ends of the fin-rays two or more rows of polygonal plates of cartilage are attached, forming the peripheral portion of the skeleton.

Horny fibres are present in large numbers, as in the pelvic fins.

\section{THE ABDOMINAL CAVITY AND VISCERA.}

Lay the dog-fish on its back on the dissecting-board, and pin it down through the fins. Deternine the positions of the pectoral and pelvic girales by feeling them through the skin, the pectoral girdle being a little in front of the pectoral fins, and the pelvic girdle a short distance in front of the cloacal aperture.

Open the abdominal cavity by a median ventral incision, extending from the pelvic girdle behind to the pectoral girdle in front. Cut through the body-wall transversely, just behind the pectoral girdle, and pin the two flaps down to the dissecting-board. Cut through the pelvic girdle in the median plane, and prolong the cut backwards to the level of the cloacal aperture, but keeping a little to its right side. Wash or sponge out the abdominal cavity.

The cclom or body-cavity extends forwards into the head in the embryo, but in the adult is reduced to the pericardial cavity which lodges the heart, and the abdominal cavity in which lie the principal viscera. The abdominal cavity is a wide space extending from the level of the pectoral girdle backwards as far as, or slightly beyond, the cloacal aperture, where it communicates with the exterior through the abdominal pores (p. 199).

Pass a seeker from the abdominal cavity through one of the abdominal pores to the exterior.

Identify the positions and relations of the following organs before proceeding further with the dissection. 
1. The liver is a very large solid organ of a brownish yellow colour, attached by a median suspensory ligament to the anterior wall of the abdominal cavity. It consists of two lobes of about equal size, which are united in front, and extend back along the sides of the abdominal cavity for the greater part of its length.

2. The stomach is a wide U-shaped tube lying between the lobes of the liver, and extending about two-thirds the length of the abdominal cavity.

3. The spleen is a large brownish red body attached to the loop of the stomach, and sending a long narrow lobe along its distal limb.

4. The intestine is a wide and nearly straight tube, running along the right side of the abdominal cavity from the stomach to the cloaca.

5. In the female the ovary is single, and attached by a delicate median fold of peritoneum to the wall of the abdominal cavity. The contained ova give its surface a tuberculated appearance.

In the male the testes are a pair of elongated solid bodies of a whitish colour, lying along the dorsal region of the anterior two-thirds of the abdominal cavity. Their hinder portions fuse together.

6. The peritoneum is the smooth membrane lining the abdominal cavity, and reflected downwards from the mid-dorsal line as a double sheet-the mesenterywhich covers the alimentary canal and other viscera.

The mesentery is well developed at the hinder end of the abdominal cavity, but is interrupted by one or more large fenestræ opposite the greater part. of the length of the intestine.

7. The kidneys are covered by the peritoneum, but can be recognised as a pair of slightly elevated longitudinal ridges, one on each side of the mid-dorsal line of the abdominal cavity, and extending nearly its whole length. 


\section{DISSECTION OF THE DIGESTIVE SYSTEM.}

In dissecting the alimentary canal, be careful not to injure the blood-vessels in relation with it; these lie for the most part. in the mesentery.

\section{A. The Alimentary Canal.}

1. The mouth and pharynx. The detailed examination of these parts is best deferred until the circulatory system is dissected.

2. The cesophagus is a short wide tube, which enters the abdominal cavity at its anterior end and expands almost at once into the stomach.

Pass the handle of a scalpel or seeker through the mouth, and down the osophagus into the stomach.

3. The stomach is $U$-shaped. Its proximal limb, which is very wide and directly continuous with the oesophagus, extends back nearly to the hinder end of the abdominal cavity: it then turns sharply forwards to form the distal limb, which is shorter and much narrower.

4. The intestine is separated from the distal limb of the stomach by a slight pyloric thickening. Its proximal part is about equal in width to the stomach, alongside which it lies; but it soon dilates to form the wide colon, the surface of which is marked spirally by blood-vessels, corresponding to the line of attachment of the spiral valve. At its hinder end the intestine narrows to form the rectum, which, after a course of about an inch and a half, opens to the exterior at the cloacal aperture.

a. The spiral valve.

Open the intestine along its whole length by a longitudinal incision along its right side. Wash out its contents. Carefully cut away with scissors the ventral wall from the several turns of the spiral valve, and turn it over to the left side, so as to fully expose the cavity of the intestine. Wash freely. 
The spiral valve is a membranous fold, attached along one edge to the inner surface of the intestine, round which it runs spirally. The fold is about an inch and a half wide at the anterior end, but gradually diminishes in width towards the posterior end, and disappears at the commencement of the rectum. The first turn of the spiral is a long one, but the succeeding ones, of which there are usually seven or eight, are only about a quarter of an inch apart. The general appearance of the valve is that of a series of cones one within another, the apices of the cones being usually directed forwards, but sometimes at the hinder end backwards.

The valve serves to retard the passage of food down the intestine, and to increase the extent of its absorptive surface.

\section{B. The Glands.}

1. The liver has been described above.

a. The gall-bladder is a large irregular sac imbedded in the anterior part of the left lobe of the liver, close to the median plane.

b. The bile-duct leaves the gall-bladder between the two lobes of the liver, and almost in the median plane. It receives ducts from the lobes of the liver, and runs back along the ventral margin of the mesentery to the intestine, along which it runs for a short distance to open into the commencement of the colon. The bile-duct has a total length of about three inches.

Find the duct along the edge of the fold of mesentery between the liver and the intestine: make an incision in its walls, and inject it with a coloured fluid such as Prussian blue: follow it forwards to the liver and gall-bladder, and backwards to its opening into the intestine.

2. The pancreas is a whitish, laterally compressed body about an inch and a half long, lying in the angle 
between the distal limb of the stomach and the intestine. Its anterior end is expanded and gives off a small ventral lobe, which is closely applied to the intestine.

a. The pancreatic duct runs forwards through the pancreas close to its ventral border. It leaves the gland at the posterior angle of its ventral lobe, and at once enters the ventral wall of the intestine about an inch beyond the pylorus : it runs in the wall of the intestine for about half an inch, and opens just to the outer side of the line of attachment of the spiral valve.

Find the duct along the ventral border of the pancreas: trace it to the intestine: slit open the intestine, and trace the duct backwoards in its wall as a white ridge ending. in a very small papilla. It is easier to follow after injection with a. coloured fuid.

3. The rectal gland is a thick-walled tube, about three quarters of an inch long, lying in the abdominal cavity dorsal to the rectum. From its hinder end a duct. runs forwards along the edge of the mesentery to open into the dorsal wall of the rectum.

Cut the gland across about the middle of its length. Note the thickness of its walls and the small size of its cavity. Pass a bristle down the gland and duct into the rectum; and" slit open the rectum to see the opening of the duct.

\section{DISSECTION OF THE RESPIRATORY SYSTEM.}

The dog-fish breathes by gills, which are vascular folds of the mucous membrane of the pharynx, supported by the branchial arches. Respiration is effected by streams of water, which, entering at the mouth, pass between the branchial arches, over the gills, and out through the gill-clefts.

\section{The gills.}

Expose the gills on the left side by prolonging the external apertures upwards and downwards with scissors. 
The gills are series of closely set, soft, and highly vascular folds of mucous membrane, radiating outwards from the cartilaginous branchial arches, and supported by the gill-rays.

Gills are borne on the posterior surface of the hyoid arch, and on both the anterior and posterior surfaces of the four following arches. The inner borders of the branchial arches have cartilaginous processes, which hinder the passage of food to the gill-clefts. The last branchial arch bears no gill.

\section{The pseudobranchiæ.}

Slit up the spiracle on the left side in the same way as the gill-clefts.

The pseudobranch is a rudimentary gill, consisting of about half a dozen small parallel folds, on the anterior wall of the spiracle. Its presence shows that the spiracle is really a gill-cleft.

\section{DISSECTION OF THE CIRCULATORY SYSTEM.}

\section{A. The Pericardial Cavity and Heart.}

\section{The pericardial cavity.}

Lay the dog-fish on its back on the dissecting board, and win it down through the fins. Open the pericardial cavity by a mid-ventral incision in front of the pectoral girdle, taking care not to injure the heart. Cut away the ventral wall of the cavity so as to expose it fully. Cut away also the middle half-inch of the pectoral girale, taking care not to injure the sinus venosus, which lies along its dorsal border.

The pericardial cavity is median, and lies between the gills; it is triangular in shape, with the apex directed forwards, and is almost completely filled by the heart, of which the globular ventricle is the most prominent portion. Its ventral wall is stiffened by the anterior prolongation of the pectoral girdle, and its dorsal wall by the wide basi-branchial plate and the fifth cerato-branchials. 
2. The heart is a single tube twisted on itself in an $\mathbf{S}$-shape, and dilated to form a series of chambers separated by transverse constrictions.

Determine the position and general characters of the chambers in order from behind forvards, pressing them aside when necessary, but making no further dissection.

a. The sinus venosus is a thin-walled transversely placed tube, attached along its whole length to the posterior and dorsal wall of the pericardial cavity. It receives laterally the veins returning blood from the body, and opens in front into the auricle by a median aperture.

To expose the sinus venosus, press the ventricle slightly forwards.

b. The auricle is a large thin-walled sac, occupying the dorsal half of the pericardial cavity. It is triangular in shape, with its posterior angles produced into processes which, in the natural position of the parts, project laterally at the sides of the ventricle.

To expose the auricle, push the globular ventricle to one side.

c. The ventricle is a thick-walled globular sac lying ventral to the auricle, and forming the most conspicuous part of the heart.

d. The conus arteriosus is a cylindrical tube, running forwards from the ventricle to the anterior end of the pericardial eavity.

3. The pericardio-peritoneal canal is a median passage about an inch long, opening in front into the hinder end of the pericardial cavity dorsal to the sinus venosus, and running back along the ventral surface of the oesophagus, to open behind into the abdominal cavity. Its anterior opening is large and conspicuous; the opening into the abdominal cavity, which may be double, is smaller and slit-like. 


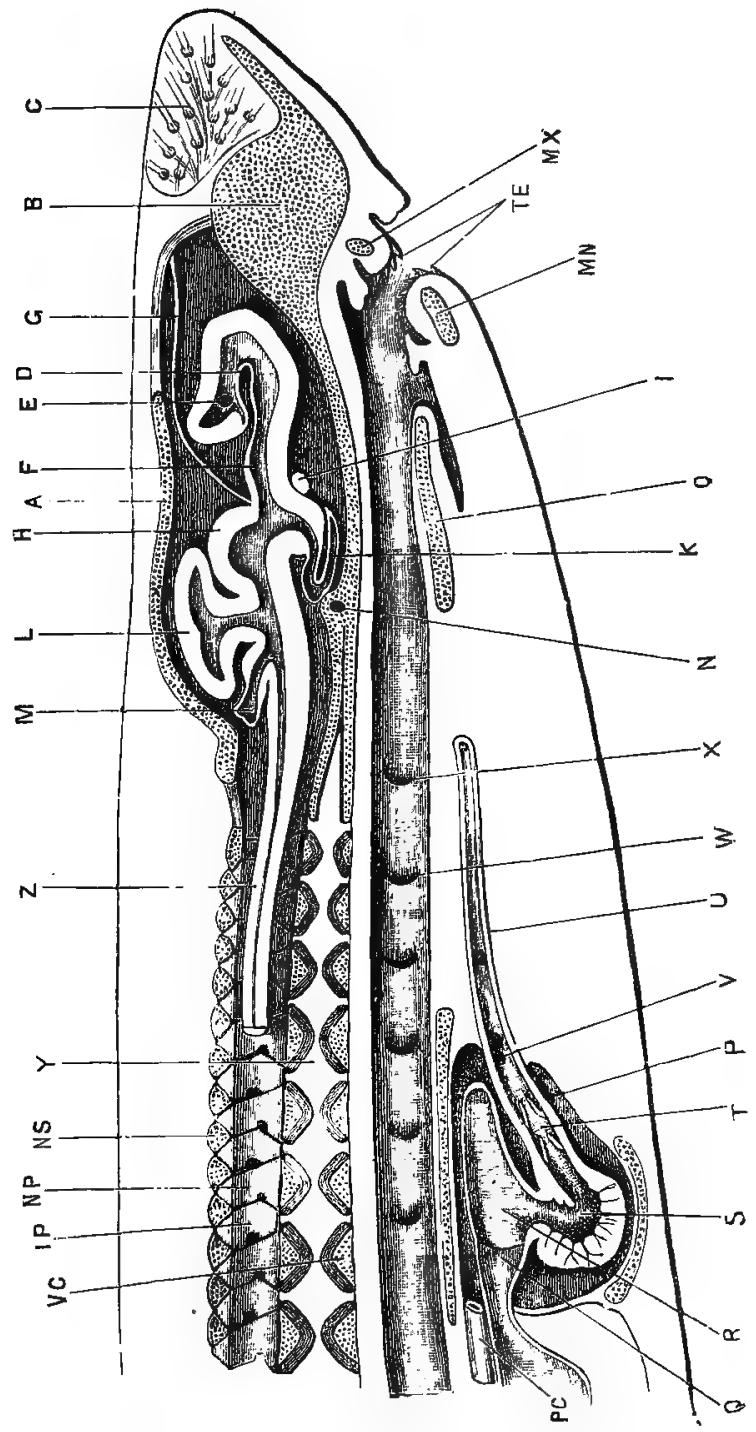


Find the pericardial opening in the middle line, immediately dorsal to the sinus venosus. Insert a seeker, and pass it down the canal into the abdominal cavity.

\section{B. The Venous System.}

The venous system of the dog-fish consists of three great divisions ; (1) the veins opening into the sinus venosus, which return blood from all parts of the body to the heart; (2) the hepatic portal system, which conveys to the liver the blood from the alimentary canal, pancreas, and spleen; (3) the renal portal system, which conveys to the kidneys the blood from the part of the body behind the cloaca.

1. The veins opening into the sinus venosus.

These veins are most readily traced by following them back from the sinus venosus, and they will be described in this way, though it must be remembered that the flow of blood in them during life is towards the sinus venosus. They are chiefly remarkable for their enormous size in places, the dilated portions being spoken of as sinuses.

To dissect the veins, slit open the ventral wall of the sinus venosus transversely, and wash out the contained blood: then follow the veins with a seeker, slitting them open along their ventral walls, and washing out the blood. If preferred, they may be injected with plaster of Paris, or with a coloured solution such as Prussian blue suspended in water; or they may be inflated with air.

FIG. 49.-Scyllium canicula. A longitudinal and vertical section through the head and anterior part of the body. The section of the brain is taken a little to one side of the median plane, and the spinal cord has been cut across a short way behind the brain. The cut surfaces of the cartilaginous skeleton are dotted. (A. M. M.)

A, roof of the cranium. B, internasal septum. C, mucous canals. D, fold of the wall of the hemisphere in which lies the choroid plexus. F, aperture leading from the cerebral vesicle to the olfactory vesicle. F, third ventricle. G, stalk of the pineal body. H, optic lobe. I, optic chissma. IP, intervertebral neural plate. $\mathbf{K}$, pituitary body. $\mathbf{L}$, cerebellum. $\mathbf{M}$, restiform body. MN, lower jaw MX, upper jaw. N, interorbital sinus. NP, vertebral neural plate. IN $\mathbf{S}$, neural spine. $\mathbf{O}$, basi-hyal, supporting the tongue. $\mathbf{P}$, pericardial cavity. $\mathbf{P C}$, pericardio-peritoneal canal. $\mathbf{Q}_{\text {, sinus venosus. }}$ $\mathbf{R}$, auricle. $\mathbf{S}$, ventricle. $\mathbf{T}$, conus arteriosus. $\mathbf{T E}$, teeth. U, cardiac aorta. $\boldsymbol{V}$, aperture of afferent branchial vessel. $\boldsymbol{\nabla} \mathbf{C}$, vertebral centrum. $\mathbf{W}$, first branchisl cleft. $\mathbf{X}$, spiracle, or hjo-mandibular cleft. $\mathbf{Y}$, notochord. $\mathbf{Z}$, spinal cord. 


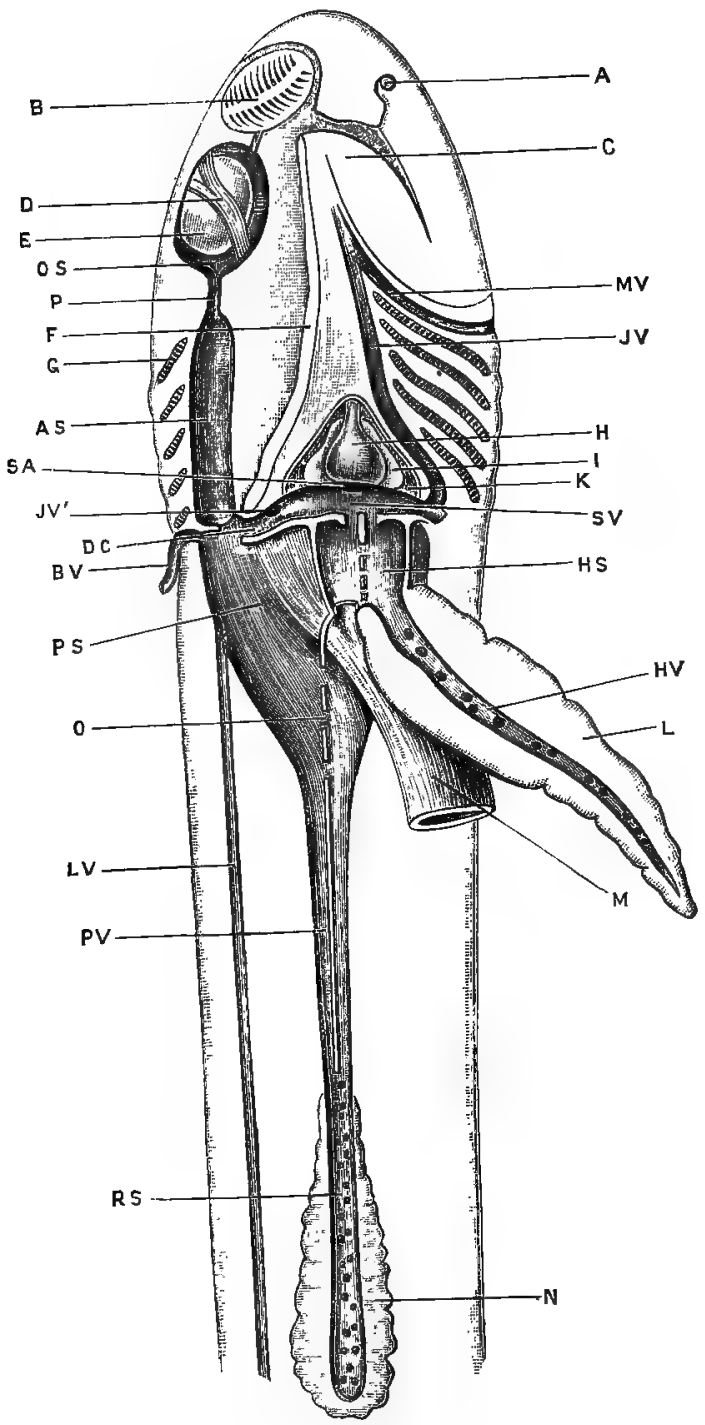


a. The hepatic sinuses open into the sinus venosus by a pair of apertures in its posterior wall, close to the median plane and near the dorsal surface. The apertures lead into a pair of wide sinuses, separated from each other by an imperfect septum, and lying immediately behind the pericardium, between it and the liver, and ventral to the cosophagus.

Slit open one of the hepatic sinuses, and follow it back into the corresponding lobe of the liver, noting its large size, and the numerous veins opening into it.

b. The Cuvierian sinuses are a pair of rather narrow tubes, forming lateral continuations of the sinus venosus, the wall of the pericardiun marking the boundary between the two. Each is about a quarter of an inch long, and curves upwards from the sinus venosus round the side of the cosophagus, lying in a notch in the posterior border of the fifth cerato-branchial cartilage. Each Cuvierian sinus receives about the middle of its course the inferior jugular sinus, and at its dorsal end the great anterior and posterior cardinal sinuses.

i. The inferior jugular sinus, which is of small size, can be traced forwards and inwards along the outer wall of the pericardial cavity; and then forwards, in the floor of the mouth, ventral to the gill-arches.

FIG. 50.-Seyllium canicula. Dissection of the venous system from the ventral surface. The right half of the lower jaw, the right lobe of the liver, and the alimentary canal from the œsophagus backward.s have been removed. (A. M. M.)

A, nostril. A.S, anterior eardinal sinus. B, right olfactory organ. $\mathbf{B V}$, subclavian vein. C, left half of lower jaw. D, fifth nerve crossing the orbit. DC, Cuvierian sinus. F, right eye. $\mathbf{F}$, cut edge of floor of mouth. $\mathbf{G}$, first branchial cleft. H, ventricle. His, left hepatic sinus. HV, left hepatic vein. I, auricle. JV, left inferior jugular sinus. JV', opening of right inferior jugular sinus into sinus venosus. $\mathbf{K}$, pericardial cavity. $\mathbf{I}$ left lobe of liver. $\mathbf{L V}$, right lateral vein. $\mathbf{M}$, cesophagus, cut across. $\vec{M} \mathbf{V}$, hyoidean sinus. $\mathbf{N}$, kidney or metanephros. $\mathbf{O}$, communication between the right and left posterior cardinal sinuses. OS, orbital sinus. $\mathbf{P}$, postorbital sinus. PS, posterior cardinal sinus. PV, posterior cardinal vein. RS, apertures of renal veins into posterior cardinal veins. SA, aperture from sinus venosus to auricle. $\mathbf{S} \nabla$, sinus venosus. 
ii. The anterior cardinal sinus opens into the Cuvierian sinus by a small aperture placed immediately behind the fifth cerato-branchial cartilage, and guarded by .a very perfect valve of which the ventral flap is much the larger.

From this opening the sinus extends forwards as a wide irregular space, situated immediately above the gill-arches. In front. of the first gill this sinus communicates with the orbit by the narrow post-orbital sinus. This passes above the spiracle and below the ear, and opens in front into the large orbital sinus which surrounds the eyeball and its muscles, and is usually filled with blood-clots. The orbital sinuses of the two sides communicate with each other by the inter-orbital sinus, which runs across in the floor of the skull, between the hinder parts of the orbits.

Just in front of the first gill the anterior cardinal sinus receives the large hyoidean sinus, which lies in a groove on the outer surface of the hyo-mandibular cartilage, and may be followed downwards and inwards to the floor of the mouth, where it communicates. with the inferior jugular sinus.

Find the opening of the left anterior cardinal sinus into the Cuvierian sinus; insert a seeker into it, turn the fish over, and lay open the cardinal sinus along its whole length by slitting through its dorsal wall; wash out the blood-clots, and follow it forwards to the orbit. Follow the hyoidean sinus, from the anterior end of the cardinal sinus, downwards along the hyomandibular cartilage to the floor of the mouth.

iii. The posterior cardinal sinus extends back along the dorsal surface of the abdominal cavity close to the median plane, its dorsal wall being firmly united to the body-wall, and its ventral surface covered by the peritoneum. Its 
anterior end forms a very large thin-walled sac, three-quarters of an inch or more in width. In front it lies at the side of the œsophagus, and close to the hepatic sinus; further back it lies dorsal to the cesophagus, and alongside its fellow of the other side, with which it communicates freely. Behind this point, i.e. about two inches behind the pericardium, the sinuses of the two sides narrow considerably, and run back side by side to the hinder end of the abdominal cavity, lying between the kidneys, from which they draw blood by numerous renal veins. The right sinus extends to the hinder end of the kidneys, but theleft usually commences further forwards.

Slit open the ventral walls of both sinuses at their anterior ends, and wash out the blood; note the enormous size of the sinuses and their free communication with each other, dorsal to the cesophagus. Pass a seeker backwards along the right sinus to its commencement between the posterior ends of the kidneys (fig. 50); but do not lay it open in this hinder part, lest the reproductive organs and the cloaca should be injured.

At its anterior end, immediately to the outer side of its opening into the Cuvierian sinus, the posterior cardinal sinus receives the subclavian vein, which returns blood from the pectoral fin and adjacent parts of the body-wall, and runs in a groove along the inner surface of the pectoral girdle. A little way further back it receives the lateral vein, which runs in the body-wall alongside the lateral line; and further back still, just behind the point where the csophagus passes between the two posterior cardinal sinuses, each of these latter has on its rentral surface the opening of the genital sinus, which surrounds the reproductive gland. 


\section{The renal portal system.}

The blood from the tail is returned by the caudal vein, which lies below the caudal artery in the hæmal arches of the vertebræ. Opposite the hinder end of the kidneys the caudal vein divides into the right and left renal portal veins, which run forwards along the dorsal edges of the right and left kidneys respectively, receiving branches from the body-wall, and supplying the kidneys along their whole length.

The dissection of the renal portal system should be postponed till after that of the urino-genital system, in order to avoid damaging the cloaca.

Cut across the tail about two inches behind the cloaca. Find the caudal vein lying in the hamal arches of the vertebra, ventral to the caudal artery; trace the vein forwards with a seeker, or inject it, and then dissect it out.

3. The hepatic portal system.

The hepatic portal vein is formed by the union of two veins, one from the main lobe of the spleen and the other from the intestine. It runs forwards in the mesentery along the dorsal border of the pancreas; and, receiving veins from the pancreas and from the stomach, enters the liver a little to the right of the median plane.

Find the hepatic portal vein at the point where it enters the liver, a little to the right of the junction of the two lobes, and follow it backwards, turning aside the viscera as may be necessary, but not cutting anything.

\section{The Arterial System.}

The arterial system may conveniently be described under three heads: (1) the afferent branchial vessels, which carry the venous blood from the heart to the gills to be purified; (2) the efferent branchial vessels, which collect the purified blood from the gills ; and (3) the dorsal aorta, which is formed by the union of the efferent branchial vessels, and conveys arterial blood to the body generally. 


\section{The afferent branchial vessels.}

The afferent branchial vessels are most readily followed by injecting them from the conus arteriosus, but this must not be done if the heart is to be dissected in the same specimen.

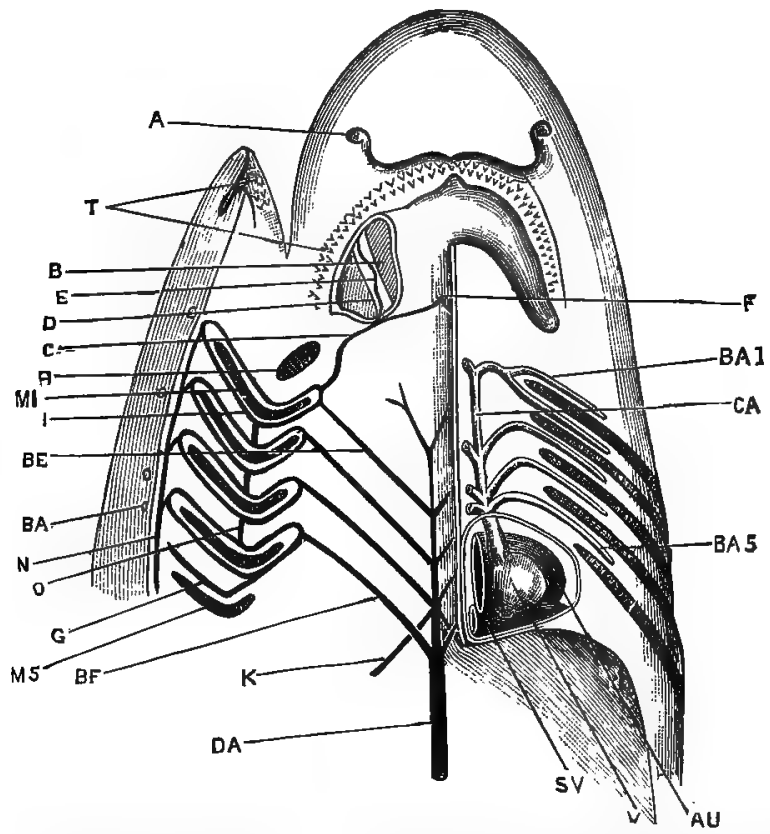

Fia. 51.--Scyllium canicula. Dissection of the arterial system from the ventral surface. The floor of the mouth has been cut through close to the median plane, and the right half turned outwards. (c. H. H.)

$\mathbf{A}$, nostril. AD, auricle. $\mathbf{B}$, right orbit. $\mathbf{B A}$, cut end of hindmost branchial artery of the right side. $B A$, first branchial artery of the left side. BA 5, fifth branchial artery of the left side. BE, first efferent branchial trunk, or epibranchial artery, of the right side. BF, fourth efferent branchial trunk, or epibranchial artery, of the right side. C. carotid artery. $\mathbf{C A}$, cardiac aorta. $\mathbf{D}$, the fifth or trigeminal nerve. DA, dorsal aorta. H, external carotid artery. F, internal carotid artery at the point where it enters the cranial cavity. $\mathbf{G}$, the hindmost of the efferent branchial vessels, $\mathbf{H}$, spiracle. I, efferent branchial loop of the first branchial cleft. $\mathbf{K}$, subclavian artery. $\mathbf{M} 1$, first branchial cleft of the right side, $\mathbf{M} 5$, fifth branchial cleft of the right side. $\mathbf{N}$, longitudinal hypo-branohial artery. $\mathbf{O}$, connectingbranch between the efferent branchial loops. SV, sinusvenosus. T, teeth. V, ventricle. 
Trace the cardiac aorta forwards from the conus arteriosus, and follow its branches on the left side to the gills, removing as much of the muscles of the under surface of the head and of the gills as is necessary to expose the vessels thoroughly.

a. The cardiac aorta is a direct continuation forwards of the conus arteriosus. It is a median vessel lying beneath the floor of the mouth and pharynx, and deeply placed in the muscles of the under surface of the head. It is little more than an inch in length, and gives off laterally the five pairs of branchial arteries.

b. The branchial arteries arise in pairs from the cardiac aorta, and carry the blood from it to the gills. The two hindmost pairs arise close together, or sometimes by a common trunk, immediately in front of the pericardial cavity. The third pair arise about a quarter of an inch further forwards. At its anterior end, about midway between the pericardial cavity and the mouth, the cardiac aorta divides into right and left branches, each of which again divides into the two anterior branchial arteries of its side.

The first or most anterior branchial artery runs along the outer border of the hyoid arch, extending nearly to its dorsal end, and giving off branches to the gill borne by it.

The four hinder branchial arteries follow similar courses along the outer borders of the first four branchial arches, extending almost to their dorsal ends, and giving off anterior and posterior branches to the gills borne by these arches.

\section{The efferent branchial vessels.}

The efferent branchial vessels can readily be injected from the caudal artery in the following manner. Cut across the tail about a couple of inches behind the cloaca. Find the caudal artery in the hamal arches of the vertebral column, lying immediately below the vertebral centra, and dorsal to the 
caudal vein. Inject the caudal artery with plaster of Paris, coloured with carmine, and carefully strained so as to remove any particles large enough to plug the vessels. Lay the animal in cold water for an hour.

Insert one of the blades of a strong pair of scissors into the mouth, and cut back horizontally along the left side through the gill-arches, as far back as the last gill-arch; from the hinder end of this incision cut transversely across the floor of the pharynx, behind the heart, to the corresponding point on the opposite side. Turn the floor of the mouth, with the heart, over to the right side.

Wash the mouth and pharynx thoroughly. Dissect off the mucous membrane of the roof of the mouth; find the efferent branchial vessels running inwards and backwards from the gill-sitts, and follow them, on the right side, outwards to the gills, and inwards to the dorsal aorta in the middle line.

The efferent branchial vessels form loops, one of which runs round the margin of each of the first four branchial clefts, and receives the arterial blood from the gills of that cleft. A single vessel, i.e. a half-loop, runs along the anterior border of the fifth branchial cleft, and opens into the loop of the fourth cleft. The successive loops communicate with one another by short horizontal branches about the middle of their length.

From the ventral ends of the loops small arteries arise, which supply the floor of the mouth and adjacent parts.

From the dorsal ends of the loops, four main efferent trunks, or epibranchial arteries, arise on each side: these run backwards and inwards in the roof of the mouth to the middle line, where they unite together in pairs to form the median dorsal aorta.

a. The carotid artery is a small vessel which arises on each side from the dorsal end of the efferent vessel of the hyoidean gill, just in front of the origin of 
the first efferent trunk. It runs forwards and inwards across the hyo-mandibular cartilage, and across the ventral surface of the skull opposite the hinder border of the orbit; and divides, about one-third of an inch from the middle line, into external and internal carotid arteries. Of these the former runs forwards and outwards across the floor of the orbit, and supplies the jaws and snout; while the latter, continuing its course in a groove in the ventral surface of the skull, unites with its fellow to form a median vessel, which passes through a foramen in the skull into the cranial cavity, and then divides into right and left arteries supplying the brain.

b. The hyoidean artery arises from the anterior limb of the first loop near its ventral end, and runs forwards and outwards to the spiracle, where it gives branches to the pseudobranch. It then continues forwards and inwards across the floor of the orbit, crossing the external carotid artery, and, entering the skull by a small hole $\frac{1}{8}$-inch in front of the inter-orbital foramen, divides into branches supplying the brain.

\section{The dorsal aorta and its branches.}

The aorta is a median vessel, formed by the union of the efferent branchial vessels of the two sides. The anterior pair of these vessels unite about the level of the inner openings of the fourth gill-clefts, and the succeeding ones open in pairs into the aorta so formed.

The dorsal aorta so formed runs back the whole length of the body, lying in the trunk just below the vertebral column, and in the tail, where it becomes the caudal artery, in the hæmal arches of the vertebræ. It gives off branches supplying the whole of the body behind the head.

The anterior part of the aorta should be dissected between the kidneys; the posterior part, which is deeply placed between 
and behind the kidneys, is best seen by dissecting one of the kidneys from the body-wall and turning it inwards.

The principal branches of the dorsal aorta are as follows.

i. The subclavian arteries are a pair of vessels, which arise from the dorsal surface of the aorta between the third and fourth pairs of efferent branchial vessels, and run outwards

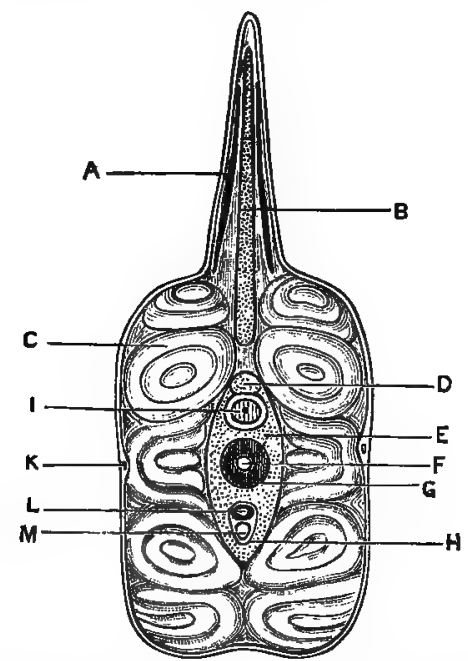

Fig. 52.- Seyllium canicula. Transverse section passing through the posterior dorsal fin. (A. ir. m.)

A, horny fibre. B, fin.ray of the dorsal fin. C, myotomes. D, neural spine. $\mathbf{E}$, base of neural arch. $\mathbf{F}$, notochord. $\mathbf{G}$, caloified portion of centrum. $\mathbf{H}$, hæmal arch. $\mathbf{I}$, spinal cord. $\mathbf{K}$, lateral vein. $\mathbf{L}$, caudal artery. $\mathbf{M}$, caudal vein.

and backwards in the body-wall and along the hinder border of the pectoral girdle to the pectoral fins, which they supply.

ii. The cœliac artery is a large median vessel arising from the ventral surface of the aorta, about half an inch behind the openings of the last pair of efferent branchial vessels. It runs backwards in the mesentery close to the dorsal 
surface of the stomach, and divides into two main branches, one of which supplies the proximal end of the stomach and the liver, and the other the first part of the intestine and the pancreas.

iii. The mesenteric artery is a median vessel arising from the aorta about an inch and a half behind the coliac artery. It supplies the intestine and rectum, and gives branches to the genital organs.

iv. The lieno-gastric artery is a median vessel arising from the aorta immediately behind the mesenteric artery. It supplies the loop of the stomach between its proximal and distal limbs, and also the spleen and part of the pancreas.

v. The posterior mesenteric artery is a small median vessel arising from the aorta about an inch and a half behind the lieno-gastric, and running backwards in the mesentery to the rectal gland, which it supplies.

vi. The parietal arteries are small paired vessels arising at intervals along the whole length of the aorta, and supplying the body-walls.

vii. The renal arteries are small paired vessels arising from the aorta along the greater part of its length, and supplying the kidneys, which they enter from the dorsal surface.

The dissection of the renal arteries should be postponed until after the dissection of the reproductive organs and cloaca.

To see the arteries, dissect one kidney away from the bodywall and turn it inwards, so as to expose its dorsal surface.

viii. The iliac arteries are a pair of small vessels leaving the aorta near the hinder end of the body-cavity, and running outwards in the bodywalls to the pelvic fins, which they supply. 


\section{Dissection of the Heart.}

Remove the floor of the mouth, with the heart and pericardium. Pin it down in a dish under water, with the ventral surface upwards. Open and examine the several cavities of the heart in the order given below, washing out the contained blood by a stream of water.

Open the ventricle by a horizontal incision, and continue the cut forwards along the right side of the conus arteriosus to its anterior end. Turn the ventral portions of both ventricle and conus over to the left side.

1. The conus arteriosus contains two sets of valves, each set consisting of three valves.

The posterior set is just in front of the ventricle, and the anterior and larger set about the middle of the length of the conus. Each valve is thick in its central part, thin and membranous at the edges.

2. The ventricle has very thick, muscular and spongy walls, and a small, nearly globular, central cavity. The opening from the auricle is a transverse slit guarded by a two-lipped valve, and is placed in the dorsal wall of the ventricle near its hinder end, and slightly to the left side.

\section{The auricle.}

Cut across the conus at its anterior end; open the auricle by a horizontal incision along its lateral and anterior borders, turn the ventral wall back, and wash out the blood from the cavity.

The walls of the auricle are thin, with radiating muscular bands; the cavity is very large and triangular in shape. The opening leading from the sinus venosus is a vertical slit in the posterior wall; and the transverse aperture leading to the ventricle is in the ventral wall, and slightly to the left of the median plane.

4. The sinus venosus has already been laid open. Its walls are thin, with a very feebly developed muscular network. The sinu-auricular aperture is a median 
vertical slit in the anterior wall, to the left of the ventral end of which is a small aperture leading into the coronary sinus, which returns to the sinus venosus the blood from the walls of the heart.

\section{DISSECTION OF THE RENAL AND REPRODUCTIVE SYSTEMS.}

These two systems have already been seen in part, but may now be examined more fully. They are originally independent of each other, but in the course of development become associated, owing to the genital ducts being formed from parts of the kidney-ducts.

The kidneys are paired organs, lying side by side along the whole length of the dorsal surface of the abdominal cavity. Each consists at an early stage of its development of a number of convoluted tubules, placed one behind another, and opening at one end into the abdominal cavity: at their other ends they open into a longitudinal duct, the segmental duct, which runs the whole length of the kidney, and opens in front into the abdominal cavity and behind into the cloaca.

During development, each kidney becomes divided into three parts ; an anterior part or pronephros, which is rudimentary or absent in the dog-fish and other Elasmobranchs, but largely developed in most ordinary fish; a middle portion or mesonephros, including the greater part of the length of the primary kidney; and a posterior portion or metanephros, formed from its hinder part.

A corresponding division of the duct takes place, so that in place of the single segmental duct, three ducts are formed; a pronephric or Millerian duct, which opens in front into the abdominal cavity, but, owing to the absence of a pronephros, loses all connection with the kidney; a mesonephric or Wolffian duct, which runs from the mesonephros to the cloaca; and a metanephric duct or ducts, which convey the excretion of the metanephros to the cloaca.

In the female the right and left pronephric ducts unite in front, so as to open into the abdominal cavity by a single 
median aperture, and become converted into the oviducts; the mesonephros is small and unimportant; while the metanephros and its ducts form the functional kidney and ureters of the adult.

In the male the pronephric ducts are rudimentary and functionless; the mesonephric tubules become connected with the testis, and act as vasa efferentia, conveying the spermatozoa to the mesonephric duct which acts as vas deferens. The metanephros and metanephric ducts, as in the female, become the functional kidney and ureters.

TABLE SHOWING THE GMBRYONIC AND ADULT CONDITIONS OF THE KIDNEYS AND THEIR DUCTS.

\begin{tabular}{|c|c|c|c|}
\hline Embryo & & Female & Male \\
\hline $\begin{array}{l}\text { Pronephros } \\
\text { Pronephric duct } \\
\text { Mesonephros } \\
\text { Mesonephric duct. } \\
\text { Metanephros } \\
\text { Metanephric ducts }\end{array}$ & 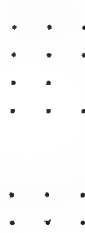 & $\begin{array}{c}\text { Absent. } \\
\text { Oviduct. } \\
\text { Rudimentary. } \\
\text { Small in front, } \\
\text { dilating behind to } \\
\text { form urinary sinus. } \\
\text { Kidney. } \\
\text { Ureters. }\end{array}$ & $\begin{array}{c}\text { Absent. } \\
\text { Rudimentary. } \\
\text { Mesonephros. } \\
\text { Vas deferens, } \\
\text { urino-genital sinus } \\
\text { and sperm-sac. } \\
\text { Kidney. } \\
\text { Ureters. }\end{array}$ \\
\hline
\end{tabular}

\section{A. The Female.}

1. The ovary, which is single, is an elongated, soft, lobulated mass, varying a good deal in size, but usually about two or three inches long in specimens of the size described. It lies in the abdominal cavity a little to the right of the median plane, and is attached to the dorsal wall by a narrow fold of peritoneum, which is very readily torn.

The surface of the ovary is tuberculated, owing to the contained ova.

2. The oviducts, which are formed from the pronephric or Miillerian ducts of the embryo, are a pair of tubes running along the dorsal wall of the abdominal cavity, ventral to the kidneys, and not far from the median plane. Their anterior ends curve inwards round the sides of the cesophagus, and unite immediately in 


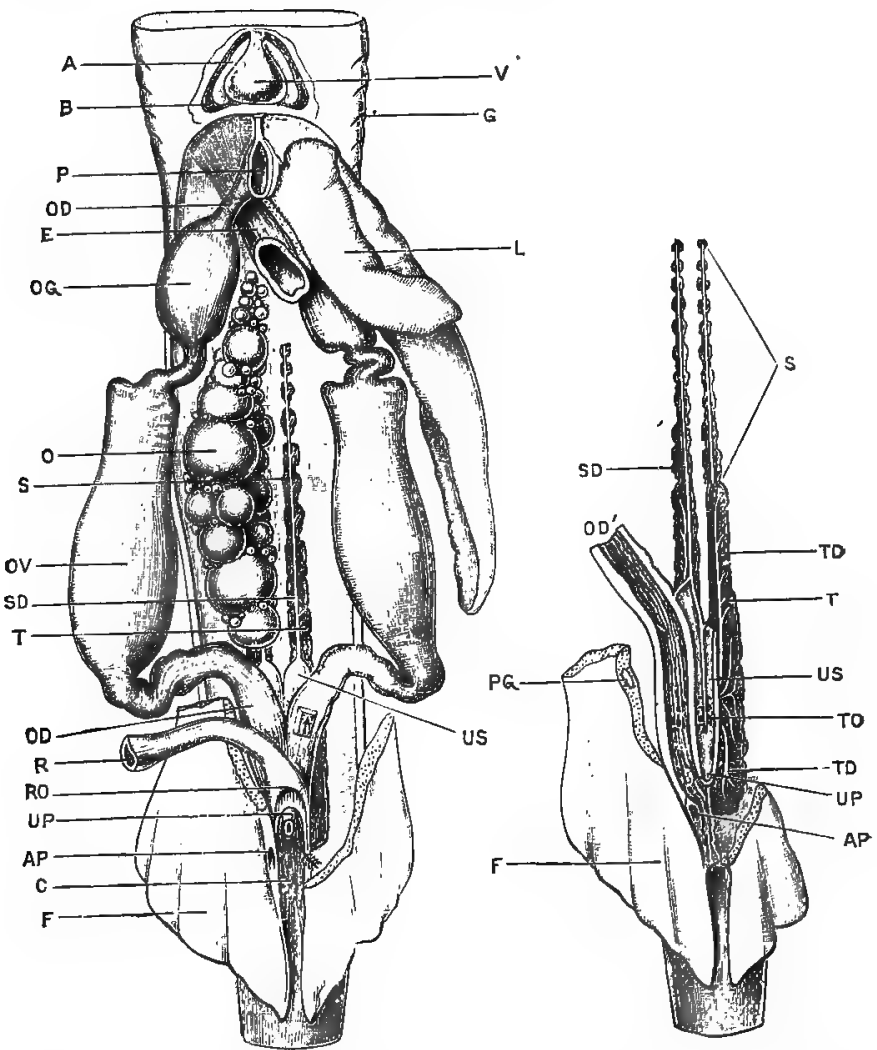

Frg. 53.-Scyllium canicula. Dissection of a female from the ventral surface, to show the renal and reproductive systems. The right. lobe of the liver and the alimentary canal from the cesophagus to the rectum have been removed. An arrow is passed up the left oviduct, which is cut open to show its point. (A. Mr, M.)

FIG. 54,-Further dissection of the renal and reproductive systems. The left oviduct is removed; the right oriduct and the urino-genital sinuses of both sides are laid open. (A. M. M.)

A, auricle. AP, abdominal pore. B, pericardial cavity. $\dot{C}$, cloaca. 포, cesophagres. $\mathbf{F}$, pelvic fin, $\mathbf{G}$, gill-slit, $\mathbf{L}$, liver. $\mathbf{O}$, ovary. $\mathbf{O} \mathbf{D}$, oviduct. OD', oviduct laid open. OG, oviducal gland. $O \bar{V}$, ripe orum in the oviduct. $\mathbf{P}$, abdominal opening of oviduct. $\mathbf{P} \mathbf{G}$, pelvic girdle. $\mathbf{R}$, rectum. $\mathbf{R O}$, opening of rectum into cloaca. $\mathbf{S}$, mesonephros. $\mathbf{S D}$, mesonephric or Wolffian duct. T, metanephros. TD, metanephric ducts or ureters. $\mathbf{T O}$, openings of ureters into urinary sinus. $\mathbf{U P}$, urinary papilla. $\mathbf{U} \mathbf{S}$, urinary sinus. $V$, ventricle. 
front of the liver, to open into the abdominal cavity by a median ventral slit, through which the ova enter the oviducts from the abdominal cavity.

About a third of its length from the anterior end, each oviduct expands to form the thick-walled oviducal gland, which secretes the horny capsules of the eggs. Behind the glands the oviducts are large and dilatable, with longitudinally folded walls : their posterior ends unite, and open by a large median aperture into the dorsal wall of the shallow cloaca, immediately behind the rectum. This aperture is closed in the young female, before fertilisation and oviposition have occurred, by a thin membrane, the hymen.

Find the anterior opening of the oviducts in the suspensory ligament of the liver, immediately behind the pericardial wall : insert a seeker through it into the oviducts, and follow one of these back to the cloaca, slitting open its ventral wall.

3. The kidneys extend nearly the whole length of the abdominal cavity, lying dorsal to the peritoneum, and one at each side of the vertebral column. The anterior half or more of each kidney, the mesonephros, is rudimentary, and appears as an ill-defined tract of soft yellowish-brown degenerate gland-tissue, arranged in patches corresponding to the vertebræ.

The metanephros, which forms the posterior third. or so of the kidney, is a well-developed, compact, laterally compressed, and lobulated mass of a brownish colour, extending back as far as the level of the cloaca.

Dissect off the peritoneum from the ventral surface of one of the kidneys, so as to expose it along its whole length. At the hinder end dissect the metanephros along its outer side from the body-wall, so as to see its. full size.

4. The mesonephric or Wolffian ducts are a pair of straight narrow tubes lying on the ventral surface of the kidneys along their whole length. Their posterior 
ends are enlarged to form a pair of tubular sacs, the urinary sinuses, which run back side by side, unite together posteriorly, and open into the cloaca at the apex of a small papilla in the posterior margin of the opening of the oviducts.

Find the opening at the apex of the urinary papilla: pass a seeter through the opening and forwards along the urinary sinus of one side: slit up the ventral wall of the sinus along its whole length.

5. The metanephric ducts or ureters are slender tubes, usually four to six in number on each side, which run from the enlarged metanephros to open into the dorsal surface of the urinary sinus by four to six slit-like apertures lying along the septum between the two sinuses. The anterior ureter is much longer than the others.

Find the ureters, lying on the ventral surface of the meta. nephros, and trace them back to the urinary sinus. Slit up the sinus, unless this has already been done, and examine its dorsal wall to see the openings of the ureters.

6. The cloaca is a shallow depression between the pelvic fins. In the female there are three openings into it: the rectum in front, the genital aperture in the middle, and the urinary sinus behind.

\section{B. The Male.}

1. The testes are a pair of soft elongated bodies lying along the dorsal wall of the abdominal cavity, and in the adult united with each other at their hinder ends. Like the ovary they are attached to the body-wall by delicate folds of peritoneum which are very readily torn, so that in many specimens the testes appear to lie free in the abdominal cavity.

2. The vasa efferentia are a number of very slender ducts which arise on each side from the dorsal surface of the testis, and run forwards in the fold of peritoneum to the mesonephros, which they enter at its anterior end. 
3. The kidneys. Each kidney is, as in the female, divided into an anterior part or mesonephros, forming about half of its entire length; and a posterior part or metanephros. The mesonephros differs from that of the female in being a well-developed lobulated glandular body along its whole length. The metanephros, as in the female, is a laterally compressed mass of considerable size, especially thick at its hinder end.

Dissect off the peritoneum from the ventral surface of one of the tidneys, so as to expose it along its whole length. At the hinder end dissect the metanephros from the body-wall along its outer side, so as to see its full size.

4. The mesonephric or Wolffian duct is a thick-walled tube running in a very sinuous course along the ventral surface of the whole length of the mesonephros, from which it receives numerous segmentally arranged tubules. Opposite the junction of mesonephros and metanephros it dilates rather suddenly into a wide thin-walled tube, the vesicula seminalis, which runs straight back along the ventral surface of the metanephros to open into the urino-genital sinus, just in front of the cloaca. The walls of the vesicula seminalis are marked by close-set transverse lines, corresponding to internal foldings of its lining membrane.

The mesonephrie duct acts as vas deferens, conveying to the exterior the spermatozoa from the testis, as well as acting as the excretory duct for the mesonephros.

Dissect off the mesonephric duct on one side from the ventral surface of the mesonephros, noting the tubules it receives from this; and follow it back to the cloaca.

5. The sperm sac is a thin-walled tube lying along the ventral surface and the outer side of the vesicula seminalis, to which it is closely attached, and which 


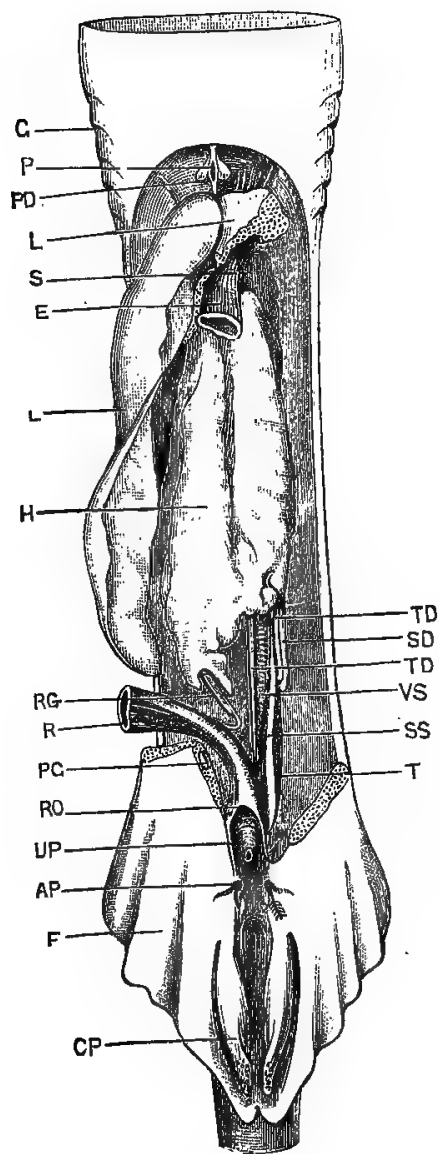

Fig. 55.

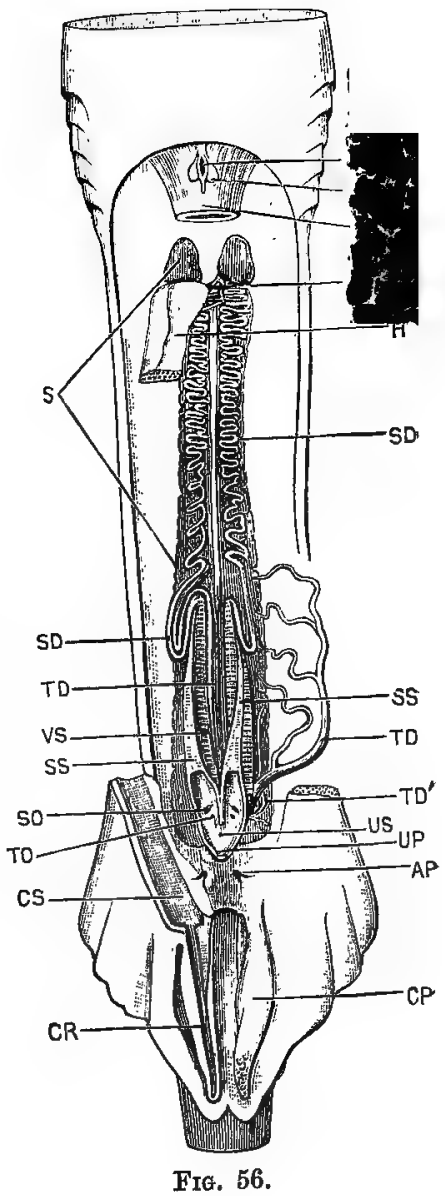


it about equals in length. It ends blindly in front, and dilates behind to form the urino-genital sinus.

Dissect away. the sperm sac from the mesonephric duct, and follow it back to the urino-genital sinus.

6. The urino-genital sinus of each side is the posterior end of the sperm sac, into which open the vesicula seminalis and the ureter. About a quarter of an inch behind these openings the sinuses of the two sides unite, and then open into the cloaca by a small aperture at the apex of the urino-genital papilla, which is situated immediately behind the opening of the rectum.

Find the opening at the apex of the urino-genital papilla: pass a seeker through the opening, and forwards along the urino-genital sinus and sperm sac of one side: slit up the ventral wall of the sinus and sac along their whole length: examine, and pass a seeker into the orifices of the vesicula seminalis and ureter.

7. The metanephric ducts are usually five in number on each side; they arise from the outer border of the ventral surface of the metanephros at intervals along its length. The four anterior ducts, after a short

Frg. 55.- Soyllium canicula. Dissection of a male from the ventral surface, to show the renal and reproductive systems. The left lobe of the liver and the alimentary canal from the cosophagus to the rectum have been removed. The excretory organs of the left side only are shown. An arrow is passed through the left abdominal pore into the abdominal eavity. (A. M. M.)

Fra. 56.-Further dissection of the renal and reproductive organs. The liver and the greater part of the testis have been removed. The right metanephric duct is shown in its natural position; the left duct has been displaced outwards. The urino-genital sinuses of both sides have been laid open. (A. M. M.)

AP, abdominal pore. CP, clasper. $C R$, groove in clasper. CS, sac continuous with groove in clasper. E, csophagus. F, peivic fin. G, gill-slit. $\mathbf{H}$, testis. I, liver. $\mathbf{P}$, abdominal opening of pronephric ducts. $\mathbf{P} \mathbf{D}$, rudimentary pronephric duct. $\mathbf{P G}$, pelvic girdle. $\mathbf{R}$, rectum. $\mathbf{R G}$, rectal gland. RO, opening of rectum into cloaca. S, mesonephros. SD, mesonephric duct or vas deferens. SO, opening of mesonephric duct into urino-genital sinus. SS, sperm sac. T, metanephros. TD, metanephric duct or ureter. TD ducts from hinder part of metanephros, joining the ureter as it reaches the urino-genitel sinus. TO, opening of ureter into urino-genital sinus. UP, urino-genital papilla. US, urino-genital sinus. VE, vasa efferentia. VS, vesicula seminalis, the dilated hinder end of the mesonephric duct. 
course, unite to form a wide thin-walled tube, the ureter, which runs back along the inner side and dorsal surface of the vesicula seminalis to open into the urino-genital sinus; just before reaching the sinus the ureter is joined by the fifth or most posterior duct, from the hinder end of the kidney. The number and arrangement of the ureters are subject to individual variation.

The metanephric ducts are most readily followed by injecting them with a coloured fluid from the ureter, which is a large wide tube, readily found along the inner side of the mesonephric duct.

8. The cloaca is the shallow depression between the pelvic fins into which, in the male, the rectum opens in front and the urino-genital sinus behind.

9. The pronephric or Mullerian ducts are rudimentary in the male, their anterior ends alone being present. A small pocket-like sac in the suspensory ligament of the liver, between it and the pericardium, represents the abdominal opening of the oviducts of the female. From this sac the ducts can be traced a short distance round the sides of the csophagus.

10. The claspers (see p. 198).

VIII. DISSECTION OF THE NERVOUS SYSTEM!

For this purpose a dog-fish should be taken which has been hardened in spirit, the roof of the skull having been previously removed so as to allow the spirit free access to the brain. The specimen must be moistened from time to time to prevent the nerves drying up, or else should be dissected in water or weak spirit.

The nervous system consists of, (1) a central portion, the brain and spinal cord, lodged within the skull and vertebral column respectively ; and (2) a peripheral portion, the nerves themselves, which run from the brain and spinal cord to all parts of the body. 
From the mode of its development the central nervous system is tubular; and it retains this character throughout life. The central canal is of very small size in the spinal cord, but widens out considerably in the brain, its local dilatations being spoken of as the ventricles of the brain.

\section{A. The Dorsal Surface of the Brain.}

Remove the skin from the dorsal surface of the head. Dissect away the eyelids on the right side so as to open up the orbit. Slice away the roof of the cranium so as to expose the brain fully, slicing through the auditory capsule, which lies immediately behind the orbit, on the right side, but leaving it uninjured on the left.

The brain is large and nearly fills the cranial cavity: it is narrow between the orbits, but expands considerably in front of them. As seen from above, the following parts are visible in order from before backwards ( $c f$. fig. 49, p. 224).

1. The prosencephalon is the most anterior and the largest division of the brain. It is a smooth, somewhat globular mass, lying in the expanded anterior part of the cranial cavity, between the olfactory capsules and the orbits. It is marked in front by a shallow median groove; and is sharply separated behind from the succeeding part of the brain. It corresponds to the cerebral hemispheres of higher vertebrates.

2. The olfactory lobes are a pair of large oval masses, arising by stout stalks from the sides of the prosencephalon, their distal surfaces being closely applied to the olfactory capsules.

3. The thalamencephalon is the narrow portion of the brain immediately behind the prosencephalon; its roof is very thin, so that the cavity, or third ventricle, can be seen through it. From the hinder part of the roof arises the pineal body, a slender tubular process which extends forwards over the prosencephalon, and ends in a slightly dilated knob attached to the membranous roof of the skull some little distance in front of the brain. 
4. The optic lobes are a pair of closely apposed oroidal protuberances, placed in the narrowest part of the cranial cavity opposite the middle of the orbits. Their posterior halves are overlapped and concealed by the cerebellum.

5. The cerebellum is a long flattened oval body arising from the dorsal surface of the brain immediately behind the optic lobes, and extending forwards over the optic lobes and backwards for an equal distance over the medulla. Its surface is slightly grooved.

6. The medulla oblongata is the part of the brain behind the cerebellum. Its roof is very thin, and liable to be torn away so as to expose its cavity, the fourth ventricle. Its sides are thickened, especially in front, where they give origin to the roots of the fifth, seventh, and eighth cerebral nerves: immediately above these roots, and below the posterior projection of the cerebellum, the medulla is produced into a pair of large lateral wing-like processes, the restiform bodies, which are united with each other beneath the overhanging cerebellum.

\section{B. The Orbit.}

This should be examined before the dissection of the nerves is proceeded with. If the eyelids have been removed as directed above, no further dissection is necessary. Wash out the blood-clots from the orbital sinus with a stream of water, avoiding injury to the nerves.

1. The eyeball is almost hemispherical in shape, its outer or corneal surface being very flat.

2. The eye-muscles, by which the movements of the eye are effected, are six narrow muscular bands which arise from the skull-wall and are inserted into the eyeball : they are arranged in two groups.

a. The recti muscles are four in number. They arise close together, near the hinder end of the orbit, and diverge outwards to be inserted into the eyeball. 
i. The rectus superior runs outwards and forwards to be inserted into the dorsal surface of the eyeball.

ii. The rectus internus, or anterior, runs forwards between the skull-wall and the eyeball, and is inserted into the anterior surface of the latter. iii. The rectus externus, or posterior, is the hindmost of the eye-muscles, and runs directly outwards bebind the eyeball, to be inserted into its posterior surface.

iv. The rectus inferior runs outwards and forwards beneath the eyeball, and is inserted into its ventral surface.

The origin of the rectus inferior can be seen from above, between the other recti muscles; to see its insertion, turn the eyeball up.

b. The oblique muscles are two in number. They arise close together at the anterior end of the orbit, and run backwards and outwards to the eyeball.

i. The obliquus superior is inserted into the dorsal surface of the eyeball, just in front of the insertion of the rectus superior.

ii. The obliquus inferior is inserted into the ventral surface of the eyeball, just in front of the insertion of the rectus inferior.

To see the obliquus inferior, turn the eyeball up.

\section{The Cranial Nerves.}

These should be dissected before the brain is removed, in order that their entire course may be seen.

Dissect the nerves on the right side, leaving the left side for the dissection of the sense-organs.

The ophthalmic branches of the fifth and seventh nerves, which run through the orbit close to the skull-wall, and dorsal to all the eye-muscles, should be identified at once; and care must be taken not to injure them. 
1. The olfactory nerves arise on each side in two large bundles from the anterior surface of the olfactory lobe, and pass at once through the sieve-lile membrane separating the cranial cavity from the nose. They end in the folds of the large olfactory organ.

Dissect the olfactory nerves from the dorsal surface, taking care to preserve the ophthalmic branches of the fifth and seventh nerves, which run forwards across the olfactory lobe, but separated from it by the cartilaginous cranium.

2. The optic nerve enters the orbit through a foramen in the side-wall of the skull, near the ventral surface, and about midway between the origins of the recti and obliqui muscles. In the orbit it is enclosed in a very tough connective-tissue sheath, and runs straight out to the eyeball.

Press the eye-muscles aside to see the optic nerve between the recti and obliqui. The course of the nerve within the skull will be seen when the brain is removed.

3. The third nerve, or motor oculi, is a rather slender nerve which, arising from the ventral surface of the brain below the optic lobes, runs outwards and slightly backwards to the skull-wall, which it perforates about a quarter of an inch behind the optic nerve, and immediately in front of the origins of the recti muscles.

In the orbit it divides at once into three branches, of which the most anterior supplies the rectus internus, and the middle one the rectus superior. The posterior branch passes downwards between the rectus superior and rectus externus close to their origins, and then forwards, ventral to the rectus inferior, which it supplies, to the obliquus inferior, in which it ends. As it crosses the rectus inferior it is joined by a branch from the fifth nerve.

Gently press away the optic lobe from the stoull-wall so as to see the nerve within the skull, and also the point at which 
it enters the orbit. In the orbit the branches to the rectus internus and rectus superior are readily dissected from above. To trace the branch to the obliquus inferior place the fish on its side, and turn the eyeball upwards so as to expose the nerve from below.

4. The fourth or pathetic nerve is a very slender nerve which arises from the dorsal surface of the brain, in the angle between the optic lobe and the cerebellum, and almost in the median plane. From the brain, it runs forwards and outwards to the skull-wall, which it perforates a little in front of the middle of the orbit, dorsal to the optic nerve, and immediately ventral to the ophthalmic branches of the fifth and seventh nerves. In the orbit it runs outwards and forwards to the posterior border of the obliquus superior, in which it ends.

Though very slender, the fourth nerve is easy to trace. Lift up the front end of the cerebellum to see its origin between the cerebellum and the optic lobes; trace it forwards within the skull, and in the orbit outwards to the obliquns superior.

5. The fifth, seventh, and eighth nerves arise very close together from the side of the medulla at its widest part, opposite the posterior part of the cerebellum. The eighth or auditory nerve, which is the hindmost of the three, enters the auditory capsule at once; while the fifth and seventh nerves pass outwards through the skull-wall, by a foramen at the posterior and inner angle of the orbit, immediately behind the origins of the recti muscles.

Gently press away the medulla from the skull-wall until the nerve-roots come into view, and then carefully cut away the skull-wall and the auditory capsule until they are fully exposed. 
a. The fifth or trigeminal nerve has three main branches.

i. The ophthalmic branch arises from the anterior border of the root of the nerve, close to the brain. It runs forwards and upwards for about a quarter of an inch within the skull, and then perforates the skull-wall to enter the orbit, above and a little in front of the origins of the recti muscles.

In the orbit it runs forwards close to the skull-wall, alongside of and immediately below the ophthalmic branch of the seventh nerve, and dorsal to all the eye-muscles. At the anterior and inner angle of the orbit it passes through a canal between the olfactory capsule and the cranium, crosses the olfactory lobe, and continuing its course straight forwards divides into branches, which supply the mucous canals of the dorsal surface of the snout.

Gently press away the brain from the skull to see the root and the part of the nerve within the cranium, taking care not to confound it with the ophthalmic branch of the seventh nerve which lies immediately dorsal to it. In the orbit dissect from the side, and in front of the orbit from above.

The main stem of the fifth nerve on entering the orbit runs forwards and outwards across its floor as a broad riband-like band, easily mistaken for a muscle. Near the outer margin of the orbit this divides into the maxillary and mandibular branches.

Turn the eye up; trace the nerve across the floor of the orbit, and follow its branches to their distribution.

ii. The maxillary branch, which is the anterior and larger of the two, turns over the upper jaw at the anterior border of the orbit, and divides into branches which run forwards to supply the mucous canals of the under surface of the snout. 
iii. The mandibular branch, the posterior and smaller of the two, crosses the upper jaw, and then curving round the angle of the mouth, where it lies very close to the surface, runs forwards along the lower jaw. It supplies the muscles moving the lower jaw.

6. The sixth nerve is very slender, and arises from the ventral surface of the medulla near the median plane, and a short way behind the roots of the fifth and seventh nerves; it passes out through the same foramen with these nerves, lying below them and separated from them by dense connective tissue. It supplies the rectus externus.

The part of the sixth nerve within the skull may be seen by cutting through the roots of the fifth and seventh nerves, and pressing the brain aside. In the orbit the nerve can be seen along the inferior border of the rectus externus.

7. The seventh or facial nerve, like the fifth, has three main branches.

i. The ophthalmic branch arises from the dorsal edge of the medulla, immediately behind the cerebellum. It runs forwards for about a quarter of an inch within the skull, and then enters the orbit through a foramen above and a little behind the origins of the recti muscles. In the orbit it runs forwards close to the skullwall, alongside of and dorsal to the ophthalmic branch of the fifth nerve, which it accompanies to its distribution.

The root is large and readily seen on pressing the brain away from the skull. The dissection is the same as for the corresponding branch of the fifth nerve.

The main stem of the seventh nerve enters the orbit immediately behind the fifth, and divides almost at once into two main branches.

Turn the eye up and dissect from the side, following the branches to their distribution. 
ii. The palatine nerve is a slender nerve which runs forwards and outwards across the floor of the orbit, immediately behind the main stem of the fifth nerve: it then turns forwards beneath this nerve and runs along the upper jaw, sending branches to the roof of the mouth.

iii. The hyoidean or post-spiracular nerve is a much stouter nerve, which runs straight outwards along the posterior wall of the orbit towards the spiracle. Continuing its course outwards and backwards, in close contact with the anterior wall of the auditory capsole, it passes along the posterior border of the spiracle, where it lies just beneath the skin, and then runs downwards and backwards along the anterior border of the hyoidean arch, giving branches to the muscles of these parts.

Close to its origin it gives off a number of small pre-spiracular branches to the anterior wall of the spiracle and to the pseudobranch.

8. The eighth or auditory nerve, immediately after its origin, enters the auditory capsule through a hole in its inner wall, and divides into branches supplying the several parts of the internal ear.

Slice away the auditory capsule down to the level of the root of the auditory nerve, and trace the nerve into it.

9. The ninth or glosso-pharyngeal is a slender nerve which arises from the side of the medulla, about an eighth of an inch behind the auditory nerve and nearer the ventral surface. It at once enters a foramen on the inner side of the auditory capsule, and passing horizontally outwards and backwards along a canal in the floor of the capsule, emerges at its posterior and outer angle, opposite the upper end of the first branchial cleft, where it divides into two branches. 
i. The anterior or hyoidean branch is the smaller of the two, and runs down the posterior border of the hyoidean arch.

ii. The posterior or branchial branch is larger, and runs down the anterior border of the first branchial arch.

Press away the medulla from the skull-wall to see the root of the nerve, about an eighth of an inch behind the auditory nerve : slice away the auditory capsule horizontally to expose the nerve in the canal, and then follow the branches along the branchial arches.

10. The pneumogastric or vagus is a large nerve, which arises by a number of roots from the side of the hinder part of the medulla, the most anterior root being immediately behind and dorsal to that of the glosso-pharyngeal.

The nerve, formed by the union of these roots, runs outwards and backwards through the skullwall, emerging at its hinder end between the cranium and the auditory capsule. It then runs horizontally backwards parallel to the vertebral column, and a short distance to its outer side, lying along the inner wall of the anterior cardinal sinus, and giving off numerous branches.

Press away the medulla from the skull-wall to see the roots of origin of the vagus. Slice away horizontally the hinder end of the skull to expose the nerve in this part of its course. Lay open the anterior cardinal sinus by slitting up its dorsal wall; wash out the contained blood, and identify the branches of the vagus running along its inner wall and across its floor. Dissect these branches out, and follow them to their distribution.

i. The branchial nerves, which are four in number, run backwards and outwards in the floor of the anterior cardinal sinus to the upper ends of the four hinder, i.e. second to fifth, branchial clefts. 
Each then divides into a smaller anterior and a larger posterior branch, which supply the two borders of the corresponding eleft, in a manner precisely similar to that in which the glossopharyngeal supplies the first branchial cleft.

ii. The visceral nerves are continuations backwards of the trunk from which the branchial nerves arise ; they give off several branches, of which the most important are the cardiac nerves to the heart, and the gastric nerves to the stomach.

iii. The nerve of the lateral line arises from the vagus near its root: it lies dorsal to the branchial nerves, and behind these it runs along the inner surface of the body-wall to the hinder end of the body, parallel to the lateral line, the sense-organs of which it supplies.

\section{Dissection of the Brain.}

\section{Removal of the brain.}

Cut across the medulla a short way behind the roots of the vagus. Cut through the olfactory nerves so as to separate the olfactory lobes from the nasal capsules; and cut through the several cranial nerves one by one, about midway between the brain and the skull-wall. Turn out the brain from the cranium, taking care not to damage the pituitary body: this latter lies on the under surface of the brain, opposite to the optic lobes, and in a hollow of the sluull-floor, to which it is firmly attached, and from which it must be dissected away.

Examine the skull after removal of the brain, and identify the several nerve-stumps and their foramina of exit.

\section{The ventral surface of the brain. (Cf. p. 247.)}

Place the brain in spirit, with the ventral surface upwards, and identify the following structures.

a. The prosencephalon is marked by a shallow median groove; posteriorly it is directly continuous with the thalamencephalon. 
b. The olfactory lobes have much the same appearance as from the dorsal surface.

c. The optic chiasma is formed by the crossing of the optic nerves on the under surface of the thalamencephalon. Beyond the chiasma the nerves diverge outwards towards the foramina in the skull-wall which lead to the orbits.

d. The infundibulum consists of a pair of prominent oval swellings, sometimes called lobi inferiores, on the under surface of the thalamencephalon, immediately behind the optic chiasma.

e. The pituitary body is a thin-walled sac immediately behind the infundibulum, and attached to it. It is produced laterally into two thin-walled pouches, the sacci vasculosi; and it has along its ventral surface a median tubular body of unknown function, the anterior end of which is connected with the infundibulum, and the posterior end, in the natural condition of the parts, attached to the floor of the skull.

f. The roots of the third nerves arise from the ventral surface of the brain close to the middle line, and opposite the junction of the infundibulum with the pituitary body.

Lift up the pituitary body and infundibulum so as to expose the third nerves fully.

g. The medulla is narrow in front: it widens considerably opposite the roots of the fifth and seventh nerves; and then narrows again as it passes back into the spinal cord.

h. The roots of the sixth nerves arise from the ventral surface of the medulla, near the middle line, and a short distance behind the level of the roots of the fifth and seventh nerves.

\section{The cavities of the brain.}

Bisect the brain by a median vertical incision along its whole length. Slice away the inner surface of one half of the prosencephalon until the cavity within it is fully exposed. 
The brain, as already noticed, is tubular, and its eavity is continued into all the principal lobes or processes, which may be regarded as formed by foldings of its wall, or rather by unequal growth at various parts. The roof of the brain varies greatly in thickness at different parts, but the floor is nearly uniform throughout ( $c f$. fig. 49, p. 224.).

a. The lateral ventricles are a pair of large laterally compressed cavities in the prosencephalon, separated from each other by a median vertical septum. Posteriorly they open into the cavity of the thalamencephalon, and on their outer sides they are prolonged into the olfactory lobes.

The posterior wall of the prosencephalon, just in front of and above the thalamencephalon, is produced on each side into a very thin-walled fold, which projects forwards into the lateral ventricle nearly to its anterior end. Between the two layers of the fold are numerous blood. vessels forming the choroid plexus.

b. The third ventricle is the cavity within the thalamencephalon: it communicates in front with the lateral ventricles, above with the very narrow cavity of the stalk of the pineal body, behind with the cavity beneath the optic lobes, and below with the infundibulum.

c. The pituitary body is a sac with very thin walls, standing in this respect in marked contrast to the infundibulum, which has thick walls. Its cavity communicates in front with that of the infundibulum, and behind with that of the tubular body which lies on its ventral surface.

d. The optic lobes, cerebellum, and restiform bodies are all hollow outgrowths from the dorsal surface of the brain. Their walls are of moderate thickness, excepting the dorsal ends of the restiform bodies, which are very thin. 
e. The fourth ventricle is the cavity in the medulla, beneath and behind the cerebellum: its sides and floor are thick, its roof very thin.

\section{E. The Spinal Cord and Nerves.}

Slice away the roof of the neural canal horizontally, so as to expose the spinal cord from the dorsal surface.

Press away the cord gently from the side of the canal, to expose the roots of the spinal nerves.

1. The spinal cord lies in the neural canal of the vertebral column. It is flattened dorso-ventrally; marked by median longitudinal grooves or fissures along its dorsal and ventral surfaces; and traversed throughout its whole length by a narrow central canal, continuous in front with the fourth ventricle of the brain.

2. The spinal nerves arise in pairs from the sides of the spinal cord. Each nerve arises by two roots, a dorsal or sensory root, and a ventral or motor one: of these the dorsal root is some distance in front of the corresponding ventral one, the successive dorsal and ventral roots alternating at about equal intervals along the sides of the spinal cord.

Each dorsal root arises as a single trunk from the spinal cord, and passes out from the neural canal through a notch in the hinder margin of the intervertebral neural plate. The corresponding ventral root arises by three or more rootlets from the spinal cord, and passes out from the neural canal through a smaller notch in the hinder margin of the vertebral neural plate. Outside the vertebral column the two roots unite to form the spinal nerve.

\section{DISSECTION OF THE SENSE-ORGANS.}

\section{A. The Nose.}

Dissect out one of the olfactory organs from its capsule: cut it across transversely about the middle; wash it, and examine its structure. 
The olfactory organ is a sac, the walls of which are thrown into a large number of transverse folds attached in pairs to a median septum. Each fold is covered by a soft mucous membrane, the surface of which is increased by secondary foldings. The olfactory nerves enter the organ from the dorsal surface, and spread out in the folds.

The great size of the olfactory organ, and of the nerves it receives, is very characteristic of Elasmobranch fishes.

\section{B. The Eye.}

Remove one of the eyes by cutting across the eye-muscles and optic nerve. Snip off the remains of the muscles, and divide the eye into an inner and an outer half by cutting all round it equatorially. Place the two halves under water in a dissecting dish.

The eye is hollow; its walls consist of three concentric layers, and within its cavity, near the outer side, is the spherical lens, about the size of a pea.

1. The sclerotic, or outermost coat of the eye, is thin and cartilaginous. It invests the whole of the eye except the part covered by the cornea.

2. The cornea is an oval patch on the outer surface of the eye, through which the light enters. It is about half an inch long and a quarter of an inch broad. It consists of transparent connective tissue, and is continuous at its edges with the sclerotic.

3. The choroid is a black opaque membrane, lying within the sclerotic. It adheres closely to the sclerotic, and is firmly attached to the line of union of sclerotic and comea. Beyond this line it is continued into the iris, which lies free behind the cornea, and is perforated in its centre by an elongated oval slit, the pupil, through which alone light can get to the interior of the eye.

The inner surface of the choroid, especially in the inner half of the eye, is covered by a glistening silvery membrane, the tapetum. 
4. The retina is a soft pulpy membrane lying on the inner surface of the choroid, and readily separable from it except at the point of entrance of the optic nerve, to which it adheres firmly; and with which it is continuous. It is the only part of the eye sensitive to light.

5. The lens is a solid spherical body, suspended in the eye immediately behind the pupil. During life it is transparent, and serves to bring the rays of light to a focus on the retina.

The spherical shape of the lens, together with the flatness of the cornea, is very characteristic of aquatic animals.

\section{The Ear.}

The ear consists of a membranous sac, or vestibule, filled with fluid, and produced into three curved tubes, the semicircular canals, which lie in planes at right angles to one another, and open into the vestibule at both ends.

The whole sac is lodged within the cartilaginous auditory capsule, which is firmly united with the side of the hinder end of the skull.

Scrape clean the hinder end of the skull so as to expose the left auditory capsule fully. Note, on the dorsal surface; the prominent ridges lodging the anterior and posterior vertical semicircular canals, the former running backwards and inwards from the hinder margin of the orbit, and the latter backwards and outwards to the posterior end of the skull. Note also, at the inner side of the point of meeting of the two canals, a hole leading downwards and outwards into a canal, the aqueductus vestibuli.

Hold the skull in the left hand and carefully pare away the cartilage with a sharp scalpel until the several parts of the auditory organ are fully exposed. Owing to the transparency of the cartilage, the parts can be seen before they are met with, and the dissection, though requiring care, is not difficult. 
1. The vestibule is a laterally compressed sac, lying in the lower and inner part of the auditory capsule. It is partially divided into two; contains milky calcareous concretions; and communicates with the dorsal surface of the skull by the aqueductus vestibuli, which is a persistent relic of the involution of the dorsal integument from which the ear is developed.

Pass a seeker down the aqueductus vestibuli into the vestibule.

2. The semicircular canals are three in number. They open at both ends into the vestibule, and each is dilated at one end into an oval ampulla, which receives a branch of the auditory nerve.

i. The anterior vertical canal has its ampulla at the anterior end close to the orbit.

ii. The posterior vertical canal is the longest of the three, and forms almost a complete circle. Its ampulla is at its posterior and inferior end, on the ventral wall of the skull.

iii. The horizontal canal lies in the wing-like lateral process of the auditory capsule, behind the orbit: its ampulla is at its anterior end, close to that of the anterior vertical canal.

3. The auditory nerve enters the auditory capsule on its inner side, and at once divides into branches, which can be traced to the vestibule and to the ampullæ of the semicircular canals.

\section{The Mucous Canals.}

These are long tubes opening by one end on the surface of the head, while the other end is closed and dilated into an oval ampulla, which is subdivided internally by partitions, and receives nerve-branches.

The ampullæ are collected together into a large mass at the anterior end of the snout, in front of the cranial cavity; and two smaller masses at the sides of the head, between the nose and the orbit. From these masses the tubes diverge to their external openings, which are arranged in rows on the 
dorsal and ventral surfaces of the head. The tubes are filled with a transparent gelatinous matter, which can readily be squeezed out from their openings on pressure.

Their function is not known with certainty, but they are generally believed to be sense organs of some kind. The nerves supplying them are the ophthalmic branches of the fifth and seventh nerves, and the maxillary division of the fifth.

Squeeze the head of a fresh specimen, so as to make the gelatinous matter exude, and so render the openings visible.

Remove the skin from the dorsal and ventral surfaces of the head, an operation much facilitated by dipping the head into hot water for a few moments : identify the masses of ampullee and the nerves in connection with them, and trace the tubes outwards and backwards to their openings. Some of the tubes which run along the dorsal surface of the skull, between the cranium and the orbit, are some inches in length.

Cut out a small piece of the mass of ampulla: : put it on a slide in a drop of water: separate the parts with needles: cover, and examine under the microscope. Note the ampulla, their internal divisions, the nerves supplying them, and the tubes. 


\section{Chapter XII. \\ THE SKELETON OF THE RABBIT.}

\section{Lepus cuniculus.}

IN the early stages of development the skeleton of the rabbit consists entirely of cartilage. This primary cartilaginous skeleton agrees in general plan with the skeleton of the dogfish, and may be taken as representing a corresponding stage in the evolution of mammals.

The skeleton of the adult rabbit is made up of bone, together with a small amount of cartilage. The bones are of two chief kinds.

(1.) Cartilage-bones are so called from their arising in direct relation with the pre-existing cartilage. They make up the greater part of the adult skeleton.

(2.) Membrane-bones are developed independently of the primary cartilaginous skeleton, and are almost confined to the head. They are to be regarded as dermal bones which have lost their connection with the skin, and become in many cases firmly united with the underlying cartilage and cartilage-bones: they are morphologically equivalent to the bony plates forming the bases of the scales in the dog-fish.

The sesamoid bones form a third class. These are of small size and little morphological importance, and are developed within the tendons of certain of the limb-muscles, close to the joints : they modify the action of these muscles by altering the direction in which they pull.

The teeth of the rabbit are cutaneous structures, comparable to the scales or teeth of the dog-fish; they are, 
however, in the adult so intimately associated with the bones of the jaws that they may conveniently be described with the skeleton. The hairs and claws, which are special developments of the epidermis, or surface-layer of the skin, will be described in the next chapter.

The rabbit's skeleton may be divided, like that of the dogfish, into axial and appendicular portions, which will be dealt with successively.

The following points in the rabbit's skeleton should be noticed as characteristic of the great majority of mammals, and as separating them from lower vertebrates.

Epiphyses are present at the ends of the vertebral centra, as well as at the ends of the long bones of the limbs; and the successive vertebræ are connected by inter-vertebral ligaments : the cervical vertebræ are seven in number. The sternum is transversely jointed. In the skull, there is a completely ossified occipital ring, with two condyles : the cranial and olfactory cavities are separated by a bony cribriform plate: there is not a distinct para-sphenoid on the under surface of the skull : the lower jaw articulates directly with the squamosal : there are three or four small but distinct auditory ossicles, of which the incus probably represents the quadrate of lower vertebrates. The teeth form a single row round the margin of each jaw, and are arranged in groups. In the shoulder girdle, the coracoid. is reduced to a small process of the scapula; . and there is no inter-clavicle. The ankle-joint is situated between the tibia and fibula above, and the proximal tarsal bones below: the fourth and fifth carpalia, and the corresponding tarsalia, fuse to form a single bone in each limb: the preaxial digit, corresponding to the thumb, has two phalanges, while the others have each three. In the rabbit the first digit of the hind limb, corresponding to the great toe in man, is rudimentary: in other mammals it bas two phalanges like the thumb.

Examine the prepared skeletons and make drawings to scale of the several parts. 


\section{THE AXIAL SKELETON.}

\section{A. The Vertebral Column or 'backbone.'}

This consists of a series of bony rings or vertebræ, placed end to end so as to form a tube, which stiffens the back, and lodges and protects the spinal cord. The successive vertebræ are movably articulated with one another, and their centra are separated by intervertebral dises of fibro-cartilage, except in the sacrum, where a certain number are fused together to support the pelvis.

The vertebræ fall readily into five groups, named as follows from before backwards:-

(1.) The cervical or neck vertebræ.

(2.) The thoracic vertebræ, which bear movably articulated ribs.

(3.) The lumbar vertebræ, which are the largest of the series, and bear no ribs.

(4.) The sacral vertebræ, which are fused together and support the pelvis.

(5.) The caudal or tail vertebræ.

1. General characters of a vertebra.

Examine, as a type, the second lumbar vertebra.

a. The vertebra is tubular, the cavity or neural canal being occupied during life by the spinal cord.

b. The centrum or body is the thickened ventral wall of the tube, forming the floor of the neural canal. It corresponds in position to the notochord of the embryo, which it replaces ( $c f$. p. 200).

c. The epiphyses are two thin plates of bones applied to the ends of the centrum, with which they fuse in old age.

d. The neural arch forms the sides and roof of the neural canal, and presents at each end, close to the centrum, a pair of intervertebral notches for the passage of the spinal nerves, the posterior notches being much the deeper. 
e. The neural spine or spinous process is a median dorsal crest, projecting upwards and forwards from the top of the neural arch.

f. The hypapophysis is a median ventral process from the under surface of the centrum, projecting. downwards and forwards.

g. The transverse processes are a pair of large flattened. processes with expanded ends, projecting forwards, downwards, and outwards from the sides of the centrum.

h. The zygapophyses or articular processes are borne on the two ends of the neural arch : they articulate with those of the adjacent vertebræ, so as to limit. torsion and lateral displacement of the vertebral column.

i. The pre-zygapophyses, or anterior articular processes, project upwards from the anterior end of the neural arch. The articular surfaces. are concave, and turned inwards towards each other; above them the processes extend upwards to form the stout metapophyses.

ii. The post-zygapophyses, or posterior articular processes, project backwards from the top of the hinder end of the neural arch. The articular surfaces are slightly convex, and face outwards. and downwards.

j. The anapophyses are a pair of small, backwardly directed processes from the hinder end of the neural arch, below the post-zygapophyses and above the intervertebral notches.

\section{The cervical vertebræ.}

These are seven in number in the rabbit, as in almost all other mammals. As a group they are characterised by the shortness of their centra; the small size of their spinous processes; and the fusion of the ribs with the vertebræe to form the perforated 


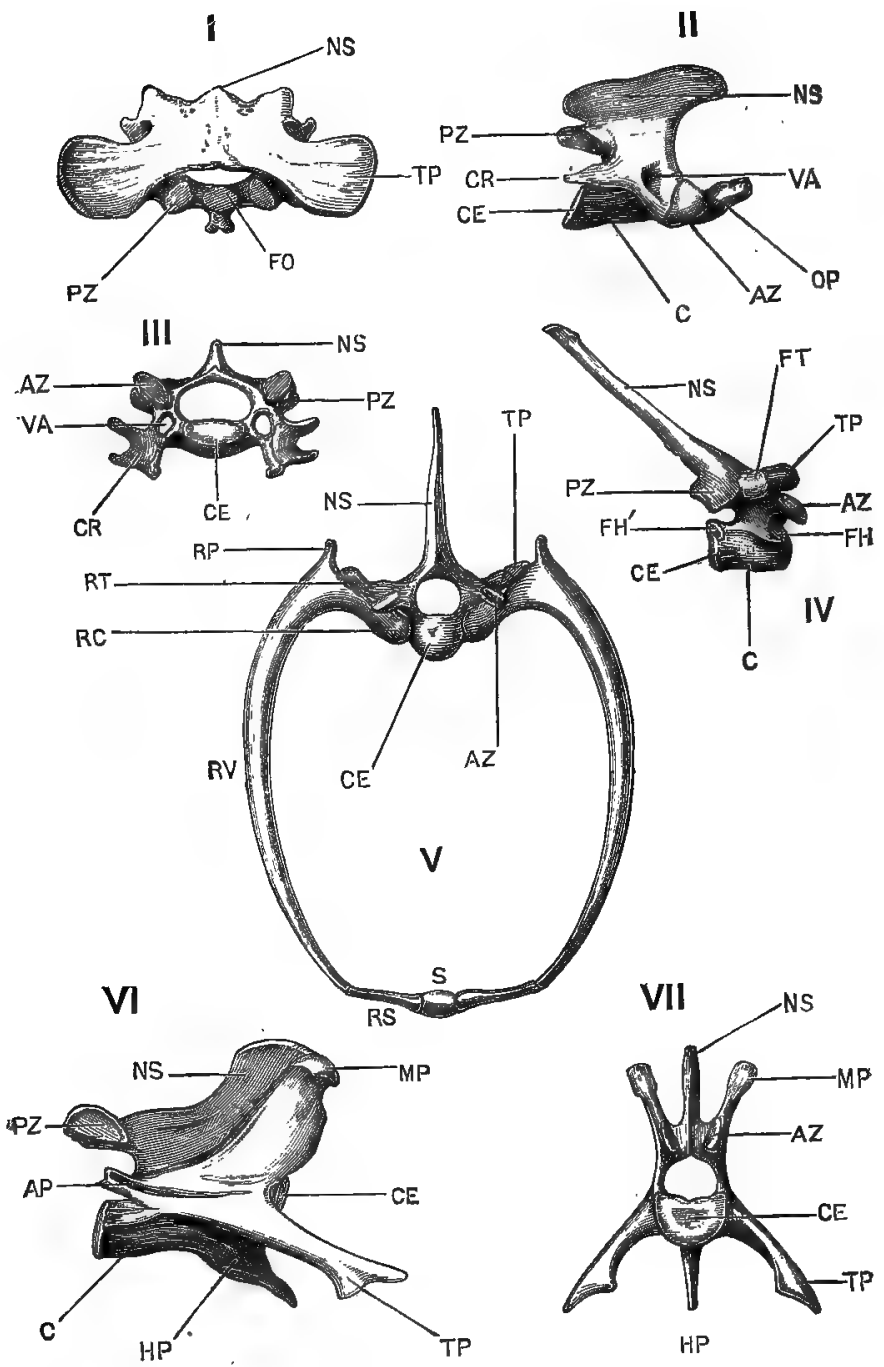


transverse processes, through which the vertebral arteries run.

a. The first, or atlas vertebra, differs very markedly from all the others : its more important characters. are as follows.

i. The neural canal is very large, and is divided into two parts by a transverse ligament, which is usually preserved in the dried skeleton: of these, the upper and larger is the true neural canal, giving passage to the spinal cord ; while the lower and smaller one lodges the odontoid process of the second vertebra.

ii. The centrum is probably represented by the odontoid process, which has become detached from the atlas, and fused with the centrum of the second vertebra. The mid-ventral portion of the atlas is very narrow, and is produced backwards into a short process.

iii. The neural spine is a very insignificant ridge, most prominent in front.

iv. The transverse processes are broad horizontal lamellæ, perforated at their bases by longitudinal vertebrarterial canals. In front of each transverse process the canal is continued upwards as a groove to a foramen in the neural areh, through which the vertebral

Frg. 57.-Lepus cuniculus. Selected vertebræ. (A. M. M.)

I. First cervical vertebra, or atlas, from the dorsal surface. II. Second cervical vertebra, or axis, from the right side. III. Fifth cervical vertebra; anterior surface. IV. Fourth thoracic vertebra, from the right side. V. Fourth thoracic vertebra, and fourth pair of ribs; anterior surface. VI. Second lumbar vertebra, from the right side. VII. Second lumbar vertebra; anterior surface.

AP, anapophysis. AZ, anterior or pre-zygapophysis. $\mathbf{C}$, centrum. CE, epiplyysis of centrum. CR, cervical rib. FH, facet for capitulum or head of the fourth rib. $\mathbf{F H}$ ', facet for capitulum of the fifth rib. $\mathrm{FO}$, facet for odontoid process. FT, facet for tubercle of fourth rib. $\mathbf{E P}$, hypapophysis. MP, metapophysis. NS, neural spine, or spinous process. $\mathbf{O P}$, odontoid process. PZ, post-zygapophysis. RC, capitulum or head of rib. 'RP, process of rib for attachment of ligaments. RS, sternal portion of rib. RT, tubercle of rib. $\mathbf{R V}$, vertebral portion of rib. $\mathbf{S}$, sternum. TP, transverse process. VA, vertebrarterial canaj. 
artery enters the neural canal and the first spinal nerve leaves it.

Along the inner and hinder border of each transverse process is a deep groove, corresponding to an intervertebral notch, for the passage of the second spinal nerve.

v. The articular surfaces. The anterior end of the atlas has two very large, deeply concave surfaces, for articulation with the condyles of the skull : the posterior end has two much smaller facets for the second vertebra.

b. The second or axis vertebra.

i. The centrum is broad and flat, and is produced in front into the conical odontoid process, which is probably the centrum of the atlas vertebra. The suture between the odontoid process and the body of the axis is readily seen in young rabbits.

ii. The neural spine forms a large vertical crest, very prominent in front, and bifid behind.

iii. The transverse processes are small, backwardly directed spines, perforated at their bases by the vertebrarterial canals.

iv. The anterior articular surfaces are large and convex, and situated at the sides of the anterior end of the centrum.

v. The post-zygapophyses project backwards from the hinder edge of the neural arch, their articular surfaces facing downwards and outwards.

c. The remaining cervical vertebræ:- third to seventh. i. The centra are short, broad from side to side, thin from above downwards, and obliquely truncated at the ends. The centrum of the seventh vertebra bears at its posterior end a pair of facets, which form parts of the articular 
surfaces for the heads of the first pair of thoracic ribs.

ii. The neural spines are small, excepting that of the seventh vertebra.

iii. The transverse processes are perforated at their bases by the vertebrarterial canals: beyond these they divide into dorsal and ventral portions, of which the latter, the inferior lamellæ, form prominent bony plates, except in the seventh vertebra, in which they are absent.

iv. The zygapophyses are borne on the sides of the neural arches; the anterior facing upwards and inwards, the posterior downwards and slightly outwards.

\section{The thoracic vertebræ.}

These are usually twelve, sometimes thirteen, in number, and are characterised by bearing movably articulated ribs. The neurapophyses are long, especially in front: that of the tenth or anticlinal vertebra is nearly vertical, those in front of it sloping backwards, and those behind forwards. The posterior two or three thoracie vertebræ are larger than the others, and gradually approach the lumbar vertebræ in size and shape.

a. A typical thoracic vertebra:-any one of the first six.

i. The centrum is short, and thick from above downwards. On each side in front is a small facet, which, with a similar one on the hinder edge of the vertebra next in front, forms the articular surface for the head of the rib. At the posterior end are a similar pair of facets for the ribs of the succeeding vertebra.

ii. The neural arch is notched, both before and behind, for the passage of the spinal nerves.

iii. The transverse processes are short, stout, and horizontal, and bear on their under surfaces 
the articular facets for the tubercles of the ribs.

iv. The neural spine is long and slender, and slopes obliquely backwards.

v. The zygapophyses. The pre-zygapophyses face upwards and outwards, the post-zygapophyses downwards and inwards.

b. The posterior three or four thoracic vertebræ.

i. The centra are larger than in the anterior thoracic vertebræ: the facet for the head of each rib is confined to the anterior end of the centrum, the posterior end having no articular surface.

ii. The neural spines are lower than in the anterior vertebræ, but longer from before backwards.

iii. The transverse processes are placed further back, and have no articular facets for the ribs.

iv. Metapophyses appear in the ninth thoracic vertebra, and increase considerably in size in the succeeding ones.

4. The lumbar vertebræ.

These are usually seven in number: the most anterior one may, however, bear ribs, in which case it is reckoned as a thirteenth thoracic vertebra, and the lumbar vertebræ become reduced to six.

The characters of a typical lumbar vertebra have already been described: as a group they are characterised by their large size, and the great development of their processes, especially the transverse processes. They increase in size from before backwards, the last but one being the largest. The first two have hypapophyses: the last one has a short stout centrum, and a stout crest-like neural spine.

5. The sacral vertebræ.

Strictly speaking, the sacral vertebræ are those of which the expanded ribs directly support the 
pelvis; and of these the rabbit has only one, or at most two. A certain number of the succeeding vertebræ, which are fused with one another and with the last true sacral vertebra, are, however, commonly included under the same name, the whole fused mass being spoken of as the sacrum. Reckoned in this way the rabbit has three or more sacral vertebræ, the lines of boundary between which can easily be made out, in spite of their fusion.

a. The first sacral vertebra is much the largest, and is produced laterally into a pair of large wing-like expansions which support the pelvis, and are regarded as modified ribs fused with the vertebra. The neural spine is large and nearly vertical.

b. The hinder sacral vertebræ are much smaller, and decrease in size from before backwards. Their number is variable, increasing with age owing to fusion with the anterior caudal vertebræ.

The anterior outer border of the second sacral vertebra just touches the pelvis, but it is doubtful whether any true costal element is present in it.

\section{The caudal vertebræ.}

These are about sixteen in number : they decrease in size from before backwards, gradually losing all their processes, and ultimately the neural arches as well, the hindmost vertebræ being reduced to mere rod-like centra.

\section{B. The Ribs and Sternum.}

These form, with the thoracic vertebræ, the skeletal framework of the wall of the thorax, which plays an essential part in the mechanism of respiration, besides protecting the thoracic viscera, and giving origin to some of the extrinsic muscles of the fore-limbs.

1. The ribs are curved bony rods, movably artieulated with the vertebræ above, and connected at their lower ends with the sternum. There are in the rabbit twelve, or sometimes thirteen, pairs. 
a. A typical rib, such as the fifth, consists of two portions of very unequal size, joined together almost at right angles.

i. The vertebral portion, which is the larger part of the rib, is a flattened rod of bone, strongly curved above, nearly straight below. The dorsal end or capitulum articulates with a concave surface, furnished partly by the centrum of the corresponding vertebra, and partly by that of the vertebra next in front. A short way beyond the capitulum, and on the dorsal surface of the rib, is the tubercle or surface for articulation with the transverse process of the corresponding vertebra; and immediately beyond the tubercle is a short vertical projection for the attachment of ligaments.

ii. The sternal portion is a short bar of calcified or imperfectly ossified cartilage, connecting the lower end of the vertebral portion with the sternum.

b. The ribs in general. The ribs increase in length from the first, which is very short, to the sixth, behind which they diminish. The first nine pairs of ribs have both capitular and tubercular articulations with the vertebræ: in the remaining three (or four) pairs, which are more slender than the anterior ones, the tubercles are absent, and the capitular articulations, which alone are present, are confined to the corresponding vertebræ.

The sternal portions of the first seven pairs articulate directly with the sternum : the remaining ones do not reach the sternum, though those of the eighth and ninth pairs are closely connected with the ribs in front of them.

2. The sternum is an elongated, laterally compressed. median rod, situated in the ventral wall of the thorax, and divided transversely into segments or sternebræ. 
It is developed in connection with the ventral ends of the ribs, and consists at first of right and left halves.

The anterior segment or manubrium is much the largest, and is produced ventrally and in front into a prominent keel.

Behind the manubrium come four segments of about equal length, then a very short segment, and finally the xiphisternum, a long slender bony rod ending behind in a horizontal plate of cartilage.

The first pair of ribs articulate with the sides of the manubrium, and the succeeding six pairs between the several sternebræ.

\section{The Skull.}

In the adult rabbit, the original cartilaginous skull is almost completely replaced by bone. In addition to these cartilage-bones, membrane-bones are largely developed; and the two groups of bones, though primitively distinct, become intimately and immovably united to form a complex mass, in which the several morphological components, and even the boundaries of individual bones, are difficult to determine, and of which the essential composition can only be ascertained by a study of the embryonic stages.

In the following description the membrane-bones are described in connection with the cartilage-bones with which they are most closely associated, and their names are printed in italies. Though it is convenient to deal with the membrane bones in this way, it must be borne in mind that, inasmuch as they are primitively independent of the cartilaginous skeleton, any attempt to group them according to the divisions of this primary skeleton must be more or less arbitrary.

1. The cranium proper or brain-case forms the posterior part of the skull; and is situated almost completely behind the orbits, or deep cup-like sockets for the eyeballs. The anterior or facial part of the skull is not in a line with the base of the cranium, but is deflected through about $60^{\circ}$. 
The bones of the cranium proper are arranged in a series of segments or rings, one in front of the other: of these the hindmost or occipital segment is the most obvious, and is very readily detached from the rest of the skull as a bony ring.

Care must be taken not to confuse these segments with vertebræ, with which they do not correspond.

a. The occipital segment consists entirely of cartilagebones, which may fuse together inseparably.

i. The basi-occipital is a median flat bone, forming the floor of the hinder end of the cranial cavity. It forms the ventral boundary of

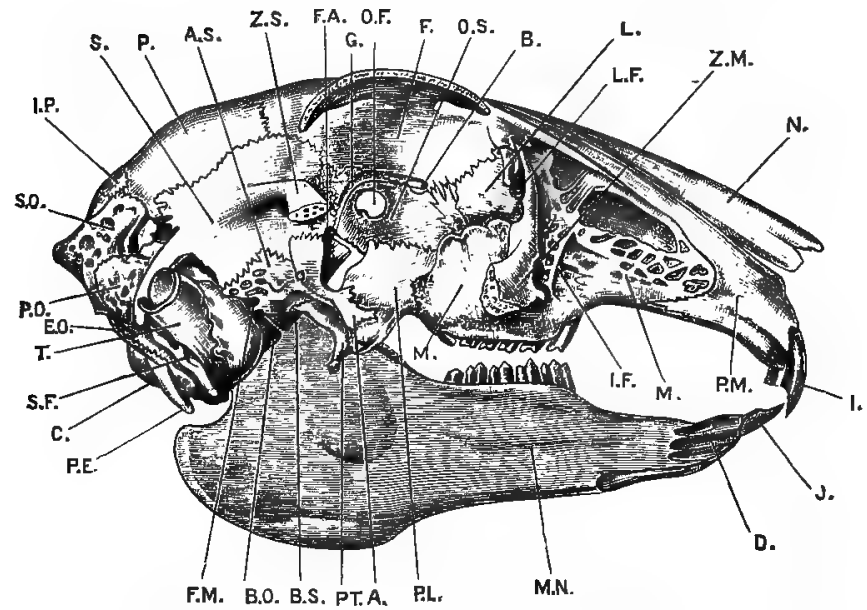

FIG. 58.--Iepus cuniculus. The skull from the right side. The middle portion of the zygomatic arch and the right half of the mandible have been removed. (A. M. M.)

A, external pterygoid process of ali-sphenoid. AS, ali-sphenoid, B, internal orbital foramen. BO, basi-occipital. BS, basi-sphenoid. C, occipital condyle. D, mandibular symphysis. $\mathbf{F O}$, ex-occipital. $\mathbf{F}$, frontal. $\mathbf{F}^{\prime} \mathbf{A}$, foramen lacerum anterius. FM, foramen lacerum medium. $\mathbf{G}$, orbital groove, for ophthalmic division of trigeminal nerve. $I$, anterior upper ineisor. If, infra-orbitgl foramen. IP, inter-parietal. J, lower incisor. $\mathbf{I}$, lacrgmal bone. IF, lacrymal foramen. M, maxilla. MN, mandible. IN, nasal bone. OF', optic foramen. OS, orbito-sphenoid. $\mathbf{P}$, parietal. $\mathbf{P} \mathbf{F}$, par-occipital process of ex-occipital. $\mathbf{P L}$, palatine bone. PM, pre-maxilla. PO, periotic. $\mathbf{P T}$, pterygoid. S, squamosal. SF, stylo-mastoid foramen. SO, supra-occipital. $\mathbf{T}$, tympanic bone. ZM, zygomatic process of maxilla, cut short. ZS, zygomatio process of squamosal, cut short. 
the foramen magnum, and a small part of each occipital condyle.

ii. The ex-occipitals bound the foramen magnum laterally, and form the greater part of the condyles. Each is produced at its outer side into a long, downwardly directed par-occipital process, which is closely applied to the posterior surface of the tympanic bulla.

iii. The supra-occipital is a large median bone, which completes the occipital segment above. The whole bone is markedly cancellous, and its outer surface is raised into a prominent shieldshaped elevation, giving attachment to the muscles raising the head.

b. The parietal segment consists of both cartilagebones and membrane-bones. It is situated in front of the occipital segment, with which it is in contact above and below; while at the sides the two segments are separated by the interposition of the auditory capsules and the squamosal bones.

i. The basi-sphenoid is a median bone placed directly in front of the basi-occipital, with which it is connected by a thin plate of cartilage. Seen from below it is triangular, with the apex directed forwards. On its upper or dorsal surface is a depression, the sella turcica: this lodges the pituitary body, and is bounded behind by the large overhanging posterior clinoid process; while its floor remains unossified as the pituitary foramen.

ii. The ali-sphenoids are a pair of wing-like bones ankylosed with the sides of the basi-sphenoid, and forming parts of the floor of the cranial cavity. From the ventral surface of each ali-sphenoid there projects downwards a vertical plate of bone, the external pterygoid 
process, which is placed nearly transversely to the longitudinal axis of the skull, and is connected along its inner edge with the palatine bone: it is perforated at its base by three foramina, of which the innermost and largest transmits the internal maxillary artery and vein, and the two outer ones, branches of the mandibular division of the fifth nerve.

Between the ali-sphenoid and the anterior end of the basi-sphenoid on each side is a large slit-like notch, the sphenoidal fissure or foramen lacerum anterius, through which the third, fourth, and sixth nerves, and the first and second divisions of the fifth pass out from the cranial cavity into the orbit.

At the posterior border of each ali-sphenoid is a deep notch for the passage of the third division of the fifth nerve.

iii. The parietals are a pair of flattened membrane-bones, articulating with each other in the mid-dorsal line at the sagittal suture, and forming a great part of the roof of the cranial cavity.

From the posterior and outer angle of each parietal a thin plate runs down on the inner surface of the squamosal, which latter bone separates the parietal from the ali-sphenoid.

iv. The interparietal is a small median bone in the roof of the skull, between the parietals and the supra-occipital.

c. The frontal segment surrounds the anterior end of the brain : it is placed in front of the parietal segment, with which it articulates closely.

i. The pre-sphenoid is a median, laterally compressed bone in front of the basi-sphenoid, and connected with it by cartilage. It forms the lower margin of the optic foramen, a large 
aperture through which the optic nerves enter the orbits from the cranial cavity, and the two orbits communicate with each other. The upper and posterior border of the presphenoid is produced backwards into the small anterior clinoid processes, which bound the sella turcica in front. In front of the optic foramen the pre-sphenoid is continued forwards as a pair of vertical laminæ, diverging from each other at an acute angle, and forming parts of the inner walls of the orbits.

ii. The orbito-sphenoids are a pair of lamellar bones, which are fused with the pre-sphenoid. They surround the optic foramen, behind which they form part of the wall of the cranium and of the orbits, uniting suturally with the ali-sphenoids and with the frontals and squamosals.

iii. The frontals are a pair of large membrane-bones completing the frontal segment. They unite suturally with each other in the mid-dorsal line, with the parietals behind, and with the orbito-sphenoids and pre-sphenoids below.

The frontals form the roof and sides of the anterior part of the cranial cavity, and meet each other on its floor in front: they extend forwards into the region of the nose. The outer surface of each frontal forms the upper part of the wall of the orbit, and bears a prominent crescentic supra-orbital process, in front of which it sends forwards a slender process between the maxilla and premaxilla.

d. The ethmoidal region lies entirely in front of the cranial cavity, and is in close relation with the olfactory organs.

i. The mes-ethmoid is a median vertical plate of cartilage, which extends forwards and down- 
wards in front of the pre-sphenoid, separating the right and left olfactory cavities from each other.

Its upper and posterior border is ossified and expanded laterally into the cribriform plate, which is placed obliquely across the anterior end of the cranial cavity, in the plane of the base of the skull, and is perforated by a number of holes through which the olfactory nerves pass from the brain to the nose.

2. The sense-capsules. Each of the organs of special sense has, as in the dog-fish, a protecting investment of cartilage or strong connective tissue. In the case of the olfactory and auditory organs these capsules are closely connected with the cranium, and are more or less extensively ossified. Membrane-bones are also developed in immediate relation with the sensecapsules, and may conveniently be considered with them.

a Bones in relation with the olfactory capsules., The olfactory capsules are of very large size in the rabbit, and occupy a great part of the facial portion of the skull. They are so closely connected with the ethmoidal region and with the upper aw, that certain of the bones may be referred, with equal propriety, to either of these divisions.

i. The nasals are a pair of elongated membranebones, forming the roof of the nasal cavities. They unite suturally with the frontals behind, and with each other in the median plane.

i. The ethmo-turbinals are intricately folded laminæ of bone, fused to the lower surface of the cribriform plate of the ethmoid, and occupying the upper and posterior part of each nasal cavity.

iii. The maxillo-turbinals are a pair of somewhat similar, but more delicate and more complex 
bones, lying in the anterior portion of the nasal cavities, in front of the ethmo-turbinals. iv. The naso-turbinals are a pair of thin laminæ of bone, folded to form pouches, and fused with the inner surfaces of the nasal bones.

v. The vomers are a pair of slender blade-like bones, fused with each other along their ventral margins, and embracing the lower edge of the mes-ethmoid cartilage. Behind they are expanded laterally, and connected with the ethmo-turbinals.

\section{b. Bones in relation with the optic capsules.}

The lacrymals are a pair of small lamellar bones forming part of the anterior walls of the orbits, and loosely wedged in between the frontals and maxillæ. Each is notched at its outer border for the passage of the lacrymal duct, which is supported by a process of the bone extending forwards into the nose.

The lacrymals correspond to the most anterior of a series of small bones, placed round the borders of the orbits in bony fishes.

c. Bones in relation with the auditory capsules (cf. fig. 60, p. 312).

i. The periotics are the bones which replace the cartilaginous capsules enclosing the organs of hearing in the embryo. Each ossifies from three centres; but the several bones-pro-otic, epiotic, opisthotic-early unite to form an irregular mass, which fits loosely into the side-wall of the cranium, between the occipita segment and the squamosal, and which readily falls out from the dried skull.

In each periotic there may be distinguished a dense petrous portion, enclosing the essential part of the organ of hearing; and a porous mastoid portion, which forms its outer and 
posterior part, and is produced downwards into the mastoid process, lying immediately in front of the par-occipital process of the exoccipital bone.

On the inner surface of each periotic, as seen in a bisected skull, there are two depressions. Of these the upper and larger one, the floccular fossa, lodges the floccular lobe of the cerebellum. The lower one is divided by a ridge of bone into two, of which the anterior one is the opening of the aqueductus Fallopii, transmitting the facial nerve; and the posterior one is that of the meatus auditorius internus, through which the auditory nerve enters the internal ear.

The anterior border of the periotic is produced inwards into a prominent crest of bone which projects into the cranial cavity, and, together with a similar but smaller ridge on the supra-occipital, separates the cerebral and cerebellar fossæ from each other.

On the outer surface of the petrous portion of the periotic, and seen only after removal of the tympanic bone, are two small apertures. Of these the anterior and more conspicuous one is the fenestra ovalis, in which the stapes often remains wedged; while the posterior and more irregular shaped one is the fenestra rotunda, which is close to the posterior edge of the bone, and overhung by the mastoid process. Immediately below the fenestra ovalis is a rounded swelling, the promontory, within which the cochlea lies.

ii. The tympanics are a pair of somewhat flaskshaped bones, imperfect on their inner sides, and closely applied to the outer surfaces of the periotics. The expanded body of the flask, or bulla, encloses the tympanic cavity; and 
the upwardly directed neck, or meatus auditorius externus, supports the cartilage of the large external ear. Within the bulla, at the base of the meatus, is an incomplete bony ring, across which the tympanic membrane is stretched during life.

The Eustachian tube leaves the tympanic cavity by an aperture at the antero-inferior angle of the tympanic bone, between it and the periotic ; and passes forwards to the posterior nasal chamber (p. 311).

The bony canal by which the facial nerve leaves the skull runs along the posterior border of the tympanic, between it and the mastoid process of the periotic, and opens below at the stylomastoid foramen.

On the ventral surface of the tympanic bone, near its inner border, is a conspicuous foramen, through which the internal carotid artery enters the skull.

iii. The auditory ossicles are most conveniently examined with the bones of the auditory capsules, on account of their close connection with the organs of hearing; though, morphologically, they belong to the mandibular and hyoidean arches.

They form on each side a chain of small bones, stretching across the tympanic cavity from the tympanic membrane to the fenestra. ovalis.

$a$. The malleus consists of a small body and two processes. The larger process, or manubrium, is blade-like, and is attached along the greater part of its length to the tympanic membrane, the tip being near the centre of the membrane. The shorter process, which is anterior, articulates with a 
small fossa on the inner surface of the tympanic bone.

$\beta$. The incus is a minute, somewhat pyriform bone: its head bears in front a saddleshaped articular surface for the body of the malleus, and is produced behind into a short process, which articulates with a fossa on the outer surface of the periotic bone, just above the fenestra ovalis.

The narrow end or stalk of the incus projects downwards: its tip is bent inwards, and has attached to it a very small bony dise, the os orbiculare.

$\gamma$. The stapes is a very small stirrup-shaped bone, articulating by its arch with the os orbiculare, and attached by its basal plate to the membrane closing the fenestra ovalis.

The morphological value of the auditory ossicles has been much debated, and is not yet determined with certainty. The incus and malleus are probably developed from the cartilage of the mandibular arch; the incus being derived from the uppermost part of the arch, and corresponding to the quadrate bone of other vertebrates; while the malleus is formed from the part of the arch below the incus.

The stapes is still more difficult to determine; it is probably developed from the uppermost part of the cartilage of the hyoidean arch.

3. The jaws. The cartilaginous maxiliary and mandibular arches of the embryo, corresponding to the upper and lower jaws of the dog-fish, disappear almost completely during development; the jaws of the adult rabbit consisting of membrane-bones developed around and in front of these arches.

a. Bones in relation with the maxillary arch. These are very closely connected with the cranium and with the olfactory capsules. 
i. The pterygoids are a pair of narrow vertical plates of bone, attached to the base of the skull at the junction of the basi-sphenoid with the ali-sphenoids. The posterior border of each pterygoid is free, and ends below in the curved hamular process, while the anterior border unites with the palatine and ali-sphenoid. On the outer side of the pterygoid, between it and the external pterygoid process of the ali-sphenoid, is the triangular pterygoid fossa.

ii. The palatines are a pair of nearly vertical plates of bone, attached above to the ventral border of the pre-sphenoid, and behind to the pterygoids and ali-sphenoids, thus completing the anterior boundaries of the pterygoid fossæ. They form the side-walls of the posterior narial passage, which they separate from the orbits. In front they are produced inwards into the horizontal palatal processes, which meet each other in the median plane in the roof of the mouth, opposite the grinding teeth, forming the posterior third of the bridge of bone supporting the hard palate.

iii. The maxilla are a pair of large irregular bones forming a large part of the skeleton of the upper jaw and of the face.

The body, or alveolar portion, of each maxilla projects into the front part of the orbit, and is excavated by the alveoli or sockets for the grinding teeth. Its inner surface is covered by the palatine behind; and in front is produced inwards into the horizontal palatal process, which meets its fellow in the median plane, and forms the anterior part of the bony bridge supporting the hard palate.

From the outer side of the body arises the stout zygomatic process, which is prolonged 
upwards into the orbital process, forming the anterior boundary of the orbit. In front, the maxilla is prolonged into the large fenestrated facial plate, which forms the lateral bound. ary of the olfactory chamber, and is perforated just above the first grinding tooth by the infra-orbital foramen, for the passage of the maxillary division of the trigeminal nerve. iv. The premaxilla are a pair of bones, forming the anterior part of the upper jaw, and lodging the incisor teeth : they articulate with the maxillæ behind, and with each other in the median plane in front.

The upper and posterior angle of each premaxilla is produced backwards into a long nasal process, which lies along the outer border of the nasal bone, and above the maxilla and the anterior process of the frontal.

On the under surface of the skull, bounded laterally by the premaxillæ and maxillæ, and behind by the hard palate, is a large triangular aperture, divided longitudinally into the slit-like anterior palatine foramina by the palatal processes of the premaxillæ, a pair of thin, longitudinally rolled laminæ attached in front to the premaxillæ, and enclosing the accessory organs of smell known as Jacobson's organs.

v. The jugals are a pair of laterally compressed bars forming the greater part of the length of the zygomatic arches, which bound the orbits below. Their anterior ends are fused with the zygomatic processes of the maxillæ, and posteriorly they articulate along their dorsal edges with the squamosals.

b. Bones in relation with the mandibular arch.

The uppermost part of the mandibular arch gives rise to the incus and malleus, which have 
already been described in connection with the auditory capsule (p. 283).

i. The squamosals are a pair of slightly curved bony plates, which complete the side-walls of the cranial cavity, lying in front of the periotics, and articulating with the parietals, frontals, orbito-sphenoids, and ali-sphenoids.

From the hinder border of each squamosal a slender post-tympanic process arches backwards over the external auditory meatus, lying along the outer surface of the periotic.

From the outer surface of the squamosal arises a stout zygomatic process, which bears on its under surface the articular facet for the mandible: beyond the facet it bends downwards, to articulate by an expanded end with the jugal bone, and so complete the zygomatic arch.

ii. The mandible, or lower jaw, consists of a pair of somewhat triangular plates of bone, firmly united together in front, and articulating by their posterior and upper angles with the zygomatic processes of the squamosals.

The anterior portion of each half or ramus is stout; it lodges an incisor tooth in front, and further back, on its upper margin, the grinding teeth. The hinder part of the ramus is much thinner: its postero-inferior border, or angle, is rounded, and inflected along its margin ; its posterior border, above the angle, is deeplynotched : its upper border is thickened to form the longitudinally elongated condyle, which articulates with the squamosal in such a way as to allow free movement of the jaw backwards and forwards, but very restricted movement from side to side: its anterior border is deeply grooved, the outer lip or coronoid process being inflected. 
On the inner surface of each ramus, just behind the grinding teeth, is the inferior dental foramen, through which the mandibular nerve enters to supply the teeth.

4. The hyoid bone lies in the floor of the hinder part of the mouth, between the rami of the mandible.

It consists of a median body, and two pairs of backwardly projecting cornua, of which the posterior are the larger.

The anterior cornua are the ventral ends of the hyoidean arches, the dorsal ends of which are probably represented by the stapes: the posterior cornua are the lower ends, and only persistent parts, of the first pair of branchial arches.

\section{Apertures on the surface of the skull.}

After the several bones have been examined, the positions of the more important foramina should be determined, most of which have already been seen. Except when otherwise stated, the several apertures are paired.

a. Foramina for the passage of cranial nerves.

i. The optic foramen is a large round median hole in the orbito-sphenoids: it puts the two orbits in communication with the crania: cavity and with each other, and transmits the optic nerves.

ii. The foramen lacerum anterius, or sphenoidal fissure, is a vertical slit below and behind the optic foramen, and between the basi-sphenoid and ali-sphenoid. Through it the third, fourth, and sixth nerves, and the ophthalmic and maxillary divisions of the fifth, enter the orbit from the cranial cavity. In most mammals the maxillary division of the fifth nerve leaves the skull by a separate aperture, the foramen rotundum, which in the rabbit is confluent with the sphenoidal fissure. 
iii. The internal orbital foramen perforates the inner wall of the orbit, about a quarter of an inch in front of the optic foramen, and at the anterior end of a groove which curves back over the optic foramen towards the sphenoidal fissure. Through it the nasal branch of the ophthalmic division of the fifth nerve passes from the orbit into the cranial cavity, which it enters just behind the lower edge of the cribriform plate.

iv. The infra-orbital foramen is on the side of the face, in front of the zygomatic process of the maxilla : it transmits the infra-orbital branch of the maxillary division of the fifth nerve from the orbit to the face.

$v$. The anterior palatine foramen or foramen incisivum is a large slit-like aperture, extending from the bony bridge of the palate almost to the incisor teeth, and imperfectly separated from its fellow by the palatal processes of the premaxillæ. Through it pass the naso-palatine branch of the maxillary division of the fifth nerve, and the naso-palatine canal.

vi. The posterior palatine foramen is a small hole in the bony palate, at the junction of its maxillary and palatine portions: it transmits the anterior palatine branch of the maxillary division of the fifth nerve, and the bloodvessels of the palate.

vii. The foramen lacerum medium is an irregular aperture on the under surface of the skull, between the ali-sphenoid and the periotic, just in front of the tympanic bulla. Its anterior part, which represents the separate foramen ovale of other mammals, transmits the mandibular division of the fifth nerve.

viii. The stylomastoid foramen is a small aperture about the middle of the posterior border of 
the tympanic bone, between it and the mastoid process of the periotic. Through it the main trunk of the seventh, or facial, nerve leaves the skull.

ix. The foramen lacerum posterius is an ixregular fissure on the under surface of the hinder end of the skull, between the occipital condyle and the tympanic bulla. It transmits the glossopharyngeal, pneumogastric, and spinal accessory nerves, and the internal jugular vein.

$x$. The condylar foramina are a couple of holes running obliquely through the ex-occipital, just in front of the condyle. They transmit the two divisions of the hypoglossal nerve, and are best seen through the foramen magnum.

b. Other important apertures in the skull.

i. The anterior narial opening is at the anterior end of the skull, and is bounded by the nasals and the premaxillæ. It is single in the dried skull, but divided in the natural condition by the vertical cartilaginous septum narium.

ii. The lacrymal foramen is formed by a deep notch in the outer border of the lacrymal bone, completed in front by the orbital process of the maxilla. It leads into the canal along which the lacrymal duct runs into the nose.

iii. The pituitary foramen is a median hole, perforating the floor of the pituitary fossa in the centre of the basi-sphenoid: in the natural condition it is closed by membrane.

iv. The carotid foramen perforates the ventral surface of the tympanic bone near its inner border, close to the occipital condyle: through it the internal carotid artery enters the skull.

v. The opening of the Eustachian canal is on the under surface of the skull, at the anterior and 
inner angle of the tympanic bone, immediately behind and to the inner side of the foramen lacerum medium: through it the Eustachian tube passes from the tympanic cavity to the posterior narial chamber.

vi. The external auditory aperture is the large upwardly directed opening of the neck of the tympanic bone, round the edge of which the cartilage of the external ear is attached.

vii. The foramen magnum is the large median hole at the hinder end of the skull, surrounded by the bones of the occipital segment. It is traversed by the central nervous system, and marks the boundary between the brain and the spinal cord.

6. The teeth.

The teeth are cutaneous structures, developed from the mucous membrane of the mouth. They consist of an extremely hard outer layer of enamel, which is epidermal in origin ; and a "central portion of dentine, which is developed from the dermis. The dentine is hollow, its cavity containing the pulp of the tooth, which receives vessels and nerves through the large aperture at its base. Surrounding the lower part of the tooth, and filling up the folds of its surface, is a thin layer of bone, the crusta petrosa or cement.

It is characteristic of the teeth of the rabbit that the aperture at the base of each does not become constricted, but remains wide open, the teeth continuing to grow throughout life; whereas in most mammals the base of the tooth sooner or later becomes narrowed to form a root or fang, the aperture becoming almost obliterated, and the growth of the tooth ceasing entirely.

The teeth of the rabbit do not form a continuous series as in man, but the front teeth or incisors are 
separated by a great gap or diastema from the group of grinding teeth in the hinder part of the mouth. The rabbit has no teeth corresponding to the canine teeth of other mammals.

a. The teeth of the upper jaw.

i. The incisors are two pairs of teeth at the front of the upper jaw, lodged in alveoli in the premaxillæ.

a. The anterior incisors are long, and curved almost into semicircles, the greater part of each being lodged in an alveolus which extends almost to the hinder end of the bone. Along the anterior surface the enamel is thick, and folded so as to form a longitudinal groove. Behind and at the side it is much thinner, and hence wears away more rapidly, rendering the eutting edge chisel-shaped.

$\beta$. The posterior incisors, which are lodged in alveoli immediately behind the anterior ones, are much smaller, and slightly curved.

ii. The grinding teeth form a set of six teeth on each side, borne by the maxilla, and divisible into two groups.

a. The premolars are the three anterior grinders of each side, and are distinguished from the posterior ones by the fact that, like the incisors, they are preceded in early life by 'milk' or deciduous teeth, which in the rabbit are shed before birth.

Each premolar is flattened from before backwards, and each of the two hinder ones has a deep groove along its outer surface which carries the enamel almost across the tooth, giving rise to a prominent ridge on the grinding surface. 
$\beta$. The molars are the three posterior grinders on each side; they differ from the premolars in having no deciduous predecessors. The first two are similar to the premolars; the last is small and not grooved.

\section{b. The teeth of the lower jaw.}

i. The incisors are two in number, and project forwards from the anterior end of the mandible. They are similar to the large upper incisors, but are less strongly curved and are not grooved: the alveoli in which they are lodged extend back in the jaw as far as the anterior grinders.

ii. The grinding teeth form a set of five on each side, placed opposite to those of the upper jaw, and like these divisible into two groups. They differ from the upper grinders in being curved so as to be convex instead of concave outwards.

$a$. The premolars are the two anterior grinders on each side. They are somewhat similar to the upper ones, but narrower from side to side. The outer surface of the first, which is the larger of the two, is marked by two deep longitudinal grooves, and that of the second by a single groove. The inner surface of each is less deeply grooved than the outer.

$\boldsymbol{\beta}$. The molars are the three hinder grinders on each side; they are similar to the second premolars, but have no deciduous predecessors : the posterior one is smaller and simpler than the other two.

II. THE APPENDICULAR SKELETON.

The appendicular skeleton comprises the bones of the two pairs of limbs, and the girdles which support them. The 
bones of the limbs are mostly elongated, and provided with epiphyses at their ends.

\section{A. The Pectoral Girdle.}

This lies outside the ribs, and is attached to the axial skeleton by muscles and ligaments only. In the rabbit, as in nearly all other mammals, it is very incomplete : the dorsal or scapular portion on each side is well developed, but the ventral or coracoid portion is almost entirely absent, being represented merely by a small knob of bone fused with the scapula : the clavicles also are imperfect.

i. The scapula is a triangular plate of bone, the apex of which is directed downwards and forwards, and is expanded and hollowed on its ventral surface to form the shallow glenoid cavity, with which the head of the humerus articulates.

Of the three borders, the anterior or coracoid border is nearly straight; the posterior or glenoid is slightly concave; and the dorsal or suprascapular, which is the shortest of the three, is convex and supports the cartilaginous suprascapula.

The spine is a prominent ridge running along the outer surface of the scapula, nearly parallel to and a short distance behind the coracoid border. It is continued ventrally into a free, downwardly directed process, the acromion, from the posterior border of which the long metacromion projects backwards.

The coracoid border is continuous below with the coracoid process, which overhangs the glenoid cavity, and is produced inwards into a blunt hook. This is the sole representative of the coracoid portion of the pectoral girdle, and is a distinct bone in the young rabbit. 
ii. The elavicle is a slender curved membrane-bone, rather less than an inch in length, and formed by ossification in the ligament running from the scapula to the sternum.

Small cartilaginous nodules occur, one at the outer and two at the inner end of the clavicle.

\section{B. The Fore-Limb.}

The fore-limb of the rabbit corresponds to the arm of man, and may be divided, like this, into arm, forearm, wrist and hand. The relative positions of these several divisions are considerably altered from the primitive condition, and are best understood by comparing them with the human arm in the following manner.

Extend the right arm horizontally outwards from the shoulder, with the palm of the hand vertical and the thumb upwards : there may now be distinguished a ventral or flexor surface ; a dorsal or extensor surface ; a preaxial border, along which are the thumb and the radius; and a postaxial border, along which are the little finger and the ulna.

Next bend the elbow nearly at right angles, so that the fingers point horizontally forwards, and bring the whole arm down to the side, rotating it through $90^{\circ}$, so that the preaxial border becomes external and the postaxial border internal. Now pronate the hand, turning it through $180^{\circ}$, so that the thumb is brought to the inner side, and the palm directed downwards. Place the rabbit's skeleton in front of you, with the vertebral column vertical, the head directed upwards, and the limbs pointing away from you, and the right fore-limb will correspond in position to your own right arm. Note that the preaxial border is on the outer side in the arm, and on the inner side in the hand, while in the forearm the two bones cross each other obliquely. The hand in the rabbit is fixed permanently in the prone position.

1. The arm contains only a single bone.

i. The humerus is an elongated bone, articulating by its enlarged proximal head with the glenoid 
cavity of the scapula, and at its distal end, by the trochlea, with the bones of the forearm.

At the proximal end of the bone, in front of the head, is the longitudinal bicipital groove, for the tendon of the biceps muscle: this is bounded on its inner side by the lesser tuberosity ; and on its outer side by the greater tuberosity, a large roughened projection continued as a ridge down the anterior surface of the bone as far as its middile.

The trochlea at the distal end of the bone is an oblique pulley-like surface, divided in front by a ridge, and articulating with the radius and ulna to form the elbow-joint. Immediately above the trochlea, in front and behind, are the supra-trochlear fossæ, which communicate with each other through the supra-trochlear foramen: the posterior or olecranon fossa is much the larger of the two, and lodges the olecranon process of the ulna when the arm is extended.

2. The forearm contains two bones, of about equal size, immovably articulated, though not ankylosed, with each other.

i. The radius, or preaxial bone, lies in front of the ulna at the proximal end of the forearm, but in the distal half lies along its inner or preaxial side.

It is an elongated bone, slightly convex forwards. Its proximal end or head articulates with both grooves of the trochlea of the humerus, and also with the anterior surface of the ulna: its distal and larger end articulates with the bones of the wrist.

ii. The ulna, or postaxial bone of the forearm, has a greatly enlarged proximal end, on the anterior surface of which is the sigmoid notch 
for articulation with the trochlea, and also two small articular surfaces for the radius. Behind the elbow-joint, the bone forms the large, laterally compressed olecranon process. The distal end of the ulna is small, and articulates with the carpus.

3. The wrist. In the rabbit the wrist or carpus consists of nine small bones, of which one, the pisiform, is commonly regarded as a sesamoid bone or ossification in the tendon of a muscle, but by some anatomists is considered to be a rudiment of a sixth digit. The arrangement of the remaining eight can only be understood by comparison with more primitive types.

The typical carpus consists of nine bones, arranged in a proximal row of three, articulating with the radius and ulna; a distal row of five, each of which supports one of the digits; and a central bone wedged in between the proximal and distal rows. In the rabbit the arrangement is as follows.

a. The proximal row of carpals consists of the three typical bones.

i. The radiale, or scaphoid, is placed on the inner or preaxial side, and articulates with the radius.

ii. The intermedium, or semilunar, is the middle bone of the three, and articulates with the radius.

iii. The ulnare, or cuneiform, is the outer or postaxial bone of the proximal row, and articulates with the ulna.

b. The central bone.

i. The centrale is a small bone, articulating with the distal surfaces of the intermedium and radiale, and appearing to belong to the distal row of carpals. 
c. The distal row of carpals consists of four bones, the two outermost of the typical carpus having fused to form a single bone.

i. The first carpale, or trapezium, is on the inner or preaxial side of the wrist; it articulates with the radiale, and supports the first metacarpal.

ii. The second carpale, or trapezoid, articulates with the radiale and centrale, and supports the second metacarpal.

iii. The third carpale, or os magnum, is very small : it articulates with the intermedium and centrale, and supports the third metacarpal.

iv. The fourth and fifth carpalia are fused to form the unciform bone, which is much larger: it articulates with the ulnare, and supports the fourth and fifth metacarpals.

d. The pisiform is a small bone on the posterior or flexor surface of the carpus, and articulates with the distal end of the ulna and with the ulnare.

4. The hand of the rabbit has five digits, as in man: the bones of each consist of a proximal metacarpal, followed by the phalanges, the last of which is conical and slightly curved, and supports the claw.

The pollex, or preaxial digit, corresponding to the thumb in man, is much shorter than the others, and has only two phalanges, while each of the other digits has three.

Sesamoid bones are developed in the tendons of the flexor muscles, opposite the metacarpo-phalangeal and certain other articulations.

\section{The Pelvic Girdle.}

The pelvic girdle, which supports the hind-limb, consists of two halves, which, though primitively nearly at right angles to the vertebral column, are in the adult rabbit almost parallel 
to it. They are firmly united to the sacrum in front, and with each other in the ventral symphysis behind.

Each half, or os innominatum, consists of three bonesilium, ischium, and pubes-and presents about the middle of its outer surface a deep cup-shaped acetabular cavity for the femur, in the formation of which all the three bones take part. i. The ilium is the anterior and dorsal member of the girdle, corresponding to the scapula in the pectoral girdle. It is expanded and bladelike in front, and narrowed behind. On its inner or sacral surface, a little way in front of the acetabulum, is a roughened crescentic surface for articulation with the sacrum; while its outer surface is divided by a longitudinal ridge into ventral or iliac, and dorsal or gluteal fossæ. The ilium forms about half of the acetabulum, the lines of boundary between it and the ischium and pubes being easily seen in the young skeleton.

ii. The ischium forms the posterior and dorsal portion of the os innominatum, and is separated from the pubes by the large oval obturator foramen. It forms about a third of the acetabulum, behind which it bears on its inner surface the sharp, backwardly directed ischial spine. Its posterior part is flattened, the margin being thickened to form the ischial tuberosity, which is raised on its outer surface into a prominent hooked process. The ischium corresponds to the coracoid in the shoulder-girdle.

iii. The pubes is the smallest bone of the three. Its acetabular portion ossifies separately in the rabbit, and remains throughout life as a small cotyloid bone, completing the margin of the acetabulum below. The pubes unites along its ventral border with its fellow of the opposite side to form the anterior part of the 
symphysis, the hinder portion of which is formed by the ischium: the line of union between these two bones is visible only in very young skeletons.

\section{The Hind-Limb.}

The hind-limb is much larger than the fore-limb, and like this is divisible into four main portions.

In the hind-limb the departure from the primitive condition is much less marked than in the fore-limb. The whole limb has rotated forwards through about a right angle, so that the preaxial border is directed inwards, and the postaxial outwards ; the original dorsal surface being turned forwards, and the ventral surface backwards.

1. The thigh, like the arm, contains a single bone, the femur. Certain sesamoid bones in connection with the knee-joint may be considered with it.

i. The femur is an elongated bone, consisting of a cylindrical shaft and two enlarged extremities.

The proximal end bears on its inner or preaxial side the prominent rounded head, which articulates with the acetabulum to form the hip-joint. To the outer side of the head, and forming the end of the bone, is a large rough process, the great trochanter: between this and the head, on the posterior surface of the bone, is a deep pit, the trochanteric or digital fossa.

On the inner or preaxial side of the bone, immediately below the head, is a rough ridge, the lesser trochanter; opposite to which on the outer or postaxial side is the prominent third trochanter.

The lower or distal end of the bone bears two large condyles which articulate with the tibia. These are separated from each other 
by the intercondylar notch, which is continuous with a deep groove on the front of the bone, along which the patella slides.

ii. The patella, or knee-pan, is a large sesamoid bone in the tendon of the extensor muscles of the leg. It is connected by ligaments with the tibia, and slides in the groove on the lower end of the femur.

At the back of the knee-joint are smaller sesamoids, the fabellæ.

2. The leg contains two bones, which are very unequal in size, and in the adult are fused together distally.

i. The tibia, or preaxial bone of the leg, is a stout and straight bone, the longest in the body. Its enlarged proximal end, which is triangular in section, bears on its upper surface two oval surfaces for articulation with the condyles of the femur. Below the head is the prominent cnemial crest, extending some distance down the anterior surface of the bone.

The distal end of the tibia, which is indistinguishably fused with the lower end of the fibula, bears an articular surface for the astragalus.

ii. The fibula, or postaxial bone, is very slender : its proximal end is distinct, and articulates with a facet on the outer surface of the head of the tibia.

Its distal portion is completely fused with the tibia, and bears at its end an articular surface for the calcaneum.

3. The tarsus consists, in the rabbit, of six bones, arranged, like the bones of the wrist, in a proximal and a distal row, with a central bone between them. The typical tarsus exactly corresponds to the typical carpus in number and arrangement of bones. 
a. The proximal row of tarsals consists of two bones.

i. The astragalus, which probably corresponds to the tibiale and intermedium of the typical tarsus fused together, is placed on the inner or preaxial side of the tarsus: it articulates by a prominent pulley-like surface with the distal end of the tibia.

ii. The calcaneum, or fibulare, is the largest bone of the tarsus: it lies along the outer side of the astragalus, articulating with the fibula, and is produced backwards into the large calcaneal process or heel.

b. The central bone.

i. The navicular, or centrale, is placed immediately in front of the astragalus. It sends forwards a large process along the ventral surface of the foot.

c. The distal row of tarsals consists of three bones, the innermost or preaxial bone of the typical tarsus being absent, together with the digit which it supports, though probably represented by the process at the proximal end of the second metatarsal; while the two outer tarsalia, like the corresponding carpalia, fuse together.

i. The meso-cuneiform, or second tarsale, is very small : it articulates with the navicular, and supports the second metatarsal.

ii. The ecto-cuneiform, or third tarsale, lies to the outer side of the meso-cuneiform. It articulates with the navicular, and supports the third metatarsal.

iii. The cuboid, formed by the fusion of the fourth and fifth tarsalia, is a much larger bone, on the outer side of the foot: it articulates with the calcaneum and navicular, and supports the fourth and fifth metatarsals. 
4. The foot of the rabbit has only four toes, the preaxial digit of the typical foot, corresponding to the great toe in man, having almost completely disappeared.

Each of the four digits consists of a long metatarsal bone, followed by three phalanges, the last of which bears a claw.

The second (apparent first) metatarsal has at its proximal end a backwardly directed process, which articulates with the navicular: this is a distinct bone in the young rabbit, and probably represents the entocuneiform and the metatarsal of the lost first toe or hallux. 


\section{Chapter XIII.}

\section{DISSECTION OF THE RABBIT.}

The rabbit may be taken as a type of the Mammalia; and the following points should be noted as characteristic of the great majority of the members of the group.

The distinguishing features of the skeleton have been noted in the preceding chapter.

Externally, the body is covered with hair: the urinogenital and anal apertures are distinct from each other: and there are large external ears.

As regards the viscera:-Mammary glands are present, opening to the exterior on teats placed on the ventral surface of the body. The heart has four chambers: there is a single aortic arch in the adult, formed from the fourth left arch of the embryo: the blood is hot, and the red corpuscles nonnucleated. There is a complete diaphragm; and the lungs, which are not attached to the ribs, are completely surrounded by pleuræ. The ureters open directly into the bladder. In the brain there is a corpus callosum, connecting the hemispheres; and corpora quadrigemina in place of corpora bigemina or optic lobes. The cochlea is coiled spirally.

The ova are extremely small, and are retained within the uterus during almost the whole period of their development. The embryo receives a constant supply of nutriment direct from the mother by means of the placenta, a structure in which the blood-vessels of the embryo and of the mother are brought into very close relation, so that diffusion can readily take place from one to the other.

In the following account the several systems are described 
one by one, in order that each may be studied in its entirety as far as practicable, and the mutual relations of its several parts demonstrated. This method is the best, and should be followed whenever possible. If, however, it is intended to perform the whole dissection on a single rabbit, it will be found advisable to somewhat modify the order of proceeding.

For dissection, rabbits should be taken that are not less than six months old, or the reproductive organs will not be fully developed. They may be killed by pouring a few drops of solution of cyanide of potassium into the mouth, or else by chloroform, or by drowning.

The brain should be removed at once and put into strong spirit. (See p. 350.)

\section{EXTERNAL CHARACTERS.}

The whole body is covered with hairs, of variable colour; and is obviously divided into head, neck, trunk, a short tail, and two pairs of limbs.

\section{A. The Head.}

Note the general form of the ihead. Determine the position of the mandible, the zygomatic arches, the supra-orbital processes of the frontals, and other parts of the stoull, by feeling through the skin.

1. The mouth is a small transverse aperture on the under surface of the front of the head, bounded by the soft hairy lips. The upper lip is divided by a median cleft, which connects the mouth with the nostrils, and leaves the upper incisors exposed.

2. The nostrils are a pair of oblique slits at the end of the hairy snout.

3. The eyes are large, and placed at the sides of the head. Each eye is protected by an upper and a lower eyelid, fringed with scanty eyelashes; and by a third eyelid, or nictitating membrane, a hairless fold of opaque white skin, which lies within the other eyelids, and can be pulled across the eyeball from the anterior angle or canthus. 
4. The vibrissæ or whiskers are long, stiff, tactile hairs, at the sides of the snout, and above and below the eyes.

5. The external ears or pinnæ are of great size and freely movable. Each is folded longitudinally, and is supported by cartilage at its base, where it surrounds the external auditory aperture.

\section{B. The Trunk.}

This is divided into an anterior portion or thorax, stiffened laterally by the ribs; and a posterior part or abdomen.

Feel through the skin, and determine the positions of the vertebral column and ribs.

1. The anus is a small median aperture beneath the base of the tail.

2. The perinæal pouches are a pair of hairless depressions of the skin, in front of and at the sides of the anus: into them open the ducts of the perinæal glands, the secretion of which has the strong smell characteristic of the rabbit.

3. The urino-genital aperture is situated in both sexes just in front of the anus.

a. In the male the aperture is placed at the end of the long cylindrical penis, which can be retracted within a loose sheath of skin, the prepuce.

b. In the female the opening, or vulva, is slit-like and larger than in the male; and its anterior margin is produced into a small fleshy process, the clitoris, which corresponds to the penis in the male.

4. [The scrotal sacs, found in the male only, are a pair of prominent oval pouches of skin, at the sides of the penis, into which the testes descend.]

5. [The teats or mammæ of the female are four or five pairs of small papillæ, perforated by the openings of the ducts of the mammary or milk-glands. They are arranged at intervals along the ventral surface of the thorax and abdomen; the most anterior pair 
being about two and a half inches apart and an inch behind the elbow, and the hindmost pair opposite the thighs and rather more than an inch apart.]

\section{The Limbs.}

1. The fore-limbs, as far as the elbows, are closely applied to the sides of the thorax, and hidden by the skin of the trunk. The several bones of the shoulder-girdle and limb, which have already been studied on the skeleton, can be readily identified by feeling them through the skin. The palmar surface of the paw is hairy: the innermost digit or pollex is shorter than the others, not reaching the ground. All the digits bear claws.

2. The hind-limbs are much longer than the fore-limbs. The pelvic girdle and the several bones of the limb can be felt through the skin. In the foot the innermost digit or hallux is absent, and the remaining four are long and clawed. The plantar surface or sole of the foot is hairy.

\section{DISSECTION OF THE BUCCAL CAVITY.}

Remove the skin from the head and neck. Lay the rabbit on its right side: insert one blade of the scissors into its mouth, and cut back through the cheek on the left side between the upper and lower teeth: continue the cut backwards across the jaw with bone forceps and scissors: turn the left ramus of the lower jaw down, breaking the two rami loose at the symphysis, so as to expose the buccal cavity fully; and wash freely.

1. The roof of the mouth.

a. The palate, or median part of the roof, is long and narrow, and separates the buccal from the nasal cavity. Its anterior half, the hard palate, is raised into hard, transverse ridges, against which the tongue works: the posterior half, or the soft palate, is smooth and soft, and ends behind in a free notched border, nearly opposite the angle of the jaw. 


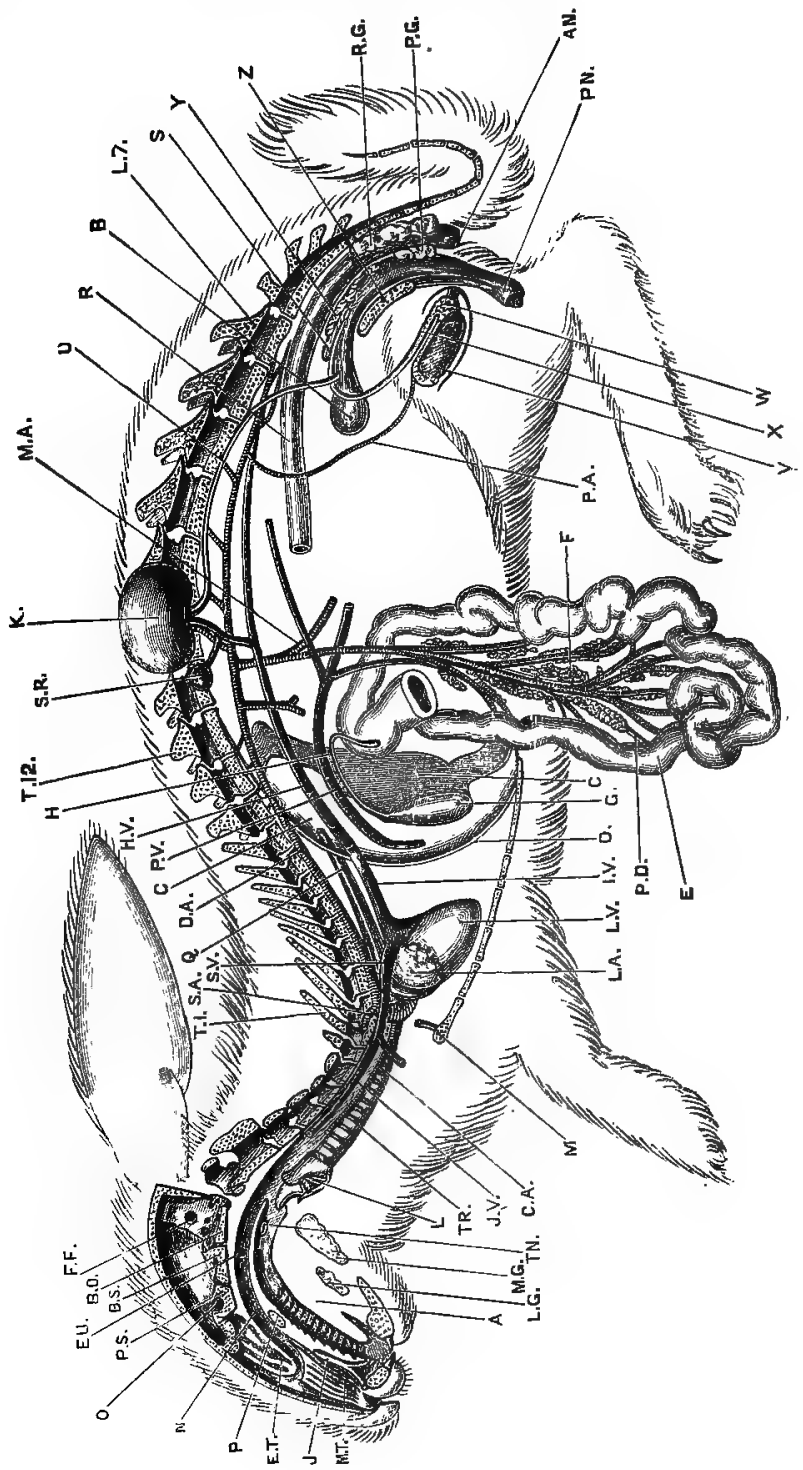


b. The upper teeth, comprising the incisors at the front of the palate, and the grinders at the sides opposite the junction of hard and soft palates, have already been described (p. 292).

c. The naso-palatine canals, which connect the nasal and buccal cavities, open in a pair of grooves running backwards from the small posterior incisors, and about an eighth of an inch behind these.

d. The tonsils are a pair of small pits at the sides of the posterior part of the soft palate, near its hinder border. The outer wall of each is much thickened, forming a papilla which nearly closes the opening.

\section{The floor of the mouth.}

The tongue is an elongated muscular mass, attached along the greater part of its length to the floor of the mouth, and produced in front into a free flattened tip.

The surface of its hinder part is hard and slightly elevated; that of the anterior part is softer and dotted over with small white taste

Fig. 59.-Lepus cuniculus.-Diagrammatic view from the left side. The head and vertebral column are represented in longitudinal section; the lungs, the left half of the liver, the stomach, the greater part of the intestine, and the brain and spinal cord have been removed, and the loop of the duodenum spread out. (A.M. M.)

$\mathbf{A}$, tongue. $\mathbf{A N}$, anus. $\mathbf{B}$, bladder. $\mathbf{B O}$, basi-ocoipital. $\mathbf{B S}$, basisphenoid. $\mathbf{C}$, liver. $\mathbf{C A}$, carotid artery. $\mathbf{D}$, diaphragm. $\mathbf{D A}$, aorta. $\mathbf{E}$, duodenum. FT, ethmo-turbinal. FU, aperture of Eustachian tube. F, pancreas. Fi, floccular fossa. G, gall-bladder. H, bile-duct. HV, opening of hepatic vein into posterior vena cava. IV, posterior vena cava. J, organ of Jacobson. JV, left external jugular vein. 'K, kidney, turned up. $\mathbf{I}$, larynx, bisected. IA, left auricle. IG, sublingual gland. IV, left ventricle. I 7, seventh lumbar vertebra. M, manubrium sterni, with the sternal portion of the first rib. MA, anterior mesenteric artery. MG, submaxillary gland. MT, maxillo-turbinal. $\mathbf{N}$, posterior nasal chamber. $\mathbf{O}$, optic foramen. $\mathbf{P}$, hard palate. $\mathbf{P A}$, spermatic artery. $\mathbf{P D}$, parcreatic duct. $\mathbf{P G}$, peringal gland. $\mathbf{P N}$, penis. PS, pre-sphenoid. $\mathbf{P V}$, portal vein. $\mathbf{Q}$, cesophagus. $\mathbf{R}$, hinder part of the rectum. RG, rectal gland. $\mathbf{S}$, uterus masculinus. SA, left subclavian artery. SR, suprarenal body, displaced. $\mathbf{S V}$, left anterior vena cava. TN, right tonsil. TR, trachea. T1, first thoracic vertebra. T 12, twelfth thoracic vertebra. $\mathbf{U}$, ureter. $\mathbf{V}$, testis. $\mathbf{W}$, epididymis. $\mathbf{X}$, vas deferens. $\mathbf{Y}$, prostate. $\mathbf{Z}$, pelvic symphysis. 
papillæ, which are especially numerous at and under the tip.

At the sides of the tongue, opposite the last .molar teeth, are a pair of oval patches, the papillæ foliatæ, the surfaces of which are crossed obliquely by close-set parallel ridges, in which are imbedded numerous taste-bulbs.

About a quarter of an inch above and behind the papillæ foliatæ are a pair of circumvallate papillæ, which are small and white, and surrounded by circular grooves.

b. The lower teeth, already described with the skeleton (p. 293), are placed opposite the corresponding teeth of the upper jaw, and bite against them.

3. The sides of the mouth are smooth and soft, with the exception of a hairy patch on each side, between the incisor and molar teeth.

4. The pharynx is a continuation backwards of the buccal cavity, beyond the margin of the soft palate. The mouth and the posterior narial chamber open into it in front; posteriorly it is continued back into the œsophagus; and ventrally it communicates through the glottis and trachea with the lungs.

To expose the pharynx, extend the cut backwards about an inch beyond the tongue.

a. The glottis is a large opening in the floor of the pharynx, a short way behind the tongue, leading into the trachea, or windpipe.

b. The epiglottis is a thin, bilobed, cartilaginous flap, forming the anterior boundary of the glottis, and projecting into the pharynx behind the tongue, opposite the free edge of the soft palate. During the act of swallowing it folds back over the glottis, and closes its entrance.

5. The posterior nasal chamber lies above the soft palate, which separates it from the buccal cavity. 
Lay open the nasal chamber by slitting up the soft palate in the median line, from behind forwards.

a. The orifices of the Eustachian tubes are a pair of oval apertures at the sides of the roof of the chamber, about the middle of its length.

The Eustachian tubes connect the tympanic cavities with the posterior nasal chamber. The tympano-Eustachian passage is the modified hyo-mandibular cleft of the embryo, corresponding to the spiracle of the dog-fish. Its connection with the ear is of a purely secondary character, and it forms no part of the essential organ of hearing.

Pass a seeker backwards and outwards, along one of the Eustachian tubes, into the tympanic cavity.

b. The organs of Jacobson are a pair of tubular bodies enclosed in cartilage, and lying in the floor of the anterior part of the nasal cavity. Each communicates in front with the mouth by the naso. palatine canal, and with the nostril by a more dorsally placed opening.

Cut away with bone-forceps the left side of the nasal chamber, so as to lay open the cavity completely. Remove the mesethmoid cartilage, and note the arrangement of the turbinal bones, and the relations of Jacobson's organs.

6. The salivary glands are large and paired.

Dissect from the left side.

a. The parotid gland, the largest of the salivary glands, is a soft pinkish mass lying in front of and below the external auditory meatus, between this and the hinder edge of the mandible.

The duct of the parotid, or Stenonian duct, leaves the gland at its anterior border, and runs forwards just beneath the skin, parallel to and about a quarter of an inch below the zygomatic arch. It opens into the mouth by a small aper- 


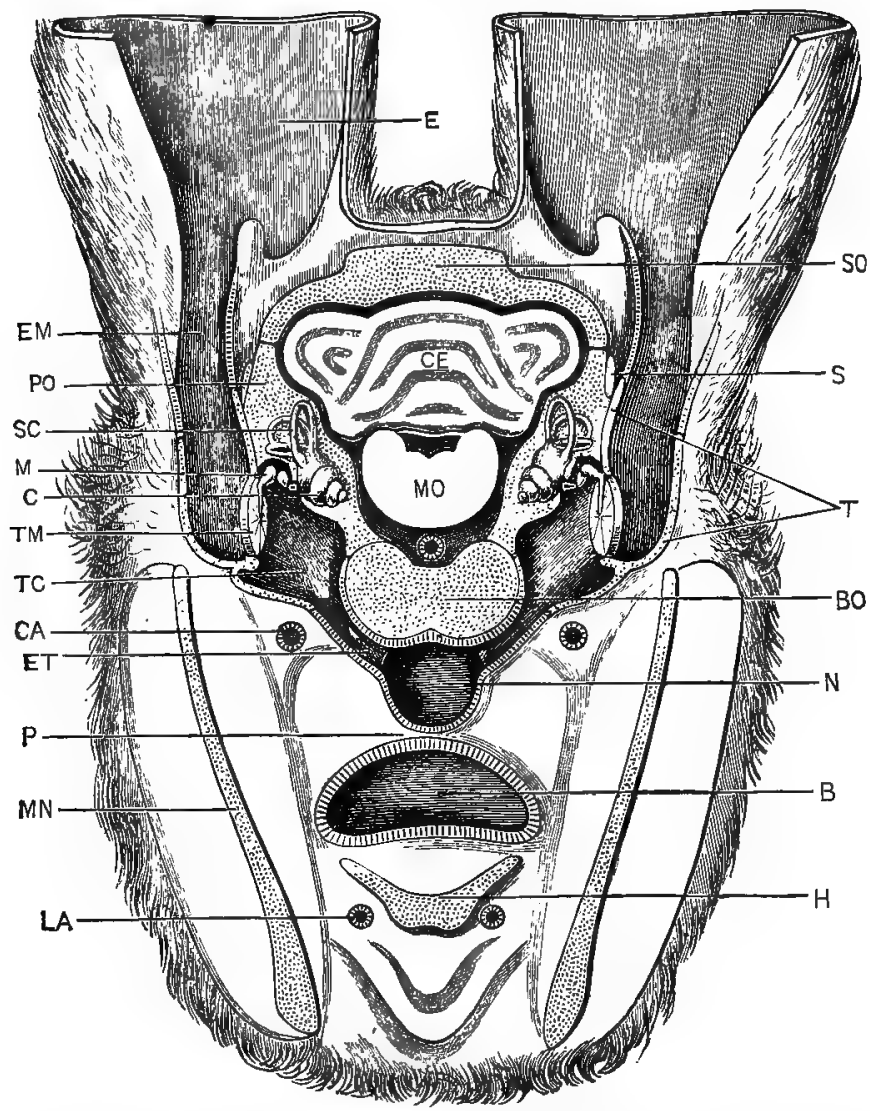

Fig. 60. Lepus cuniculus.-Diagrammatic section across the head to show the relations of the internal ear, tympanic cavity and membrane and the auditory ossicles. The section is drawn as seen from the front, and is taken along a line joining the reference letters SO and MN in fig. $58, p .276$. The external ears are cut short close to their bases, and the floccular lobes of the cerebellum, which lie between the three semicircular canals on each side, are omitted entirely. (A. M. M.)

B, buccal cavity. BO, basi-occipital. C, cochlea, CA, right external carotid artery. $\mathbf{C D}$, cerebellum. $\mathbf{E}$, external ear or pinna. $\mathbf{F} \mathbf{M}$, external auditory meatus. FT, Fustachian tube. H, body of the hyoid. LA, right lingual artery. M, malleus; to its inner side are seen the incus and stapes. MNV, mandible. MO, medulla oblongata. N, posterior nasal chamber. $\mathbf{P}$, soft palate. PO, periotic. S, post-tympanic process of squamosal. SC, anterior vertical semicircular canal. SO, supra-occipital. T, tympanic bone. TC, tympanic carity. TM, tympanic membrane. 
ture on the inner side of the cheek, opposite the second upper premolar tooth.

b. The infra-orbital gland is an irregular lobulated mass, about three-quarters of an inch long, lying below and in front of the eye, partly above the zygomatic arch and partly hidden by it.

The duct runs downwards to open into the mouth, close to the Stenonian duct.

c. The submaxillary gland is a compact, reddish, ovoidal mass, lying close to its fellow between the angles of the mandible, and in front of the larynx.

The duct, Wharton's duct, leaves the outer side of the gland near its hinder end, and runs forwards along the inner side of the jaw to open into the floor of the mouth, midway between the lower incisors and the root of the tongue, the openings of the ducts of the two sides being about an eighth of an inch apart.

Dissect from the ventral surface. To follow the duct into the mouth, turn the halves of the lower jaw aside.

d. The sublingual gland is an elongated, flattened, reddish body, about three-quarters of an inch long, lying along the inner side of the ramus of the mandible, between this and Wharton's duct.

The ducts open separately on the floor of the mouth.

\section{THE ABDOMINAL VISCERA.}

Remove the skin from the trunk and limbs. Lay the rabbit on its back, and fasten it down to the dissecting-board by pins through the limbs.

[A. The mammary glands in the female lie immediately beneath the skin. They are yellowish, fatty-looking, dendri- 
form masses, covering the ventral surface of the whole length of the thorax and abdomen; their ducts may be readily traced, converging to the teats.]

B. The Abdominal Viscera in situ.

Open the abdominal cavity'by a median incision through its ventral wall, from the hinder end of the sternum to the pubic symphysis, taking care not to injure the viscera. From the anterior end of the incision cut outwards for about two inches on each side, just behind the ribs, and pin out the flaps right and left. Note the positions of the following viscera, without disturbing them in any way.

1. The liver is a large dark-red body at the anterior end of the abdominal cavity, extending further back on the left side than on the right.

2. The stomach lies immediately behind the liver and is partially covered by it: its surface is smooth and of a bluish white colour.

3. The duodenum is a narrow pinkish tube, a small part of which is seen to the right of the stomach.

4. The small intestine is a long, narrow, much convoluted tube, part of which is seen at the left side of the abdominal cavity, behind the liver.

5. The cæcum is a very wide tube, of a dark colour, coiled on itself and marked by a spiral constriction. In the natural position of the parts it covers almost all the other viscera behind the stomach. On the right side the cæcum ends in the pale fleshy vermiform appendix.

6. The colon is a wide tube, with markedly sacculated walls, running obliquely across the abdomen between the folds of the cæcum.

7. The rectum is a white tube, dilated at intervals by the fæcal pellets: small parts of it are seen to the right of the stomach, and at the hinder end of the abdomen. 
8. The bladder is a thin-walled sac at the posterior end of the abdomen, just in front of the pubic symphysis.

Turn the alimentary canal aside, without cutting or tearing anything, to expose the following parts.

9. The spleen is an elongated dark-red body lying behind the stomach, and attached to its left or cardiac end.

10. The kidneys are a pair of compact oroid bodies attached to the dorsal wall of the abdomen; the right one being immediately behind the liver, and the left one about an inch and a half further back. In front of each kidney is a small, round, yellow adrenal body.

11. The diaphragm is a muscular partition forming the anterior wall of the abdomen, and separating it from the thorax. To see it the liver should be pressed back.

12. The peritoneum is a glistening membrane which lines. the abdominal cavity. The mesentery is a double layer of peritoneum, reflected from the dorsal wall of the abdomen, which supports and encloses at its margin the alimentary canal : between its two layers the vessels and nerves pass to and from the various organs. The omentum is a special fold of peritoneum, extending backwards from the stomach and loaded with fat.

13. The cœliac ganglia are a pair of closely apposed pinkish bodies situated in the mesentery, near its. dorsal margin and opposite the right adrenal body. They receive right and left splanchnic nerves from the main sympathetic trunks of the thorax, and send numerous branches to the alimentary canal.

\section{DISSECTION OF THE DIGESTIVE SYSTEM.}

In this section the abdominal portion of the digestive system will alone be considered: the buccal cavity and pharynx have already been dissected; and the cesophagus will be described with the neck and thorax. 


\section{A. External Characters of the Digestive System.}

\section{The stomach.}

Expose the stomach fully, by turning the liver forwards, and the intestine over to the animal's left.

The stomach is much dilated at its left or cardiac end; narrower towards the right or pyloric end; and markedly curved, the concavity being directed forwards. Near the left end of its anterior border is the cardia, or opening of the oesophagus ; and from the thick-walled pyloric end a small aperture, the pylorus, leads to the duodenum.

2. The duodenum, or first portion of the intestine, runs from the pylorus along the right side of the abdominal cavity almost to its hinder end: it then turns back on itself, forming a U-shaped loop. In the mesentery connecting the two limbs of the loop are the large duodenal vessels.

\section{The pancreas.}

Turn the duodenal loop over to the left side without injuring the mesentery, and spread it out over the other viscera.

The pancreas is a diffuse fat-like gland of a pinkish colour, scattered in the mesentery of the duodenal loop. It lies mainly around the large vessels of the loop, and is more compact at its anterior end.

The pancreatic duct commences in front, and runs backwards through the gland, receiving smaller ducts from its several lobes; it opens into the inner side of the distal limb of the duodenal loop, about three inches beyond the bend.

Open the duodenum, opposite to the entrance of the pancreatic duct; wash out the contents; note the aperture of the duot, and insert a bristle into it.

4. The liver is a large solid organ, thick in the middle and thinning towards the edges. Its anterior surface is 
convex and fits against the diaphragm, to which it is attached by a median vertical fold of peritoneum, the suspensory ligament: its posterior surface is concave, and lies against the stomach.

a. The lobes of the liver.

i. The left central lobe lies against the left half of the diaphragm.

ii. The left lateral lobe lies between the left central lobe and the stomach, to the cardiac end of which it is applied. It is the largest of the lobes, and is partially subdivided.

iii. The Spigelian lobe fits into the concave anterior border of the stomach, and is much the smallest of the lobes.

iv. The right central lobe lies against the right half of the diaphragm, and is grooved on its posterior surface for the gall-bladder.

v. The caudate lobe lies along the right side and dorsal surface of the pyloric end of the stomach. Its posterior margin is hollowed to fit the right kidney : its inner and anterior border is connected by a bridge of liver-tissue with the Spigelian lobe.

b. The gall-bladder is an elongated thin-walled sac, rather less than an inch in length, of a dark green or brown colour, and lying in the groove in the posterior surface of the right central lobe of the liver.

c. The bile-duct is a narrow tube, about two inches long, running backwards from the smaller and dorsal end of the gall-bladder to open into the dorsal surface of the duodenum, about a third of an inch beyond the pylorus. It receives ducts from the several lobes of the liver, and lies in the mesentery immediately to the right of the large portal vein. 
Open the duodenum opposite to the opening of the bile-duct; wash out its contents; find the aperture of the duct on a small papilla, and insert a fine seeker or bristle into it.

The bile-duct is most readily traced from the duodenum forwards, and may with advantage be first injected with plaster of Paris from a point about half an inch from the duodenum.

Cut through the mesentery of the small and large intestines, leaving the duodenal loop and its mesentery untouched; lay out the alimentary canal on the dissecting-board, so as to show the relations and proportions of its several parts. Take care not to damage the splanchnic ganglia. Avoid all unnecessary injury to the blood-vessels, and ligature any that bleed.

5. The small intestine, which is directly continuous with the duodenum, is about seven or eight feet in length, and of uniform diameter throughout.

a. Peyer's patches are slightly thickened oval spots, granular in appearance, and about a third of an inch in diameter, which occur at intervals along the whole length of the small intestine, on the side opposite to the attachment of the mesentery.

b. The sacculus rotundus is the dilated distal end of the small intestine, opening into the side of the cæcum about an inch from its proximal end. Its walls have the structure of Peyer's patches.

\section{The cæcum and vermiform appendix.}

The cæcum is a large thin-walled diverticulum of the alimentary canal, at the junction of the small intestine and colon. It is about twenty inches long and an inch or more in diameter, and is marked externally by a spiral constriction which runs twenty to thirty times round it. The small intestine opens into the side of the cæcum about an inch from its proximal end ; a large Peyer's patch in the wall of the cæcum being continuous with the sacculus rotundus.

Proximally, the cæcum passes directly into the colon, while distally it ends blindly in the thickwalled finger-like vermiform appendix. This latter 
is about four inches long, and its walls resemble Peyer's patches in appearance and structure.

In the rabbit the cæcum is of enormous size, as in most herbivorous mammals with simple stomachs. 7. The colon is about a foot and a half in length. The first part of it is very markedly sacculated, the sacculations lying in three longitudinal rows, separated by smooth areas of unequal width. Towards the rectum the walls become smooth.

The rectum or terminal portion of the intestine is a narrow tube, about two and a half feet in length, pale in colour, and presenting a moniliform appearance, owing to the contained fæcal pellets. At its hinder end it passes through the pelvic cavity to terminate at the anus.

\section{B. Internal Structure of the Alimentary Canal. The stomach.}

Remove the stomach from the body, cutting across the cesophagus and duodenum close to it, and taking care not to injure the vessels of the liver. Slit up the stomach along its posterior surface: wash, and examine it under water.

The wall of the stomach consists of an outer peritoneal investment; a middle muscular layer, thickened at the pyloric end; and an inner layer of mucous membrane, which is raised into irregular longitudinal folds. The pyloric orifice is narrowed by a prominent muscular rim.

\section{The small intestine.}

Cut out a small piece from the wall, and examine its inner surface under water.

The villi are minute tag-like processes of the mucous membrane, covering its surface, and giving it a velvety appearance.

Peyer's patches present a honeycombed appearance, the depressions being occupied by smooth convex lymph-follicles, bordered by villi. 


\section{The cæcum.}

Remove the cacum from the body, cutting across the smalt intestine and the colon about an inch from it. Take the cacum to the sink, and slit it open longitudinally: wash freely, and examine under water.

The inner surface is papillose, and raised along the line of the external constriction into a prominent. spiral fold, a quarter of an inch or more in width. The opening from the sacculus rotundus is small and circular ; that into the colon is much larger.

Cut the vermiform appendix across to see the thickness of its wall; and slit it open to examine its inner surface, which is similar to that of Peyer's patches, but with smaller follicles.

4. The colon.

Slit open part of the colon; wash; and examine under water, noting the papillose surface and the sacculations of the wall.

\section{The rectum.}

Examine a part of the rectum in a similar manner, and note its smooth mucous membrane.

\section{THE THORACIC VISCERA.}

Open the thorax by a transverse.incision just in front of the diaphragm: cut through the ribs along each side, and remove the front wall of the thorax as a triangular piece, taking great care not to injure the vessels and other structures beneath it.

Identify and examine the following parts without any further dissection.

1. The thymus is a pale, soft, glandular-looking body in the anterior part of the thorax. It is very variable in size, being larger in the young animal, and sometimes extending back so as to overlap the heart.

2. The heart, enclosed in the thin transparent pericardium, lies in the middle of the thorax : it is dark in colour 
and conical in form ; the apex being directed backwards and slightly to the left ; and the base, with the roots of the great vessels, forwards.

3. The lungs are a pair of pink spongy bodies at the sides of the thorax, and filling the greater part of its cavity. They collapse when the thorax is opened.

Find the trachea in the midale line of the neck, recognising it by its transverse cartilaginous rings. Free it from the surrounding parts about the middle of its course; make a longitudinal incision in it; insert a blowpipe, and pass a ligature loosely round the trachea behind it; inflate the lungs through the blowpipe, and when they are fully distended tie the ligature firmly, so as to retain the air in the lungs.

The lungs lie quite free in the thoracic cavity, except at their roots, where the blood-vessels and - bronchi enter them. The left lung is divided into two lobes; and the right into four, of which the smallest and hindmost lies across the median plane behind the heart, closely applied to the œsophagus.

4. The diaphragm is a thin partition, separating the thoracic cavity from the much larger abdominal cavity. Its marginal portion is muscular, the fibres arising from the inner surface of the hinder ribs and from the vertebral column, and converging to be inserted into the thinner, tendinous, central portion. The muscular margin is specially developed in the dorsal region, forming two stout pillars arising from the lumbar vertebræ.

The diaphragm is arched so as to present a very convex surface towards the thorax: by contraction of its muscular portion, in the act of inspiration, this convexity is diminished and the thoracic cavity thereby enlarged. The small size of the thoracic relatively to the abdominal cavity should be noticed.

5. The phrenic nerves are a pair of white cords lying between the heart and the lungs, and readily seen on pressing these apart. Posteriorly they divide into 
branches supplying the muscular portion of the diaphragm.

Their origin from the fourth cervical nerves will be seen in the dissection of the neck (p. 349).

6. The pleuræ. Each side of the thorax is lined by a glistening membrane, the pleura, which is reflected over the lung at its root in the same way as the peritoneum is reflected over the abdominal viscera. The right and left pleural sacs are separated from each other by the mediastinal cavity, the greater part of which is occupied by the heart and pericardium, the posterior part remaining vacant.

During life the lungs are distended so as to almost fill the thoracic cavity, the visceral layer of the pleuræ, covering the lungs, being closely applied to the parietal layer lining the thorax, so that the pleural cavities are practically obliterated.

Each pleura being an air-tight sac, the lungs follow the movements of the ribs and diaphragm: so that enlargement of the thoracic cavity causes expansion of the lungs, and hence inspiration.

7. The esophagus is a narrow muscular tube, running through the thorax immediately ventral to the vertebral column, and readily seen on raising the left lung.

\section{DISSECTION OF THE CIRCULATORY SYSTEM.}

\section{A. The Heart and the Roots of the Great Vessels.}

Remove the thymus and the parietal layer of the pericardium, and carefully clean the base of the heart and the roots of the great vessels, avoiding injury to the nerves.

1. The heart. The ventricular portion of the heart, which forms almost the whole of the exposed ventral surface, is marked by a groove which runs from the base obliquely backwards and to the right, indicating the division into right and left ventricles. 
The auricles lie at the base of the heart, on its dorsal aspect, the auricular appendices being the only parts of them visible on the ventral surface.

a. The right ventricle forms the right side of the ventricular portion, but does not reach to the apex. It is soft to the touch.

b. The left ventricle forms the left side and apex of the heart; and is firmer than the right ventricle, owing to the greater thickness of its walls.

c. The left auricle is anterior to the left ventricle, and is largely concealed by the great vessels. It is produced ventrally into the ear-like auricular appendix.

d. The right auricle is dorsal to the base of the right ventricle, and is produced in front into the auricular appendix.

2. The roots of the great vessels.

a. Opening into the right auricle.

i. The right anterior vena cava returns blood from the right side of the head, neck, and thorax, and from the right fore-limb. It enters the thorax at its anterior end, and runs back along the inner side of the right lung, to open into the right auricle by an aperture in its dorsal wall.

ii. The left anterior vena cava returns blood from the left side of the head, neck, and thorax, and from the left fore-limb. It runs back along the inner side of the left lung, and crosses between the heart and the root of the left lung, to open into the left side of the right auricle.

iii. The posterior vena cava returns blood from all parts of the body behind the diaphragm. It enters the thorax through an aperture in the 
diaphragm and runs forwards, slightly to the right of the median plane, to open into the posterior end of the right auricle.

b. Arising from the right ventricle.

i. The pulmonary artery conveys the venous blood from the heart to the lungs. It arises from the anterior border of the right ventricle in the mid-ventral line, and arches over to the dorsal surface of the left auricle, where it divides into the right and left pulmonary arteries, supplying the two lungs.

c. Opening into the left auricle.

i. The pulmonary veins return the blood from the lungs to the heart. There are two main veins on each side, which converge to open into the dorsal surface of the left auricle.

d. Arising from the left ventricle.

i. The aorta conveys arterial blood from the heart to all parts of the body. It arises from the middle of the base of the heart, dorsal to the root of the pulmonary artery, runs forwards about half an inch, and then arches over to the left side, and runs back along the left side of the vertebral column. Its thoracic portion is readily seen on turning aside the left lung.

The ductus arteriosus is a ligamentous band, connecting the aorta with the pulmonary artery, just before the division of the latter into right and left branches. During embryonic life it forms a tubular connection between the two vessels.

\section{B. The Veins.}

The veins form three distinct systems, communicating with one another only through capillaries, and containing different kinds of blood. 
(1). The first of these systems consists of the three venæ cavæ, with the veins opening into them: these bring to the right auricle venous blood from the body generally, and from the liver.

(2). The second or portal system collects the blood from the walls of the alimentary canal, and from the pancreas and spleen, and conveys it to the liver.

(3). The third or pulmonary system conveys arterial blood from the lungs to the left auricle.

In dissecting the veins it is convenient to follow them from the larger to the smaller trunks, and they will be described in this order; it must be remembered, however, that the flow of blood is in the reverse direction. Care must be taicen not to out the veins, as the blood obscures the dissection, and the vessels themselves when empty are difficult to follow, owing to the thinness of their walls. If by chance a large vein is cut it must be ligatured immediately to stop the bleeding.

1. The system of the venæ cavæ.

a. The right anterior vena cava has already been seen. It is formed by the union of the jugular and subclavian veins, immediately in front of the first rib.

i. The azygos cardinal vein is a median vein which runs forwards in the mid-dorsal line of the thorax, lying close to the vertebral column and along the right side of the aorta. It receives branches from the posterior seven or eight intercostal spaces of both sides of the body. Opposite the level of the auricles it passes round the right side of the esophagus and trachea, to open into the vena cava close to the auricle.

ii. The right anterior intercostal vein is a small vein returning blood from the anterior four or five intercostal spaces of the right side, and opening into the vena cava just in front of the azygos vein. 
iii. The right internal mammary vein runs forwards on the inner surface of the ventral thoracic wall, near the middle line, and opens into the vena cava opposite the first rib.

iv. The right subclavian vein returns blood from the fore-limb and shoulder.

v. The right external jugular vein is a large vein running along the side of the neck. It is formed in front by the union, just behind the angle of the jaw, of the anterior and posterior facial veins, returning blood from the face and ear.

vi. The right internal jugular vein is a small vein returning blood from the brain, and running down the neck alongside the trachea, to open into the right external jugular vein close to its union with the subclavian.

b. The left anterior vena cava resembles the right, except that it receives no azygos vein, and that its course in the thorax is somewhat different, as already described (p. 323).

c. The posterior vena cava is a large median vein, commencing at the hinder end of the abdomen, and running forwards close to the vertebral column and to the right (or left) of the aorta. In front, it leaves the abdominal wall and becomes imbedded in the dorsal surface of the liver, beyond which it enters the thorax through an aperture in the central tendon of the diaphragm, and runs for wards, as already described (p. 323), to open into the right auricle.

The following are the principal veins opening into the posterior vena cava.

In dissecting these veins, take care not to damage the arteries, which accompany and roughly correspond to them, or the renal and reproductive organs. 
i. The phrenic veins are small veins in the substance of the diaphragm, opening into the vena cava as it passes through.

ii. The hepatic veins are large vessels returning blood from the several lobes of the liver, and opening into the portion of the vena cava imbedded in the liver. There are four chief ones.

To see the hepatic veins, ligature the vena cava, both in front of and behind the liver: cut across the vena cava, remove the liver, and lay it on the dissecting-board with the dorsal surface upwards: slit open the vena cava; wash out the contained blood; note the openings of the hepatic veins, and follow these with a seeker into the several lobes of the liver.

iii. The renal veins are a pair of large veins running inwards from the kidneys to the vena cava, the right and shorter one being about three-quarters of an inch in front of the left.

Each renal vein receives a small vein from the corresponding adrenal body, and a much larger one from the dorsal wall of the abdomen.

iv. The spermatic veins in the male [or ovarian veins in the female] are a pair of small veins returning blood from the testes [or ovaries], and opening into the vena cava near the hinder end of the abdomen. The left one frequently opens into the renal vein, or the ilio-lumbar vein.

v. The ilio-lumbar veins are paired, and roturn blood from the hinder part of the abdominal walls, opening into the vena cava about the level of the anterior border of the thigh. The left one often receives the spermatic [or ovarian] vein, and opens into the renal vein.

vi. The external iliac veins are a pair of large veins returning blood from the hind-limbs, and opening into the vena cava at the hinder end of the abdomen, about an inch in front of 
the pubic symphysis. They receive small veins from the bladder [and in the female from the uterus].

They are direct continuations of the femoral veins, which lie along the inner or preaxial borders of the thighs.

vii. The internal iliac veins return blood from the backs of the thighs, and, running forwards through the pelvic cavity, unite about half an inch behind the external iliacs to form the commencement of the posterior vena cava.

2. The portal system consists of the veins returning blood from the whole length of the alimentary canal from the stomach backwards, and also from the pancreas and spleen. These veins lie in the mesentery, and converge to form the main trunk, or portal vein, which lies close alongside the posterior vena cava and, entering the liver, divides into branches supplying its several lobes.

The portal system can readily be seen in a freshly killed animal on opening the abdomen and turning the viscera aside. The veins are of large size and usually gorged with blood, and care must be taken not to injure them, as they bleed very freely.

The following are the main trunks which unite to form the portal vein.

i. The lieno-gastric vein returns blood from the walls of the stomach and from the spleen.

ii. The duodenal vein returns blood from the duodenum and pancreas.

iii. The anterior mesenteric vein is formed by the union of veins from the whole length of the small intestine, and from the cæcum, the colon, and the greater part of the length of the rectum. 
iv. The posterior mesenteric vein lies in the mesorectum or hindmost part of the mesentery, and returns blood from the terminal portion of the rectum.

3. The pulmonary system consists of the pulmonary veins, which have already been described (p. 324).

\section{The Arteries.}

The arteries form two distinct systems, of which one, the aortic system, conveys arterial blood from the left ventricle to all parts of the body; while the other, or pulmonary system, carries venous blood from the right ventricle to the lungs. The arteries are thicker-walled, and hence paler in colour than the veins.

The aortic system should be dissected first. Clean the aorta along its whole length, and follow the arteries arising from it to their distribution.

1. The aorta arises from the base of the left ventricle, and, turning towards the left side to form the arch of the aorta, runs backwards through the thorax and abdomen. It lies on the ventral surface of the vertebral column, and divides about an inch in front of the level of the pubic symphysis into the two common iliac arteries.

2. Arteries arising from the aorta in the thorax.

a. The innominate artery arises from the commencement of the arch of the aorta: it gives off, close to its origin, the left carotid artery, and then runs forwards a short distance and divides into the right subclavian and right carotid arteries.

i. The right subclavian artery runs outwards just in front of the first rib: it gives branches to the shoulder, and runs on as the brachial artery into the right fore-limb. Its principal branches are the following. 
$a$. The vertebral artery arises from the subclavian just beyond its origin, and passing dorsalwards enters the vertebrarterial canal of the cervical vertebræ, along which it passes, supplying the spinal cord and the brain.

$\beta$. The internal mammary artery arises from the subclavian just before it leaves the thorax, and runs backwards along the inner surface of the ventral wall of the thorax.

ii. The right carotid artery runs forwards alongside the trachea, supplying the neck. Opposite the angle of the jaw it divides into (1) the internal carotid artery, which enters the skull through the carotid foramen to supply the brain; and (2) the external carotid artery, which supplies the right side of the head and face.

iii. The left carotid artery corresponds to the right carotid in its course and distribution.

b. The left subclavian artery arises from the left side of the arch of the aorta: its course and branches correspond to those of the right subclavian.

c. The intercostal arteries are a series of small paired arteries, arising from the dorsal surface of the aorta, and running outwards on the inner surface of the thoracic walls, one behind each rib.

3. Arteries arising from the aorta in the abdomen.

a. The cœliac artery is a large median vessel, arising from the ventral surface of the aorta, about half an inch behind the diaphragm. It runs in the mesentery, and divides into the hepatic artery supplying the liver, and the lieno-gastric artery supplying the stomach and spleen.

b. The anterior mesenteric artery is a large median vessel, arising from the aorta about three-quarters 
of an inch behind the cœliac artery. It divides into numerous branches, which run in the mesentery to supply the duodenum, pancreas, small intestine, cæcum, and colon.

c. The renal arteries are paired, the right one arising just behind the anterior mesenteric artery, and the left a little further back. Each gives a small branch to the muscles of the back, and then runs outwards to the kidney.

d. The spermatic arteries, in the male, are a pair of very small arteries, which arise about a couple of inches behind the left renal artery, and run backwards and outwards along the dorsal surface of the abdomen to enter the epididymes at their anterior ends.

The ovarian arteries, in the female, correspond in origin to the spermatic, but run outwards and slightly forwards to the ovaries.

e. The posterior mesenteric artery is a median vessel, arising from the aorta about an inch in front of its division into the common iliacs. It supplies the hinder part of the rectum.

f. The lumbar arteries are small median arteries, arising from the dorsal surface of the aorta, and dividing into right and left branches supplying the body-walls.

g. The median sacral artery is a small vessel arising from the dorsal surface of the aorta, shortly before its division, and running along the ventral surface of the sacrum to the tail. It is to be regarded as the posterior continuation of the aorta.

h. The common iliac arteries, formed by the bifurcation of the aorta, run backwards and outwards to the hind-limbs, along which they are continued as the femoral arteries. In the abdomen each gives off the following branches. 
i. The ilio-lumbar artery runs outwards along the posterior part of the dorsal wall of the abdomen.

ii. The internal iliac artery runs backwards along the dorsal wall of the pelvic cavity.

iii. The vesical artery is a small branch which runs backwards to the bladder. In the female it also supplies the uterus.

4. The pulmonary artery has already been described (p. 324).

Follow the branches of the pulmonary artery into the lungs.

D. The Thoracic Duct.

The thoracic duct is a slender thin-walled tube running forwards through the thorax, and Iying immediately to the left of and dorsal to the aorta. Between the third and fourth ribs it expands somewhat, and then turning inwards traverses the thymus and opens into the left anterior vena cava close to the left subclavian vein.

The thoracic duct receives lymphatic vessels from almost all parts of the body, and places these in communication with the venous system.

\section{E. Dissection of the Heart.}

Cut across the great vessels, about half an inch from the heart. Remove the heart, and pin it down under water with the dorsal surface upwards, passing the pins through the ventricles and the arch of the aorta. Clean carefully the roots of the several vessels.

1. The auricles.

Cut away the outer walls of both auricles so as to expose their cavities. Wash out the contained blood.

a. The right auricle. The wall of the auricle is thin; that of the auricular appendix is thicker, and is marked internally by muscular ridges.

i. The orifices of the venæ cavæ.

The right anterior vena cava opens into the anterior part of the auricle. 
The left anterior vena cava opens into the left side of the posterior end of the auricle: just before its opening it receives the coronary vein, returning blood from the substance of the heart.

The posterior. vena cava opens into the dorsal surface of the auricle, in front of the left anterior vena cava.

ii. The Eustachian valve is a membranous fold, between the orifices of the left anterior vena cava and the posterior vena cava. During foetal life it directs the blood from the posterior vena cava through the foramen ovale into the left auricle.

iii. The septum auricularum is a thin partition between the right and left auricles. In it is an oval patch, the fossa ovalis, close to the opening of the pasterior vena cava. This is thinner than the rest of the septum, and is perforated in the embryo by the foramen ovale.

iv. The right auriculo-ventricular aperture is a wide crescentic opening leading into the right ventricle.

b. The left auricle. The wall of the auricle is thin; that of the auricular appendix is thicker, and is marked by a network of muscular ridges.

i. The apertures of the pulmonary veins. The veins of the two sides unite as they enter the dorsal surface of the auricle.

ii. The left auriculo-ventricular aperture is a large circular funnel-like opening, leading into the left ventricle.

\section{The ventricles.}

Cut across the ventricles about one-third of an inch from the apex; note the shape of the cavities. 
The cavity of the right ventricle is crescentic in section: its wall is thin, embracing and half-surrounding the left ventricle.

The left ventricle has very thick walls, and a small irregular cavity.

a. The right ventricle.

Remove the auricles, and cut short the aorta and pulmonary artery. Remove the outer wall of the right ventricle, by longitudinal incisions through its dorsal and ventral walls, and a transverse incision across its base a short distance behind the anterior border. Avoid injury to the valve.

i. The tricuspid valve guards the right auriculoventricular aperture, and is formed by three membranous flaps attached round its margin. The free borders of the flaps project into the ventricle, and are connected by tendinous strings, the chordæ tendineæ, to muscular processes of the wall of the ventricle, the larger of which are known as musculi papillares.

ii. The orifice of the pulmonary artery is at the left anterior angle of the ventricle, and is guarded by three pocket-like semilunar valves, the free edges of which are directed away from the ventricle.

Note the position of the valves from above; slit up the pulmonary artery between two of them, and examine them.

b. The left ventricle.

Remove the outer wall in the same manner as that of the right ventricle.

i. The mitral valve, guarding the left auriculoventricular aperture, resembles the tricuspid valve, except that there are only two flaps, and the musculi papillares are much larger. 
ii. The columnæ carneæ are muscular ridges projecting into the cavity of the ventricle, and mainly longitudinal in direction.

iii. The orifice of the aorta is at the base of the ventricle, immediately to the right of the mitral valve. It is guarded by three semilunar valves, one of which is dorsal, and the other two ventro-lateral in position. Immediately beyond the two latter valves the coronary arteries, which supply the heart, arise from the aorta.

\section{DISSECTION OF THE RENAL AND REPRODUCTIVE SYSTEMS.}

In the rabbit, as in the dog-fish, certain parts of the primitive kidneys and their ducts lose their excretory function, and become converted into accessory genital organs and genital ducts. Owing to the close connection of the two systems, they may, in the adult, be conveniently considered together, though it must be remembered that the connection is merely a secondary one ( $c f$. pp. 238-239).

In the male rabbit, of the three divisions into which the primitive kidney is divided, the pronephros and its duct are absent or rudimentary; the mesonephros acquires an intimate connection with the testis, and becomes converted into the epididymis, its duct becoming the vas deferens.

In the female, the pronephros is absent, but its duct becomes the oviduct; the mesonephros and its duct are absent, or rudimentary.

In both sexes alike, the metanephros and its duct become the kidney and ureter respectively.

The bladder of the rabbit arises as a ventral diverticulum of the rectum, and is at first entirely independent of the kidney-ducts, which open into the dorsal wall of the rectum, a condition retained throughout life in the frog. During development the ducts shift round so as to open into the bladder, which then separates from the rectum and acquires 
a separate opening to the exterior, through which the urinary and genital products leave the body.

It is convenient to describe the two sexes separately, as they differ very greatly; and to include in the account certain glands in the neighbourhood of the rectum.

\section{A. The Male Rabbit.}

\section{The renal system.}

a. The kidneys are a pair of dark red bodies about an inch and a quarter long, imbedded in fat in the dorsal wall of the abdomen, outside the peritoneum. Each is of a flattened ovoid shape, with a notch, the hilus, on its inner side where the ureter and blood-vessels enter and leave it.

Bisect one of the kidneys longitudinally, from its outer edge; and turn the ventral half inwards, so as to expose the. cut surfaces.

i. The cortical substance forms the superficial layer of the kidney; the Malpighian bodies, which are confined to it, give it a dotted appearance.

ii. The medullary substance, forming the deeper part of the kidney, is radially striated, and consists mainly of the non-glandular parts of the tubules. The inner surface of the medullary substance is raised into a number of conical papillæ or pyramids, on which the tubules open.

iii. The pelvis is the expanded anterior end of the ureter, which lies in the cavity of the kidney, and receives the urine from the apertures on the pyramids.

b. The ureters are a pair of slender tubes, running back from the kidneys along the dorsal wall of the abdomen, a short distance from the middle line. At the hinder end of the abdomen, they turn inwards and open into the bladder, opposite the anterior border of the pubic symphysis. 
c. The bladder is a thin-walled muscular sac, of which the anterior end, in front of the pubic symphysis, can alone be seen at this stage of the dissection.

\section{The reproductive system.}

The testes, in early life, lie against the dorsal wall of the abdomen, close to the kidneys. Before maturity, however, they separate from the dorsal wall, and, slipping down to the ventral wall, pass along the inguinal canals into the scrotal sacs.

a. The scrotal sacs are a pair of muscular pouch-like diverticula of the abdominal wall, lying ventral to the pelvis and close to the median plane.

Slit up one of the scrotal sacs along its ventral surface to expose the testis lying in it.

b. The testes.

The testes are a pair of elongated ovoid bodies, of a pink colour, varying greatly in size at different times, and about an inch and a half in length when fully developed. They are attached to the bottoms of the scrotal sacs, but can be readily pulled back into the abdominal cavity.

c. The epididymes are a pair of irregular masses of convoluted tubes, lying along the inner edges of the testes. Each epididymis consists of (1) a soft pinkish body, the caput epididymis, in close contact with the anterior end of the testis: (2) a narrow band, extending backwards from the caput. along the testis: and (3) a larger, more conical, and much convoluted body, the cauda epididymis, lying at the posterior end of the testis, and connected by a short cord, the gubernaculum, with the bottom of the scrotal sac.

The epididymes are the modified mesonephra of the embryo, the tubules of which grow into the testes, and so form the vasa efferentia, through which the spermatozoa escape from the testes. 
The spermatic arteries and veins, which supply the testes, enter the epididymes at their anterior ends.

d. The vasa deferentia are a pair of whitish tubes one of which arises from each cauda epididymis and runs forwards, alongside the testis, to enter the abdominal cavity through the inguinal canal. Within the abdomen it loops round the ureter from the outer to the inner side, and runs back to the neck of the bladder. The hinder part of its course is sinuous, but further forwards it becomes straighter.

The vasa deferentia are the modified mesonephric ducts of the embryo.

Dissect both scrotal sacs from the abdominal wall, and turn them forwards with the testes and vasa deferentia. Determine with the finger the boundaries of the pubes and ischia. Cut longitudinally through the muscles ventral to the pelvis, about half an inch from the middle line on each side, and down to the bone. Scrape clean the descending rami of the pubes and ischia, and cut through them with bone-forceps in front of, and behind, the obturator foramen on each side. Remove the pelvic symphysis, which is now isolated; carefully dissecting it from the underlying parts. Note the corpora cavernosa, a pair of hard ligamentous bodies running backwards from the hinder border of the ischia to the penis; and out them across close to the ischia.

Inflate the bladder and urino-genital canal by a blowpipe inserted into the terminal aperture of the penis, and tie a ligature round the penis half an inch from its end, to prevent the air escaping.

Clean the various structures in the order given below.

e. The urino-genital canal, or urethra, is the continuation backwards of the neck of the bladder, through the pelvic cavity. It is a straight tube, with thin and very vascular walls, which lies immediately dorsal to the pelvic symphysis, and ventral to the 
rectum. Behind the symphysis it is continued into the penis.

f. The penis is an elongated body, projecting from the ventral surface of the animal, immediately behind the pelvic symphysis and in front of the anus. It is traversed by the urino-genital canal, through which the urinary and genital products are discharged. The posterior or dorsal wall of the penis is very vascular, and forms the corpus spongiosum; while its ventral or anterior surface is stiffened by the corpora cavernosa. The free end of the penis is soft, and obliquely truncated; and is invested by a loose sheath of skin, the prepuce. The external aperture of the urethra is a slit-like opening on its posterior surface.

g. The uterus masculinus is a large median sac, slightly bilobed in front, attached to the dorsal surface of the urino-genital canal, into which it opens, and extending forwards along the dorsal surface of the bladder to about the middle of its length.

h. The posterior ends of the vasa deferentia are slightly dilated, and run back, side by side, along the dorsal surface of the neck of the bladder, between it and the uterus masculinus. They open separately into the ventral wall of the uterus masculinus, just in front of its communication with the urino-genital canal, and opposite the hinder end of the obturator foramen.

Slit up the uterus masculinus along one side with scissors. Note the large aperture leading into the urino-genital canal, and, just in front of this, the small paired openings of the vasa deferentia.

i. The prostate is a series of glandular masses, placed around the dorsal surface and sides of the uterus masculinus and vasa deferentia, and opening by 
short ducts into the urino-genital canal. There are four or five lobes, the largest of which is an anterior lobe, closely applied to the dorsal wall of the uterus masculinus, and bilobed in front: behind this is a smaller posterior lobe, sometimes divided into two: a pair of still smaller lateral lobes lie in the angle between the vasa deferentia and the uterus masculinus.

k. Cowper's glands are a pair of glandular bodies, varying greatly in size, and placed on the dorsal wall of the urino-genital canal, behind the prostate.

1. The rectum is a white tube, with rather thick muscular walls, lying dorsal to the urino-genital organs, and close to the ventral surface of the sacrum.

m. The perinæal glands are a pair of elongated glandular bodies, dark in colour, and about threequarters of an inch long. They lie at the sides of the penis, and in the anterior walls of the perinæal sacs, into which their ducts open.

n. The rectal glands are a pair of pale yellowish bodies, much larger than the perinæal glands, and lying along the sides and posterior surface of the last two inches of the rectum.

Slit up with scissors the urino-genital canal along its whole length, in the mid-ventral line.

o. The aperture of the uterus masculinus is a large crescentic opening in the dorsal wall of the urino-genital canal.

p. The verumontanum is a small vascular papilla, immediately behind the aperture of the uterus masculinus.

q. The apertures of the ducts of the prostatic glands are small slit-like openings at the sides of the verumontanum. 


\section{B. The Female Rabbit.}

1. The urinary system is the same as in the male, except that the ureters open into the bladder much further forwards close to its anterior end.

\section{The reproductive system.}

Remove the pelvic symphysis as in the male (p. 338); and inflate the vagina and bladder with a blowpipe, inserted into the vulva. Ligature the vestibule about an inch in front of the vulva, to prevent the air escaping.

a. The ovaries are a pair of oval bodies, of a pale yellowish colour, and about three-quarters of an inch long, attached to the dorsal wall of the abdomen, behind the kidneys and about an inch in front of the crests of the ilia.

On their surfaces the Graafian follicles, each of which contains an ovum, are visible as small rounded semi-transparent projections.

b. The oviducts are formed from the pronephric ducts of the embryo. Their anterior ends are narrow, and form the Fallopian tubes: the middle portions are wider, and become the uteri, within which the young are developed; and the posterior ends unite to form a median tube, the vagina.

i. The Fallopian tubes are a pair of narrow, slightly convoluted tubes, about the size of the ureters. They lie along the free edges of the broad ligaments, a pair of folds of peritoneum suspending them from the dorsal wall of the abdomen.

The anterior ends of the Fallopian tubes form wide, membranous, funnel-shaped mouths, lying along the outer sides of the ovaries, and attached to their anterior ends.

ii. The uteri, which are continuous with the posterior ends of the Fallopian tubes, are a pair of thick-walled tubes, which vary enormously 
in size according to the presence or absence of embryos, and the stage of their development. The two uteri open by separate apertures into the anterior end of the vagina.

iii. The vagina is a very wide median tube, commencing a little in front of the crests of the ilia, and extending straight back to unite, within the pelvic carity, with the neck of the bladder, at the commencement of the vestibule.

c. The vestibule or urino-genital canal is a wide median tube, commencing opposite the hinder end of the obturator foramen, and running back through the pelvic cavity, ventral to the rectum, to the vulva. Its walls are very vascular, and correspond to the corpus spongiosum in the male.

d. The clitoris is a small rod-like body, corresponding to the penis of the male, and lying in the anterior or ventral wall of the vestibule, just within its external opening. It ends in a soft conical body, the glans clitoridis, and is connected with the ischia by two corpora cavernosa similar to those of the male, but of smaller size.

e. Cowper's glands are small, and lie on the dorsal wall of the vestibule, opposite the hinder border of the pelvic symphysis.

f. The perinæal and rectal glands are similar to those of the male (p. 340).

Slit up with scissors the vestibule and hinder part of the vagina along one side. Note the great vascularity of the walls, the position and relations of the clitoris, and the opening of the bladder into the vestibule.

Slit up the anterior part of the vagina along the midventral line, to expose the openings of the uteri into the vagina. Note the very prominent lips of these openings. 


\section{DISSECTION OF THE NECK.}

A special dissection of the neck is desirable on account of the great importance of the structures contained in it, more particularly the nerves.

Lay the rabbit on its back on a dissecting-board; and pin back the head so as to extend the neck strongly. Make a median ventral incision through the skin of the neck, and dissect the skin away from the underlying parts, taking care not to damage these.

\section{A. The Hyoid Bone.}

The body of the hyoid is a transverse bar of bone, deeply placed between the rami of the mandible, and about midway between the hinder end of the mandibular symphysis and the angles of the jaw.

The posterior cornua of the hyoid are a pair of slender rods of bone, about half an inch long, running backwards and outwards from the outer angles of the body of the hyoid, parallel to the angles of the jaw.

Determine the position and shape of the hyoid by feeting with the finger.

\section{B. The Trachea.}

The trachea or windpipe is a straight tube, running down the neck almost in the middle line. It is only partially exposed at present, but will be seen better in the course of the dissection. Its anterior end, which is placed almost immediately behind the hyoid, is dilated, and surrounded on the ventral and lateral surfaces by the wide thyroid cartilage, which forms a prominent swelling in the throat, placed between the rami of the mandible and readily identified by the finger. Behind this is the annular cricoid cartilage, which is wide dorsally and narrow ventrally. Behind the cricoid the trachea narrows slightly, and is surrounded by a series of cartilaginous rings, incomplete along the mid-dorsal line. The trachea runs backwards along the neck, and, entering the thorax, divides about the level of the middle of 
the heart into the right and left bronchi, which enter the right and left lungs respectively.

\section{The Thyroid Body.}

The thyroid is a soft vascular body, consisting of a pair of lateral lobes at the sides of the anterior part of the trachea, connected by a narrow median lobe, which runs across the ventral surface of the trachea a short way behind the thyroid cartilage.

\section{The Muscles of the Neck.}

A few of the more prominent of the ventral muscles of the neck are alone described here.

1. The sterno-mastoid muscles are a pair of narrow but strong muscular bands, which arise from the anterior end of the sternum, close to the middle line, and run obliquely forwards and outwards, across the front of the neck, to be inserted into the mastoid processes of the skull immediately behind the ears.

Cut through the right sterno-mastoid close to its origin from the sternum, and turn it forwards. Near its anterior end, note, and carefully preserve, a branch of the spinal accessory nerve, which enters the muscle on its inner side, about half an inch behind the angle of the jaw.

2. The sterno-hyoid muscles are a pair of broad but thin muscular bands, running along the ventral surface of the neck. They arise from the dorsal surface of the anterior end of the sternum, where the two are fused, and run forwards along the neck. A little behind the angle of the jaw, the two muscles diverge slightly from each other, and are inserted into the bases of the posterior cornua of the hyoid. Their hinder ends are covered by the sterno-mastoids.

3. The stylo-hyoid muscles are a pair of short muscles, running almost transversely across the neck, opposite the angles of the jaws. They arise from the under surface of the tympanic bullæ, close to the insertions 
of the sterno-mastoid muscles, and are inserted into the tips of the posterior cornua of the hyoid.

4. The mandibular muscles are a pair of slender muscles, triangular in shape, which arise from the tips of the par-occipital processes of the ex-occipital bones by long thin tendons, running forwards and inwards, dorsal to the stylo-hyoid muscles, and parallel to the rami of the mandible. The anterior part of each is muscular, and is inserted into the inner surface of the ramus of the mandible, close to the symphysis.

5. The sterno-thyroid muscles are a pair of thin bands of muscle, lying immediately dorsal to the sterno-hyoid muscles, and seen on dissecting along the outer borders of the latter. They are fused posteriorly, and arise from the sternum as a median band in common with the sterno-hyoid : further forwards the muscles diverge, and run along the ventral surface and sides of the trachea, to be inserted into the sides of the thyroid cartilage.

\section{E. The Blood-vessels of the Neck.}

The two most important, on each side, are the following :

1. The external jugular vein has already been seen.

2. The carotid artery runs forwards along the outer borders of the sterno-hyoid and sterno-thyroid muscles.

Clean the carotid artery carefully, taking great care to avoid injuring the nerves which lie close alongside it, and a nerve which crosses its ventral surface about the level of the thyroid cartilage.

\section{F. The Nerves of the Neck.}

Expose and clean the following nerves on either the right or the left side of the neck.

1. The spinal accessory nerve, the eleventh cranial nerve, leaves the skull at the foramen lacerum posterius, between the auditory capsule and the ex-occipital bone, in company with the pneumogastric and glossopharyngeal nerves. Outside the skull it runs almost 
vertically downwards for about half an inch, and then divides into branches distributed to the sternomastoid and other muscles.

The branch to the sterno-mastoid has already been seen (p. 344). Follow this back to its junction with the main stem of the nerve, and then trace this latter up to the base of the skoull.

2. The hypoglossal nerve, the twelfth cranial, is a stout nerve which leaves the skull by the condylar foramina, immediately behind the foramen lacerum posterius, and lies at first very close to the pneumogastric, glosso-pharyngeal, and spinal accessory nerves. Outside the skull it runs downwards and inwards for about half an inch, and then turns forwards, crossing ventral to the carotid artery at its point of division into internal and external carotids. It then runs forwards and inwards, along the dorsal surface of the stylo-hyoid muscle, and parallel to the posterior cornu of the hyoid, to the base of the tongue, the muscles of which it supplies.

The hypoglossal nerve is readily found, where it turns forwards between the carotid artery and the stylo-hyoid muscle; and should be followed from this point backwards to the skull, and forwards to the tongue.

a. The ramus descendens noni is a branch of the hypoglossal, which arises from the main nerve just before it reaches the carotid artery. It runs back along the outer border of the carotid for about half an inch, giving branches to some of the muscles of the neck. It then crosses the carotid, about the level of the lower border of the thyroid cartilage, and runs back on the sternothyroid muscle, to which as well as to the sternohyoid it sends branches.

The descendens noni is readily found crossing the carotid about the point named above, and should then be traced back- 
wards along the sterno-thyroid muscle, and forwards to the point at which it leaves the main stem of the hypoglossal.

3. The pneumogastric or vagus nerve, the tenth cranial, is a stout nerve which leaves the skull by the foramen lacerum posterius, presenting a very distinct swelling near its origin. It runs downwards and backwards until it reaches the carotid artery, and then straight backwards along the neck, lying immediately to the outer side of, and slightly dorsal to, the carotid artery. Entering the thorax, it runs alongside the csophagus to the stomach, where it ends.

Its principal branches are described below.

Find the pneumogastric nerve in the neck, along the outer side of the carotid artery. Follow it along the neck to the thorax, and forwards to the stoull.

a. The superior laryngeal nerve is a small nerve, arising from the pneumogastric opposite the upper border of the thyroid cartilage. It runs almost directly inwards, passing dorsal to the carotid artery, and ends in branches distributed to the mucous membrane of the larynx (p. 350), and to the crico-thyroid muscle.

The sole difficulty in dissecting the superior laryngeal nerve lies in the possibility of confusing it with the descendens noni. This is avoided if it be remembered that, while both nerves cross the carotid artery, the descendens noni lies ventral to the artery, and the superior laryngeal dorsal to it.

i. The depressor nerve is a very slender branch of the superior laryngeal nerve, which runs backwards along the neck; lying dorsal to the carotid artery, and along the inner side of the main stem of the pneumogastric nerve, very close to the sympathetic nerve-trunk. It ends. in the heart.

Find the depressor nerve where it leaves the superior laryngeal nerve, about the level of the posterior end of the 
thyroid cartilage, and follow it back along the neck. Its dissection in the thorax is difficult.

b. The inferior or recurrent laryngeal nerve arises, on the right side, from the pneumogastric nerve at the posterior end of the neck. It loops round the subclavian artery, and then runs forwards along the neck, lying alongside the trachea, and dorsal to the carotid artery, or slightly to its inner side. It supplies all the muscles of the larynx, except the crico-thyroid.

On the left side, the nerve is not given off until. after the pneumogastric has entered the thorax. It loops round the arch of the aorta, and then runs forwards along the neck to the larynx.

Find the nerve, as it loops round the subclavian artery, or on the left side the arch of the aorta; and follow it forwards along the neck to the larynx.

The curious course of the recurrent laryngeal nerves is explained by the shifting backwards of the heart, which occurs during development. As this shifting involves the roots of the great vessels, these nerves become pulled out into loops.

c. Branches of the pneumogastric to the heart, lungs, and esophagus. These are of small size, and are given off in the thorax : the cardiac branches end in a plexus, lying between the roots of the aorta and pulmonary artery.

Turn the lungs and heart over to one side: follow the pneumogastric back through the thorax, alongside the cesophagus, and trace out its branches.

4. The cervical sympathetic nerve lies alongside the trachea, to the inner side of the carotid artery, and in close proximity to the depressor nerve.

About the level of the angle of the jaw, and dorsal to the stylo-hyoid muscle, it has a small oval swelling, the superior cervical ganglion and at the pos- 
terior end of the neck, a short distance in front of the subclavian artery on either side, there is a similar swelling, the inferior cervical ganglion.

The sympathetic nerves are of a pale pinkish colour, and are not nearly so easy to see as the white cords of the pneumogastric and its branches.

5. The great auricular nerve is a large branch of the third spinal nerve, given off immediately after the nerve emerges from the spinal canal, between the second and third cervical vertebræ. It runs vertically upwards to the base of the ear, lying along the posterior border of the parotid gland, and about half an inch behind the angle of the jaw : it then continues its course along the outer surface of the pinna of the ear, as far as its tip, lying just beneath the skin.

The nerve can readily be felt as a cord running along the pinna, or seen by holding the ear up to the light, and looking through it.

Having determined its position in one of these ways, cut down upon it, and follow it to the tip of the ear, and downwards to its point of emergence from the vertebral canal.

6. The phrenic nerve is a branch of the fourth cervical nerve, which arises just beyond the point of emergence of this nerve from the neural canal, and runs back alongside the vertebral column. Entering the thorax it continues its course backwards, lying between the heart and the lungs on either side, and ends in the diaphragm, which it supplies with motor fibres.

In the neck it crosses the roots of the hinder cervical nerves almost at right angles. It receives a small branch from the fifth cervical nerve, and sometimes one from the sixth, as it passes them.

Press the heart and right lung gently apart to expose the phrenic nerve. Follow the nerve back to the diaphragm, and forwards along the neck to its origin. 


\section{G. The Larynx.}

The larynx, or organ of voice, is formed by the anterior part of the trachea. The thyroid and cricoid cartilages form its outer wall, and it is overhung in front by the epiglottis.

Make a median longitudinal section through the anterior part of the trachea.

1. The thyroid cartilage forms the most anterior ring of the trachea : it is about half an inch in length along the mid-ventral line, but is incomplete dorsally.

2. The cricoid cartilage, or second ring of the trachea, is a complete ring, lying immediately behind the thyroid. It is narrow ventrally, but wide dorsally; and articulates with the postero-dorsal angles of the thyroid cartilage.

3. The arytenoid cartilages are a pair of cartilaginous nodules, articulated to the anterior edge of the dorsal surface of the cricoid cartilage.

4. The ventricles of the larynx are a pair of shallow lateral depressions of the mucous membrane of the inner surface of the larynx.

5. The vocal cords are a pair of folds of mucous membrane, strengthened by elastic ligaments, and forming the posterior lips of the ventricles. They are attached dorsally to the arytenoids, and at their ventral ends to the thyroid cartilage. It is by their vibration that the voice is produced.

6. The glottis is the narrow vertical slit between the vocal cords, through which the air passes to and from the lungs.

7. The false vocal cords are folds of mucous membrane similar to the vocal cords, which form the anterior boundaries of the ventricles.

\section{DISSECTION OF THE BRAIN.}

The brain should be removed directly after the rabbit is killed, and placed at once in strong spirit, as it decomposes rapidly. It is well to put a loose pad of cotton-wool in the 
bottom of the bottle, and place the brain upon it, in order to prevent flattening from contact with the glass, and to ensure free access of the spirit to all parts of the surface.

To remove the brain, first stin the head; then expose the brain by snipping away, with the bone-forceps, the roof and sides of the skull, beginning at the foramen magnum, and working forwards. Take special care in the region of the ears to avoid injury to the floccular lobes of the cerebellum, which are lodged in the floccular fossa of the periotic bones (p. 282).

Having completely removed the roof and sides of the cranium, and cut through the dura mater, a tough fibrous membrane which lines the cranial cavity, cut across the spinal cord about half an inch behind the foramen magnum, and turn out the brain from behind forwards, lifting it up gently with the handle of a scalpel.

Note and identify the several nerve-roots as you do so, and cut through them in order, close to the skull-wall, so as to leave as much of the roots as possible attached to the brain.

Place the brain in spirit at once, handling it with great care, as it is very soft and easily damaged. Leave it in spirit for three or four days, and then dissect it as described below.

The several divisions of the rabbit's brain are the same as those of the brain of the dog-fish, the most important differences between the two being, (1) the great size of the cerebral hemispheres, which overlap and conceal the dorsal and lateral surfaces of the thalamencephalon and optic lobes ; (2) the greater size and more complicated structure of the cerebellum; (3) the much greater development of the transverse commissures, connecting the two halves of the brain with each other.

Remove the hardened brain from spirit, and examine it in a small dish of water or weak spirit.

\section{A. The Brain-membranes.}

1. The dura mater is the tough connective-tissue membrane which lines the cranial cavity. The greater part or the whole of it is left in the skull on removal of the brain. It projects into the cranial cavity as a median 
vertical fold, the falx cerebri, which lies between the hemispheres; and a transverse fold, the tentorium, which separates the hemispheres from the cerebellum.

2. The pia mater is a much thinner and very vascular layer of connective tissue, which closely invests the brain, and conveys blood-vessels to and from the brain substance.

\section{B. External Characters of the Brain.}

Strip off the pia mater from the brain with fine forceps as fully as possible, taking great care on the base of the brain not to drag away the nerve-roots.

1. The dorsal surface of the brain.

a. The cerebral hemispheres, which form the anterior two-thirds of the brain, are a pair of broad triangular bodies, pointed in front and closely apposed to each other along the median plane.

Their surfaces are smooth and convex : they are marked by a few shallow grooves, or sulci; and they are divided by rather more conspicuous grooves at their outer edges into anterior or frontal, and posterior or parietal lobes.

The two hemispheres are connected with each other by a large transverse commissure, the corpus callosum, which is readily seen on gently separating the hemispheres with the handle of a scalpel.

b. The olfactory lobes are a pair of large club-shaped bodies, projecting forwards from beneath the anterior ends of the hemispheres.

c. The thalamencephalon is covered by the hemispheres, and cannot be seen from the dorsal surface.

d. The corpora quadrigemina, or optic lobes, are two pairs of rounded elevations on the dorsal surface of the brain, and almost completely covered by the hemispheres. 
e. The cerebellum is a large transversely elongated mass, lying behind the hemispheres. It is divided into a large median lobe, the vermis, and two lateral lobes, to the outer sides of which are the small rounded floccular lobes.

The whole surface of the cerebellum is marked by close-set folds, mainly transverse in direction. f. The medulla oblongata is the part of the brain beneath and behind the cerebellum. It is some-

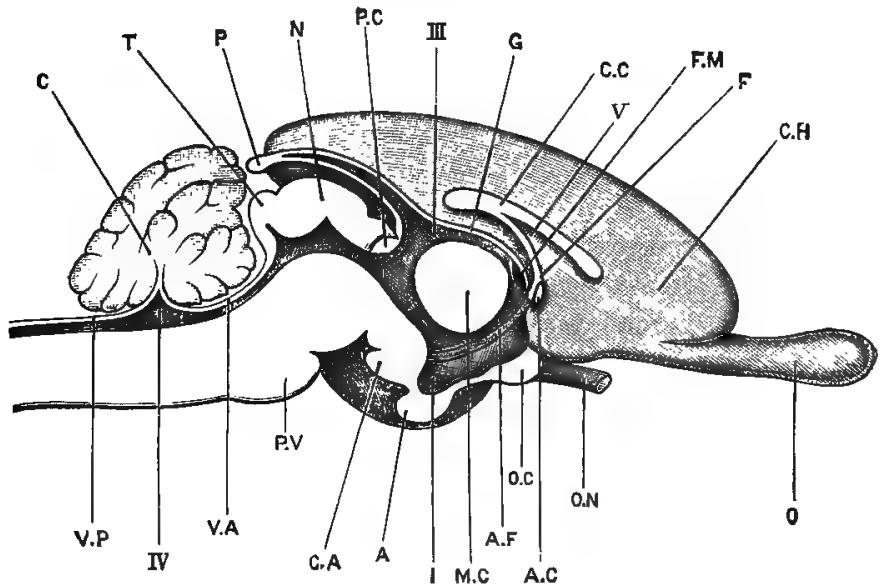

Frg. 61.-Lepus cuniculus. A longitudinal and vertical section of the brain, taken in the median plane. (A. M. M.)

$\mathbf{A}$, pituitary body. AC, anterior commissure. AF, anterior pillar of the fornix, seen in the wall of the third rentricle. $\mathbf{C}$, cerebellum. CA, corpus albicans. CC, corpus callosum. CH, inner surface of left cerebral hemisphere. $\mathbf{F}$, body of the fornix. FM, foramen of Monro. $\mathbf{G}$, velum interpositum. $\mathbf{I}$, infundibulum. MC, middle commissure. $\mathbf{N}$, anterior lobe of corpora quadrigemina, or ' nates.' $O$, olfactory lobe. OC, optic chiasma. ON, left optic nerve. $\boldsymbol{P}$, pineal body. $\mathbf{P O}$, posterior commissure. $\mathbf{P V}$, pons $\mathbf{V}$ arolii. $\mathbf{T}$, posterior lobe of corpora quadrigemina, or 'testis.' $\mathbf{V} \mathbf{A}$, velum medulle anterius, or valve of Vieussens. $\boldsymbol{V} \mathbf{P}$, velum medulla posterius.

III, third ventricle. IV, fourth ventricle. V, fifth ventricle.

what flattened dorso-ventrally, is widest in front, and gradually narrows behind, passing into the spinal cord.

i. The velum medullæ anterius, or valve of Vieussens, is a thin transparent membrane, 
connecting the hinder border of the optic lobes with the cerebellum, and roofing over the anterior part of the fourth ventricle. It lies beneath the anterior part of the cerebellum, which must be gently pressed backwards to expose it.

ii. The velum medullæ posterius is a thin transparent membrane, forming the roof of the posterior part of the fourth ventricle: it is covered in front by the cerebellum.

g. The pineal body is a small rounded median body, lying on the optic lobes, and connected by a stalk with the roof of the thalamencephalon.

It is visible on the dorsal surface, in the angle between the hinder ends of the cerebral hemispheres and the cerebellum, but is very liable to be torn away with the dura mater. It has recently bcen shown to be a degenerate eye.

2. The ventral surface of the brain.

a. The cerebral hemispheres, as seen from below, are closely apposed in front, but diverge posteriorly. About the middle of its length the inner border of each hemisphere presents a notch, which is continued outwards across the surface of the hemisphere as a shallow groove, the Sylvian fissure, separating the frontal lobe from the temporal.

b. The olfactory lobes lie, in their hinder portions, along the under surface of the frontal lobes of the hemispheres, extending back as far as the Sylvian fissures. In front, they project some distance beyond the hemispheres.

c. The infundibulum is a median rounded elevation, lying between the temporal lobes, about the middle of the length of the brain : at its apex is the rounded vascular pituitary body. This latter is usually left behind in the pituitary fossa in 
the base of the skull when the brain is removed: in this case there is at the apex of the infundibulum a slit-like aperture, leading into the third ventricle.

d. The optic chiasma is a transverse band of nervefibres, lying immediately in front of the infundibulum. From its anterior border the optic nerves arise, and from its outer ends the optic tracts may be followed backwards towards the optic lobes by gently pressing aside the temporal lobes.

e. The corpus mammillare, or corpus albicans, is a small rounded median elevation, immediately behind the infundibulum.

f. The pons Varolii is a stout band of transverse nerve-fibres, which crosses the ventral surface of the medulla, opposite the posterior ends of the hemispheres. It connects the two sides of the cerebellum with each other.

g. The crura cerebri are two large bands of nerve-fibres, immediately in front of the pons Varolii, which run forwards, diverging from each other, and connect the medulla oblongata with the hemispheres.

h. The medulla oblongata, as seen from the ventral surface, is broad in front and gradually narrows behind. It is marked by a median ventral fissure, bordered by two narrow bands, the ventral pyramids. At the outer sides of the pyramids, immediately behind the pons Varolii, are a pair of oblong patches of transverse nerve-fibres, the corpora trapezoidea.

\section{The Roots of the Oerebral Nerves.}

There are twelve pairs of cerebral or cranial nerves in the rabbit, all of which, with the exception of the fourth pair, arise from the ventral surface or from the sides of the brain. 


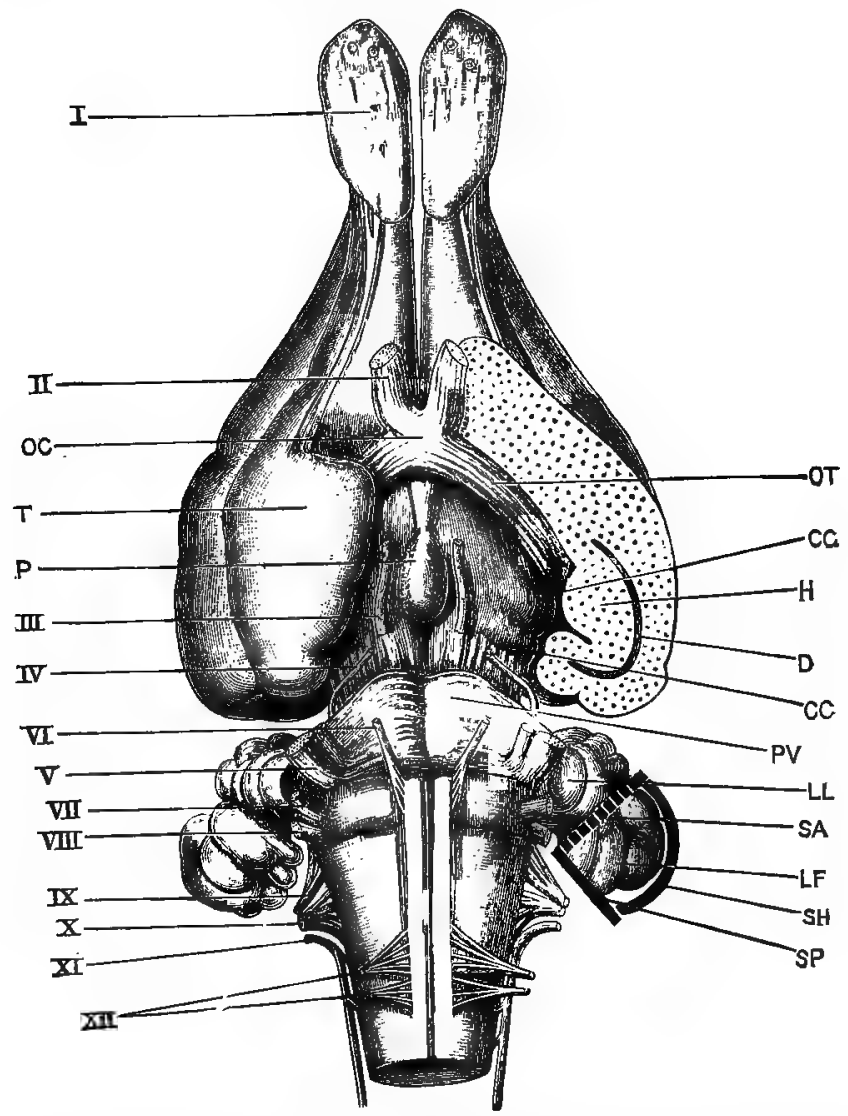

Frc. 62.-Iepus cuniculus. -The brain from the ventral surface. The greater part of the left temporal lobe has been sliced off horizontally. The planes of the three semicircular canals of the left side are indicated by the thick lines surrounding the floccular lobe of the cerebellum; $\times 2$. (A. M. Mr.)

CC, crus cerebri. CG, corpus geniculatum, D, descending cornu of left lateral ventricle. H, hippocampus major. IF, floccular lobe of cerebellum. LI, lateral lobe of cerebellum. OC, optic chiasma. OT, optic tract. $\mathbf{P}$, pituitary body. $\mathbf{P V}$, pons Varolii. SA, anterior vertical semicircular canal. SH, horizontal semicircular canal. SP, posterior vertical semicircular canal. T, temporal lobe of cerebral hemisphere.

I, olfactory lobe, with roots of olfactory nerves. II, optic nerve. III, third nerve or motor oculi. IV, fourth nerve. V, trigeminal nerve. VI, sixth nerve or abducens. VII, facial nerve. VIII, auditory nerve. IX, glosso-pharjngeal nerve. $\mathbf{X}$, pneumogastric nerre. $\mathbf{X} \mathbf{I}$, spinal accessory nerve. XII, hypoglossal nerve. 
Identify, and clean the nerve-roots on one side of the brain, in the order given below, taking great care not to drag them away from the brain.

1. The olfactory nerve arises, by many roots, from the ventral surface of the anterior end of the olfactory lobe. These roots are almost necessarily removed in taking out the brain.

2. The optic nerve is a stout nerve, running forwards from the anterior border of the optic chiasma, and then curving outwards.

3. The oculo-motor nerve is small, and arises from the inner border of the crus cerebri, close to the middle line, and immediately behind the corpus mammillare.

4. The pathetic nerve is very small. It arises from the dorsal surface of the valve of Vieussens, close to the middle line, and just behind the optic lobes. It then runs round the side of the brain to the ventral surface, lying between the cerebellum and optic lobe.

Find the nerve on the ventral surface, and follow it round the side of the brain to its origin.

5. The trigeminal nerve is very large, and arises by two closely apposed roots from the side of the hinder border of the pons Varolii. Of the two roots the inner and smaller one is motor, the larger outer one sensory in function.

6. The abducent nerve is very slender, and arises from the ventral surface of the medulla, close to the middle line, and immediately behind the pons Varolii.

7. The facial nerve arises from the outer side of the anterior end of the corpus trapezoideum, immediately behind the trigeminal nerve.

8. The auditory nerve is large, and arises from the side of the medulla, immediately behind and to the outer side of the facial nerve.

9 and 10. The glosso-pharyngeal and pneumogastric nerves arise close together, by many slender rootlets, from 
the side of the medulla, close behind, and a little above the root of the auditory nerve.

11. The spinal accessory nerve arises from the side of the medulla and spinal cord by about ten rootlets, the most anterior of which is just behind the pneumogastric, and the hindmost as far back as the fifth spinal nerve.

12. The hypoglossal nerve arises by a number of rootlets from the ventral surface of the medulla, close to the middle line, and to the outer side of the ventral pyramid.

\section{The Structure of the Brain.}

The brain is, from the mode of its formation, tubular; its cavity being direetly continuous with the central canal of the spinal cord. From a very early period it presents a series of dilatations or vesicles, partially separated from one another by constrictions. Of these vesicles, the first or most anterior one is the fore-brain, or thalamencephalon, from which the cerebral hemispheres arise as hollow outgrowths; the second vesicle is the mid-brain or mesencephalon; and the succeeding ones, of which there are at least four or five, are spoken of collectively as the hind-brain. The cavities of the vesicles remain in communication with one another throughout life, and are spoken of in the adult as the ventricles of the brain.

\section{Dissection of the brain.}

\section{a. The cerebral hemispheres.}

Gently press the hemispheres apart, and note the position of the corpus callosum.

Then, with the back of a knife, peel off the portion of the right hemisphere lying above the corpus callosum. Keep the brain wet with spirit.

i. The corpus callosum is a broad horizontal band of fibres connecting the two hemispheres.

ii. The structure of the hemisphere.

$\alpha$. The outer layer or cortex is a superficial investment of grey matter, about an eighth of an inch thick. 
$\beta$. The central or medullary portion consists of white matter, i.e. of medullated nerve-fibres. The fibres of the corpus callosum spread out into, and are continuous with it.

iii. The lateral ventricle, or cavity of the hemisphere.

Cut through the corpus callosum on the right side, so as to open the lateral ventricle beneath it. Lift up the corpus callosum, and carefully out it away, so as to expose the ventricle fully, leaving a narrow strip along its inner edge.

The lateral ventricle, opposite the middle of the hemisphere, is wide from side to side, but shallow dorso-ventrally. Anteriorly, it extends forwards as a narrow vertical slit, the anterior cornu, which lies nearer the inner than the outer surface of the hemisphere.

Posteriorly, the lateral ventricle curves backwards and inwards towards the hinder end of the hemisphere as the posterior cornu ; and from its outer side, a little behind the middle of the hemisphere, a descending cornu runs outwards and downwards, and then curves round in the temporal lobe to the ventral region, running forwards and inwards almost to the anterior end of the lobe, and ending opposite the infundibulum.

Open up the descending cornu along its whole length, by carefully cutting away the outer and ventral walls of the temporal lobe.

iv. The hippocampus major is a prominent convex ridge, projecting into the floor and inner side of the descending cornu, along its whole length.

v. The corpus striatum is a thickened part of the wall of the hemisphere, forming the outer side and floor of the anterior part of the lateral 
ventricle. It lies in front of the hippocampus major, from which it is separated by a deep groove, running obliquely backwards and outwards across the floor of the ventricle.

Cut away carefully the outer wall of the anterior cormu of the lateral ventricle, including the corpus striatum.

vi. The septum lucidum is the thin vertical inner wall of the hinder part of the anterior cornu. It is placed opposite the corpus striatum, and ventral to the corpus callosum.

vii. The foramen of Monro is a small hole immediately beneath the hinder border of the septum lucidum, through which the lateral ventricle communicates with the third ventricle, or cavity of the thalamencephalon.

viii. The posterior pillar of the fornix is a narrow band, lying along the anterior edge of the hippocampus major. Posteriorly it follows the hippocampus into the descending cornu; while anteriorly it becomes continuous with its fellow of the opposite side in the median plane, forming the body of the fornix, immediately above the foramen of Monro.

ix. The choroid plexus is a thin vaseular fold of the inner wall of the hemisphere, projecting into the descending cornu in front of the hippocampus major, and closely applied to the anterior surface of the latter along its whole length.

$x$. The 'fifth ventricle' is a median vertical cleft between the right and left hemispheres. It lies below the corpus callosum and above the fornix, and is bounded laterally by the inner walls of the two hemispheres (septum lucidum).

It is merely a part of the space between the two hemispheres, partially enclosed by the 
corpus callosum and the fornix ; and is not, properly speaking, a 'ventricle' at all.

To expose the fifth ventricle from above, carefully remove the narrow strip of the corpus callosum that has been left along the inner side of the hemisphere, and the fifth ventricle will be seen as a narrow cleft between the two hemispheres.

xi. The hippocampal sulcus is a deep groove on the surface of the temporal lobe. It gives rise to the inwardly projecting fold of the hemisphere which forms the hippocampus major.

Remove the temporal lobe completely, and the hippocampal sulcus will be seen on its inner surface.

b. The fore-brain, or thalamencephalon.

i. The optic thalami are a pair of large lateral thickenings of the sides of the fore-brain, placed immediately in front of the optic lobes, and overlapped by the temporal lobes of the hemispheres. They are separated by grooves from the corpora striata in front, and the optic lobes behind.

On the outer and ventral surface of each optic thalamus, near its anterior end, is a rounded elevation, the corpus geniculatum.

ii. The optic tracts are a pair of narrow bands, which can be traced backwards and upwards from the outer ends of the optic chiasma, over the surface of the optic thalami, to the anterior borders of the optic lobes.

Take hold of the anterior end of the right optic tract with the forceps, and strip it off the optic thalamus, so as to see its origin from the optic lobe.

iii. The velum interpositum is a thin membrane, forming the roof of the third ventricle. On its upper surface is a plexus of blood-vessels, continuous with the choroid plexuses of the lateral ventricles. 
iv. The pineal body is connected by a tubular stalk with the hinder end of the velum interpositum.

Remove the velum interpositum, so as to open the third ventricle.

v. The third ventricle, or cavity of the fore-brain, is a median vertical cleft between the optic thalami. It is very narrow from side to side, and communicates through the foramina of Monro with the lateral ventricles.

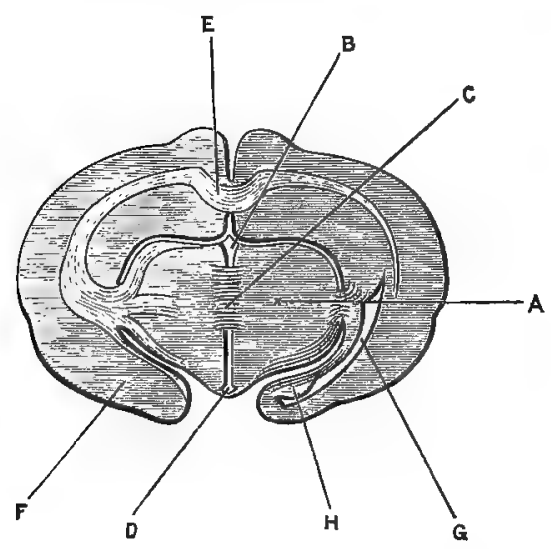

FIG. 63.-Lepus cuniculus. Transverse section of the brain, passing through the middle commissure of the third ventricle. The shading indicates diagrammatically the distribution of white and grey matter. The section is taken somewhat obliquely, so as to show different parts on the two sides. (A. M. M.)

A, optic thalamus, forming the side-wall of the third ventricle. $\mathbf{B}$, part of the third rentricle above the middle commissure. C, middle commissure. $\mathbf{D}$, infundibulum. $\mathbf{E}$, corpus gallosum. $\mathbf{F}$, temporal lobe of the left cerebral hemisphere. G, descending cornu of the right lateral ventricle. H, hippocampus major,

c. The mid-brain, or mesencephalon.

i. The corpora quadrigemina, or optic lobes, form the dorsal portion of the mid-brain, and are now well seen. 
ii. The crura cerebri form the base of the midbrain : after removal of the optic tracts they can be traced forwards and outwards from the anterior border of the pons to the optic thalami.

\section{Longitudinal section of the brain (fig. 61, p. 353).}

Divide the brain into right and left halves by a median longitudinal section. Place the left half in a small dish of spirit, and examine its inner surface.

If the dissection has been properly performed, the left half of the brain will be uninjured. If it has been damaged, a second brain should be taken, and bisected as directed above.

a. The cerebral hemisphere.

i. The corpus callosum is seen cut across, and its boundaries can easily be determined.

ii. The body of the fornix is a thin median band lying below the corpus callosum. It is continuous with this latter behind, but in front diverges from it considerably, the space between the two being the fifth ventricle.

b. The fore-brain, or thalamencephalon.

i. The third ventricle, though very narrow from side to side, is of considerable depth from the dorsal to the ventral surface. Owing to its narrowness, its boundaries are difficult. to determine.

The outline of the third ventricle can be rendered more obvious by adding a little ink or other colouring matter to the spirit in which the brain is examined.

ii. The commissures of the third ventricle are three bands of transverse fibres, connecting its sides together. Of the three the middle one alone really crosses the cavity of the ventricle.

a. The anterior commissure is a small band of transverse nerve-fibres, in the substance of 
the anterior wall of the ventricle, and immediately in front of the fornix.

$\beta$. The middle commissure is a very large band of fibres, connecting the two optic thalami across the ventricle. It is nearly circular in outline, and crosses the ventricle about its middle, filling up the greater part of it.

$\gamma$. The posterior commissure is a small band of transverse fibres in the roof of the hinder end of the third ventricle, just in front of the optic lobes.

iii. The velum interpositum is the thin membrane

which forms the roof of the third ventricle.

iv. The stalk of the pineal body is a tubular pro-

longation, upwards and backwards, of the roof

of the ventricle, just in front of the posterior commissure.

v. The lamina terminalis is the thin anterior wall of the ventricle.

vi. The optic chiasma is a thickening in the floor of the anterior end of the ventricle.

vii. The infundibulum is a funnel-like depression of the floor of the ventricle, about the middle of its length : to its apex the pituitary body is attached.

viii. The corpus albicans is a rounded swelling on the ventral surface of the floor of the ventricle, in the hinder wall of the infundibulum.

ix. The foramen of Monro is a vertical slit-like opening at the anterior end of the ventricle, between and slightly above the anterior and middle commissures. It leads from the third to the lateral ventricle.

$x$. The anterior pillar of the fornix is a slender band of nerve-fibres in the side-wall of the ventricle, running backwards and downwards from the body of the fornix, beneath the 
middle commissure, to the corpus albicans; in which it ends.

c. The mid-brain, or mesencephalon.

i. The Sylvian aqueduct, or iter a tertio ad quartum ventriculum, is the cavity or ventricle of the mid-brain.

ii. The optic lobes, or corpora quadrigemina, have moderately thick walls, and form the roof of the Sylvian aqueduct, which is prolonged a short distance into them.

iii. The crura cerebri, which form the floor of the Sylvian aqueduct, are of great thickness.

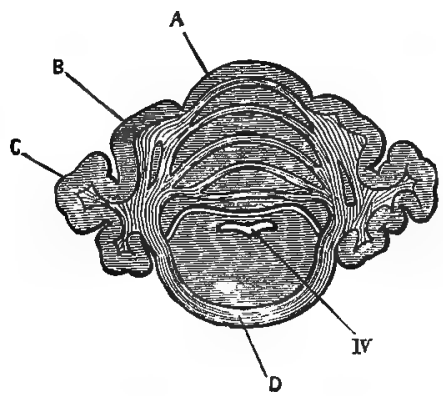

Frg. 64.-Lepus cuniculus.-Transverse section of the brain, passing through the widest part of the cerebellum. The shading indicates diagrammatically the distribution of white and grey matter. (A. … …)

A, vermis, or middle lobe of the cerebellum. B, lateral lobe of the cerebellum. C, floceular lobe of the cerebellum. D, transverse fibres of the pons Varolii. IV, fourth ventricle.

d. The hind-brain.

i. The fourth ventricle, or cavity of the hindbrain, is moderately wide from side to side, but shallow from above downwards. It has a very thin roof, but thick floor and sides. ii. The velum medullæ anterius, or valve of Vieussens, is a thin membrane extending from the optic lobes to the cerebellum, and forming the roof of the anterior half of the fourth ventricle. 
iii. The velum medullæ posterius is a thin membrane, forming the roof of the posterior half of the fourth ventricle.

iv. The cerebellum is attached to the dorsal surface, at the junction of the vela medullæ anterius and posterius. It consists of an outer layer of grey matter, which is very deeply folded, and a central much-branched mass of white matter, the section having a tree-like appearance, which has given rise to the name arbor vitæ.

v. The pons Varolii is seen, in transverse section, crossing the ventral surface of the anterior part of the hind-brain, opposite the velum medullæ anterius.

\section{Transverse sections of the brain.}

If another brain can be obtained, it should be cut with a razor into a series of transverse sections, about an eighth of an inch thick, and the sections arranged in order in a dish of spirit.

Such a series of sections affords very instructive views of the mutual relations of the several parts of the brain. 


\section{Chapter XIV.}

\section{THE SKELETON OF THE FOWL.}

Gallus bankiva, var. domesticus.

THe fowl's skeleton is, on account of its larger size, more convenient for study than that of the pigeon. The differences between the two are of but slight importance, and the following description will practically apply to either.

The bird's skeleton consists essentially of the same parts as the rabbit's, but presents certain special characteristics, of which the more important are : (1) the great lightness of all the bones, many of which contain large air-cavities; (2) the marked tendency of bones, primitively distinct, to fuse with one another in the adult; (3) the modifications in the limbgirdles and limbs, fitting it for flight and bipedal locomotion.

As in the case of the rabbit's skeleton, the names of the membrane-bones are printed in italics, those of the cartilagebones in thick type.

\section{THE AXIAL SKELETON.}

The axial skeleton, as in other vertebrates, is divisible into skull and vertebral column.

\section{A. The Vertebral Column.}

As compared with the rabbit's, the vertebral column of the bird is distinguished by, (1) the great length and flexibility of the neck, the cervical portion being nearly equal in length to the whole of the rest of the column; (2) the rigidity of the thoracic and abdominal portions of the column, owing to the fusion of their component vertebræ; (3) the great length of 


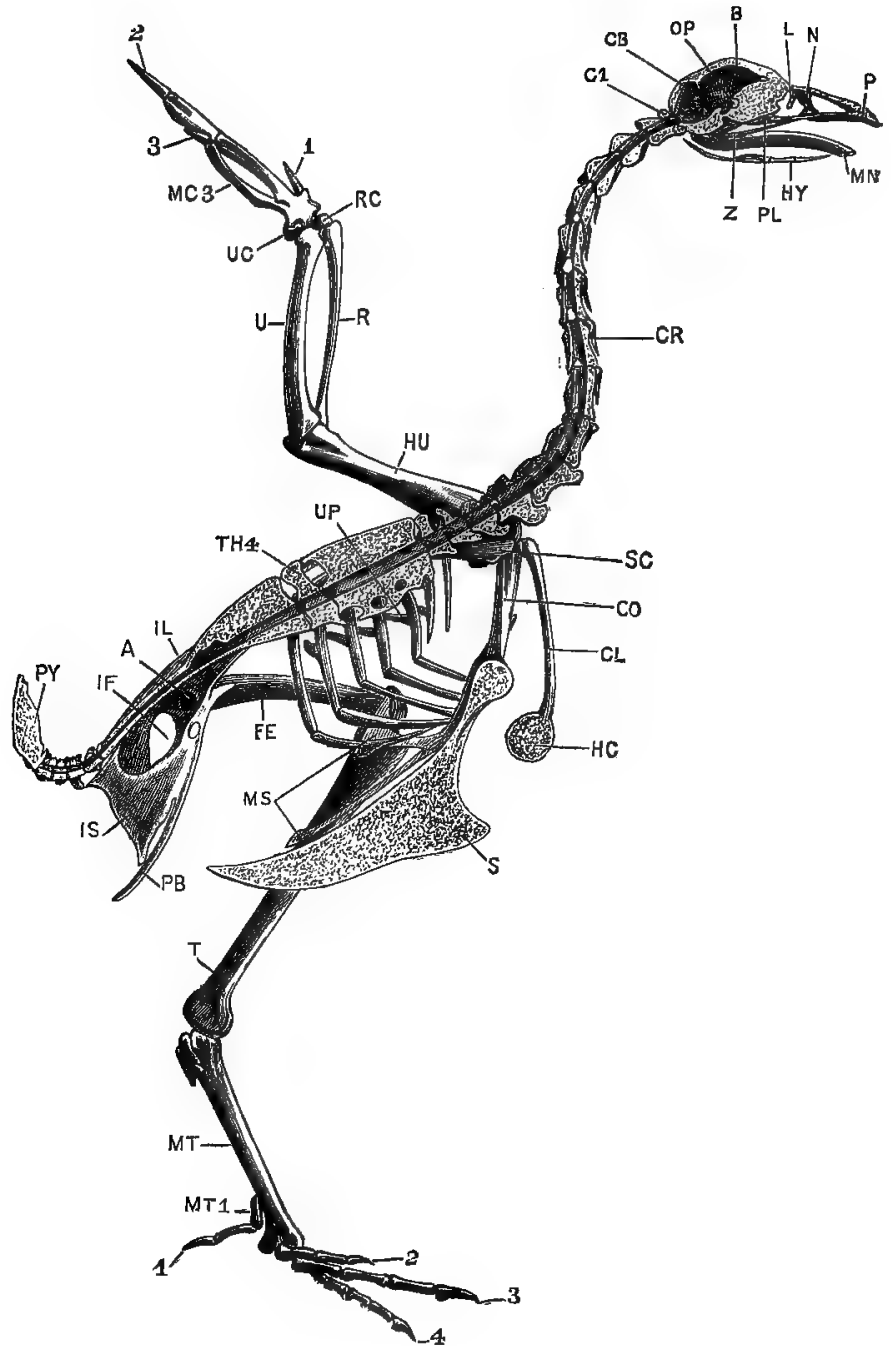


the vertebral column, with which the pelvis is connected; (4) the small number of the caudal vertebræ, and the fusion of the posterior ones to form the pygostyle; (5) the absence of epiphyses.

The column may be divided into cervical, thoracic, sacral, and caudal regions; the boundaries between these being ill-defined, and the sacral portion being further divisible into three sub-sections.

1. The cervical vertebræ. The distinction between cervical and thoracic vertebræ is an arbitrary one, of no morphological value, and is not easy to determine in the fowl. It is usual to count as the first thoracic vertebra, the most anterior one which bears a rib articulating with the sternum, and to name all the vertebræ in front of this one cervical. Reckoned in this way there are sixteen cervical vertebræ, the two hindmost of which bear well-developed and movably articulated ribs, which, however, do not reach the sternum.

In the following description the vertebral column is supposed to be placed horizontally.

a. A typical cervical vertebra : any one from the sixth to the tenth.

The parts of the vertebra are the same as in the rabbit, except that there are no terminal epiphyses. The chief peculiarities are the following:-

i. The vertebra is very long and light, as compared with the cervical vertebra of a rabbit.

FIG. 65.-Gallus bankiva, var. domesticus. The left half of the skeleton. The skull, vertebral column, and sternum are bisected in the median plane. (с. н. н.)

A, acetabulum. B, cerebral fossa. CB, cerebellar fossa. CL, clavicle. $\mathbf{C O}$, coracoid. $\mathbf{C R}$, cervical rib. $\mathbf{C} \mathbf{1}$. first cervical vertebra. $\mathbf{H} \mathbf{F}$, femur. HC, hypocleidium.' HU, humerus. HY, hyoid. IF, ilio-scistic foramen. II, ilium. IS, ischium. I, lacrymal bone. IMC3, metacarpal bone of third digit. MN, mandible. MS, metosteon. MT, tarso-metatarsus. MT 1, metatarsal bone of first digit. $\mathbf{N}$, nasal bone. OP, optic foramen. $\mathbf{P}$, premaxilla, $\mathbf{P B}$, pubes. $\mathbf{P I}$, palatine bone. $\mathbf{P} \mathbf{Y}$, pygostyle. $\mathbf{R}$, radius. $\mathbf{R}$, radial carpal bone. $\mathbf{S}$, keel of the sternum. SC, scapula. $\mathbf{T}$, tibiotarsus. TH 4, fourth thoracic vertebra. U, ulna. UC, ulnar carpal bone. $\mathbf{U P}$, uncinate process of rib. $\mathbf{Z}$, infra-orbital bar.

$1,2,3,4$, the first, second, third, and fourth digits. 
ii. The centrum is slender. The terminal articular surfaces are saddle-shaped, the anterior one being convex from above downwards, and concave from side to side; while the posterior surface, which is larger and more prominent, is concave from above downwards, and convex from side to side. These saddleshaped articular surfaces are very characteristic of the cervical vertebræ of birds.

iii. The neural arch is shorter than the centrum, and deeply notched in the middle line, both in front and behind. The neural arch is also notched behind, on both sides, for the passage of the spinal nerves.

iv. The neural spine is rudimentary in the middle cervical vertebræ.

v. The transverse processes and cervical ribs. At the anterior end of the vertebra an irregular process projects outwards at each side, which is perforated at its base by a large foramen, and is produced at its hinder border into a sharp, backwardly directed process.

This process is really a rib, which, as in the cervical vertebræ of the rabbit, is fused with the vertebra; the foramen at its base, which transmits the vertebral artery, corresponding to the notch between the capitulum and tuberculum of the rib.

vi. The zygapophyses.

The anterior zygapophyses are borne on the upper surfaces of the transverse processes: their articular surfaces are flat, and look upwards and inwards.

The posterior zygapophyses project backwards from the posterior border of the neural arch, their articular surfaces looking downwards and outwards. 
b. The atlas, or first vertebra, is very short and ringlike. Its ventral portion is thickened, resembling a centrum, and is notched above to receive the odontoid process of the axis : its anterior surface is deeply cupped to articulate with the occipital condyle of the skull. The posterior border of the neural arch is produced backwards into zygapophyses, articulating with the second vertebra.

c. The axis or second vertebra is, with the exception of the atlas, the smallest of the series: it has a blunt neural spine, but no transverse processes, and no ribs. The centrum is produced in front into the slender odontoid process.

d. The remaining cervical vertebræ. The third and fourth cervical vertebræ are shorter than the succeeding ones, and have short thick ribs, and lateral wing-like ridges placed obliquely along their sides. The middle cervicals are the longest of the series, and the hinder ones are shorter and more massive. The last cervical vertebra is fused with the first thoracic.

The neural spines are present, though small, in the anterior cervical vertebræ; well developed in the hinder ones, but almost absent in the middle of the region.

The hypapophyses are well developed in the hindmost half-dozen cervical vertebræ.

The ribs are more than half the length of the vertebræ in the middle of the neck, but are blunt and inconspicuous in the twelfth to the fourteenth vertebræ. In the fifteenth the ribs are well developed, and movably articulated with the vertebra; and in the sixteenth they are still larger, and resemble the thoracic ribs, but do not meet the sternum.

2. The thoracic vertebræ are five in number. The three anterior ones are fused together and with the last 
cervical vertebra; their centra, arches, and various processes being confluent, and the neural spines and hypapophyses forming continuous dorsal and ventral ridges.

The fourth thoracic vertebra is free; and the fifth is fused with the first sacral vertebra, its transverse processes abutting against and fusing with the ilia.

3. The sacrum consists of fourteen or fifteen vertebræ, fused together and supporting the ilia. It may be divided into the following regions.

a. The three anterior sacral vertebræ are firmly fused together, and with the last thoracic vertebra. They have stout transverse processes, which abut against the ilia, and which in the first one or two are single, but in the third are divided into dorsal and ventral portions, the latter being very stout and projecting directly outwards from the broad centrum. The neural spines of these vertebræ are fused to form a vertical crest of bone, which is continuous in front with that of the last thoracic vertebra, and fused along its edge with the dorsal edges of the ilia.

b. Behind these come four or five shorter vertebræ, with broad centra fused together. Their transverse processes, which have no ventral elements, are firmly connected together to form bony plates, which support the ilia along their outer margins.

Between the bodies of these vertebræ and the ilia, are the deep hollows in which lie the middle lobes of the kidneys.

c. Next come seven vertebræ, the centra of which are compressed laterally in the anterior ones, and dorso-ventrally in the hindmost two or three. The transverse processes, except in the last, are double; the dorsal elements fusing to form bony 
plates, continuous with those of the vertebræ in front of them. The ventral elements of the transverse processes are rod-like in the case of the first four or five vertebræ, and are regarded as ribs, because they ossify independently of the centra : in the hinder vertebræ they are smaller. The first pair of these ribs are much stouter than the rest: they are placed in the adult opposite the acetabula, with which they are connected by bony ridges running across the inner surfaces of the ilia, and separating the fossæ for the middle and posterior lobes of the kidneys from each other. In the embryo, and in the young chick, these ribs are some distance behind the acetabula, and are no larger than those of the succeeding vertebræ.

It is very doubtful how many of these vertebræ, and which ones, correspond to the sacral vertebræ of other vertebrates, usually two in number. Huxley considers that group (b) are the true sacral vertebræ, on the ground that the spinal nerves associated with them unite to form the sacral plexus. Gegenbaur and others hold that two or more of the vertebræ in group (c) are the true sacral ones, on account of their possessing separately ossified ribs.

4. The caudal vertebræ consist of four or five free ones, and a terminal pygostyle. This latter, which supports the rectrices, or tail feathers, is much larger than the others, and triangular in shape. It is formed by the fusion of a number of vertebræ, which are distinct in the embryo.

\section{B. The Ribs and Sternum.}

1. The ribs.

a. The cervical ribs. As already noticed, the last two cervical ribs of each side are alone movably articulated, the remainder being fused with the transverse processes of the corresponding vertebræ. 
Of these two, the first is small, but has distinct capitular and tubercular articulations : the second is larger and bears on its posterior border a backwardly projecting uncinate process, which overlaps the next rib.

b. The thoracic ribs are five on each side, and slightly increase in size from before backwards: each consists of vertebral and sternal portions, meeting at an angle.

i. The vertebral portions are flattened and slightly curved bars of bone, which articulate by capitular and tubercular surfaces with the centra and transverse processes of their respective vertebræ. Uncinate processes project backwards from the posterior borders of all, except the last, or sometimes the last two.

ii. The sternal portions are slender, nearly straight rods of bone, which run forwards and downwards, from their junctions with the vertebral portions, to the side of the anterior part of the sternum, with which they articulate.

2. The sternum, or breastbone, is very large, and projects. back far beyond the ribs, and over the greater part of the wall of the abdomen. Its ventral surface is raised into a prominent vertical crest or keel, which gives origin to the muscles of flight.

i. The body of the sternum is a somewhat spoonshaped plate of bone: it is wide and stout in front, and perforated on its concave dorsal surface by apertures, through which the airsacs communicate with the cavity of the bone: it is narrow in the middle portion of its length, but widens somewhat behind.

ii. The manubrium is a small vertical plate of bone, projecting from the anterior end and ventral surface of the body.

iii. The carina, or keel, is a very prominent triangular 
crest, projecting downwards from the ventral surface of the sternum, and slightly thickened along its anterior border, which is concave.

iv. The metostea are a pair of large processes, projecting backwards from the sides of the anterior end of the body of the sternum. Each divides, close to its origin, into two large flattened processes, the external and middle xiphoid processes, which are expanded at their hinder ends.

v. The costal surfaces are the lateral borders of the body, in front of the metostea: they are notched for the sternal ends of the ribs.

vi. The costal processes project forwards and outwards, in front of the costal surfaces.

vii. The coracoid grooves are a pair of deep transverse grooves at the anterior end of the body, continuous with each other, across the median plane, through a hole in the base of the manubrium. With them the ventral ends of the coracoid bones articulate.

\section{The Skull.}

The chief characteristics of the bird's skull are:-(1) its great lightness; (2) the very marked tendency of the bones of the cranium to fuse together, most of the cranial sutures being closed, and the outlines of the bones obliterated, by the end of the first year; (3) the large size of the orbits, which are separated from each other by a thin vertical interorbital septum ; (4) the prolongation of the face forwards into a long conical toothless beak.

The skull consists of the same essential parts as that of the rabbit, from which, however, it differs in the following important points : (1) there is only a single occipital condyle, for articulation with the atlas vertebra; (2) the base of the skull is strengthened by a large membrane-bone, the basitemporal ; (3) the orbits are placed almost entirely in front of the cranium, instead of below it, and are separated from 
each other only by the thin interorbital septum; (4) the lower jaw is connected with the squamosal by a movably articulated bone, the quadrate, which probably corresponds to either the incus or the malleus of the rabbit; (5) there is only a single auditory ossicle, the columella; (6) there are no teeth.

The fowl's skull may be readily divided into cranial and facial portions which are loosely connected together, and capable of slight movement upon each other. The cranial portion consists of the cranium proper, or brain-case, and the auditory capsules: the facial part is formed by the jaws and the olfactory capsuleș.

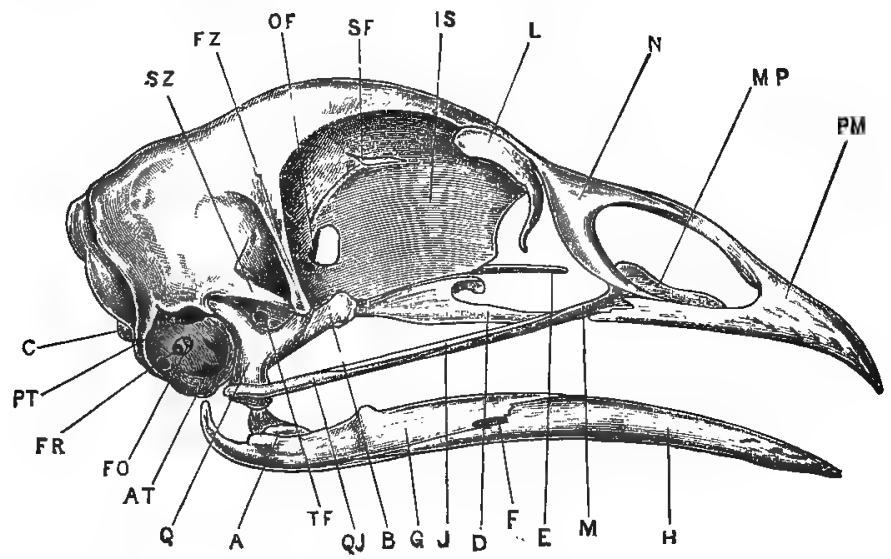

Fra. 66.-Gallus bankiva. The skull from the right side. (A. м. м.)

A, articular surface of the mandible. AT, anterior tympanic recess, leading to Eustachian tube. B, pterygoid. C, occipital condyle. D, palatine. $\mathbf{E}$, rostrum. $\mathbf{E}$, mandibular foramen. $\mathbf{F} \mathbf{O}$, fenestra ovalis. $\mathbf{F R}$, fenestra rotunda. FZ, zygomatio process of frontal bone. G, supra-angular. H, dentary. IS, inter-orbital septum. J, jugal. I, lacrymal. M, maxilla. MP, maxillo-palatine process of maxilla. N, nasal. OE, optic foramen. PM, premaxilla. PT, posterior tympanic recess. Q, quadrate. QJ, quadrato-jugal. SF, olfactory foramen. SZ, zygomatic process of squamosal. TF, foramen for fifth nerve.

1. The cranial portion of the skull is a somewhat conical box of bone, the base of which forms the hinder end of the skull, the apex being directed forwards. In the young skull the outlines of the component bones 
can be readily followed, but in the adult they are indistinguishable. The following are the main points to be noticed.

a. The hinder end of the cranium.

i. The foramen magnum is a large, nearly circular aperture, in the middle of the posterior end of the skull, through which the spinal cord enters the cranial cavity.

ii. The occipital condyle is a rounded $\mathrm{knob}$, immediately below the foramen magnum, into which it projects slightly. It is notched in the mid-dorsal line, and has below it a deep pit-like depression.

iii. The foramen of exit for the hypoglossal nerve, on each side, is a small aperture, immediately to the outer side of the occipital condyle, and slightly ventral to it.

iv. The foramen of exit for the pneumogastric and glosso-pharyngeal nerves, on each side, is a larger hole, about an eighth of an inch to the outer side of that for the hypoglossal nerve, and midway between the condyle and the rim of the tympanic cavity.

v. To the outer side of the above foramen, and between it and the tympanic rim, is a group of three or four larger holes: some of these lead into the tympanic cavity; while the most ventrally situated one is the entrance to the carotid canal, which traverses the base of the skull, and lodges the carotid artery.

vi. The lambdoidal crest is a prominent ridge of bone surrounding the dorsal half of the hinder end of the skull, and separating the occipital from the parietal region.

b. The ventral surface of the cranium.

i. The base of the skull is wide behind, where it is covered by the broad transverse basi-temporal, 
a membrane-bone which forms part of the boundaries of the tympanic cavities, and probably corresponds to the posterior part of the para-sphenoid of the frog.

In front of the basi-temporal, the base of the skull is narrow, and formed by the rostrum, or thickened ventral border of the interorbital septum.

ii. The anterior openings of the Eustachian tubes are a pair of small holes, close to the median plane, and opening into a deep notch between the anterior end of the basi-temporal bone and the rostrum.

iii. The foramina of exit for the carotid canals are a pair of small holes, near the middle line, and immediately to the outer sides of the Eustachian apertures. They are connected with each other by a canal, traversing the base of the skull.

Pass a bristle along the carotid canal of one side, and through the transverse canal from side to side.

c. The side of the cranium.

i. The tympanic cavity is a hemispherical depression, at the side of the hinder end of the skull, bounded above by the squamosal and below by the basi-temporal. The tympanic membrane is attached just within its prominent lip.

Within the tympanic cavity can be seen the following.

u. The fenestral recess is an oval depression in the middle of the cup, divided by a partition into an upper hole, the fenestra ovalis, and a lower, the fenestra rotunda. The former of these lodges during life the inner end of the auditory ossicle, or columella, a slender bar of bone and cartilage, the outer 
end of which is attached to the tympanic membrane.

$\beta$. The posterior opening of the Eustachian tube is a funnel-like depression, at the ventral and anterior corner of the tympanic cavity. The tube into which it leads passes forwards and inwards, between the basi-temporal and the true base of the cranium, to open, as already seen, at the base of the skull, close to the middle line.

Pass a bristle along the Eustachian tube of one side, from behind forwards.

$\gamma$. The anterior tympanic recess is a slit-like depression, immediately above the posterior opening of the Eustachian tube, with which it is often continuous.

$\delta$. The posterior tympanic recess is a large depression, close to the dorsal border of the tympanic cavity, and just above the fenestral recess.

ii. The zygomatic process of the squamosal is a thin bar of bone, which projects forwards almost horizontally from the anterior and upper edge of the tympanic cavity. Its distal end meets, and often fuses with, the post-orbital process of the frontal bone.

iii. The articular surface for the quadrate is a depression on the side of the skull, immediately in front of the tympanic cavity, between it and the zygomatic process of the squamosal.

iv. The foramen of exit for the trigeminal nerve is a round hole in the side of the skull, below and in front of the articular surface for the quadrate.

v. The orbit is a very large depression in the side of the skull, which lodges the eyeball: it is bounded above by the frontal bone; in front, 
by the frontal and laerymal bones, the latter of which is produced downwards at its anterior end into a slender curved process. Behind, the orbit is bounded by the frontal with its post-orbital process, and by the alisphenoid ; and on the inner side it is separated from the orbit of the other side by the interorbital septum, a thin vertical plate of bone, formed by the coalescence of the pre-sphenoid and orbito-sphenoid behind, and the mesethmoid in front and above. The lower edge of the septum is thickened, forming the rostrum, and is covered by a membrane-bone, the para-sphenoid.

Below, the orbit is very incomplete; its outer margin is formed by the slender zygomatic arch, and its floor, nearer the middle line, is formed in part by the palatine and pterygoid bones.

vi. The foramen of exit for the olfactory nerves is a median opening, at the anterior end of the cranium, and is continued forwards as a horizontal slit, between the inter-orbital septum and the frontal bones.

vii. The optic foramen is a large median hole in the orbito-sphenoid bones, opening into the posterior part of the orbits, and continued forwards as a semicircular notch in the interorbital septum.

viii. The foramina of exit for the third and fourth nerves are small holes, immediately to the outer side of the optic foramen, and sometimes confluent with it.

d. The dorsal surface of the cranium.

The dorsal surface of the cranium is rounded, and,formed almost entirely by the parietal and frontal bones. It is broad behind, narrower in 
front; and separated by a sharp, almost transverse line from the facial part of the skull. The anterior ends of the frontal bones diverge slightly from each other, and allow the mesethmoid to come to the surface between them.

2. The facial portion of the skull lies below and in front of the cranial portion, with which it is only loosely connected. It consists of, (1) the olfactory capsules, and the bones associated with them; (2) the skeletal elements of the jaws, and the hyoid apparatus.

a. The olfactory capsules and associated bones. The olfactory capsules themselves remain in a cartilaginous condition throughout life, and are therefore absent in macerated skulls. They are separated only by a thin vertical septum, and almost completely fill the conical space which, in the dried skull, lies between the anterior end of the orbit and the tip of the beak. Each olfactory capsule has its cavity complicated by a spirally rolled lamina of cartilage, which projects inwards from its outer wall.

In relation with the olfactory capsules are the following bones.

i. The nasals are a pair of thin lamellar bones, which cover the sides of the hinder ends of the olfactory capsules. Behind, they are fused with the anterior ends of the frontals; while their anterior borders are divided by deep notches into superior and inferior processes, which embrace the external narial openings, and are closely connected with processes of the premaxilla.

ii. The vomer is a slender median bony rod, which lies below the hinder part of the olfactory capsules, and immediately in front of the para-sphenoid. It is paired in some birds. 
b. The upper jaw. The bones of the upper jaw, as seen from the ventral surface, form on each side two bony arcades, which are fused together in front to form the beak, and are closely approximated behind, but widely separated from each other along the greater part of their course. The inner arcade is formed by the pterygoid and palatine bones, which articulate with the rostrum : the outer arcade consists of the slender sub-orbital bar and the premaxilla.

The upper jaw is connected with the anterior end of the frontals, and with the mesethmoid. Posteriorly, it is slung up to the side of the skull, just in front of the tympanic cavity, by the quadrate bones.

i. The pterygoid is a short stout bone, forming the hinder part of the inner arcade. It articulates at its outer and posterior end with the inner surface of the quadrate, and at its inner and anterior end with the rostrum, along which it can slide.

ii. The palatine is a slender horizontal bar of bone lying in front of the pterygoid, and forming the greater part of the inner arcade. Behind, it articulates with the pterygoid, and is expanded into a broad lamella, which articulates with the rostrum along its inner edge. In front, the palatine is wedged in between the maxilla and the premaxilla.

iii. The premaxilla is a triradiate bone, the anterior part of which is ankylosed with its fellow in the median plane to form the tip of the beak, while the three processes diverge backwards.

The ascending or nasal process, which is much the longest of the three, is a long slender bar of bone, which runs backwards and upwards, closely applied to its fellow of 
the opposite side, and along the inner side of the nasal bone, to the mesethmoid, with which it is connected. The bone is very thin and elastic at its hinder end, so as to allow of a certain amount of movement of the facial on the cranial portion of the skull.

The outer or maxillary process runs backwards and slightly outwards, forming part of the margin of the upper jaw. The nasal and maxillary processes of the premaxilla, together with the superior and inferior processes of the nasal bone, surround the large aperture at the side of the beak in which the external narial opening lies.

The inner or palatine process, the smallest of the three, is a narrow plate of bone which extends horizontally backwards, a short distance from the median plane. It forms part of the roof of the mouth, and is connected behind with the anterior end of the palatine bone.

iv. The maxilla is a slender rod of bone, lying behind and to the inner side of the maxillary process of the premaxilla, and forming part of the sub-orbital bar. Its anterior end is expanded into a thin horizontal lamina of bone, the maxillo-palatine process, which projects inwards towards the median plane, lying dorsal to the palatine bone.

v. The jugal is a very slender rod, which forms the middle portion of the sub-orbital bar. It lies dorsal to the other two components of the bar, and its exact limits are almost impossible to determine in the adult.

vi. The quadrato-jugal is the hindmost of the three components of the sub-orbital bar. Its posterior end is thickened, and articulates with 
the outer side of the distal end of the quadrate. In front it extends forwards as far as the maxilla, being overlapped for a considerable part of its length by the jugal.

c. The mandibular arch is divided into two portions of very unequal length, placed almost at right angles to each other. The proximal part is the quadrate bone, which connects both the upper and lower jaws with the cranium : the distal portion, forming the lower jaw, is of much greater length, is fused with its fellow in front, and is composed of several bones firmly united together.

i. The quadrate, which forms the suspensorium, is a stout triradiate bone. Its proximal or dorsal arm articulates with a cup-like depression in the squamosal, at the anterior and upper border of the tympanic cavity, between this and the base of the zygomatic process.

The distal or ventral arm, which is the stoutest of the three, is expanded transversely at its lower end, and forms the articular surface for the lower jaw: it also articulates at its outer edge with the quadrato-jugal, and along its inner margin with the pterygoid.

The third or anterior arm of the quadrate is a more slender process, which projects forwards and inwards into the hinder part of the orbit, lying parallel to and above the pterygoid.

ii. The mandible is a long, laterally compressed plate of bone, thickened and expanded at its hinder end, where it articulates with the quadrate, and firmly united with its fellow in the median plane in front to form the lower jaw. Like the upper jaw, it bears no teeth, but is covered by a horny epidermic sheath.

The mandible really consists of five bones, one of which is developed in the primary 
cartilaginous arch, while the others are formed around it.

a. The articular forms the expanded posterior end of the mandible. On its dorsal surface is the elongated and slightly concave articular surface for the quadrate: behind this the bone is produced backwards and upwards into the curved posterior articular process ; and on the inner side into a similar but stouter internal articular process.

The articular is continuous in front with Meckel's cartilage, the cartilaginous bar of the mandibular arch, which persists, even in the adult, along the greater part of the length of the mandible, ensheathed in membrane-bones.

$\beta$. The angular is a splint-like bone which underlies the articular, and extends forwards along the ventral edge and inner surface of the proximal half of the lower jaw.

$\gamma$. The supra-angular forms the dorsal part of the posterior third of the mandible, along both outer and inner surfaces. Its dorsal border is raised into a small coronoid process, about the middle of its length.

$\delta$. The dentary is the largest of the bones of the jaw, and forms the distal half of each ramus: it is fused with its fellow in front at the symphysis ; and posteriorly it extends back along the ventral border of the supraangular, between this and the angular, as far as the articular.

¿. The splenial is a thin lamina of bone, applied to the inner surface of the jaw, along the middle half of its length. 
d. The columella and the hyoid apparatus. These may conveniently be considered together, as the columella is, according to most authorities, the uppermost part of the hyoid arch, just as the quadrate is of the mandibular.

i. The columella is a minute and slender rod of bone, the proximal end of which is expanded into an oval plate, fitted into the fenestra ovalis of the tympanic cavity; while the outer or distal end, which is cartilaginous and divided into three diverging arms, is attached to the tympanic membrane. It serves to convey the sound-vibrations from the tympanic membrane to the membrane closing the fenestra ovalis, and so to the internal ear.

Owing to its small size and slight attachment, the columella is often lost in macerated skulls : it may, however, sometimes be found projecting from the fenestra ovalis.

ii. The hyoid apparatus is a bony framework, lying in the floor of the mouth, and supporting the tongue. It consists of a median jointed rod of bone and cartilage, formed of glosso-hyal, basi-hyal, and basi-branchial ; to the sides of which are attached in front the short ceratohyals, and further back, the elongated and jointed thyro-hyals, the ventral portions of the first branchial arches.

II. THE APPENDICULAR SKELETON.

\section{A. The Pectoral Girdle.}

The pectoral girdle is stout, and is firmly connected with the sternum to give support to the wings. It differs markedly from that of the rabbit in the large size of the coracoid, which is almost perpendicular to the scapula; and in the completeness of the clavicle. 
i. The scapula is a long flattened blade-like bone, which in the natural condition of the parts lies above the ribs, a short distance from and almost parallel to the vertebral column. It is connected by muscles with both the ribs and the vertebræ. Its anterior end, which is expanded and firmly connected with the coracoid, bears on its outer surface a shallow depression, forming part of the glenoid cavity ; and is produced forwards, at its inner border, into a process which helps to form the canal for the tendon of the second pectoral muscle.

ii. The coracoid is a stout straight bone, running from the shoulder to the anterior end of the sternum. Its upper end is connected behind and at its inner side with the scapula, and

- bears on its outer surface a cup-shaped depression, which forms the greater part of the glenoid cavity.

Above the glenoid cavity, the coracoid is produced upwards and inwards into a strong process, which, with the corresponding process of the scapula, completes the foramen triosseum through which the tendon of the second pectoral muscle passes to its insertion into the back of the humerus. This process also articulates with the clavicle.

The lower end of the coracoid is broad and flattened, and bears a transversely elongated surface, articulating with the anterior end of the sternum.

iii. The elavicles are a pair of slender curved bones, whose upper ends are slightly expanded, and connected, as described above, with the coracoids; while their lower ends are fused together to form a median, laterally compressed plate.

The two clavicles together form the furcula or 'merrythought.' 


\section{B. The Fore-Limb or Wing.}

The wing of the bird corresponds to the fore-limb of the rabbit, but differs from it in several respects, the more important of which are, (1) the complete absence of the two. outer digits; and, (2) the fusion of the distal row of carpal bones with the three persistent metacarpals to form a compound bone, the carpo-metacarpus.

When the wing is extended for flight its surfaces and borders correspond to those of the primitive vertebrate limb, the preaxial border being directed forwards, the postaxial backwards, and the dorsal and ventral surfaces upwards and downwards respectively. When the wing is folded, in the position of rest, the three main divisions-arm, fore-arm, and hand-are bent on each other like the letter $\mathbf{Z}$, the elbow pointing backwards, and the wrist forwards. Owing to a slight rotation at the shoulder-joint, and a slight obliquity of the elbow-joint, the dorsal surface of the arm now looks inwards and upwards; that of the fore-arm outwards and slightly upwards; while in the hand, which is bent on the fore-arm in a position of extreme abduction, the dorsal surface looks almost directly outwards.

1. The arm has only a single bone.

i. The humerus is an elongated, slightly curved bone, expanded at both ends.

The proximal end, or head, presents a large, convex, vertically elongated surface for articulation with the glenoid cavity, bordered by two tuberosities, which are preaxial and postaxial respectively.

The preaxial tuberosity is small, but is continued along the bone as the prominent forwardly directed deltoid ridge: this gives insertion to the great pectoral and deltoid muscles; while a small tubercle at its proximal end, and slightly on the extensor surface, marks the insertion of the tendon of the second pectoral muscle, or elevator of the wing. 
The postaxial or greater tuberosity is much larger, and has on its extensor surface a deep pit, the pneumatic foramen, leading to an aircavity in the shaft of the bone.

The distal end of the humerus bears a trochlear articular surface for the radius and ulna; the radial surface being preaxial, and placed obliquely to the long axis of the bone; the ulnar surface being postaxial, and transverse to the axis.

2. The fore-arm has two bones, which are separate from each other along their whole length.

i. The radius is slender and nearly straight. Its proximal end, or head, presents a terminal cup-shaped articular surface for the preaxial condyle of the humerus; and its distal end articulates with the carpus.

ii. The ulna is rather longer and much stouter than the radius, and is slightly curved. Its proximal and larger end has a large articular surface for the postaxial tubercle of the humerus, beyond which it projects as the blunt olecranon process. Its distal end articulates with the carpus, and with the radius.

3. The wrist consists, in the embryo, of two rows of carpals, a proximal row of two and a distal row of three. Of these the two proximal carpals persist in the adult, while those of the distal row fuse with the metacarpals to form the compound carpo-metacarpus.

a. The proximal row of carpals consists of two small irregular bones.

i. The radiale lies between the end of the radius and the manus.

ii. The ulnare is a larger, more irregular bone, which articulates proximally with the ulna, 
and is notched distally to receive the carpometacarpus. It is very freely movable.

4. The manus. In the embryo there are four separate metacarpals, corresponding to those of the thumb and next three digits of man. In the adult the first three fuse together, and with the distal row of carpals, to. form the carpo-metacarpus; while the fourth either disappears completely, or, according to some authorities, fuses with the third.

a. The carpo-metacarpus is an elongated bone, in which the three component metacarpals can readily be distinguished.

i. The first metacarpal, belonging to the first digit or thumb, is very short and stumpy, forming a small projection on the radial side of the proximal end of the bone.

ii. The second metacarpal, that of the second digit or fore-finger, is a stout straight bone, enlarged. at its ends, and giving off near its proximal end a process from its ulnar side.

iii. The third metacarpal is slightly curved, and is more slender than the second, with which it is fused at both ends.

b. The phalanges.

i. The pollex, or first digit, has two phalanges, the terminal one being small.

ii. The second digit has three phalanges, the proximal one being large and lamellar, and the distal one very small.

iii. The third digit has only a single phalanx.

\section{The Pelvic Girdle.}

The pelvic girdle consists of the same three elementsilium, ischium, and pubes-as the mammalian pelvis, from which, however, it differs widely in form. The three component bones are firmly united together to form the os innominatum; 
and at the junction of the three is the acetabulum, or socket for the head of the femur, which is perforated at its bottom.

i. The ilium is an elongated lamellar bone, which extends a considerable distance both in front of and behind the acetabulum. Its inner border is connected along nearly its whole length with the sacral vertebre and their processes. The outer surface of the anterior part is concave, that of the posterior part convex : the inner surface of the bone forms, with the adjacent vertebræ, two deeply concave depressions in which lie the kidneys. On the outer surface, immediately behind and above the acetabulum, is a projection, the antitrochanter, which bears on its outer surface an articular facet for the great trochanter of the femur.

ii. The ischium is a flattened lamellar bone, projecting almost horizontally backwards from the acetabulum, of which it forms about a third. It is separated from the ilium, just behind the acetabulum, by the oval ilio-sciatic foramen, behind which the two bones are fused together.

iii. The pubes is a long slender bar of bone, which projects downwards and backwards, parallel to the lower border of the ischium. It forms a very small part of the ventral border of the acetabulum, in front of which it extends forwards as a blunt pre-pubic process, corresponding to the pubes of mammals.

Behind the acetabulum, the pubes and ischium are separated by the slit-like obturator foramen, behind which the two bones are fused for a short distance. The hinder end of the pubes is thickened, and extends some distance beyond the ischium. 


\section{The Hind-Limb.}

In the bird's leg there is the same tendency to fusion of the bones which is characteristic of all parts of the skeleton. There are no free tarsals, the proximal row having fused with the tibia, and the distal row with the metatarsals.

The position of the limb is the same as in the rabbit; the knee pointing forwards, and the preaxial border being internal along the whole length of the limb.

1. The thigh has only a single bone.

i. The femur has a cylindrical, slightly curved shaft, and enlarged ends. The proximal end is produced on its inner side into a rounded head, articulating with the acetabulum. On the outer or postaxial side of the head is an irregular process, the great trochanter, between which and the head, at the proximal end of the bone, is the articular surface for the anti-trochanter of the ilium.

The distal end of the femur presents, in front, a deeply grooved pulley-like surface for the patella, a sesamoid bone in the tendon of the extensor muscle of the leg: this surface is bounded laterally by two prominent condylar ridges, which articulate with the tibia; the outer or postaxial condyle having on its posterior surface a deeply grooved facet for the upper end of the fibula.

2. The leg.

i. The tibio-tarsus is a large, stout, nearly straight bone, considerably longer than the femur: it is formed by fusion of the tibia with the proximal row of tarsals. The proximal extremity of the bone is much enlarged, and bears at its end two surfaces which articulate with the condyles of the femur: on its extensor surface is the prominent cnemial crest, into which the tendon of the extensor 
muscle of the thigh is inserted, beyond the patella.

The distal end of the bone has a prominent pulley-like articular surface for the tarsometatarsus.

The fibula is a slender bone, closely applied to the outer or postaxial surface of the tibiotarsus, with which it is often fused. Its proximal end is enlarged, and articulates with the femur; the rest of the bone is very slender, and usually does not reach to the ankle.

3. The ankle-joint is between the proximal and distal rows of tarsals : these at a very early age fuse with the tibia and metatarsus respectively, so that in the adult there are no free tarsal bones.

4. The pes. The first four digits are present: the meta. tarsal of the first is small and imperfect; those of the next three are large, and are fused with one another and with the distal tarsal row. The fifth or postaxial digit, corresponding to the little toe in man, is present in the embryo, but disappears early.

a. The tarso-metatarsus is a stout straight bone, about as long as the femur, and formed by the fusion of the distal tarsal row with the second, third, and fourth metatarsals. Its proximal end is enlarged, and bears terminally two cup-shaped facets for the tibio-tarsus: on its posterior surface is a prominent ridge, deeply grooved behind, and traversed at its base by a longitudinal canal, for the passage of the tendons of the flexor muscles of the toes. Behind the ridge, two sesamoid bones are usually present.

In the shaft of the bone, the three metatarsals which compose it can readily be distinguished. They do not lie quite parallel to one another, the middle one being slightly posterior to the 
other two at its proximal end, and anterior distally.

At the distal end of the bone, the three metatarsals separate from one another, and each ends in a pulley-like surface for the corresponding digit.

b. The first metatarsal is incomplete, its distal end alone being present. It is a small irregular nodule of bone, attached by ligament to the inner and posterior surface of the tarso-metatarsus, a short distance above its distal end.

c. The digits. The four preaxial digits are present. In many domestic fowls, notably Dorkings, an extra or supernumerary toe is present on the inner side of the foot. The true fifth, or postaxial digit, is always absent.

i. The hallux, or great toe, is connected with the first metatarsal, and directed backwards. It consists of two phalanges, the last one bearing a claw.

ii. The second toe is directed forwards and slightly inwards. It has three phalanges, the last one clawed.

iii. The third toe is the largest, and is directed forwards. It has four phalanges, the terminal one clawed.

iv. The fourth toe is directed forwards and outwards. It has five phalanges, and the distal one is clawed. 


\section{CHAPTER XV.}

\section{DISSECTION OF THE PIGEON. Columba livia.}

BrRDs are in many respects the most highly differentiated vertebrates. While agreeing in their essential morphological. characters with the other air-breathing vertebrates, and more especially with reptiles, they present a number of very special characters, chiefly connected with the power of flight.

The modifications in the skeleton have been noticed in the preceding chapter : in the other systems the most noteworthy features are:-(1) the development of feathers, which are very highly specialised products of the epidermis : (2) the great size of the pectoral muscles, by which flight is effected; (3) the prolongation of the bronchi through the lungs into spacious thinwalled air-sacs, lying between the viscera, and prolonged into several of the bones; (4) the persistence of the fourth right. aortic arch, instead of as in mammals the fourth left, to form the arch of the aorta ; (5) the lateral, instead of dorsal, position of the optic lobes of the brain; and (6) in the female, the disappearance, during development, of the ovary and oviduct of the right side.

Physiologically, the most interesting points are :-the high temperature of the blood, which exceeds that of mammals, varying between $100^{\circ}$ and $110^{\circ} \mathrm{F}$. in different birds; and the modifications in the mechanism of respiration, expiration being the active movement, and inspiration almost purely passive.

Birds are oviparous, and the eggs, which are of large size, are protected by calcareous shells. The eggs are fertilised in the upper part of the oviduct before the shell is formed, and. 
hence commence to develop before they are laid. The egg contains within itself sufficient nutriment for the development of the chick up to the time of hatching: and all that is necessary to ensure development is that the egg, after it is laid, should be kept at about the temperature of the parent's body. This is in most birds effected by incubation, a task usually fulfilled by the hen bird, but sometimes wholly or in part by the cock.

Pigeons should be killed with chloroform, otherwise the lice with which they are invariably infested may prove a great nuisance. Before the dissection is commenced, the bird should be thoroughly plucked, with the exception of one of the wings, on which the feathers may be left till later.

\section{EXTERNAL CHARACTERS.}

Excepting the lower joints of the legs and the toes, nearly the whole surface of the body is covered with feathers.

\section{A. The Main Divisions of the Body.}

1. The head is elongated antero-posteriorly, and produced in front into a pointed beak, encased in a horny epidermal sheath.

At the base of the upper beak is a naked swollen patch of skin, the cere.

At the sides of the head are the large eyes, each provided with upper and lower eyelids, and with a well-developed third eyelid, or nictitating membrane, a semi-transparent fold of skin which can be flicked across the eye from its anterior angle with great rapidity.

2. The neck is very long and flexible.

3. The trunk is deep dorso-ventrally, and somewhat compressed from side to side. Along the mid-ventral line is a prominent ridge, formed by the ventral border of the carina, or keel of the sternum.

Posteriorly the trunk ends in a short blunt tail. 


\section{B. The Limbs.}

\section{The wing.}

Identify the several bones of the wing by feeling them through the skin. Note also the varying positions of the surfaces of the several divisions of the wing, when it is extended and folded respectively (p. 388).

The ala spuria, or bastard wing, is a small tuft. of feathers attached to the phalanx of the first digit.

2. The leg.

The toes and the tarso-metatarsal region are, except in some domesticated breeds, devoid of feathers and covered with large horny epidermal scales. The ends of the toes are provided with horny claws.

\section{The Feathers.}

The possession of feathers, which are specially modified epidermal structures, is one of the most characteristic features of the bird.

\section{Structure of a feather.}

Pull out one of the large feathers from the wing or tail; and note in it the following points.

a. The scapus, or stem, is a stiff axial rod, running the whole length of the feather. It consists of two dissimilar portions.

i. The calamus, or quill, is the tubular, semi. transparent proximal portion, the base of which is inserted in the skin. At the proximal end of the quill there is a small aperture, the inferior umbilicus; and at its distal end, between the quill and the vane, there is a second aperture, the superior umbilicus.

ii. The rachis, or shaft, is the distal solid portion of the stem. It is a tapering, flexible, elastic rod, square in transverse section, and grooved along its ventral surface. 
b. The vexillum, or vane, is the flattened portion of the feather, attached along the sides of the rachis. It is made up of barbs and barbules.

i. The barbs are a series of narrow, elastic laminæ, attached by their bases along the two sides of the rachis.

ii. The barbules are much smaller processes, which form fringes along the sides of the barbs: they bear hooklets, which hold together the adjacent barbs, and so give the feather that resistance to the air on which the bird's power of flight depends.

c. The after-shaft is a miniature feather, attached to the ventral surface of the base of the rachis, and overhanging the superior umbilicus. It is usually very small, but is in some birds as large as the primary feather.

2. Varieties of feathers.

a. The quill feathers are the large feathers of the wings and tail : they are of two kinds.

i. The remiges, or wing-quills, have the inner or posterior half of the vane much broader than the outer or anterior half. They are divided into groups in accordance with the bones to which they are attached (fig. 67, p. 406).

The primary quills are those attached to the bones of the manus. They are eleven in number, of which six are attached to the second metacarpal, and are hence called metacarpal quills : one, ad-digital, is attached to the phalanx of the third digit; two, middigital, to the proximal phalanx of the second digit ; and two, pre-digital, one of which is small, are attached to the distal phalanx of the second digit.

The secondary quills are those attached to the ulna. 
ii. The rectrices, or tail-quills, have the two halves of the vane about equal in size. They are used by the bird for steering during flight.

They are twelve in number in the wild rock-pigeon, but vary in domestic breeds.

b. The coverts are the smaller feathers which cover the bases of the quills of the wings and tail.

c. The contour feathers are the short soft feathers covering the body generally. The barbules are less perfectly developed than in the quill-feathers, so that the barbs readily separate from one another, especially at the base of the vane.

d. The filoplumes are the minute rudimentary feathers left in the skin after the bird has been plucked. Each consists of a hair-like stem, with a very rudimentary vane at its apex.

\section{Arrangement of the feathers.}

The feathers are attached to certain definite areas of the skin, known as pterylæ, the intervening tracts or apteria being devoid of feathers. This arrangement is not very obvious in the pigeon, in which the feathers are more uniformly distributed than in many birds. Apteria may, however, be seen in the mid-ventral and mid-dorsal regions of the body, and along the outer surfaces of the proximal segments of the wings.

\section{External Apertures.}

a. Median apertures.

i. The mouth is a wide slit-like opening in the long pointed beak.

ii. The cloacal aperture is a transverse slit, with prominent lips, on the ventral surface of the body, below the base of the tail.

b. Paired apertures.

i. The nostrils are a pair of oblique slit-like apertures, between the beak and the cere. 
ii. The external auditory apertures are a pair of circular openings, below and slightly behind the posterior or outer angles of the eyes. Each is surrounded by a circlet of small, backwardly directed feathers-the auriculars.

Each leads inwards, and slightly backwards and downwards, to the tympanic cavity.

\section{DISSECTION OF THE PECTORAL MUSCLES.}

Remove the skin from the ventral surface of the thorax and from one shoulder, so as to fully expose the first pectorat muscle. Clean this muscle along its whole length, defining its boundaries carefully.

1. The first or great pectoral muscle is large and triangular, and forms the greater part of the side of the breast. It arises from the whole length of the ventral half of the keel of the sternum, from the whole length of the clavicle, and from the lateral part of the body of the sternum. Its fibres run forwards and outwards, converging to a broad flat tendon, which is inserted into the greater tuberosity of the humerus. From the anterior border of the muscle, near the shoulder, a slip is sent off to the skin.

The great pectoral is the main depressor of the wing, and the most important of the muscles of flight.

Cut through the great pectoral muscle; transversely to its fibres, and about the middle of its length. Turn the two halves of the muscle aside, so as to clearly define its origin and insertion: then cut away the muscle entirely.

2. The second pectoral muscle is a smaller and more deeply placed muscle, similar in shape to the first pectoral, and completely covered by it.

It arises from the dorsal part of the side of the keel of the sternum, and from the inner part of the 
ventral surface of its body. Its fibres run forwards and outwards, converging to be inserted into a long tendon, which passes through the foramen triosseum, formed by the scapula, coracoid, and clavicle, and reaches the back of the shoulder-joint, where it is inserted into the dorsal surface of the humerus, near the greater tuberosity.

Owing to the course taken by its tendon, the muscle elevates the wing, and so antagonises the great pectoral muscle.

To see the insertion of the tendon of the second pectoral muscle, remove or push aside the deltoid muscle, which covers the back of the shoulder-joint. Test the action of the muscle by pulling the tendon.

3. The third pectoral muscle is a small triangular muscle, arising from the outer border of the posterior twothirds of the coracoid, and from the costal process of the sternum. Its fibres run forwards and outwards, to be inserted into the postaxial border of the head of the humerus. It aids the great pectoral muscle in depressing the wing.

\section{DISSECTION OF THE AIR-SAGS.}

The air-sacs are large but very thin-walled sacs, lying mainly along the sides and dorsal surface of the body-cavity. They communicate, as already noticed, with the bronchi, and they play a very important part in the mechanism of respiration of the bird.

Expiration is effected by the thoracic and abdominal muscles, which compress the thorax and abdomen, driving. the air from the air-sacs, through the lungs and trachea.

Inspiration is effected by the elastic expansion of the thorax and abdomen on relaxation of the muscles: this. expansion causes an inrush of air along the trachea, through the lungs and into the air-sacs, which are thus filled with fresh air. 
A complete dissection of the air-sacs is not easy to perform, and involves a good deal of damage to other structures. The main features in their distribution and relations can, however, be readily determined, as described below. The dissection of the air-sacs must precede that of the other systems, as if they are once cut into, the walls of the sacs collapse, and can no longer be inflated.

The air-sacs are nine in number; a median interclavicular, a pair of cervical or pre-bronchial, two pairs of thoracic or intermediate, and a pair of abdominal or posterior.

Make a median ventral incision through the skin of the neck, and pin out the flaps. Note the cesophagus, a wide fleshy median tube; and the trachea, which lies to the left of the cesophagus, and is readily recognised by the close-set cartilaginous rings by which it is surrounded.

Separate the trached from the surrounding tissues in the hinder part of the neck, and pass a ligature round it. Slit open the trachea about the middle of its length: pass a blowpipe down the trachea through the slit, and tie it firmly in. Inflate the lungs and air-sacs through the blow-pipe, and tighten the ligature round the trachea to prevent the air escaping.

Make a mid-ventral incision through the skin from the posterior edge of the sternum to the cloaca, and a transverse incision along the posterior border of the sternum. Carry the incisions through the muscles of the abdominal walls, taking care not to injure the viscera beneath, and reflect the flaps .outwards.

1. The posterior or abdominal air-sacs are a pair of large sacs, with very thin transparent membranous walls, which lie along the dorsal wall of the pelvic cavity, ventral to the kidneys but dorsal to the intestine. At the hinder end of the abdomen they come close up to the ventral surface when inflated.

Each communicates with the posterior border of the corresponding lung, near its outer angle.

2. The posterior thoracic air-sacs are a pair of rather smaller sacs, lying along the outer sides of the anterior 
ends of the abdominal sacs. They lie mainly behind the lungs, but overlap the ventral surfaces of their hinder ends.

Each communicates with the outer and posterior angle of the corresponding lung.

3. The anterior thoracic air-sacs cover the ventral surfaces of the anterior two-thirds of the lungs, and extend back so as to overlap the posterior thoracic sacs. They lie at the sides of the thorax, and are in extensive contact with the ribs and with the pericardium.

Each communicates with the ventral surface of the lung, rather in front of the middle of its length, and not far from the median plane of the body.

4. The interclavicular air-sac is a median sac, formed by the fusion of two originally distinct ones. It consists of, (1) a median portion, which surrounds the hinder end of the trachea, and can readily be seen in the angle between the two clavicles; and (2) lateral processes, one of which runs forwards along each side of the trachea, while others pass outwards, beneath the coracoid, and open into large axillary air-sacs in the neighbourhood of the shoulderjoints, which communicate with the cavities of the humeri.

The interclavicular air-sac opens into the ventral surface of each lung, immediately in front of the point of entrance of the main bronchus.

5. The cervical air-sacs are much smaller, and lie in front of the lungs at the base of the neck, alongside the vertebral column.

They communicate with the anterior angles or apices of the lungs.

IV. DISSECTION OF THE DIGESTIVE SXSTEM.

\section{A. The Buccal Cavity.}

Open the mouth sightly: cut through the cheek, parallei to the jaws, for about half an inch on each side; and turn 
down the floor of the mouth so as to expose the cavity fully. Note the following structures.

1. The posterior narial apertures are a pair of long narrow apertures, lying side by side in the roof of the mouth. The anterior two-thirds of their length are hidden by a pair of prominent folds of the mucous membrane of the palate.

2. The aperture of the Eustachian tubes is a median open. ing, behind the posterior narial apertures.

Pass a bristle through the aperture, and along the right and left Eustachian tubes to the tympanic cavities.

3. The tongue is triangular, ending in front in a sharp, forwardly directed point; and produced behind into a pair of backwardly directed processes, fringed with fine horny papillæ.

4. The glottis is an oval aperture, with tumid lips, in the floor of the mouth, just behind the tongue.

5. The entrance to the cesophagus is large and wide, and situated behind the glottis.

\section{B. The Abdominal Viscera in situ.}

Lift up the hinder end of the sternum, and cut through its sides about midway between the dorsal and ventral surfaces. Cut through the coracoids and clavicles about the middle of their length. Detach the sternum with a scalpel from the underlying parts, and remove it entirely.

1. The falciform ligament is a median vertical sheet of peritoneum, connecting the dorsal surface of the sternum with the underlying viscera : it is continuous in front with the pericardium, and behind with the omentum.

2. The heart, which is still enclosed in the pericardium, is large and conical, with the apex directed backwards. It lies in the anterior part of the thorax.

3. The lungs are spongy bodies lying at the sides of, and dorsal to, the heart, and very largely concealed by it.

4. The liver is a solid, lobed organ, of a dark-red colour, lying behind, and at the sides of, the heart. 
5. The great omentum is a fold of mesentery loaded with fat, covering the viseera behind the liver. It is continuous in front with the falciform ligament. by it.

Lift up to the great omentum to expose the organs covered

6. The gizzard is a large firm body, lying immediately behind the left lobe of the liver, and imbedded in a deep groove in its substance.

7. The duodenum is a loop of intestine, lying along the right side of the abdominal cavity, and embracing the pancreas.

8. The intestine forms a convoluted mass in the hinder part of the abdomen.

\section{The Alimentary Canal.}

Unravel the intestine by cutting through the mesentery close to it, but taking care not to touch the duodenal loop.

Note the veins in the mesentery, which collect the blood from the alimentary canal, and converge to form the portal vein, which enters the liver in the notch between its right and left lobes.

Note also, and preserve, the coccygeo-mesenteric vein, which runs in the mesenterial fold connecting the rectum with the dorsal wall of the abdomen.

1. The œsophagus commences at the hinder part of the buccal cavity, and runs back through the neck and thorax to the stomach. In the neck it is thin-walled, and dilates behind to form a large bilobed sac, the crop. In the thorax, where it is much narrower, and has thick muscular walls, the osophagus lies between the lungs and dorsal to the heart.

2. The stomach, or proventriculus, lies dorsal to the left lobe of the liver: it is rather wider than the œsophagus, and has thick glandular walls, in which the gastric juice is secreted. Attached to the right 


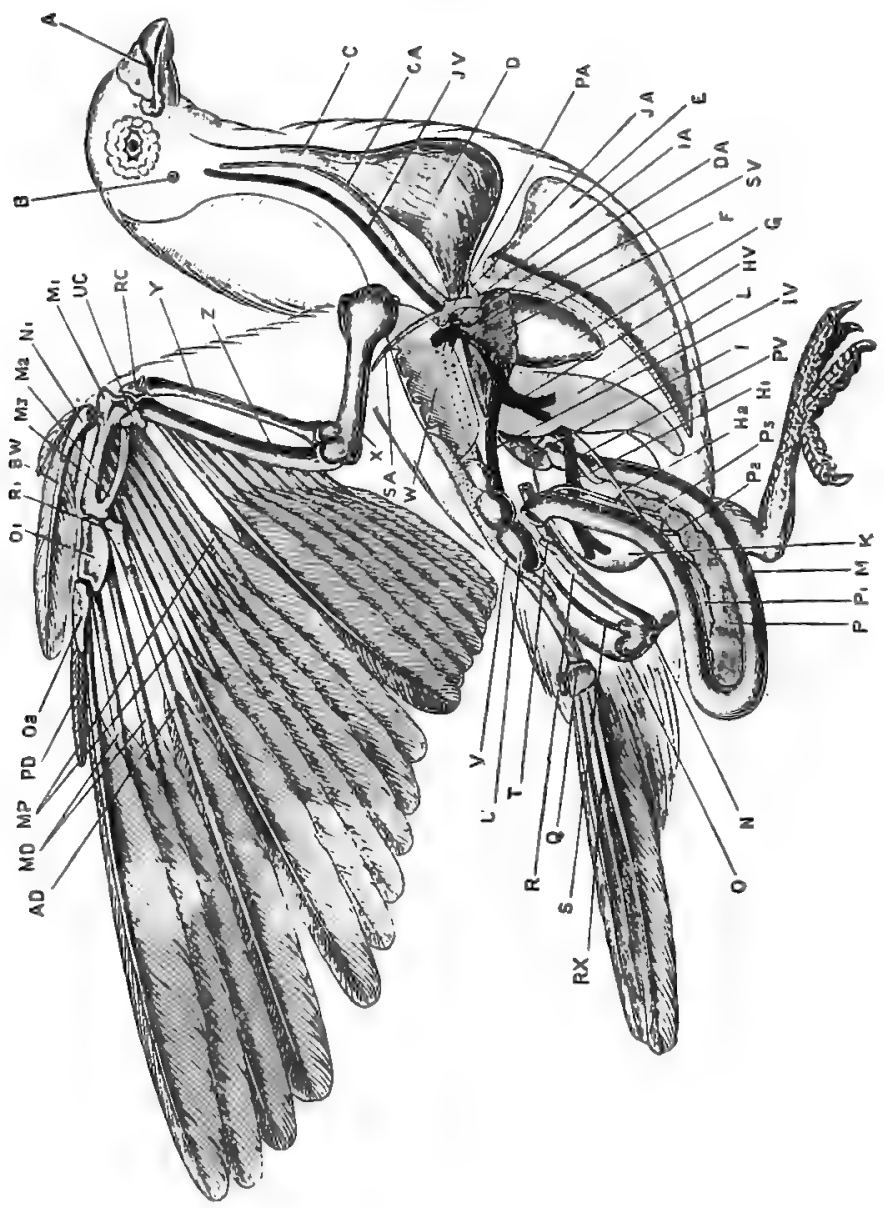


side of the proventriculus is a small red body, the spleen.

3. The gizzard is a large hard body, somewhat flattened. laterally, and with very thick muscular walls, the muscular fibres radiating outwards from a bluish tendon in the centre of each lateral surface. It forms a powerful mill for grinding the food.

The proventriculus opens into the gizzard at its dorsal border, and rather to the left side; while the duodenum leaves it close to the same spot, but more to the right side.

Split open the gizzard along its ventral edge, and wash out the contents. Note the following points :-

a. The great thickness of the muscular walls.

b. The thick horny epidermis which lines the cavity of the gizzard, and in which small stones are usually found imbedded.

c. The openings of the proventriculus and duodenum, placed side by side at the dorsal edge.

4. The duodenum is a $\mathrm{U}$-shaped loop, formed by the first part of the intestine. The pancreas lies between the two limbs of the loop, the further dissection of which

Fia. 67.-Columba livia.-Dissection of the male from the right side. Half the liver and the greater part of the intestine have been removed. In the right wing, the bones and the arrangement of the primary and secondary feathers are shown. The reproductive organs are omitted. (А. м. $\mathbf{m}$.)

A, nostril. AD, ad-digital primary feather. B, external auditory meatus. $\mathbf{B W}$, bastard wing. $\mathbf{C}$, cesophagus. $\mathbf{C A}$, right carotid artery. $\mathbf{D}$, crop. $\overrightarrow{D A}$, aorta. $\mathbf{E}$, keel of the sternum. $\mathbf{E}$, right apricle. $\mathbf{G}$, right ventricle. HV, hepatic vein. H 1 , left bile-duct. H 2 , right bile-duct. I, distal end of stomach. IA, right innominate artery. IV, posterior vena cava. JA, left innominate artery. JV, right jugular vein. $\mathbf{K}$, gizzard. $\mathbf{L}$, liver. $\mathbf{M}$, proximal limb of duodenum. MD, the two mid-digital primary feathers. MP, the six metacarpal primary feathers. M1, first metacarpal. M2, second metacarpal. M 3, third metacarpal. $\mathbf{N}$, cloacal aperture. N 1, first digit of manus. $O$, bursa Fabricii. $O 1$, proximal phalanx of second digit of manus. $\mathbf{O}$, distal phalanx of second digit of manus. $P$, pancreas. $\mathbf{P A}$, right pectoral artery. $\mathbf{P D}$, pre-digital primary feather. $\mathbf{P V}$, portal vein. $\mathbf{P} 1$, first pancreatic duct. $\mathbf{P} 2$, second pancreatic duct. $\mathbf{P} 3$, third pancreatic duct. $\mathbf{Q}$ pygostyle. $\mathbf{R}$, rectum. $\mathbf{R C}$, radial carpal bone. $\mathbf{R X}$, rectrices or tail-feathers. $\mathbf{R} 1$, third digit of manus. $\mathbf{S}$, ureter. SA, right subolavian artery. SV, right anterior vena cava, T, rectal diverticulum. $\mathbf{U}$, kidney. UC, ulnar carpal bone. $\mathbf{V}$, pelvisa $\vec{W}$, lung.

$\mathbf{X}$, humerus. $\mathbf{Y}$, radius. $\mathbf{Z}$, ulna. 
will be postponed until the liver and pancreas are examined.

5. The small intestine is a much convoluted tube, of nearly uniform diameter, and about two feet and a half in length.

6. The rectum, or terminal portion of the intestine, is about an inch and a half long. Its commencement is marked by a pair of small cæcal processes, the rectal diverticula. Posteriorly, the rectum opens into the cloaca, the examination of which will be deferred until the kidneys have been dissected.

\section{The Liver.}

The liver is divided into a larger right, and a smaller left lobe, the surfaces of which are hollowed and grooved to receive the heart, duodenum, and gizzard.

The bile-ducts are two in number, and open into the duodenum.

1. The left bile-duct is a wide tube, about an inch long, which, emerging from the notch between the right and left lobes of the liver, runs backwards to open into the proximal limb of the duodenum, about half an inch beyond the gizzard.

2. The right bile-duct is longer and narrower. It leaves the liver near the dorsal margin of the right lobe, and runs back to open into the distal limb of the duodenum, about the junction of its middle and distal thirds.

\section{E. The Pancreas.}

The pancreas is a compact pinkish gland lying in the loop of the duodenum, along nearly the whole of its length, and grooved by it.

The pancreatic ducts are three in number, and all open into the distal limb of the duodenum.

The first two ducts leave the right border of the gland about the middle of its length, and run forwards, parallel to 
each other, to open close together into the distal limb of the duodenum, about the middle of its length, and close to the right bile-duct.

The third pancreatic duct is longer than the others. It leaves the gland near its anterior end, and runs forwards to open into the distal limb of the duodenum, close to its junction with the small intestine.

Pin out the duodenum on a dissecting-board, and dissect the bile-ducts and pancreatic ducts. The dissection is best performed under water, and from the dorsal surface.

\section{DISSECTION OF THE CIRCULATORY SYSTEM.}

\section{A. The Heart.}

The heart of the bird is of large size, and is divided into four chambers, those of the right and left sides being completely separated from each other. The right side of the heart receives the blood which is brought back by the veins from all parts of the body, and drives it to the lungs to be aërated : from the lungs it is returned to the left side of the heart, and thence driven all over the body through the arteries. The right side of the heart therefore contains venous blood, and the left side arterial blood.

Open the pericardial cavity so as to expose the heart fully; and dissect away the connective tissue and fat from the vessels at the base of the heart.

The heart is conical in shape, with the apex directed backwards and slightly to the left side. A line of fat, which encircles it, marks externally the division into auricular and ventricular portions.

1. The auricular portion is anterior in position, and forms the base of the cone. It is thin-walled, dark in colour, and distinctly divided into right and left auricles, the division being indicated externally by a line of fat. 
2. The ventricular portion is posterior, and forms the apex of the cone. It is much thicker-walled than the auricular portion, and paler in colour. The division between right and left ventricles is not obvious. externally.

\section{B. The Veins,}

The veins should be dissected before the arteries, as they lie for the most part nearer the surface. Great care must be taken not to cut or tear them, as bleeding occurs freely, and is apt to be very troublesome. If a complete dissection is required, the venous system should be injected from either the femoral or the jugular vein.

1. Veins opening into the right auricle. The right auricle receives the venous blood from the whole of the body by three large veins, the venæ cavæ.

a. The right anterior vena cava returns blood from the right side of the head and neck, the right wing, and the right side of the breast.

It is a short wide vein, about a quarter of an inch in length, opening into the right anterior angle of the right auricle: it is formed by the union of three principal veins.

i. The right jugular vein runs along the right side of the neck, close to the vertebral column, and dorsal to the œsophagus.

Anteriorly it unites with the left jugular vein, on the under surface of the base of the skull. In its course down the neck it receives veins from the side of the neck and from the crop ; and at the posterior end of the neck it is joined by the vertebral vein.

ii. The right brachial vein returns blood from the wing.

'iii. The right pectoral vein is the largest of the three. It returns blood from the pectoral muscles, and receives also the internal mam- 
mary vein, which runs along the inner surface of the thoracic wall.

b. The left anterior vena cava returns blood from the left side, by veins corresponding to those opening into the right vena cava. At the base of the heart, it runs transversely across the dorsal surface of the left auricle, to open into the left border of the right auricle.

To see the opening of the left vena cava into the auricle, lift up the apex of the heart and turn it forwards.

c. The posterior vena cava returns blood from the posterior part of the body, including the liver.

It is formed by the union of the two iliac veins a short distance behind the liver. The vein, so formed, runs forwards through the substance of the right lobe of the liver, close to its dorsal surface : it receives the hepatic veins as it leaves the liver, and then runs forwards as a short wide vessel to open into the posterior surface of the right auricle.

Turn the heart forwards, to expose the posterior vena cava as it enters the right auricle. Follow it back through the liver, slitting it up and washing out the contained blood. Note the openings of the hepatic veins, and follow them with a seeker into the liver. Follow the vena cava back, behind the liver, to the point where it is formed by the union of the right and left iliac veins.

2. The veins of the kidneys.

The veins in connection with the kidneys are rather complicated, and are best dissected in specimens injected from the femoral vein. Their relations may, however, be determined by slitting them open and following them with a seeker.

The kidneys are a pair of elongated three-lobed bodies, attached to the ventral surface of the ilia, immediately behind the lungs. 
a. The posterior mesenteric vein is a median vein, running backwards in the mesentery supporting the rectum : it collects the blood from the hinder part of the small intestine, and from the rectum and cloaca. It receives a very small median caudal vein from the tail; and divides, opposite the posterior end of the kidneys, into right and left hypogastric veins.

b. The hypogastric vein, on each side, receives the internal iliac vein from the inner surface of the pelvic cavity, and enters the kidney at its posterior end; it then runs forwards through the posterior and middle lobes of the kidney, to open into the femoral vein, in the groove between the middle and anterior lobes.

c. The femoral vein, on each side, returns the blood from the leg. Entering the kidney at its outer side, between the middle and anterior lobes, it receives, as just noticed, the hypogastric vein : a little further on, the renal vein, returning blood from the two hinder lobes of the kidney, joins it to form the iliac vein.

d. The iliac vein, formed in this way by the junction of the femoral, hypogastric, and renal veins, leaves the kidney at its inner border, between its anterior and middle lobes; and then runs forwards, uniting opposite, the anterior end of the kidney with the iliac vein of the other side; to form the posterior vena cava.

\section{The hepatic portal system.}

The portal vein lies in the omentum, between the two bile-ducts, and on reaching the liver divides into two branches, one supplying each lobe. It conveys to the liver the blood from the greater part of the length of the alimentary canal, and is formed by the union of three chief veins. 
a. The gastro-duodenal vein returns the blood from the right side of the gizzard, from the duodenum and from the last loop of the small intestine.

b. The anterior mesenteric vein returns blood from the greater part of the length of the small intestine.

c. The posterior mesenteric vein returns blood from the hinder part of the small intestine, and from the rectum. The blood in the anterior part of this vein flows forwards to the portal vein, that in the hinder part backwards to the hypogastric veins.

4. Veins opening into the left auricle.

The pulmonary veins, which are extremely short, open into the posterior surface of the left auricle.

Turn the heart forwards: open the left auricle: wash out the contents; and pass a seeker along the pulmonary veins into the lungs.

\section{The Arteries.}

1. The pulmonary artery is a single trunk, which arises from the anterior end of the right ventricle, close to the ventral surface and a little to the left of the median plane. It divides soon after its origin into right and left arteries.

a. The left pulmonary artery is short, and passes direct to the left lung, which it enters in front of the pulmonary vein, and ventral to the left bronchus.

b. The right pulmonary artery passes dorsal to the other arterial trunks, to enter the right lung in front of the bronchus.

2. The aorta arises from the base of the left ventricle, lying between the two auricles, and to the right of, and slightly dorsal to, the pulmonary artery. It gives off almost immediately the very large right and left innominate arteries, and then continues its course as the dorsal aorta. 
a. The left innominate artery is a short wide artery, which divides, after a course of about a quarter of an inch, into two vessels of very unequal size.

i. The left carotid artery, which is much the smaller of the two, runs forwards along the neck, lying close to its fellow of the opposite side, in a groove along the ventral surface of the vertebral column.

About half an inch from its origin it gives off the vertebral artery, which runs forwards along the side of the neck, in the vertebrarterial canal of the cervical vertebræ.

Opposite the angle of the jaw, the carotid artery divides into external and internal carotid arteries.

a. The external carotid artery supplies the tongue, the muscles of the jaws, and other parts of the head.

$\beta$. The internal carotid artery enters the skull by a foramen at its base, and supplies the brain.

ii. The subclavian artery, which is much the larger of the two branches of the innominate, runs outwards, and divides after a course of not more than an eighth of an inch into the brachial and pectoral arteries.

a. The brachial artery runs straight outwards to the wing, giving a branch to the shoulderjoint.

$\beta$. The pectoral artery is a large vessel, very little smaller than the innominate itself: it runs outwards and backwards, looping round the outer side of the sternal end of the coracoid, to enter the deeper surface of the great pectoral muscle, in which it divides into numerous branches. 
b. The right innominate artery has branches exactly corresponding to those of the left innominate.

c. The dorsal aorta, which is rather smaller than either of the innominate arteries, arches over to the right side, crosses the right pulmonary artery, and then runs backwards along the mid-dorsal line of the thorax and abdomen. Its principal branches are as follows.

i. The cœliac artery is a median vessel which arises about the level of the auriculo-ventricular septum of the heart. It runs back in the mesentery, and divides into branches which supply the stomach, gizzard, and part of the intestine.

ii. The anterior mesenteric artery is a median vessel, which arises about a quarter of an inch behind the cœliac artery, and runs backwards in the mesentery, dividing into branches which supply the greater part of the length of the intestine.

iii. The anterior renal arteries are a pair of small vessels, which arise from the aorta opposite the anterior ends of the kidneys, and supply their anterior lobes. From the artery of the left side a branch is given off to the ovary.

iv. The femoral arteries are paired, and arise opposite the anterior lobes of the kidneys. They pass outwards, dorsal to the kidneys, to supply the extensor muscles of the thigh. Each gives off a pubic artery, which runs backwards along the ventral border of the pubes.

v. The sciatic arteries are a pair of larger vessels, which arise opposite the middle lobes of the kidneys : they pass outwards and backwards, between the middle and posterior lobes of the kidneys, and through the ilio-sciatic foramina, 
to supply the flexor muscles of the thighs, and the muscles of the legs.

Each sciatic artery gives off a middle renal artery to the middle lobe of the kidney, and a posterior renal artery to the posterior lobe.

vi. The posterior mesenteric artery is a median vessel, which arises opposite the hinder ends of the kidneys, and supplies the rectum and cloaca.

vii. The internal iliac arteries are a pair of vessels arising about the same level as the posterior mesenteric artery, and running outwards and backwards along the hinder part of the pelvis.

viii. The caudal artery is the terminal portion of the dorsal aorta, which runs along the ventral surface of the caudal vertebræ.

\section{Dissection of the Heart.}

The heart may be dissected in situ, or the vessels may be cut across about a quarter of an inch from it, and the heart removed and placed in a dissecting-dish under water.

1. The auricles.

Slit up the outer walls of the two auricles with scissors, and wash out the contained blood.

a. The right auricle.

\section{i. The openings of the venæ cavæ.}

Pass a seeker into these, and note their positions relatively to the cavities of the heart.

ii. The Eustachian valve is a muscular fold, projecting into the cavity of the auricle, on the right side of the opening of the posterior vena cava.

iii. The septum auricularum is a thin muscular partition, dividing the right and left auricles 
from each other. Near its centre is a thin oval patch, the fossa ovalis, marking the position of the foramen ovale of the embryo.

iv. The right auriculo-ventricular aperture is a large crescentic opening in the posterior wall of the auricle.

b. The left auricle.

i. The openings of the pulmonary veins are in a small recess of the dorsal wall of the auricle.

ii. The left auriculo-ventricular aperture is circular.

\section{The ventricles.}

Cut across the ventricles transversely, about the middle of their length. Note the shape of the cavities, and the thickness of their walls.

a. The right ventricle wraps round the right side of the left ventricle. Its outer wall is thin, and its cavity crescentic in transverse section.

i. The right auriculo-ventricular valve is a muscular flap, hanging into the ventricular cavity, on the right side of the auriculo-ventricular aperture. It is connected by chordæ tendineæ with the wall of the ventricle.

ii. The aperture of the pulmonary artery is at the extreme anterior end and left side of the ventricle.

Pass a seeker from the ventricle along the pulmonary artery, and lay it open with scissors.

a. The semilunar valves are three crescentic pocket-like flaps, guarding the entrance to the artery.

b. The left ventricle has very thick spongy walls, except at the apex, where it is thin. Its cavity is somewhat irregular in shape, but is more or less circular in transverse section. 
i. The left auriculo-ventricular or mitral valve is formed by two membranous flaps, which hang into the ventricle. Their hinder borders are attached by chordæ tendineæ to muscular processes of the ventricular wall, the musculi papillares.

ii. The aperture of the aorta is at the anterior end of the ventricle, and at its right side. In the natural position of the parts, it lies dorsal to the aperture of the pulmonary artery.

Pass a seeker from the ventricle along the aorta, and lay it open with scissors along its right side.

a. The semilunar valves are three pocket-like flaps, similar to those of the pulmonary artery.

VI. DISSECTION OF THE RESPIRATORY SYSTEM.

Remove the heart, if this has not already been done, and also the alimentary canal and liver. Clean the trachea and the ventral surface of the lungs.

1. The trachea, or wind-pipe, is a tube which commences in front at the glottis, runs back along the neck, and divides in the thorax into two bronchi, entering the right and left lungs respectively.

It is surrounded by a series of closely set rings, which are ossified ventrally, and cartilaginous along the dorsal surface.

In the anterior part of the neck the trachea is ventral to the csophagus; further back it lies along its left side. In the thorax, where it again lies ventral to the cesophagus, it divides into the two bronchi, which run outwards and backwards to enter the lungs on their ventral surface and near their anterior ends. 
2. The lungs are a pair of spongy bodies, attached to the dorsal and dorso-lateral walls of the anterior part of the thorax. They are covered ventrally by the pleura, an anterior continuation of the peritoneum. Their inner edges lie close together, separated from each other in the median plane by the projecting centra of the thoracic vertebræ, and by the dorsal aorta.

Remove the pleura from the ventral surface of one lung; and separate the lung from the thoracic wall, noting its close attachment to this wall, and the indentations of its surface caused by the ribs.

3. The syrinx, or 'lower larynx,' is the organ of voice of the pigeon, and is formed by the dilated hinder end of the trachea, and the commencement of the bronchi.

Cut across the trachea about an inch in front of its bifurcation. Make two lateral incisions, with scissors, along the sides of the trachea and bronchi, and turn down the ventral wall.

i. The tympanum is the cavity of the lower end of the trachea and commencement of the bronchi.

ii. The membrana tympaniformis interna is the mucous membrane lining the inner wall of the commencement of each bronchus.

iii. The membrana semilunaris is a delicate vertical fold of mucous membrane, extending forwards into the tympanum from the angle of bifurcation of the trachea: by its vibration the voice is produced.

iv. The pessulus is a slender bar of cartilage running across the tympanum, in the substance of the membrana semilunaris, from the dorsal to the ventral surface. 


\section{The structure of the lungs.}

Pass a seeker along one of the bronchi, and slit it open with scissors. Follow the branches of the bronchus through the lungs, and note the openings of the various air-sacs described above (p. 402).

\section{DISSECTION OF THE RENAL AND REPRODUCTIVE SYSTEMS.}

These may conveniently be considered together, because, as in the rabbit, the genital ducts are in both sexes formed from what were originally parts of the excretory system.

In the male the vas deferens is formed from the mesonephric duct: in the female the oviduct is formed from the pronephric duct. In both sexes the kidneys and ureters of the adult are formed from the hindmost division of the kidneys of the embryo, i.e. the metanephra, and the metanephric ducts.

\section{A. The Male Pigeon.}

\section{The renal system.}

a. The kidneys are a pair of solid three-lobed bodies, firmly imbedded in the ventral wall of the pelvis: and sacrum immediately behind the lungs. Their ventral surfaces are covered by peritoneum.

b. The ureters are a pair of straight narrow tubes. Each arises in the substance of the anterior lobe: of its kidney, emerges between the anterior and middle lobes, and runs backwards along the inner side and ventral surface of the middle and posterior lobes, and then straight back to the dorsal wall of the cloaca.

Slit up one of the ureters, and follow it into the anterior lobe of the kidney, noting the openings of smaller ducts into. it at intervals along its length.

c. The adrenals are a pair of small, elongated, yellowish bodies, attached to the ventral surface of the 
anterior ends of the kidneys, alongside the iliac veins. They have no physiological connection with the kidneys.

\section{The reproductive system.}

a. The testes are a pair of oval bodies, attached by a fold of peritoneum to the inner borders of the anterior lobes of the kidneys, and lying in contact with their ventral surfaces.

b. The vasa deferentia emerge from the inner sides of the posterior ends of the testes, and run back along the outer borders of the ureters as a pair of sinuous tubes with opaque walls.

Their hinder ends are dilated into small vesiculæ seminales, which open into the cloaca.

3. The cloaca is a shallow cup-like depression into which the rectum, ureters, and genital ducts open.

a. The rectum opens into the ventral wall of the cloaca.

Slit up the rectum along one side, and note its opening into the cloaca.

b. The urino-genital pouch is a saccular diverticulum of the dorsal wall of the cloaca, separated from the rectum by a transverse fold. The ureters open into it dorsally and close to the middle line; and to their outer sides are the openings of the vasa deferentia, on the apices of a pair of small papillæ.

c. The posterior chamber of the cloaca is a thin-walled dilatation of the dorsal wall of the cloaca, behind the urino-genital pouch, and close to the external cloacal opening : into its dorsal surface a glandular diverticulum, the bursa Fabricii, opens in young birds, but is usually absent in the adult.

\section{B. The Female Pigeon.}

1. The renal system is the same as in the male. 


\section{The reproductive system.}

a. The ovary. In the embryo a pair of ovaries are present, but of these the right one disappears during development, and the left alone persists. This is a large irregular-shaped body, suspended in a fold of peritoneum opposite, and partly in front of, the anterior lobe of the left kidney. Numerous ova in various stages of development project from its surface.

b. The oviducts. The left oviduct is a wide convoluted tube, thin-walled in front, thick behind, and lying along the left side of the pelvic cavity. In front it opens into the body-carity by a long oblique slit-like mouth, which is in close contact with the ovary. Posteriorly it opens into the cloaca.

The right oviduct is rudimentary: it has the form of a slender tube, opening behind into the cloaca, and usually not more than half an inch in length. The egg, when discharged from the ovary, consists of the 'yolk' only: the 'white' is an albuminous substance formed round the yolk by the walls of the middle portion of the oviduct; and the shell is secreted by the hindmost part of the oviduct shortly before the egg is laid.

3. The cloaca is similar to that of the male, but of larger size.

a. The rectum has the same relations as in the male.

b. The urino-genital pouch. The openings of the ureters are on the anterior wall of the pouch, about an eighth of an inch apart. The openings - of the oviducts are to the outer sides of those of the ureters, the opening of the left oviduct being much larger than that of the rudimentary right one.

c. The posterior chamber is the same as in the male. 


\section{EXAMINATION OF THE BRAIN}

\section{A. Removal of the Brain.}

Cut through the skin of the top of the head along the middle line, and turn the flaps aside. Expose and scrape clean the bones of the roof of the skull. Slice off with a scalpel the skull-roof, taking care not to injure the brain, which lies very close to the bone.

Cut away, bit by bit, the roof and sides of the skull, with the scalpel and stout scissors, so as to expose the brain thoroughly. Remove the neural arches of the first two vertebre, and divide the spinal cord transversely. Turn out the brain carefully, cutting across the several nerve-roots one by one. Place the brain in a bottle of strong spirit, with a pad of loose cotton-wool at the bottom, and leave it for two or three days until it is thoroughly hardened. Examine it in weak spirit.

\section{B. External Characters of the Brain.}

\section{The dorsal surface.}

a. The cerebral hemispheres are a pair of large pyriform bodies, closely applied to each other in the median plane. Their surfaces are nearly smooth, and their anterior ends bluntly pointed.

b. The olfactory lobes are a pair of small conical bodies, projecting forwards from the anterior ends of the hemispheres.

c. The pineal body is a small oval body, immediately behind the hemispheres, and in the angle between them.

d. The optic lobes are a pair of smooth ovoid bodies at the sides of the brain, behind and rather below the hemispheres.

e. The cerebellum is a median elongated oval body, marked by a number of transverse fissures. In front it is in contact with the hemispheres; behind, it overlaps the medulla; and laterally, it lies above the optic lobes. 
f. The medulla oblongata is thick and wide: its anterior end is covered by the cerebellum, and posteriorly it is continuous with the spinal cord.

2. The ventral surface of the brain.

a. The optic chiasma, formed by the crossing of the optic nerves, lies between and slightly in front of the optic lobes.

b. The infundibulum is a small median process, immediately behind the optic chiasma. It is connected with the pituitary body, which is usually left in the skull when the brain is removed.

\section{Bisection of the Brain.}

Divide the brain into right and left halves by a median longitudinal section, so as to expose its cavities.

a. The third ventricle, or cavity of the fore-brain, is of considerable extent from before backwards, and dorso-ventrally; but is very narrow from side to side. It opens in front by lateral apertures, the foramina of Monro, into the lateral ventricles of the hemispheres. Its roof is produced upwards into the stalk of the pineal body, and its floor is depressed to orm the infundibulum.

b. The Sylvian aqueduct, or cavity of the mid-brain, is small, but communicates laterally with large cavities in the optic lobes.

c. The fourth ventricle, or eavity of the hind-brain, is wide from side to side, but shallow dorsoventrally.

\section{A. The Eye.}

\section{THE SENSE-ORGANS.}

1. The eyelids.

The upper eyelid is but slightly movable; the lower one very freely so.

The nictitating membrane, or third eyelid, is a fold at the anterior angle of the eye, lying within 
the other eyelids, between them and the eyeball. It can be pulled obliquely downwards and backwards over the front of the eyeball with great rapidity.

\section{The muscles of the eyeball.}

Open the orbit by removing its dorsal wall with strong scissors, taking care not to damage the nictitating membrane.

a. The muscles moving the eyeball are essentially the same as those of the skate or rabbit.

Remove the eyeball completely, cutting through the muscles and the optic nerve as far as possible from the eyeball.

b. The muscles of the nictitating membrane are two in number, and lie close to the inner or orbital surface of the eyeball.

Snip away with scissors the recti and oblique muscles.

i. The quadratus, or bursalis muscle, is an oblong sheet of muscle, lying in close contact with the inner surface of the eyeball. Its fibres arise along the dorsal edge of the eyeball and run downwards, ending just above the optic nerve in a free border, which forms a tubular tendinous sheath for the tendon of the pyramidalis muscle.

ii. The pyramidalis is a small muscle, somewhat triangular in shape. It arises from the inner surface of the ventral part of the eyeball, below, and a little anterior to, the entrance of the optic nerve. From this origin its fibres run upwards and backwards, converging to form a long thread-like tendon, which, passing through the tubular sheath of the quadratus, runs down on the inner surface of the eyeball, and then under it, to be inserted into the ventral border of the nictitating membrane.

By the combined action of these two muscles the rapid movement of the nictitating membrane is effected. 


\section{The structure of the eye.}

Place the eye under water, and divide it by an equatoriat incision into two halves.

The general structure of the eye is the same as that of other vertebrates; but the following points are of special interest.

a. The pecten is a vascular pigmented fold, which projects into the cavity of the eye from its orbital surface, ventral to the entrance of the optic nerve. It is folded longitudinally like a fan; and its function is unknown.

b. The lens is, as in terrestrial animals generally, much flatter than that of the dog-fish.

c. The sclerotic plates are a ring of small flat bones, overlapping one another, and surrounding the eye, just behind the junction of the cornea and the sclerotic.

\section{B. The Ear.}

1. The tympano-Eustachian passage.

This is the modified hyo-mandibular cleft of the. embryo, corresponding to the spiracle of the dog-fish. Its connection with the ear is of a purely secondary character, and it forms no part of the essential organ of hearing.

a. The external auditory meatus is the outer part of this passage, from the exterior to the tympanic cavity. The external aperture is a circular hole on the side of the head, behind and below the posterior border of the orbit.

Cut away the lower jaw. Cut away the external meatus carefully with scissors, so as to expose the tympanic membrane.

b. The tympanic membrane is a thin transparent septum, separating the external meatus from the tympanic cavity. 
c. The columella is a small rod of bone and cartilage, lying on the inner side of the tympanic membrane, but readily seen through it. It lies almost horizontally, its free anterior end forming a slight projection in the centre of the membrane.

The columella is formed from the uppermost. part of the cartilage of the hyoidean arch, and probably corresponds to part, or the whole of, the hyo-mandibular cartilage of the dog-fish.

Cut across the tympanic membrane in front of the columella, to open up the tympanic cavity.

d. The tympanic cavity is the middle, dilated portion of the tympano-Eustachian passage. On its inner wall is the fenestra ovalis, into which the posterior end of the columella is inserted.

e. The Eustachian tube is the innermost division of the tympano-Eustachian passage. It is a narrow tube, leading from the anterior and lower angle of the tympanic eavity, and running forwards and inwards between the proper base of the skull and the basi-temporal bone. The Eustachian tubes of the two sides unite, and open into the roof of the mouth by a median aperture, just behind the posterior border of the narial opening, and in front of the transverse ridge forming the hinder border of the palate.

\section{The auditory organ.}

The essential organ of hearing is imbedded in the side-wall of the skull. It is, however, easy to dissect, as the layer of bone immediately investing it is very firm and compact, and so preserves the shape of the organ, while the rest of the bone is soft and spongy, and easily removed.

Remove the hinder part of the side of the sluull, containing the auditory organ; and carefully pick away with scalpel and 
forceps the outer spongy layer of bone, until the shape of the organ can be clearly seen.

a. The anterior vertical semicircular canal lies, in the natural position of the parts, close alongside the posterior border of the optic lobe and the side of the cerebellum.

b. The posterior vertical semicircular canal lies almost immediately below the anterior vertical canal.

c. The horizontal semicircular canal lies opposite the lower border of the optic lobe, and the hinder end of the cerebellum. 


\section{APPENDIX.}

\section{LIST OF REAGENTS.}

I, REAGENTS FOR KILLING, HARDENING, AND PRESERVING.

OSMIIC ACDD, 2 per cent. solution.

Preparation.-Dissolve 1 grm. osmic acid crystals in 50 c.c. distilled water. Keep in the dark.

Use.-For killing microscopic animals and for staining fats. Before using, it is to be diluted with 1 to 3 times its bulk of water.

PICRIC ACID, saturated solution.

Preparation.-Saturate water with picric acid by shaking it up with the crystals. Allow to stand: decant before use. Use.-Hardening reagent.

PICRIC ACID, Kleinenberg's solution.

Preparation.-To 250 c.c. saturated solution of picric acid add c.o. strong sulphuric acid: filter: make up to 1 litre with water.

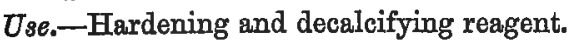


CORROSIVE SUBLIMATE, solution.

Preparation.-Saturate water with mercuric chloride: 1 litre dissolves about 70 grms.

Use.-(1) As a hardening reagent it is used cold.

(2) For killing small animals it may be used hot or cold.

\section{ABSOLUTE ALCOHOL.}

Use.-Hardening reagent, but most used for dehydrating specimens before putting them into oil of cloves or turpentine.

ALCOHOL (Spirit).

It is used of the following strengths: 90 per cent., 70 per cent., 50 per cent., 30 per cent.

Preparation.-90 per cent. alcohol is the ordinary 'methylated spirit.' The weaker solutions are obtained by diluting it with water.

Use.-(1) For preserving specimens; not weaker than 70 per cent.

(2) Hardening reagent; 90 per cent.

(3) For dissolving the intercellular substance of certain tissues, so that the cells may be isolated by teasing; 30 per cent.

CHROMIC ACID, 10 per cent. solution.

Preparation.-Dissolve 10 grms. chromic acid crystals in 100 c.c. water.

Use.-With or without addition of nitric acid for decalcifying bone, etc. As a hardening reagent it must be reduced to a 1 per cent. or 5 per cent. solution by addition of water before use; and osmic acid may be added to it.

\section{IIÜLLER'S FLUID.}

Preparation.-Dissolve 25 grms. potassium bichromate and 10 grms. sodium sulphate in 1 litre water.

Use.-Hardening reagent. It also dissolves intercellular substance, so that the cells may be separated by teasing.

ACETIC ACID, 1 per cent. solution.

Preparation.-Dissolve 10 c.c. glacial acetic acid in water: make up to 1 litre with water.

Use.-For clearing tissues, and especially for rendering nuclei more distinct. 


\section{REAGENTS USED IN STAINING.}

\section{ALUM CARMINE.}

Preparation.-Dissolve 200 grms. ammonia alum in water: boil 10 to 20 minutes with excess of carmine : filter : make up to 1 litre with water: add a fow drops of carbolic acid to preserve from mould.

Use.-Stain for hardened tissues. It may be diluted with 4 times its bulk of water or less.

BORAX CARMINE (Grenacher's).

Preparation.-Dissolve 12.5 grms. carmine and 20 grms. borax in 500 c.c. water : add 500 c.c. 70 per cent. alcohol : allow to stand two or three days: filter.

Use.-Stain for tissues which have been hardened. May be used warm $\left(50^{\circ} \mathrm{C}\right.$.) or cold. The tissues should afterwards be treated with acid alcohol. For staining objects which are to be mounted whole, it should be diluted with several times its bulk of 70 per cent. alcohol, and the treatment with acid alcohol should be prolonged.

\section{ACID ALCOHOL.}

Preparation.-To 100 c.c. of 70 per cent. alcohol add 25 c.c. hydrochloric or nitric acid.

Use.-For differentiating stained specimens, and for removal of surplus stain.

\section{PICROCARMINE.}

Preparation.-Dissolve 10 grms. carmine in 40 c.c. strong ammonia and 2 litres water. Add 50 grms. picric acid. Shake well a few minutes: allow to stand: and decant. Allow to stand a few days, stirring occasionally. Evaporate to dryness over a water bath. To every 2 grms. of dry residue add 100 c.c. water.

Use.-Stain. It may be used with advantage for specimens hardened with osmic acid or otherwise. Subsequent immersion for a short time in acid alcohol improves the effect in the case of some tissues. If the object be dehydrated rapidly, or by means of alcohol containing picric acid, a double-stained effect may be obtained, some tissues being stained yellow and others red. 


\section{HAMANN'S CARMINE.}

Preparation.-Dissolve 30 grms. carmine in 240 c.c. strong ammonia: add glacial acetic acid drop by drop till neutral: allow to stand four weeks: decant and filter.

The deposit may be redissolved and the solution neutralised as before, the product being preferable to the solution first obtained.

Use.-Stain for hardened tissues. It may with advantage be used warm. $t$ is especially useful for animals like the leech, which are liable to become too hard if treated with borax carmine and acid alcohol.

HIMATOXYLIN, Kleinenberg's solution.

Preparation.-Make two solutions, (a) and (b), as follows.

(a) Saturate 70 per cent. alcohol with crystallised calcium chloride, and add powdered alum to saturation.

(b) Saturate 70 per cent. alcohol with alum.

Mix 100 c.c. solution (a) with 800 c.c. solution (b) : allow to stand a week: decant and add a few drops of saturated solution of hæmatoxylin in absolute alcohol.

The solution should be of a deep violet-blue colour. If it be reddish, add a solution of sodium carbonate (not bicarbonate) drop by drop till the violet-blue colour is obtained.

Use.-Stain. The specimen must be previously placed in strong alcohol. It will not give satisfactory results with tissues which contain acid.

\section{MAGENTA.}

Preparation.-Dissolve 6 grm. crystallised magenta (roseine) in 1 litre water. Add 6 c.c. absolute alcohol.

Use.-Stain. Used for fresh preparations. Not fitted for specimens of which it is desired to make permanent preparations.

GOLD CHLORIDE, 1 per cent. solution.

Preparation.-Dissolve 1 grm. gold chloride in 100 c.c. water. Use.-Stain for nerve-tissues. It may be diluted with an equal bulk of water. 


\section{CLEARING REAGENTS,}

(i.e. REAGENTS FOR REMOVING ALCOHOL OR PARAFFIN, AND RENDERING THE OBJECT PERMEABLE BY BALSAM).

\section{OIL OF CLOVES.}

Use.-To remove alcohol from an object before mounting it in balsam. The object must be left in the oil till it becomes transparent: if left too long the object may become brittle.

\section{TURPENTINE.}

Use.-To remove paraffin from sections (see Introduction, p. $x \times x)$.

\section{CREasOTE AND TURPENTINE.}

Preparation.-Mix 100 c.c. creasote with 400 c.c. turpentine: allow to stand till clear.

Use.-Same as turpentine, but must not be used for sections cemented down with shellac.

\section{MOUNTING MEDIA.}

SALT SOLUTION, $\cdot 75$ per cent.

Preparation.-Dissolve 7.5 grms. common salt in 1 litre water. Use.-Living or fresh tissues are usually examined in it.

GLYCERIN.-Used either pure, or diluted with an equal bulk of water.

Use.-For mounting microscopical objects.

\section{GLYCERIN JELLY.}

Preparation.-Soak gelatin in water till soft : pour off excess of water: melt, and while still fluid but cool add oneeighth of its bulk of white of egg : mix thoroughly : boil : strain through flannel : to strained fluid while still hot add half its volume of a mixture of equal parts of glycerin and camphor water. 
Use.-For mounting microscopical objects which are too transparent when mounted in balsam. (See Introduction, p. $x \times i \dot{x}_{\text {.) }}$

\section{FARRANT'S MEDIUM.}

Preparation.-Dissolve 400 grms. white gum arabic in 400 c.c. water without heat, stirring occasionally: add 200 c.c. glycerin: strain through clean flannel if necessary. Keep in a stoppered bottle with a piece of camphor.

Use.-Same as glycerin-jelly, but it is used cold, and objects may be transferred to it direct from water. It soon hardens at the edges: ringing with varnish is therefore unnecessary.

\section{CANADA BALSAM.}

Preparation.-May be used as bought from the chemist, or diluted to a suitable consisténcy with benzol or turpentine. Still better, evaporate to dryness : powder and dissolve in chloroform, benzol, or tarpentine.

Use.-The most useful of all media for mounting stained objects. (For mode of using, see Introduction, p. xxx.)

\section{MICROTOMIST'S MEDIA.}

\section{GUM-WATER.}

Preparation.-Dissolve gum-arabic in 3 times its weight of water without heat: allow to stand: strain if necessary. Use.-For impregnating objects to be cut by the freezing microtome.

\section{PARAFFIN.}

Melting point about $56^{\circ} \mathrm{C}$.

Use.-For imbedding objects to be cut into sections.

\section{SOFT PARAFFIN.}

Melting point about $40^{\circ} \mathrm{C}$.

Use-(1) To mix with hard paraffin when used in a cold room (see Introduction, p. xxviii).

(2) To smear the sides of the block that is being cut, so as to ensure the sections cohering by their edges into a continuous ribbon. 


\section{CEMENTS FOR FIXING DOWN SECTIONS CUT IN PARAFFIN.}

\section{SHELLAC AND ALCOHOL.}

Preparation.-Dissolve perfectly dry bleached shellac in absolute alcohol: allow to stand two or three weeks: decant. Keep in a bottle with a cap ground to fit. Dilute with absolute alcohol when it becomes too thick to flow evenly.

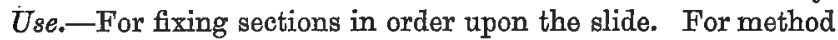

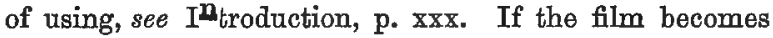
white and opaque on drying, warm it to $60^{\circ} \mathrm{C}$. till the opacity disappears.

\section{COLLODION AND OIL OF CLOVES.}

Preparation.-Mix equal parts of collodion and oil of cloves.

Use.-Same as above. The slide is smeared very slightly with the mixture and the sections laid upon the film while still moist. Then proceed as with shellac and cloves.

If the solution becomes too stiff dilute with ether.

\section{GLYCERIN AND ALBUMEN.}

Preparation.-Mix equal volumes of fresh white of egg and glycerin. Keep a piece of camphor in the bottle.

Use.-Same as above. After clearing, the slide must be placed. in absolute alcohol for a few minutes and then returned to turpentine.

If desired, sections may be stained after they are fixed to the slides. To do this, clear as before, transfer successively to absolute alcohol, 90 per cent. and 70 per cent. spirit; then stain, and transfer to the alcohol in the reverse order (70, 90 per cent., absolute) : clear and mount.

\section{INJECTION FLUIDS.}

\section{CARMINE INJECTION FLUID.}

Preparation.-Rub up carmine in water : add ammonia drop by drop till the carmine is dissolved: filter: evaporate at a gentle heat, stirring constantly to get rid of the ammonia. Rub up the residue with camphor water.

Use.-For injecting small animals and parts of animals. It must be well shaken before use. 


\section{PRUSSIAN-BLUE INJECTION FLUID.}

Preparation.-To a solution of ferric chloride add a few drops of hydrochloric acid and sufficient potassium ferrocyanide to cause complete precipitation. Shake before use.

\section{PLASTER OF PARIS INJECTION.}

Preparation.-Rub up fine plaster of Paris with water, in a mortar, to the consistency of thin cream. Colour to the required tint with carmine injection fluid or with French blue. Strain through fine muslin.

$\bar{U}_{8 e}$.-For injecting blood-vessels, etc., of rabbits and other large animals. It must be used immediately after preparation. (See Introduction, p. xxi.)

\section{GELATIN INJECTION.}

Preparation.-Soak gelatin in water till thoroughly soft. Pour off the superfluous water: melt and stir into it carmine injection fluid, or other insoluble colouring substance, and a few drops of carbolic acid; or if preferred the gelatin may be soaked in camphor water, and carbolic acid is then unnecessary. Stir till almost cold. The in. jection is solid when cold.

Use.-For injection of specimens of which it is proposed to make sections. It is to be melted, and kept well stirred during injection. If the specimen is large or difficult to inject, the whole operation must be performed in a vessel of water as hot as the hand can bear it.

\section{WHITE-OF-EGG INJECTION.}

Preparation.-Rub up fresh white of egg with carmine injection, or other colouring fluid which will not cause coagula. tion of the albumen.

Use.-For injecting specímens of which sections are to be cut. It is used fresh, and the specimen is then placed in alcohol, or corrosive sublimate, or in hot water.

\section{GUM INJECTION.}

Preparation.-Make a thick mucilage of gum-arabic and cold water.

Use.-Inject cold, previously colouring as desired. Plunge the specimen into alcohol, and the injection will solidify. 


\section{IN DEX.}

Abdomen : Crayfish, 129 ; Cockroach, 156

Abdominal appendage : Crayfish, 130-131; Cockroach, 159

Abdominal pore: Dog-fish, 199, 217

Abdominal viscera : $\log -$ fish $_{2} 217-218$; Rab6it, 313-315; Pigeon, 404-405

A.cetrbulum : Rabbit, 299 ; Forol, 391

Acromion : Rabbit, 294

Adrenal body : Rabbit, 315 ; Pigeon, 420

After-shaft : Pigeon, 398

Air-sacs : Pigeon, 401-403

Alm cordis : Orayfish, 142

Ala spuria : Pigeon, 397

Albumen gland : Snail, 108, 117

Ali-sphenoid : Rabbit, 277; Fowl, 380

Alimentary canal, see Digestive System

Alternation of generations : Liver-fiuke, 26

Alveolus : Rabbit, 285

AMCEBA, 1-3

A.MPHIOXUS, 170-195 ; external characters, 170-171 ; skeletal system, 173-174; muscular system, 174-175; digestive and respiratory systems, 175-177; atrial cavity, 177-178; collom, 178 ; circulatory system, 179-180 ; excretory system, 180 ; nervous system, 181-183 ; sense organs, 183 ; reproductive

Anapophysis, see Vertebra system, 183-184; transverse sections, 184-195

Angular bone : Forol, 385

Ankle, see Tarsus

Ankle-joint : Fonol, 393

Annulus : Leech, 38; Earthworm, 57

Anodonta, see Mussel

Antenza: Crayfish, 135 ; Cockroach, 157

Antennule : Crayfish, 135

Anti-trochanter : Fowol, 391

Anus : Paramecium, 6 ; Vorticella, 10 ; Leech, 39 ; Earthworm, 58; Mussel, 84, 96; Snail, 105 ; Crayfish, 145 ; Cockroach, 157, 163 ; Amphioxus, 171, 195 ; Rabbit, 306

Aorta : Mfussel, 89-90; Snail, 123; Rabbit, 324, 329; Pigeon, 413, 415 cardiac : Amphioxus, 179, 190; Dog-fish, 232

dorsal : Amphioxus, 179, 190, 193; Dog-fish, 233, 234 ; Pigeon, 415

Aortic arch : Amphioxus, 179

Aperture, see also Foramen

auditory : Crayfish, 135 ; Rabbit, 291, 306 ; Pigeon, 400

oloscal : Mussel, 83 ; Dog-fish, 198 ; Pigeon, 399

excretory : Liver-fhike, 29 ; Leech, 39 ; Earthwoorm, 58; Mussel, 92, 99; Snail, 112 ; Crayfish, 135

exhalent : Mussel, 83

external : Leech, 39 ; Earthworm, 58, 59 ; Mressel, 84 ; Snail, 105-106 ; Cockroach, 157; Dog-fish, $198-199$; Pigeon, 399-400

genital : Liver-fluke, 27,32; Leech, 39; Earthworm, 58; Mussel, 92, 99 ; Snail, 105 ; Crayfish, 133 ; Cockroach, 157

hyoidean: Amphioxus, 175

inhalent : Mussel, 83

of Eustachian canal : Rabbit, 290 ; Foul, 378, 379 
Aperture, of Eustachian tube : Rabbit, 311; Pigeon, 404 on surface of skull : Rabbit, 288-291 ; see also Foramen posterior narial : Pigeon, 404 reno-pericardial : Mussel, 93; Snail, 111 respiratory : Snail, 105, 109 ; Cockroach, 157 urino-genital : Rabbit, 306

Appendage : Crayfish, 125, 128, 129-136; Cockroach, 157-159 abdominal : Crayfish, 130-131 ; Cockroach, 159 hend : Crayfish, 134-136 ; Cockrouch, 157-159

Apteria : Pigeon, 399 thoracic : Crayfish, 131-134; Cockroach, 159

Aqueductus Fallopii : Rabbit, 282 vestibuli : $D o g-f i s h, 206,261-262$

Arbor vitæ : Rabbit, 366

Arm, see Fore-limb

Arteries : Mussel, 89-90; Snail, 123-124; Crayfish, 151-152; Dog-fish, 230-236 ; Rabbit, $329-332$; Pigeon, 413-416; see also Aorta

Artery, brachial : Rabbit, 329 ; Pigeon, 414

branchial : Dog-fish, 232

carotid : Dog-fish, 233 ; Rabbit, 329, 345 ; Pigeon, 414

caudal: Dog-fish, 234 ; Pigeon, 416

coeliac : Dog-fish, 235 ; Rabbit, 330 ; Pigeon, 415

coronary : Rabbit, 335

epibranchial : Dog-fish, 233

external carotid: Dog-fish, 234; Rabbit, 330 ; Pigeon, 414

femoral : Pigeon, 415

hyoidean : Dog-fish, 234

iliac: Dog-fish, 236; Rabbit, 331, 332 ; Pigeon, 416

ilio-lumbar : Rabbit, 332

inferior (posterior) mesenteric : Dog-fish, 236 ; Rabbit, 331 ; Pigeon, 416

innominate: Rabbit, 329 ; Pigeon, 413,414

intercostal : Rabbit, 330

internal carotid : Dog-fish, 234; Rabbit, 330 ; Pigeon, 414

internal mammary : Rabbit, 330

lieno-gastric : Dog-fish, 236

lumbar : Rabbit, 331

mesenteric : Dog-fish, 236 ; Rabbit, 330, 331 ; Pigeon, 415, 416

ovarian : Rabbit, 331

parietal : Dog-fish, 236

pectoral : Pigeon, 414

pubic : Pigeon, 415

pulmonary : Rabbit, 324 ; Pigeon, 413

renal : Dog-fish, 236 ; Rabbit, $\mathbf{3 3 1}$; Pigeon, 415, 416

sacral : Rabbit, 331

sciatic : Pigeon, 415-416

spermatic : Rabbit, 331

subclavian : Dog-fish, 235 ; Rabbit, 329, 330 ; Pigeon, 414

vertebral : Rabbit, 330 ; Pigeon, 414

vesical : Rabbit, 332

Arthro-branchia: Chayfish, 137, 138

Arthrodial membrane: Crayfish, 128

Articular bone : Fowl, 385

Articular process, see Vertebra

Arytenoid cartilage : Rabbit, 350

Astacus, see Crayfish

Atlas vertebra: Rabbit, 269-270; Fow , 371

Atrial cavity: Amphioxus, 177, 186, 189-190, 192, 193

Atrial epithelium : Amphioxus, 188-189

Atrial fold : $\Delta$ mphioxus, 177

Atrial pore: Amphioxus, 171

Auditory aperture, see Aperture

Auditory capsule : Dog-fish, 206 ; Rabbit, 281-282

Auditory organ: Mrussel, 94; Snail, 121 ; Crayfish, 150; Dog-tish, 261-262 ; Pigeon, $426-428$

Auditory ossicle : Rabbit, 283-284; Fonot, 378, 386 ; Pigeon, 427

Auricle: Mussel, 89, 100 ; Snail, 1111 ; Dog-fish, 223, 237; Rabbit, 323-324, 332-333 ; Pigeon, $416-417$

Auricular feathers : Pigeon, 400

Axis vertebra: Rabbit, 270; Fowl, 371 
Backbone, see Vertebral column

Barb : Pigeon, 398

Barbule : Pigeon, 398

Basi-branchial : Dog-fish, 213

Basi-hyal : Dog-fish, 212

Basi-occipital : Rabbit, 276

Basipodite, see Appendage

Basi-pterygium : Dog-fish, 214

Basi-sphenoid : Rabirit, 277

Basi-temporal : Fowl, 377

Beak : Pigeon, 396

Bile-duct: Mussel, 96 ; Snail, 116 ; Crayfish, 144; Dog-fish, 220 ; Rabbit, 317 ; Pigeon, 408

Birds, characters of, 367,395

Bladder : Rabbit, $\mathbf{3 1 5}, 337$

Blatta, see Cockroach

Blood : Leech, 43 ; Earthworm, 62

Blood-sinus: Leech, 43-44, 54; Snail, 124 ; Crayfish, 139-141; see also Sinus and Vein

Blood-vessel : Leech, 43-44, 54-55 ; Earthworm, 62-63, 74, 75; Mussel, 89-90; Snail, 123-

Body-cavity, see Coelom 124 ; see also Circulatory System, Artery, Vein

Bojanus, organs of : Mussel, 91-92

Bone, cartilage : Rabbit, 264,275 membrane : Rabbit, 264, 275 sesamoid : Rabbit, 264

Botryoidal tissue : Leech, 55

Brain : Dog-fish, 247-248, 256-259 ; Rabbit, 350-366 ; Pigeon, 423-424

Brain-case, see Cranium

Brain, cavity of, see Ventricle

Brain-membrane : Rabbit, 351-352

Branchia, see Gill

Branchial arch : Dog-fish, 212-213

Branchisl bar : Amphioxus, 176, 188

Branchial chamber : Mussel, 82, 102 ; Crayfish, 136-137

Branchial vessels : Dog-fish, 230-234

Branchio-cardiac canal : Crayfish, 142

Branchio-cardiac groove : Crayfish, 129

Branchiostegite: Crayfish, 129, 137

Bronchus : Rabbit, 344

Buccal cavity : Earthoorm, 59 ; Cockroach, 162; Amphioxus, 175, 186 ; Rabbit, 307-313 Pigeon, 403-404

Buceal mass : Snail, 113, 121-122

Budding : Hydra, 20

Bulla, tympanic : Rabbit, 277, 282

Bursa Fabricii: Pigeon, 421

Byssus : Mussel, 97

Crecum : Rabbit, 314, 318, 320

Calamus : Pigeon, 397

Canal, branchio-cardiac : Crayfish, 142

carotid : Forol, 377, 378

central : Amphioxus, 186

interorbital : $D o g-f i s h, 207$

mucous : $D$ og- $f i s h, 199,262-263$

naso-palatine : Rabbit, 309

neural, see Vertebra.

of Laurer : Liver-fiuke, 31

pericardio-peritoneal : Dog-fish, 223

reno-pericardial : Mussel, 93 ; Snail, 111

semicircular : Dog-fish, 206, 262; Pigeon, 428

urino-genital : Rabbit, 338, 342

vertebrarterial, see Vertebra

Capitulum: Rabbit, 274

Carspace : Crayfish, 128

Cardia : Rabbit, 316

Cardo : Cockroach, 158

Carina: Fowl, 374

Carpopodite, see Appendage

Carpo-metacarpus : Fout, 388, 389, 390

Carpus : typical, 297; Rabbit,297-298; Fovol, 389 
Cartilage-borie : Rabbit, 264, 275

Centrum : Dog-fish, 201 ; see also Vertebra

Cephalo-thorax : Crayfish, 128

Cerato-branchial : $D o g-f i s h, 213$

Cerato-hyal : Dog-fish, 212

Cercaria : Liver-fiuke, 36

Cerci : Cockroach, 157, 159

Cere : Pigeon, 396

Cerebellum : Dog-fish, 248, 258 ; Rabbit, 353, 366 ; Pigeon, 423

Cerebral hemispheres : Dog-fish, 247 ; Rabbit, 352, 354, 358-361, 363 ; Pigeon, 423

Cervical canal : Crayfish, 137

Cervical groove : Crayfish, 128

Chelæ: Crowfish, 133

Chiasma, optic: Dog-fish, 257 ; Rabbit, 355, 364 ; Pigeon, 424

Chlorophyll : Hydra, 23

Chordæ tendineæ: Rabbit, 334 ; Pigeon, 417, 418

Choroid : Dog-fish, 260

Choroid plexus : Dog- $A 3 h, 258$; Rabbit, 360

Cilia: Paramecium, 5 ; Opalina, 7 ; Vorticella, 10

Cingulum : Earthworm, 57, 73

Circulation, course of : Mussel, 90 ; Craufish, 139

Circulatory system : Leech, 43-44, 54-55 ; Earthoorm, 62-63 ; Musset, 88-90, 99, 100 ; Snail, 122-124; Crayfish, 139-142 ; Cockroach, 161; Amphioxts,

Cirrus : Amphioxus, 171 179-180; Dog-fish, 222-238; Rabbit, 322-335; Pigeon, 409-418

Cirrus-sac : Liver-fluke, 31

Clasper : Dog-fish, 198, 215

Clavicle : Rabbit, 295 ; Fospl, 387

Clinoid process : Rabbit, 277,279

Clitellum: Earthoorm, 57,73

Clitoris : Rabbit, 306, 342

Cloaca : Dog-fish, 242, 246; Pigeon, 421, 422

Cloacal aperture : Mussel, 83 ; Dog-fish, 198 ; Pigeon, 399

Cloacal chamber : Mussel, 82, 87

Clypeus : Cockroach, 154

Cnemial crest : Rabbit, 301 ; Fonol, 392

Gnidocil : Eydra, 18

Cochlea : Rabbit, 282, 304

CoCkroACH, 153-169; external characters, 154-157 ; appendages, 157-161 ; circulatory system, 161; digestive system, 162-164; exoretory system, 164; respiratory system, 164-165; nervous system, 165-167; sense-organs, 167 ; reproductive system, $167-169$

Cocoon : Leech, 37 ; Earthuorm, 56, 66

Coelom : Leech, 43-44, 54; Earthoorm, 59, 73 ; Mussel, 93 ; Amphioxus, 178-179, 190, 192, 193,195

Coelomic canal : Amphioxus, 178, 190, 192

Collar : Snail, 105

Colon : Cockroach, 163 ; Dog-Ash, 219 ; Rabbit, 314, 319, 320

Columba, see Pigeon

Columella : Snait-shell, 106 ; Fotol, 376, 386; Pigeon, 427

Columnæ carnea : Rabbit, 335

Commissure, of brain : Rabbit, 363-364

Condyle, of skull : Dog-fish, 209 ; Rabbit, 277 ; Fowt, 377

Conjugation : Paramecium, 6 ; Porticelli, 11

Conus arteriosus : Dogfsh, 223, 237

Coracoid : Fonol, 387

Coracoid process : Rabbit, 294

Cornea: Crayfish, 149 ; Cockroach, $167 ;$ Dog-fish, 260

Coronoid process : Rabbit, 287 ; Fonol, 385

Corpus or Corpora

albicans : Rabbit, 355,364

callosum : Rabbit, 352,363

cavernosa : Rabbit, 338, 339, 342

geniculatum: Rabbit, 361

mammillare : Rabbit, 355,364

quadrigemina : Rabbit, 304, 352, 362, 365

spongiosum : Rabbit, 339

striatum : Rabbit, 359

trapezoidea : Rabbit, 355 
Cotyloid bone: Rabbit, 299

Covert feathers: Pigeon, 399

Coxa : Cockroach, 159

Coxopodite, see Áppendage

Cranium : Dog-fish, 203; Rabbit, 275-280 ; Forol, 376-381

CRAYFISH, 125-152 ; external characters, 126-129 ; appendages, 129-136 ; gills, 136-138 ; ciroulatory system, 139-142 ; reproductive system, 142-143; digestive system, 143-146; excretory system, 147; nervous system, 147-148;

Cribriform plate : Rabbit, 280 sense-organs, 149-151; injected specimen, 151-152

Gricoid cartilage : Rabbit, 343,350

Crop : Leech, 42-43 ; Earthworm, 60 ; Snail, 115 ; Cocksoach, 162 ; Pigeon, 405

Crura cerebri : Rabbit, $355,363,365$

Cutaneous blood-vesseIs : Leech, 44

Cuticle : Paramecium, 5 ; Vortícella, 10 ; Liver-Aluke, 27, 33; Leech, 51 ; Eartherm, 57 72 ; Mussel, see Shell; Snail, see Shell ; Crayfish, 125; Cockroach, 153

Dactylopodite, see Appendage

Dart-sac : Snail, 118

Dentary bone: Fovol, 385

Dermis : Leech, 51 ; Earthworm, 73 ; Amphioxus, 185

Development : Liver-fluke, 32-36 ; Mussel, 76, 97 ; Crayfish, 126 ; Dog-fish, 196, 238-239 ;. Rabbit, 304 ; Bird, 395-396

Diaphragm : Rabbit, 315,321

Digestive system : Liver-fukke, 27-29; Redia, 35 ; Cercaria, 36 ; Leech, 41-43, 54 ; Earthworm, 59-62, 73-74; Mrussel, 95-96 ; \$nail, 113-116 ; Crayfish, 143-. 146; Cockroach, 162-164; Amphioxus, 175-177 ; Dogfish, 219-221; Rabbit, 315-320; Pigeon, 403-409

Digit : Rabbit, 298, 303; Fowt, 390, 394

Dise : Vorticella, 8

Distomum, see Liver-fluke

DOG-FISH, 196-263; external characters, 197-200; skeleton, 200-217 ; abdominal cavity and viscera, 217-218; digestive system, 219-221; respiratory system, 221-222 ; circulatory system, 222-238; renal and reproductive systems, 238-246 ; nervous system, 246-259; sense-organs, 259-263

Duct, bile : Mussel, 96 ; Snail, 116; Crayfish, 144; Dog-fish, 220; Rabbit, 317-318; Pigeon 408

common genital : Sncil, 117

hermaphrodite : Snail, 117

lacrymal : Rabbit, 281

mesonephric : Dog-fish, 238-239, 241, 243 ; Rabbit, 335; Pigeon, 420

metanephric: Dog-fish, 238-239, 242, 245-246; Rabbit, 335 ; Pigeon, 420

Mutllerian : Dog-fish, 238, 239, 246

of salivary receptacles: Cockroach, 163

ovarian : Liver-fiuke, 31

pancreatic : Dog-fish, 221 ; Rabbit, 316 ; Pigeon, 408-409

pronephric: Dog-fish, 238, 239, 246 ; Rábbit, 335; Pigeon, 420

salivary : Snail, 116 ; Cockroach, 162, 163; Rabbit, 311-313

segmental : Dog-fish, 238

Stenonian : Rabbit, 311

vitellarian : Liver-fuke, 31

Wharton's: Rabbit, 313

Wolflian : Dog-fish, 238-239, 241, 243

Ductus arteriosus : Rabbit, 324

Ductus ejaculatorius : Liver-fluke, 30 ; Cockroach, 167

Duoednum : Rabbit, 314, 316 ; Prgeon, 405, 407

Dura mater : Rabbit, 351

Ear, see Auditory Organ

Earthworm, 56-75 : external characters, 57-59 ; digestive system, 59-62 ; circulatory system, 62-63 ; excretory system, 63-65 ; reproductive system, $65-70$;: nervous system, 70-72; transvers sections, 72-75

Eedysis : Crayfish, 126 ; Cockrouch, 153

Fetoderm: $B y d 7^{\prime} a, 16,17-19,22-23,24$

Ectosarc: Ameeba, 2 ; Paramecium, 4 ; Opalina, 7 ; Vorticella, 10

Eggg, see Orum

Elytra : Cockroach, 155-156

Embryo: Liver-fluke, 33 ; Mussel, 76, 97 ; Crayfish, 126 ; Rabbit, 304

Ehncystment: Vorticella, 12 
Endoderm : $H y d r \alpha, 17,19,23,24$

Endophragmal skeleton : Crouyfish, 147

Endopodite: Crayfish, 129 ; see also Appendage

Endosarc: Amobba, 2; Paramecium, 6; Opalina, 7 ; Vorticella, 11

Epi-branchial : Dog-fish, 213

Epicranium : Cockroach, 154

Epidermis : Leech, 51; Earthworm, 72; Amphioxus, 185

Epididymis : Leech, 49; Rabbit, 337

Epiglottis : Rabbit, 310

Epimera : Craufish, 127, 137

Epiotic: Rabbit, 281

Epiphragm : Snail, 103

Epiphysis, of vertebra : Rabbit, 266

Epipleural cavity : $\Delta m p$ hioxus, 177

Epipleural fold: Amphioxus, 177

Epipodite : Craufish, 130

Eithmoidal region : Rabbit, 279

Ethmo-turbinal : Rabbit, 280

Eustachian tube : Rabbit, 311 ; Pigeon, 427

Eustachian valve : Rabbit, 333; Pigeon, 416

Ex-occipital : Rabbit, 277

Frxopodite : Crayfish, 128 ; see also Appendage

Frosikeleton : Crayjish, 126-128

Extra-branchial : Dog-fish, 213

Eye : Leech, 50; Snail, 121; Crayfish, 149 ; Cockroach, 154, 167 ; Amphioxus, 183 ; Dog-

Eyelid : Pigeon, 424 fish, 248, 260-261; Rabbit, 305 ; Pigeon, 424-426

Eye-muscle, see Muscle

Fabella: Rabbit, 301

Fallopian tube: Rabbit, 341

Falx cerebri : Rabbit, 352

Frsciola, see Liver-fluke

Fat-body: Cockroach, 162

Feathers: Pigeon, 397-399

Femur : Cockroach, 159; Rabbit, 300; Fow 392

Fenestra : Cockroach, 154, 167

Fenestra ovalis : Rabbit, 282 ; Foovl, 378,386

Fenestra rotunda: Rabbit, 282; Fotol, 378

Fenestral recess : Forol, 378

Fibula : Rabbit, 301 ; Fowl, 393

Filoplume, 399

Fin : Amphioxus, 171; Dog-fish, 197-198, 214-217

Fin-ray : Dog-fish, 214, 216

Fission : Amceba, 3; Paramecium, 6; Opalina, 7 ; Vorticella, 11 ; Hydra, 20

Flagella: Bydra, 19

Flagellum: Snail, 118

Floccular fossa : Rabbit, 282

Floccular lobe : Rabbit, 353

Fontanelle : Dog-fish, 205

Food: Amoeba, 3; Paramecium, 6; Opalina, 7; Vorticella, 10; Hydra, 13 ; Leech, 37 ; Earthworm, 56 ; Mussel, 95 ; Snail, 103 ; Crayfish, 126 ; Cockroach, 153 ; Dogfish, 196

Foot : Hyära, 15 ; Arussel, 83 ; Snail, 104 ; Rabbit, 303

Foramen, anterior palatine : Rabbit, 286, 289

condylar : Rabbit, 290

for internal carotid artery : Dog-fish, 209 ; Rabbit, 283, 290

ilio-sciatic : Fowl, 391

incisivum : Rabbit, 289

inferior dental : Rabbit, 288

infra-orbital : Rabbit, 286, 289

internal orbital : Rabbit, 289

laerum anterius : Rabbit, 278, 288

lacerum medium : Rabbit, 289

lacerum posterius : habbit, 290

Jacrymal : Rabbit, 290

magnum: Dog-fish, 209 ; Rabbit, 291 ; Fovl, 377

obturator : Rabbit, 299 ; Forol, 391

of Monro: Rabbit, 360,364 ; Pigeon, 424 
Foramen, optic : Rabbit, 278,288

orbito-nasal : $D o g-f i s h, 207$

ovale, 289 ; Rabbit, 333 ; Pigeon, 417

pituitary : Rabbit, 290

posterior palatine : Rabbit, 289

rotundum; 288

stylomastoid : Rabbit, 283, 289

triosseum: Pigeon, 387, 401

Foramina of exit for cranial nerves

for Ist cranial nerve (olfactory) : Dog-fish, 209 ; Rabbit, 280 ; Fovol, 380

- for 2nd cranial nerve (optic) : Dog-fish, 207; Rabbit, 278, 288; Foul, 380

for 3rd cranial nerve (oculo-motor): Dog-fish, 207; Rabbit, 278, 288; Fovol, 380

for 4th cranial nerve (pathetic) : Dog-fish, $207 ; R \pi b b i t, 278,288 ; F o v t, 380$

for 5th cranial nerve (trigeminal) : Dog-fish, 207; Foovl, 379

for ophthalmic branch of 5th: Dog-fish, 205, 207; Rabbit, 278, 288

for maxillary branch of 5th : Rabbit, 278, 286, 288

for mandibular branch of 5 th : Rabbit, 278,289

for 6th cranial nerve (abducent) : Dog-fish, 207; Rabbit, 278, 288

for 7 th cranial nerve (facial) : Dog-fish, 207 ; Rabbit, 283, 289

for ophthalmio branch of 7 th : Dog-fish, 205, 207

for 8th cranial nerve (auditory) : Rabbit, 282

for 9th cranial nerve (glosso-pharyngeal): Dog-fish, 208; Rabbit, 290; Foul, 377

for I0th cranial nerve (pneumogastric) : Dog-fish, 209; Rabbit, 290; Fool, 377

for 11th cranial nerve (spinal accessory) : Rabbit, 290

Fore-brain : Rabbit, 358, 361, 363

for 12 th cranial nerve (hypoglossal) : Rabbit, $290 ;$ Fovol, 377

Fore-limb : Rabbit, 295-298,307; Fowt, 388-390

Fornix : Rabbit, 360, 363, 364

Fossa ovalis : Rabbit, 3333 ; Pigeon, 417

FowL, skeleton of, 367-394; vertebral column, 367-373; ribs and sternum, 373-375 ; skull, 375-386 ; appendicular skeleton, 386-394

Frontal bone : Rabbit, 279 ; Fowl, 379-380, 381

Frontal segment : Rabbit, 278-279

Funnel, nephridial : Leech, 46; Earthworm, 64, 65

Eunnel, seminal : Earthworm, 68

Furcula : Fowl, 387

Gailea : Cockroach, 158

Gall-bladder : Dog-fish, 220 ; Rabbit, 317

Gallus, see Frowl

Ganglion, see Nerve-ganglion

Gastric mill : Crayfish, 145-146

Gastrula : Liver-fiuke, 35

Gemmation : Hydra, 20

Gena : Cockrouch, 154

Generations, alternation of : Liver-fuke, 26

Genital sinus : Liver-fiuke, 32

Germ-cell : Liver-fluke, 33

Giant-fibres : Ecrithworm, 74

Gill : Mussel, 83, 85-88, 98, 102 ; Crayfish, 136-138 ; Dog-fish, 221-222

Gill-arch : Amphioxus, 176, 188; see also Branchial arch

Gill-ray : $D o g-f i s h, 212$

Gill-slit : Amphioxus, $176 ;$ Dog-fish, 199

Gizzard : Earthetorm, 60; Cockroach, 162 ; Pigeon, 405, 407

Gland, albumen : Snail, 108, 117

clitellar : Leech, 51

colleterial : Cockroach, 169

Cowper's : Rabbit, 340, 342

digestive : Crayfish, 144 ; Cockrouch, 163-164; Dog-fish, 220

epidermal : Ieech, 51

green : Craufish, 147

hermaphrodite : Snail, 108, 116-117

infra-0rbital : Rabbit, 313

mammary : Rabbit, 306, 313

mucous : Leech, 51; Snail, 118

mushroom-shaped : Cockroach, 167

cssophageal : Eart hicorm, 60

oviducal : Dog-fish, 241 
- Gland, parotid: Rabbit, 311

pedal : Snail, 121

perinæal : Rabbit, 306, 340, 342

prostatic : Rabbit, $339-340$

rectal : Dog-fish, $221 ;$ Rabbit, 340, 342

salivary : Leech, 42, 51 ; Snail, 115 ; Cockroach, 163; Rabbit, 311-313

shell : Liver-fluke, 31

sublingual : Rabbit, 313

submaxillary : Rabbit, 313

Jolk : liver-fiuke, 31

Glochicia : Mussel, 97

Glottis : Rabbit, 310,350 ; Pigeon, 404

Gonapophysis : Cockroach, 159-161, 167, 169

Grafian follicle : Rabbit, 341

Green gland : Crayfish, 147

Growth : Mussel-shell, 77, 79 ; Snail-shell, 106 ; Crayfish, 126

Gubernaculum : Rabbit, 337

Hæmal arch : Dog-fish, 201-202

Himal process : $D \circ g-f i s h, 202$

Hæmal spine: Dog-fish, 202

Hæmoglobin : Earthworm, 62

Hallux : Rabbrit, 303 ; Fowl, 394

Hamular process, Rabbit, 285

Hend : Rabbit, 298

Head : Snail, 104; Crayfish, 128; Cockroach, 154; Rabbit, 305-306 ; Pigeon, 396

Heart : Earthworm, 63 ; Mussel, 89, 100 ; Snail, 111 ; Crayfish, 141 ; Cockroach, 161 ; Dog-

Helix, see Snail fish, 223, 237 ; Rabbit, 320, 322-323, 332-335; Pigeon, 404, 409-410, 416-418

Hepatic Cæce : Cockroach, 164

Hepatic cells : Earthworm, 62, 74

Hermaphrodite duct : Snail, 117

Hermaphrodite gland : Snail, 108, 116

Hind-brain : Rabbit, 353, 365-366

Hind-limb : Rabbit, 300-303, 307; Fowl, 392-394

Hinge of mussel-shell, 78

Hippocampus major : Rabbit, 359

Hirudo, see Leech

Humerus : Rabbit, 295-296; Fowl, 388-389

HYDRA, 13-25; general appearance, 15 ; structure, $16-20$; reproduction, $20-22$; teased specimens, $22-23$; transverse sections, $24-25$

Hydra, species of, 13

Hymen : Dog-fish, 241

Hyoid bone : Rabbit, 288, 343 ; Fowl, 386

Hyoidean arch : Dog-fish, 212

Hyomandibular cartilage : Dog-fish, 212

Hyostylic skull : Dog-fish, 212

Hypapophysis, see Vertebra.

Hyperbranchial groove: Amphioxus, 176, 189

Hypo-branchial : Dog-fish, 213

Hypobranchial groove: Amphioxus, 176, 189, 192

Hypostome: Eydra, 15

Ileum : Cockroach, 163

Ilium : Rabbit, 299 ; Font, 391

Incisor : $R a b b i t, 292,293$

Incus : Rnbbit, 284

Infundibulum : Dog-fish, 257 ; Rabbit, 354, 364; Pigeon, 424

Injection : Liver-fiuke, 26-27

Integument : Leech, 51 ; Eartheorm, 72-73; Shatil, 107 ; Amphioxus, 185

Internasal septum : $D_{0 g}-f_{i s h}, 205$

Interorbital canal : Dog-fish, 207

Interorbital septum : Forol, 375, 380

Interparietal : Rabbit, 278

Interstitial cell : Hydra, 17, 21, 22, 24

Intestine : Lives-fluke, 29 ; Leech, 43 ; Earthoorm, 62, 73 ; Mussel, 96 ; Snail, 108, 115 ; Crayfish, 144 ; Cockroach, 163; Amphioxus, 176, 193; Dog-fish, 218, 219 ;

Ischiopodite, see Appendage

$$
\text { - Rabbit, 314, 318-320; Pigeon, 405, 407-408 }
$$


Ischinm: Rabbit, 299 ; Fowl, 391

Iter a tertio ad quartum ventriculum : Rabbit, 365

Jacobson's organ : Rabbit, 286, 311

Jaw : Leech, 41 ; Snail, 122 ; Crayfish, 134; Dog-fish, 211 ; Rabbit, 284-288 ; Fovol, 382-385

Jugal : Rabbit, 286; Fonot, 383

Kidney : Arussel, 91-93, 99, 100 ; Snail, 107, 111; Crayfish, 147; Amphioxus, 180 ; Dog-fish, 218, 238-239, 24I, 243 ; Rabbit, 315, 336 ; Pigeon, 420

Killing, mode of : Vorticella, 12 ; Hyara, 24; Liver-fluke, 26-27 ; Leech, 37 ; Earthworm, 56, 72 ; Snail, 103 ; Crayfish, 126 ; Cockroach, 154; Rabbit, 305 ; Pigeon, 396

Labial cartilage : Dog-fish, 213

Labial palp : Irussel, 84 ; Cockroach, 159

Labium : Cockroach, 158-159

Labrum : Cockroach, 154

Tacinia : Cockroach, 158

Lacrymal bone: Rabbit, 281 ; Fowl, 380

Ineunar tissue : Mussel, 88

Lambdoidal crest : $F o w l, 377$

Lamella, inferior, see Vertebra

Lamina terminalis : Rabbit, 364

Lancelet, see Amphioxus

Larynx: Rabbit, 350 ; Pigeon, 419

Lateral line: Dog-fis $h, 197$

LEECE, 37-55 ; external characters, 38-39 ; digestive system, 41-43 ; colom and bloodvessels, 43-44; excretory system, 45-48; reproductive system, 48-49; nervous system, 49-50; sense-organs, 50 ; transverse sections, 50-55

Leg : Crayfish, 131; Cockroach, 159; see also Hind-limb

Lens: $D o g-f i s h, 261$; Pigeon, 426

Lepus, see Rabbit

Life-history : Liver-fluke, 32-36 ; Crayfish, 126 ; Cockrocch, 153

Iigament, broad : Rabbit, 341

ethmo-palatine : $D \circ o_{-}-i s h, 211$

falciform : Pigeon, 404

of jaws: Dog-fish, 211

of mussel-shell, 77,78

pre-spiracular: Dog-fish, 211

Ligula : Cockroach, 159

suspensory of liver : Dog-fish, 218 ; Rabbit, 317

Limb, see Fore-limb, Hind-limb, Appendage, Leg

Limnæa truncatula, 32, 36

Lingua : Cockrouch, 162

Lip : Snail, $105 ;$ Rabbit, 305

Liver: Mussel, 96 ; Snait, 108, 116 ; Crayfish, 144; Amphioxus, 177, 192; Dog-fish, 218 ; Rabbit, 314, 316-317 ; Pigeon, 404, 408

LTVER-FLUKR, 26-36; external characters, 27 ; alimentary canal, 27-29; excretory system, 29,35 ; reproductive system, 29-32; nervous system, 32 ;

Lobi inferiores : $D$ og- - ish, 257 life-history, 32-36

Locomotion : Amœeba, 3 ; Paramecium, 3 ; Vorticella, 11 ; Hydra, 13 ; Leech, 37 ; Earthworm, 56; Mussel, 76, 83 ; Crayfish, 125 ; Amphioxus, 170, 174

Lumbricus, see Farthworm

Lung : Snail, 108-110; Rabbit, 321; Pigeon, 404, 419, 420

Lymphatios : Rabbit, 332

Malleus : Rabbit, 283

Malpighian body : Rabbit, 336

Malpighian tube : Cockroach, 164

Mammæ: Rabbit, 306

Mammalian skeleton, characters of, 265

Mammals, characters of, 304

Mandible: Crayfish, 135; Cockroach, 158; Rabbit, 287-288 ; Fowl, 384-385

Mandibular arch: Dog-fish, 211; Rabbit, 286-288; Fowl, 384-385

Mantle: Mussel, 81-82, 98 ; Snail, 108

Mantle-cavity : Mussel, 82; Snail, 108-110

Manubrium mallei : $R a b b i t, 283$

Manubrium sterni : Rabbit, 275; Fotol, 374

Manus : Rabbit, 298; Fovl, 390

Mastoid portion : Rabbit, 281 
Mastoid process : Rabbit, 282

Maxilla : Crayfish, 134; Cockroach, 158-159; Rabbit, 285; Fotcl, 383

Maxillary arch : Rabbit, 284-286

Maxillipede : Crayfish, 133-134

Maxillo-palatine process : Fowl, 383

Maxillo-turbinal : Rabbit, 280

Meatus auditorius externus : Rabbit, 283 ; Pigeon, 426

Meatus auditorius internus: Rabbit, 282

Meckel's cartilage : Fowl, 385

Mediastinum : Rabbit, 322

Membrane-bone : Rabbit, 264, 275

Mentum : Cockroach, 159

Meropodite, see Appendage

Mesencephalon : Rabbit, 358, 362-363, 365

Mesenteron : Crayfish, 143, 144; Cockroach, 162, 163

Mesentery : Dog-fish, 218 ; Rabbit, 315

Mesethmoid : Rabbit, 279-280; Fowl, 380

Mesoglœe, see Supporting lamella

Mesonephros: $\log _{-j}$ fish $238,241,243$; Rabbit, 335

Mesonotum : Cockroach, 156

Meso-pterygium : Dog-fish, 216

Mesothorax: Cockrouch, 155

Metacarpus : Rabbui, 298; Fonol, 390

Metaoromion : Rabbit, 294

Metanephros : Dog-fish, 238, 241, 243 ; Rabbit, 335 ; Pigeon, 420

Metapleural canal : Amphioxus, 179, 190, 192,193

Metapleural fold : Amphioxus, 171

Metapophysis, see Vertebra

Meta-pterygium : Dog-fish, 216

Metastoma : Crayfish, 135

Metatarsus : Rabbit, 303; Fotol, 393-394

Metathorax : Cockrouch, 156

Metosteon : Fowl, 375

Mid-brain : Rabbit, 358, 362 363, 365

Mid-gut, see Mesenteron

Molar : Rabbit, 293

MoruIa : Liver-ficke, 33

Mouth : Paramecium, 5; Hydira, 15 ; Liver-fluke, 27 ; Leech, 39, 41 ; Earthworm, 58, 59 ; Mussel, 84, 95 ; Snail, 105 ; Crayfish, 129, 144 ; Cockroach. 157; Amphioxus, 171, 186 ; Dog-fish, 198 ; Rabbit, 305 ; Pigeon, 399 ; see also Buccal cavity

Mucous canal : Dog-fish, 199, 262-263

Muscle, bursalis : Pigeon, 425

columellar : Snail, 108

mandibular : Rabbit, 345

of eyeball : Dog-fish, 248-249; Pigeon, 425

of neck : Rabbit, 344-345

of nictitating membrane: Pigeon, 425

pectoral : Pigeon, 400-401

pyramidalis : Pigeon, 425

quadratus : Pigeon, 425

retractor penis : Snail, 118

sterno-hyoid : Rabbit, 344

sterno-mastoid: Rabbit, 344

sterno-thyroid : Rabzit, 345

stylo-hyoid: Rabbit, 344

Muscle-process : Hydra, 22, 23, 24

Muscular impression : Irussel-shell, 78-79

Muscular system : Leech, 53-54; Earthworm, 73 ; Mussel, 81 ; Amphioxus, 174-175, 185186 ; see chlso Muscle

Musculi papillares : Rabbit, 334 ; Pigeon, 418

Mushroom-shaped giand : Cockroach, 167

MUSSEL, 76-102; shell, 77-80 ; external characters, $81-84$; gills, 85-88; circulatory system, 88-90; excretory system, 91-93; nervous system, $93-95$; digestive system, 95-96; reproductive system, 96-97; transverse sections, 97-102

Myophan striation : Paramecium, 4; Opalina, 7 ; Vorticella, 10

Myotome: Amphioxus, 171, 174, 185

Nacreous layer : Mrussel-shell, 80; Snail-shell, 107

Nasal bone : Rabbit, 280; Fowol, 381 
Nasal cartilage : Dog-fish, 209

Nasal chamber : Rabbit, 310-311

Naso-turbinal : Rabbit, 281

Neok : Cockroach, 155 ; Rabbit, 343-350 ; Pigeon, 396

Nematocyst: Hydra, 18, 19, 23, 24 ; discharge of, 19

Nephridia : Leech, 45-48, 55 ; Edrthworm, 63-65, 74

Nephrostome : Earthuorm, 64, 65

Nerve, 1st cranial (olfactory): Dog-fish, 250

2nd cranial (optic) : $\log _{-}-i s h, 250$

3rd cranial (oculo-motor): Dog-fish, 250

4th cranial (pathetic) : Dog-fish, 251

5th cranial (trigeminal) : $\log _{-} f i s h, 251-253$

6th cranial (abducent): $D \circ g-f i s h, 253$

7th cranial (facial): $D \circ g-f i s h, 251,253-254$

8th cranial (auditory): Dog-fish, 251, 254, 262

9th crenial (glosso-pharyngeal) : Dog-fish, 254-255

10th cranial (pneumogastric or vagus) : Dog-fish, 255-256; Rabbit, 347-348

11th cranial (spinal sccessory) : Rabbit, 345

12th cranial (hypoglossal) : Rabbit, 346

antennary : Cockroach, 165

anditory : Snail, 120 ; Crayfish, 151 ; see also 8th cranial nerve

branchial : Dog-fish, 255

buccal : Snail, 120

cardiac : Dog-fish, 256 ; Rabbit, 348

cranial: Dog-fish, 249-256

depressor : Rabbit, 347

descendens noni : Rabbit, 346

gastric : $D o g-f i s h, 256$

great euricular : Rabbit, 349

intermediary : Leech, 55

mandibular : $\operatorname{Dog}_{-} f i s h, 253$

maxillary : Dog-fish, 252

of lateral line : $D o g-f i s h, 256$

of neck : Rabbit, $345-349$

ophthalmic: Dog-fish, 252, 253

optic: Crayfish, 150; Cockroach, 165 ; see also 2nd cranial nerve

palatine : Dog-fish, 254

phrenic : Rabbit, 321. 349

post-spiracular : Dog-fish, 254

pre-spiracular : Dog-fish, 254

pulmonary : Rabbit, 348

recurrent laryngeal : Rabbit, 348

spinal : $D$ og-fish, 259

splanchnic : Rabbit, 315

superior laryngeal : Rabbit, 347

sympathetic, cervical : Rabbit, 348

visceral : Crayfish, 148 ; Cookroach, $166 ;$ Dog-fish, 256

Nerve-cells : Hydra, 19; Leech, 55; Earthworm, 74; Snail, 120

Nerve chain, see Nervous system

Nerve-collar : Liver-fluke, 32 ; Leech, 49 ; Earthworm, 70

Nerve-commissure : Mussel, 94

Nerve-connective: Mussel, 94-95; Crayfish, 147-148; Cockroach, 166

Nerve-cord : see Nervous system

Nerve-ganglia :

buccal : Snail, 120

cerebral : Nussel, 94 ; Snati, 119 ; Cockroach, 165

coliac : Rabbit, 315

frontal : Cockroach, 166

inferior cervical : Rabbit, 349

optic : Cray/sh, 150

parieto-splanchnic : Mussel, 95

pedal : Mrussel, 94 ; Snail, 120

post-cesophageal : Crayfish, 148 ; Cockroach, 166

pre-cesophageal : Crayfish, 147 ; Cockroach, 165

sub-cesophageal : Leech, 50; Snail, 120; Cockroach, 166

superior cervical : Rabbit, 348

supra-œsophageal : Leech, 49; Earthoorm, 70 ; Snail, 119 ; Cockroach, 165

visceral : Mussel, 95, 102

viscero-pleural : Snail, 120 
Nerve-roots :

cranial : Dog-fish, 250-255 ; Rabbit, 355-358

spinal : Amphioxus, 182-183, 187 ; Dog-fish, 259

Nerrous system : Hydra, 19 ; Liver-fluke, 32; Leech, 49-50, 55 ; Eavthworm, 70-72, 74 ; Miussel, 93-95; Snail, 119-120; Crayfish, 147-148; Cockroach, 165167 ; Amphioxus, 181-183; Dog-fish, 246-259 ; Rabbit, 315, 345-349, 350-366; Pigeon, 423-424

Neurat arch : Dog-fish, 202 ; see also Vertebra

Neural canal, see Vertebra

Neural plate: $D o g-f i s h, 202$

Neural process : Dog-fish, 202

Neural spine: $D_{0 g} / h s h, 202$; see also Vertebra

Nictitating membrane: Rabbit, 305; Prgeon, 396, 424, 425

Nose, see Olfactory organ

Nostril : Dog-fish, 198 ; Rabbit, 305 ; Pigeon, 399

Notochord: Amphioxus, 173, 185; Dog-fish, 200, 201, 209

Nucleolus: Paramecium, 6

Nucleus: Amabr, 3 ; Paramecium, 6 ; Opalina, 7 ; Vorticella, 11; Snait-shell, 106

Occipital condyle : Dog-fish, 209 ; Rabbit, 277 ; Fowt, 377

Occipital segment : Rabbit, 276-277

Odontoid process : Rabbit, 270 ; Fowl, 371

Odontophore : Snail, 113, 122

Esophageal pouches : Earthuorm, 60

Wsophagus: Liver-fuke, 29 ; Leech, 42 ; Earthworm, 60 ; Mussel, 96 ; Snail, 115 ; Crayfish, 144; Cockroach, 162 ; Dog-fish, 219 ; Rabbit, 322 ; Pigeon, 405

Olecranon process : Rabbit, 297 ; Fowl, 389

Olfactory capsule : Dog-fish, 203 ; Rabbit, 280 ; Fowl, 381

Olfactory lobe: Dog-fish, 247; Rabbit, 352, 354 ; Pigeon, 423

Olfactory organ : Craufish, 151; Amphioxus, 183; Dog-fish, 259-260

Omentum: Rabbit, 315 ; Pigeon, 405

OPALINA, 7

Opisthotic : Rabbit, 281

Optic chiasma: Dog-fish, 257 ; Rabbit, 355, 361 ; Pigeon, 424

Optic foramen: Rabbit, 288

Optic lobe: Dog-fish, 248, 258 ; Rabbit, 352, 365 ; Pigeon, 423

Optic thalami : Rabbit, 361

Optic tract: Rabbit, 355,361

Oral groove: Paramecium, 5

Orbit: Dog-fish, 206-208, 248-249; Foul, 379-380

Orbito-sphenoid : Rabbit, 279; Fonol, 380

Os innominatum : Rabbit, 299; Fowl, 390-391

Os orbiculare : Rabbit, 284

Ossicle, auditory : Rabbit, 283-284

Ossicles, of stomach : Crayfish, 145-146

Otocyst : Mussel, 94; Snail, 121

Orarian duct : Liver-fluke, 31

Ovary : Hydra, 21; Liver-fiuke, 31 ; Leech, 49 ; Earthoorm, 65, 69 ; Miussel, 97 ; Crayksh, 143 ; Cockroach, 168; Amphioxus, 183-184; Dog-fish, 218, 239; Rabbit, 341; Pigeon, 422

Oviduct: Liver-ghuke, 32 ; Leech, 49 ; Earthroorm, 66; Snail, 118 ; Crayfish, 143 ; Cock-

Ovisac: Leech, 49 roach, 169 ; Dog-fish, 239-241; Rabbit, 341 ; Pigeon, 422

Orum : Hydra, 22; tiver-fluke, 32 ; Mussel, 97 ; Snail, 117; Cockroach, 168; Amphioxus, 184 ; Dog-fish, 196 ; Rabbit, 304 ; Bird, 395 ; Pigeon, 422

Palatal process : Rabbit, 285, 286

Palate : Rabbit, 307

Palatine bone: Rabbit, 285 ; Forol, 382

Pallial cavity: Mussel, 82

Pallial line : Mrussel-shell, 79

Palp, labial : Mussel, 84; Cockroach, 159

mandibular: Croyfish, 135

maxillary : Cockroach, 158

Palpiger : Cockroach, 159

Pancreas : Dog-fish, 220; Rabbit, 316; Pigeon, 405, 408

Papilla, circumvallate : Rabbit, 310

foliata : Rabbit, 310

urino-genital : Dog-fish, 245 
Paraglossa : Cockroach, 159

Parameicium, 3-7

Paranucleus: Paramecium, 6

Para-sphenoid: Foot, 380

Parietal bone : Rabbit, 278 ; Fout, 380

Parietal segment : Rabbit, $277-278$

Par-occipital process : Rabbit, 277

Patella : Rabbit, 301 ; Fovol, 392

Pearl, 80

Pecten : Pigeon, 426

Pectoral fin : $D$ og-fish, 216

Pectoral girdle : Dog-fish, 215; Rabbit, 294-295 ; Fool, 386-387

Pelvic fin : Dog-fis $h$, 214-215

Pelvic girdle : Dog-fish, 214 ; Rabbit, 298-300; Fovl, 390-391

Pelvis of kidney : Rabbit, 336

Penis : Liver-fluke, 31 ; Leech, 49 ; Snail, 118 ; Rabbit, 306, 339

Pericardial cavity : Mrussel, 88, 99, 100 ; Snait, 110; Dog-fish, 222

Pericardial sinus : Crayfish, 141

Pericardium : Snail, 108 ; Rabbtt, 320

Peringeal pouch : Rabbit, 306

Periostracum : Mucssel-shell, 80 ; Snail-shell, 107

Periotic : Rabbit, 281

Periplaneta, see Cockroach

Peristome: Vorlicella, 8

Peritoneum : Earthworm, 73 ; Dog-fish, 218 ; Rabbit, 315

Pes: Rabbit, 303; Fowl, 393-394

Pessulus : Pigeon, 419

Petrous portion : Rabbit, 281

Peyer's patch : Rabbit, 318, 319

Phalanges : Rabbit, 298, 303 ; Fotol, 390, 394

Pharyngo-branchial : Dog-fish, 213

Pharynx: Vorticella, 10; Liver-fluke, 27 ; Leech, 42 ; Earthworm, 60 ; Snail, 113 ;

Pia mater: Rabbit, 352 Amphioxus, 176, 188-189, 191 ; Rablit, 310

PIG DON, 395-428; external characters, $396-400$; pectoral muscles, 400-401; air-sacs, 401-403 ; digestive system, 403-409; circulatory system, 409-418; respiratory system, 418-420; renal and reproductive systems, 420-422; brain, 423-424; sense-organs, 424-428

Pigmented canals : Amphioxus, 180

Pineal body : Dog-fish, 247 ; Rabbit, 354, 362, 364; Pigeon, 423, 424

Pinna : Rabbit, 306

Pituitary body : Dog:fish, 257, 258 ; Rabbit, 354 ; Pigeon, 424

Pituitary foramen : Rabbit, 277, 290

Pituitary fossa, see Sella turcica

Placenta : Rabbit, 304

Pleura: Rabbit, 322 ; Pigeon, 419

Pleurobranchia : Crayfish, 137, 138

Pleuron: Crayfish, 127

Podical plate: Cockroach, 156

Podobranohia : Crayfish, 137, 138

Pollex : Rabbit, $298 ;$ Fonol, 390

Pons Varolii : Rabbit, 355,366

Pore, dorsal : Earthworm, 59

Post-axial border of limb: Rabbil, 295, 300 ; Fowbl, 388

Post-orbital groove : Dog-fish, 208

Post-zygapophysis, see Vertebra

Pre-axial border of limb : Rabbit, 295, 300; Foul, 388, 392

Premaxilla : Rabbit, 286 ; Fowl, 382-383

Premolar : Rabbit, 292, 293

Prepuce : Rabbit, 306, 339

Pre-sphenoid : Rabbit, 278-279; Fowl, 380

Pre-zygapophysis, see Vertebra

Primary quill : Pigeon, 398

Prismatic layer : Mussel-shell, 80

Proctodcoum : Crayfish, 143, 144; Cockroach, 162, 163

Pronephros: Dog-fish, 238 ; Rabbit, 335

Pronotum : Cockroach, 155

Pro-otic : Rabbit, 281

Propodite, see Appendsge 
Pro-pterygium : Dog-fish, 216

Prosencephalon : $D o g-f i s h, 247,256$

Pyostate : Rabbit, 339-340

Prostomium : Leech, 38 ; Earthoorm, 58

Prothorax : Cockroach, 155

Protopodite : Crayfish, 128; see also Appendage

Protozos, 1-12

Proventriculus : Cockroach, 162 ; Pigeon, 405

Pseudobranchia : $D \circ g-f i s h, 222$

Pseudopodia, 1-3

Pterygoid bone: Rabbit, 285; Fowl, 382

Pterygoid fossa : Rabbit, 285

Pterygoid process : Rabbit, 277-278

Pterylæ: Pigeon, 399

Pubes : Rabbit, 299 ; Fowl, 391

Pulvillus : Cockroach, 159

Pupil : Dog-fish, 260

Pygostyle : Fowl, 373

Pylorus : Rabbit, 316

Pyramid of kidney : Rabbit, 336

Quadrate : Fowl, 384

Quadrato-jugal : Fowl, 383

Quill : Figeon, 397

Quill-feathers : Pigeon, 398

RABBIT, 264-366 ; vertebral column, 266-273 ; ribs anđ sternum, 273-275 ; skull, 275291 ; teeth, 291-293; appendicular skeleton, 293-303; external characters, 305-307 ; buccal cavity, 307-313; abdominal viscera, 313-315 ; digestive system, 315-320 ; thoracic viscera, 320-322; circulatory system, 322-335 ; renal and reproductive systems, 335-342; dissection of neck, 343-350; dissection of brain, 350-366

Rachis : Pigeon, 397

Radius : Rabbit, 298 ; Fowl, 389

Radula: Snail, 1.22

Receptaculum ovorum : Earthworm, 66, 69

Receptaculum seminis, see Spermatheca

Rectal diverticula : Pigeon, 408

Rectrices : Fonot, 373 ; Pigeon, 399

Rectum : Mrussel, 88, 96; Snail, 108, 112; Cockroach, 163 ; Dog-fish, 219 ; Rabbit, 314, 319,

Redia, 33-35 320,340 ; Prigeon, 408, 421, 422

Rejuvenescence : Paramecium, 7

Remiges : Pigeon, 398

Reno-pericardial canal : Snail, 111

Reproduction : Amoba, 3; Paramecium, 6 ; Opalina, 7; Vorticella, 11 ; Hydra, 20-22 ; Liver-fluke. 32-36 ; Snail, 116

Reprodrotive system : Liver-fluke, 29-32; Leech, 48-49, 55; Earthworm. 65-70; Mussel, 96-97; Shail, 116-119; Crayfish, 142-143 ; Cockroach, 167-169; Amphioxus, 183-184, 193 ; Dog-fish, 238-246; Rabbit, 335-342; Pigeon, 420-422

Respiration : Leech, 51; Mussel, 87 ; Crayfish, 136, 139 ; Cockroceh, 164 ; Amphioxus, 176 ; Dog-fish, 221; Pigeon, 401

Respiratory system : Snail, 108-110; Cockroach, 164 ; Amphioxus, 175-176 ; Dog-fish, 221-222; Pigeon, 418-420; see also Gill, Lung

Restiform bodies : Dog-fish, 248,258

Retina : Dog-fish, 261

Rib : Dog-fish, 201 ; Rabbit, 273-274; Fowl, 373-374

Rostrum : Crayfish, 128 ; Dog-fish, 205; Fowl, 378, 380

Sacci vasculosi : Dog-fish, 257

Sacculus rotundus : Rabbit, 318

Srcrum : Rabbit, 272-273 ; Fowl, 372-373

Salivary receptacle : Cockroach, 163

Scales : Dog-jish, 199-200

Scaphocerite : Crayfish, 135

Scsphognathite : Crayfish, 134, 139

Scapula : Rabbit, 294 ; Fowl, 387

Scapus : Pigeon, 397 
Sclerite: Crayfish, 145 ; Cockroach, 155

Sclerotic : Dog-fish, 260

Solerotic plate : Pigeon, 426

Sorotal sac : Rabbit, 306, 337

Scyllium, see Dog-fish

Secondary quills : Pigeon, 388

Seotion, transverse : Hydra, 24; Leech, 50-55 ; Earthworm, 72-75 ; Mussel, 97-102 ;

Segment : Leech, 37 Amphioxus, 184-195

Segmental organ, see Nephridia

Segmentation : Leech, 37; Earthuorm, 56 ; Crayfish, 125 ; Cockroach, 153

Segmentation of skull : Rabbit, 276

Sella turcica : Rabbit, 277

Semiciroular canal : Dog-fish, 206, 261, 262 ; Pigeon, 428

Seminal funnel : Earthworm, 68

Sense-capsules : Dog-fish, 203 ; Rabbit, 280-284

Sense-organs: Leech, 50; Mussel, 94; Snail, 121; Crayfish, 149-151; Cockroach, 167 ; Amphiodrus, 183 ; Dog-fish, 259-263 ; Pigeon, 424-428

Septum : Earthworm, 69, 74 ; Amphioxus, 174

Septum auricularum : Pigeon, 416-417

Septum, interorbital : Fowl, 375, 380

Septum lucidum : Rabbrit, 360

Septum marium : $D o g-f i s h, 205$; Rabbit, 290

Sesamoid bone : Rabbit, 264

Setæ: Earthwoorm, 57, 73

Shell : Mussel, 77-80 ; Snail, 103-104, 106-107

Shell-gland : Liver-fiuke, 31

Sinus, blood : Leech, 43-44, 54 ; Snail, 124 ; Craytush, 139

anterior cardinal : Dog-fish, 227, 228

coronary : Dog-fish, 238

Cuvierian, Dog-fish, 227

genital : $D$ og- $f$ ish, 229

hepatic: $D$ og- $f i s h, 227$

hyoidean : $D o g-f i s h, 228$

inferior jugular : Dog-fish, 227

inter-orbital : $D_{0}$ g- $_{-13}, 228$

orbital : Dog-fish, 207, 228

posterior cardinal : Dog-fish, 227, 228

post-orbital : Dog-fish, 228

venosus : $D_{0} g_{-f i s h}, 225,237$

Sinus, genital : Liver-fiuke, 32

urinary : Dog-fish, 242

urino-genital : Dog-fish, 245

Skate, 196

Skeleton : Amphioxus, 173-174, 185 ; Dog-fish, 200-217 ; Rabbit, 264-303 ; Fowl, 367-394

Skeleton, appendicular : Rabbit, 293-303 ; Fonol, 386-394

axial : Rabbit, 266-291; Fowl, 367-386

branchial : Amphioxus, 174

buccsl : Amphioxus, 173, 185

endophragmal : Crayfish, 147

of fins : Amphioxus, 173, 185; Dog-fish, 214-217

mammalian, 265

visceral : $D 0 g_{-}$fish, 209-213

Skin, see Integument

Skull : Dog-fish, 203-209 ; Rabtrit, 275-291 ; FFowl, 375-386

SNAII, 103-124; external characters, 103-108; mantle cavity, 108-112; digestive system, 113-116 ; reproductive system, 116-119 ; nervous system, 119-120 ; gense-organs, 121 ; buccal mass, 121-122 ; circulatory system, 122-124

Somite : Leech, 37

Spermathece : Earthworm, 66, 69 ; Snail, 119 ; Cackroach, 169

Spermatoblast : Earthworm, 70

Spermatophore : Leech, 49 ; Snail, 118

Spermatospore : Eavthworm, 70

Spermatoz0a: Hydra, 21; Leech, 48; Elarthoorm, 69-70; Snail, 117; Crayfish, 143 ;

Sperm sac: Dog-fish, 243 Amphioxus, 184

Sphenoidal fissure : Rabbit, 278

Spinal cord : Amphioxus, 181, 186 ; Dog-thh, 259

Spinous process, see Vertebra 
Spiracle : Cockroach, 157 ; Dog-fish, 198

Spiral valve : Dog-fish, 219-220

Spleen : Dog-fish, 218; Rabbit, 315 ; Pigeon, 407

Splenial : Fow , 385

Sporocyst, 33

Squamosal : Rabbit, 287 ; Fowl, 378, 379

Stapes: Rabbit, 284

Sternebra : Rabbit, 274

Sternum : Crayfish, 127, 129 ; Rabbit, 274-275 ; Fowl, 374-375

Stigmata : Cockroach, 157, 164

Stipes: Cockroach, 158

Stomach : Leech, 43 ; Mussel, 96 ; Snail, 115: Crayfish, 144 ; Amphioxus, 177 ; Dog-fsh, 218, 219; Rabbit, 314, 318, 319; Pigeon, 405

Stomatodoum ; Crayfish, 143 ; Cockroach, 162

Submentum : Cockroach, 159

Sucker : Ziver-fluke, 27 ; Leech, 38

Sulcus : Rabbit, 352, 361

Supporting lamella: Eydra, 17, 19, 23, 24

Supra-angular : Fow h, 385

Supra-branchial chamber : Mussel, 82, 87, 98, 100, 102

Supra-occipital : Rabbrit, 277

Supra-orbital crest : Dog-fish, 205

Supra-orbital process : Rabbit, 279

Supra-scapula : Rabbit, 294

Suspensorium : Dog-fish, 212

Suspensory fold of pharynx : Amphioxus, 178, 189

Swimmeret, see Abdominal appendage

Sylvian aqueduct : Rabbit, 365; Pigeon, 424

Sylvian fissure : Rabbit, 354

Symphysis pubis : Rabbit, 299-300

Syrinx : Pigeon, 419

Tapetum : Dog-fish, 260

Tarso-metatarsus : Fowl, 393-394

Tarsus : Cockroach, 159 ; Rabbit, 301-302

Teat : Rabbit, 306

Teeth : Crayfish, 144, 146 ; Cockroach, 162; Dog-fish, 200, 211, Rabbit, 264-265, 291-293

Telson : Crayfish, 129

Tentacle: Hydra, 15-16, 17-19; Mussel, 83 ; Snati, 104 ; Amphioxus, 171

Tentorium; Cockroach, 165 ; Rabbit, 352

Tergum : Crayfish, 127

Testis : Hydra, 21 ; Liver-fuke, 29 ; Leech, 48, 55 ; Larthworm, 68, 69 ; Mussel, 97 ; Crayfish, 142; Cockroach, 167 ; Amphioxus, 184;Dog-fish, 218, 242; Rabbit, 337; Pigeon, 421

Thalamencephalon : Dog- $f i s h, 247$; Rabbit, 352, 358, 361-362, 363-364

Thoracic appendage : Crayfish, 131-134; Cockroach, 159

Thoracic duct : Rabbit, 332

Thoracic viscera : Rabbit, 320-322

Thorax : Crayfish, 129 ; Cockroach, 155-156

Thread-cell : Hydra, 18, 19, 23, 24; see also Trichocyst

Thymus : Rabbit, 320

Thyroid body : Rabbit, 344

Thyroid cartilage : Rabbit, 343,350

Tibia : Cockroach, 159; Rabbit, 301

Tibio-tarsus : Font, 392-393

Tongue : Cockroach, 162 ; Rabbit, 309-310 ; Pigeon, 404

Tonsil : Rabbit, 309

Traches: Rabbit, 321, 343; Pigeon, 418

Tracheal tube : Cockroach, 164-165

Transverse process : Dog-fish, 201 ; see also Vertebra

Trichocyst: Paramecium, 5

Trochanter: Cockroach, 159; Rabbit, 300 ; Fout, 392

Tubercle : Rabbit, 274

Tympanio bone: Rabbit, 282-283

bulla: Rabbit, 277, 282

cavity : Rabbit, 282; Fonol, 378-379; Pigeon, 427

membrane : Rabbit, 283 ; Fowl, 378 ; Pigeon, 426

Tympano-Eustachian passage : Pigeon, 426-427

Typhlosole: Earthworm, 62,73, 74; Mussel, 96, 98

Typical segment : Crayfish, 126-128 
Vlna : Rabbit, 296-297; Fonol, 389

Dmbilícus, of feather : Pigeon, 397

Umbo: Mussel-shell, 77 of snail-shell, 106

Uncinate process : Fowl, 374

Ureter : Mfussel, 92, 99, 100 ; Snail, 112 ; Crawfish, 147; Dog-fish, 242, 246 ; Rabbit, 336 ; Pigeon, 420

Urethra : Rabbrit, 338

Urino-genital pouch : Pigeon, 421, 422

Uterus: Liver-fiuke, 32 ; Rabbit, 341

Uterus masculinus : Rabbit, $339, \mathbf{3 4 0}$

Vacuole : Hydra, 23, 24

Vacuole, food: Amceba, 2 ; Paramecium, 6; Vorticella, 11 pulsating : Amabba, 3 ; Paramecium, 5 ; Porticella, 11

Vagina : Liver-fluke, 31 ; Leech, 49 ; Snail, 118 ; Rabbit, 342

Vagus, see Nerve, 10th cranial

Valve, Eustachian : Rabbit, 333 ; Pigeon, 416

mitral : Rabbit, 334 ; Pigeon, 418

of Vieussens : Rabbit, 353,365

semilunar : Rabbit, 334, 335; Pigeon, 417, 418

spiral : Dog-fish, 219-220

tricuspid: Rabbit, 334

Vane : Pigeon, 398

Vas deferens: Liver-fulke, 29 ; Leech, 48, 55 ; Earthworm, 69 ; Snahl, 118 ; Crayfish, 143 ; Cockroach, 167 : Dog-fish, 243 ; Rabbit, 338, 339 ; Pigeon, 421

Vas efferens : Leech, 48 ; ERavthworm, $69 ;$ Dog-fish, 242 ; Rabbit, 337

Vein, azygos cardinal : Rabbit, 325

brachial : Pigeon, 410

caudal : Dog-fish, 230 ; Pigeon, 412

coccygeo-mesenteric : Pigeon, 405

coronary : Rabbit, 333

duodenal : Rabbit, 328

facial : Rabbit, 326

femoral : Rabbit, 328 ; Pigeon, 412

gastro-duodenal : Pigeon, 413

hepatic: Amphioxus, 180, 193 ; Rabbit, 327 ; Pigeon, 411

hypogastric: Pigeon, 412

iliac : Rabbit, 327, 328; Pigeon, 411, 412

ilio-lumbar : Rabbit, 327

intercostal : Rabbit, 325

internal iliac: Pigeon, 412

internal mammary : Rabbit, 326 ; Pigeon, 410-411

jugular : Rabbit, 326 ; Pigeon, 410

lateral : $D \circ g-f i s h, 229$

lieno-gastric: Rabbit, 328

mesenteric : Rabbit, 328, 329 ; Pigeon, 412, 413

ovarian : Rabbit, 327

pectoral : Pigeon, 410

phrenic : Rabbit, 327

portal : Amphioxus, 180, 192 ; Dog-fish, 230 ; Rabbu, 328 ; Pigeon, 405, 412-413

pulmonary : Snail, 110; Rabbit, 324; Pigeon, 413

renal : Dog-fish, 229 ; Rabbit, 327 ; Pigeon, 412

renal portal : $\operatorname{Dog}_{-}$ish, 230

spermatic : Rabbit, 327

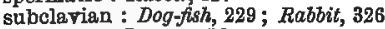

vertebral : Pigeon, 410

Veins: Mussel, 90; Snaill, 124; Dog-fish, 225-230; Rabbit, 324-329; Pigeon, 410-413; dee also Sinus

Veins, of kidney : Pigeon, 411-412

Velum : Amphioxus, 175

Velum interpositum : Rabbit, 361, 364

Velum medullie : Rabbit, 353-354, 365-366

Vena cava: Mussel, 90, 99, 100

Vena cava, anterior : Rabbit, 323, 325, 326; Pigeon, 410, 411

posterior : Rabbit, 323, 326 ; Pigeon, 411

Ventricle, of brain : Dog-fish, 258; Rabbit, 358

lateral : Dog-fish, 258; Rabbit, 359 ; Pigeon, 424

third : Dog-fish, 247, 258; Rabbit, 362, 363 ; Pigeon, 424 
Ventricle, fourth : Dog-fsh, 248, 259 ; Rabbil, 365 ; Pigeon, 424

fifth : Rabbii, 360

Ventricle of heart : Mussel, 89, 100; Snail, 111; Dog-fish, 223, 237; Rabbit, 323, 333-335 ;

Ventricle of larynx : Rabbit, 350 Pigeon, 417-418

Ventricle of nervous system : Amphioxus, 181

Vermiform appendix : Rabbit, 314, 318, 320

Vermis cerebelli : Raboit, 353

Vertebra, anticlinal : Rabbit, 271 caudal : Rabbit, 273 ; Fovol, 373 cervical : Rabbit, 267-271; Fovol, 369-371

lumbar : Rabbit, 272

sacral : Rabbit, 272-273; Fowl, 372-373

thoracic : Rabbit, 271-272 ; Fowl, 371-372

typical : Rabbit, 266-267

Vertebral column : Dog-fish, 201-203 ; Rabbit, 266-273 ; Foul, 367-373

Vertebral column, detelopment of : Dog-fish, 200

Vertebrarterial canal, see Vertebra

Verumontanum : Rabbit, 340

Vesicula seminalis: Liver-fluke, 30; Earthtoorm, 66-68; Cockroach, 167; Dog-fish, 243 ;

Vessel, see Blood-vessel Pigeon, 421

Vestibule: Vorticella, 8 ; Rabbit, 342

Vestibule of ear: $D o g-f i s h, 262$

Vexillum : Pigeon, 398

Vibrissa : Rabbit, 306

Villi : Rabbit, 319

Visceral hump : Snail, 107-108

Visceral mass: Mussel, 83, 08

Visceral nervous system : Crayfish, 148 ; Cockroach, 166

Visceral skeleton : Dog-fish, 209-213

Vitellarium : Liver-fuike, 31

Vocal cord : Rabbit, 350

Vomer : Rabbit, 281; Fowl, 381

VORTICELLA : 8-12

Vulva: Rabbit, 306

Wing : Cockroach, 156 ; Forol, 388-390; Pigeon, 397

Wrist, see Carpus

Xiphisternum : Rabbit, 275

Xiphoid process : Fonol, 375

Yolk-cell : Liver-fluke, 33

Yolk-gland : Liver-fluke, 31

Yolk-reservoir : Liver-ftuke, 31

Zygapophysis, see Vertebra

Zygomatic arch : Rabbit, 286

Zygomatic process : Rabbit, 286, 287 ; Fout, 379

PRINTED BT

GPOTTISWOODE AND CO, NEW-BTRETT SQUARE

LONDON 
BY THE SAME AUTHOR.

Second Fdition, Revised and Illustrated. Crown 8vo, 3s, 6d.

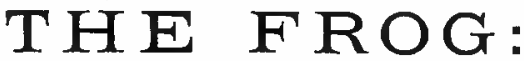

\section{AN INTRODUCTION TO ANATOMY AND HISTOLOGY.}

By A. MILNES MARSHALL, M.D., D.Sc., M.A.,

Fellow of St. John's College, Cambridge; Beyer Professor of Zoology in Owens College;

Frxaminer in Zoology and Comparative Anatomy in the Universities of Cambridge

and London, and in the Victoria University.

A COURSE of PRACTICAL HISTOLOGY. By EDWARD AlberT SOBÄHFR, Assigtant-Professor of Physiology, University College. With numerous Illustrations. Crown 8vo. 10s. $6 \alpha$.

ELEMENTS of HUMAN PHYSIOLOGY. By Dr. L. Hermann, Professor of Phygiology in the Univeraity of Zurich. Second Edition. Entirely recast from the Sixth German Edition, with very copious additions, and many additional Woodouts, by ARTHUR GAMGEE, M.D., F.R.S., Brackenbury Professor of Physiology in Owens College, Manohester, and Examiner in Physiology in the University of Edinburgh. Demy 8vo. $16 s$.

The FUNCTIONS of the BRAIN. By DAVID FrrRIER, M.D., LL.D., F.R.S., Professor of Forensic Medicine, King's College; Physician to King's College Hospital; Physician to the National Hospital for the Paralysed and Epileptic. Second Fdition, Re-written, with many New Illustrations, 8vo. 18s.

The LOCALISATION of CEREBRAL DISEASE. By DAVID F'ERRIER, M.D., F.R.S., Assistant-Physician to King's Oollege Hospital ; Professor of Forensic Medicine, King's College. With numerous Illustrations, 8vo. 7s. 6d.

The DESCRIPTIVE ATLAS of ANATOMY. A Representation of the Anatomy of the Human Body. In 92 Royal 4to. Plates, containing 550 Illustrations. Introducing Heitamann's Figures, considerably modified, and with many Original Drawings from Nature. By NoBLE SMrTH, F.R.O.S. Fdin., L,R.O.P. Lond., Surgeon to the All Saints' Children's Hospital and Orthopedic Surgeon to the British Home for Incurables. Bound in half-leather, price $25 s$.

POST-MORTEM HANDBOOK: or, How to Conduct Post-Mortem Examinations for Clinical and Medico-legal Purposes. By Thomas Harris, M.D. Lond ", M.R.O.P., Pathological Registrar to the Manchester Royal Infirmary, AasistantLecturer and Demonstrator of Pathology in the Owens College, and Honorary AssistantPhysician to the Manchester Hospital for Consumption and Discases of the Throat. With Illustrations. Crown 8vo. 3s, 6d.

A DIRECTORY fOr the DISSECTION of the HUMAN BODY. By JoHN ClmLAND, M.D., F.R.S., Professor of Anatomy in the University of Glasgow. Second Edition. Fop. 8vo. 3s.6d.

A DICTIONARY Of PRACTICAL SURGERY. By VARIOUS BRITISH Hospital SuRgeoNs. Edited by CHRISTOPHER HEATH, F.R.O.S., Hulme Profesgor of Clinical Surgery in University College, London, Surgeon to University College Hospital, Member of the Council and Court of Fxaminers of the Royal College of Burgeons of Fngland. In 2 vols. of about 1,000 pages each. Second Eldition. Royal 8vo. price 32s., bound in cloth ; or in half-morocoo, marbled edges, 42s.

SURGerY : its Principles and Practice. By Timothy Holmes, M.A. Cantab., F.R.C.S., Surgeon to St. George's Hospital. Fourth Edition. With 418 Illustrations, chiefly by Dr. WESTMACOTT. Royal 8vo. 30s.

An INDEX of SURGERY : being a Concise Classification of the Main Facts and Theories of Surgery for the Uøe of Senior Students and others. By O. B. KreTLEY, F.R.O.S, Surgeon to the West London Hospited, and to the Surgical-Aid Society. Fourth Edition. Orown 8vo. 10s, 6a.

The SURGERY of DEFORMITIES. A Manual for Students and Practitioners. By NOBLE SMITH, F.R.O.S. Edin., L.R.O.P. Lond, Surgeon to the All Saints' Children's Hospital, Senior Surgeon and Surgeon to the Orthopædio Department of the Farringdon Dispensary, and Orthopæedic Surgeon to the British Home for Incurables. With 118 Illustrations. Orown 8vo. 10s, $6 d$. 
ANTISEPTIC SURGERY : it PRINCIPLes, Practice, History, and RugulTs. By W. Watson CHerNe, M.B., F.R.C.S, Assistant-Surgeon to King's College Hospital, and Demonstrator of Surgical Pathology in King's College. With 145 Illinstrations. 8vo. $21 s$.

MANUAL of the ANTISEPTIC TREATMENT of WOUNDS. For Students and Practitioners. By W. WATson CHEYNR, M.B., F.R.O.S., Assistant-Surgeon to King's College Hospital, Surgeon to the Paddington Green Children's Hospital, \&c. With Illustrations. Crown 8vo.4s. 6d.

A MANUAL of DENTAL SURGERY and PATHOLOGY. By ALFRED ColkmaN, L.R.C.P., F.R.C.S. Exam., L.D.S., \&c. ; Senior Dental Surgeon and Lecturer on Dentrl Surgery to St. Bartholomew's and the Dental Hospital of London; Member of Board of Examiners in Dental Surgery, Royal College of Surgeons ; Iate President Odontological Society of Great Britgin. With 388 Illustrations. Crown 8vo. 12s. 6d.

A TREATISE on the THEORY and PRACTICE of MEDICINE. By JOHN SYRE BRISTOWF, M.D. Lond., Fellow and formerly Censor of the Royal College of Physicians; Senior Physician to, and Joint Lecturer on Medicine at, St. Thomas's Hospital; President of the Society of Medical Offlcers of Health; Examiner in Medicine to the Royal College of Surgeons; formerly Examiner in Medicine to the University of London, and Lecturer on General Pathology and on Physiology at St. Thomas's Hospital. Fifth Edition, 8vo. 21s.

CLINICAL MANUAL for the STUDY of MEDICAL CASES. Edited by J. Finlayson, M.D., Physician and Lecturer on Clinical Medicine in the Glasgow Western Infirmary. ' With Special Chapters by Prof. GAIRDNER on the Physiognomy of Disease; Dr. Arexandm RoBerison on Insanity; Dr. JogmpH CoAps on the Throat ; Professor Stephanson on Disorders of the Female Organs ; and Dr. SAMson GramkiL on Physical Diagnosis. Second Edition, Revised and Enlarged, with many new Illustrations, crown 8vo. 12s, 6d.

An INDEX of SYMPTOMS as an AID to DIAGNOSIS. By RALPH WINNINGTON LFFTwCH. M.D., late Assistant-Physician to the East London Children's Hospital. Fcp. 8vo. 5s.

* Each Symptom is followed by an alphabetical list of the Diseases in which it occurs.

DISEASES of the BONES : their Pathology, Diagnosis, and Treatment, By THOMas JoNes, F.R.C.S. Eng., B.S. Lond., Surgeon to the Manchester Royal Infirmary; Lecturer on Practical Surgery in the Owens College, Victoria University ; Consulting Surgeon to the Children's Hospital, Pendlebury, Manchester. With 7 Chromo-Lithographic Plates, 9 Ftchings, and 77 Woodcuts. 8vo. 12s. 6d.

A PRACTICAL TREATISE on URINARY and RENAL DISEASES, inclading Urinary Deposits. Illustrated by numerous Cases and Engravings. Fourth Fdition, Revised and Enlarged. By Sir WIILAM RoBerTs, M.D., F.R.S., Fellow of the Royal College of Physicians, London; Professor of Medicine at the Victoria University; Consulting Physioian to the Manchester Royal Infirmary. Assisted by RoBenT MAGURr, M.D. Lond., Member of the Royal College of Phygiciana, London; Physician to Out-patients, St. Mary's Hospital, London; late Pathologist to the Manchester Royal Infirmary. Small 8vo. 12s.6d.

LECTURES on DIETETICS and DYSPEPSIA. Delivered at the Owens College School of Medicine in February and March 1885. By Sir WuLlam RoBirts, M.D., F.R.S., Fellow of the Royal College of Physicians, London; Professor of Medicine at the Victoria University; Consulting Physician to the Manchester Royal Infirmary. Second Edition. Small 8vo.'3s.

A TREATISE on the SCIENCE and PRACTICE of MIDWIFERY. By W. S. PLA YFAIR, M.D., F.R.C.P., Physician-Accoucheur to H.I. and R.H. the Duchess of Edinburgh ; Professor of Obstetric Medicine in King's College; Physician for the Diseases of Women and Children to King's College Hospital ; Consulting Physician to the General Lying-in Hospital, and to the Evelina Hospital for Obildren; late President of the Obstetrical Society of London; Bxaminer in Midwifery to the University of London, and to the Royal College of Physicians. 2 vols.-demy 8vo. Sixth Edition. With 181 Illustrations and 6 Plates. 28s.

A SYSTEM of OBSTETRIC MEDICINE and SURGERY, Theoretical and Clinical. For the Stadent and Practitioner. By ROBRRT BARNES, M.D., Obstetric Physician to the St. George's Hospital ; Consulting Physician to the Ohelsea Hospital for Women, \&c. : and FANCOURT BARNEs, M.D., Physician to the Royal Maternity Oharity and to the British Lying-in Hospital; Obstetric Physician to the Great Northern Hospital ; Physician to the Chelsea Hospital for Women. 2 vols. 8vo. with numerous Illustrations. Vol. I., 18s.; Vol, II., 20s. 



